GEOPHYSICAL WELL-LOG DATA BASE FOR

FHE GULF COAST AQGIFER SRSTEMS,

SOUTH-CENTRAL UNITED STATES

By TeRry A. WILSON AND R. L. HOSMAN

U.S. GEOLOGICAL SURVEY

OPEN-FILE REPORT 87-677 


\section{DEPARTMENT OF THE INTERIOR \\ DONALD PAUL HODEL, Secretary \\ U.S. GEOLOGICAL SURVEY}

Dallas L. Peck, Director

For additional information write to:

Project Chief

U.S. Geological Survey

Gulf Coast RASA

N. Shore Plaza Bldg., Rm. 104

55 North Interregional Hwy. Austin, Texas 78702
Copies of this report can be purchased from:

U.S. Geological Survey Books and Open-File Reports Federal Center, Bldg. 810 Box 25425

Denver, Colorado 80225 


\section{CONTENTS}

Page

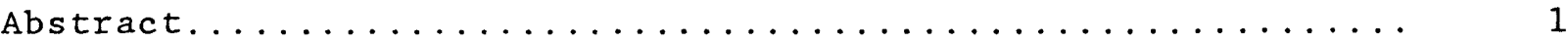

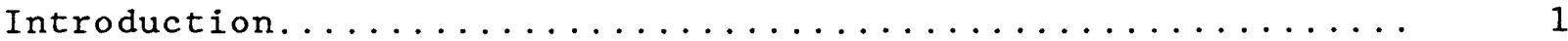

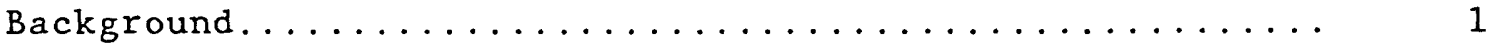

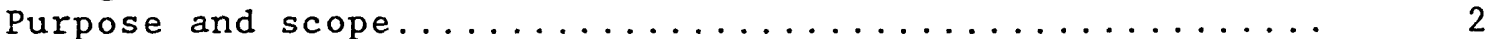

Creation of geophysical we11-log database.............. 2

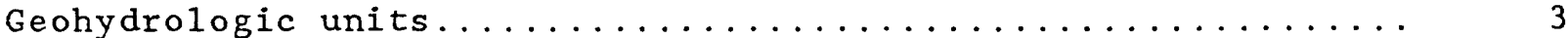

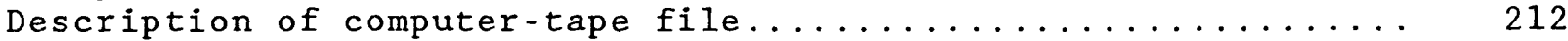

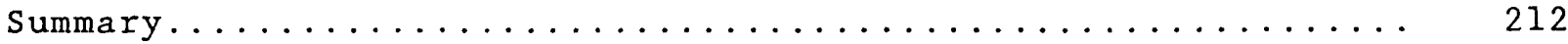

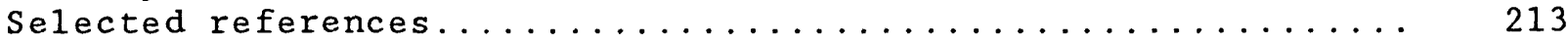

\section{ILLUSTRATIONS}

[P1ate is in pocket]

Plate 1. Map showing location of project area and wells for which data are tabulated, Coastal Plain and adjacent Continental Shelf, south-central United States.

\section{TABLES}

Table 1. Relation among aquifer systems, regional mode1layers, and aquifers and confining units.........

2. Log number and sequence number, well location (degrees), interval logged, and land-surface altitude by state and county...............

3. Log number and well location (county), company, and well name, by $\log$ sequence number............

4. Layer number, altitude of top, thickness, sand percentage of layer; and average dissolved-solids concentration and temperature of water in layer;

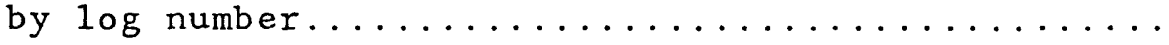

5. Statistical summary of thickness, altitude of top,

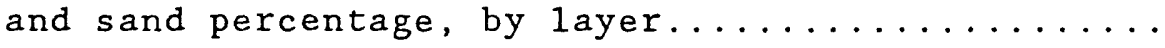




\section{CONVERSION FACTORS}

For those readers interested in metric (International system) units, the factors for converting inch-pound units to metric units are given below:

Multiply Inch-pound units

by

To obtain metric units

foot (ft)

0.3048

1.609

meter ( $m$ )

mile (mi)

2.590

kilometer $(\mathrm{km})$

square mile

National Geodetic Vertical Datum of 1929 (NGVD of 1929)--a geodetic datum derived from a general adjustment of the first-order level nets of both the United States and Canada, formerly called "Mean Sea level of 1929." 


\title{
GEOPHYSICAL WELL-LOG DATABASE FOR
}

\author{
THE GULF COAST AQUIFER SYSTEMS, \\ SOUTH-CENTRAL UNITED STATES
}

By Terry A. Wilson and R.L. Hosman

\begin{abstract}
As part of the U.S. Geological Survey's Regional Aquifer-System Analysis (RASA) program, the Gulf Coast RASA was begun to investigate ground-water flow systems in the Coastal Plain of the south-central United States. Three regional aquifer systems are identified in the area. The Mississippi embayment aquifer system and the Texas coastal uplands aquifer system are composed predominantly of Eocene sediments; the coastal lowlands aquifer system is composed predominantly of Oligocene and younger sediments. Automated data-processing techniques were chosen to facilitate a study of this magnitude. A computer database of information derived from almost 1,000 geophysical well logs was constructed to support the delineation of the geohydrologic framework of the study area and the simulation of ground-water flow. The database contains entries pertaining primarily to physical characteristics of the hydrologic units.
\end{abstract}

\section{INTRODUCTION}

\section{Background}

The U.S. Geological Survey began a program in 1978 to investigate regional ground-water systems throughout the Nation. A major objective of the Regional Aquifer-System Analysis (RASA) program is to provide an understanding of ground-water flow systems on a regional scale (Bennett, 1979). Natural hydrologic boundaries, rather than political boundaries, have been used to determine areas to be studied. The Gulf Coast RASA study area consists of about 230,000 square miles onshore in Louisiana and parts of Alabama, Arkansas, Florida, Illinois, Kentucky, Mississippi, Missouri, Tennessee, and Texas. The study area contains an additional 60,000 square miles offshore on the Continental Shelf, as the aquifers extend beyond the coastline beneath the Gulf of Mexico. The extent of the Gulf Coast RASA study area is shown on plate 1 . 
Three regional aquifer systems are delineated in the Gulf Coast RASA study area (Grubb, 1984). The Mississippi embayment aquifer system and the Texas coastal uplands aquifer system are composed predominantly of Eocene sediments; the coastal lowlands aquifer system is composed predominantly of oligocene and younger sediments. Each of these systems consists of thousands of feet of sediments, which contain numerous aquifers and confining units. Some of these geohydrologic units are regionally extensive, whereas others are of local importance. In order to quantitatively describe the groundwater hydrology, each of these systems was subdivided into discrete geohydrologic units. It became obvious early in this study that timely and accurate completion of work of this magnitude would require the techniques of automated data processing. Therefore, a computer database of information derived from geophysical well logs was constructed to support the delineation of the geohydrologic framework of the study area and the simulation of ground-water flow.

\section{Purpose and Scope}

The purpose of this report is to describe the contents of a database containing machine-readable information derived from geophysical well logs, present a summary of the data, and provide computer-generated tables of the data used for constructing maps of the geohydrologic units in the study area. These data also are being used in conjunction with computer-based interpolation techniques to construct data arrays for ground-water flow models.

Most of the data regarding physical characteristics of geologic and geohydrologic units studied in the Gulf Coast RASA were derived from geophysical well logs. Some of the data were also used in making water-quality calulations. The logs were almost entirely electrical or induction-electrical surveys of wells drilled by the petroleum industry. A few gamma ray and single-point electrical logs of water wells and nonpetroleum test wells were used where other data were not available. Oil-test logs generally were obtained from commercial sources, and logs of water wells and nonpetroleum tests were available from U.S. Geological Survey files.

\section{CREATION OF GEOPHYSICAL WELL-LOG DATABASE}

The logs used in the study were selected on the basis of two criteria: spatial distribution and interval logged. Because of the size of the study area, approximately 290,000 square miles, a density of one log per 400 square miles (20-mile spacing) was chosen as one that would produce representative regional data and still keep the data base at a manageable size. It was possible to maintain this distribution in nearly the entire area, owing to the fact that petroleum exploration is widespread throughout the Coastal Plain. 
Where possible, the interval logged also was given consideration if more than one $\log$ was available for a given site. The top of the logged interval may be tens, hundreds, or even thousands of feet below land surface, and the bottom of the log varies with the depth of the hole. Log selection was determined by best overall coverage.

Data obtained from the geophysical logs to create the database included depths to the top and the thickness of geologic and hydrologic units. Other geohydrologic unit data include: (1) Thickness and depth of sands more than $20 \mathrm{ft}$ thick in aquifer units and thickness and depth of major clays in confining units; (2) spontaneous-potential and formation-resistivity values for sands greater than $20 \mathrm{ft}$ thick in the aquifers, and average resisitivity values for sands greater than $20 \mathrm{ft}$ thick interbedded in the confining layers; and (3) cumulative thickness of all sands thicker than $20 \mathrm{ft}$ in aquifer units and of all interbedded sands in confining units. Estimates of dissolved-solids concentrations in water were derived from calculations involving density, resistivity, and temperature of the drilling mud; resistivity and temperature of the mud filtrate; and bottom hole temperature in a procedure described by Weiss (1987).

The data were obtained directly from almost 1,000 geophysical logs, coded, and entered into a computer file designed to facilitate retrieval for listings or processing for interpretations and calculations. Machine-generated data lists, maps and graphs based on these files were used to construct the geohydrologic framework.

\section{GEOHYDROLOGIC UNITS}

Prior to this study, regional aquifers had been recognized insofar as the geologic units comprising them were recognized. However, geologic nomenclature is not uniform throughout the study area, and local names do not lend themselves to a regional perspective. The following designations were chosen to identify major geohydrologic units of regional extent (R.L. Hosman and J.S. Weiss, U.S. Geological Survey, written commun., 1986). The units are listed in sequence by associated model layer numbers that are used in multilayer simulation of ground-water flow in the aquifer systems and are not in descending order of units, the normal U.S. Geological Survey standard for geohydrologic units: 
Regional mode1

layer number
Hydrologic unit

McNairy-Nacatoch aquifer

Lower Wilcox aquifer

Middle Wilcox aquifer

Lower Claiborne-upper Wilcox aquifer

Middle Claiborne aquifer

Upper Claiborne aquifer

Zone E (lower Miocene-upper Oligocene deposits)

Zone D (middle Miocene deposits)

Zone C (lower Pliocene-upper Miocene deposits)

Zone B (lower Pleistocene-upper Pliocene deposits)

11

Mississippi River Valley alluvial aquifer and Zone A (Holocene-upper Pleistocene deposits)

Midway confining unit

13

Lower Claiborne confining unit

14

Middle Claiborne confining unit

15

Vicksburg-Jackson confining unit

16

Zone E confining unit

17

Zone D confining unit

The relation of these hydrologic units to aquifer systems in the study area is shown in table 1 in descending order. 
Table 1.--Relation among aquifer systems, regional model layers, and aquifers, permeable zones, and confining units

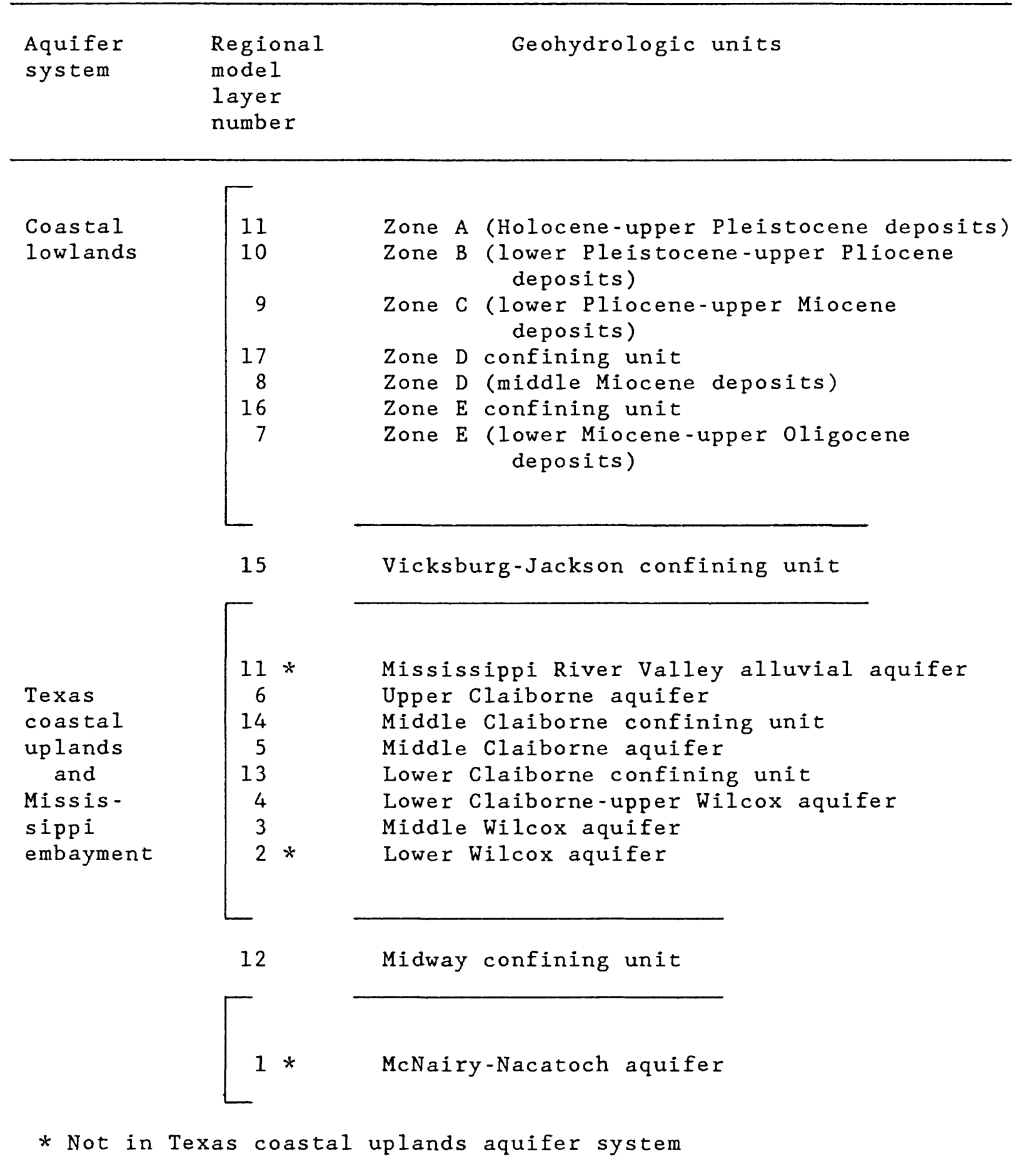

The data in each of the log files are listed in tables 2, 3, and 4 . A statistical summary of the data contained in table 4 is presented in table 5. Preceding each table is a glossary-type explanation of the data presented in that table. 
Explanation of data in table 2:

State--State in which the logged well was drilled.

County--County or parish in which the logged well was drilled. Offshore logs are listed following county (parish) lists.

Log number--An alphanumeric system that uses the two-letter state abbreviation followed by a two-letter county (parish) abbreviation followed by a two-digit sequential number. For example, the second well numbered in Baldwin County, Alabama, would be ALBL02. Offshore numbers are composed of the single letter initial of the state and a three-digit API (American Petroleum Institute) number followed by a two-digit sequential number.

Log sequence number--Wells for which logs were used in this study were numbered sequentially for convenience in this report. The numbers begin at the northernmost well in the project area and end at the southernmost.

Latitude--Latitude of the logged well, in degrees to four decimal places.

Longitude--Longitude of the logged well, in degrees to four decimal places.

Depth logged from-- The depth to the top of the logged interval, in feet. On electrical and induction-electrical logs it generally begins at the bottom of the uppermost string of casing. (The file contains some data for the interval between land surface and the top of the log. These data generally were derived from drillers' or geophysical logs of nearby test holes or water wells, or from published reports.)

Depth logged to--The bottom of the logged interval, in feet. It generally represents the bottom of the hole at the time of logging. If the bottom of the logged interval is within an aquifer system included in this study, it also is the maximum depth for which data exists in the file. If the bottom of the logged interval is below the base of the lowest aquifer system being studied, no data will have been entered into the file for depths below the base of that system. The bases of the aquifer systems are the Vicksburg-Jackson confining unit, the Midway confining unit, and the top of the geopressured zone. The geopressured zone is a zone of abnormally excessive fluid pressures that occurs in some Gulf Coast sediments. It is considered the base of the flow system for purposes of this study.

Land-surface altitude--The altitude of the land surface at the logged well, in feet above sea level. For wells drilled offshore in the Gulf of Mexico, this number is the altitude of the sea floor. 


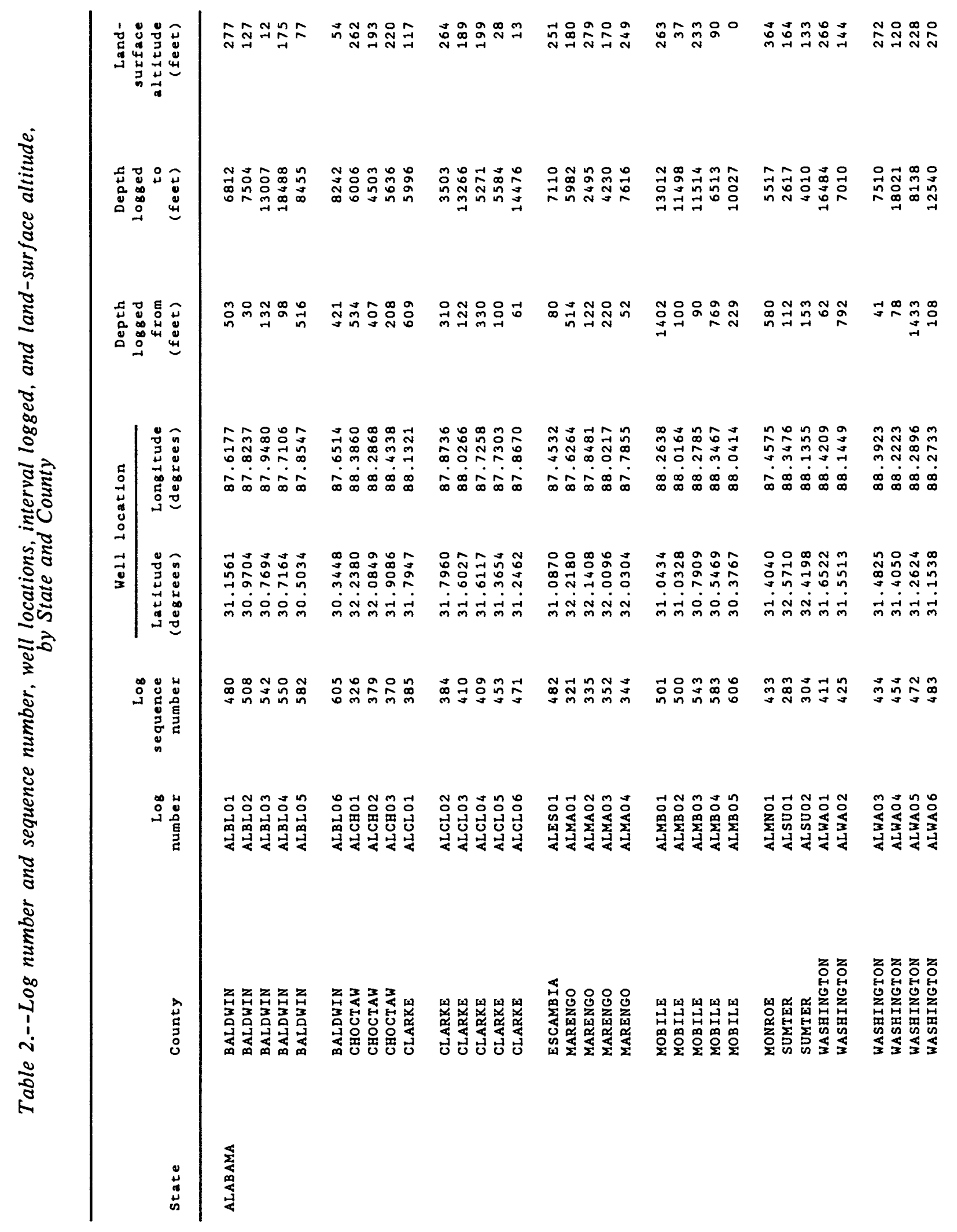




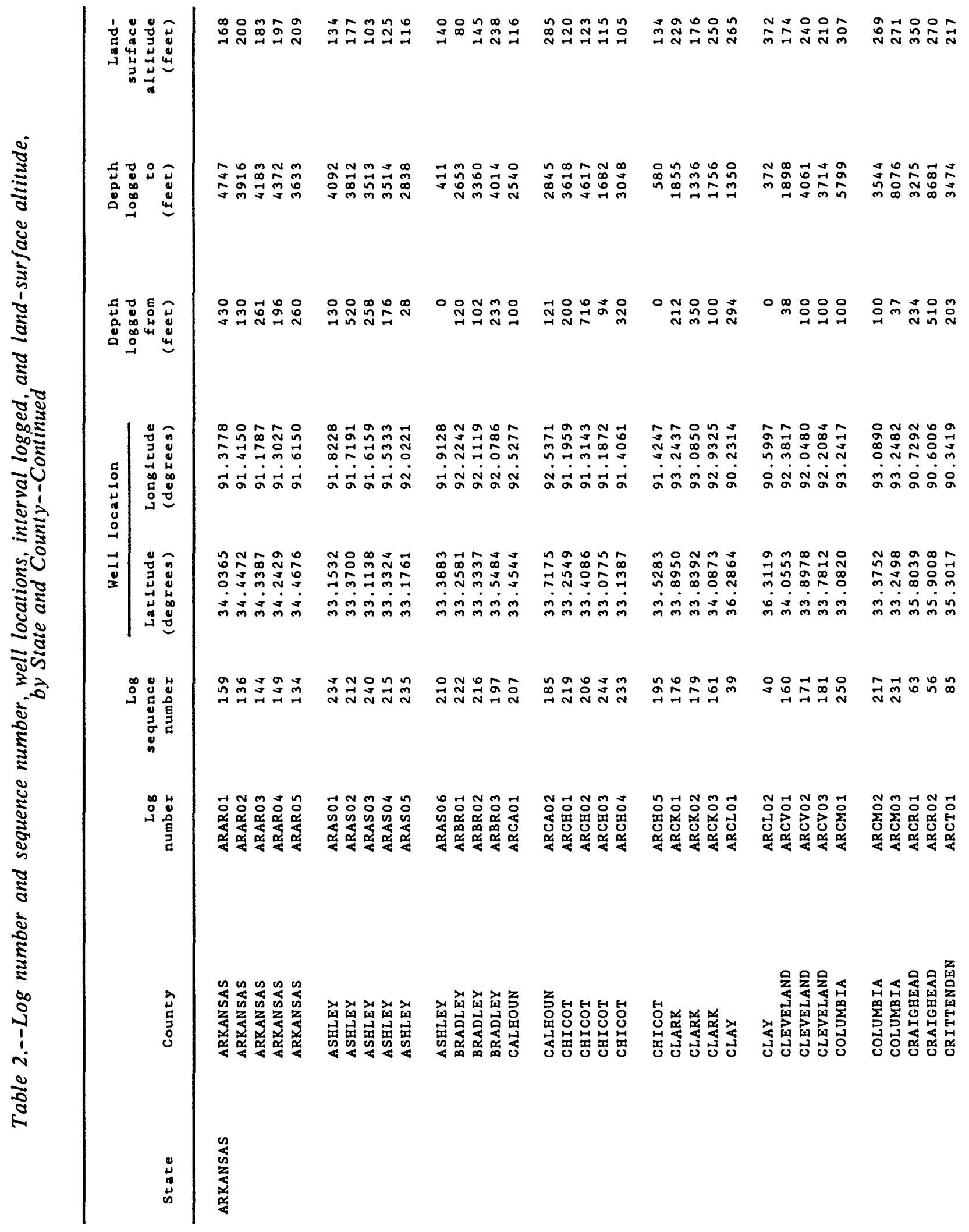




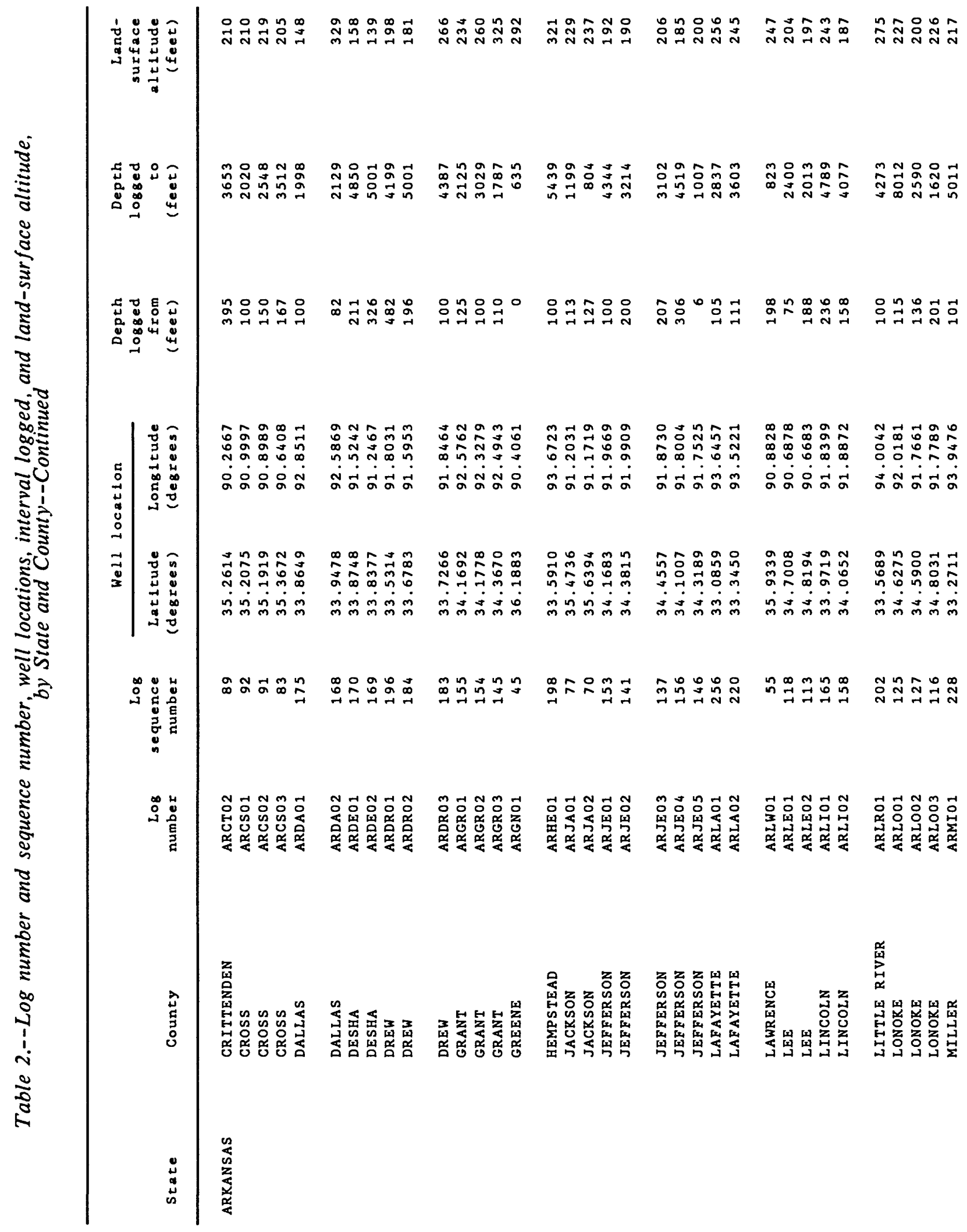




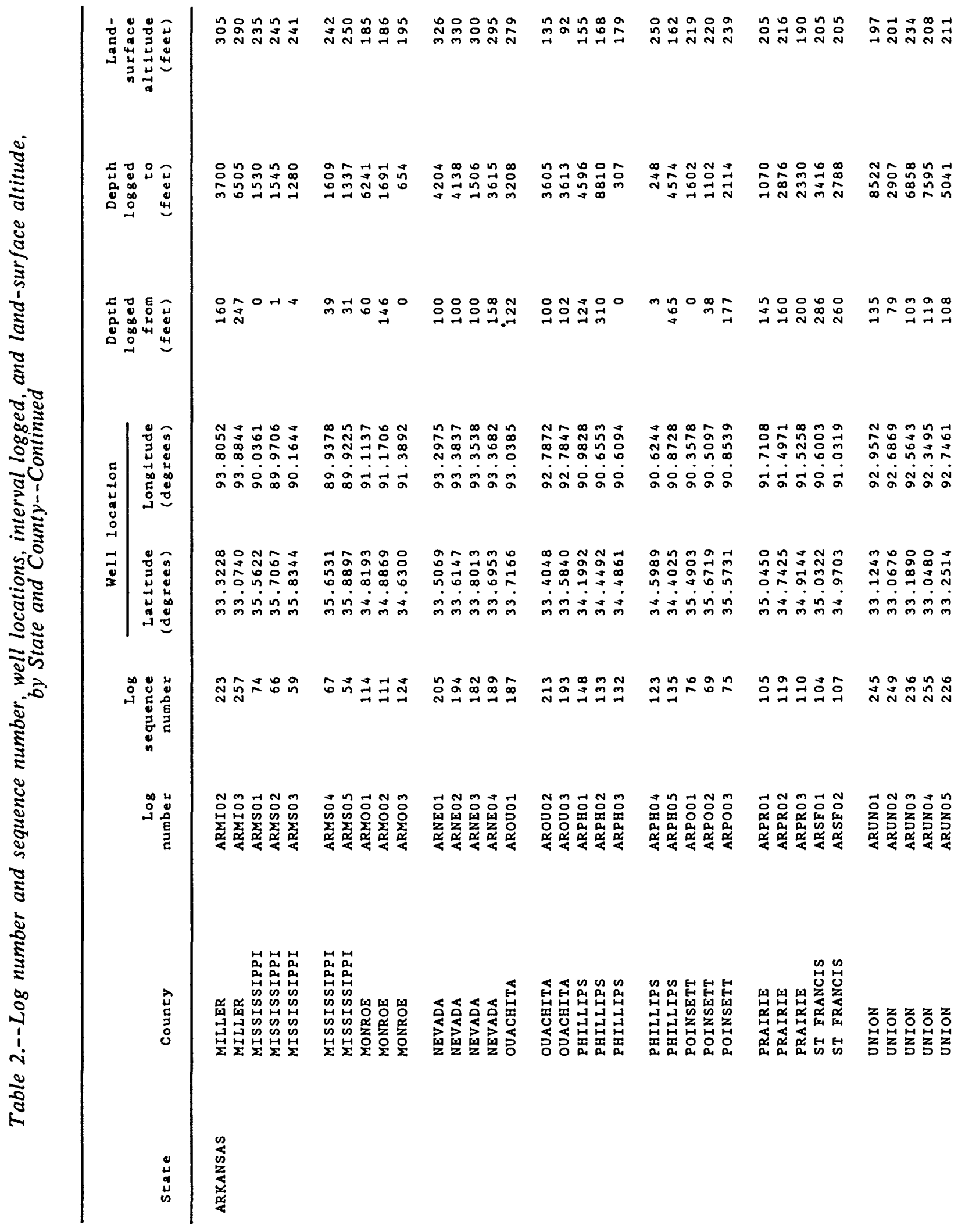




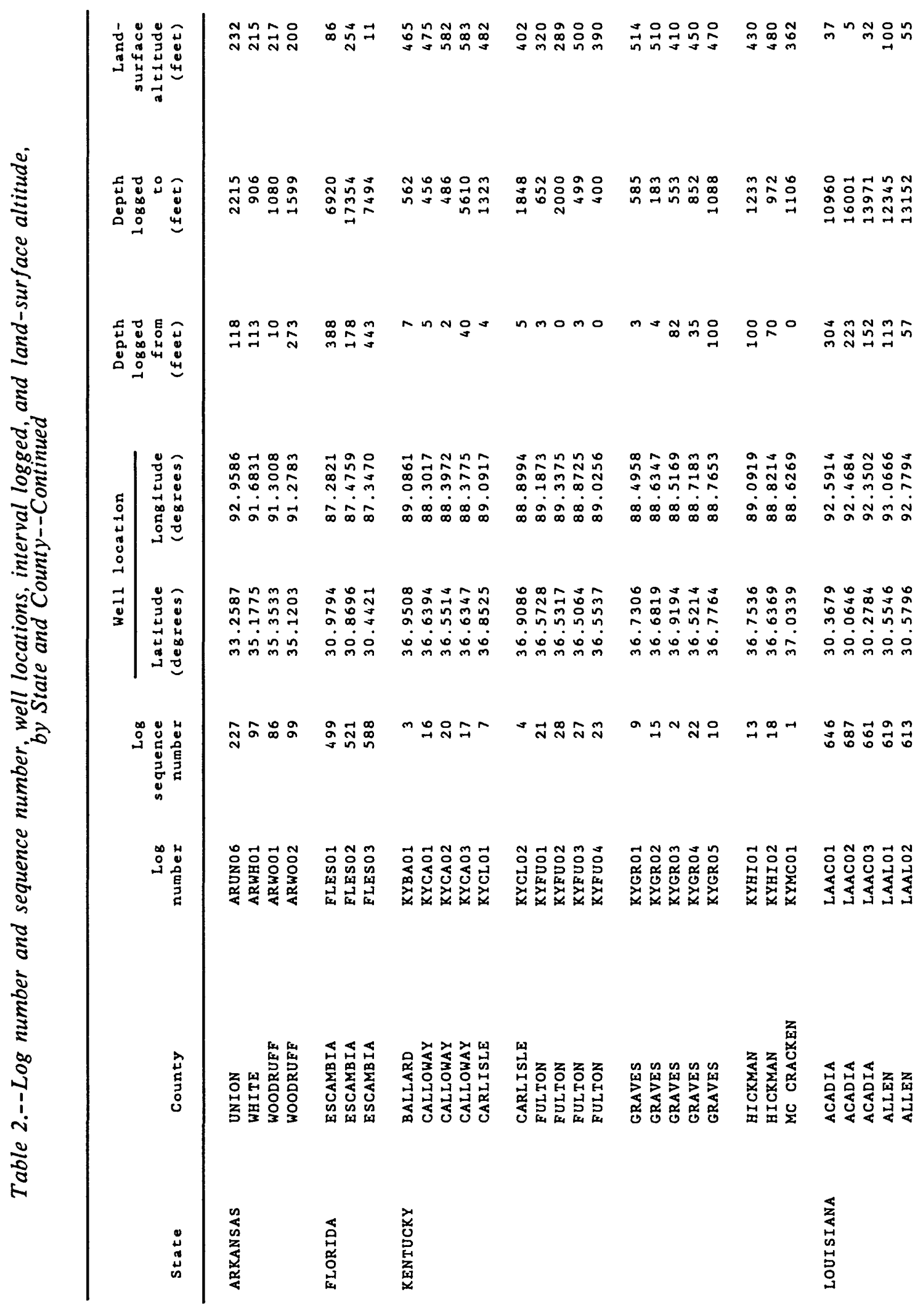




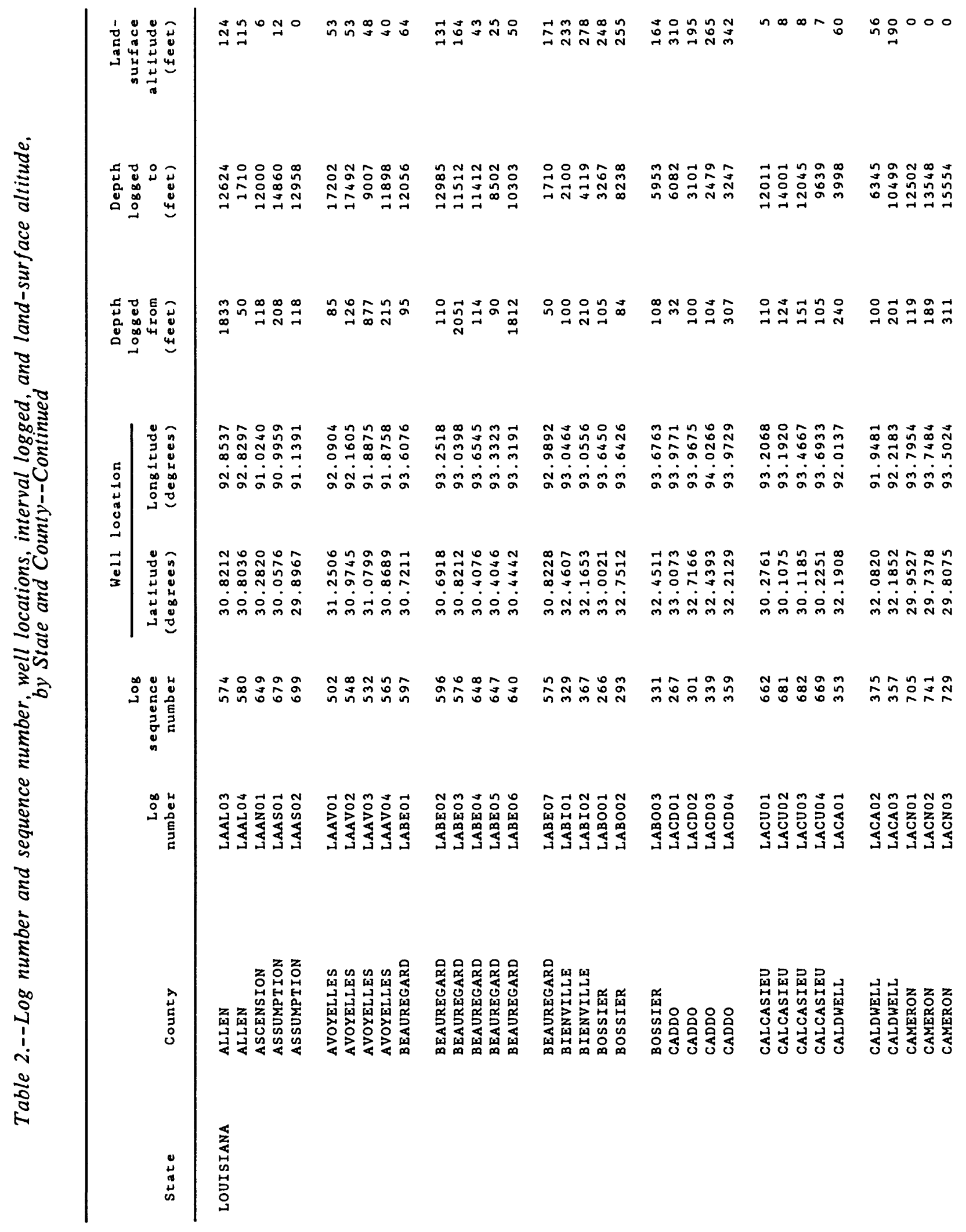




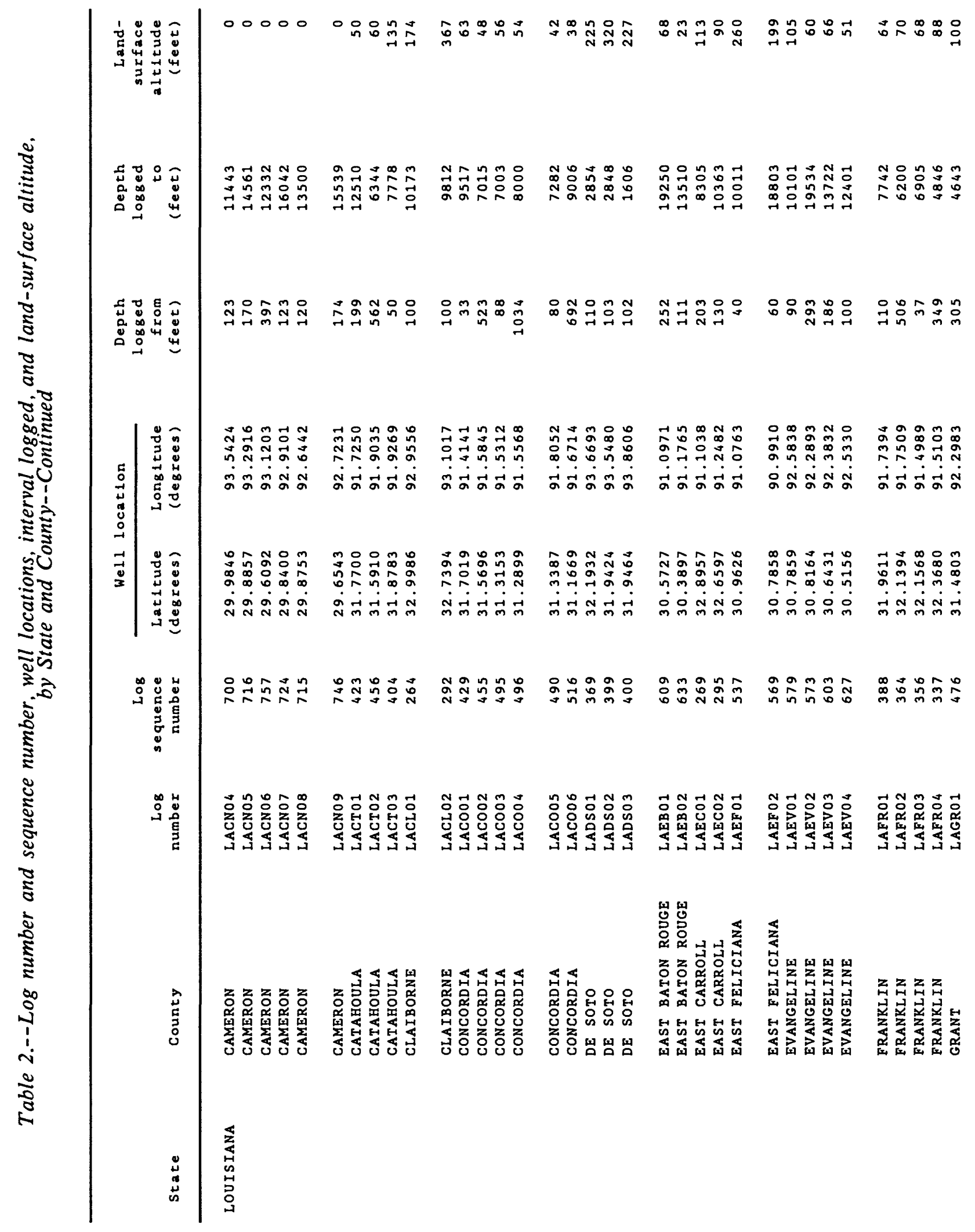




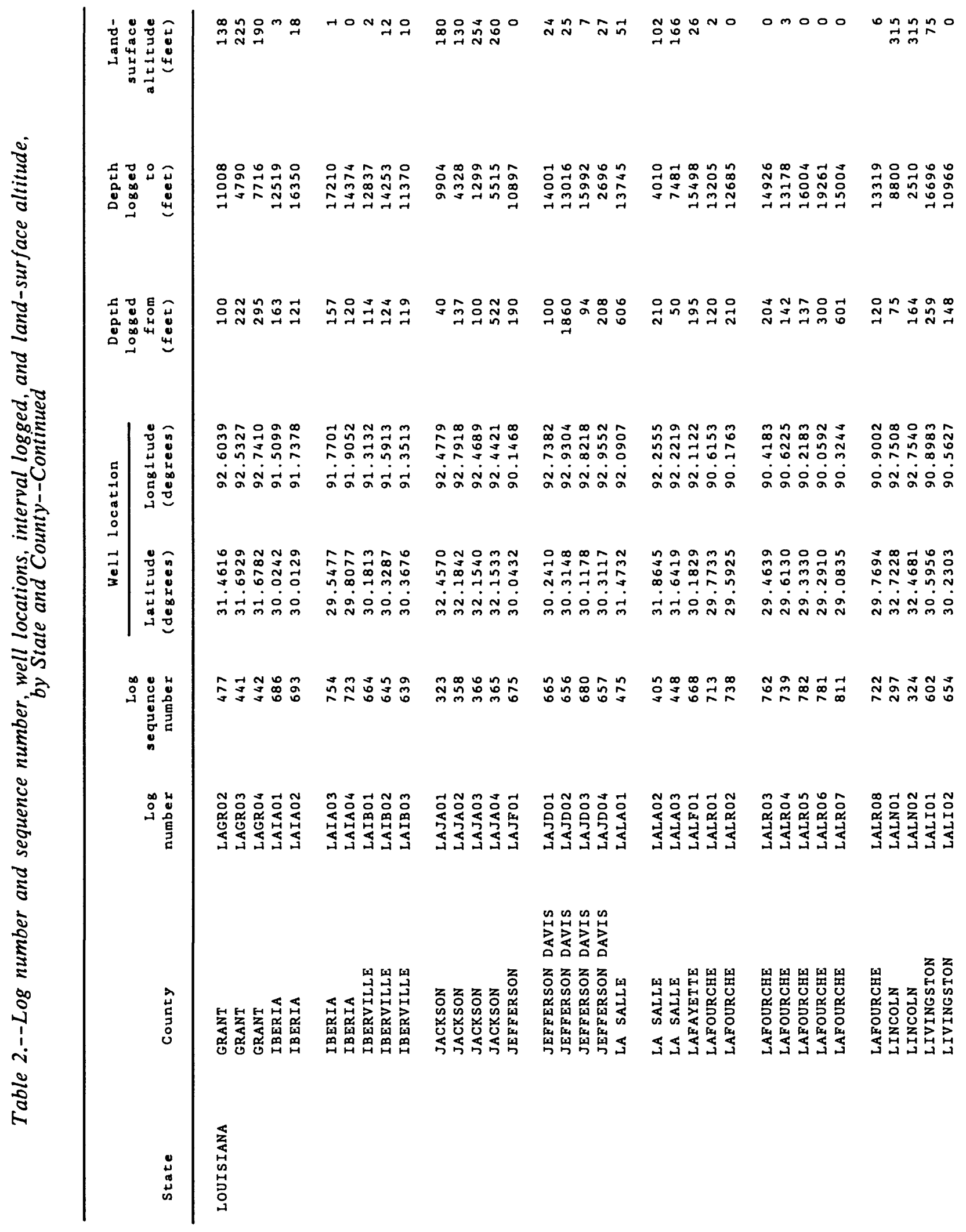




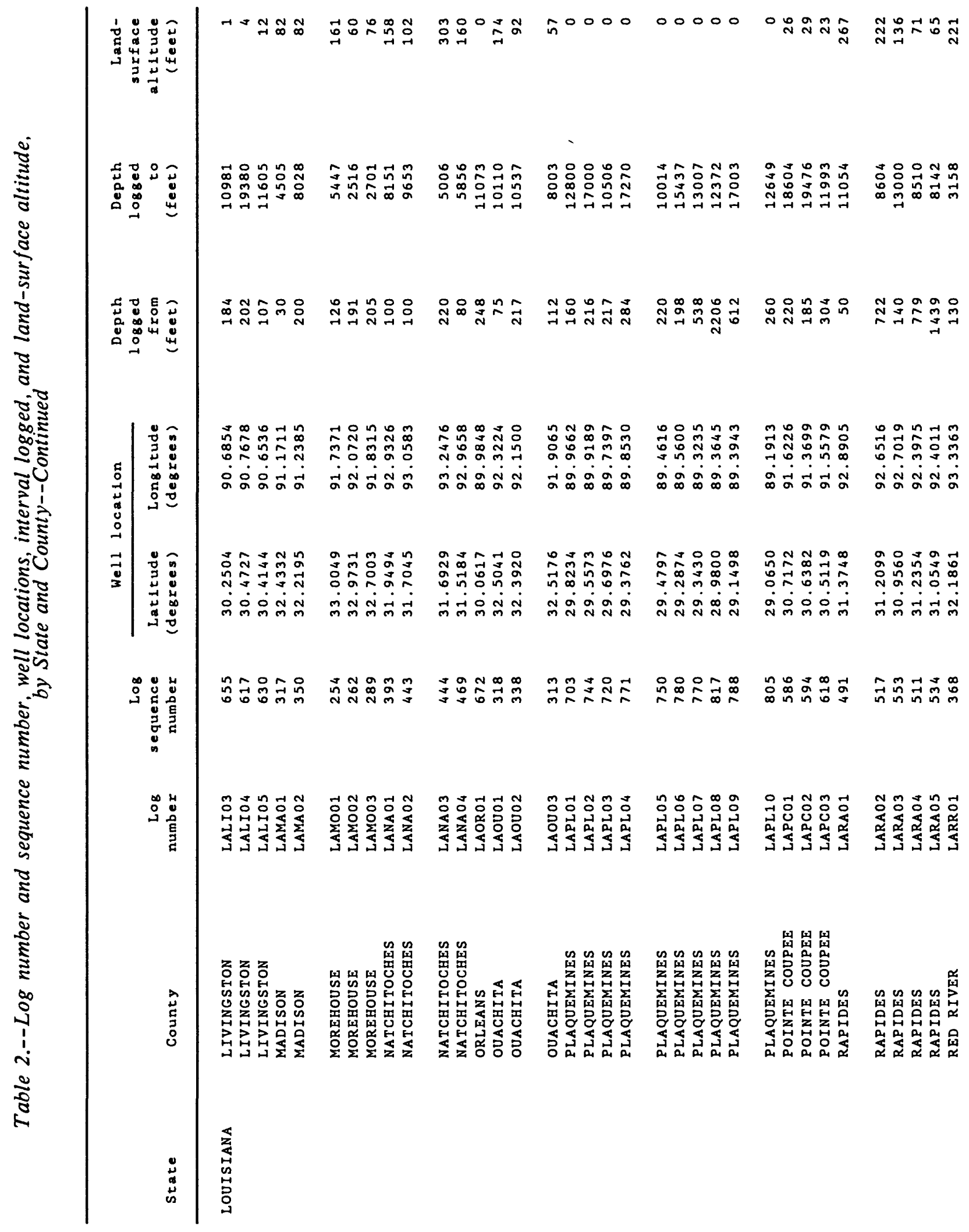




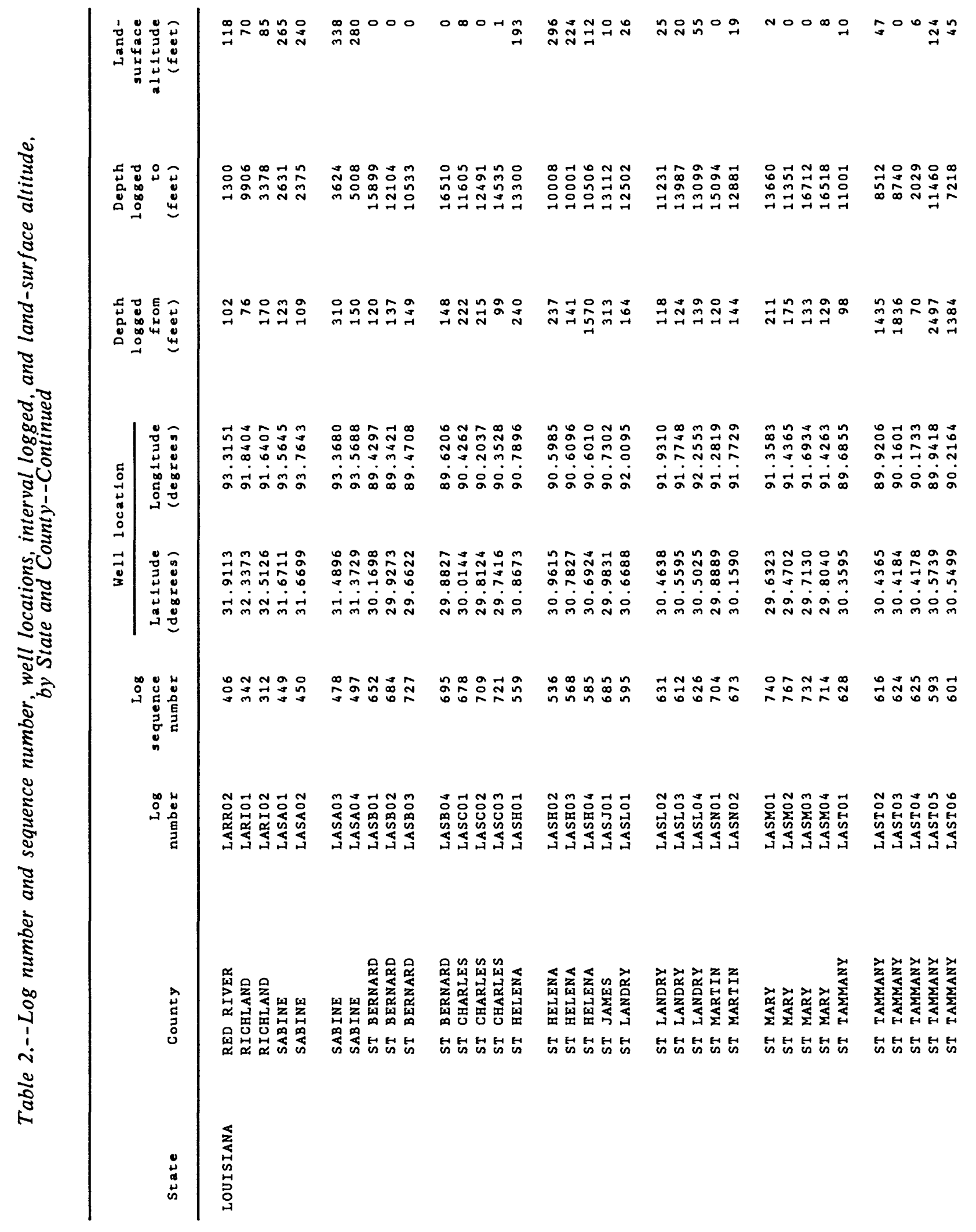




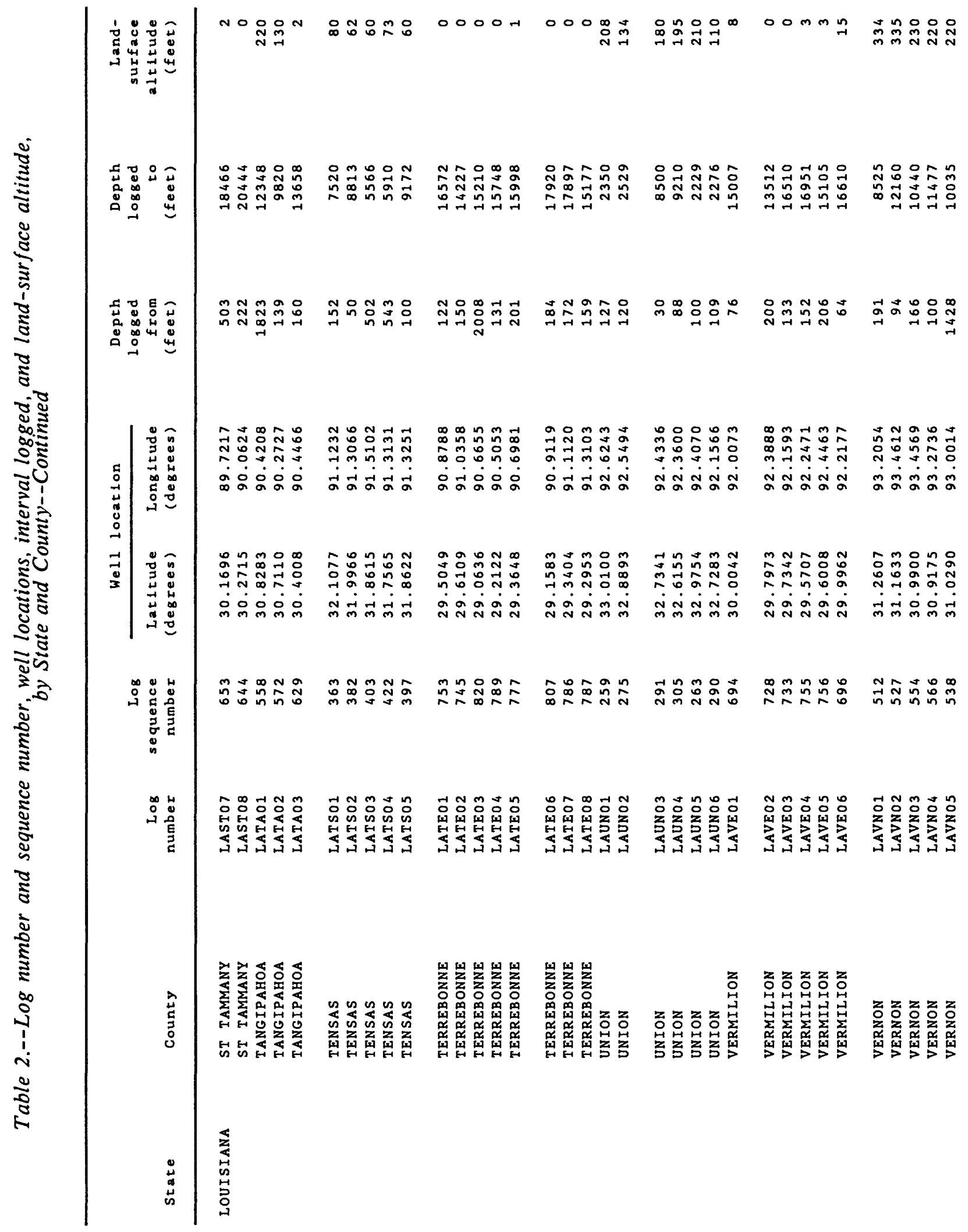




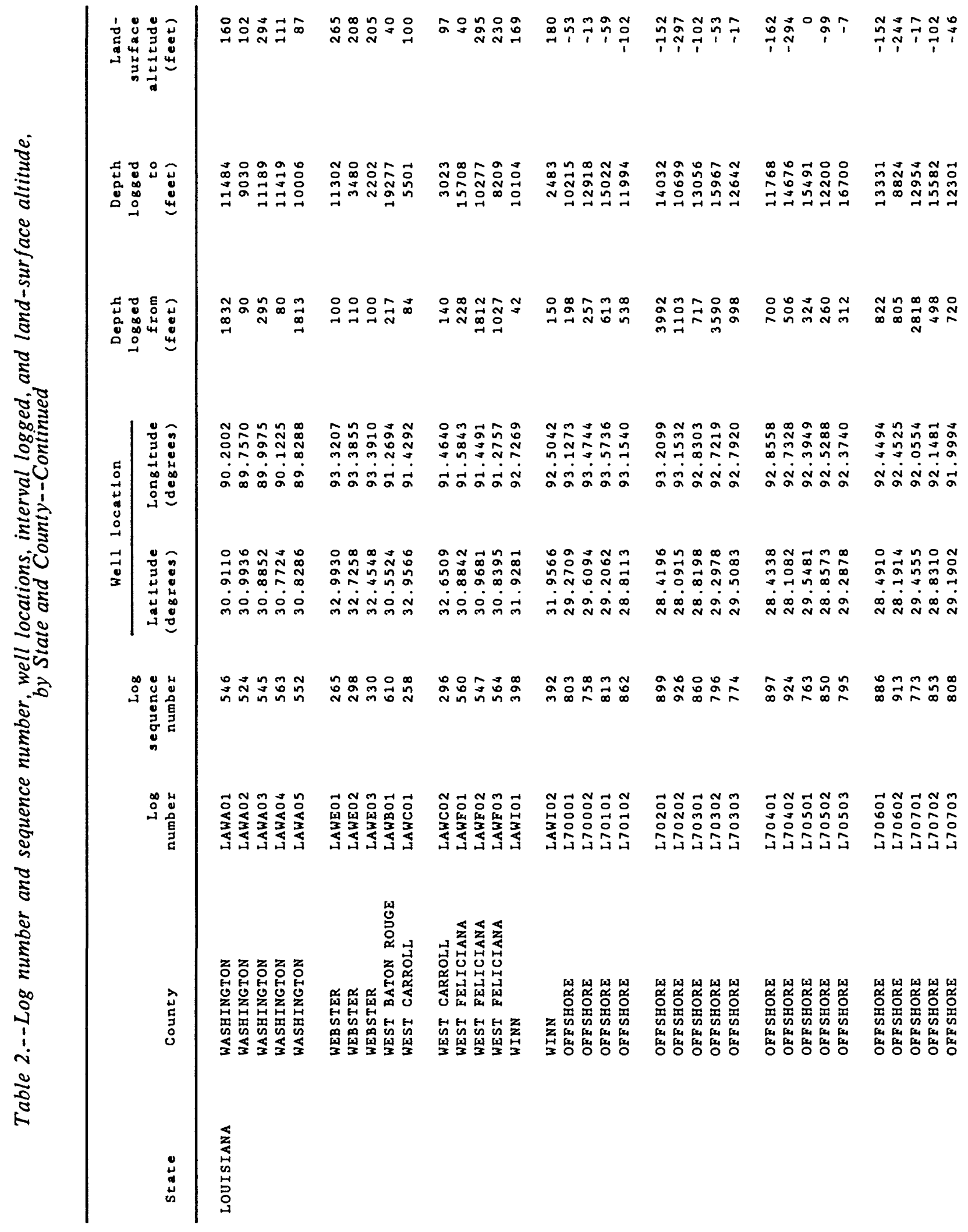




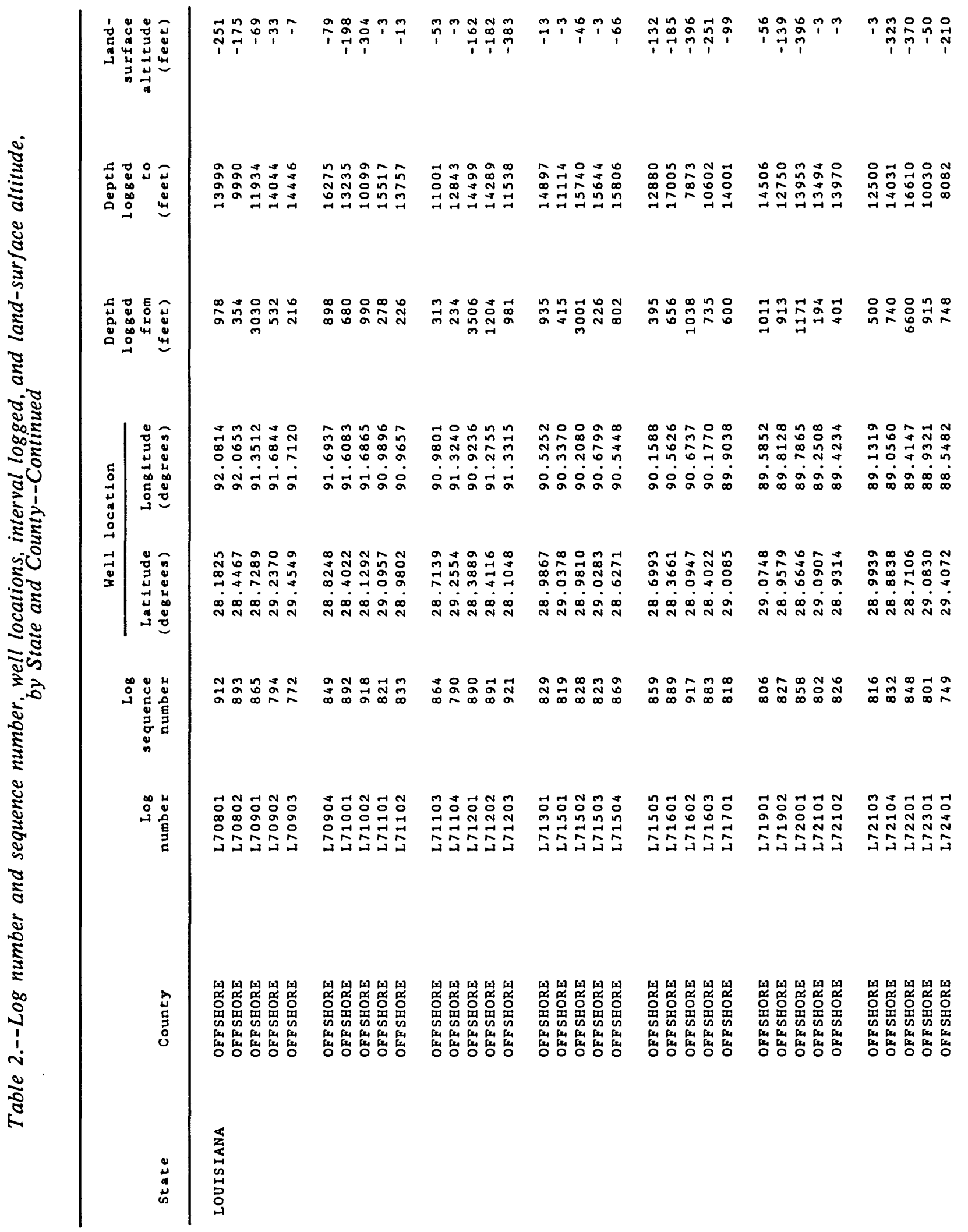




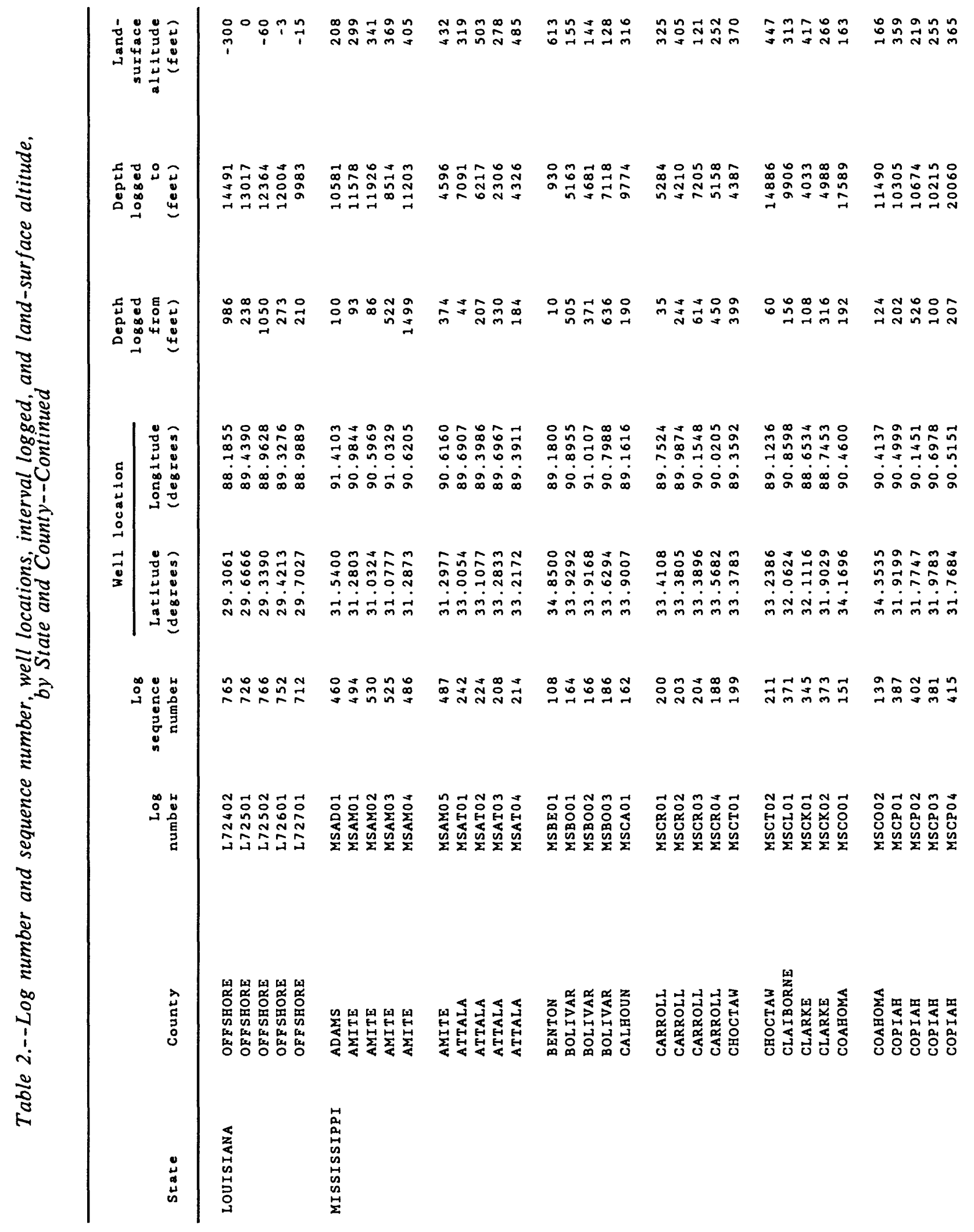




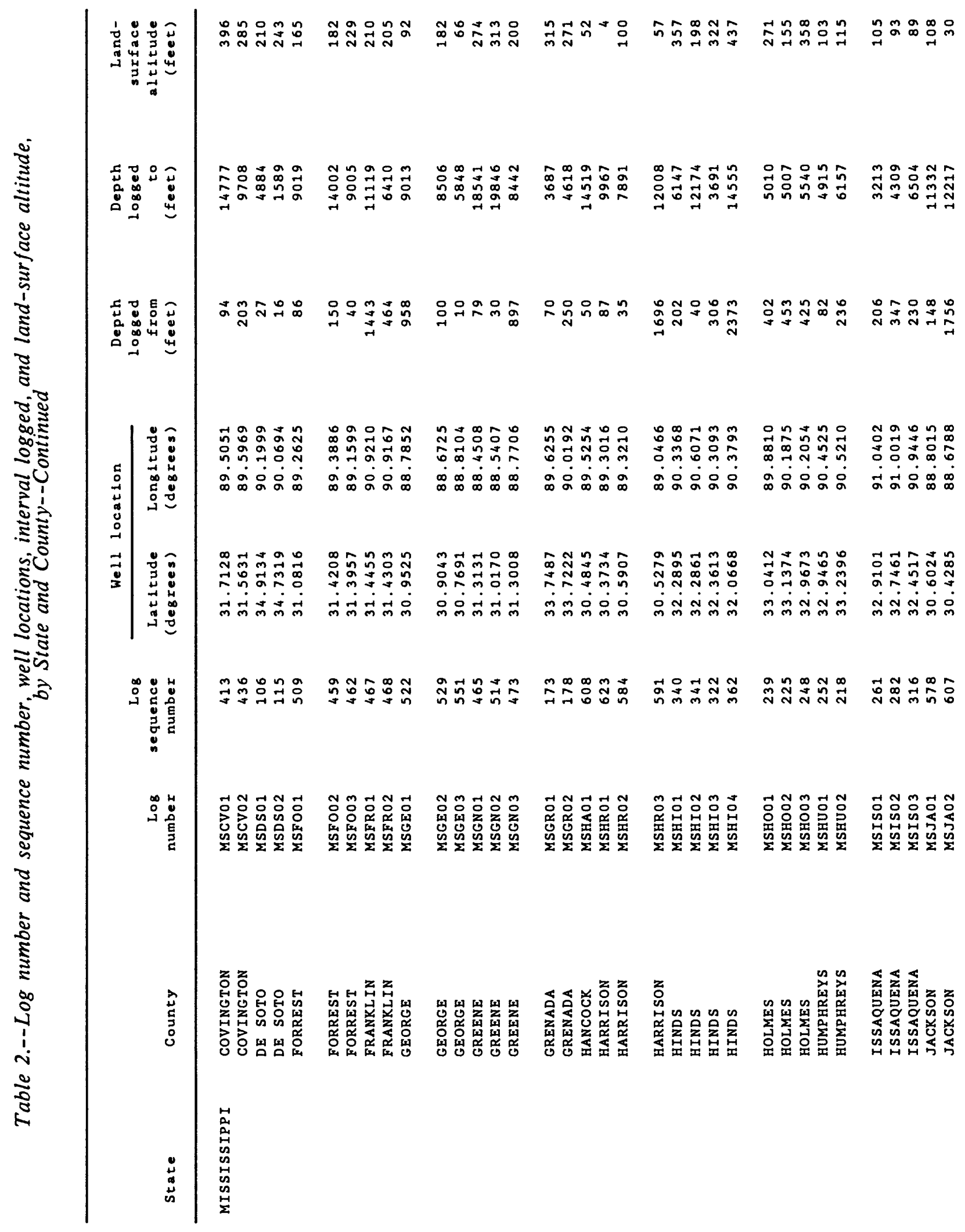




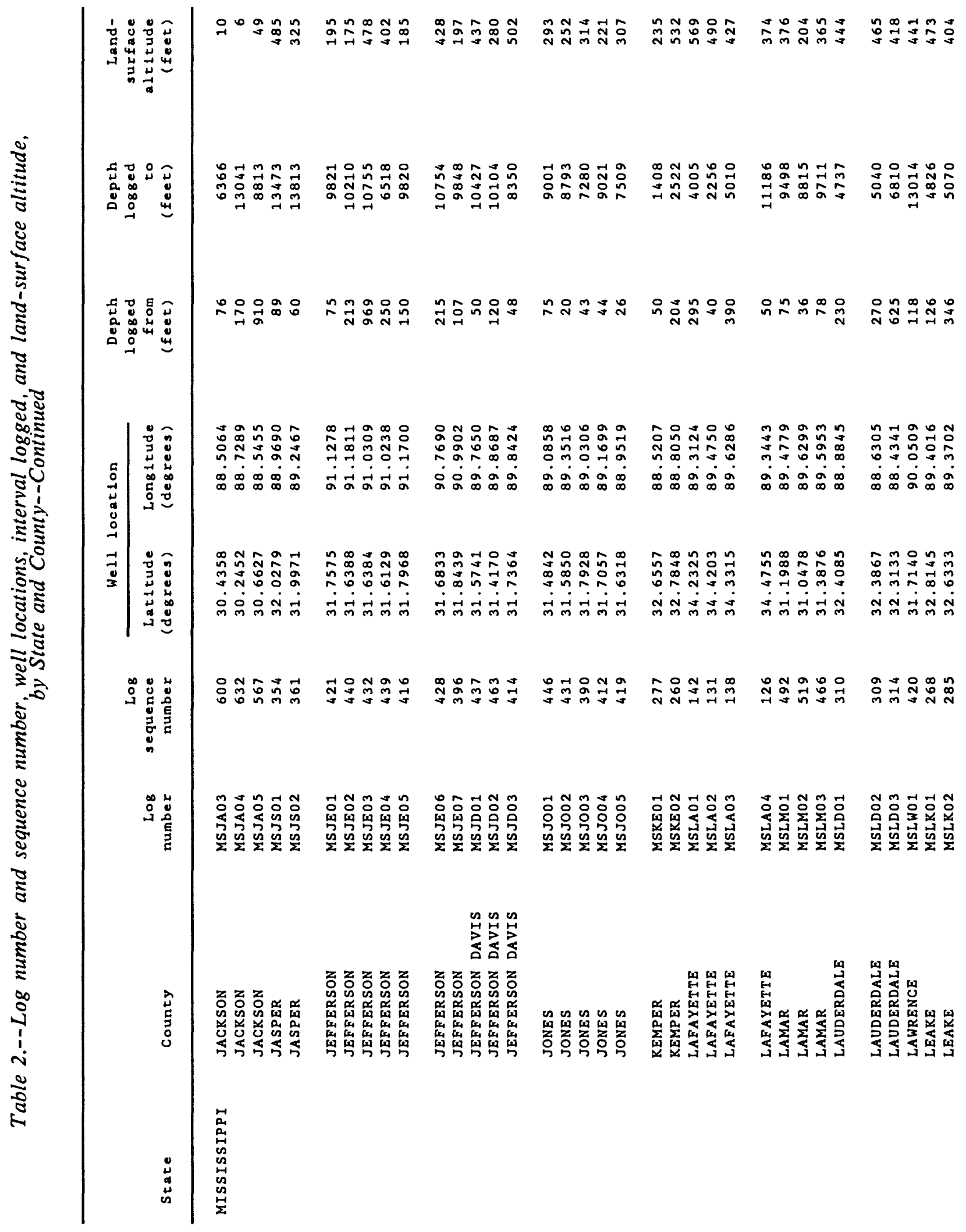




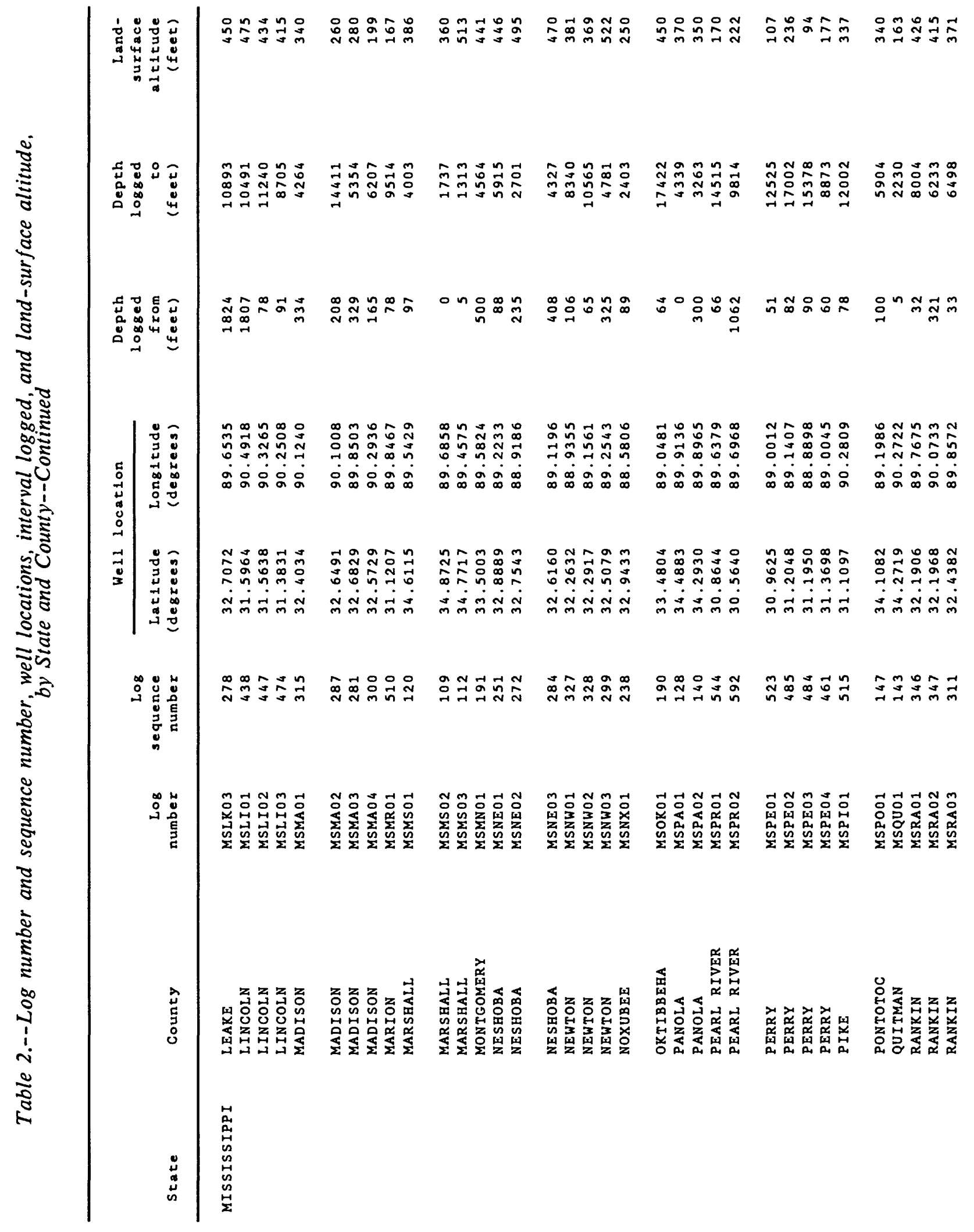




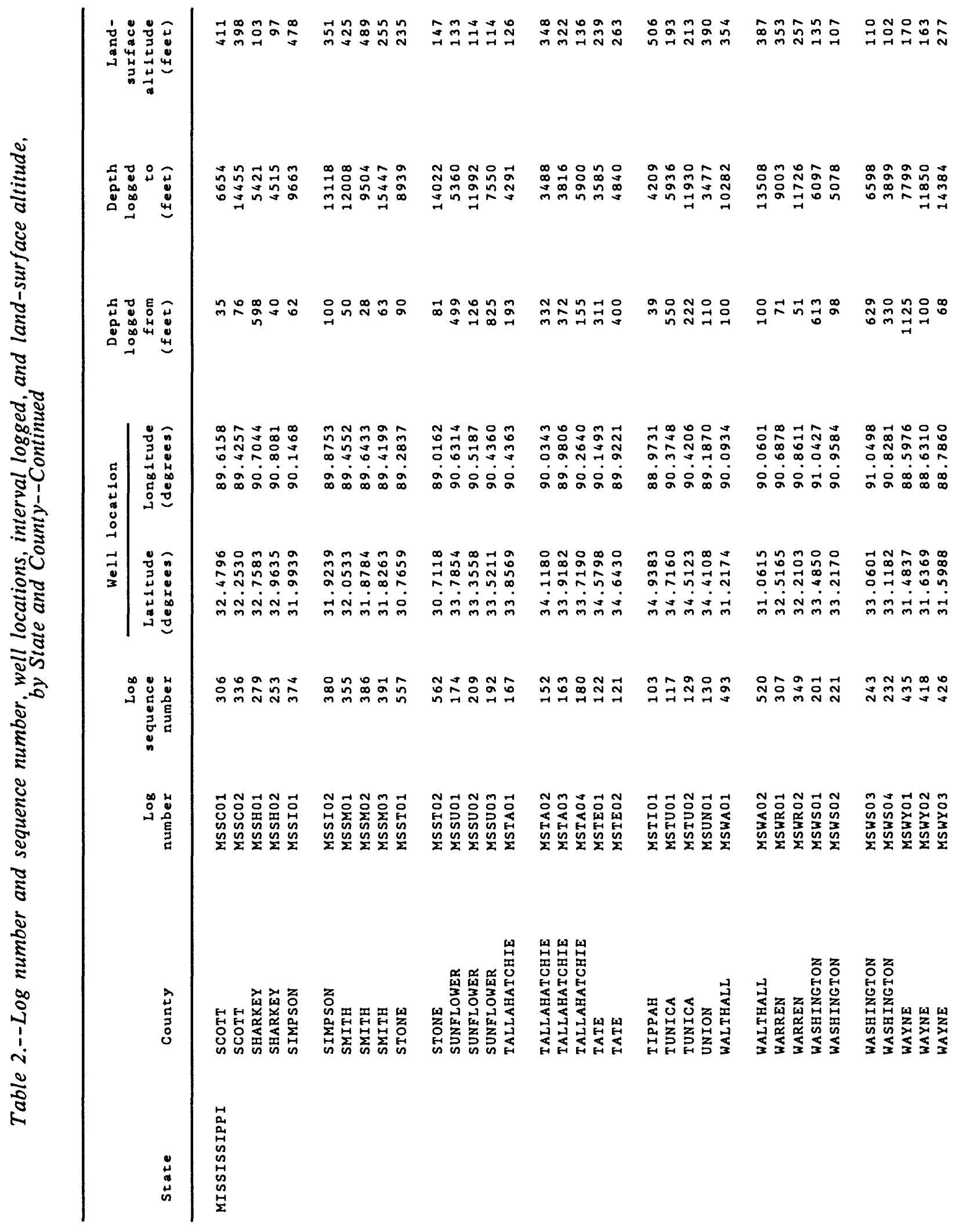




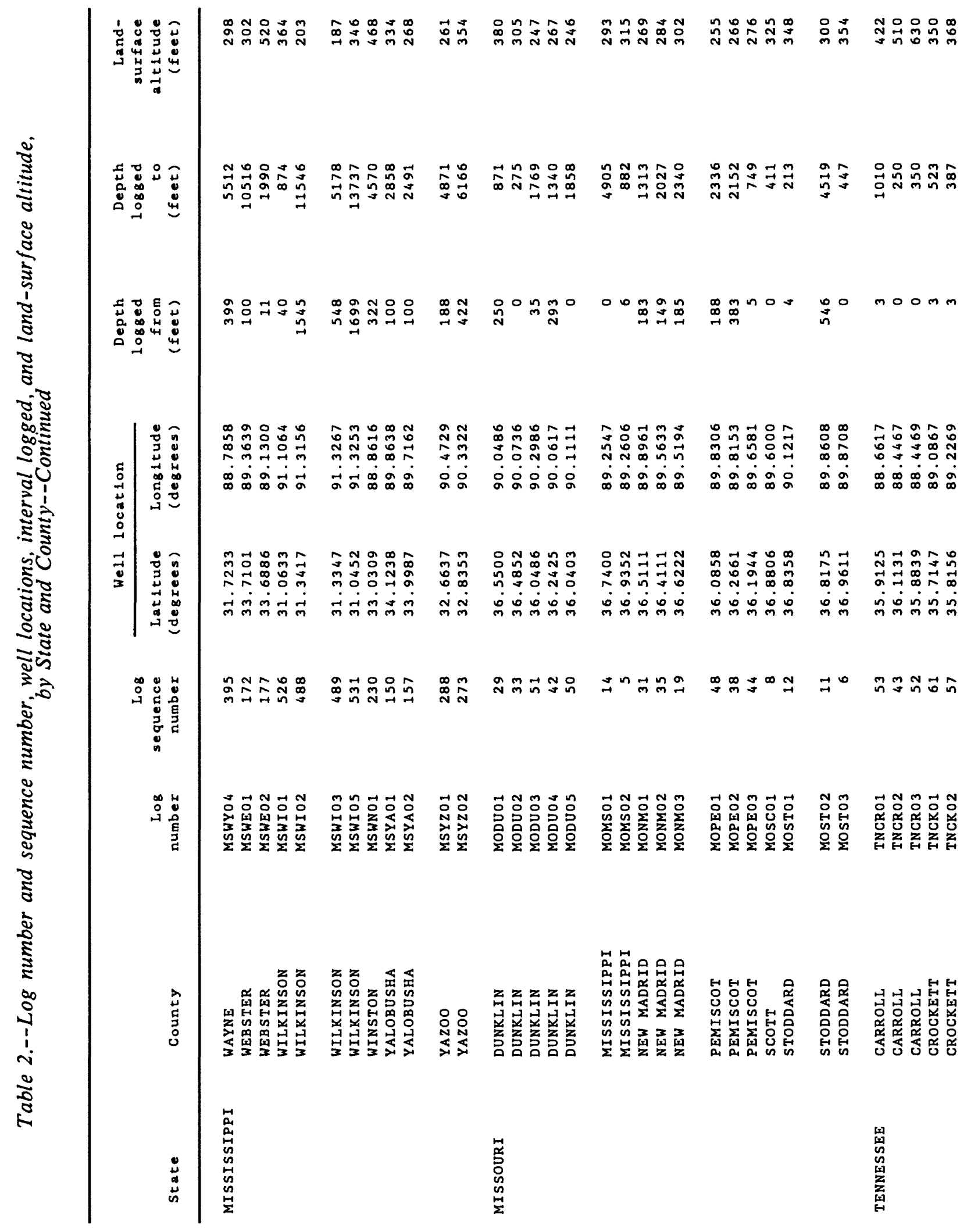




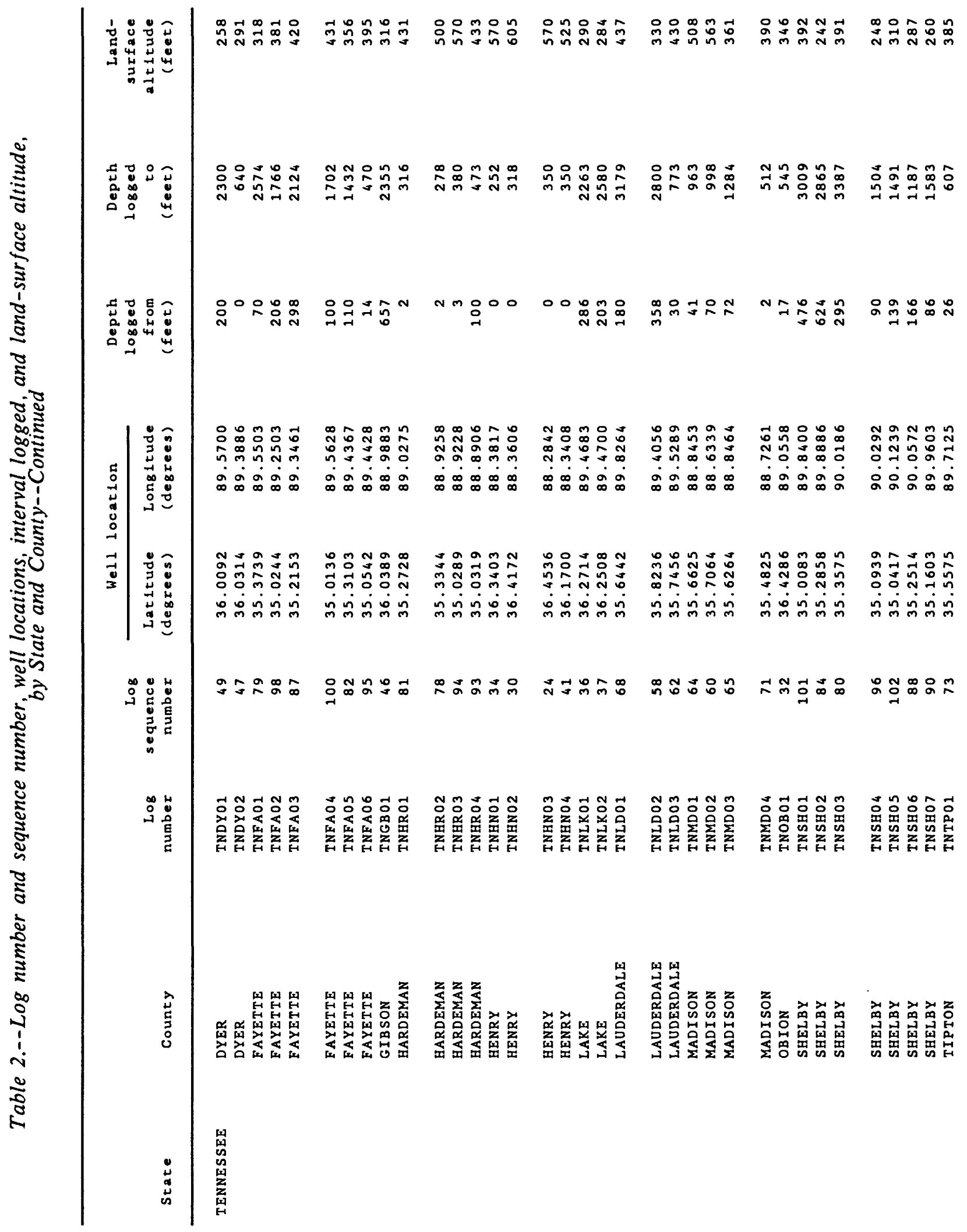




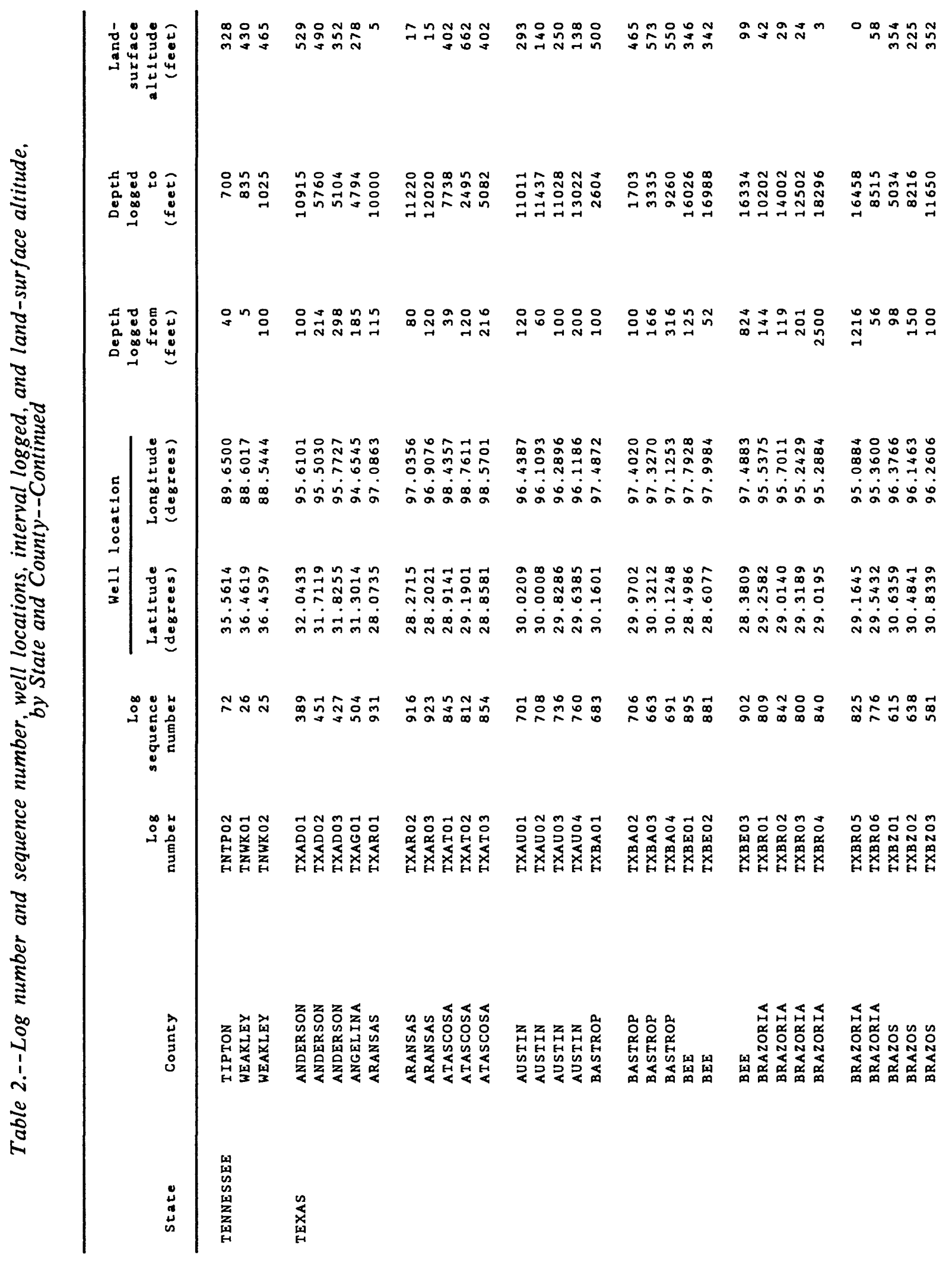




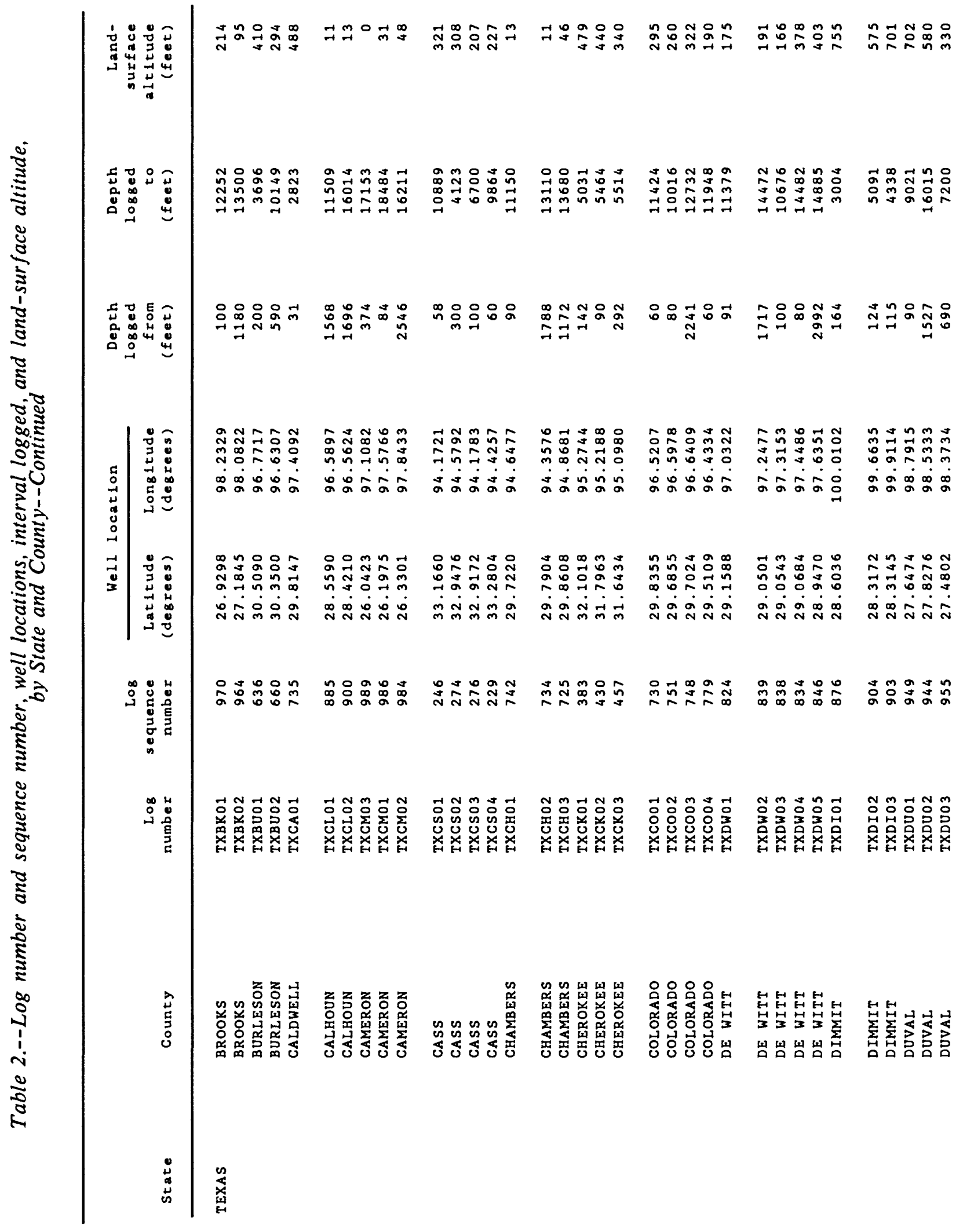




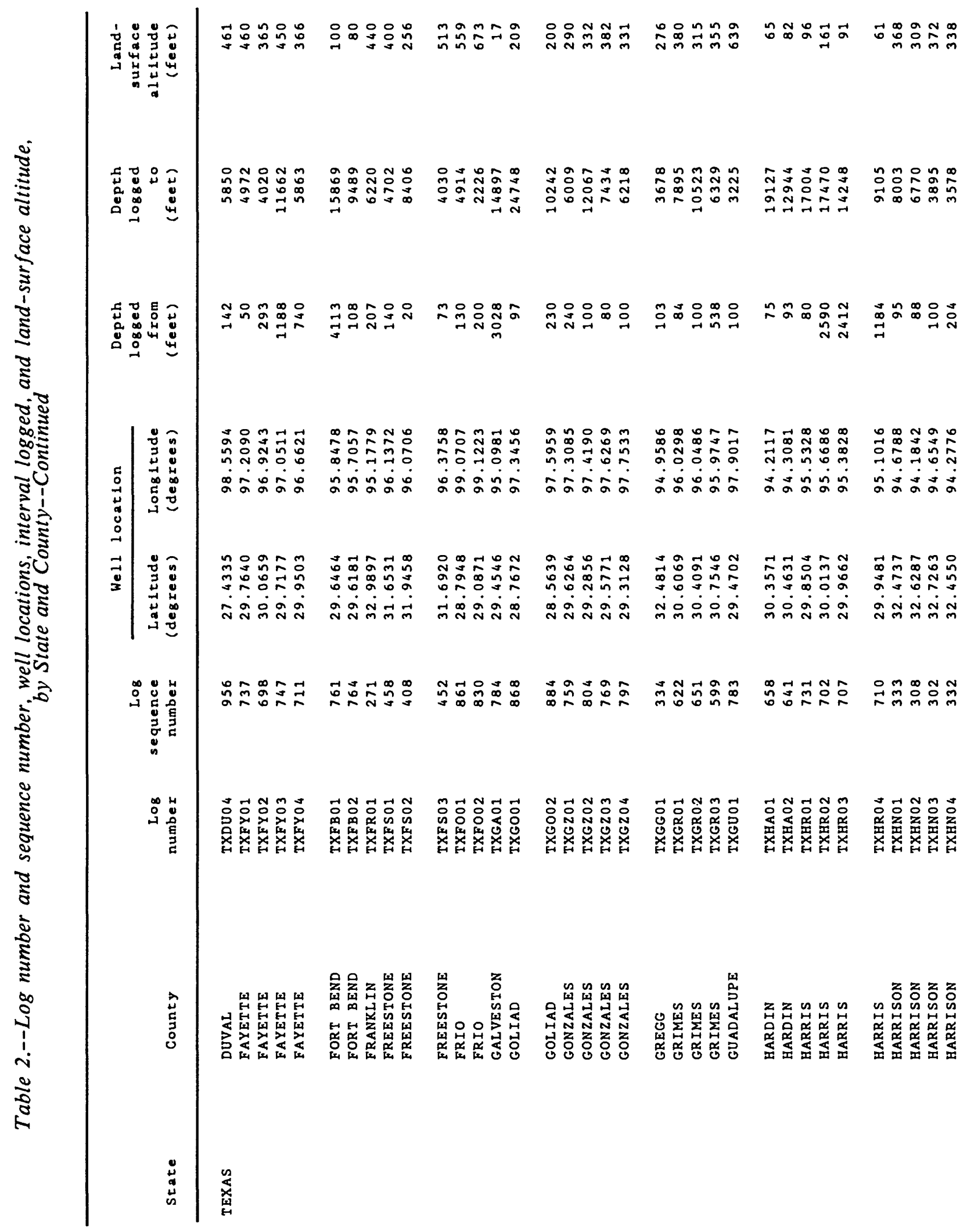




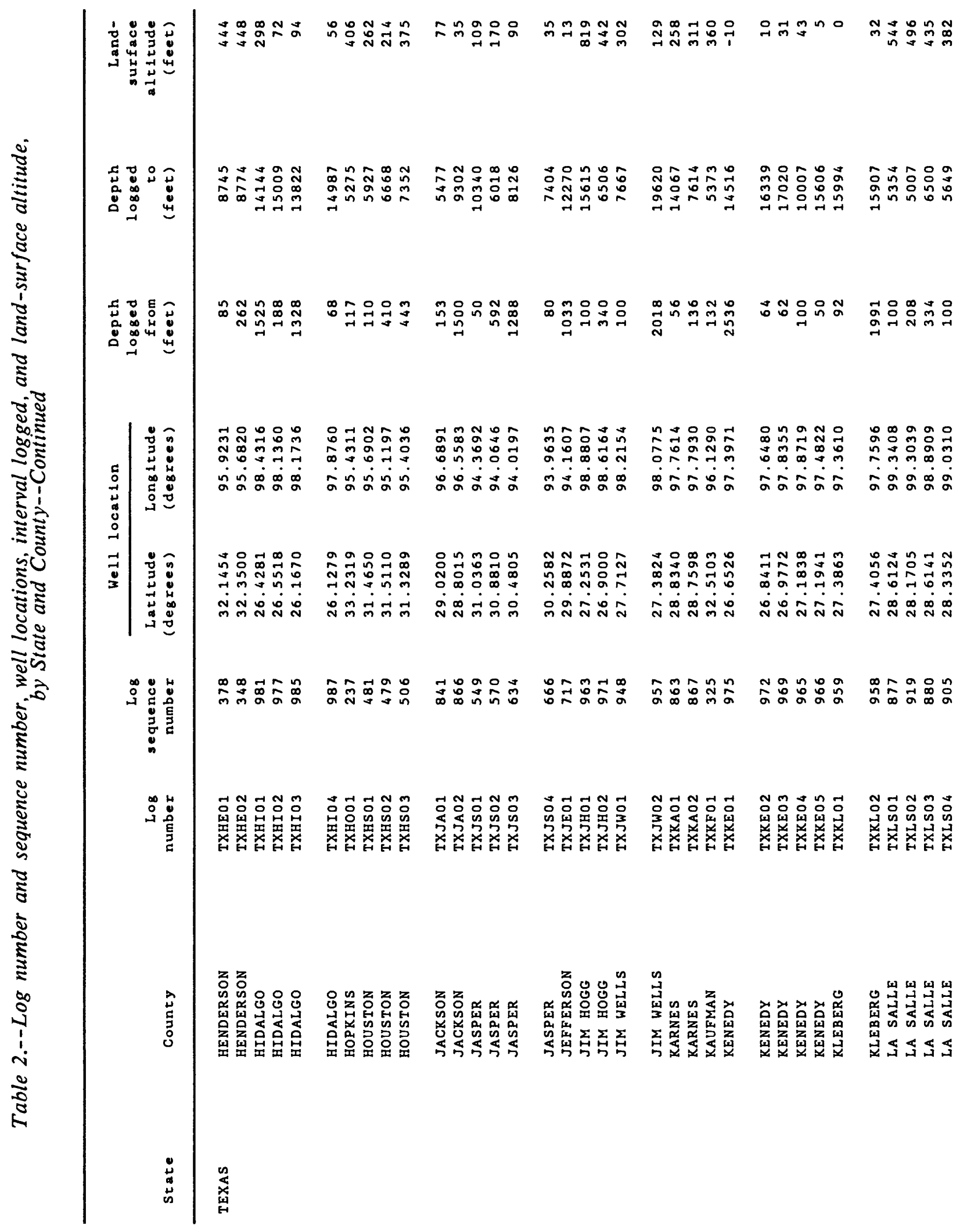




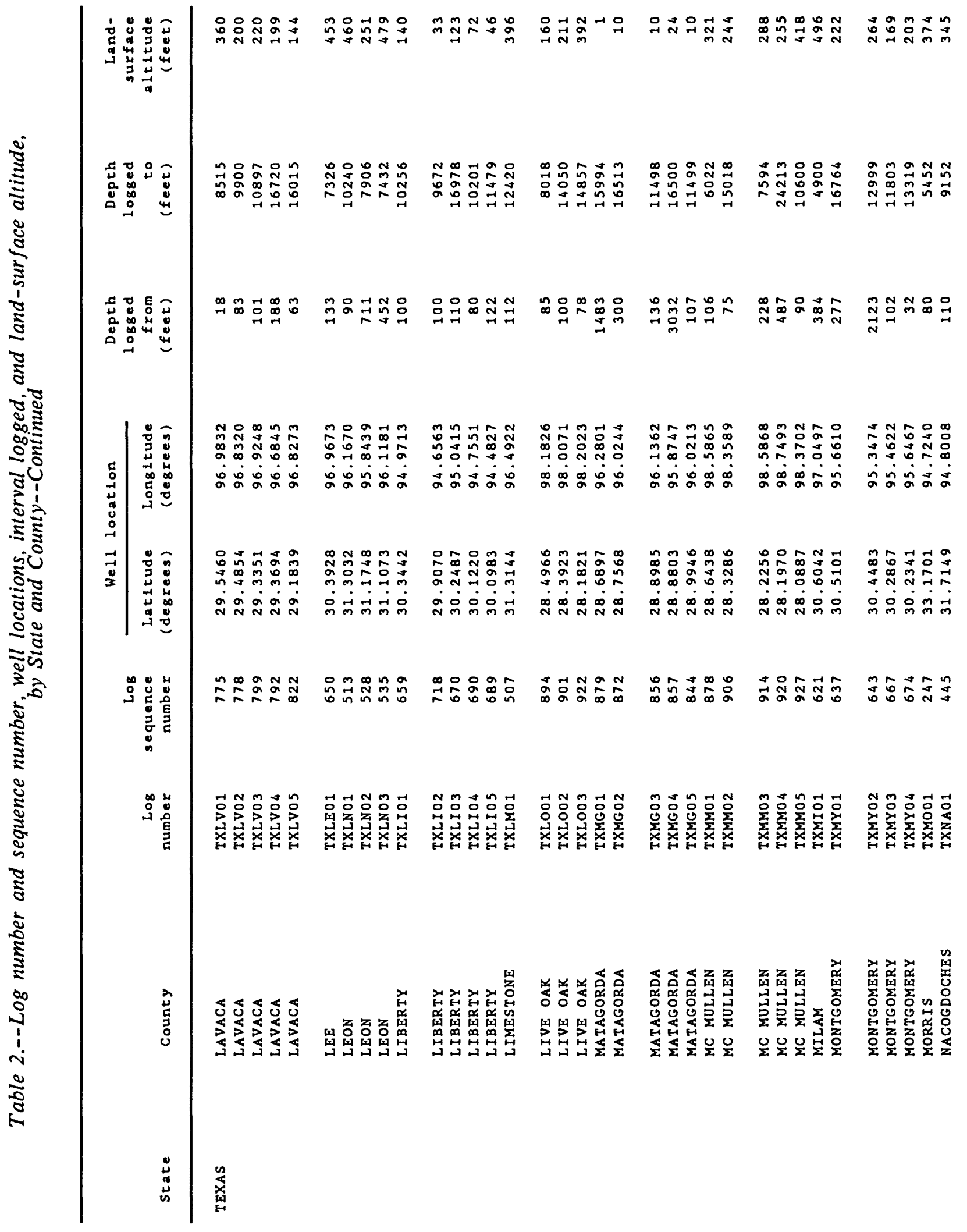




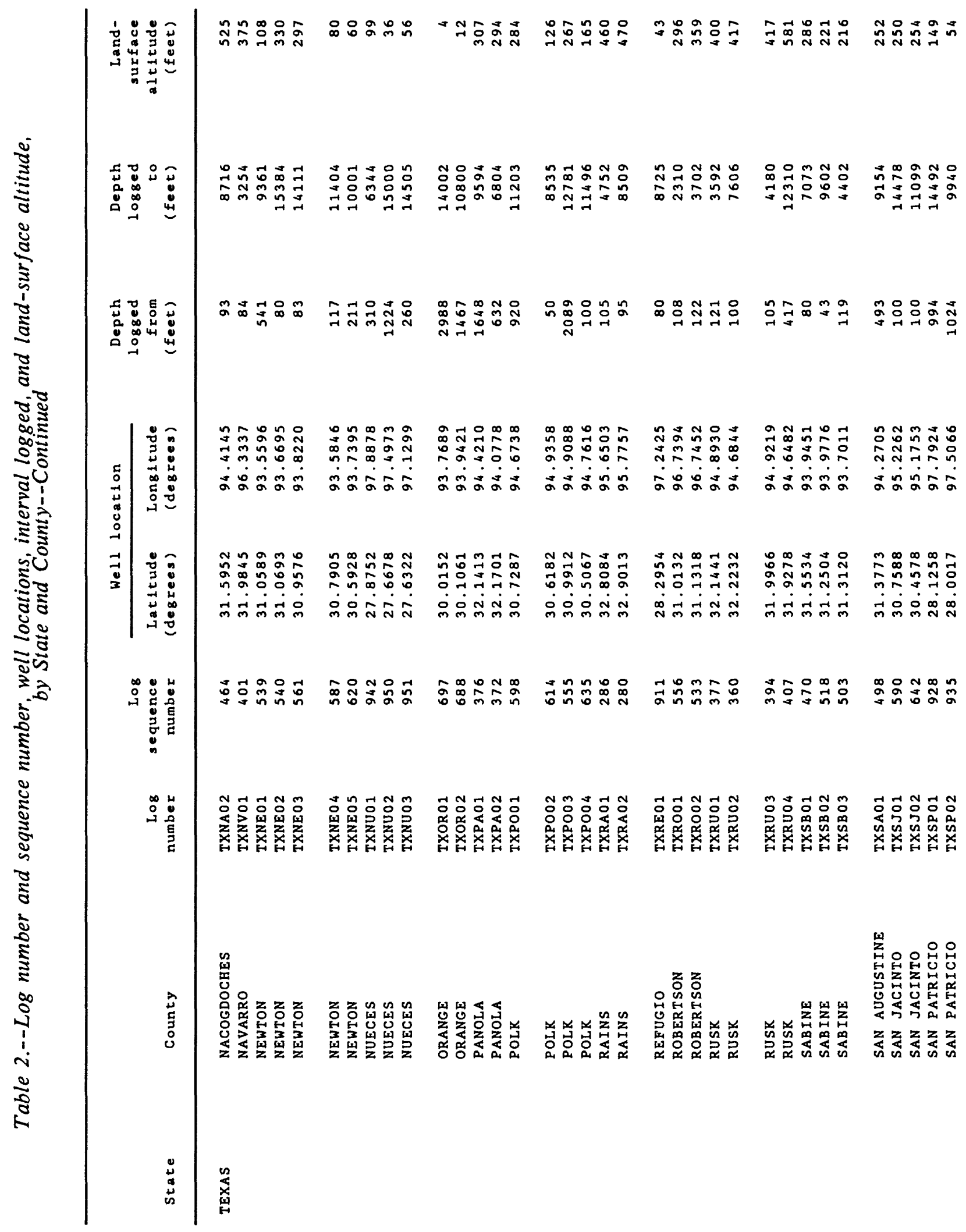




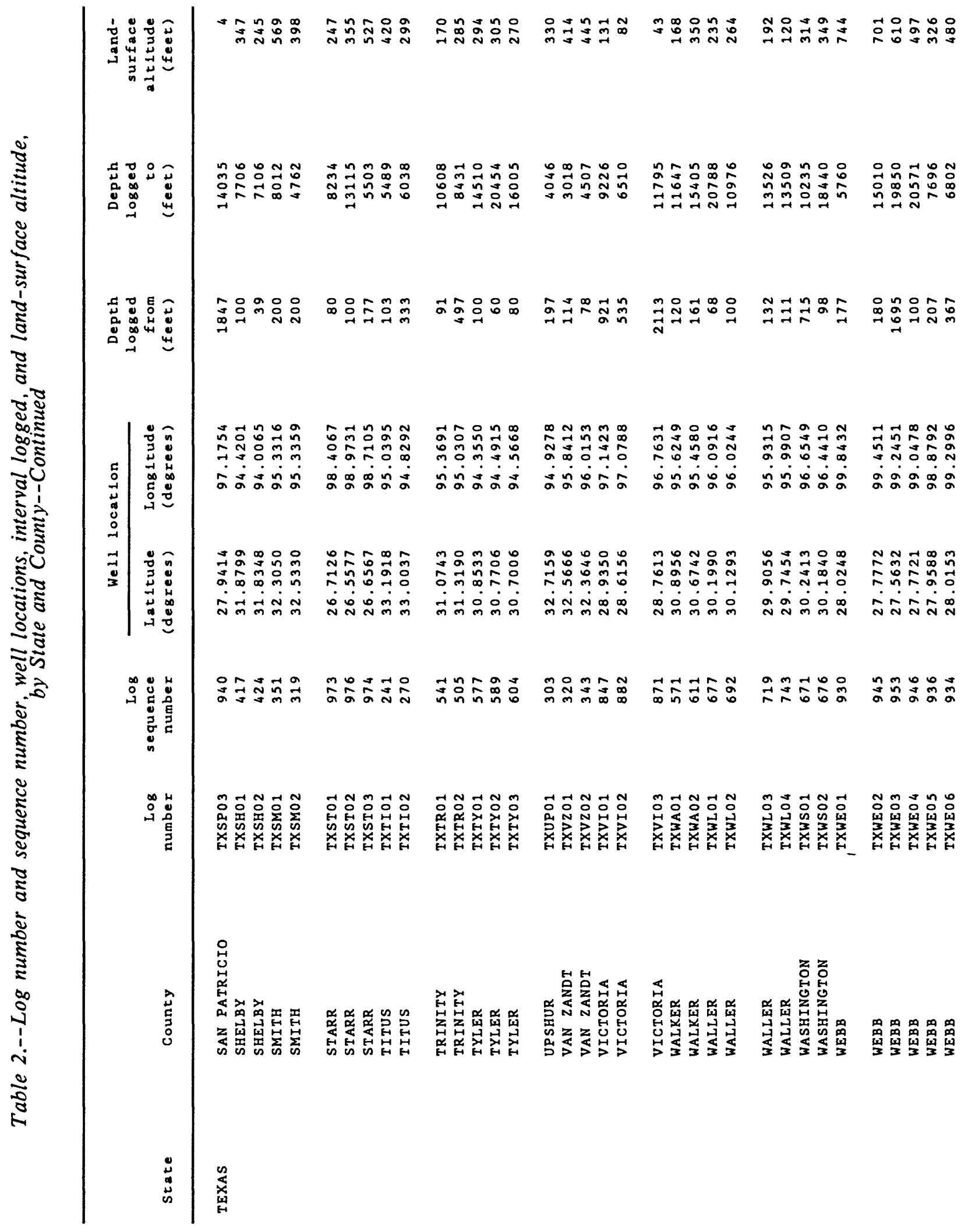




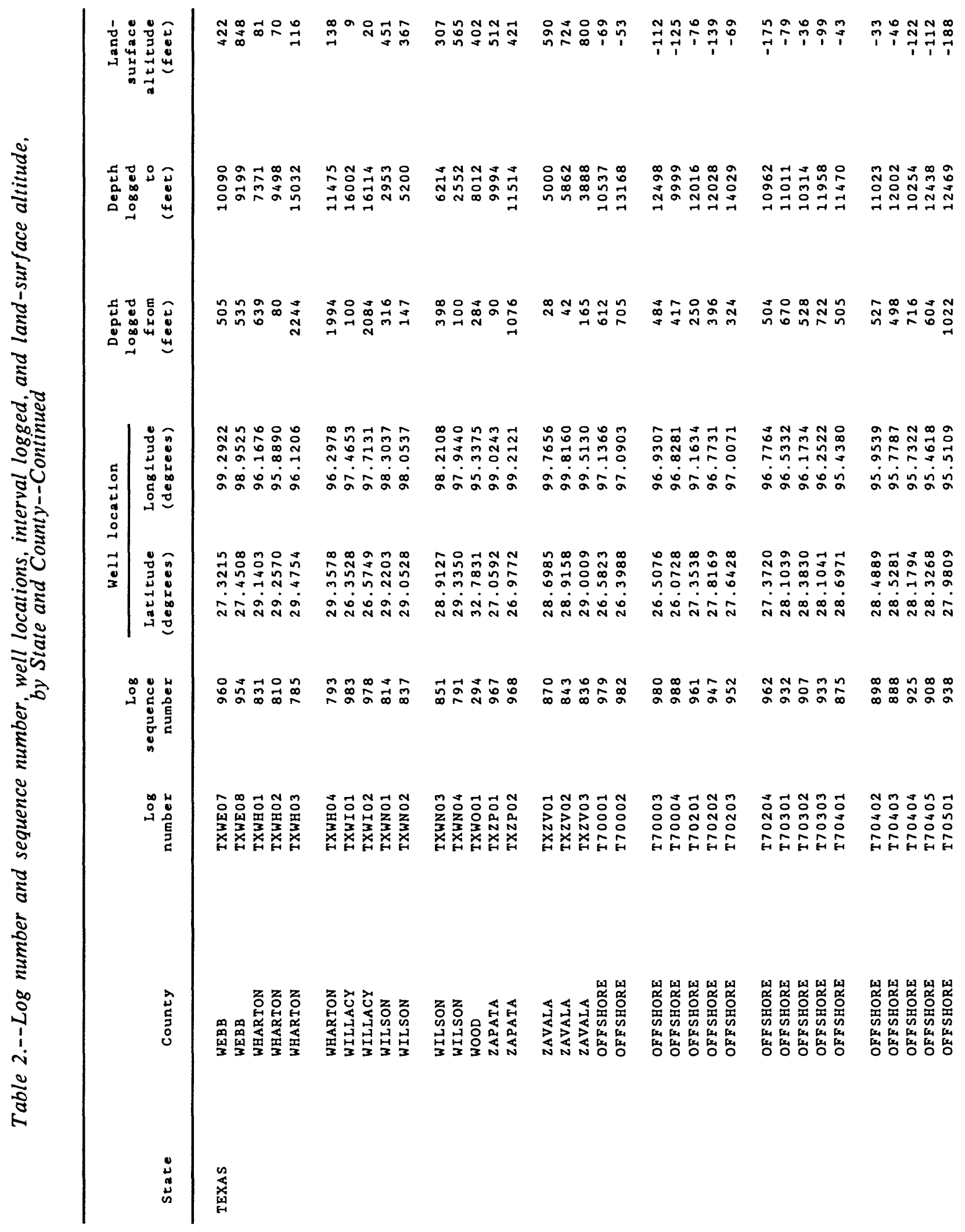




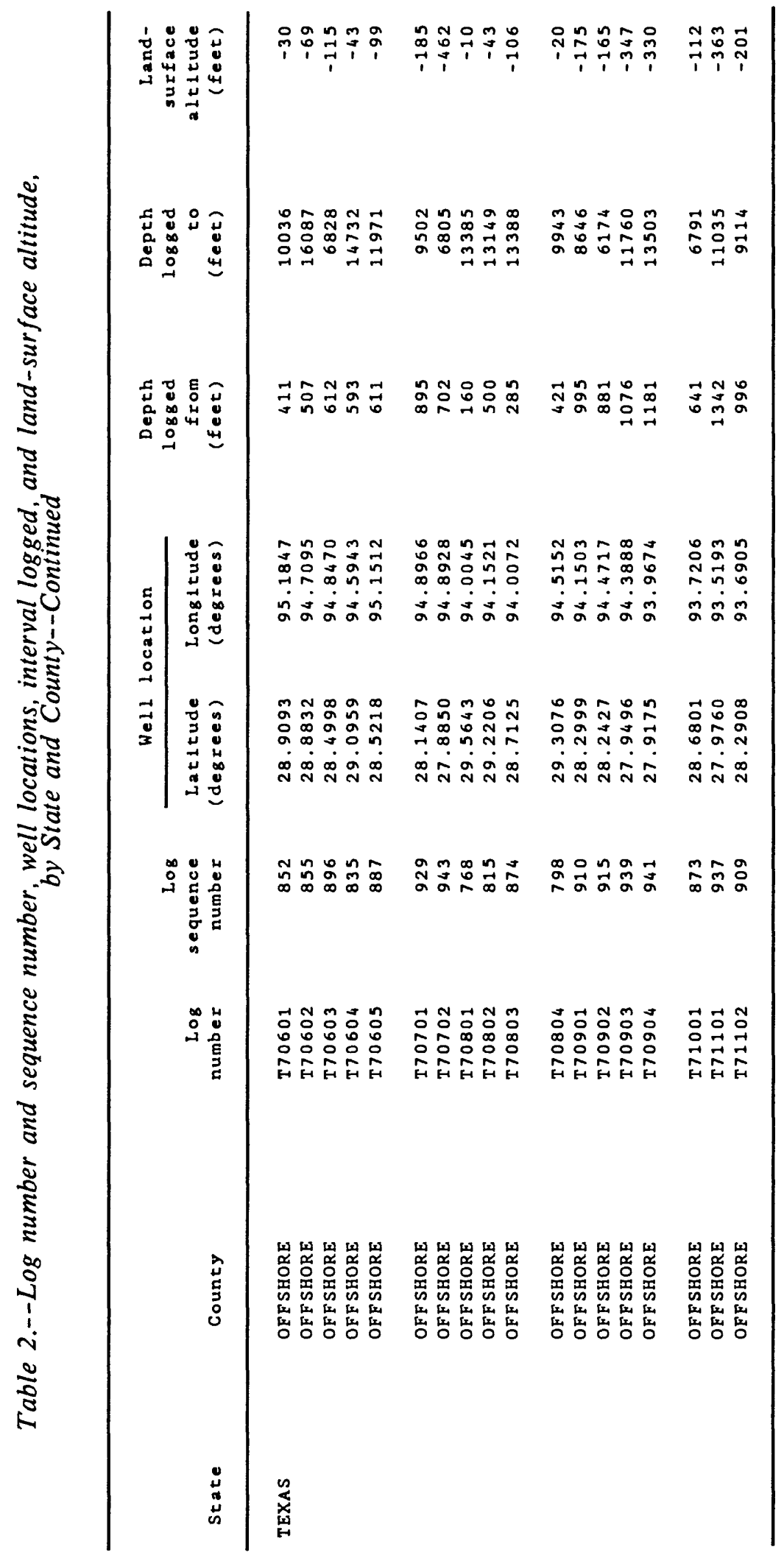


Explanation of data in table 3 :

Log sequence number--Wells for which logs were used in this study were numbered sequentially for convenience in this report. The numbers begin at the northernmost well in the project area and end at the southernmost.

Log number-An alphanumeric system that uses the two-letter State abbreviation followed by a two-letter county (parish) abbreviation followed by a two-digit sequential number. For example, the first well numbered in Ballard County, Kentucky, would be KYBA01. Offshore numbers are composed of the single letter initial of the State and a three-digit API (American Petroleum Institute) number followed by a two-digit sequential number.

County-County (parish) in which the logged well was drilled. Offshore logs are listed following county (parish) lists.

Company name--The name of the organization or individual that drilled the well, such as Little Egypt Oil Co.

Well name--Based on the owner of the mineral rights to the land (generally the landowner) on which the well is drilled and, in most instances, sequential well number such as Delaney, No. 1. 


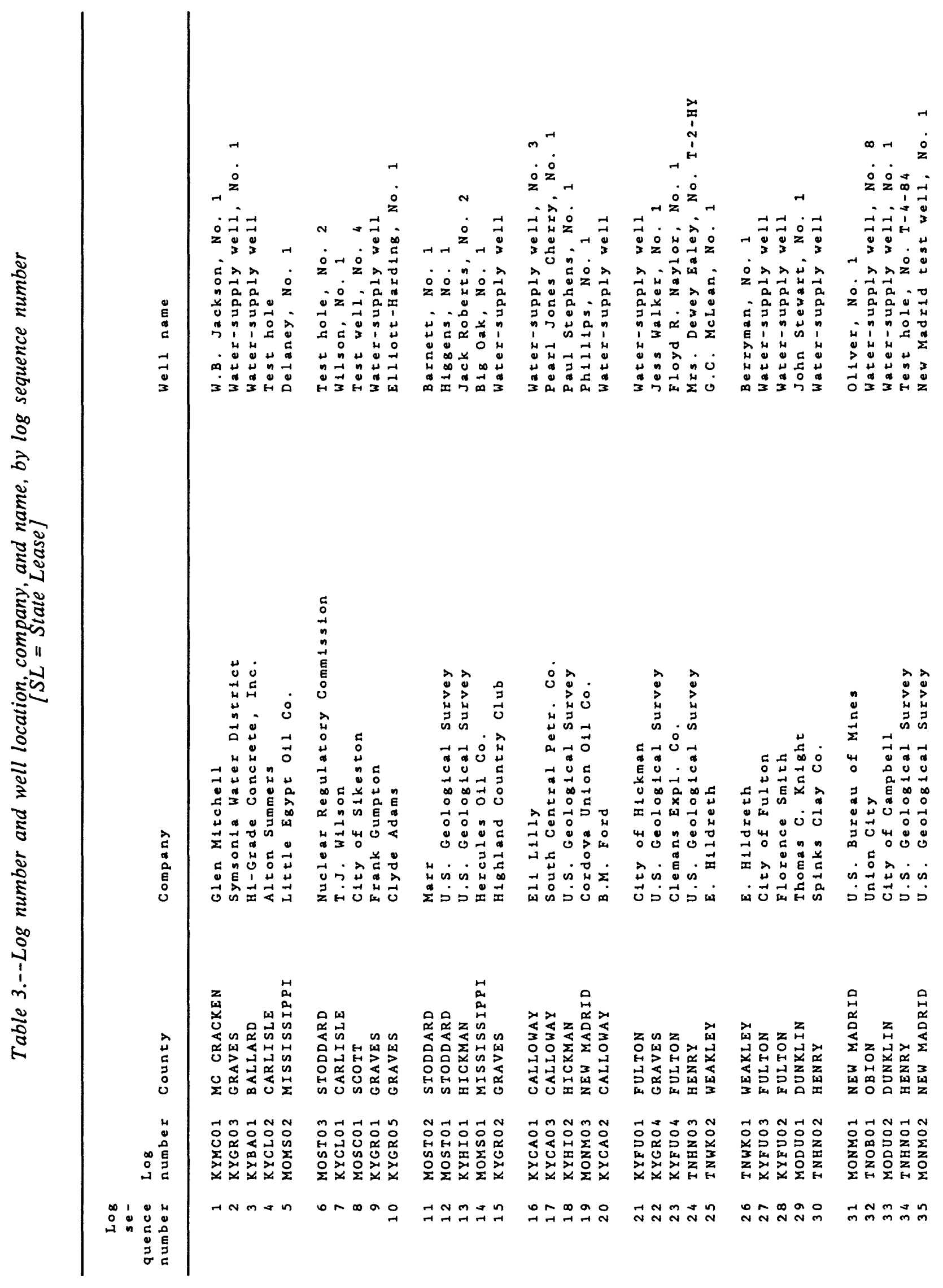




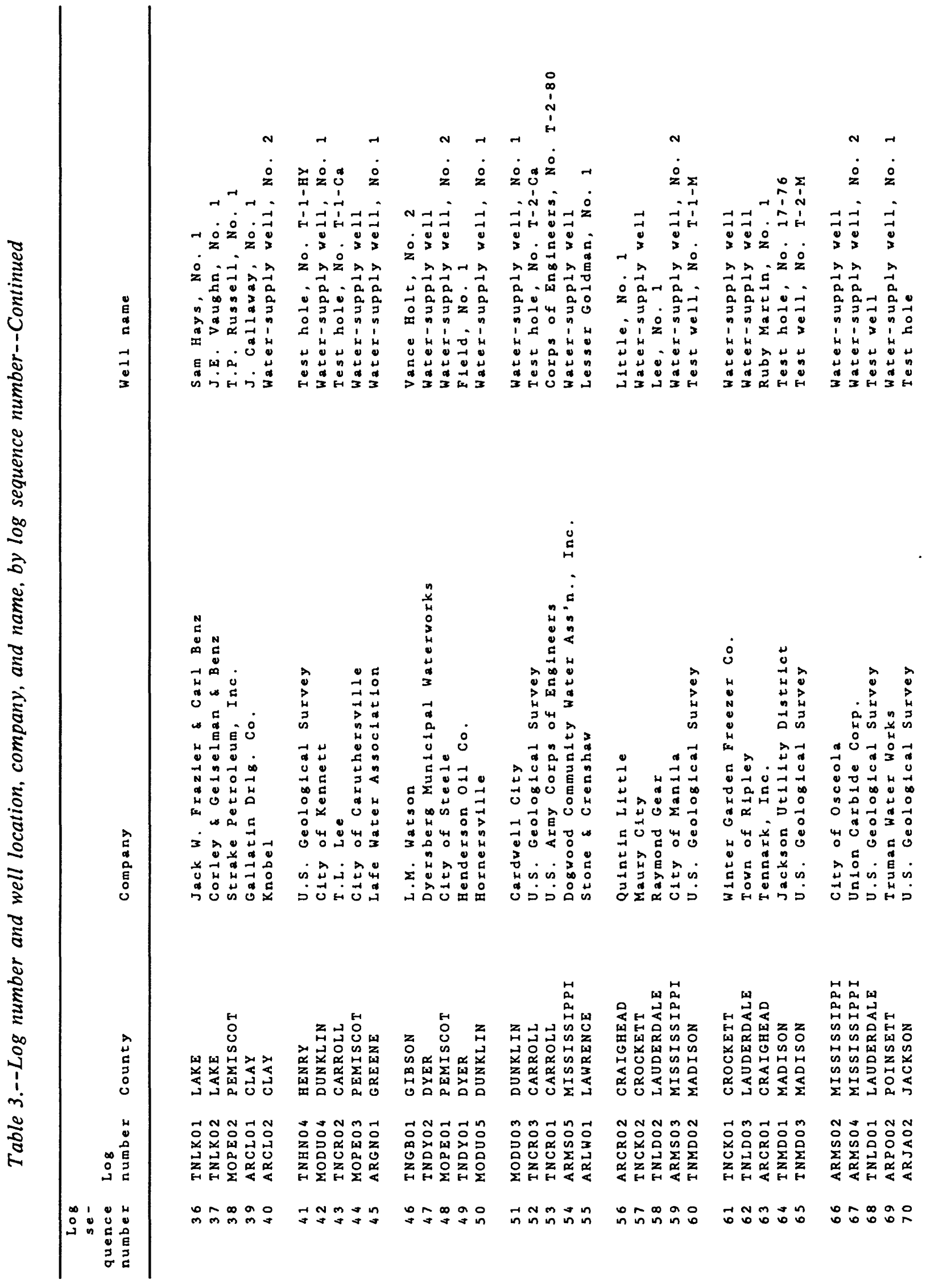




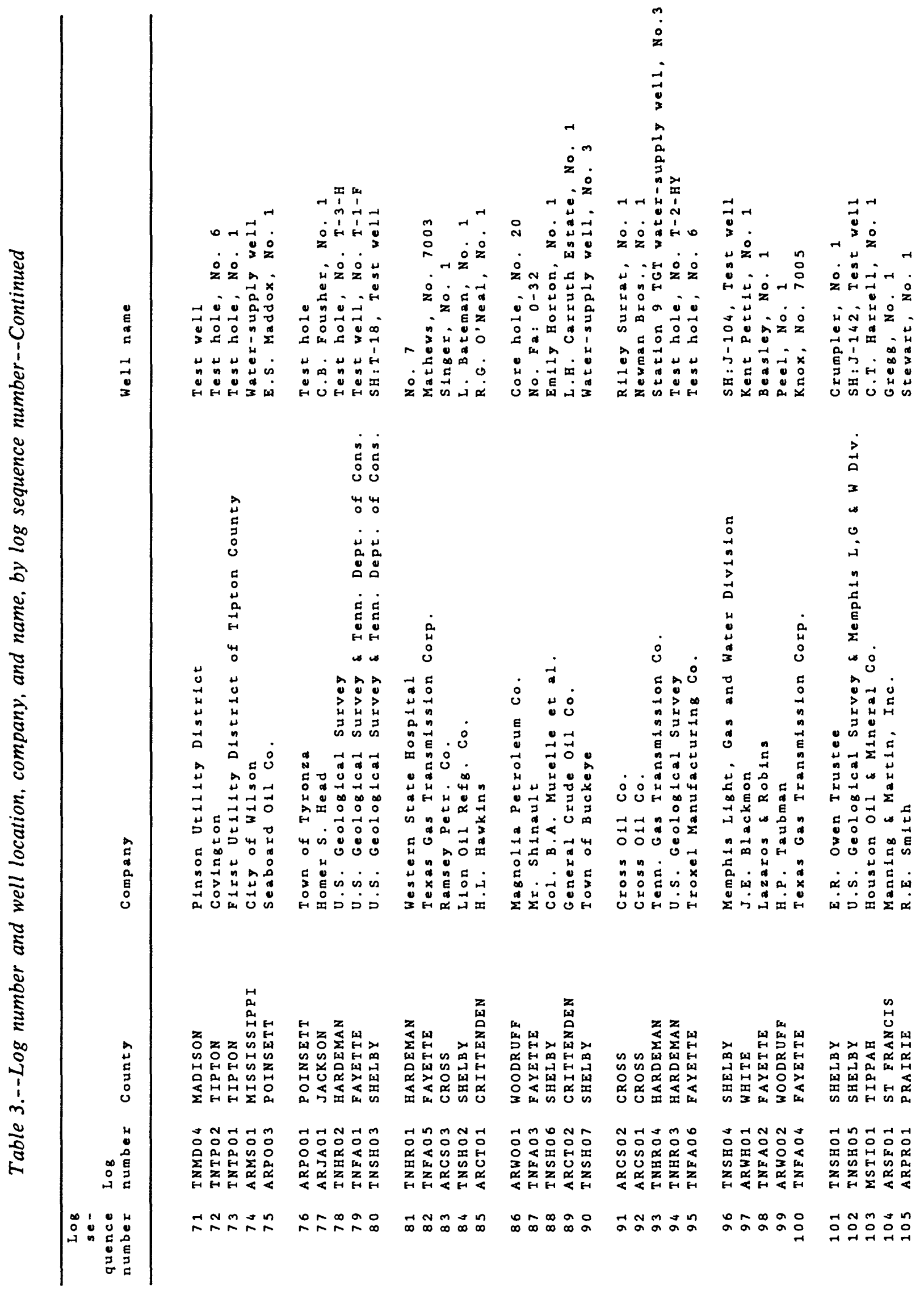




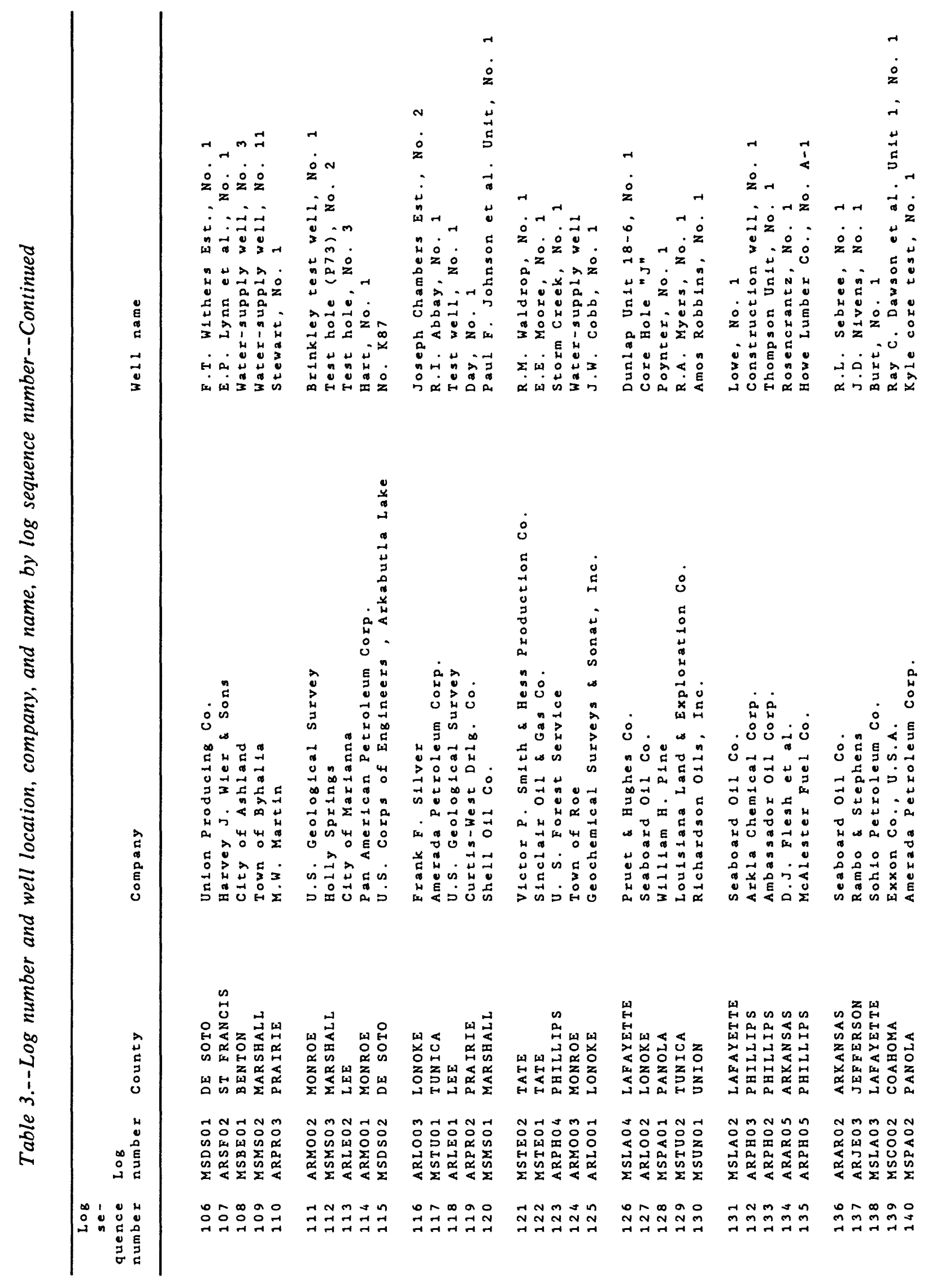




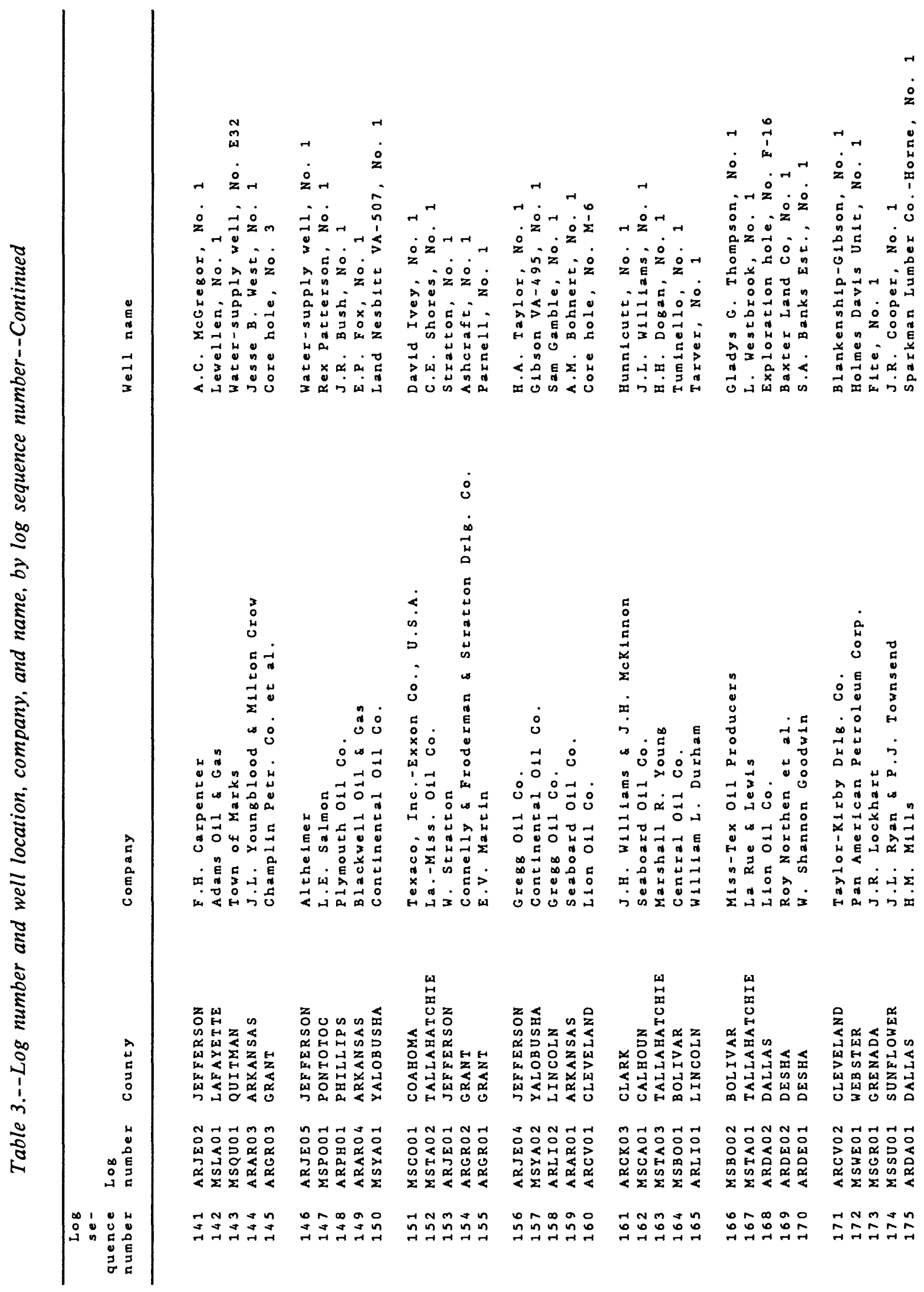




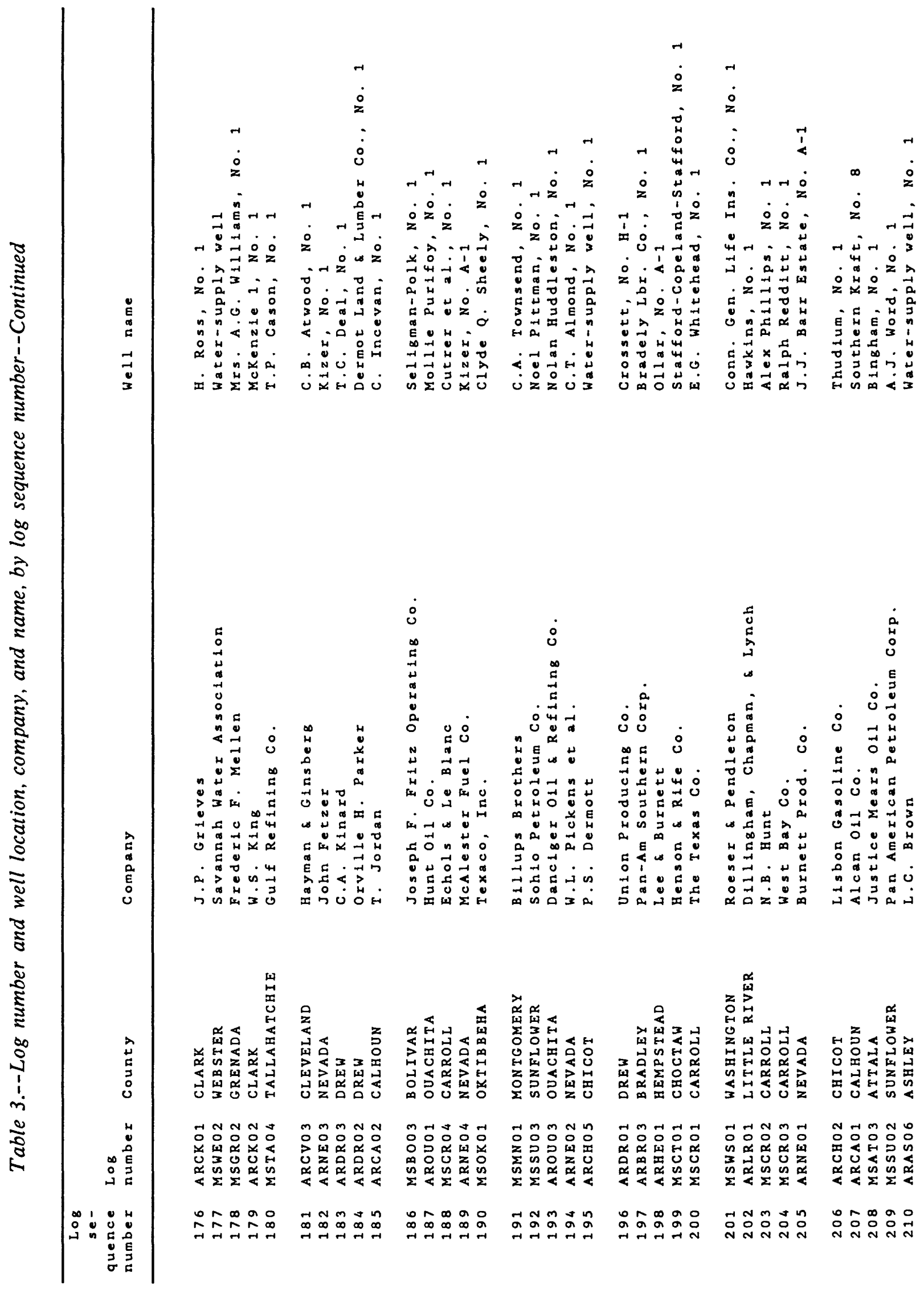




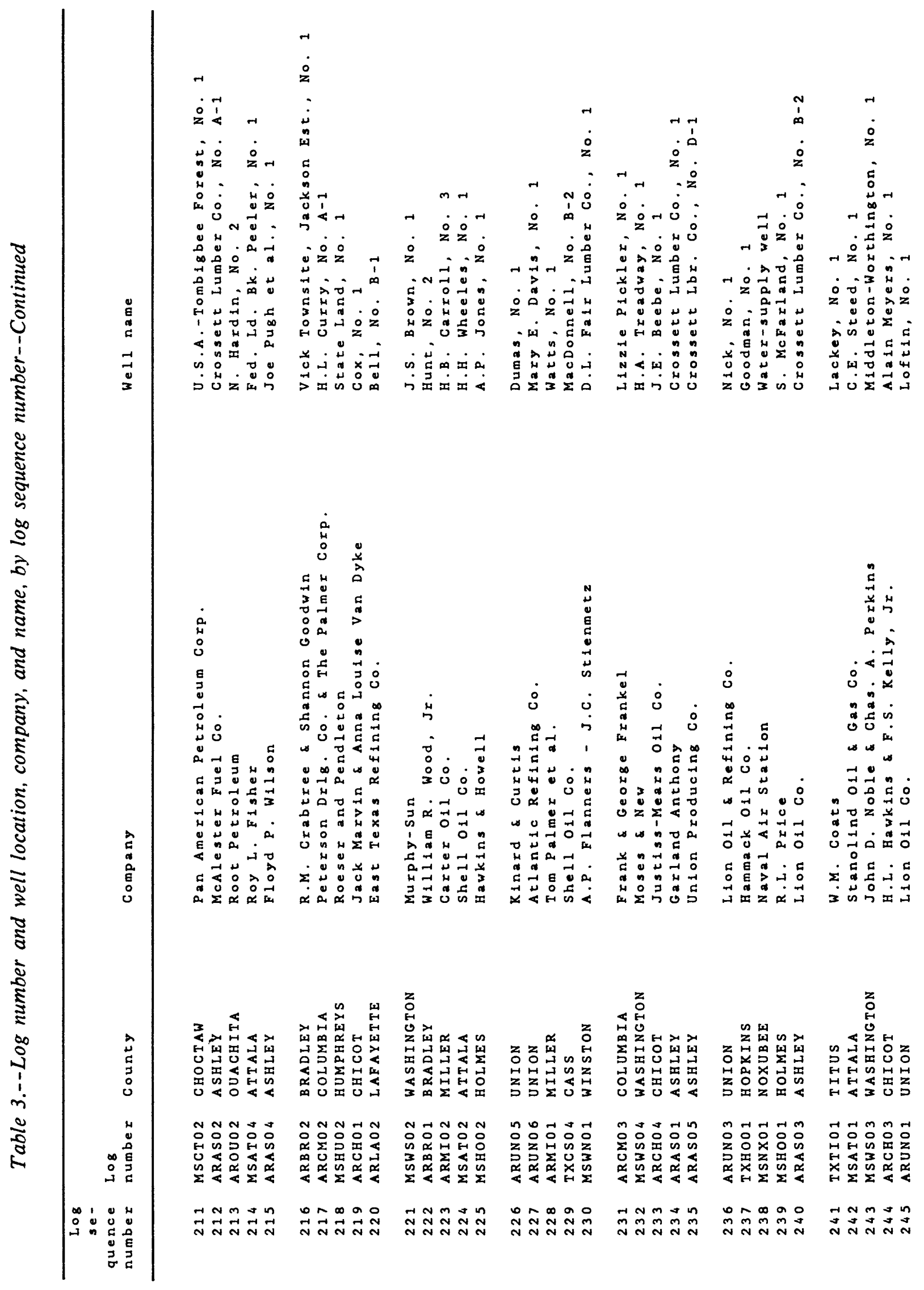




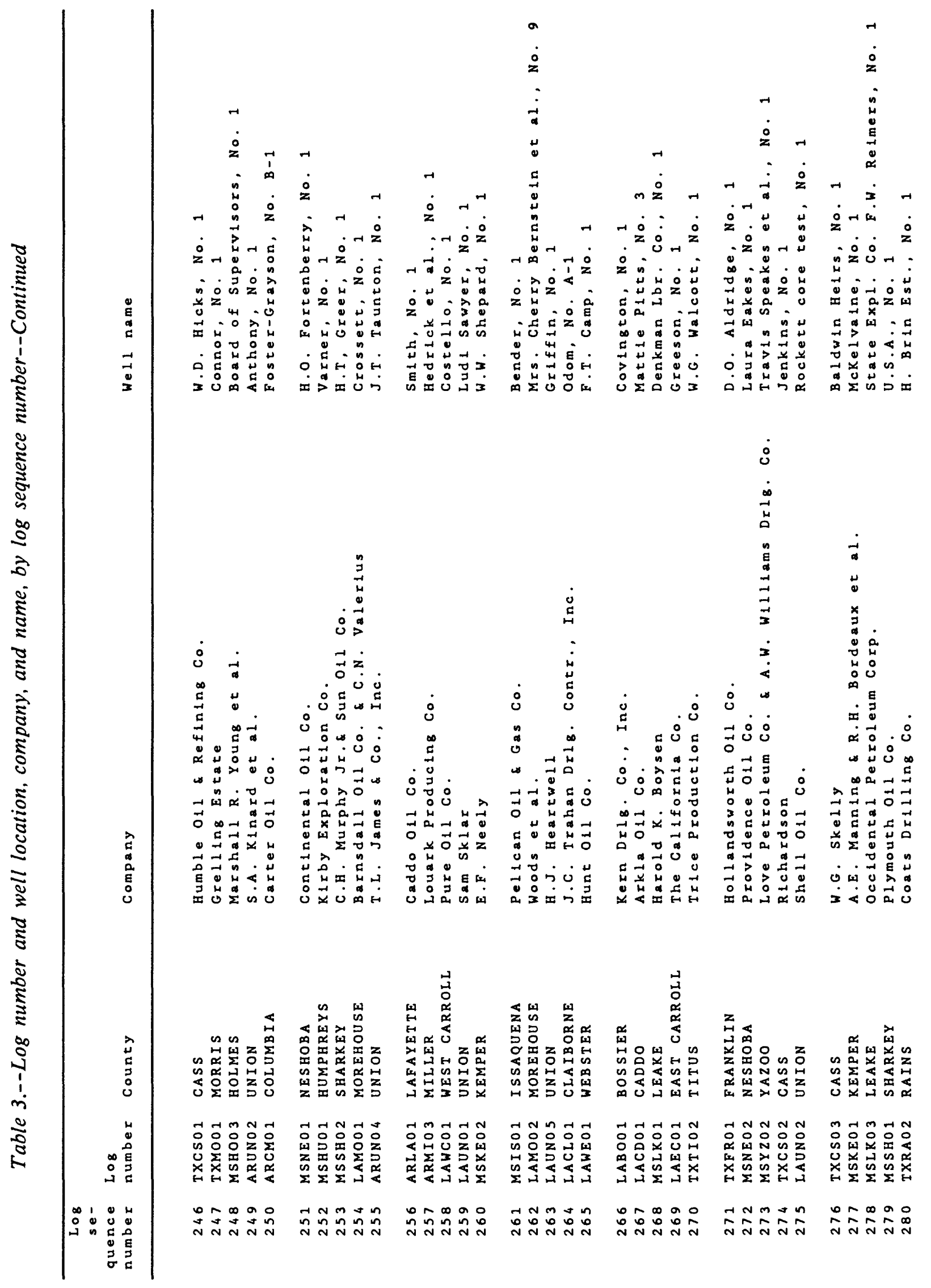




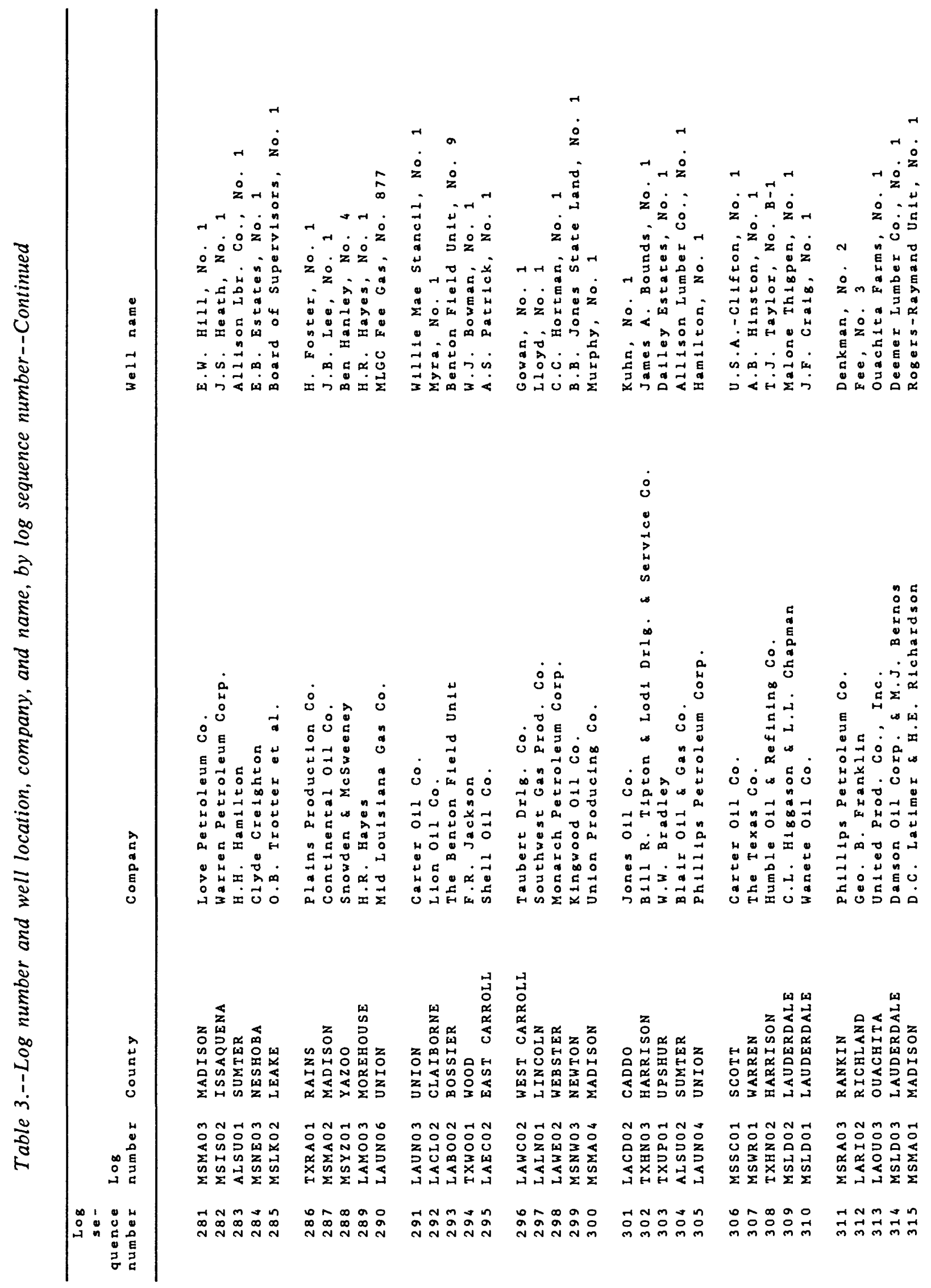




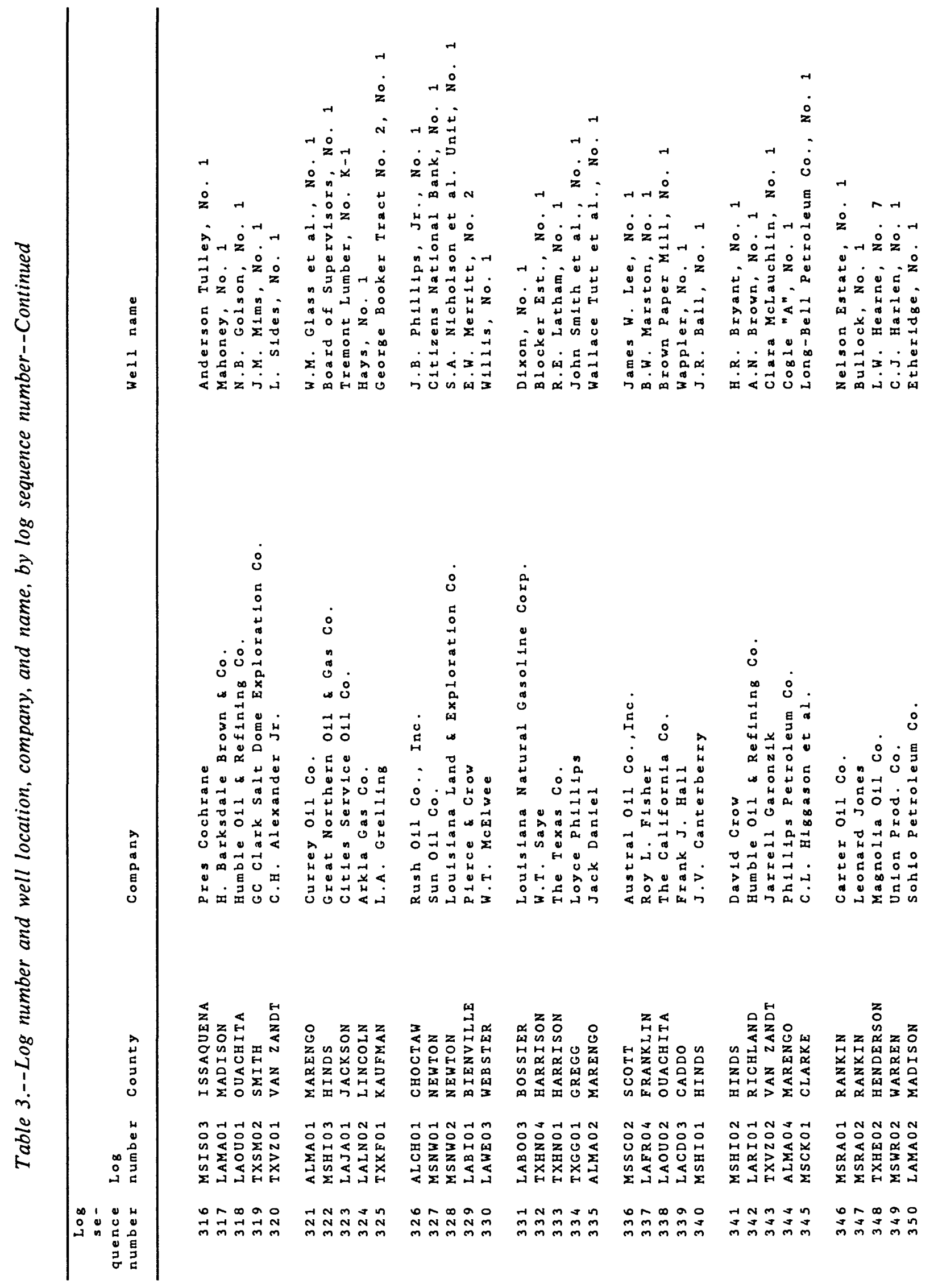




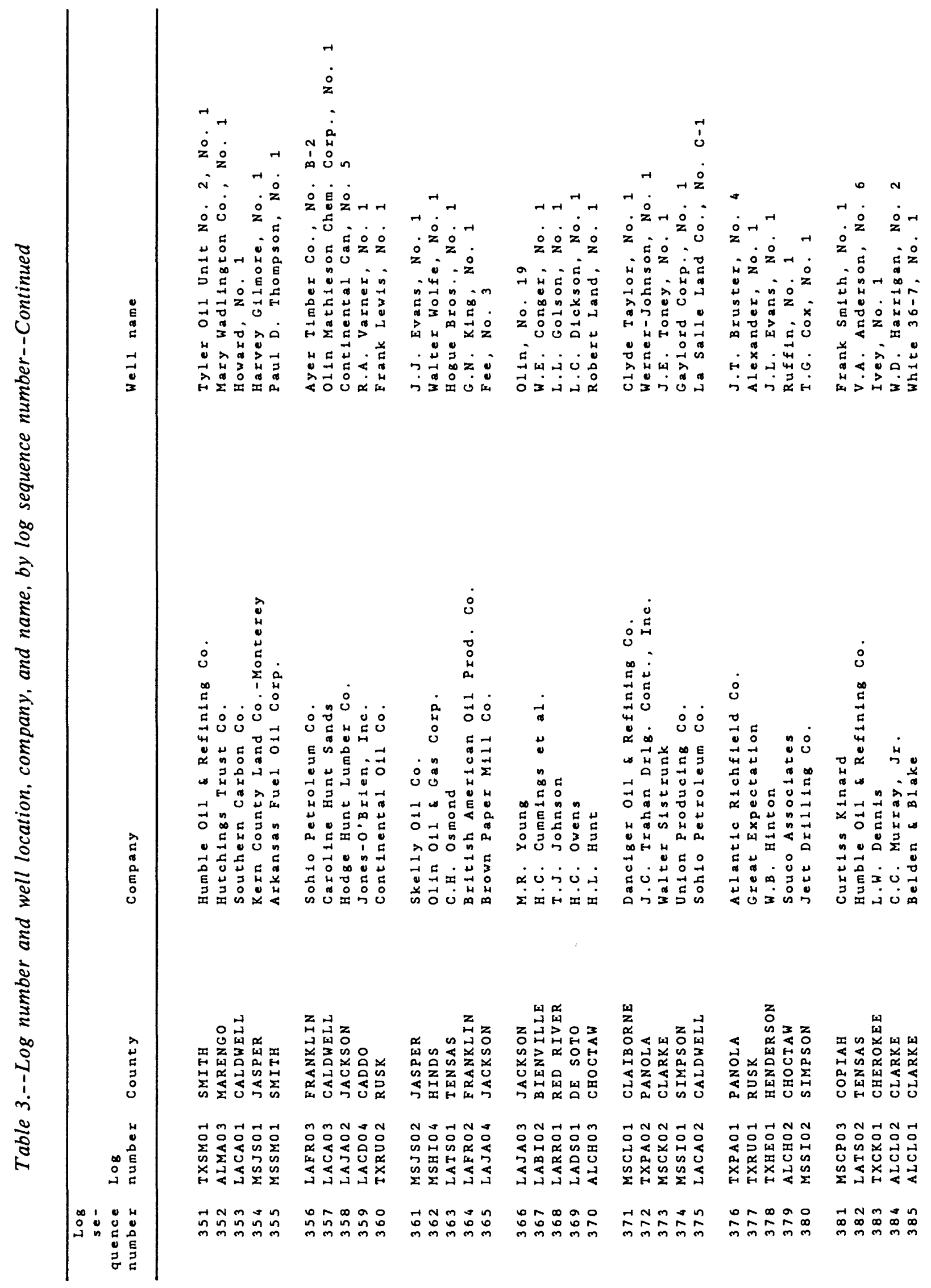




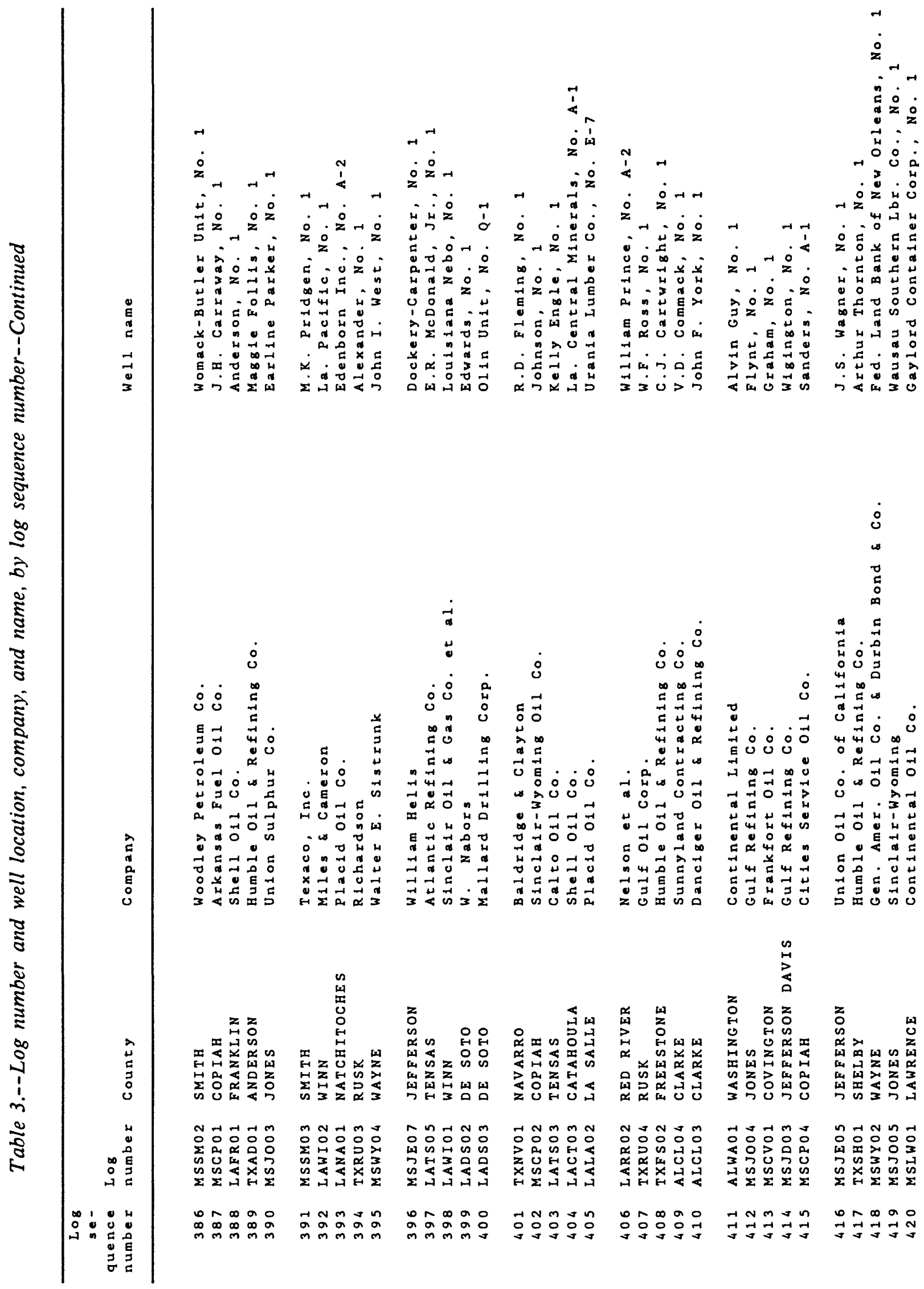




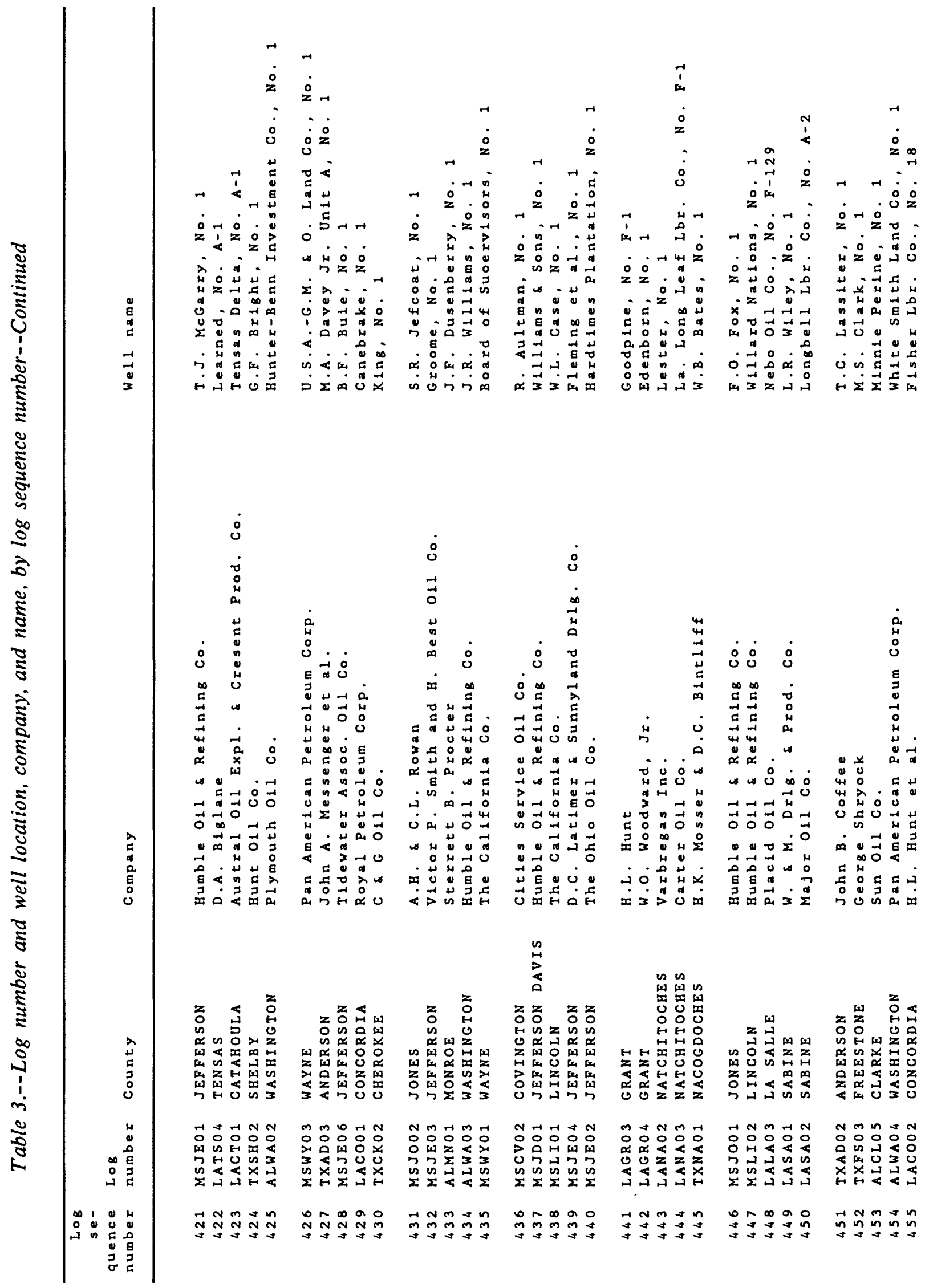




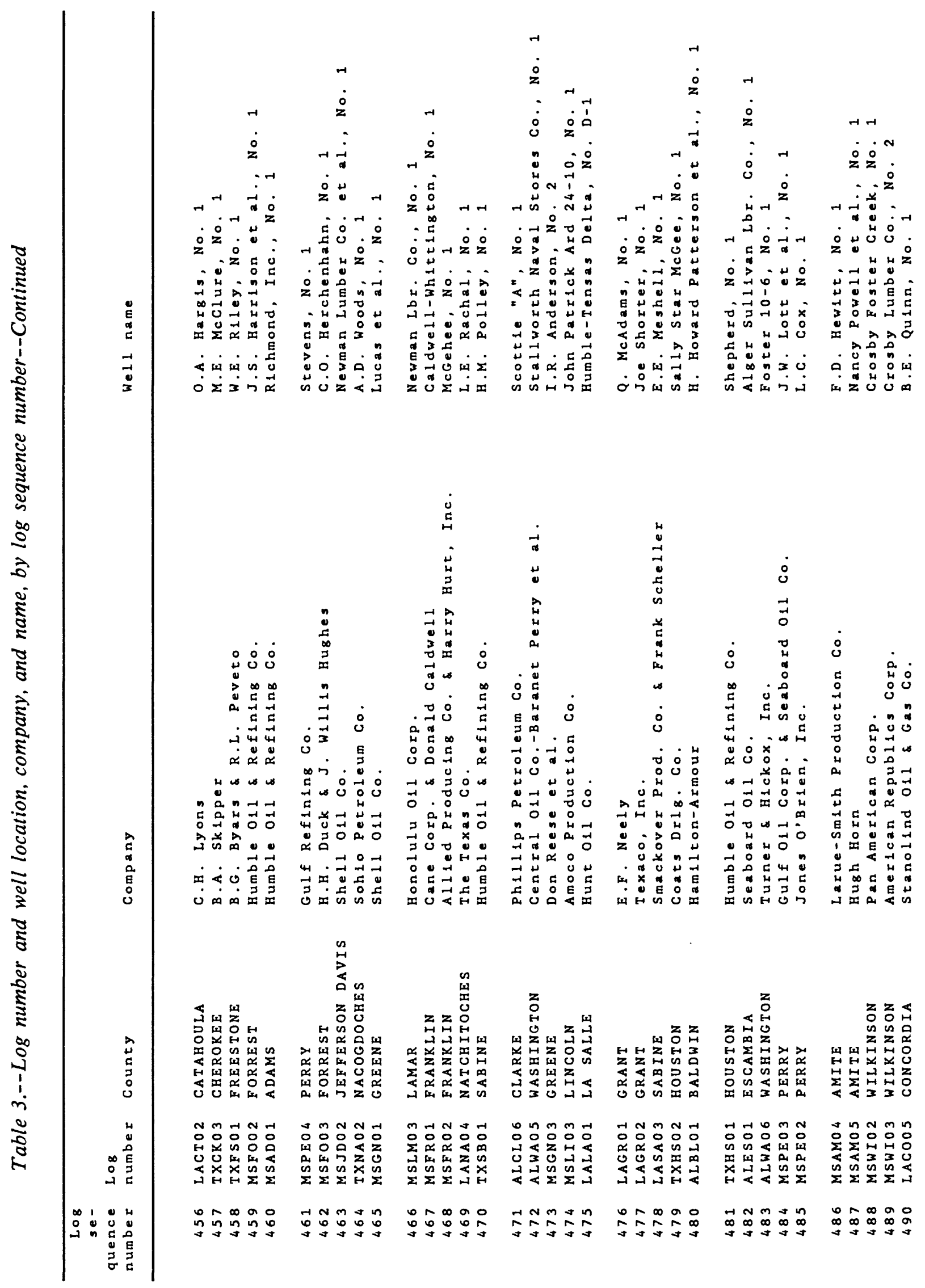




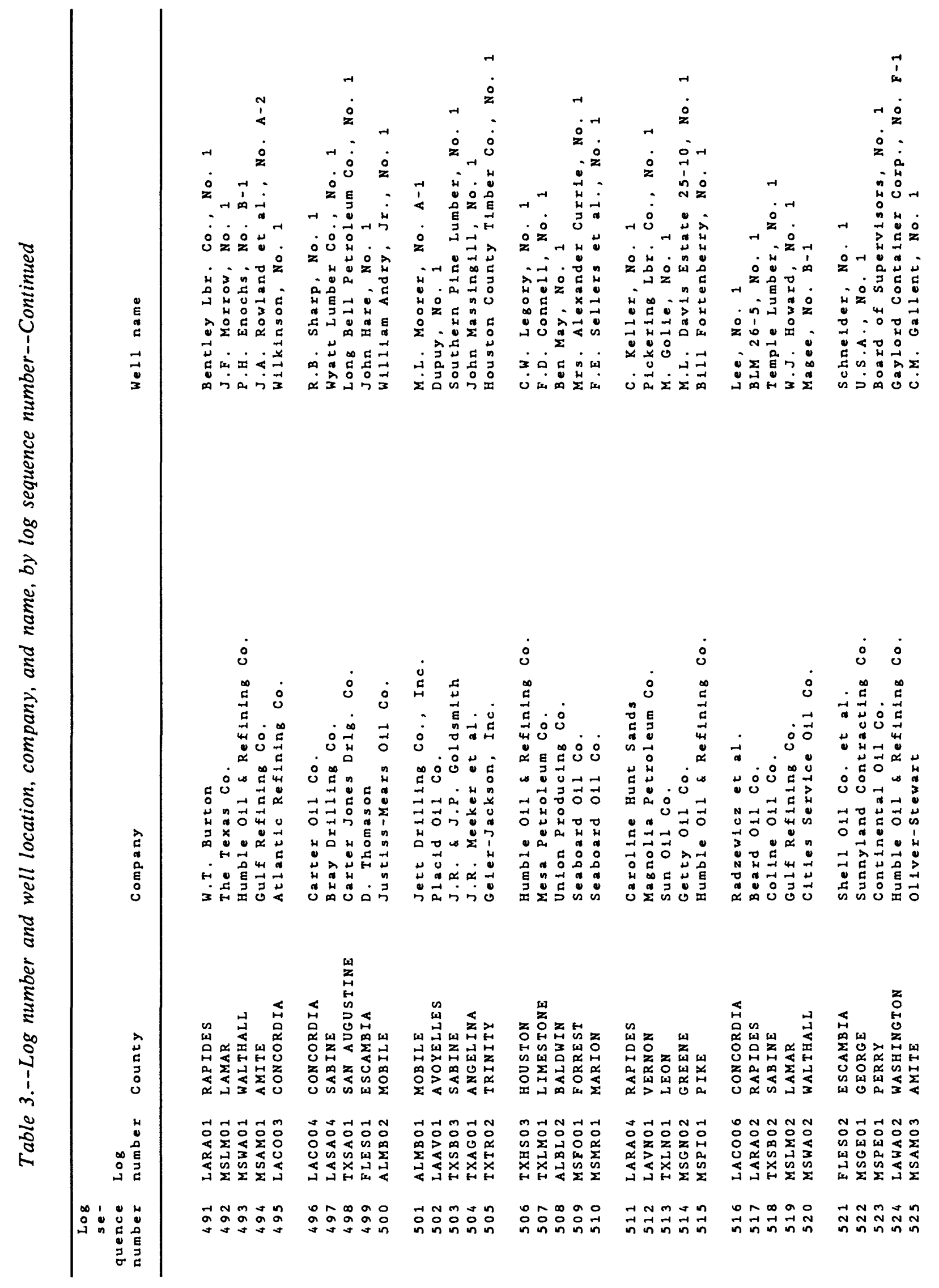




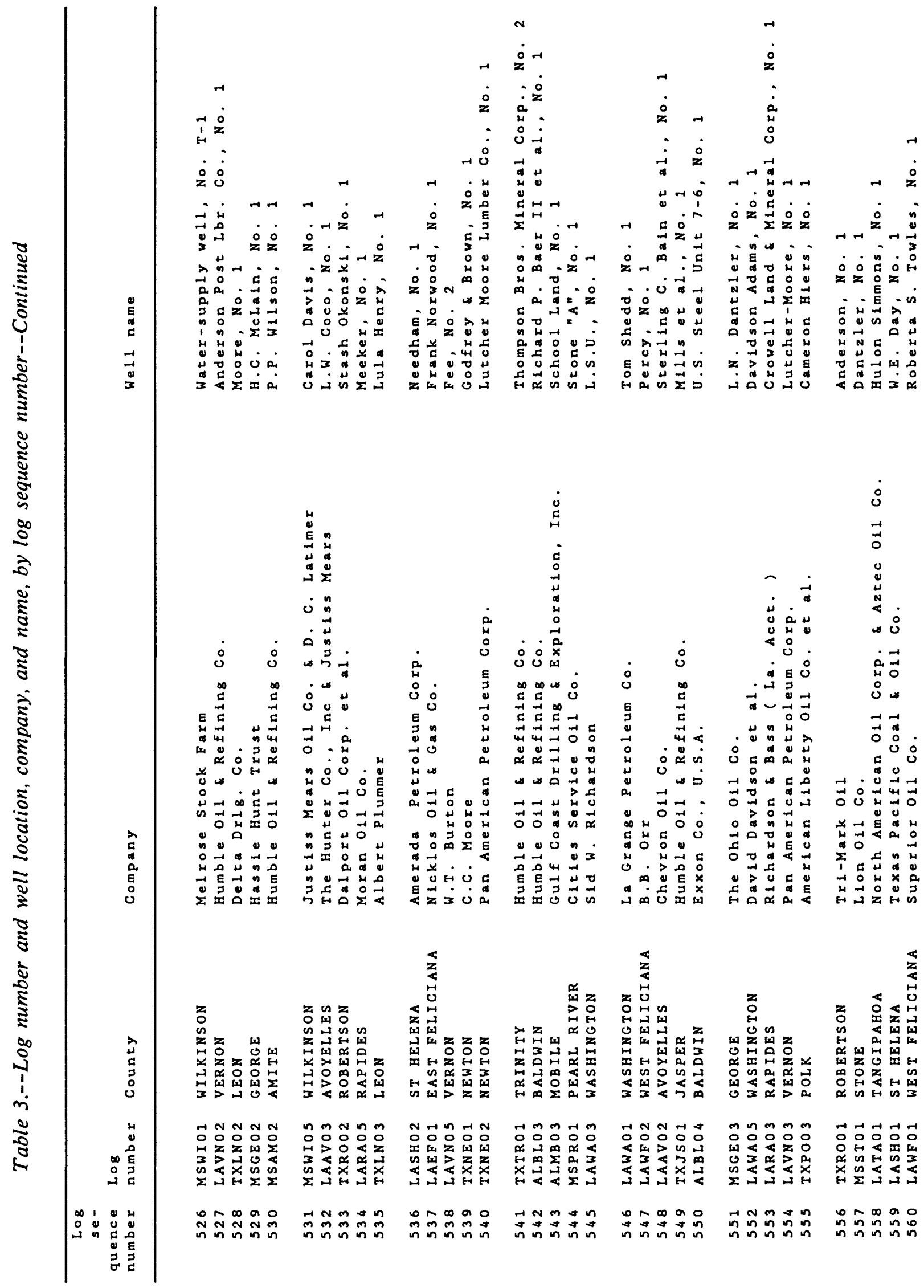









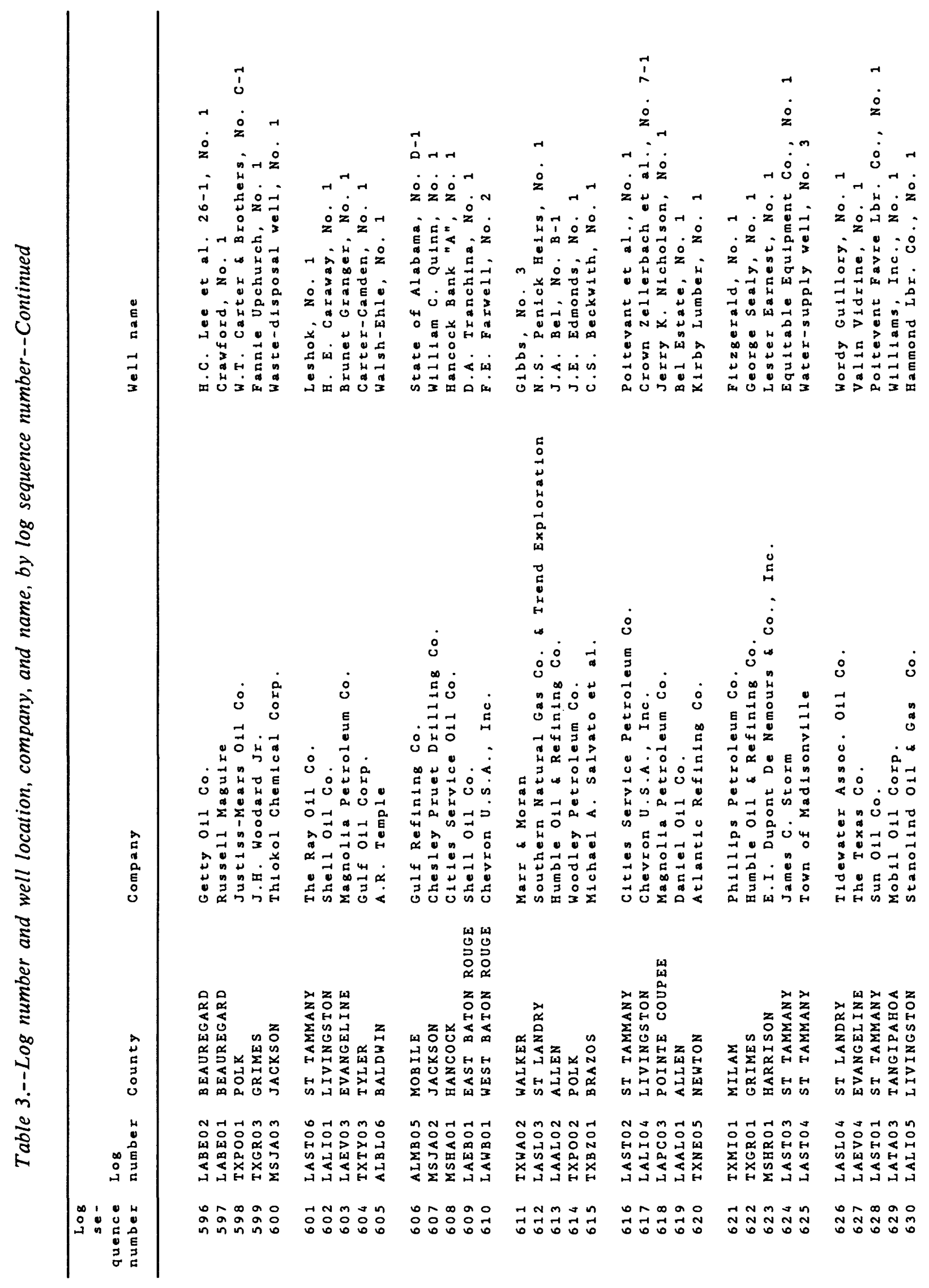




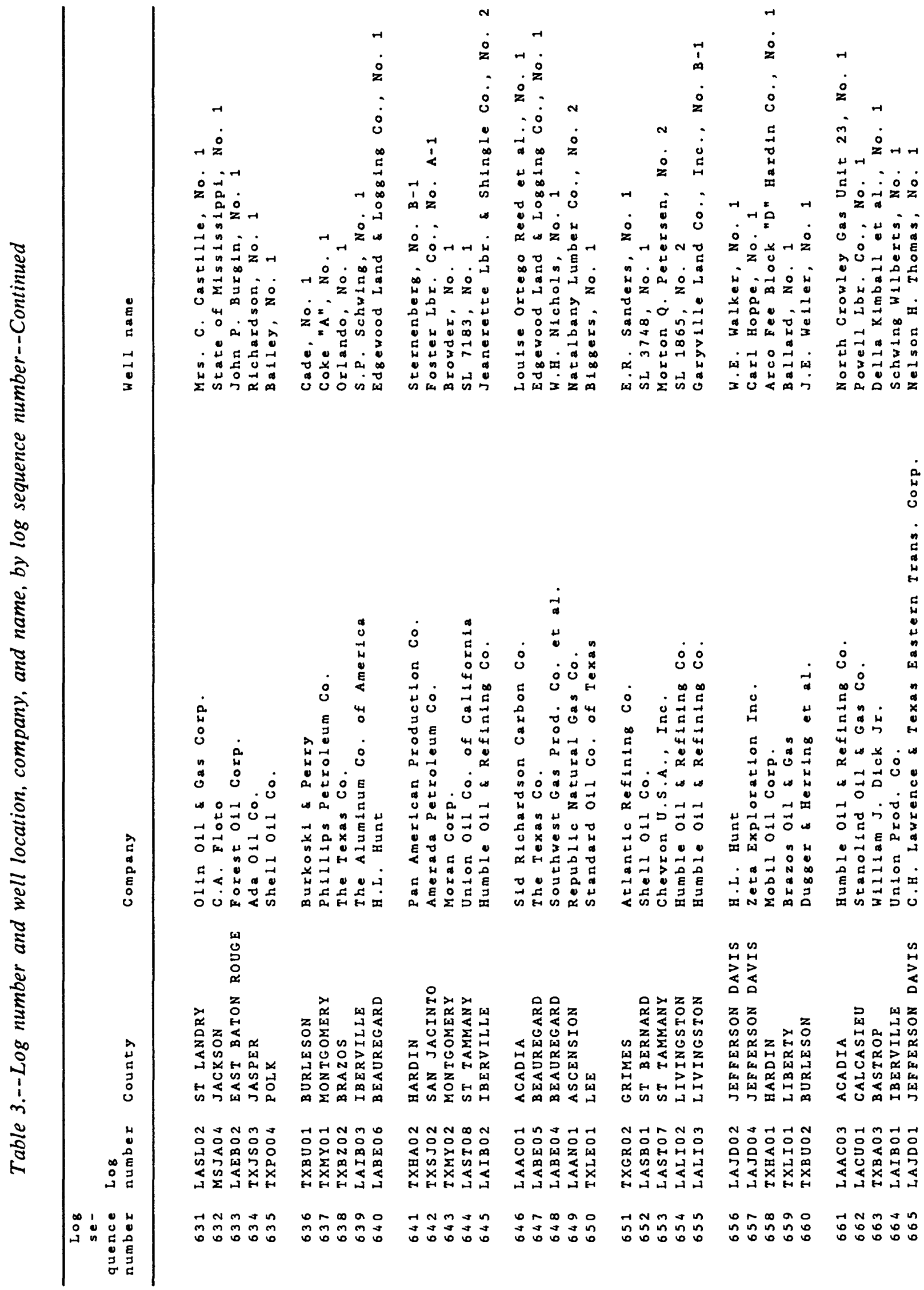




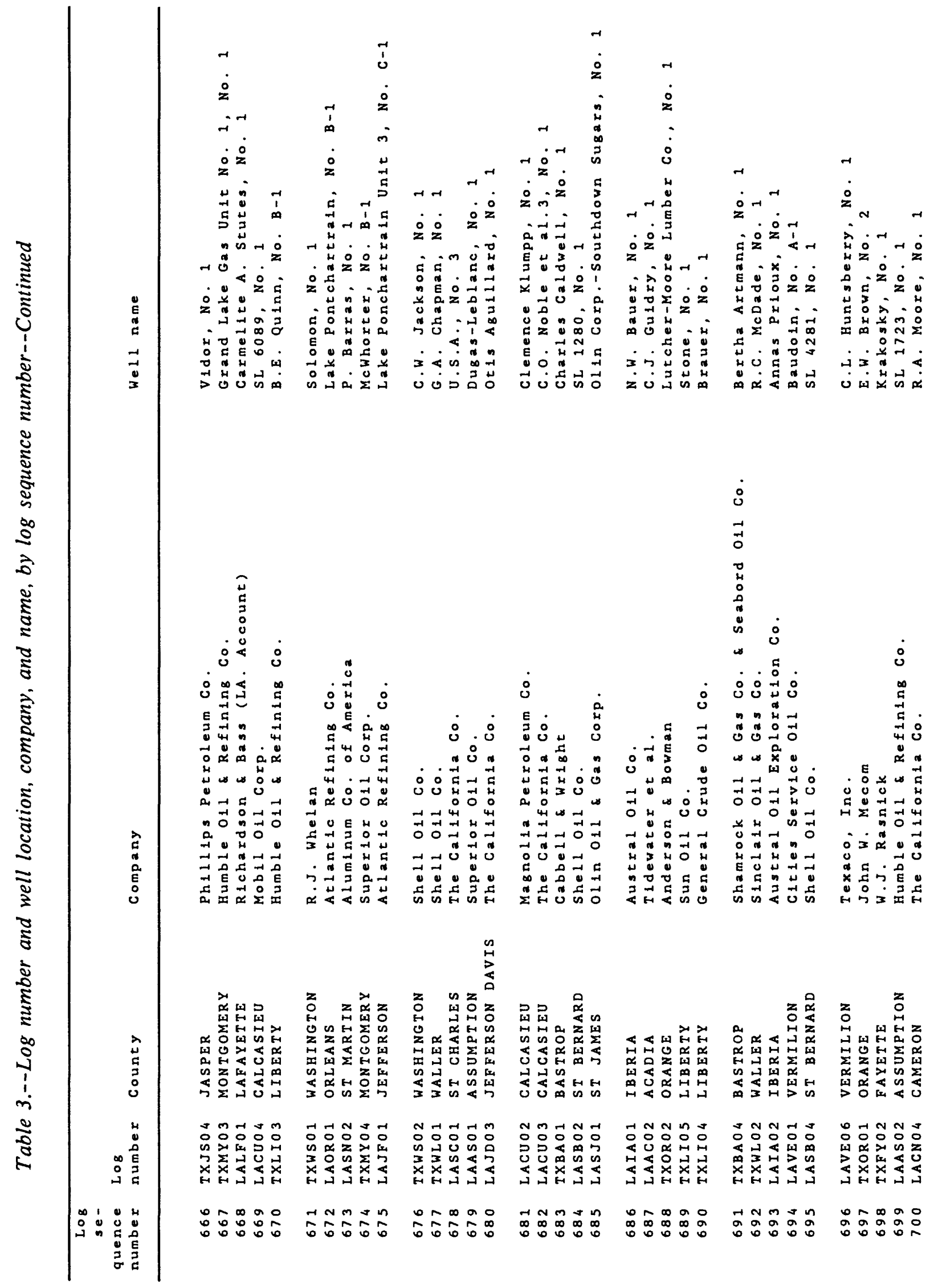




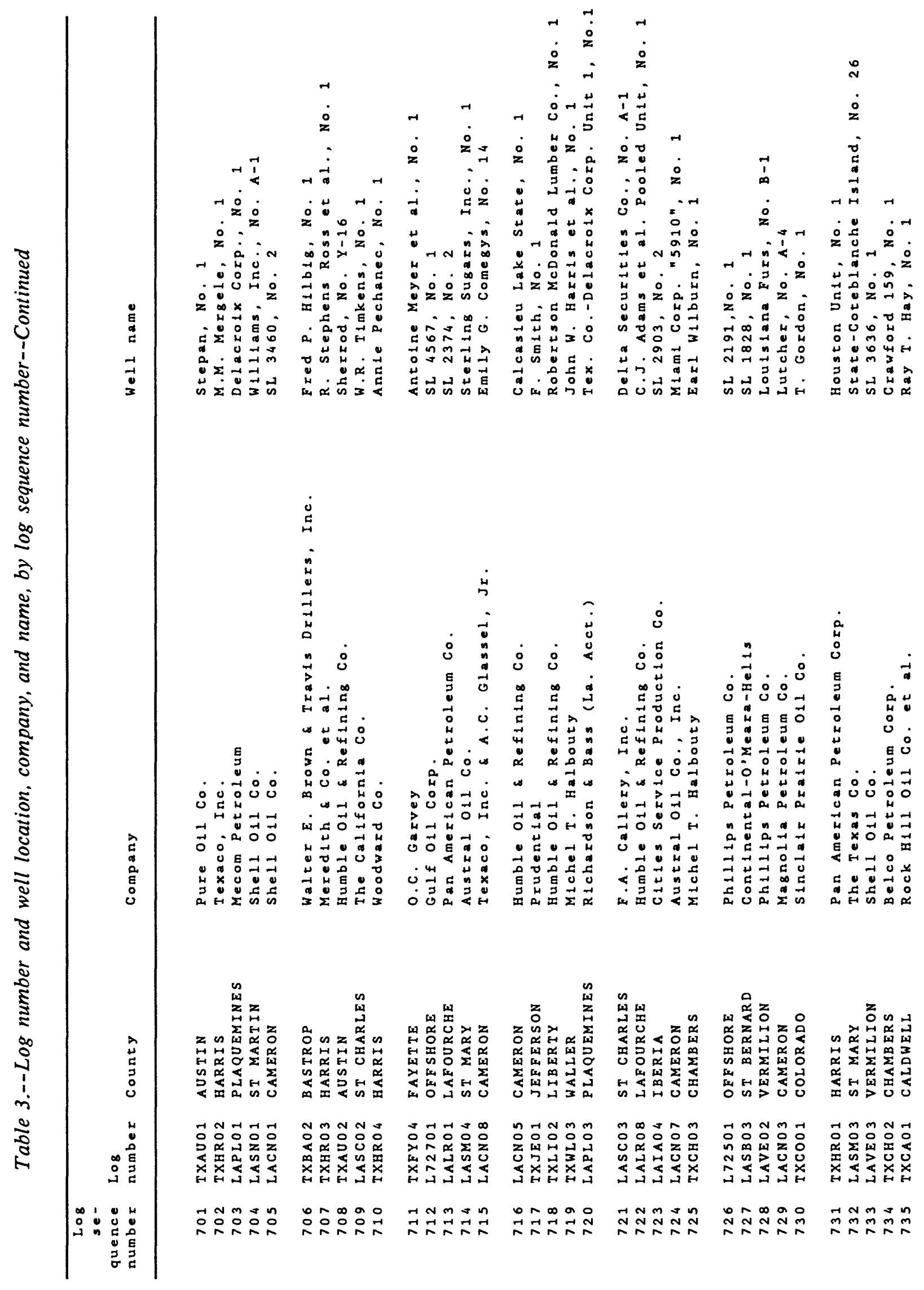




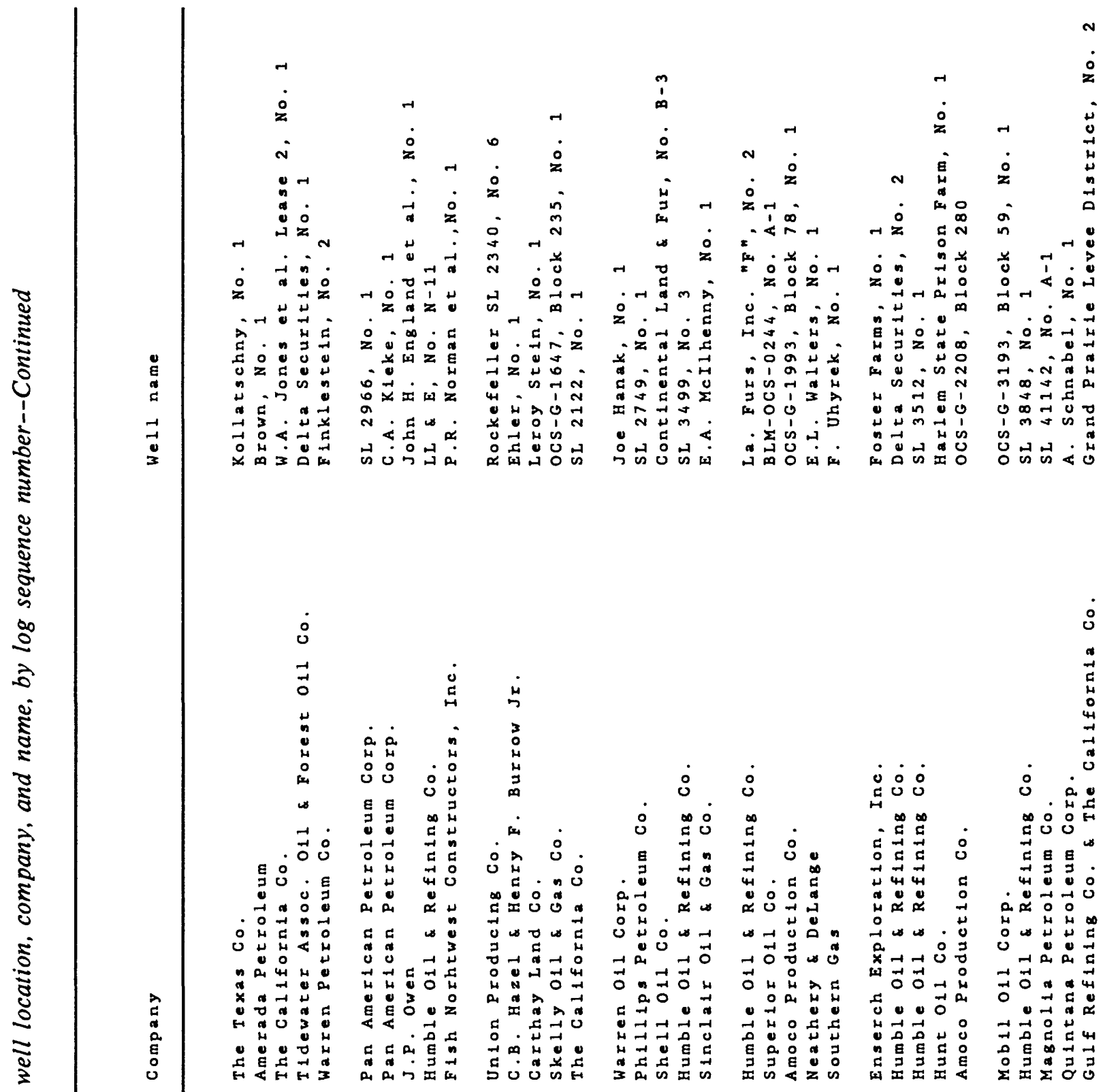




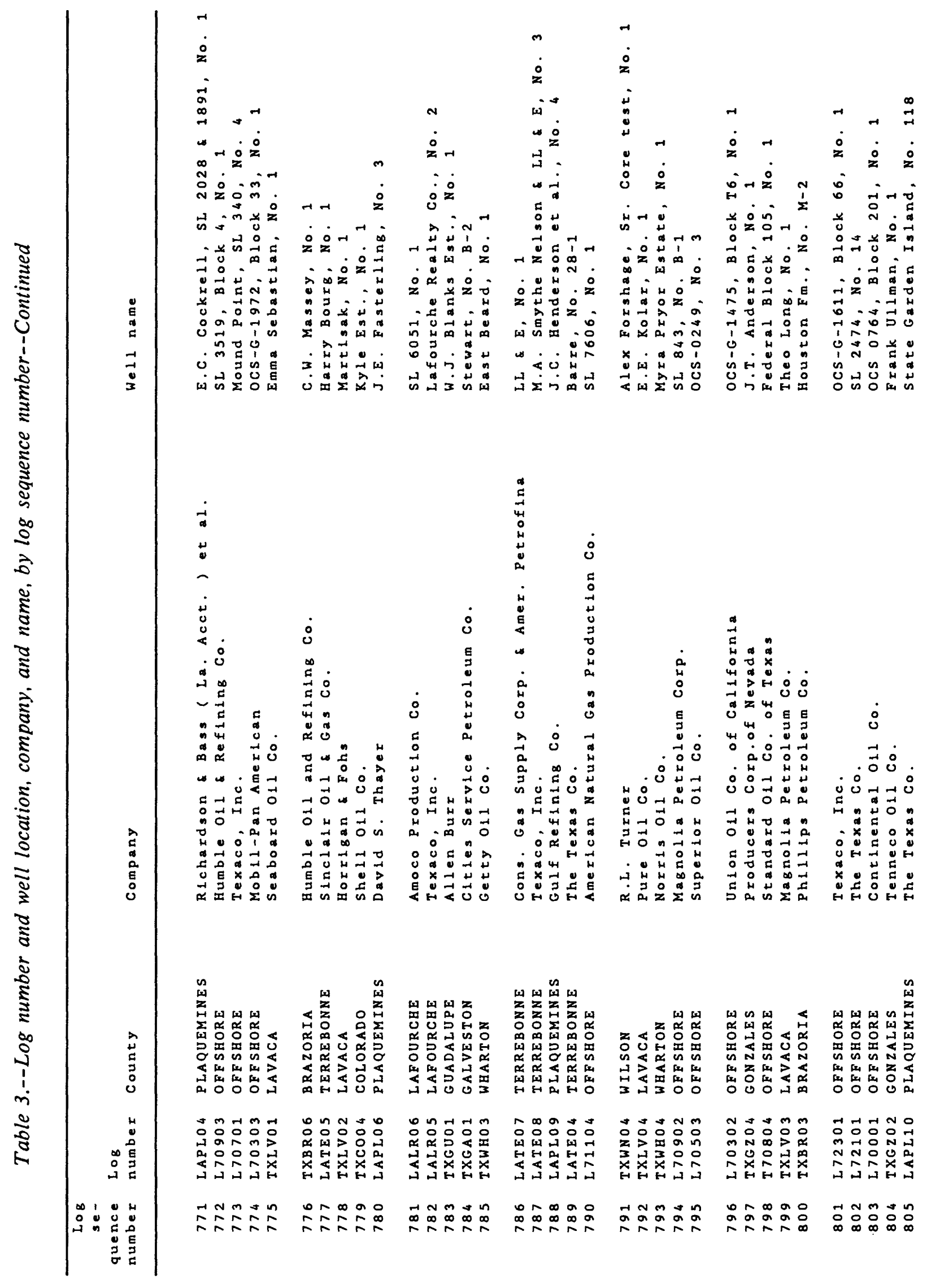




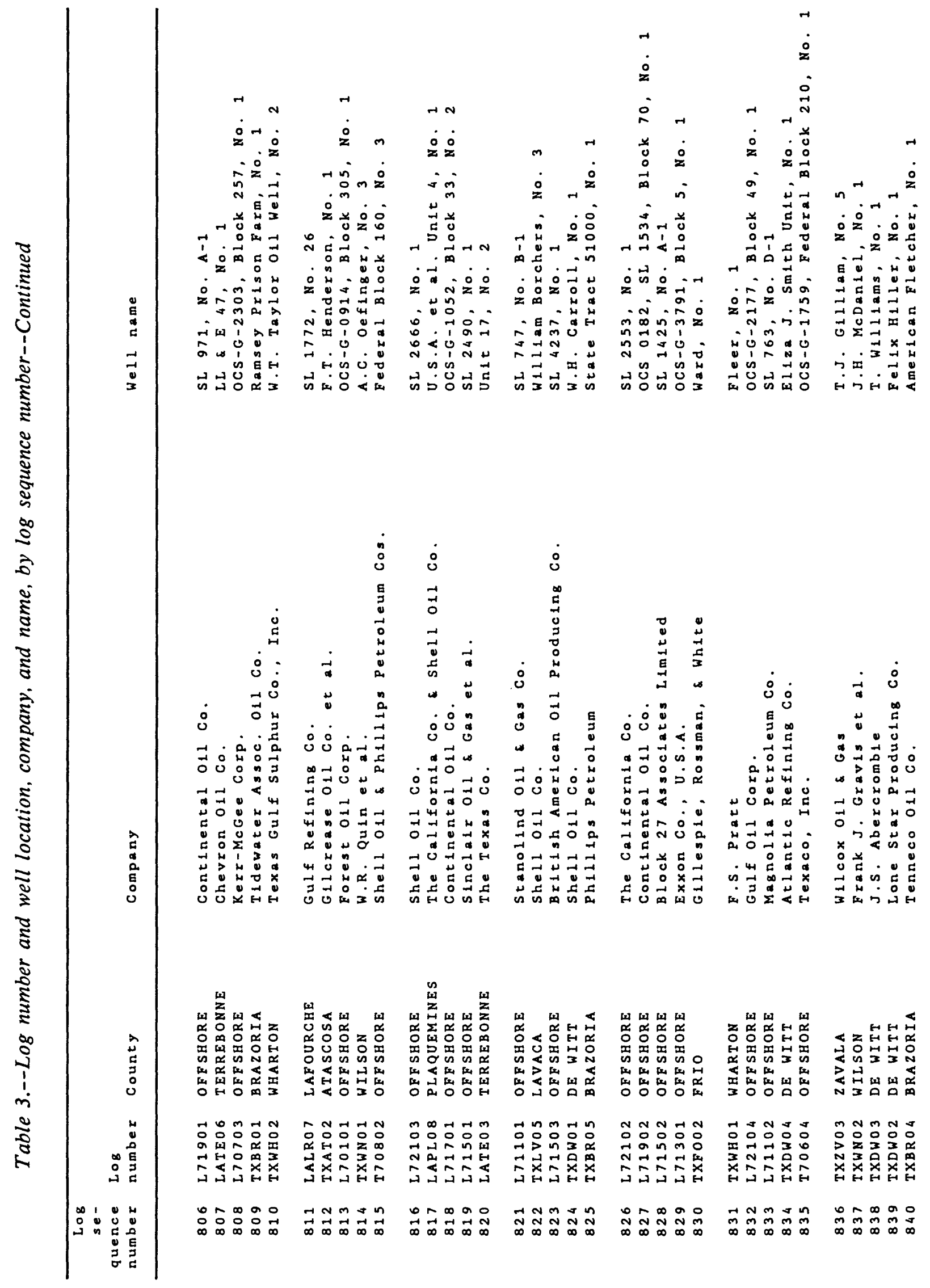




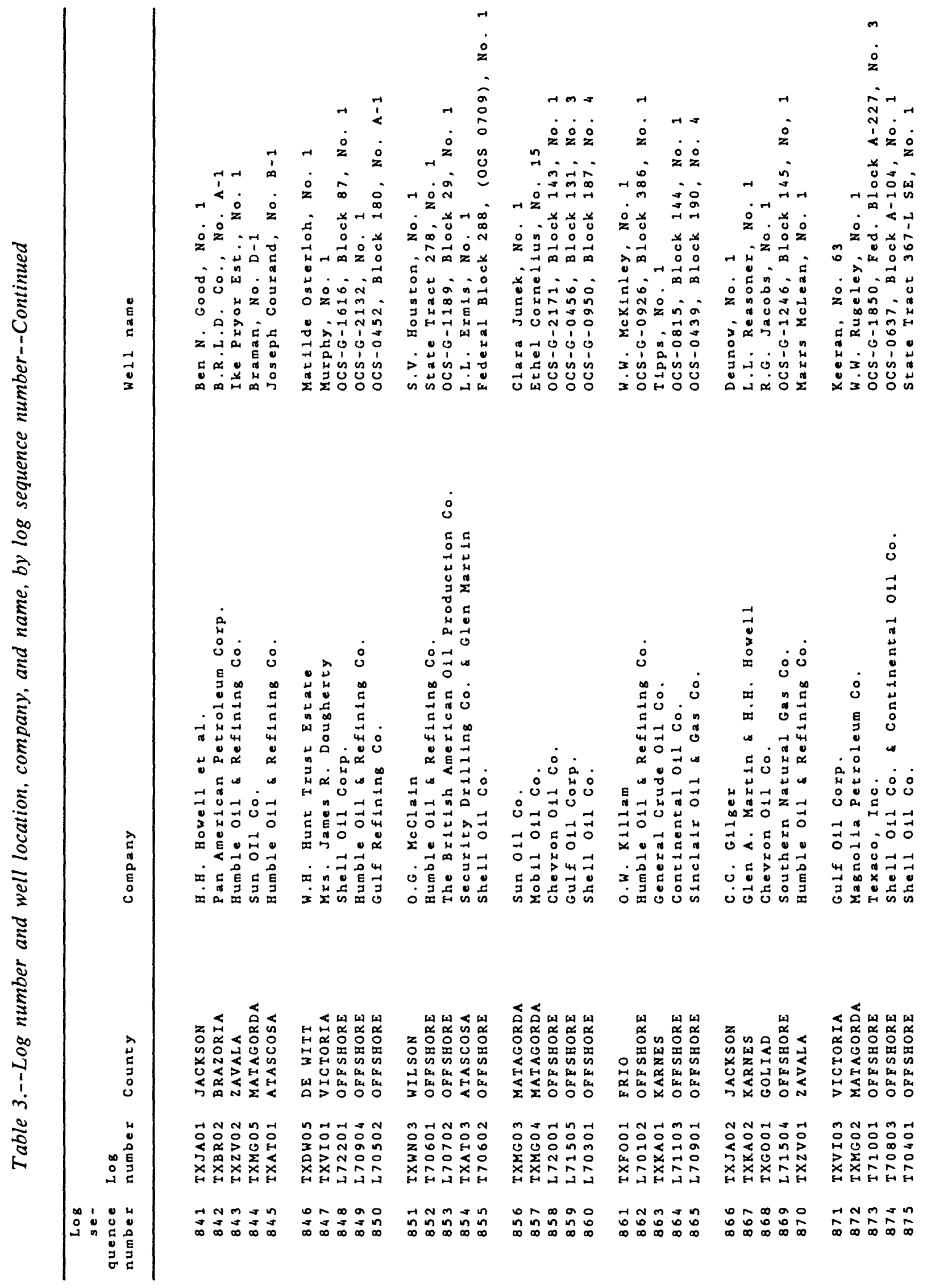




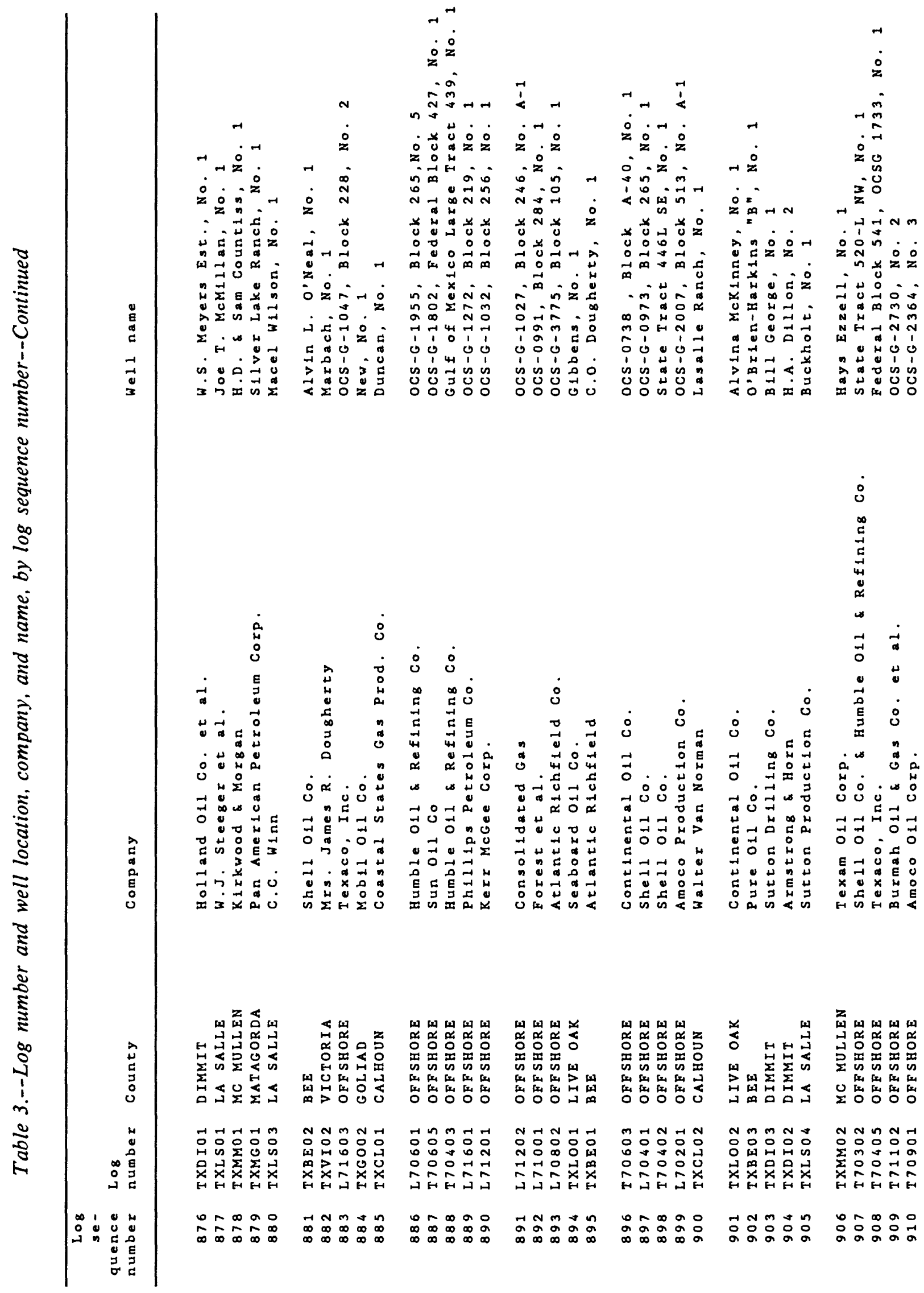




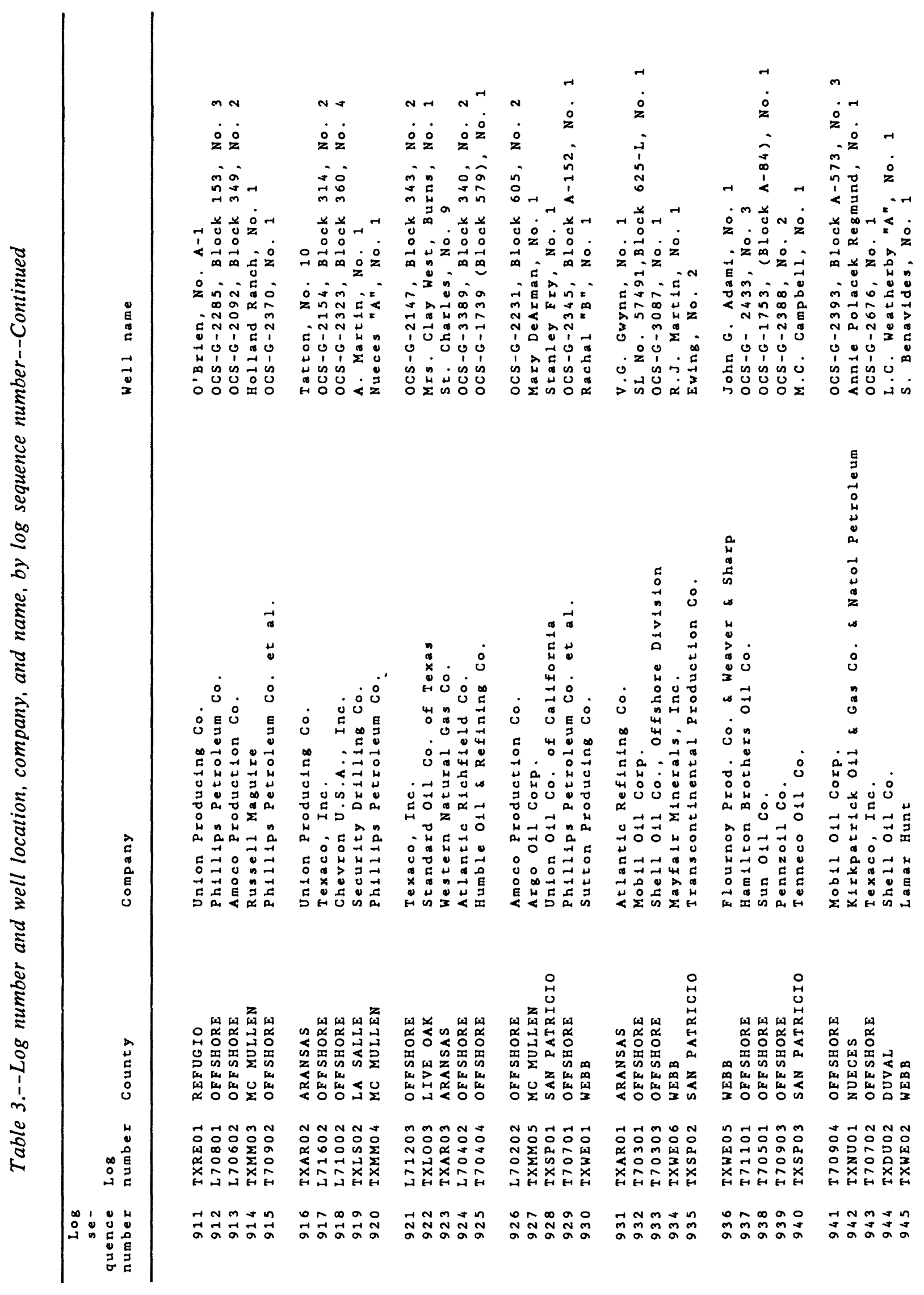




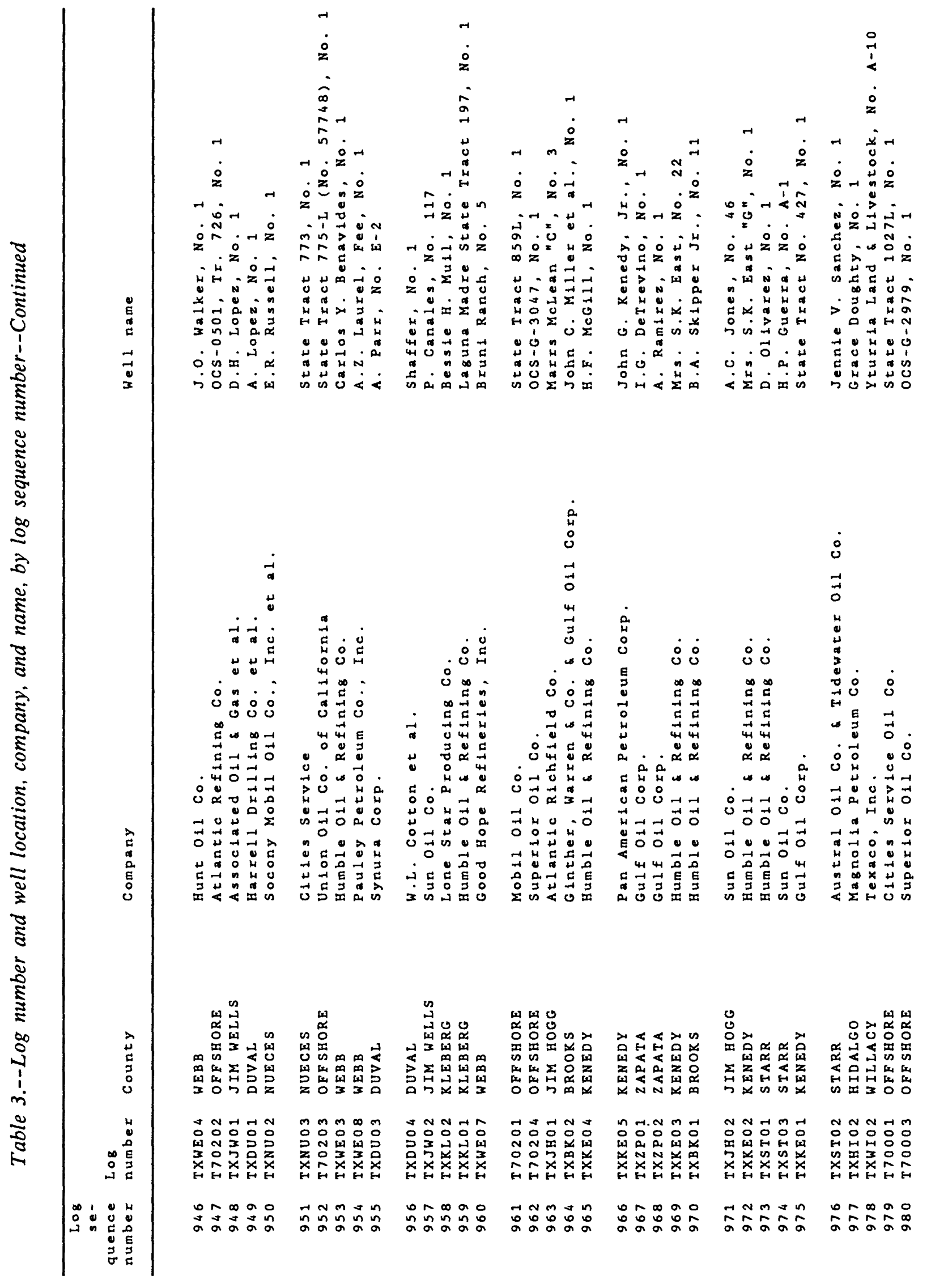




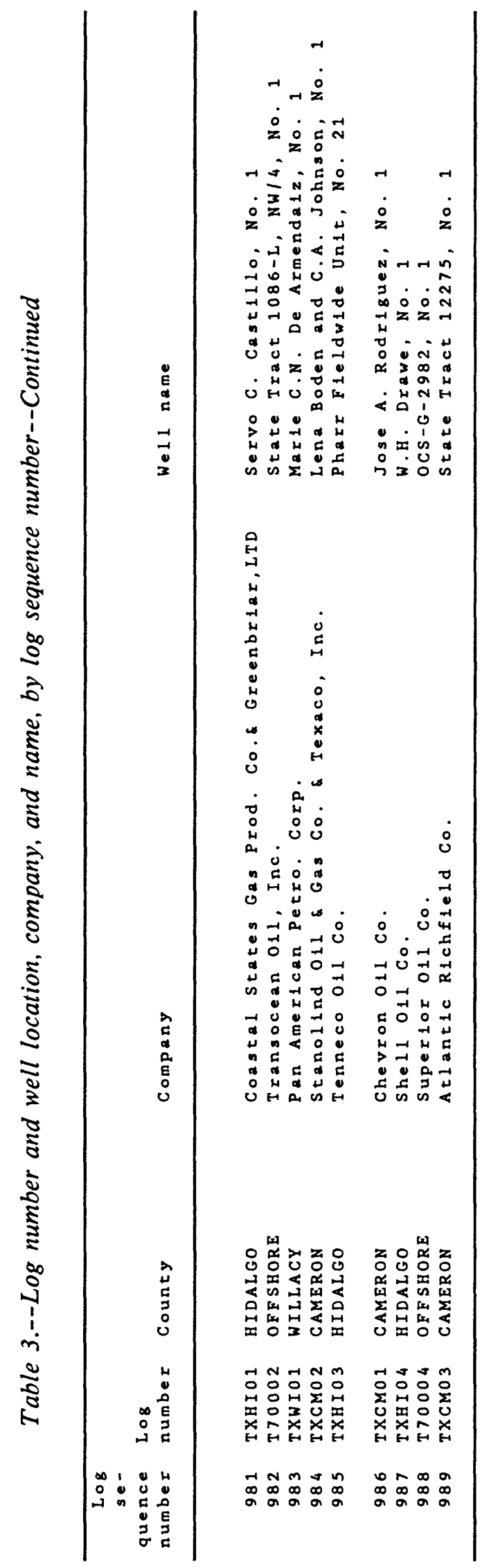


Explanation of data in table 4:

Log number--An alphanumeric system that uses the two-letter state abbreviation followed by a two-letter county (parish) abbreviiation followed by a two-digit sequential number. For example, the second well numbered in Baldwin County, Alabama, would be ALBL02. Offshore numbers are composed of the single letter initial of the State and a three-digit API (American Petroleum Institute) number followed by a two-digit sequential number.

Layer number--Sequential number of layer, beginning with lowermost aquifer and numbering aquifers upward (1-11), then lowermost confining unit and numbering confining units upward $(12-17)$.

Altitude of top--Altitude of top of a given layer in feet, relative to sea level.

Thickness--Total thickness of given layer in feet.

Sand percentage--Percentage of the total thickness of sand in a given layer.

Average dissolved solids--Average dissolved-solids concentration in water in a given layer as calculated from data obtained from geophysical well logs.

Average temperature--Average temperature of water in a given layer as determined by temperature-gradient and bottom-hole temperature data. 
Table 4--Layer number; altitude of top, thickness, and sand percentage of layer; and average dissolved-solids concentration and temperature of water in layer; by log number.

[Average dissolved-solids concentration in water in thousands of milligrams per liter; average water temperature in degrees Celsius]

\begin{tabular}{|c|c|c|c|c|c|c|}
\hline $\begin{array}{l}\mathrm{L} \circ \mathrm{g} \\
\text { number }\end{array}$ & $\begin{array}{r}\text { Layer } \\
\text { number }\end{array}$ & $\begin{array}{r}\text { Altitude } \\
\text { of top } \\
(f e e t)\end{array}$ & $\begin{array}{r}\text { Thick- } \\
\text { ness } \\
\text { (feet) }\end{array}$ & $\begin{array}{r}\text { Sand } \\
\text { per- } \\
\text { centage }\end{array}$ & $\begin{array}{l}\text { Dis- } \\
\text { solved } \\
\text { solids }\end{array}$ & $\begin{array}{l}\text { Tem- } \\
\text { per- } \\
\text { ature }\end{array}$ \\
\hline \multirow[t]{7}{*}{$A L B L 01$} & 2 & -2525 & 230 & 90 & $<10$ & 39 \\
\hline & 3 & -990 & 1535 & 10 & -- & 33 \\
\hline & 5 & -415 & 575 & 25 & $<10$ & 26 \\
\hline & 7 & 105 & 460 & -- & - & - \\
\hline & 8 & 277 & 172 & -- & -- & 20 \\
\hline & 12 & -2755 & 685 & 0 & -- & - \\
\hline & 15 & -355 & 60 & 0 & -- & - \\
\hline \multirow[t]{6}{*}{$A L B L O 2$} & 3 & -1573 & 1410 & 9 & 17 & 36 \\
\hline & 5 & -993 & 580 & 26 & $<10$ & 29 \\
\hline & 7 & -166 & 687 & 17 & $<10$ & 24 \\
\hline & 8 & 127 & 293 & 41 & $<10$ & 21 \\
\hline & 12 & -2983 & 1225 & 0 & -- & - \\
\hline & 15 & -853 & 140 & 0 & -- & -- \\
\hline \multirow[t]{7}{*}{$A L B \perp 03$} & 2 & -3832 & 216 & 42 & 30 & 48 \\
\hline & 3 & -2281 & 1551 & 10 & 32 & 42 \\
\hline & 5 & -1598 & 683 & 26 & 17 & 34 \\
\hline & 7 & -408 & 970 & 30 & -- & 27 \\
\hline & 8 & 12 & 420 & 27 & -- & 22 \\
\hline & 12 & -4048 & 835 & 0 & -- & -- \\
\hline & 15 & -1378 & 220 & 0 & -- & -- \\
\hline \multirow[t]{6}{*}{$A L B L O 4$} & 3 & -2242 & 1667 & 9 & 62 & 39 \\
\hline & 5 & -1505 & 737 & -- & -- & -- \\
\hline & 7 & -244 & 1055 & 50 & $<10$ & 26 \\
\hline & 8 & 175 & 419 & 40 & $<10$ & 22 \\
\hline & 12 & -3909 & 820 & 0 & -- & -- \\
\hline & 15 & -1299 & 206 & 0 & -- & -- \\
\hline \multirow[t]{7}{*}{$A L B L O 5$} & 3 & -2623 & 1680 & 14 & 47 & 41 \\
\hline & 5 & -2263 & 360 & -- & -- & -- \\
\hline & 7 & -683 & 1400 & 26 & -- & 29 \\
\hline & 8 & -93 & 590 & 83 & -- & 24 \\
\hline & 9 & 77 & 170 & -- & -- & 21 \\
\hline & 12 & -4303 & 870 & 0 & -- & - \\
\hline & 15 & -2083 & 180 & 0 & -- & -- \\
\hline \multirow[t]{7}{*}{$A L B L O 6$} & 3 & -2796 & 520 & 36 & -- & 43 \\
\hline & 5 & -2461 & 335 & - & -- & -- \\
\hline & 7 & -781 & 1495 & 5 & $<10$ & 32 \\
\hline & 8 & -136 & 645 & 62 & $<10$ & 24 \\
\hline & 9 & 54 & 190 & -- & - & 21 \\
\hline & 12 & -3316 & 1755 & 1 & -- & $\cdots$ \\
\hline & 15 & -2276 & 185 & 0 & -- & -- \\
\hline \multirow[t]{3}{*}{ ALCHOL } & 2 & -206 & 260 & 36 & $<10$ & 20 \\
\hline & 3 & 262 & 468 & -- & - & 17 \\
\hline & 12 & -466 & 430 & 0 & -- & -- \\
\hline
\end{tabular}


Table 4--Layer number; altitude of top, thickness, and sand percentage of layer; and average dissolved-solids concentration and temperature of water in layer; by log number--Continued.

\begin{tabular}{|c|c|c|c|c|c|c|}
\hline $\begin{array}{l}\text { Log } \\
\text { number }\end{array}$ & $\begin{array}{r}\text { Layer } \\
\text { number }\end{array}$ & $\begin{array}{r}\text { Altitude } \\
\text { of top } \\
(f e e t)\end{array}$ & $\begin{array}{r}\text { Thick- } \\
\text { ness } \\
\text { (feet) }\end{array}$ & $\begin{array}{r}\text { Sand } \\
\text { per- } \\
\text { centage }\end{array}$ & $\begin{array}{l}\text { Dis- } \\
\text { solved } \\
\text { solids }\end{array}$ & $\begin{array}{l}\text { Tem- } \\
\text { per- } \\
\text { ature }\end{array}$ \\
\hline \multirow[t]{3}{*}{$A L C H O 2$} & 2 & -420 & 235 & 51 & $<10$ & 22 \\
\hline & 3 & 193 & 613 & -- & $<10$ & 19 \\
\hline & 12 & -655 & 705 & 0 & - & $\cdots$ \\
\hline \multirow[t]{8}{*}{$A L C$ HO 3} & 2 & -1609 & 335 & 67 & $<10$ & 32 \\
\hline & 3 & -339 & 1270 & 22 & $<10$ & 25 \\
\hline & 4 & -69 & 270 & 54 & $<10$ & 19 \\
\hline & 5 & 131 & 175 & -- & $<10$ & 16 \\
\hline & 6 & 220 & 39 & -- & -- & 15 \\
\hline & 12 & -1944 & 780 & 8 & -- & -- \\
\hline & 13 & -44 & 25 & 0 & -- & - \\
\hline & 14 & 181 & 50 & -- & $\cdots$ & - \\
\hline \multirow[t]{7}{*}{$A L C L O 1$} & 2 & -1672 & 310 & 34 & $<10$ & 33 \\
\hline & 3 & -322 & 1350 & 20 & $<10$ & 27 \\
\hline & 4 & -122 & 200 & -- & -- & 21 \\
\hline & 5 & 78 & 175 & -- & -- & 20 \\
\hline & 12 & -1982 & 690 & 0 & $\ldots$ & $\ldots$ \\
\hline & 13 & -97 & 25 & -- & -- & -- \\
\hline & 14 & 117 & 39 & -- & -- & -- \\
\hline \multirow[t]{9}{*}{$\mathrm{ALCLO} 2$} & 2 & -961 & 270 & 56 & $<10$ & 28 \\
\hline & 3 & -316 & 645 & 34 & $<10$ & 24 \\
\hline & 4 & -116 & 200 & -- & -- & 21 \\
\hline & 5 & 44 & 130 & -- & -- & 20 \\
\hline & 6 & 224 & 150 & -- & -- & 18 \\
\hline & 12 & -1231 & 610 & 0 & -- & -- \\
\hline & 13 & -86 & 30 & 0 & -- & -- \\
\hline & 14 & 74 & 30 & -- & -- & - \\
\hline & 15 & 264 & 40 & -- & -- & - \\
\hline \multirow[t]{3}{*}{$A L C L O 3$} & 2 & -385 & 235 & 28 & $<10$ & 23 \\
\hline & 3 & 189 & 574 & 21 & $<10$ & 20 \\
\hline & 12 & -620 & 660 & 0 & -- & - \\
\hline \multirow[t]{9}{*}{$A L C L O 4$} & 2 & -1250 & 360 & 69 & $<10$ & 32 \\
\hline & 3 & -580 & 670 & 15 & $<10$ & 27 \\
\hline & 4 & -345 & 235 & 89 & $<10$ & 23 \\
\hline & 5 & -130 & 175 & 40 & $<10$ & 21 \\
\hline & 6 & 160 & 250 & -- & -- & 19 \\
\hline & 12 & -1610 & 610 & 0 & -- & -- \\
\hline & 13 & -305 & 40 & 0 & -- & -- \\
\hline & 14 & -90 & 40 & -- & -- & -- \\
\hline & 15 & 199 & 39 & -- & -- & -- \\
\hline \multirow[t]{8}{*}{$A L C L O 5$} & 2 & -2031 & 270 & 32 & 13 & 34 \\
\hline & 3 & -961 & 1070 & 13 & - & 30 \\
\hline & 4 & -896 & 65 & 62 & $<10$ & 26 \\
\hline & 5 & -291 & 400 & 80 & - & 23 \\
\hline & 7 & 28 & 264 & -- & -- & -- \\
\hline & 12 & -2301 & 640 & 0 & -- & -- \\
\hline & 13 & -691 & 205 & 7 & -- & -- \\
\hline & 15 & -236 & 55 & 0 & -- & - \\
\hline
\end{tabular}


Table 4--Layer number; altitude of top, thickness, and sand percentage of layer: and average dissolved-solids concentration and temperature of water in layer; by log number--Continued.

\begin{tabular}{|c|c|c|c|c|c|c|}
\hline $\begin{array}{l}\text { Log } \\
\text { number }\end{array}$ & $\begin{array}{r}\text { Layer } \\
\text { number }\end{array}$ & $\begin{array}{r}\text { Alt t tude } \\
\text { of top } \\
(f \in e t)\end{array}$ & $\begin{array}{r}\text { Thlck- } \\
\text { ness } \\
(\text { feet })\end{array}$ & $\begin{array}{r}\text { Sand } \\
\text { per- } \\
\text { centage }\end{array}$ & $\begin{array}{l}\text { Dis- } \\
\text { solved } \\
\text { solids }\end{array}$ & $\begin{array}{l}\text { Tem- } \\
\text { per- } \\
\text { 2ture }\end{array}$ \\
\hline \multirow[t]{6}{*}{ ALCLO 6} & 2 & -2168 & 200 & 70 & 30 & 34 \\
\hline & 3 & -813 & 1355 & 13 & 49 & 29 \\
\hline & 5 & -373 & 440 & 64 & $<10$ & 24 \\
\hline & 7 & 13 & 306 & 47 & - - & 21 \\
\hline & 12 & -2368 & 725 & 0 & -- & -- \\
\hline & 15 & -293 & 80 & 0 & -- & -- \\
\hline \multirow[t]{7}{*}{ ALESO 1} & 2 & -2713 & 235 & 40 & $<10$ & 39 \\
\hline & 3 & -1113 & 1600 & 7 & -- & 33 \\
\hline & 5 & -568 & 545 & 8 & $<10$ & 25 \\
\hline & 7 & 2 & 470 & 34 & -- & 20 \\
\hline & 8 & 251 & 249 & 66 & -- & 18 \\
\hline & 12 & -2948 & 745 & 0 & -- & -- \\
\hline & 15 & -468 & 100 & 0 & -- & - \\
\hline$A L M A O 1$ & 12 & 180 & 392 & -- & -- & -- \\
\hline \multirow[t]{3}{*}{$A L M A O 2$} & 2 & 187 & 210 & 84 & $<10$ & - \\
\hline & 3 & 279 & 92 & -- & -- & -- \\
\hline & 12 & -23 & 345 & 0 & -- & -- \\
\hline \multirow[t]{3}{*}{$A L M A O 3$} & 2 & -88 & 260 & 67 & $<10$ & 19 \\
\hline & 3 & 170 & 258 & -- & -- & 17 \\
\hline & 12 & -348 & 690 & 0 & -- & -- \\
\hline \multirow[t]{3}{*}{$A L M A O 4$} & 2 & 171 & 250 & 42 & $<10$ & -- \\
\hline & 3 & 249 & 78 & -- & -- & -- \\
\hline & 12 & -79 & 580 & 0 & -- & -- \\
\hline \multirow[t]{7}{*}{$A L M B O 1$} & 2 & -3316 & 295 & 53 & 39 & 43 \\
\hline & 3 & -1376 & 1940 & 17 & -- & 36 \\
\hline & 5 & -976 & 400 & -- & -- & -- \\
\hline & 7 & -126 & 750 & -- & -- & 24 \\
\hline & 8 & 263 & 389 & -- & -- & 20 \\
\hline & 12 & -3611 & 805 & 0 & -- & -- \\
\hline & 15 & -876 & 100 & -- & -- & - \\
\hline \multirow[t]{7}{*}{$A L M B O 2$} & 2 & -4503 & 255 & 67 & -- & 52 \\
\hline & 3 & -1853 & 2650 & 31 & 37 & 42 \\
\hline & 5 & -1293 & 560 & -- & -- & -- \\
\hline & 7 & -333 & 685 & 37 & $<10$ & 25 \\
\hline & 8 & 37 & 370 & 95 & $<10$ & 21 \\
\hline & 12 & -4758 & 870 & 0 & -- & -- \\
\hline & 15 & -1018 & 275 & 18 & -- & -- \\
\hline \multirow[t]{10}{*}{$A L M B O 3$} & 2 & -4001 & 295 & 39 & 28 & 50 \\
\hline & 3 & -2764 & 1237 & 9 & 19 & 44 \\
\hline & 4 & -2536 & 228 & 60 & 51 & 39 \\
\hline & 5 & -1856 & 533 & 18 & 25 & 35 \\
\hline & 7 & -376 & 1270 & 62 & $<10$ & 28 \\
\hline & 8 & 154 & 530 & 52 & -- & 21 \\
\hline & 9 & 233 & 79 & -- & -- & 19 \\
\hline & 12 & -4296 & 950 & 0 & -- & -- \\
\hline & 13 & -2389 & 147 & 0 & -- & -- \\
\hline & 15 & -1646 & 210 & 0 & -- & -- \\
\hline
\end{tabular}


Table 4--Layer number; altitude of top, thickness, and sand percentage of layer: and average dissolved-solids concentration and temperature of water in layer; by log number--Continued.

\begin{tabular}{|c|c|c|c|c|c|c|}
\hline $\begin{array}{l}\text { Log } \\
\text { number }\end{array}$ & $\begin{array}{r}\text { Layer } \\
\text { number }\end{array}$ & $\begin{array}{r}\text { Altitude } \\
\text { of top } \\
(f e e t)\end{array}$ & $\begin{array}{c}\text { Thick- } \\
\text { ness } \\
(\text { feet) }\end{array}$ & $\begin{array}{r}\text { Sand } \\
\text { per- } \\
\text { centage }\end{array}$ & $\begin{array}{l}\text { Dis- } \\
\text { solved } \\
\text { solids }\end{array}$ & $\begin{array}{l}\text { Tem- } \\
\text { per- } \\
\text { ature }\end{array}$ \\
\hline \multirow[t]{9}{*}{ ALMBO 4} & 2 & -4535 & 240 & 58 & -- & -- \\
\hline & 3 & -3245 & 1290 & 4 & -- & $\cdots$ \\
\hline & 5 & -2690 & 180 & - & -- & -- \\
\hline & 7 & -1015 & 1490 & 24 & -- & - \\
\hline & 8 & -160 & 855 & 33 & -- & -- \\
\hline & 9 & 90 & 250 & -- & -- & - \\
\hline & 12 & -4775 & 930 & 0 & -- & -- \\
\hline & 13 & -2870 & 375 & 0 & -- & -- \\
\hline & 15 & -2505 & 185 & 0 & -- & - \\
\hline \multirow[t]{7}{*}{ ALMBO5 } & 3 & -3170 & 442 & 21 & -- & 44 \\
\hline & 5 & -2895 & 275 & -- & -- & - \\
\hline & 7 & -1170 & 1555 & 14 & 55 & 34 \\
\hline & 8 & -220 & 950 & 20 & -- & 25 \\
\hline & 9 & 0 & 220 & -- & -- & 21 \\
\hline & 12 & -3612 & 1998 & 2 & -- & $\cdots$ \\
\hline & 15 & -2725 & 170 & 0 & -- & $-\cdots$ \\
\hline \multirow[t]{8}{*}{ ALMNO 1} & 2 & -1239 & 205 & 99 & $<10$ & 30 \\
\hline & 3 & -634 & 605 & 10 & -- & 27 \\
\hline & 4 & -559 & 75 & 43 & $<10$ & 23 \\
\hline & 5 & 26 & 460 & -- & - - & 20 \\
\hline & 7 & 364 & 288 & -- & -- & 16 \\
\hline & 12 & -1444 & 950 & 19 & -- & -- \\
\hline & 13 & -434 & 125 & 0 & -- & -- \\
\hline & 15 & 76 & 50 & - & -- & -- \\
\hline ALSUOI & 12 & 164 & 309 & -- & -- & -- \\
\hline ALSUO2 & 12 & 133 & 232 & -- & -- & -- \\
\hline \multirow[t]{10}{*}{$A L W A O 1$} & 2 & -2230 & 270 & 54 & $<10$ & 34 \\
\hline & 3 & -840 & 1390 & 13 & $<10$ & 29 \\
\hline & 4 & -645 & 195 & 46 & $<10$ & 24 \\
\hline & 5 & -420 & 190 & 55 & $<10$ & 22 \\
\hline & 6 & -70 & 270 & 69 & $<10$ & 20 \\
\hline & 7 & 266 & 261 & 66 & -- & 18 \\
\hline & 12 & -2500 & 850 & 0 & -- & -- \\
\hline & 13 & -610 & 35 & 0 & -- & -- \\
\hline & 14 & -340 & 80 & 0 & -- & -- \\
\hline & 15 & 5 & 75 & 0 & -- & -- \\
\hline \multirow[t]{10}{*}{$A L W A O 2$} & 2 & -2730 & 240 & 54 & 56 & 37 \\
\hline & 3 & -1275 & 1455 & 20 & $<10$ & 31 \\
\hline & 4 & -1085 & 190 & 32 & $<10$ & 26 \\
\hline & 5 & -800 & 235 & 57 & $<10$ & 25 \\
\hline & 6 & -545 & 200 & 74 & $<10$ & 23 \\
\hline & 7 & 144 & 289 & -- & -- & 19 \\
\hline & 12 & -2970 & 825 & 0 & -- & -- \\
\hline & 13 & -1035 & 50 & 0 & -- & $\cdots$ \\
\hline & 14 & -745 & 55 & 0 & -- & $\cdots$ \\
\hline & 15 & -145 & 400 & -- & -- & -- \\
\hline
\end{tabular}


Table 4--Layer number; altitude of top, thickness, and sand percentage of layer; and average dissolved-solids concentration and temperature of water in layer; by log number--Continued.

\begin{tabular}{|c|c|c|c|c|c|c|}
\hline $\begin{array}{l}\text { Log } \\
\text { number }\end{array}$ & $\begin{array}{r}\text { Layer } \\
\text { number }\end{array}$ & $\begin{array}{r}\text { Altitude } \\
\text { of top } \\
\text { (feet) }\end{array}$ & $\begin{array}{r}\text { Thlck- } \\
\text { ness } \\
\text { (feet) }\end{array}$ & $\begin{array}{r}\text { Sand } \\
\text { per- } \\
\text { centage }\end{array}$ & $\begin{array}{l}\text { Dis- } \\
\text { solved } \\
\text { solids }\end{array}$ & $\begin{array}{l}\text { Tem- } \\
\text { per- } \\
\text { ature }\end{array}$ \\
\hline \multirow[t]{10}{*}{$A L W A 03$} & 2 & -2522 & 295 & 27 & 70 & 42 \\
\hline & 3 & -1047 & 1475 & 19 & 29 & 34 \\
\hline & 4 & -847 & 200 & 57 & 11 & 27 \\
\hline & 5 & -647 & 150 & 37 & $<10$ & 25 \\
\hline & 6 & -367 & 210 & 52 & $<10$ & 22 \\
\hline & 7 & 272 & 549 & 46 & -- & 18 \\
\hline & 12 & -2817 & 700 & 0 & -- & -- \\
\hline & 13 & -797 & 50 & 0 & -- & -- \\
\hline & 14 & -577 & 70 & 0 & -- & -- \\
\hline & 15 & -277 & 90 & 0 & -- & -- \\
\hline \multirow[t]{10}{*}{$\mathrm{ALWAO} 4$} & 2 & -2707 & 245 & 43 & 12 & 44 \\
\hline & 3 & -957 & 1750 & 12 & -- & 35 \\
\hline & 4 & -792 & 165 & 67 & $<10$ & 26 \\
\hline & 5 & -452 & 260 & 44 & $<10$ & 23 \\
\hline & 6 & -242 & 160 & 75 & $<10$ & 21 \\
\hline & 7 & 120 & 302 & 30 & $<10$ & 18 \\
\hline & 12 & -2952 & 820 & 0 & - & $\cdots$ \\
\hline & 13 & -712 & 80 & 0 & -- & -- \\
\hline & 14 & -402 & 50 & 0 & -- & -- \\
\hline & 15 & -182 & 60 & 0 & -- & -- \\
\hline \multirow[t]{7}{*}{$A L W A O 5$} & 2 & -3106 & 475 & 38 & 45 & 40 \\
\hline & 3 & -1191 & 1915 & 15 & 40 & 33 \\
\hline & 5 & -731 & 460 & -- & -- & 25 \\
\hline & 7 & -61 & 600 & -- & -- & 21 \\
\hline & 8 & 228 & 289 & -- & -- & 18 \\
\hline & 22 & -3581 & 915 & 0 & -- & -- \\
\hline & 15 & -661 & 70 & -- & -- & -- \\
\hline \multirow[t]{7}{*}{ ALWA 06} & 2 & -3279 & 240 & 92 & -- & 41 \\
\hline & 3 & -1539 & 1740 & 10 & -- & 35 \\
\hline & 5 & -819 & 720 & -- & -- & -- \\
\hline & 7 & -49 & 700 & 34 & $<10$ & 22 \\
\hline & 8 & 270 & 319 & 35 & -- & 18 \\
\hline & 12 & -3519 & 850 & 0 & -- & -- \\
\hline & 15 & -749 & 70 & 0 & -- & -- \\
\hline \multirow[t]{10}{*}{ ARARO 2} & 2 & -2632 & 230 & 99 & $<10$ & 38 \\
\hline & 3 & -1942 & 690 & 76 & $<10$ & 34 \\
\hline & 4 & -1694 & 248 & 99 & $<10$ & 31 \\
\hline & 5 & -347 & 815 & 96 & $<10$ & 23 \\
\hline & 6 & -72 & 235 & -- & -- & 19 \\
\hline & 11 & 168 & 158 & -- & -- & 17 \\
\hline & 12 & -2862 & 1130 & 0 & -- & -- \\
\hline & 13 & -1162 & 532 & 8 & -- & -- \\
\hline & 14 & -307 & 40 & 0 & -- & -- \\
\hline & 15 & 10 & 82 & -- & -- & -- \\
\hline
\end{tabular}


Table 4--Layer number; altitude of top, thickness, and sand percentage of layer; and average dissolved-solids concentration and temperature of water in layer; by log number--Continued.

\begin{tabular}{|c|c|c|c|c|c|c|}
\hline $\begin{array}{l}\text { Log } \\
\text { number }\end{array}$ & $\begin{array}{r}\text { Layer } \\
\text { number }\end{array}$ & $\begin{array}{r}\text { Altitude } \\
\text { of top } \\
\text { (feet) }\end{array}$ & $\begin{array}{l}\text { Thick- } \\
\text { ness } \\
\text { (feet) }\end{array}$ & $\begin{array}{r}\text { Sand } \\
\text { per- } \\
\text { centage }\end{array}$ & $\begin{array}{l}\text { Dis- } \\
\text { solved } \\
\text { solids }\end{array}$ & $\begin{array}{l}\text { Tem- } \\
\text { per- } \\
\text { ature }\end{array}$ \\
\hline \multirow[t]{10}{*}{ ARARO 2} & 2 & -2100 & 160 & 34 & - - & 35 \\
\hline & 3 & -1560 & 540 & 48 & $<10$ & 32 \\
\hline & 4 & -1260 & 300 & 99 & $<10$ & 28 \\
\hline & 5 & -340 & 405 & 99 & $<10$ & 21 \\
\hline & 6 & -50 & 110 & 99 & $<10$ & 17 \\
\hline & 11 & 200 & 115 & -- & - - & 15 \\
\hline & 12 & -2260 & 830 & 0 & -- & $\ldots$ \\
\hline & 13 & -745 & 515 & 0 & -- & -- \\
\hline & 14 & -160 & 180 & 17 & -- & - \\
\hline & 15 & 85 & 135 & 0 & -- & -- \\
\hline \multirow[t]{10}{*}{ ARARO 3} & 2 & -2372 & 145 & 99 & -- & -- \\
\hline & 3 & -1702 & 670 & 40 & -- & -- \\
\hline & 4 & -1422 & 280 & 99 & -- & - \\
\hline & 5 & -372 & 415 & 82 & -- & -- \\
\hline & 6 & -102 & 235 & 43 & -- & -- \\
\hline & 11 & 183 & 135 & -- & -- & -- \\
\hline & 12 & -2517 & 883 & 0 & -- & -- \\
\hline & 13 & -787 & 635 & 20 & -- & -- \\
\hline & 14 & -337 & 35 & 0 & -- & - \\
\hline & 15 & 48 & 150 & -- & -- & -- \\
\hline \multirow[t]{10}{*}{ ARARO 4} & 2 & -2303 & 310 & 44 & -- & -- \\
\hline & 3 & -1843 & 460 & 41 & -- & - \\
\hline & 4 & -1523 & 320 & 83 & -- & -- \\
\hline & 5 & -423 & 420 & 90 & -- & - \\
\hline & 6 & -153 & 220 & 20 & -- & -- \\
\hline & 11 & 197 & 149 & -- & -- & - \\
\hline & 12 & -2613 & 915 & 0 & -- & -- \\
\hline & 13 & -843 & 680 & 12 & -- & -- \\
\hline & 14 & -373 & 50 & 0 & -- & -- \\
\hline & 15 & 48 & 201 & 0 & -- & -- \\
\hline \multirow[t]{9}{*}{ ARARO 5} & 2 & -1911 & 125 & 52 & 30 & 33 \\
\hline & 3 & -1521 & 390 & 23 & -- & 31 \\
\hline & 4 & -1211 & 310 & 99 & $<10$ & 28 \\
\hline & 5 & -346 & 652 & 81 & $<10$ & 22 \\
\hline & 6 & 105 & 321 & 97 & $<10$ & 17 \\
\hline & 11 & 209 & 104 & -- & -- & 15 \\
\hline & 12 & -2036 & 767 & 0 & -- & - \\
\hline & 13 & -998 & 213 & 19 & -- & -- \\
\hline & 14 & -216 & 130 & 0 & -- & -- \\
\hline \multirow[t]{9}{*}{ ARASO 1} & 2 & -2006 & 140 & 99 & $<10$ & 41 \\
\hline & 3 & -1476 & 530 & 48 & $<10$ & 38 \\
\hline & 4 & -1421 & 55 & 99 & $<10$ & 35 \\
\hline & 5 & -326 & 735 & 78 & $<10$ & 27 \\
\hline & 6 & -1 & 205 & 61 & $<10$ & 21 \\
\hline & 11 & 134 & 135 & -- & -- & 19 \\
\hline & 12 & -2146 & 540 & 0 & -- & - \\
\hline & 13 & -1061 & 360 & 0 & -- & - \\
\hline & 14 & -206 & 120 & 0 & -- & $\ldots$ \\
\hline
\end{tabular}


Table 4--Layer number; altitude of top, thickness, and sand percentage of layer; and average dissolved-solids concentration and temperature of water in layer; by log number--Continued.

\begin{tabular}{|c|c|c|c|c|c|c|}
\hline $\begin{array}{l}\text { Log } \\
\text { number }\end{array}$ & $\begin{array}{r}\text { Layer } \\
\text { number }\end{array}$ & $\begin{array}{r}\text { Altitude } \\
\text { of top } \\
\text { (feet) }\end{array}$ & $\begin{array}{r}\text { Thick- } \\
\text { ness } \\
\text { (feet) }\end{array}$ & $\begin{array}{r}\text { Sand } \\
\text { per- } \\
\text { centage }\end{array}$ & $\begin{array}{l}\text { Dis- } \\
\text { solved } \\
\text { solids }\end{array}$ & $\begin{array}{l}\text { Tem- } \\
\text { per- } \\
\text { ature }\end{array}$ \\
\hline \multirow[t]{9}{*}{ ARASO2 } & 2 & -2158 & 220 & 57 & 28 & 39 \\
\hline & 3 & -1828 & 330 & 47 & -- & 36 \\
\hline & 4 & -1688 & 140 & 50 & $<10$ & 35 \\
\hline & 5 & -423 & 690 & 83 & $<10$ & 26 \\
\hline & 6 & -113 & 245 & -- & -- & 22 \\
\hline & 12 & -2378 & 600 & 0 & -- & -- \\
\hline & 13 & -1113 & 575 & 7 & -- & - \\
\hline & 14 & -358 & 65 & 0 & - & $\cdots$ \\
\hline & 15 & 177 & 290 & -- & -- & - \\
\hline \multirow[t]{10}{*}{ ARASO 3} & 2 & -2135 & 100 & 99 & 11 & 46 \\
\hline & 3 & -1610 & 525 & 53 & -- & 42 \\
\hline & 4 & -1485 & 125 & 99 & $<10$ & 38 \\
\hline & 5 & -470 & 585 & 80 & $<10$ & 29 \\
\hline & 6 & -35 & 310 & 58 & $<10$ & 22 \\
\hline & 11 & 103 & 91 & -- & -- & 19 \\
\hline & 12 & -2235 & 455 & 0 & -- & -- \\
\hline & 13 & -1055 & 430 & 0 & -- & -- \\
\hline & 14 & -345 & 125 & 0 & -- & - \\
\hline & 15 & 12 & 47 & -- & -- & -- \\
\hline \multirow[t]{10}{*}{ ARASO 4} & 2 & -2178 & 135 & 99 & -- & -- \\
\hline & 3 & -1653 & 525 & 21 & -- & -- \\
\hline & 4 & -1533 & 120 & 99 & -- & -- \\
\hline & 5 & -478 & 670 & 68 & -- & -- \\
\hline & 6 & -198 & 95 & 99 & -- & -- \\
\hline & 11 & 125 & 73 & -- & -- & -- \\
\hline & 12 & -2313 & 525 & 0 & -- & -- \\
\hline & 13 & -1148 & 385 & 23 & -- & -- \\
\hline & 14 & -293 & 185 & 0 & -- & -- \\
\hline & 15 & 52 & 250 & 0 & -- & -- \\
\hline \multirow[t]{9}{*}{ ARASOS } & 2 & -1734 & 165 & 99 & $<10$ & 41 \\
\hline & 3 & -1439 & 295 & 15 & -- & 38 \\
\hline & 4 & -1384 & 55 & 99 & $<10$ & 36 \\
\hline & 5 & -379 & 670 & 79 & $<10$ & 28 \\
\hline & 6 & 16 & 240 & 99 & $<10$ & 21 \\
\hline & 11 & 116 & 100 & -- & -- & 19 \\
\hline & 12 & -1899 & 645 & 0 & -- & -- \\
\hline & 13 & -1049 & 335 & 0 & -- & -- \\
\hline & 14 & -224 & 155 & 0 & -- & - \\
\hline \multirow[t]{3}{*}{ ARASO 6} & 6 & -60 & -- & -- & -- & -- \\
\hline & 11 & 140 & 80 & 89 & -- & $\cdots$ \\
\hline & 15 & 60 & 120 & 29 & -- & - \\
\hline \multirow[t]{9}{*}{ ARBRO 1} & 2 & -1785 & 115 & 99 & -- & -- \\
\hline & 3 & -1510 & 275 & 27 & -- & -- \\
\hline & 4 & -1370 & 140 & 99 & -- & -- \\
\hline & 5 & -460 & 615 & 66 & -- & -- \\
\hline & 6 & -10 & 295 & 96 & -- & -- \\
\hline & 11 & 80 & 90 & -- & -- & -- \\
\hline & 12 & -1900 & 380 & 0 & -- & -- \\
\hline & 13 & -1075 & 295 & 0 & -- & -- \\
\hline & 14 & -305 & 155 & 23 & -- & $\cdots$ \\
\hline
\end{tabular}


Table 4--Layer number; altitude of top, thickness, and sand percentage of layer; and average dissolved-solids concentration and temperature of water in layer; by log number--Continued.

\begin{tabular}{|c|c|c|c|c|c|c|}
\hline $\begin{array}{l}\text { Log } \\
\text { number }\end{array}$ & $\begin{array}{r}\text { Layer } \\
\text { number }\end{array}$ & $\begin{array}{r}\text { Altitude } \\
\text { of top } \\
\text { (feet) }\end{array}$ & $\begin{array}{r}\text { Thlck- } \\
\text { ness } \\
(\text { feet) }\end{array}$ & $\begin{array}{r}\text { Sand } \\
\text { per- } \\
\text { centage }\end{array}$ & $\begin{array}{l}\text { Dis- } \\
\text { solved } \\
\text { solids }\end{array}$ & $\begin{array}{l}\text { Tem- } \\
\text { per- } \\
\text { ature }\end{array}$ \\
\hline \multirow[t]{9}{*}{ ARB RO 2} & 2 & -1788 & 100 & 99 & 31 & 35 \\
\hline & 3 & -1378 & 410 & 26 & -- & 32 \\
\hline & 4 & -1278 & 100 & 99 & -- & 30 \\
\hline & 5 & -353 & 590 & 61 & $<10$ & 24 \\
\hline & 6 & -33 & 175 & 99 & $<10$ & 19 \\
\hline & 12 & -1888 & 575 & 0 & -- & - \\
\hline & 13 & -943 & 335 & 0 & -- & -- \\
\hline & 14 & -208 & 145 & 10 & -- & -- \\
\hline & 15 & 145 & 178 & 0 & -- & -. \\
\hline \multirow[t]{9}{*}{ ARB RO 3} & 2 & -1930 & 105 & 71 & $<10$ & 35 \\
\hline & 3 & -1515 & 415 & 30 & $<10$ & 33 \\
\hline & 4 & -1415 & 100 & 99 & $<10$ & 30 \\
\hline & 5 & -115 & 840 & 78 & $<10$ & 23 \\
\hline & 6 & 195 & 205 & -- & -- & 17 \\
\hline & 12 & -2035 & 700 & 0 & -- & - \\
\hline & 13 & -955 & 460 & 51 & -- & -- \\
\hline & 14 & -10 & 105 & 0 & -- & -- \\
\hline & 15 & 238 & 43 & -- & -- & -- \\
\hline \multirow[t]{8}{*}{$\mathrm{ARCAO} 1$} & 2 & -1395 & 20 & 50 & -- & 31 \\
\hline & 3 & -1137 & 258 & 45 & $<10$ & 30 \\
\hline & 4 & -1077 & 60 & 99 & $<10$ & 29 \\
\hline & 5 & -177 & 380 & 87 & $<10$ & 22 \\
\hline & 6 & 116 & 183 & 83 & $<10$ & 18 \\
\hline & 12 & -1415 & 562 & 0 & -- & -- \\
\hline & 13 & -557 & 520 & 0 & -- & -- \\
\hline & 14 & -67 & 110 & 32 & -- & - \\
\hline \multirow[t]{7}{*}{$A R C A O 2$} & 2 & -1225 & 20 & 50 & -- & 31 \\
\hline & 3 & -890 & 335 & 37 & $<10$ & 29 \\
\hline & 4 & -805 & 85 & 99 & $<10$ & 27 \\
\hline & 5 & 245 & 675 & 58 & $<10$ & 20 \\
\hline & 12 & -1245 & 580 & 0 & -- & -- \\
\hline & 13 & -430 & 375 & 44 & -- & -- \\
\hline & 14 & 285 & 40 & -- & -- & -- \\
\hline \multirow[t]{10}{*}{ ARCHO1 } & 2 & -2059 & 360 & 47 & $<10$ & 36 \\
\hline & 3 & -1724 & 335 & 31 & $<10$ & 33 \\
\hline & 4 & -1559 & 165 & 91 & $<10$ & 31 \\
\hline & 5 & -494 & 590 & 69 & $<10$ & 25 \\
\hline & 6 & -164 & 160 & 99 & $<10$ & 20 \\
\hline & 11 & 120 & 154 & -- & -- & 18 \\
\hline & 12 & -2419 & 660 & 0 & -- & -- \\
\hline & 13 & -1084 & 475 & 17 & -- & -- \\
\hline & 14 & -324 & 170 & 0 & -- & -- \\
\hline & 15 & -34 & 130 & 0 & -- & -- \\
\hline
\end{tabular}


Table 4--Layer number; altitude of top, thickness, and sand percentage of layer; and average dissolved-solids concentration and temperature of water in layer; by log number--Continued.

\begin{tabular}{|c|c|c|c|c|c|c|}
\hline $\begin{array}{l}\text { Log } \\
\text { number }\end{array}$ & $\begin{array}{r}\text { Layer } \\
\text { number }\end{array}$ & $\begin{array}{r}\text { Altitude } \\
\text { of top } \\
\text { (feet) }\end{array}$ & $\begin{array}{r}\text { Thick- } \\
\text { ness } \\
\text { (feet) }\end{array}$ & $\begin{array}{r}\text { Sand } \\
\text { per- } \\
\text { centage }\end{array}$ & $\begin{array}{r}\text { Dis- } \\
\text { solved } \\
\text { solids }\end{array}$ & $\begin{array}{l}\text { Tem- } \\
\text { per- } \\
\text { ature }\end{array}$ \\
\hline \multirow[t]{10}{*}{ ARCHO2 } & 2 & -2302 & 300 & 75 & $<10$ & 42 \\
\hline & 3 & -1877 & 425 & 18 & $<10$ & 39 \\
\hline & 4 & -1707 & 170 & 99 & $<10$ & 36 \\
\hline & 5 & -602 & 585 & 55 & $<10$ & 26 \\
\hline & 6 & -220 & 222 & -- & - - & 21 \\
\hline & 11 & 123 & 95 & -- & -- & 16 \\
\hline & 12 & -2602 & 665 & 0 & -- & -- \\
\hline & 13 & -1187 & 520 & 36 & -- & -- \\
\hline & 14 & -442 & 160 & -- & -- & -- \\
\hline & 15 & 28 & 248 & -- & -- & -- \\
\hline \multirow[t]{7}{*}{$\mathrm{ARCHO3}$} & 4 & -1546 & -- & -- & -- & -- \\
\hline & 5 & -431 & 737 & 84 & -- & -- \\
\hline & 6 & -128 & 173 & 90 & -- & -- \\
\hline & 11 & 115 & 163 & -- & -- & -- \\
\hline & 13 & -1168 & 378 & 5 & -- & -- \\
\hline & 14 & -301 & 130 & 15 & -- & -- \\
\hline & 15 & -48 & 80 & 0 & -- & -- \\
\hline \multirow[t]{10}{*}{ ARCHO 4} & 2 & -2008 & 290 & 67 & 37 & 41 \\
\hline & 3 & -1668 & 340 & 34 & $<10$ & 38 \\
\hline & 4 & -1543 & 125 & 88 & $<10$ & 35 \\
\hline & 5 & -468 & 685 & 67 & -- & 26 \\
\hline & 6 & -148 & 140 & 63 & $<10$ & 19 \\
\hline & 11 & 105 & 93 & -- & -- & 16 \\
\hline & 12 & -2298 & 490 & 0 & -- & -- \\
\hline & 13 & -1153 & 390 & 6 & -- & -- \\
\hline & 14 & -288 & 180 & 11 & -- & -- \\
\hline & 15 & 12 & 160 & -- & -- & -- \\
\hline \multirow[t]{3}{*}{ ARCHO5 } & 6 & -232 & -- & -- & -- & -- \\
\hline & 11 & 134 & 74 & 78 & -- & -- \\
\hline & 15 & 60 & 292 & 20 & -- & -- \\
\hline$A R C K O 1$ & 12 & 229 & 21 & -- & -- & -- \\
\hline \multirow[t]{3}{*}{$\mathrm{ARCKO} 2$} & 2 & -14 & 20 & -- & -- & -- \\
\hline & 3 & 176 & 190 & -- & -- & -- \\
\hline & 12 & -34 & 245 & 0 & -- & -- \\
\hline \multirow[t]{3}{*}{$\mathrm{ARCKO} 3$} & 2 & 45 & 20 & 50 & -- & 18 \\
\hline & 3 & 250 & 205 & -- & -- & 17 \\
\hline & 12 & 25 & 300 & 0 & -- & -- \\
\hline \multirow[t]{6}{*}{ ARCLO1 } & 1 & -751 & 120 & 99 & -- & -- \\
\hline & 2 & -256 & 25 & 99 & -- & -- \\
\hline & 3 & -181 & 75 & 33 & -- & -- \\
\hline & 4 & 218 & 399 & - & -- & -- \\
\hline & 11 & 265 & 47 & -- & -- & -- \\
\hline & 12 & -281 & 470 & 0 & -- & -- \\
\hline
\end{tabular}


Table 4--Layer number; altitude of top, thickness, and sand percentage of layer; and average dissolved-solids concentration and temperature of water in layer; by log number--Continued.

\begin{tabular}{|c|c|c|c|c|c|c|}
\hline $\begin{array}{l}\text { Log } \\
\text { number }\end{array}$ & $\begin{array}{r}\text { Layer } \\
\text { number }\end{array}$ & $\begin{array}{r}\text { Altitude } \\
\text { of top } \\
(f e e t)\end{array}$ & $\begin{array}{r}\text { Thick- } \\
\text { ness } \\
(\text { feet })\end{array}$ & $\begin{array}{r}\text { Sand } \\
\text { per- } \\
\text { centage }\end{array}$ & $\begin{array}{l}\text { Dis- } \\
\text { solved } \\
\text { solids }\end{array}$ & $\begin{array}{l}\text { Tem- } \\
\text { per- } \\
\text { ature }\end{array}$ \\
\hline \multirow[t]{3}{*}{$A R C L O 2$} & 1 & 100 & 100 & 87 & -- & -- \\
\hline & 11 & 372 & 171 & 93 & -- & -- \\
\hline & 12 & 201 & 101 & 0 & -- & -- \\
\hline \multirow[t]{8}{*}{ ARCMOI } & 2 & -1168 & 20 & 50 & -- & 42 \\
\hline & 3 & -748 & 420 & 33 & -- & 41 \\
\hline & 4 & -693 & 55 & 99 & -- & 40 \\
\hline & 5 & 127 & 470 & 87 & $<10$ & 30 \\
\hline & 6 & 307 & 30 & -- & - - & -- \\
\hline & 12 & -1188 & 635 & 0 & -- & -- \\
\hline & 13 & -343 & 350 & 26 & -- & -- \\
\hline & 14 & 277 & 150 & 0 & -- & -- \\
\hline \multirow[t]{6}{*}{ ARCMO2 } & 2 & -631 & 60 & 92 & $<10$ & 25 \\
\hline & 3 & -436 & 195 & 46 & $<10$ & 24 \\
\hline & 4 & -391 & 45 & 99 & $<10$ & 23 \\
\hline & 5 & 269 & 430 & 66 & $<10$ & 19 \\
\hline & 12 & -691 & 585 & 0 & - - & -- \\
\hline & 13 & -161 & 230 & 17 & -- & -- \\
\hline \multirow[t]{7}{*}{ ARCMO3 } & 2 & -919 & 40 & 63 & -- & -- \\
\hline & 3 & -679 & 240 & 19 & -- & -- \\
\hline & 4 & -549 & 130 & 81 & -- & -- \\
\hline & 5 & 166 & 470 & 70 & -- & -- \\
\hline & 12 & -959 & 610 & 0 & -- & -- \\
\hline & 13 & -304 & 245 & 10 & -- & -- \\
\hline & 14 & 271 & 105 & 0 & -- & -- \\
\hline \multirow[t]{5}{*}{ ARCRO 1} & 1 & -920 & 105 & 99 & -- & -- \\
\hline & 2 & -135 & 180 & 78 & -- & -- \\
\hline & 3 & 10 & 145 & 34 & -- & -- \\
\hline & 4 & 350 & 340 & -- & -- & -- \\
\hline & 12 & -315 & 605 & 0 & -- & -- \\
\hline \multirow[t]{5}{*}{ ARCRO2 } & 1 & -920 & 80 & 99 & -- & -- \\
\hline & 2 & -255 & 75 & 99 & -- & -- \\
\hline & 3 & -60 & 195 & -- & -- & -- \\
\hline & 4 & 270 & 330 & -- & -- & -- \\
\hline & 12 & -330 & 590 & 0 & -- & -- \\
\hline \multirow[t]{7}{*}{$\operatorname{ARCSO} 01$} & 2 & -810 & 130 & 85 & $<10$ & 31 \\
\hline & 3 & -655 & 155 & 26 & -- & 28 \\
\hline & 4 & -180 & 475 & 99 & $<10$ & 23 \\
\hline & 5 & 15 & 195 & 99 & $<10$ & 17 \\
\hline & 11 & 210 & 160 & -- & -- & 14 \\
\hline & 12 & -940 & 720 & 0 & -- & -- \\
\hline & 14 & 50 & 35 & 0 & -- & -- \\
\hline
\end{tabular}


Table 4--Layer number; altitude of top, thickness, and sand percentage of layer; and average dissolved-solids concentration and temperature of water in layer: by log number--Continued.

\begin{tabular}{|c|c|c|c|c|c|c|}
\hline $\begin{array}{l}\text { Log } \\
\text { number }\end{array}$ & $\begin{array}{r}\text { Layer } \\
\text { number }\end{array}$ & $\begin{array}{r}\text { Altitude } \\
\text { of top } \\
(\text { feet) }\end{array}$ & $\begin{array}{r}\text { Th1ck- } \\
\text { ness } \\
(\text { feet) }\end{array}$ & $\begin{array}{r}\text { Sand } \\
\text { per- } \\
\text { centage }\end{array}$ & $\begin{array}{l}\text { Dis- } \\
\text { solved } \\
\text { solids }\end{array}$ & $\begin{array}{r}\text { Tem- } \\
\text { per- } \\
\text { ature }\end{array}$ \\
\hline \multirow[t]{8}{*}{$\mathrm{ARCSO} 2$} & 2 & -945 & 140 & 99 & $<10$ & 27 \\
\hline & 3 & -735 & 210 & 26 & $<10$ & 25 \\
\hline & 4 & -355 & 380 & 99 & $<10$ & 22 \\
\hline & 5 & -70 & 285 & 65 & $<10$ & 19 \\
\hline & 6 & 75 & 55 & 99 & $<10$ & 16 \\
\hline & 11 & 219 & 144 & -- & -- & 15 \\
\hline & 12 & -1085 & 820 & 0 & -- & -- \\
\hline & 14 & 20 & 90 & 0 & -- & -- \\
\hline \multirow[t]{9}{*}{$\operatorname{ARCSO} 3$} & 1 & -2005 & 40 & 99 & 17 & 37 \\
\hline & 2 & -985 & 200 & 99 & $<10$ & 27 \\
\hline & 3 & -810 & 175 & 31 & -- & 26 \\
\hline & 4 & -270 & 540 & 99 & -- & 22 \\
\hline & 5 & -40 & 230 & 61 & -- & 18 \\
\hline & 6 & 95 & 95 & 99 & $<10$ & 16 \\
\hline & 11 & 205 & 110 & -- & - & 15 \\
\hline & 12 & -1185 & 820 & 0 & -- & -- \\
\hline & 14 & 0 & 40 & 0 & -- & -- \\
\hline \multirow[t]{9}{*}{ ARCTO1 } & 1 & -2188 & 50 & 99 & $<10$ & 33 \\
\hline & 2 & -1038 & 315 & 99 & $<10$ & 26 \\
\hline & 3 & -778 & 260 & 52 & $<10$ & 24 \\
\hline & 4 & -373 & 405 & 60 & $<10$ & 22 \\
\hline & 5 & -43 & 330 & 91 & $<10$ & 19 \\
\hline & 6 & 112 & 100 & -- & -- & 17 \\
\hline & 11 & 217 & 105 & -- & -- & 16 \\
\hline & 12 & -1353 & 835 & 0 & -- & -- \\
\hline & 14 & 12 & 55 & 0 & -- & -- \\
\hline \multirow[t]{9}{*}{ ARCTO2 } & 1 & -2290 & 40 & 99 & $<10$ & 37 \\
\hline & 2 & -1220 & 165 & 99 & $<10$ & 28 \\
\hline & 3 & -900 & 320 & 45 & $<10$ & 26 \\
\hline & 4 & -430 & 470 & 99 & $<10$ & 23 \\
\hline & 5 & -180 & 250 & 78 & $<10$ & 20 \\
\hline & 6 & 110 & 235 & -- & -- & 18 \\
\hline & 11 & 210 & 100 & -- & -- & 16 \\
\hline & 12 & -1385 & 905 & 7 &,-- & - \\
\hline & 14 & -125 & 55 & -- & -- & -- \\
\hline \multirow[t]{8}{*}{ ARCVO 1} & 2 & -1491 & 125 & 52 & -- & -- \\
\hline & 3 & -1146 & 345 & 59 & -- & -- \\
\hline & 4 & -1021 & 125 & 99 & -- & -- \\
\hline & 5 & 4 & 825 & 80 & -- & -- \\
\hline & 6 & 174 & 30 & -- & -- & -- \\
\hline & 12 & -1616 & -- & -- & -- & -- \\
\hline & 13 & -821 & 200 & 0 & -- & -- \\
\hline & 14 & 144 & 140 & 0 & -- & -- \\
\hline
\end{tabular}


Table 4--Layer number; altitude of top, thickness, and sand percentage of layer; and average dissolved-solids concentration and temperature of water in layer; by log number--Continued.

\begin{tabular}{|c|c|c|c|c|c|c|}
\hline $\begin{array}{l}\text { Log } \\
\text { number }\end{array}$ & $\begin{array}{r}\text { Layer } \\
\text { number }\end{array}$ & $\begin{array}{r}\text { Alt l tude } \\
\text { of top } \\
(f e e t)\end{array}$ & $\begin{array}{r}\text { Thtck- } \\
\text { ness } \\
(\text { feet })\end{array}$ & $\begin{array}{r}\text { Sand } \\
\text { per- } \\
\text { centage }\end{array}$ & $\begin{array}{l}\text { DIs- } \\
\text { solved } \\
\text { solids }\end{array}$ & $\begin{array}{l}\text { Tem- } \\
\text { per- } \\
\text { ature }\end{array}$ \\
\hline \multirow[t]{9}{*}{$\operatorname{ARCVO} 2$} & 2 & -2155 & 165 & 58 & 22 & 39 \\
\hline & 3 & -1700 & 455 & 37 & $<10$ & 36 \\
\hline & 4 & -1545 & 155 & 99 & $<10$ & 32 \\
\hline & 5 & -285 & 740 & 82 & $<10$ & 23 \\
\hline & 6 & 100 & 220 & 80 & $<10$ & 16 \\
\hline & 12 & -2320 & 850 & 0 & - & - \\
\hline & 13 & -1025 & 520 & 24 & $\ldots$ & -- \\
\hline & 14 & -120 & 165 & 0 & -- & - \\
\hline & 15 & 240 & 140 & -- & -- & -- \\
\hline \multirow[t]{9}{*}{$\mathrm{ARCVO} 3$} & 2 & -1810 & 35 & 99 & $<10$ & 30 \\
\hline & 3 & -1405 & 405 & 41 & $<10$ & 29 \\
\hline & 4 & -1300 & 105 & 99 & $<10$ & 27 \\
\hline & 5 & -130 & 590 & 75 & $<10$ & 21 \\
\hline & 6 & 180 & 165 & 19 & $<10$ & 17 \\
\hline & 12 & -1845 & 865 & 0 & -- & -- \\
\hline & 13 & -720 & 580 & 34 & -- & - \\
\hline & 14 & 15 & 145 & 0 & -- & - \\
\hline & 15 & 210 & 30 & -- & -- & -- \\
\hline \multirow[t]{5}{*}{ ARDAO 1} & 2 & -430 & 20 & 50 & -- & 24 \\
\hline & 3 & -155 & 275 & 71 & $<10$ & 22 \\
\hline & 4 & -40 & 115 & 65 & $<10$ & 20 \\
\hline & 5 & 148 & 188 & 99 & $<10$ & 18 \\
\hline & 12 & -450 & 445 & 0 & -- & -- \\
\hline \multirow[t]{7}{*}{$\mathrm{ARDAO} 2$} & 2 & -961 & 125 & 60 & -- & - \\
\hline & 3 & -706 & 255 & 43 & -- & $=-$ \\
\hline & 4 & -631 & 75 & 99 & -- & - \\
\hline & 5 & 109 & 495 & 87 & -- & -- \\
\hline & 12 & -1086 & - - & -- & -- & -- \\
\hline & 13 & -386 & 245 & 41 & -- & - \\
\hline & 14 & 329 & 220 & 0 & -- & - \\
\hline \multirow[t]{10}{*}{ ARDEO 1} & 2 & -2747 & 195 & 56 & 28 & 46 \\
\hline & 3 & -2157 & 590 & 28 & $<10$ & 42 \\
\hline & 4 & -1970 & 187 & 99 & $<10$ & 38 \\
\hline & 5 & -442 & 855 & 67 & $<10$ & 25 \\
\hline & 6 & -157 & 220 & 68 & $<10$ & 19 \\
\hline & 11 & 158 & 140 & -- & -- & 15 \\
\hline & 12 & -2942 & 1070 & 0 & -- & -- \\
\hline & 13 & -1297 & 673 & 25 & -- & -- \\
\hline & 14 & -377 & 65 & 0 & -- & - \\
\hline & 15 & 18 & 175 & 0 & -- & -- \\
\hline \multirow[t]{9}{*}{ ARDE 02} & 2 & -2391 & 215 & 51 & -- & - \\
\hline & 3 & -1911 & 480 & 17 & -- & $\cdots$ \\
\hline & 4 & -1726 & 185 & 84 & -- & -- \\
\hline & 5 & -181 & 890 & 46 & -- & -- \\
\hline & 6 & 19 & 150 & -- & -- & -- \\
\hline & 11 & 139 & 120 & -- & -- & -- \\
\hline & 12 & -2606 & 1130 & 0 & -- & -- \\
\hline & 13 & -1071 & 655 & 50 & -- & -- \\
\hline & 14 & -131 & 50 & -- & -- & - \\
\hline
\end{tabular}


Table 4--Layer number; altitude of top, thickness, and sand percentage of layer; and average dissolved-solids concentration and temperature of water in layer; by log number--Continued.

\begin{tabular}{|c|c|c|c|c|c|c|}
\hline $\begin{array}{l}\text { Log } \\
\text { number }\end{array}$ & $\begin{array}{r}\text { Layer } \\
\text { number }\end{array}$ & $\begin{array}{r}\text { Altitude } \\
\text { of top } \\
\text { (feet) }\end{array}$ & $\begin{array}{r}\text { Thick- } \\
\text { ness } \\
\text { (feet) }\end{array}$ & $\begin{array}{r}\text { Sand } \\
\text { per- } \\
\text { centage }\end{array}$ & $\begin{array}{l}\text { Dis- } \\
\text { solved } \\
\text { solids }\end{array}$ & $\begin{array}{l}\text { Tem- } \\
\text { per- } \\
\text { ature }\end{array}$ \\
\hline \multirow[t]{9}{*}{ ARDRO I } & 2 & -2402 & 150 & 99 & 32 & 42 \\
\hline & 3 & -1762 & 640 & 42 & $\ldots$ & 37 \\
\hline & 4 & -1682 & 80 & 99 & $<20$ & 33 \\
\hline & 5 & -482 & 665 & 56 & $<10$ & 22 \\
\hline & 6 & -92 & 310 & 52 & $<10$ & 25 \\
\hline & 12 & -2552 & 510 & 0 & - & - \\
\hline & 13 & -1147 & 535 & 0 & -- & - \\
\hline & 14 & -402 & 80 & 0 & -- & - \\
\hline & 15 & 198 & 290 & -- & -- & -- \\
\hline \multirow[t]{10}{*}{ ARDRO 2} & 2 & -2460 & 300 & 83 & 21 & 40 \\
\hline & 3 & -2000 & 460 & 47 & $<10$ & 36 \\
\hline & 4 & -1835 & 165 & 33 & $<10$ & 33 \\
\hline & 5 & -330 & 880 & 60 & $<10$ & 22 \\
\hline & 6 & -60 & 215 & 47 & $<10$ & 16 \\
\hline & 11 & 181 & 141 & -- & -- & -- \\
\hline & 12 & -2760 & 750 & 0 & -- & - \\
\hline & 13 & -1210 & 625 & 44 & - - & - - \\
\hline & 24 & -275 & 55 & 0 & -- & -- \\
\hline & 15 & 40 & 100 & 0 & -- & $\cdots$ \\
\hline \multirow[t]{10}{*}{ ARDRO 3} & 2 & -2419 & 140 & 99 & $<10$ & 41 \\
\hline & 3 & -1914 & 505 & 34 & -- & 37 \\
\hline & 4 & -1734 & 180 & 89 & $<10$ & 33 \\
\hline & 5 & -234 & 955 & 84 & $<10$ & 21 \\
\hline & 6 & 36 & 165 & 70 & $<10$ & -- \\
\hline & 11 & 266 & 25 & - & - & -- \\
\hline & 12 & -2559 & 760 & 0 & -- & -- \\
\hline & 13 & -1189 & 545 & 12 & -- & $\ldots$ \\
\hline & 14 & -129 & 105 & 32 & -- & - \\
\hline & 15 & 241 & 205 & 0 & -- & -- \\
\hline \multirow[t]{5}{*}{ ARGNO1 } & 1 & -582 & 75 & 84 & -- & $\cdots$ \\
\hline & 2 & -64 & 78 & 94 & -- & - \\
\hline & 3 & 148 & 212 & 54 & -- & - \\
\hline & 4 & 292 & 144 & 55 & -- & - \\
\hline & 12 & -142 & 440 & 0 & -- & -- \\
\hline \multirow[t]{6}{*}{ ARGRO 1} & 2 & -1010 & 20 & 50 & -- & 35 \\
\hline & 3 & -590 & 420 & 55 & $<10$ & 31 \\
\hline & 4 & -100 & 490 & 59 & $<10$ & 24 \\
\hline & 5 & 130 & 230 & 91 & $<10$ & 19 \\
\hline & 12 & -1030 & 428 & 0 & -- & - \\
\hline & 14 & 234 & 104 & $\cdots$ & -- & -- \\
\hline \multirow[t]{8}{*}{$A R G R O 2$} & 2 & -1630 & 50 & 99 & $<10$ & 29 \\
\hline & 3 & -1295 & 335 & 36 & $<10$ & 28 \\
\hline & 4 & -1035 & 260 & 94 & - & 26 \\
\hline & 5 & 20 & 885 & 67 & $<10$ & 21 \\
\hline & 6 & 260 & 130 & $\ldots$ & - & 17 \\
\hline & 22 & -1680 & 640 & 0 & -- & - \\
\hline & 13 & -865 & 170 & 26 & -- & -- \\
\hline & 14 & 130 & 110 & 0 & -- & -- \\
\hline
\end{tabular}


Table 4--Layer number; altitude of top, thickness, and sand percentage of layer; and average dissolved-solids concentration and temperature of water in layer; by log number--Continued.

\begin{tabular}{|c|c|c|c|c|c|c|}
\hline $\begin{array}{l}\text { Log } \\
\text { number }\end{array}$ & $\begin{array}{r}\text { Layer } \\
\text { number }\end{array}$ & $\begin{array}{r}\text { Altitude } \\
\text { of top } \\
\text { (feet) }\end{array}$ & $\begin{array}{r}\text { Thlck- } \\
\text { ness } \\
\text { (feet) }\end{array}$ & $\begin{array}{r}\text { Sand } \\
\text { per- } \\
\text { centage }\end{array}$ & $\begin{array}{l}\text { Dis- } \\
\text { solved } \\
\text { solids }\end{array}$ & $\begin{array}{l}\text { Tem- } \\
\text { per- } \\
\text { ature }\end{array}$ \\
\hline \multirow[t]{6}{*}{ ARGRO 3} & 2 & -890 & 35 & 99 & $<10$ & 31 \\
\hline & 3 & -560 & 330 & 41 & - & 28 \\
\hline & 4 & -65 & 495 & 44 & $<10$ & 23 \\
\hline & 5 & 200 & 265 & 45 & $<10$ & 18 \\
\hline & 12 & -925 & -- & -- & -- & -- \\
\hline & 14 & 325 & 125 & -- & -- & -- \\
\hline \multirow[t]{2}{*}{ AREEO1 } & 2 & 321 & 10 & -- & -- & 16 \\
\hline & 12 & 311 & 580 & 0 & -- & -- \\
\hline \multirow[t]{4}{*}{ ARJAO 1} & 1 & -706 & 145 & 99 & -- & -- \\
\hline & 2 & 114 & 210 & 99 & -- & -- \\
\hline & 11 & 229 & 115 & -- & -- & -- \\
\hline & 12 & -96 & 610 & 0 & -- & -- \\
\hline \multirow[t]{3}{*}{$A R J A O 2$} & 1 & -267 & 130 & 92 & $<10$ & 31 \\
\hline & 11 & 237 & 115 & -- & -- & 14 \\
\hline & 12 & 122 & 389 & -- & -- & -- \\
\hline \multirow[t]{10}{*}{ ARJEO 1} & 2 & -2623 & 165 & 99 & 11 & 41 \\
\hline & 3 & -2013 & 610 & 40 & $<10$ & 38 \\
\hline & 4 & -1698 & 315 & 99 & $<10$ & 33 \\
\hline & 5 & -563 & 835 & 86 & $<10$ & 25 \\
\hline & 6 & -173 & 290 & 41 & -- & 18 \\
\hline & 11 & 192 & 100 & - & -- & 14 \\
\hline & 12 & -2788 & 700 & 0 & -- & -- \\
\hline & 13 & -1398 & 300 & 30 & -- & -- \\
\hline & 14 & -463 & 100 & 0 & -- & -- \\
\hline & 15 & 92 & 265 & 13 & -- & -- \\
\hline \multirow[t]{9}{*}{ ARJEO2 } & 2 & -1680 & 320 & 91 & 12 & 35 \\
\hline & 3 & -1420 & 260 & 81 & $<10$ & 31 \\
\hline & 4 & -1275 & 145 & 55 & $<10$ & 29 \\
\hline & 5 & -355 & 705 & 68 & $<10$ & 22 \\
\hline & 6 & 80 & 240 & 50 & $<10$ & 14 \\
\hline & 11 & 190 & 110 & -- & -- & -- \\
\hline & 12 & -2000 & 950 & 0 & -- & -- \\
\hline & 13 & -1060 & 215 & 23 & -- & -- \\
\hline & 14 & -160 & 195 & 9 & -- & -- \\
\hline \multirow[t]{9}{*}{ ARJEO3 } & 2 & -1745 & 155 & 99 & $<10$ & 31 \\
\hline & 3 & -1395 & 350 & 24 & -- & 29 \\
\hline & 4 & -960 & 435 & 91 & $<10$ & 26 \\
\hline & 5 & -205 & 705 & 79 & $<10$ & 21 \\
\hline & 6 & 115 & 230 & 55 & $<10$ & 17 \\
\hline & 11 & 206 & 91 & -- & -- & 16 \\
\hline & 12 & -1900 & 725 & 0 & -- & - \\
\hline & 13 & -910 & 50 & 0 & -- & -- \\
\hline & 14 & -115 & 90 & 0 & -- & -- \\
\hline
\end{tabular}


Table 4--Layer number; altitude of top, thickness, and sand percentage of layer; and average dissolved-solids concentration and temperature of water in layer; by log number--Continued.

\begin{tabular}{|c|c|c|c|c|c|c|}
\hline $\begin{array}{l}\text { L } \circ g \\
\text { number }\end{array}$ & $\begin{array}{r}\text { Layer } \\
\text { number }\end{array}$ & $\begin{array}{r}\text { Altitude } \\
\text { of top } \\
\text { (feet) }\end{array}$ & $\begin{array}{r}\text { Th1ck- } \\
\text { ness } \\
\text { (feet) }\end{array}$ & $\begin{array}{r}\text { Sand } \\
\text { per- } \\
\text { centage }\end{array}$ & $\begin{array}{l}\text { D1s- } \\
\text { solved } \\
\text { solids }\end{array}$ & $\begin{array}{l}\text { Tem- } \\
\text { per- } \\
\text { ature }\end{array}$ \\
\hline \multirow[t]{10}{*}{ ARJEO 4} & 2 & -2855 & 145 & 99 & 16 & 45 \\
\hline & 3 & -2045 & 810 & 33 & - & 40 \\
\hline & 4 & -1735 & 310 & 77 & $<10$ & 34 \\
\hline & 5 & -610 & 715 & 97 & $<10$ & 25 \\
\hline & 6 & -290 & 255 & 59 & $<10$ & 19 \\
\hline & 11 & 185 & 95 & -- & -- & -- \\
\hline & 12 & -3000 & 840 & 0 & -- & -- \\
\hline & 13 & -1325 & 410 & 44 & -- & - \\
\hline & 14 & -545 & 65 & 0 & - & -- \\
\hline & 15 & 90 & 380 & 0 & -- & -- \\
\hline \multirow[t]{5}{*}{ ARJEO5 } & 5 & -585 & - & - & - & -- \\
\hline & 6 & -75 & 176 & 56 & -- & - \\
\hline & 11 & 200 & 128 & 78 & $\cdots$ & -- \\
\hline & 14 & -251 & 334 & 23 & -- & -- \\
\hline & 15 & 72 & 147 & 17 & -- & -- \\
\hline \multirow[t]{6}{*}{$A R L A 01$} & 2 & -678 & 105 & 67 & $<10$ & 27 \\
\hline & 3 & -458 & 220 & 52 & $<10$ & 26 \\
\hline & 4 & -413 & 45 & 99 & $<10$ & 24 \\
\hline & 5 & 256 & 444 & 46 & $<10$ & 19 \\
\hline & 12 & -783 & 785 & 0 & -- & -- \\
\hline & 13 & -188 & 225 & 36 & - & -- \\
\hline \multirow[t]{6}{*}{$A R L A O 2$} & 2 & -824 & 20 & 50 & $\cdots$ & - \\
\hline & 3 & -414 & 410 & 29 & $\cdots$ & $\cdots$ \\
\hline & 4 & -244 & 170 & 99 & - & - \\
\hline & 5 & 245 & 284 & 76 & -- & $\cdots$ \\
\hline & 12 & -844 & 770 & 0 & - & -- \\
\hline & 13 & -39 & 205 & 19 & $\cdots$ & $\cdots$ \\
\hline \multirow[t]{8}{*}{ ARLEOI } & 2 & -1626 & 125 & 99 & $<10$ & 36 \\
\hline & 3 & -1211 & 415 & 66 & $<10$ & 32 \\
\hline & 4 & -586 & 625 & 72 & $<10$ & 25 \\
\hline & 5 & -171 & 335 & 85 & $<10$ & 17 \\
\hline & 6 & 204 & 310 & 55 & $<10$ & - \\
\hline & 12 & -1751 & -- & -- & -- & $\cdots$ \\
\hline & 13 & -506 & 80 & 0 & -- & -- \\
\hline & 14 & -106 & 65 & 0 & $\cdots$ & $\cdots$ \\
\hline \multirow[t]{9}{*}{$A R L E O 2$} & 2 & -1620 & 120 & 63 & $<10$ & 38 \\
\hline & 3 & -1150 & 470 & 57 & $<10$ & 34 \\
\hline & 4 & -557 & 593 & 61 & $<10$ & 25 \\
\hline & 5 & -120 & 330 & 87 & $<10$ & 15 \\
\hline & 6 & 80 & 130 & 19 & - & -- \\
\hline & 11 & 197 & 117 & -- & -- & -- \\
\hline & 12 & -1740 & - & -- & $\cdots$ & -- \\
\hline & 13 & -450 & 107 & 0 & -- & $\cdots$ \\
\hline & 14 & -50 & 70 & 0 & -- & - \\
\hline
\end{tabular}


Table 4--Layer number; altitude of top, thickness, and sand percentage of layer; and average dissolved-solids concentration and temperature of water in layer; by log number--Continued.

\begin{tabular}{|c|c|c|c|c|c|c|}
\hline $\begin{array}{l}\text { Log } \\
\text { number }\end{array}$ & $\begin{array}{r}\text { Layer } \\
\text { number }\end{array}$ & $\begin{array}{r}\text { Altitude } \\
\text { of top } \\
\text { (feet) }\end{array}$ & $\begin{array}{r}\text { Thick- } \\
\text { ness } \\
\text { (feet) }\end{array}$ & $\begin{array}{r}\text { Sand } \\
\text { per- } \\
\text { centage }\end{array}$ & $\begin{array}{l}\text { Dis- } \\
\text { solved } \\
\text { solids }\end{array}$ & $\begin{array}{l}\text { Tem- } \\
\text { per- } \\
\text { ature }\end{array}$ \\
\hline \multirow[t]{9}{*}{ ARLIOI } & 2 & -2602 & 340 & 32 & 26 & 39 \\
\hline & 3 & -1977 & 625 & 18 & $<10$ & 35 \\
\hline & 4 & -1817 & 160 & 81 & $<10$ & 32 \\
\hline & 5 & -457 & 840 & 53 & $<10$ & 24 \\
\hline & 6 & -222 & 145 & 79 & $<10$ & 20 \\
\hline & 12 & -2942 & 835 & 0 & -- & $\cdots$ \\
\hline & 13 & -1297 & 520 & 22 & -. & - \\
\hline & 14 & -367 & 90 & 50 & -- & -- \\
\hline & 15 & 243 & 465 & 0 & -- & -- \\
\hline \multirow[t]{10}{*}{ ARLIO 2} & 2 & -2684 & 385 & 39 & 11 & 46 \\
\hline & 3 & -2044 & 640 & 27 & $<10$ & 40 \\
\hline & 4 & -1869 & 175 & 99 & $<10$ & 36 \\
\hline & 5 & -594 & 720 & 59 & $<10$ & 26 \\
\hline & 6 & -284 & 155 & 90 & $<10$ & 20 \\
\hline & 11 & 187 & 91 & $\ldots$ & - & 15 \\
\hline & 12 & -3069 & 740 & 0 & -- & - \\
\hline & 13 & -1314 & 555 & 21 & -. & - \\
\hline & 14 & -439 & 155 & 26 & -- & -. \\
\hline & 15 & 96 & 380 & 0 & -- & - \\
\hline \multirow[t]{7}{*}{ ARLOO1 } & 2 & -434 & 105 & 99 & $<10$ & 24 \\
\hline & 3 & -354 & 80 & 31 & -. & 23 \\
\hline & 4 & -69 & 285 & 88 & $<10$ & 20 \\
\hline & 5 & 66 & 135 & 57 & $<10$ & 18 \\
\hline & 11 & 227 & 96 & $\cdots$ & - & 16 \\
\hline & 12 & -539 & 640 & 0 & -- & -. \\
\hline & 14 & 131 & 65 & 0 & -- & -- \\
\hline \multirow[t]{8}{*}{ ARLOO2 } & 3 & -1055 & 415 & 61 & $<10$ & 34 \\
\hline & 4 & -720 & 335 & 42 & $<10$ & 29 \\
\hline & 5 & -90 & 575 & 63 & $<10$ & 23 \\
\hline & 6 & 80 & 70 & 99 & $<10$ & 17 \\
\hline & 11 & 200 & 120 & - & - & 16 \\
\hline & 12 & -1470 & 766 & 0 & -- & - \\
\hline & 13 & -665 & 55 & 0 & -- & - \\
\hline & 14 & 10 & 100 & 30 & -- & -- \\
\hline \multirow[t]{6}{*}{ ARLOO3 } & 2 & -454 & 140 & 79 & -- & - \\
\hline & 3 & -369 & 85 & 65 & -. & -. \\
\hline & 4 & -69 & 300 & 99 & -- & -. \\
\hline & 5 & 91 & 160 & 99 & -- & -. \\
\hline & 11 & 226 & 135 & -- & -- & -- \\
\hline & 12 & -594 & 566 & 0 & -- & -- \\
\hline ARLROI & 12 & 275 & 192 & 0 & -- & - \\
\hline \multirow[t]{5}{*}{ ARLWO1 } & 1 & -208 & 130 & 92 & -- & -- \\
\hline & 2 & 160 & 50 & - & -- & -- \\
\hline & 3 & 110 & 50 & - & -- & -- \\
\hline & 11 & 247 & 87 & -- & -- & - \\
\hline & 12 & 60 & 268 & -- & -- & -- \\
\hline
\end{tabular}


Table 4--Layer number; altitude of top, thickness, and sand percentage of layer; and average dissolved-solids concentration and temperature of water in layer; by log number--Continued.

\begin{tabular}{|c|c|c|c|c|c|c|}
\hline $\begin{array}{l}\text { Log } \\
\text { number }\end{array}$ & $\begin{array}{r}\text { Layer } \\
\text { number }\end{array}$ & $\begin{array}{r}\text { Altitude } \\
\text { of top } \\
\text { (feet) }\end{array}$ & $\begin{array}{r}\text { Thick- } \\
\text { ness } \\
\text { (feet) }\end{array}$ & $\begin{array}{r}\text { Sand } \\
\text { per- } \\
\text { centage }\end{array}$ & $\begin{array}{l}\text { Dis- } \\
\text { solved } \\
\text { solids }\end{array}$ & $\begin{array}{l}\text { Tem- } \\
\text { per- } \\
\text { ature }\end{array}$ \\
\hline \multirow[t]{6}{*}{ ARMIO 1} & 2 & -600 & 65 & 99 & $<10$ & 24 \\
\hline & 3 & -125 & 475 & 41 & $<10$ & 22 \\
\hline & 4 & 45 & 170 & -- & - - & 19 \\
\hline & 5 & 217 & 112 & -- & -- & 18 \\
\hline & 12 & -665 & 735 & 0 & -- & -- \\
\hline & 13 & 105 & 60 & 0 & -- & -- \\
\hline \multirow[t]{6}{*}{ ARMIO 2} & 2 & -668 & 75 & 57 & $<10$ & 27 \\
\hline & 3 & -243 & 425 & 29 & $<10$ & 24 \\
\hline & 4 & -78 & 165 & 61 & $<10$ & 21 \\
\hline & 5 & 305 & 333 & -- & -- & 18 \\
\hline & 12 & -743 & 775 & 0 & -- & -- \\
\hline & 13 & -28 & 50 & 0 & -- & -- \\
\hline \multirow[t]{6}{*}{ ARMIO 3} & 2 & -561 & 123 & 93 & -- & 23 \\
\hline & 3 & -334 & 227 & 37 & -- & 22 \\
\hline & 4 & -134 & 200 & 50 & -- & 21 \\
\hline & 5 & 290 & 364 & -- & -- & 18 \\
\hline & 12 & -684 & 938 & 0 & -- & -- \\
\hline & 13 & -74 & 60 & 17 & -- & -- \\
\hline A RMOOI & 2 & -1410 & 115 & 98 & $<10$ & 34 \\
\hline \multirow[t]{8}{*}{ • } & 3 & -1070 & 340 & 70 & $<10$ & 30 \\
\hline & 4 & -935 & 135 & 99 & $<10$ & 25 \\
\hline & 5 & -125 & 750 & 73 & $<10$ & 16 \\
\hline & 6 & 95 & 95 & 99 & $<10$ & -- \\
\hline & 11 & 185 & 90 & -- & $<10$ & -- \\
\hline & 12 & -1525 & 845 & 3 & - - & -- \\
\hline & 13 & -875 & 60 & 3 & -- & -- \\
\hline & 14 & 0 & 125 & 4 & -- & -- \\
\hline \multirow[t]{8}{*}{ ARMOO2 } & 2 & -1212 & 81 & 79 & $<10$ & 32 \\
\hline & 3 & -941 & 271 & 48 & -- & 28 \\
\hline & 4 & -448 & 493 & 83 & $<10$ & 19 \\
\hline & 5 & -153 & 295 & 63 & $<10$ & -- \\
\hline & 6 & 62 & 76 & 78 & $<10$ & -- \\
\hline & 11 & 186 & 124 & -- & - - & -- \\
\hline & 12 & -1293 & -- & -- & -- & -- \\
\hline & 14 & -14 & 139 & 17 & -- & -- \\
\hline \multirow[t]{4}{*}{ ARMOO 3} & 5 & -185 & -- & -- & -- & -- \\
\hline & 6 & 55 & 79 & 85 & -- & -- \\
\hline & 11 & 195 & 140 & 56 & -- & -- \\
\hline & 14 & -24 & 161 & 20 & -- & -- \\
\hline \multirow[t]{7}{*}{ ARMSO 1} & 2 & -1173 & -- & -- & -- & -- \\
\hline & 3 & -905 & 268 & 65 & -- & -- \\
\hline & 4 & -429 & 476 & 86 & -- & - \\
\hline & 5 & -151 & 278 & 84 & -- & -- \\
\hline & 6 & 71 & 172 & 56 & -- & -- \\
\hline & 11 & 235 & 164 & 73 & -- & -- \\
\hline & 14 & -101 & 50 & 0 & -- & -- \\
\hline
\end{tabular}


Table 4--Layer number; altitude of top, thickness, and sand percentage of layer; and average dissolved-solids concentration and temperature of water in layer; by log number--Continued.

\begin{tabular}{|c|c|c|c|c|c|c|}
\hline $\begin{array}{l}\text { Log } \\
\text { number }\end{array}$ & $\begin{array}{r}\text { Layer } \\
\text { number }\end{array}$ & $\begin{array}{r}\text { Alt teude } \\
\text { of top } \\
\text { (feet) }\end{array}$ & $\begin{array}{r}\text { Thick- } \\
\text { ness } \\
\text { (feet) }\end{array}$ & $\begin{array}{r}\text { Sand } \\
\text { per- } \\
\text { centage }\end{array}$ & $\begin{array}{l}\text { Dis- } \\
\text { solved } \\
\text { solids }\end{array}$ & $\begin{array}{l}\text { Tem- } \\
\text { per- } \\
\text { ature }\end{array}$ \\
\hline \multirow[t]{7}{*}{ ARMSO 2} & 2 & -1065 & -- & -- & -- & $\cdots$ \\
\hline & 3 & -855 & 210 & 59 & - & - \\
\hline & 4 & -345 & 510 & 83 & - & - \\
\hline & 5 & -95 & 250 & 80 & -- & -- \\
\hline & 6 & 49 & 60 & - & -- & $\cdots$ \\
\hline & 11 & 245 & 196 & 75 & -- & $\cdots$ \\
\hline & 14 & -11 & 84 & 12 & -- & - \\
\hline \multirow[t]{6}{*}{ ARMSO 3} & 2 & -879 & -- & $\cdots$ & - & - \\
\hline & 3 & -681 & 198 & 75 & -- & -- \\
\hline & 4 & -271 & 410 & 45 & -- & -- \\
\hline & 5 & -27 & 244 & 79 & -- & -- \\
\hline & 11 & 241 & 178 & -- & -- & $\cdots$ \\
\hline & 14 & 63 & 90 & 0 & -- & -- \\
\hline \multirow[t]{7}{*}{ ARMSO 4} & 2 & -1114 & -- & - & -- & -- \\
\hline & 3 & -962 & 152 & 42 & -- & -- \\
\hline & 4 & -429 & 533 & 92 & -- & -- \\
\hline & 5 & -202 & 227 & 62 & - & $\cdots$ \\
\hline & 6 & 98 & 198 & 69 & -- & - \\
\hline & 11 & 242 & 144 & -- & $\cdots$ & $\cdots$ \\
\hline & 14 & -100 & 102 & 0 & - & - \\
\hline \multirow[t]{7}{*}{ ARMSO 5} & 2 & -948 & -- & -- & -- & $\cdots$ \\
\hline & 3 & -664 & 284 & 14 & - & $\cdots$ \\
\hline & 4 & -310 & 354 & 99 & $\cdots$ & $\cdots$ \\
\hline & 5 & -89 & 221 & 81 & -- & $\cdots$ \\
\hline & 6 & 114 & 132 & 98 & -- & - \\
\hline & 11 & 250 & 136 & 94 & -- & $\cdots$ \\
\hline & 14 & -18 & 71 & 0 & -- & - \\
\hline \multirow[t]{6}{*}{ ARNEOI } & 2 & -254 & 65 & 99 & -- & 22 \\
\hline & 3 & -74 & 180 & 33 & -- & 20 \\
\hline & 4 & 6 & 80 & 81 & $<10$ & 19 \\
\hline & 5 & 326 & 145 & -- & -- & 16 \\
\hline & 12 & -319 & 545 & 0 & -- & - \\
\hline & 13 & 181 & 175 & 20 & -- & $\cdots$ \\
\hline \multirow[t]{4}{*}{ ARNEO2 } & 2 & 118 & 20 & 50 & -- & 18 \\
\hline & 3 & 263 & 145 & 38 & $<10$ & 17 \\
\hline & 4 & 330 & 67 & -- & -- & 16 \\
\hline & 12 & 98 & 525 & 0 & -- & -- \\
\hline ARNEO3 & 12 & 300 & 184 & - & -- & -- \\
\hline \multirow[t]{3}{*}{ ARNEO 4} & 2 & 176 & 20 & -- & - & $\cdots$ \\
\hline & 3 & 295 & 119 & - & -- & - \\
\hline & 12 & 156 & 377 & -- & -- & -- \\
\hline
\end{tabular}


Table 4--Layer number; altitude of top, thickness, and sand percentage of layer; and average dissolved-solids concentration and temperature of water in layer; by log number--Continued.

\begin{tabular}{|c|c|c|c|c|c|c|}
\hline $\begin{array}{l}\text { Log } \\
\text { number }\end{array}$ & $\begin{array}{r}\text { Layer } \\
\text { number }\end{array}$ & $\begin{array}{r}\text { Altitude } \\
\text { of top } \\
(f e e t)\end{array}$ & $\begin{array}{r}\text { Thick- } \\
\text { ness } \\
\text { (feet) }\end{array}$ & $\begin{array}{r}\text { Sand } \\
\text { per- } \\
\text { centage }\end{array}$ & $\begin{array}{l}\text { Dis- } \\
\text { solved } \\
\text { solids }\end{array}$ & $\begin{array}{l}\text { Tem- } \\
\text { per- } \\
\text { ature }\end{array}$ \\
\hline \multirow[t]{5}{*}{ AROU 01} & 2 & -231 & 20 & 50 & -- & $\ldots$ \\
\hline & 3 & -26 & 205 & 61 & -- & - \\
\hline & 4 & 101 & 127 & 99 & -- & - \\
\hline & 12 & -251 & 450 & 0 & -- & -- \\
\hline & 13 & 279 & 178 & -- & -- & - \\
\hline \multirow[t]{8}{*}{ AROUO2 } & 2 & -1220 & 95 & 99 & $<10$ & 30 \\
\hline & 3 & -980 & 240 & 21 & -- & 29 \\
\hline & 4 & -920 & 60 & 99 & $<10$ & 27 \\
\hline & 5 & -175 & 375 & 76 & $<10$ & 22 \\
\hline & 6 & 135 & 175 & 99 & $<10$ & 18 \\
\hline & 12 & -1315 & 655 & 0 & - - & $\ldots$ \\
\hline & 13 & -550 & 370 & 18 & -- & - \\
\hline & 14 & -40 & 135 & 7 & -- & - \\
\hline \multirow[t]{6}{*}{ AROU 03} & 2 & -808 & 65 & 99 & -- & - \\
\hline & 3 & -538 & 270 & 56 & -- & -- \\
\hline & 4 & -513 & 25 & 99 & -- & -- \\
\hline & 5 & 92 & 230 & 51 & -- & -- \\
\hline & 12 & -873 & 580 & 0 & -- & -- \\
\hline & 13 & -138 & 375 & 29 & -- & - \\
\hline \multirow[t]{9}{*}{ ARPHO 1} & 2 & -2150 & 240 & 83 & 21 & 36 \\
\hline & 3 & -1630 & 520 & 38 & $<10$ & 32 \\
\hline & 4 & -1345 & 285 & 68 & $<10$ & 29 \\
\hline & 5 & -290 & 600 & 82 & $<10$ & 21 \\
\hline & 6 & 10 & 115 & 48 & $<10$ & 17 \\
\hline & 11 & 155 & 145 & - & -- & 15 \\
\hline & 12 & -2390 & 1165 & 3 & -- & -- \\
\hline & 13 & -890 & 455 & 25 & -- & -- \\
\hline & 14 & -105 & 185 & 46 & -- & - \\
\hline \multirow[t]{9}{*}{ ARPHO2 } & 2 & -1722 & 265 & 74 & $<10$ & 32 \\
\hline & 3 & -1282 & 440 & 57 & $<10$ & 29 \\
\hline & 4 & -1017 & 265 & 99 & $<10$ & 26 \\
\hline & 5 & -162 & 480 & 65 & $<10$ & 20 \\
\hline & 6 & 28 & 30 & -- & - - & 16 \\
\hline & 11 & 168 & 140 & -- & -- & 15 \\
\hline & 12 & -1987 & 1195 & 0 & -- & - \\
\hline & 13 & -642 & 375 & 16 & -- & - \\
\hline & 14 & -2 & 160 & -- & -- & - \\
\hline \multirow[t]{2}{*}{ ARPHO 3} & 6 & 20 & -- & -- & -- & - \\
\hline & 11 & 179 & 159 & -- & -- & $\cdots$ \\
\hline \multirow[t]{4}{*}{ ARP $\mathrm{HO} 4$} & 5 & 106 & -- & -- & -- & - \\
\hline & 6 & 212 & 34 & -- & -- & $\ldots$ \\
\hline & 11 & 250 & 38 & - & -- & - \\
\hline & 14 & 178 & 72 & 0 & -- & - \\
\hline
\end{tabular}


Table 4--Layer number; altitude of top, thickness, and sand percentage of layer; and average dissolved-solids concentration and temperature of water in layer; by log number-Continued.

\begin{tabular}{|c|c|c|c|c|c|c|}
\hline $\begin{array}{l}\text { Log } \\
\text { number }\end{array}$ & $\begin{array}{r}\text { Layer } \\
\text { number }\end{array}$ & $\begin{array}{r}\text { Alt } 1 \text { tude } \\
\text { of top } \\
\text { (feet) }\end{array}$ & $\begin{array}{r}\text { Thlck- } \\
\text { ness } \\
\text { (feet) }\end{array}$ & $\begin{array}{r}\text { Sand } \\
\text { per- } \\
\text { centage }\end{array}$ & $\begin{array}{l}\text { Dis- } \\
\text { solved } \\
\text { solids }\end{array}$ & $\begin{array}{l}\text { Tem- } \\
\text { per- } \\
\text { ature }\end{array}$ \\
\hline \multirow[t]{9}{*}{$A R P B O 5$} & 2 & -2003 & 485 & 59 & 11 & 31 \\
\hline & 3 & -1438 & 565 & 46 & $<10$ & 28 \\
\hline & 4 & -1151 & 287 & 74 & $<10$ & 26 \\
\hline & 5 & -93 & 580 & 97 & $<10$ & 21 \\
\hline & 6 & 47 & 70 & -- & -- & 19 \\
\hline & 11 & 162 & 115 & -- & -- & 18 \\
\hline & 12 & -2488 & 935 & 0 & -- & -- \\
\hline & 13 & -673 & 478 & 17 & -- & -- \\
\hline & 14 & -23 & 70 & -- & -- & -- \\
\hline \multirow[t]{8}{*}{ ARPOO 1} & 2 & -1101 & 248 & 99 & -- & - \\
\hline & 3 & -1007 & 94 & 37 & -- & -- \\
\hline & 4 & -581 & 426 & 99 & -- & - \\
\hline & 5 & -351 & 230 & 99 & -- & - \\
\hline & 6 & 52 & 293 & 27 & -- & -- \\
\hline & 11 & 219 & 167 & 96 & -- & -- \\
\hline & 12 & -1349 & -- & -- & -- & -- \\
\hline & 14 & -241 & 110 & 0 & -- & -- \\
\hline \multirow[t]{5}{*}{$A R P O O 2$} & 2 & -760 & -- & -- & -- & -- \\
\hline & 3 & -560 & 200 & 49 & -- & -- \\
\hline & 4 & -140 & 420 & 88 & -- & -- \\
\hline & 5 & 88 & 228 & 64 & -- & -- \\
\hline & 11 & 220 & 132 & -- & -- & -- \\
\hline \multirow[t]{6}{*}{ ARPOO 3} & 1 & -1365 & 160 & 99 & $<10$ & 35 \\
\hline & 2 & -520 & 175 & 73 & $<10$ & 25 \\
\hline & 3 & -295 & 225 & 24 & - - & 23 \\
\hline & 4 & 91 & 386 & 68 & $<10$ & 19 \\
\hline & 11 & 239 & 148 & -- & - - & 16 \\
\hline & 12 & -695 & 670 & 0 & -- & -- \\
\hline \multirow[t]{3}{*}{$A R P R O 1$} & 2 & 105 & 105 & -- & -- & -- \\
\hline & 11 & 205 & 100 & -- & -- & -- \\
\hline & 12 & 0 & 485 & 0 & -- & -- \\
\hline \multirow[t]{8}{*}{ ARPRO 2} & 3 & -944 & 530 & 32 & -- & - \\
\hline & 4 & -704 & 240 & 73 & -- & - \\
\hline & 5 & -144 & 445 & 72 & -- & - \\
\hline & 6 & 86 & 160 & 32 & -- & - \\
\hline & 11 & 216 & 130 & -- & -- & -- \\
\hline & 12 & -1474 & 605 & 0 & -- & -- \\
\hline & 13 & -589 & 115 & 0 & -- & -- \\
\hline & 14 & -74 & 70 & 0 & -- & -- \\
\hline \multirow[t]{6}{*}{ ARPRO 3} & 2 & -595 & 155 & 95 & -- & - \\
\hline & 3 & -440 & 155 & 42 & -- & -- \\
\hline & 4 & -125 & 315 & 77 & -- & -- \\
\hline & 5 & 75 & 200 & 72 & -- & - \\
\hline & 11 & 190 & 115 & -- & -- & -- \\
\hline & 12 & -750 & 705 & 0 & -- & -- \\
\hline
\end{tabular}


Table 4--Layer number; altitude of top, thickness, and sand percentage of layer; and average dissolved-solids concentration and temperature of water in layer; by log number--Continued.

\begin{tabular}{|c|c|c|c|c|c|c|}
\hline $\begin{array}{l}\text { L०g } \\
\text { number }\end{array}$ & $\begin{array}{r}\text { Layer } \\
\text { number }\end{array}$ & $\begin{array}{r}\text { Altitude } \\
\text { of top } \\
(f e e t)\end{array}$ & $\begin{array}{r}\text { Thlck- } \\
\text { ness } \\
\text { (feet) }\end{array}$ & $\begin{array}{r}\text { Sand } \\
\text { per- } \\
\text { centage }\end{array}$ & $\begin{array}{l}\text { D1s- } \\
\text { solved } \\
\text { solids }\end{array}$ & $\begin{array}{r}\text { Tem- } \\
\text { per- } \\
\text { ature }\end{array}$ \\
\hline \multirow[t]{8}{*}{ ARSF 01} & 2 & -1215 & 400 & 71 & -- & -- \\
\hline & 3 & -1035 & 180 & 28 & -- & -- \\
\hline & 4 & -445 & 590 & 99 & -- & -- \\
\hline & 5 & -180 & 265 & 99 & -- & -- \\
\hline & 6 & 60 & 165 & -- & -- & -- \\
\hline & 11 & 205 & 145 & -- & -- & -- \\
\hline & 12 & -1615 & 730 & 5 & -- & -- \\
\hline & 14 & -105 & 75 & 0 & -- & -- \\
\hline \multirow[t]{8}{*}{ ARSFO2 } & 2 & -1085 & 170 & 76 & $<10$ & 31 \\
\hline & 3 & -940 & 145 & 62 & -- & 29 \\
\hline & 4 & -435 & 505 & 77 & $<10$ & 25 \\
\hline & 5 & -170 & 265 & 55 & $<10$ & 20 \\
\hline & 6 & 65 & 200 & 50 & $<10$ & 17 \\
\hline & 11 & 205 & 140 & -- & -- & 15 \\
\hline & 12 & -1255 & 855 & 0 & -- & -- \\
\hline & 14 & -135 & 35 & 0 & -- & -- \\
\hline \multirow[t]{8}{*}{ ARUNO 1} & 2 & -1123 & 130 & 46 & 14 & 34 \\
\hline & 3 & -913 & 210 & 31 & -- & 32 \\
\hline & 4 & -863 & 50 & 90 & $<10$ & 30 \\
\hline & 5 & -13 & 490 & 80 & -- & 23 \\
\hline & 6 & 197 & 30 & -- & -- & 18 \\
\hline & 12 & -1253 & 610 & 0 & -- & -- \\
\hline & 13 & -503 & 360 & 0 & -- & -- \\
\hline & 14 & 167 & 180 & 0 & -- & -- \\
\hline \multirow[t]{8}{*}{ ARUNO2 } & 2 & -1119 & 95 & 79 & -- & -- \\
\hline & 3 & -884 & 235 & 30 & -- & -- \\
\hline & 4 & -849 & 35 & 99 & -- & -- \\
\hline & 5 & 16 & 600 & 81 & -- & -- \\
\hline & 6 & 201 & 110 & -- & -- & -- \\
\hline & 12 & -1214 & 740 & 0 & -- & -- \\
\hline & 13 & -584 & 265 & 0 & -- & -- \\
\hline & 14 & 91 & 75 & 27 & -- & -- \\
\hline \multirow[t]{8}{*}{ ARUNO3 } & 2 & -1089 & 145 & 60 & $<10$ & 31 \\
\hline & 3 & -984 & 105 & 19 & -- & 30 \\
\hline & 4 & -932 & 52 & 99 & $<10$ & 29 \\
\hline & 5 & -87 & 530 & 77 & -- & 24 \\
\hline & 6 & 234 & 226 & 71 & $<10$ & 20 \\
\hline & 12 & -1234 & 643 & 0 & -- & -- \\
\hline & 13 & -617 & 315 & 0 & -- & -- \\
\hline & 14 & 8 & 95 & 21 & -- & -- \\
\hline \multirow[t]{8}{*}{ ARUNO4 } & 2 & -1332 & 45 & 99 & $<10$ & 40 \\
\hline & 3 & -1024 & 308 & 54 & $<10$ & 37 \\
\hline & 4 & -997 & 27 & 99 & $<10$ & 35 \\
\hline & 5 & -72 & 645 & 37 & $<10$ & 25 \\
\hline & 6 & 208 & 175 & -- & $<10$ & 17 \\
\hline & 12 & -1377 & 690 & 4 & -- & -- \\
\hline & 13 & -717 & 280 & 0 & -- & -- \\
\hline & 14 & 33 & 105 & 19 & -- & -- \\
\hline
\end{tabular}


Table 4--Layer number; altitude of top, thickness, and sand percentage of layer; and average dissolved-solids concentration and temperature of water in layer; by log number--Continued.

\begin{tabular}{|c|c|c|c|c|c|c|}
\hline $\begin{array}{l}\text { Log } \\
\text { number }\end{array}$ & $\begin{array}{r}\text { Layer } \\
\text { number }\end{array}$ & $\begin{array}{r}\text { Altitude } \\
\text { of top } \\
\text { (feet) }\end{array}$ & $\begin{array}{r}\text { Thick- } \\
\text { ness } \\
\text { (feet) }\end{array}$ & $\begin{array}{r}\text { Sand } \\
\text { per- } \\
\text { centage }\end{array}$ & $\begin{array}{l}\text { Dis- } \\
\text { solved } \\
\text { solids }\end{array}$ & $\begin{array}{l}\text { Tem- } \\
\text { per- } \\
\text { ature }\end{array}$ \\
\hline \multirow[t]{8}{*}{ ARUNO5 } & 2 & -1094 & 105 & 74 & $<10$ & 30 \\
\hline & 3 & -889 & 205 & 49 & $<10$ & 29 \\
\hline & 4 & -861 & 28 & 99 & $<10$ & 28 \\
\hline & 5 & -29 & 532 & 64 & $<10$ & 23 \\
\hline & 6 & 211 & 165 & -- & -- & 20 \\
\hline & 12 & -1199 & 720 & 0 & - & - \\
\hline & 13 & -561 & 300 & 0 & - & - \\
\hline & 14 & 46 & 75 & 20 & - & - \\
\hline \multirow[t]{7}{*}{ ARUNO 6} & 2 & -868 & 170 & 84 & -- & -- \\
\hline & 3 & -758 & 110 & 70 & - & - \\
\hline & 4 & -688 & 70 & 99 & $\ldots$ & - \\
\hline & 5 & 107 & 475 & 81 & $\ldots$ & $\cdots$ \\
\hline & 12 & -1038 & 630 & 0 & - & -- \\
\hline & 13 & -368 & 320 & 13 & $\cdots$ & - \\
\hline & 14 & 232 & 125 & -- & - & - \\
\hline \multirow[t]{2}{*}{ ARWHO 1} & 11 & 215 & 96 & -- & - & - \\
\hline & 12 & 119 & 330 & -- & -- & - \\
\hline \multirow[t]{3}{*}{ ARWOO 1} & 2 & 117 & 175 & 38 & -- & - \\
\hline & 11 & 217 & 100 & - & - & -- \\
\hline & 12 & -58 & 700 & 0 & -- & - \\
\hline \multirow[t]{5}{*}{ ARWOO 2} & 2 & -419 & 154 & 92 & $<10$ & 24 \\
\hline & 3 & -280 & 139 & 36 & -- & 22 \\
\hline & 4 & 75 & 355 & 99 & - & 18 \\
\hline & 11 & 200 & 125 & -- & - & 14 \\
\hline & 12 & -573 & 727 & 0 & - & - \\
\hline \multirow[t]{7}{*}{ FLESO1 } & 2 & -2758 & 150 & 77 & $<10$ & 38 \\
\hline & 3 & -1253 & 1505 & 23 & - & 33 \\
\hline & 5 & -593 & 660 & 33 & $<10$ & 25 \\
\hline & 7 & -103 & 430 & - & - & $\ldots$ \\
\hline & 8 & 86 & 189 & -- & $\cdots$ & 19 \\
\hline & 12 & -2908 & 690 & 0 & - & - \\
\hline & 15 & -533 & 60 & 0 & -- & $\cdots$ \\
\hline \multirow[t]{6}{*}{ FLESO2 } & 3 & -1700 & 1525 & 19 & -- & 41 \\
\hline & 5 & -945 & 755 & 14 & $<10$ & 30 \\
\hline & 7 & -65 & 740 & 55 & $<10$ & 22 \\
\hline & 8 & 254 & 319 & 99 & $<10$ & 17 \\
\hline & 12 & -3225 & 800 & 0 & - & $-\infty$ \\
\hline & 15 & -805 & 140 & 0 & - & $\cdots$ \\
\hline \multirow[t]{6}{*}{ FLESO3 } & 3 & -2240 & 1110 & 39 & - & 40 \\
\hline & 5 & -1730 & 510 & - & -- & -. \\
\hline & 7 & -480 & 1080 & 15 & $<10$ & 26 \\
\hline & 8 & 11 & 491 & - & - & 20 \\
\hline & 12 & -3350 & 1170 & 0 & - & - \\
\hline & 15 & -1560 & 170 & 0 & -- & - \\
\hline
\end{tabular}


Table 4--Layer number; altitude of top, thickness, and sand percentage of layer; and average dissolved-solids concentration and temperature of water in layer; by log number--Continued.

\begin{tabular}{|c|c|c|c|c|c|c|}
\hline $\begin{array}{l}\text { Log } \\
\text { number }\end{array}$ & $\begin{array}{r}\text { Layer } \\
\text { number }\end{array}$ & $\begin{array}{r}\text { Altitude } \\
\text { of top } \\
(f e e t)\end{array}$ & $\begin{array}{r}\text { Thlck- } \\
\text { ness } \\
\text { (feet) }\end{array}$ & $\begin{array}{r}\text { Sand } \\
\text { per- } \\
\text { centage }\end{array}$ & $\begin{array}{l}\text { Dis- } \\
\text { solved } \\
\text { solids }\end{array}$ & $\begin{array}{l}\text { Tem- } \\
\text { per- } \\
\text { ature }\end{array}$ \\
\hline \multirow[t]{3}{*}{$\mathrm{KYBAO} 1$} & 3 & 0 & -- & -- & -- & - \\
\hline & 4 & 395 & 395 & 46 & -- & -- \\
\hline & 5 & 465 & 70 & 63 & -- & -- \\
\hline \multirow[t]{2}{*}{$\mathrm{KYCAO} 1$} & 1 & 393 & 374 & 88 & -- & - \\
\hline & 12 & 475 & 82 & 26 & -- & - \\
\hline \multirow[t]{5}{*}{$\mathrm{KYCAO} 2$} & 1 & 154 & -- & -- & -- & -- \\
\hline & 2 & 368 & 26 & 50 & -- & -- \\
\hline & 3 & 484 & 116 & 15 & -- & - \\
\hline & 4 & 582 & 98 & 71 & -- & - \\
\hline & 12 & 342 & 188 & 0 & -- & -- \\
\hline \multirow[t]{5}{*}{$\mathrm{KYCAO} 3$} & 1 & 226 & 425 & 78 & $<10$ & 19 \\
\hline & 2 & 427 & 12 & 99 & -- & - \\
\hline & 3 & 435 & 8 & 99 & -- &.- \\
\hline & 4 & 583 & 148 & 75 & -- & -- \\
\hline & 12 & 415 & 189 & 8 & -- & - \\
\hline \multirow[t]{8}{*}{ KYCLO 1} & 1 & -536 & 300 & 42 & -- & - \\
\hline & 2 & -338 & 30 & 99 & -- & - \\
\hline & 3 & -300 & 38 & 39 & -- & - \\
\hline & 4 & 22 & 322 & 99 & -- & $\cdots$ \\
\hline & 5 & 152 & 130 & 99 & -- & - \\
\hline & 6 & 482 & 282 & 79 & -- & $\cdots$ \\
\hline & 12 & -368 & 168 & 0 & -- & - \\
\hline & 14 & 200 & 48 & 17 & -- & $\cdots$ \\
\hline \multirow[t]{6}{*}{$\mathrm{KYCLO} 2$} & 1 & -227 & 186 & 47 & $<10$ & 19 \\
\hline & 2 & 1 & 30 & 99 & -- & 17 \\
\hline & 3 & 157 & 156 & 32 & -- & 16 \\
\hline & 4 & 355 & 198 & 99 & -- & 15 \\
\hline & 5 & 402 & 47 & -- & -- & 14 \\
\hline & 12 & -29 & 198 & 0 & -- & - \\
\hline \multirow[t]{4}{*}{ KYFUO 1} & 4 & -130 & -- & -- & - & $\cdots$ \\
\hline & 5 & -18 & 112 & 31 & -- & -- \\
\hline & 6 & 320 & 192 & 40 & -- & - \\
\hline & 14 & 128 & 146 & 0 & -- & - \\
\hline \multirow[t]{9}{*}{ KYFUO2 } & 1 & -1341 & 328 & 51 & -- & -- \\
\hline & 2 & -871 & 170 & 99 & -- & - \\
\hline & 3 & -701 & 170 & 8 & -- & - \\
\hline & 4 & -306 & 395 & 94 & -- & - \\
\hline & 5 & -101 & 205 & 41 & -- & -- \\
\hline & 6 & 149 & 110 & 35 & -- & -- \\
\hline & 11 & 289 & 140 & 79 & -- & - \\
\hline & 12 & -1041 & 300 & 0 & -- & -- \\
\hline & 14 & 39 & 140 & 0 & -- & - \\
\hline
\end{tabular}


Table 4--Layer number; altitude of top, thickness, and sand percentage of layer; and average dissolved-solids concentration and temperature of water in layer; by log number--Continued.

\begin{tabular}{|c|c|c|c|c|c|c|}
\hline $\begin{array}{l}\text { Log } \\
\text { number }\end{array}$ & $\begin{array}{r}\text { Layer } \\
\text { number }\end{array}$ & $\begin{array}{r}\text { Altitude } \\
\text { of top } \\
\text { (feet) }\end{array}$ & $\begin{array}{r}\text { Thlck- } \\
\text { ness } \\
\text { (feet) }\end{array}$ & $\begin{array}{r}\text { Sand } \\
\text { per- } \\
\text { centage }\end{array}$ & $\begin{array}{l}\text { Dis- } \\
\text { solved } \\
\text { solids }\end{array}$ & $\begin{array}{l}\text { Tem- } \\
\text { per- } \\
\text { ature }\end{array}$ \\
\hline \multirow[t]{4}{*}{$\mathrm{XYF \cup O3}$} & 4 & 155 & -- & -- & -- & -- \\
\hline & 5 & 240 & 85 & 55 & -- & -- \\
\hline & 6 & 500 & 158 & 74 & -- & -- \\
\hline & 14 & 342 & 102 & 49 & -- & -- \\
\hline \multirow[t]{3}{*}{ KYFUO4 } & 5 & 50 & -- & -- & -- & -- \\
\hline & 6 & 390 & 104 & 96 & -- & -- \\
\hline & 14 & 286 & 236 & 9 & -- & -- \\
\hline \multirow[t]{5}{*}{ KYGRO 1} & 1 & 106 & -- & -- & -- & -- \\
\hline & 2 & 314 & 75 & 20 & -- & -- \\
\hline & 3 & 424 & 110 & 68 & -- & -- \\
\hline & 4 & 514 & 90 & -- & -- & -- \\
\hline & 12 & 239 & 130 & 0 & -- & -- \\
\hline \multirow[t]{2}{*}{$\mathrm{KYGRO2}$} & 5 & 407 & -- & -- & -- & -- \\
\hline & 14 & 510 & 103 & 51 & -- & - \\
\hline \multirow[t]{2}{*}{ KYGRO 3} & 1 & 186 & - - & -- & -- & -- \\
\hline & 12 & 410 & 224 & 0 & -- & -- \\
\hline \multirow[t]{6}{*}{ KYGRO 4} & 2 & -184 & 79 & 99 & -- & -- \\
\hline & 3 & -92 & 92 & 67 & -- & -- \\
\hline & 4 & 234 & 326 & 99 & -- & $\ldots$ \\
\hline & 5 & 360 & 126 & 97 & -- & -- \\
\hline & 12 & -263 & - - & -- & -- & -- \\
\hline & 14 & 450 & 90 & -- & -- & -- \\
\hline \multirow[t]{6}{*}{ XYGRO5 } & 1 & -307 & -- & -- & -- & -- \\
\hline & 2 & -103 & 40 & 99 & -- & - \\
\hline & 3 & -39 & 64 & 99 & -- & -- \\
\hline & 4 & 373 & 412 & 94 & -- & -- \\
\hline & 5 & 470 & 97 & -- & -- & -- \\
\hline & 12 & -143 & 164 & 0 & -- & -- \\
\hline \multirow[t]{8}{*}{ KYHIO 1} & 1 & -785 & -- & -- & -- & -- \\
\hline & 2 & -469 & 26 & 92 & -- & -- \\
\hline & 3 & -321 & 148 & 7 & -- & -- \\
\hline & 4 & 38 & 359 & 99 & -- & -- \\
\hline & 5 & 243 & 205 & 24 & -- & -- \\
\hline & 6 & 430 & 127 & -- & -- & -- \\
\hline & 12 & -495 & 290 & 0 & -- & -- \\
\hline & 14 & 303 & 60 & 20 & -- & -- \\
\hline \multirow[t]{7}{*}{ KYHIO2 } & 2 & -335 & 52 & 99 & -- & -- \\
\hline & 3 & -251 & 84 & 99 & -- & -- \\
\hline & 4 & 105 & 356 & 96 & -- & -- \\
\hline & 5 & 221 & 116 & 99 & -- & -- \\
\hline & 6 & 480 & 191 & -- & -- & -- \\
\hline & 12 & -387 & -- & -- & -- & -- \\
\hline & 14 & 289 & 68 & 0 & -- & -- \\
\hline
\end{tabular}


Table 4--Layer number; altitude of top, thickness, and sand percentage of layer; and average dissolved-solids concentration and temperature of water in layer; by log number--Continued.

\begin{tabular}{|c|c|c|c|c|c|c|}
\hline $\begin{array}{l}\text { Log } \\
\text { number }\end{array}$ & $\begin{array}{r}\text { Layer } \\
\text { number }\end{array}$ & $\begin{array}{r}\text { Altitude } \\
\text { of top } \\
\text { (feet) }\end{array}$ & $\begin{array}{r}\text { Thick- } \\
\text { ness } \\
\text { (feet) }\end{array}$ & $\begin{array}{r}\text { Sand } \\
\text { per- } \\
\text { centage }\end{array}$ & $\begin{array}{l}\text { Dis- } \\
\text { solved } \\
\text { solids }\end{array}$ & $\begin{array}{l}\text { Tem- } \\
\text { per- } \\
\text { ature }\end{array}$ \\
\hline \multirow[t]{2}{*}{ KYMCO 1} & 1 & 92 & 103 & 99 & $\cdots$ & - \\
\hline & 12 & 362 & 270 & 0 & -- & $\cdots$ \\
\hline \multirow[t]{6}{*}{$\mathrm{LAACO} 1$} & 7 & -6391 & 2224 & 36 & 97 & 59 \\
\hline & 8 & -3578 & 2813 & 64 & 92 & 46 \\
\hline & 9 & -2143 & 1435 & 51 & 31 & 35 \\
\hline & 10 & -843 & 1300 & 46 & 10 & 28 \\
\hline & 11 & 37 & 880 & 58 & $<10$ & 23 \\
\hline & 15 & -8615 & 1579 & 11 & -- & -- \\
\hline \multirow[t]{5}{*}{$\mathrm{LAACO} 2$} & 8 & -6255 & 4625 & 52 & 116 & 78 \\
\hline & 9 & -3236 & 3019 & 66 & 113 & 49 \\
\hline & 10 & -932 & 2304 & 50 & 30 & 32 \\
\hline & 11 & 5 & 937 & 79 & $<10$ & 23 \\
\hline & 16 & -10880 & 1258 & 3 & - & -- \\
\hline \multirow[t]{6}{*}{$\mathrm{LAACO} 3$} & 7 & -9365 & 2228 & 13 & 103 & 69 \\
\hline & 8 & -4457 & 4170 & 57 & 132 & 49 \\
\hline & 9 & -2280 & 2177 & 64 & 99 & 35 \\
\hline & 10 & -863 & 1417 & 44 & 30 & 28 \\
\hline & 11 & 32 & 895 & 82 & $<10$ & 23 \\
\hline & 16 & -8627 & 738 & 6 & -- & - \\
\hline \multirow[t]{9}{*}{ LAAL 01} & 3 & -11543 & $\cdots$ & $\cdots$ & -- & -- \\
\hline & 6 & -8919 & 31 & 99 & 28 & 80 \\
\hline & 7 & -3982 & 3111 & 46 & 110 & 53 \\
\hline & 8 & -1935 & 2047 & 30 & $<10$ & 36 \\
\hline & 9 & -870 & 1065 & 32 & $<10$ & 28 \\
\hline & 10 & -233 & 637 & 49 & $<10$ & 23 \\
\hline & 11 & 100 & 333 & 99 & $<10$ & 20 \\
\hline & 14 & -8950 & 2593 & 1 & -- & -- \\
\hline & 15 & -7093 & 1826 & 3 & -- & - \\
\hline \multirow[t]{7}{*}{ LAALO 2} & 3 & -12036 & 705 & 21 & -- & 100 \\
\hline & 7 & -4359 & 3207 & 46 & 65 & 65 \\
\hline & 8 & -2110 & 2249 & 53 & 44 & 55 \\
\hline & 9 & -1031 & 1079 & 36 & $<10$ & 38 \\
\hline & 10 & -251 & 780 & 34 & $<10$ & 25 \\
\hline & 11 & 55 & 306 & 66 & $<10$ & 17 \\
\hline & 15 & -7566 & 4470 & 1 & -- & -- \\
\hline \multirow[t]{11}{*}{ LAALO3 } & 3 & -9027 & -- & -- & -- & -- \\
\hline & 5 & -8235 & 227 & 34 & 52 & 75 \\
\hline & 6 & -6727 & 750 & 26 & 57 & 67 \\
\hline & 7 & -3057 & 2557 & 48 & 52 & 48 \\
\hline & 8 & -1357 & 1700 & 19 & $<10$ & 34 \\
\hline & 9 & -457 & 900 & -- & - & 25 \\
\hline & 10 & -7 & 450 & -- & -- & 21 \\
\hline & 11 & 124 & 131 & -- & - & 19 \\
\hline & 13 & -8462 & 565 & 0 & - & -- \\
\hline & 14 & -7477 & 758 & 7 & -- & -- \\
\hline & 15 & -5614 & 1113 & 0 & -- & -- \\
\hline
\end{tabular}


Table 4--Layer number; altitude of top, thickness, and sand percentage of layer; and average dissolved-solids concentration and temperature of water in layer; by log number--Continued.

\begin{tabular}{|c|c|c|c|c|c|c|}
\hline $\begin{array}{l}\text { Log } \\
\text { number }\end{array}$ & $\begin{array}{r}\text { Layer } \\
\text { number }\end{array}$ & $\begin{array}{r}\text { Altitude } \\
\text { of top } \\
(f e e t)\end{array}$ & $\begin{array}{r}\text { Thlck- } \\
\text { ness } \\
(\text { feet) }\end{array}$ & $\begin{array}{r}\text { Sand } \\
\text { per- } \\
\text { centage }\end{array}$ & $\begin{array}{l}\text { Dis- } \\
\text { solved } \\
\text { solids }\end{array}$ & $\begin{array}{l}\text { Tem- } \\
\text { per- } \\
\text { ature }\end{array}$ \\
\hline \multirow[t]{4}{*}{ LAALO 4} & 8 & -1361 & -- & -- & -- & -- \\
\hline & 9 & -461 & 900 & 28 & -- & -- \\
\hline & 10 & -11 & 450 & 31 & -- & -- \\
\hline & 11 & 115 & 126 & 99 & -- & -- \\
\hline \multirow[t]{6}{*}{ LAANO 1} & 7 & -8469 & 1211 & 40 & 134 & 66 \\
\hline & 8 & -4371 & 4098 & 58 & 106 & 53 \\
\hline & 9 & -2575 & 1796 & 41 & 61 & 38 \\
\hline & 10 & -949 & 1626 & 48 & 15 & 29 \\
\hline & 11 & 6 & 955 & 70 & $<10$ & 23 \\
\hline & 15 & -9680 & 1803 & 4 & -- & -- \\
\hline \multirow[t]{5}{*}{ LAAS 01} & 8 & -7075 & 5145 & 49 & 90 & 72 \\
\hline & 9 & -3735 & 3340 & 63 & 99 & 53 \\
\hline & 10 & -998 & 2737 & 54 & 28 & 36 \\
\hline & 11 & 12 & 1010 & 54 & $<10$ & 23 \\
\hline & 16 & -12220 & 2595 & 0 & -- & -- \\
\hline \multirow[t]{4}{*}{$\mathrm{LAASO2}$} & 8 & -8041 & 4247 & 44 & 143 & 68 \\
\hline & 9 & -3977 & 4064 & 47 & 109 & 49 \\
\hline & 10 & -1055 & 2922 & 60 & 76 & 32 \\
\hline & 11 & 0 & 1055 & 67 & $<10$ & 22 \\
\hline \multirow[t]{12}{*}{ LAAV0 1} & 3 & -4722 & 3346 & 54 & 96 & 72 \\
\hline & 4 & -4642 & 80 & 76 & 122 & 62 \\
\hline & 5 & -3740 & 713 & 46 & 79 & 58 \\
\hline & 6 & -2935 & 565 & 50 & 67 & 53 \\
\hline & 7 & -1321 & 1184 & 37 & 51 & 44 \\
\hline & 8 & -564 & 757 & 33 & 10 & 32 \\
\hline & 9 & -92 & 472 & 66 & $<10$ & 23 \\
\hline & 11 & 53 & 145 & 99 & $<10$ & 19 \\
\hline & 12 & -8068 & 1002 & 2 & -- & -- \\
\hline & 13 & -4453 & 189 & 0 & -- & -- \\
\hline & 14 & -3500 & 240 & 8 & -- & - \\
\hline & 15 & -2505 & 430 & 1 & -- & -- \\
\hline \multirow[t]{12}{*}{$\mathrm{LAAVO} 2$} & 3 & -7987 & 3535 & 39 & 50 & 73 \\
\hline & 5 & -6821 & 736 & 31 & 59 & 57 \\
\hline & 6 & -5867 & 527 & 34 & 61 & 50 \\
\hline & 7 & -2867 & 2292 & 30 & 58 & 38 \\
\hline & 8 & -1425 & 1442 & 49 & 19 & 30 \\
\hline & 9 & -517 & 908 & 66 & $<10$ & 24 \\
\hline & 10 & -102 & 415 & 56 & $<10$ & 22 \\
\hline & 11 & 53 & 155 & -- & $<10$ & 20 \\
\hline & 12 & -11522 & 1935 & 0 & -- & -- \\
\hline & 13 & -7557 & 430 & 0 & -- & -- \\
\hline & 14 & -6394 & 427 & 0 & -- & -- \\
\hline & 15 & -5159 & 708 & 0 & -- & -- \\
\hline
\end{tabular}


Table 4--Layer number; altitude of top, thickness, and sand percentage of layer; and average dissolved-solids concentration and temperature of water in layer; by log number--Continued.

\begin{tabular}{|c|c|c|c|c|c|c|}
\hline $\begin{array}{l}\text { Log } \\
\text { number }\end{array}$ & $\begin{array}{r}\text { Layer } \\
\text { number }\end{array}$ & $\begin{array}{l}\text { Altitude } \\
\text { of top } \\
\text { (feet) }\end{array}$ & $\begin{array}{r}\text { Thick- } \\
\text { ness } \\
\text { (feet) }\end{array}$ & $\begin{array}{r}\text { Sand } \\
\text { per- } \\
\text { centage }\end{array}$ & $\begin{array}{l}\text { Dis- } \\
\text { solved } \\
\text { solids }\end{array}$ & $\begin{array}{r}\text { Tem- } \\
\text { per- } \\
\text { ature }\end{array}$ \\
\hline \multirow[t]{11}{*}{ LAAVO 3} & 3 & -6471 & -- & -- & - - & -- \\
\hline & 5 & -5398 & 667 & 50 & 107 & 54 \\
\hline & 6 & -4623 & 336 & 72 & 106 & 49 \\
\hline & 7 & -2285 & 1808 & 39 & 99 & 39 \\
\hline & 8 & -1029 & 1256 & 43 & 112 & 30 \\
\hline & 9 & -343 & 686 & -- & - & 24 \\
\hline & 10 & -118 & 225 & $-\infty$ & -- & 21 \\
\hline & 11 & 48 & 166 & -- & -- & 20 \\
\hline & 13 & -6065 & 406 & 0 & -- & -- \\
\hline & 14 & -4959 & 439 & 2 & -- & -- \\
\hline & 15 & -4093 & 530 & 4 & -- & -- \\
\hline \multirow[t]{11}{*}{ LAAV0 4} & 3 & -8620 & -- & -- & -- & -- \\
\hline & 5 & -7564 & 503 & 43 & -- & 58 \\
\hline & 6 & -6794 & 223 & 35 & 84 & 53 \\
\hline & 7 & -3459 & 2713 & 57 & 79 & 43 \\
\hline & 8 & -1657 & 1802 & 61 & 40 & 33 \\
\hline & 9 & -700 & 957 & 74 & -- & 26 \\
\hline & 10 & -157 & 543 & 39 & $<10$ & 22 \\
\hline & 11 & 40 & 197 & -- & - & 20 \\
\hline & 13 & -8067 & 553 & 4 & -- & - \\
\hline & 14 & -7017 & 547 & 5 & -- & - \\
\hline & 15 & -6172 & 622 & 0 & -- & -- \\
\hline \multirow[t]{8}{*}{ LABEO 1} & 3 & -8431 & -- & -- & -- & -- \\
\hline & 6 & -5651 & 1178 & 39 & 43 & 53 \\
\hline & 7 & -2221 & 2230 & 39 & $<10$ & 37 \\
\hline & 8 & -581 & 1640 & 47 & $<10$ & 28 \\
\hline & 9 & -41 & 540 & 31 & $<10$ & 23 \\
\hline & 10 & 64 & 105 & -- & $<10$ & 22 \\
\hline & 14 & -6829 & 1602 & 6 & -- & -- \\
\hline & 15 & -4451 & 1200 & 3 & - & -- \\
\hline \multirow[t]{9}{*}{ LABEO2 } & 3 & -9445 & -- & -- & -- & -- \\
\hline & 6 & -6805 & 673 & 33 & 50 & 70 \\
\hline & 7 & -2915 & 2690 & 52 & 46 & 50 \\
\hline & 8 & -1145 & 1770 & 42 & $<10$ & 34 \\
\hline & 9 & -325 & 820 & 32 & $<10$ & 26 \\
\hline & 10 & -25 & 300 & 92 & $<10$ & 22 \\
\hline & 11 & 131 & 156 & -- & $<10$ & 20 \\
\hline & 14 & -7478 & 1967 & 1 & -- & - \\
\hline & 15 & -5605 & 1200 & 1 & -- & - \\
\hline \multirow[t]{11}{*}{$\angle A B E 03$} & 3 & -8606 & -- & -- & -- & - \\
\hline & 5 & -7922 & 116 & 98 & 36 & 87 \\
\hline & 6 & -6258 & 822 & 50 & 28 & 76 \\
\hline & 7 & -2748 & 2338 & 45 & 49 & 53 \\
\hline & 8 & -1158 & 1590 & 41 & $<10$ & 36 \\
\hline & 9 & -358 & 800 & -- & -- & 26 \\
\hline & 10 & -18 & 340 & -- & -- & 21 \\
\hline & 11 & 164 & 182 & -- & -- & 19 \\
\hline & 13 & -8038 & 568 & 2 & -- & -- \\
\hline & 14 & -7080 & 842 & 5 & -- & -- \\
\hline & 15 & -5086 & 1172 & 2 & -- & -- \\
\hline
\end{tabular}


Table 4--Layer number; altitude of top, thickness, and sand percentage of layer; and average dissolved-solids concentration and temperature of water in layer; by log number--Continued.

\begin{tabular}{|c|c|c|c|c|c|c|}
\hline $\begin{array}{l}\text { Log } \\
\text { number }\end{array}$ & $\begin{array}{r}\text { Layer } \\
\text { number }\end{array}$ & $\begin{array}{r}\text { Altitude } \\
\text { of top } \\
\text { (feet) }\end{array}$ & $\begin{array}{r}\text { Thick- } \\
\text { ness } \\
\text { (feet) }\end{array}$ & $\begin{array}{r}\text { Sand } \\
\text { per- } \\
\text { centage }\end{array}$ & $\begin{array}{l}\text { Dis- } \\
\text { solved } \\
\text { solids }\end{array}$ & $\begin{array}{l}\text { Tem- } \\
\text { per- } \\
\text { ature }\end{array}$ \\
\hline \multirow[t]{6}{*}{$\mathrm{LABEO} 4$} & 7 & -3804 & 2587 & 56 & 46 & 42 \\
\hline & 8 & -1817 & 1987 & 57 & 11 & 33 \\
\hline & 9 & -867 & 950 & 36 & $<10$ & 28 \\
\hline & 10 & -367 & 500 & 63 & $<10$ & 25 \\
\hline & 11 & 43 & 410 & 53 & $<10$ & 23 \\
\hline & 15 & -6391 & 2052 & 1 & -- & -- \\
\hline \multirow[t]{6}{*}{ L ABEO5 } & 7 & -4331 & 2244 & 55 & 59 & 47 \\
\hline & 8 & -2238 & 2093 & 49 & 30 & 37 \\
\hline & 9 & -1165 & 1073 & 30 & $<10$ & 30 \\
\hline & 10 & -495 & 670 & 59 & $<10$ & 26 \\
\hline & 11 & 25 & 520 & 61 & $<10$ & 23 \\
\hline & 15 & -6575 & -- & -- & -- & -- \\
\hline \multirow[t]{6}{*}{ LABEO 6} & 7 & -4160 & 2903 & 52 & 175 & 55 \\
\hline & 8 & -2093 & 2067 & 56 & 92 & 40 \\
\hline & 9 & -1043 & 1050 & -- & 15 & 31 \\
\hline & 10 & -413 & 630 & -- & -- & 26 \\
\hline & 11 & 50 & 463 & -- & -- & 22 \\
\hline & 15 & -7063 & -- & -- & -- & -- \\
\hline \multirow[t]{4}{*}{ L A B E 07} & 8 & -1169 & -- & -- & -- & -- \\
\hline & 9 & -369 & 800 & 25 & -- & -- \\
\hline & 10 & -19 & 350 & 32 & -- & -- \\
\hline & 11 & 171 & 190 & 94 & -- & -- \\
\hline \multirow[t]{5}{*}{$\mathrm{LABI} 01$} & 3 & -598 & 359 & 25 & $<10$ & 26 \\
\hline & 4 & -578 & 20 & 50 & -- & 25 \\
\hline & 5 & 233 & 402 & 63 & $<10$ & 22 \\
\hline & 12 & -957 & 767 & 0 & -- & -- \\
\hline & 13 & -169 & 409 & 1 & -- & -- \\
\hline \multirow[t]{6}{*}{$\mathrm{LAB} I 02$} & 2 & -884 & 38 & 79 & $<10$ & 28 \\
\hline & 3 & -39 & 845 & 45 & $<10$ & 25 \\
\hline & 4 & 16 & 55 & 18 & -- & 22 \\
\hline & 5 & 278 & 77 & -- & -- & 20 \\
\hline & 12 & -922 & 732 & 0 & -- & -- \\
\hline & 13 & 201 & 185 & -- & -- & -- \\
\hline \multirow[t]{6}{*}{$\mathrm{LABOOI}$} & 2 & -686 & 33 & 94 & $<10$ & 27 \\
\hline & 3 & -396 & 290 & 54 & $<10$ & 26 \\
\hline & 4 & -366 & 30 & 83 & $<10$ & 25 \\
\hline & 5 & 248 & 426 & 74 & $<10$ & 21 \\
\hline & 12 & -719 & 662 & 0 & -- & -- \\
\hline & 13 & -178 & 188 & 12 & -- & -- \\
\hline \multirow[t]{6}{*}{$\mathrm{LABOO2}$} & 2 & -343 & 20 & 50 & -- & 28 \\
\hline & 3 & -13 & 330 & 36 & -- & 25 \\
\hline & 4 & 32 & 45 & 99 & $<10$ & 21 \\
\hline & 5 & 255 & 43 & -- & -- & 17 \\
\hline & 12 & -363 & 655 & 2 & -- & -- \\
\hline & 13 & 212 & 180 & 8 & -- & -- \\
\hline
\end{tabular}


Table 4--Layer number; altitude of top, thickness, and sand percentage of layer; and average dissolved-solids concentration and temperature of water in layer; by log number--Continued.

\begin{tabular}{|c|c|c|c|c|c|c|}
\hline $\begin{array}{l}\text { Log } \\
\text { number }\end{array}$ & $\begin{array}{r}\text { Layer } \\
\text { number }\end{array}$ & $\begin{array}{r}\text { Alt } 1 \text { tude } \\
\text { of top } \\
(f e e t)\end{array}$ & $\begin{array}{r}\text { Thick- } \\
\text { ness } \\
(\text { feet })\end{array}$ & $\begin{array}{r}\text { Sand } \\
\text { per- } \\
\text { centage }\end{array}$ & $\begin{array}{l}\text { Dis- } \\
\text { solved } \\
\text { solids }\end{array}$ & $\begin{array}{l}\text { Tem- } \\
\text { per- } \\
\text { ature }\end{array}$ \\
\hline \multirow[t]{3}{*}{$L A B O 03$} & 2 & -52 & 20 & 50 & -- & 22 \\
\hline & 3 & 164 & 216 & 65 & $<10$ & 21 \\
\hline & 12 & -72 & 569 & 0 & -- & - \\
\hline \multirow[t]{9}{*}{$L A C A O 1$} & 2 & -2016 & 773 & 66 & -- & - \\
\hline & 3 & -1264 & 752 & 72 & -- & -- \\
\hline & 4 & -1041 & 223 & 87 & -- & - \\
\hline & 5 & -171 & 662 & 75 & -- & - \\
\hline & 6 & -11 & 60 & - & -- & -- \\
\hline & 11 & 60 & 71 & $\ldots$ & -- & - \\
\hline & 12 & -2789 & 567 & 0 & -- & -- \\
\hline & 13 & -833 & 208 & 0 & -- & -- \\
\hline & 14 & -71 & 100 & -- & -- & -- \\
\hline \multirow[t]{9}{*}{$\mathrm{LACAO} 2$} & 2 & -2902 & 704 & 56 & 41 & 41 \\
\hline & 3 & -1668 & 1234 & 75 & 33 & 35 \\
\hline & 4 & -1541 & 127 & 98 & 45 & 30 \\
\hline & 5 & -551 & 692 & 57 & 13 & 26 \\
\hline & 6 & $\cdot-11$ & 400 & 54 & $<10$ & 21 \\
\hline & 11 & 56 & 67 & - & -- & 20 \\
\hline & 12 & -3606 & 660 & 0 & -- & $\ldots$ \\
\hline & 13 & -1243 & 298 & 3 & -- & -- \\
\hline & 14 & -411 & 140 & 5 & -- & -- \\
\hline \multirow[t]{8}{*}{$\mathrm{LACAO}_{3}$} & 2 & -2114 & 490 & 62 & 30 & 39 \\
\hline & 3 & -1354 & 760 & 71 & 31 & 34 \\
\hline & 4 & -1214 & 140 & 99 & 36 & 30 \\
\hline & 5 & -214 & 734 & 59 & $<10$ & 24 \\
\hline & 6 & 190 & 246 & $=$ & $<10$ & 19 \\
\hline & 12 & -2604 & 700 & 0 & - & - \\
\hline & 13 & -948 & 266 & 0 & -- & - \\
\hline & 14 & -56 & 158 & 0 & -- & - \\
\hline \multirow[t]{6}{*}{ LACDO 1} & 2 & -511 & 58 & 33 & -- & 25 \\
\hline & 3 & -111 & 400 & 38 & $<10$ & 23 \\
\hline & 4 & -4 & 107 & 77 & $<10$ & 21 \\
\hline & 5 & 310 & 249 & 57 & $<10$ & 19 \\
\hline & 12 & -569 & 792 & 0 & - & -- \\
\hline & 13 & 61 & 65 & 0 & -- & -- \\
\hline \multirow[t]{3}{*}{ LACDO2 } & 2 & 104 & 20 & 63 & -- & 20 \\
\hline & 3 & 195 & 91 & -- & -- & 20 \\
\hline & 12 & 84 & 556 & 0 & -- & -- \\
\hline \multirow[t]{3}{*}{$\mathrm{LACDO} 3$} & 2 & 180 & 20 & -- & -- & - \\
\hline & 3 & 265 & 85 & -- & -- & - \\
\hline & 12 & 160 & 724 & 0 & -- & - \\
\hline
\end{tabular}


Table 4--Layer number; altitude of top, thickness, and sand percentage of layer; and average dissolved-solids concentration and temperature of water in layer; by log number--Continued.

\begin{tabular}{|c|c|c|c|c|c|c|}
\hline $\begin{array}{l}\text { Log } \\
\text { number }\end{array}$ & $\begin{array}{r}\text { Layer } \\
\text { number }\end{array}$ & $\begin{array}{c}\text { Altitude } \\
\text { of top } \\
\text { (feet) }\end{array}$ & $\begin{array}{r}\text { Thlek- } \\
\text { ness } \\
\text { (feet) }\end{array}$ & $\begin{array}{r}\text { Sand } \\
\text { per- } \\
\text { centage }\end{array}$ & $\begin{array}{l}\text { Dis- } \\
\text { solved } \\
\text { solids }\end{array}$ & $\begin{array}{l}\text { Tem- } \\
\text { per- } \\
\text { ature }\end{array}$ \\
\hline \multirow[t]{3}{*}{$\mathrm{LACDO} 4$} & 2 & -42 & 20 & 50 & -- & 21 \\
\hline & 3 & 342 & 384 & -- & $<10$ & 20 \\
\hline & 12 & -62 & 847 & 0 & - & $\ldots$ \\
\hline \multirow[t]{7}{*}{$\angle A C L O 1$} & 2 & -1169 & 70 & 60 & $<10$ & 32 \\
\hline & 3 & -709 & 460 & 13 & - & 29 \\
\hline & 4 & -691 & 18 & 99 & - - & 27 \\
\hline & 5 & 53 & 534 & 61 & $<10$ & 22 \\
\hline & 12 & -1239 & 567 & 1 & -- & -- \\
\hline & 13 & -481 & 210 & 7 & - & -. \\
\hline & 14 & 174 & 121 & -- & -- & -- \\
\hline \multirow[t]{7}{*}{$\mathrm{LACLO} 2$} & 2 & -930 & 20 & 50 & -- & 29 \\
\hline & 3 & -510 & 420 & 59 & $<10$ & 27 \\
\hline & 4 & -490 & 20 & 50 & -- & 25 \\
\hline & 5 & 220 & 500 & 76 & $<10$ & 20 \\
\hline & 12 & -950 & 590 & 0 & - & - - \\
\hline & 13 & -280 & 210 & 12 & -- & - \\
\hline & 14 & 367 & 147 & -- & -- & -- \\
\hline \multirow[t]{5}{*}{ LACNO 1} & 8 & -4266 & 3384 & 67 & 152 & 61 \\
\hline & 9 & -2140 & 2126 & 45 & 81 & 45 \\
\hline & 10 & -861 & 1279 & 52 & 16 & 33 \\
\hline & 11 & 0 & 861 & 54 & $<10$ & 24 \\
\hline & 16 & -7650 & 3994 & 2 & -- & - \\
\hline \multirow[t]{4}{*}{$\mathrm{LACNO} 2$} & 8 & -5430 & 4063 & 56 & 124 & 55 \\
\hline & 9 & -2731 & 2699 & 54 & 75 & 40 \\
\hline & 10 & -957 & 1774 & 58 & 22 & 30 \\
\hline & 11 & 0 & 957 & 52 & $<10$ & 24 \\
\hline \multirow[t]{5}{*}{$\mathrm{LACNO} 3$} & 8 & -5259 & 4312 & 68 & 108 & 70 \\
\hline & 9 & -2656 & 2603 & 63 & 51 & 50 \\
\hline & 10 & -931 & 1725 & 37 & - & 34 \\
\hline & 11 & 0 & 931 & 27 & $<10$ & 24 \\
\hline & 16 & -9571 & 3948 & 0 & -- & - \\
\hline \multirow[t]{7}{*}{$\mathrm{LACNO} 4$} & 7 & -9963 & 268 & 49 & 102 & 79 \\
\hline & 8 & -4485 & 3683 & 63 & 174 & 54 \\
\hline & 9 & -2262 & 2223 & 47 & 58 & 37 \\
\hline & 10 & -923 & 1339 & 59 & 29 & 29 \\
\hline & 11 & 0 & 923 & 67 & $<10$ & 24 \\
\hline & 15 & -10231 & - & - & -- & -- \\
\hline & 16 & -8168 & 1795 & 5 & -- & -- \\
\hline \multirow[t]{4}{*}{ LACNO 5} & 8 & -7837 & 6173 & 48 & 82 & 110 \\
\hline & 9 & -3872 & 3965 & 43 & 87 & 60 \\
\hline & 10 & -918 & 2954 & 60 & 27 & 44 \\
\hline & 11 & 0 & 918 & 34 & $<10$ & 24 \\
\hline
\end{tabular}


Table 4--Layer number; altitude of top, thickness, and sand percentage of layer; and average dissolved-solids concentration and temperature of water in layer; by log number--Continued.

\begin{tabular}{|c|c|c|c|c|c|c|}
\hline $\begin{array}{l}\text { Log } \\
\text { number }\end{array}$ & $\begin{array}{r}\text { Layer } \\
\text { number }\end{array}$ & $\begin{array}{r}\text { Altitude } \\
\text { of top } \\
\text { (feet) }\end{array}$ & $\begin{array}{r}\text { Thick- } \\
\text { ness } \\
\text { (feet) }\end{array}$ & $\begin{array}{r}\text { Sand } \\
\text { per- } \\
\text { centage }\end{array}$ & $\begin{array}{l}\text { Dis- } \\
\text { solved } \\
\text { solids }\end{array}$ & $\begin{array}{l}\text { Tem- } \\
\text { per- } \\
\text { ature }\end{array}$ \\
\hline \multirow[t]{4}{*}{ IACNO6 } & 8 & -8191 & 2464 & 59 & 129 & 73 \\
\hline & 9 & -4051 & 4140 & 40 & 119 & 57 \\
\hline & 10 & -950 & 3101 & 59 & 66 & 45 \\
\hline & 11 & 0 & 950 & 88 & $<10$ & 25 \\
\hline \multirow[t]{4}{*}{ LACNOZ } & 8 & -7444 & 2639 & 67 & 127 & 70 \\
\hline & 9 & -3899 & 3545 & 52 & 105 & 49 \\
\hline & 10 & -977 & 2922 & 54 & 21 & 32 \\
\hline & 11 & 0 & 977 & 69 & $<10$ & 24 \\
\hline \multirow[t]{4}{*}{ L ACNO8 } & 8 & -7408 & 4260 & 47 & -- & 74 \\
\hline & 9 & -3926 & 3482 & 59 & 75 & 53 \\
\hline & 10 & -1046 & 2880 & 54 & 32 & 35 \\
\hline & 11 & 0 & 1046 & 94 & $<10$ & 25 \\
\hline \multirow[t]{4}{*}{ LACNO9 } & 8 & -8933 & 5232 & 24 & 81 & 80 \\
\hline & 9 & -4699 & 4234 & 39 & 55 & 56 \\
\hline & 10 & -1085 & 3614 & 50 & 23 & 37 \\
\hline & 11 & 0 & 1085 & 70 & $<10$ & 25 \\
\hline \multirow[t]{11}{*}{ LACOO 1} & 2 & -6092 & 410 & 52 & 46 & 76 \\
\hline & 3 & -3421 & 2671 & 54 & 57 & 67 \\
\hline & 5 & -2145 & 854 & 64 & 34 & 53 \\
\hline & 6 & -1354 & 620 & 60 & 12 & 42 \\
\hline & 7 & -177 & 710 & 22 & $<10$ & 25 \\
\hline & 8 & -117 & 60 & 82 & $<10$ & 20 \\
\hline & 11 & 63 & 180 & 48 & $<10$ & 18 \\
\hline & 12 & -6502 & 991 & 0 & -- & -- \\
\hline & 13 & -2999 & 422 & 0 & -- & -- \\
\hline & 14 & -1974 & 171 & 0 & -- & -- \\
\hline & 15 & -887 & 467 & 1 & -- & - \\
\hline \multirow[t]{10}{*}{$\mathrm{LACOO2}$} & 2 & -6809 & -- & -- & -- & - \\
\hline & 3 & -3889 & 2920 & 52 & 45 & 55 \\
\hline & 5 & -2614 & 898 & 60 & 26 & 40 \\
\hline & 6 & -1824 & 627 & 39 & 27 & 34 \\
\hline & 7 & -447 & 808 & 46 & 13 & 25 \\
\hline & 8 & -147 & 300 & -- & -- & 21 \\
\hline & 11 & 48 & 195 & -- & -- & 20 \\
\hline & 13 & -3512 & 377 & 0 & -- & -- \\
\hline & 14 & -2451 & 163 & 0 & -- & -- \\
\hline & 15 & -1255 & 569 & 0 & -- & -- \\
\hline \multirow[t]{10}{*}{ LACOO3 } & 3 & -4821 & -- & -- & -- & -- \\
\hline & 5 & -3481 & 906 & 48 & 39 & 46 \\
\hline & 6 & -2944 & 447 & 58 & 48 & 40 \\
\hline & 7 & -1266 & 1205 & 34 & 30 & 32 \\
\hline & 8 & -440 & 826 & 51 & $<10$ & 25 \\
\hline & 9 & -146 & 294 & 59 & $<10$ & 21 \\
\hline & 11 & 56 & 202 & 99 & $<10$ & 20 \\
\hline & 13 & -4387 & 434 & 7 & - & -- \\
\hline & 14 & -3391 & 90 & 6 & -- & -- \\
\hline & 15 & -2471 & 473 & 3 & -- & -- \\
\hline
\end{tabular}


Table 4--Layer number; altitude of top, thickness, and sand percentage of layer; and average dissolved-solids concentration and temperature of water in layer; by log number--Continued.

\begin{tabular}{|c|c|c|c|c|c|c|}
\hline $\begin{array}{l}\log \\
\text { number }\end{array}$ & $\begin{array}{r}\text { Layer } \\
\text { number }\end{array}$ & $\begin{array}{c}\text { Altitude } \\
\text { of top } \\
\text { (feet) }\end{array}$ & $\begin{array}{r}\text { Thick- } \\
\text { ness } \\
\text { (feet) }\end{array}$ & $\begin{array}{r}\text { Sand } \\
\text { per- } \\
\text { centage }\end{array}$ & $\begin{array}{l}\text { Dis- } \\
\text { solved } \\
\text { solids }\end{array}$ & $\begin{array}{l}\text { Tem- } \\
\text { per- } \\
\text { ature }\end{array}$ \\
\hline \multirow[t]{10}{*}{ L ACOO 4} & 3 & -4926 & -- & -- & -- & $\cdots$ \\
\hline & 5 & -3556 & 930 & 58 & 32 & 43 \\
\hline & 6 & -3076 & 380 & 60 & 44 & 39 \\
\hline & 7 & -1322 & 1280 & 34 & 42 & 31 \\
\hline & 8 & -451 & 871 & 45 & 17 & 25 \\
\hline & 9 & -151 & 300 & -- & -- & 21 \\
\hline & 11 & 54 & 205 & $\ldots$ & -- & 20 \\
\hline & 13 & -4486 & 440 & 0 & -- & -- \\
\hline & 14 & -3456 & 100 & 0 & -- & -- \\
\hline & 15 & -2602 & 474 & 0 & -- & $\cdots$ \\
\hline \multirow[t]{10}{*}{$\mathrm{LACOO5}$} & 3 & -4600 & - & -- & -- & - \\
\hline & 5 & -3386 & 903 & 54 & - & 45 \\
\hline & 6 & -2728 & 518 & 49 & -- & 39 \\
\hline & 7 & -1096 & 1152 & 27 & -- & 30 \\
\hline & 8 & -396 & 700 & 44 & $<10$ & 24 \\
\hline & 9 & -166 & 230 & 60 & $<10$ & 21 \\
\hline & 11 & 42 & 208 & 86 & $<10$ & 20 \\
\hline & 13 & -4289 & 311 & 2 & -- & - \\
\hline & 14 & -3246 & 140 & 4 & $\ldots$ & $\ldots$ \\
\hline & 15 & -2248 & 480 & 5 & -- & $\cdots$ \\
\hline \multirow[t]{13}{*}{ LACOO6 } & 2 & -7878 & 936 & 57 & 65 & 78 \\
\hline & 3 & -5715 & 2163 & 44 & 47 & 67 \\
\hline & 5 & -4428 & 912 & 26 & 63 & 54 \\
\hline & 6 & -3971 & 239 & 72 & 61 & 48 \\
\hline & 7 & -1962 & 1496 & 36 & 53 & 38 \\
\hline & 8 & -1001 & 961 & 42 & 25 & 30 \\
\hline & 9 & -501 & 500 & 69 & $<10$ & 25 \\
\hline & 10 & -178 & 323 & -- & - & 22 \\
\hline & 11 & 38 & 216 & -- & - & 20 \\
\hline & 12 & -8814 & -- & -- & -- & - \\
\hline & 13 & -5340 & 375 & 5 & -- & - \\
\hline & 14 & -4210 & 218 & 21 & $\ldots$ & - \\
\hline & 15 & -3458 & 513 & 2 & $\cdots$ & $\cdots$ \\
\hline \multirow[t]{11}{*}{ LACTOL } & 2 & -5285 & 410 & 75 & 30 & 57 \\
\hline & 3 & -3162 & 2123 & 62 & 31 & 50 \\
\hline & 4 & -3035 & 127 & 97 & 36 & 45 \\
\hline & 5 & -1816 & 906 & 68 & -- & 39 \\
\hline & 6 & -1032 & 610 & 43 & - & 31 \\
\hline & 7 & -32 & 492 & 23 & -- & 22 \\
\hline & 11 & 50 & 82 & -. & -- & 19 \\
\hline & 12 & -5695 & 627 & 0 & -- & -- \\
\hline & 13 & -2722 & 313 & 0 & -- & $\cdots$ \\
\hline & 14 & -1642 & 174 & 0 & $\ldots$ & - \\
\hline & 15 & -524 & 508 & 0 & -- & $\cdots$ \\
\hline
\end{tabular}


Table 4--Layer number; altitude of top, thickness, and sand percentage of layer; and average dissolved-solids concentration and temperature of water in layer; by log number--Continued.

\begin{tabular}{|c|c|c|c|c|c|c|}
\hline $\begin{array}{l}\text { Log } \\
\text { number }\end{array}$ & $\begin{array}{r}\text { Layer } \\
\text { number }\end{array}$ & $\begin{array}{r}\text { Altitude } \\
\text { of top } \\
\text { (feet) }\end{array}$ & $\begin{array}{r}\text { ThIck- } \\
\text { ness } \\
(\text { feet ) }\end{array}$ & $\begin{array}{r}\text { Sand } \\
\text { per- } \\
\text { centage }\end{array}$ & $\begin{array}{l}\text { Dis- } \\
\text { solved } \\
\text { solids }\end{array}$ & $\begin{array}{l}\text { Tem- } \\
\text { per- } \\
\text { ature }\end{array}$ \\
\hline \multirow[t]{12}{*}{ L ACTO2 } & 2 & -5138 & 840 & 41 & -- & -- \\
\hline & 3 & -3465 & 1673 & 50 & -- & - \\
\hline & 4 & -3295 & 170 & 74 & - & -- \\
\hline & 5 & -2213 & 778 & 56 & -- & -- \\
\hline & 6 & -1435 & 660 & 50 & -- & - \\
\hline & 7 & -253 & 692 & 32 & -- & - \\
\hline & 8 & -43 & 210 & -- & -- & -- \\
\hline & 11 & 60 & 103 & -- & -- & - \\
\hline & 12 & -5978 & -- & -- & -- & - \\
\hline & 13 & -2991 & 304 & 6 & -- & 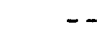 \\
\hline & 14 & -2095 & 118 & 0 & -- & -- \\
\hline & 15 & -945 & 490 & 1 & -- & - \\
\hline \multirow[t]{10}{*}{ LACTO3 } & 2 & -4196 & 624 & 57 & 72 & 56 \\
\hline & 3 & -2526 & 1670 & 64 & 54 & 47 \\
\hline & 4 & -2410 & 116 & 99 & 76 & 40 \\
\hline & 5 & -1306 & 818 & 66 & 36 & 33 \\
\hline & 6 & -546 & 606 & 64 & 17 & 26 \\
\hline & 7 & 135 & 106 & 46 & $<10$ & 19 \\
\hline & 12 & -4820 & 644 & 0 & -- & $\ldots$ \\
\hline & 13 & -2124 & 286 & 7 & -- & $\ldots$ \\
\hline & 14 & -1152 & 154 & 0 & -- & - \\
\hline & 15 & 29 & 575 & 1 & -- & -- \\
\hline \multirow[t]{7}{*}{ LACUOI } & 7 & -7589 & 1470 & 44 & 161 & 73 \\
\hline & 8 & -3464 & 3355 & 43 & 73 & 49 \\
\hline & 9 & -1744 & 1720 & 29 & 26 & 35 \\
\hline & 10 & -704 & 1040 & 45 & $<10$ & 28 \\
\hline & 11 & 5 & 709 & 76 & $<10$ & 23 \\
\hline & 15 & -9059 & 924 & 1 & -- & -- \\
\hline & 16 & -6819 & 770 & 4 & -- & - \\
\hline \multirow[t]{6}{*}{ LACUO2 } & 7 & -10077 & 709 & 22 & 74 & 82 \\
\hline & 8 & -4472 & 4262 & 52 & 81 & 64 \\
\hline & 9 & -2302 & 2170 & 44 & -- & 46 \\
\hline & 10 & -1002 & 1300 & 47 & $<10$ & 33 \\
\hline & 11 & 8 & 1010 & 59 & $<10$ & 24 \\
\hline & 16 & -8734 & 1343 & 1 & -- & - \\
\hline \multirow[t]{7}{*}{ LACUO3 } & 7 & -8178 & 685 & 37 & 82 & 64 \\
\hline & 8 & -3718 & 3602 & 57 & 93 & 49 \\
\hline & 9 & -1883 & 1835 & 44 & 62 & 35 \\
\hline & 10 & -773 & 1110 & 51 & 21 & 28 \\
\hline & 11 & 8 & 781 & 65 & $<10$ & 23 \\
\hline & 15 & -8863 & 2013 & 3 & -- & -- \\
\hline & 16 & -7320 & 858 & 7 & -- & -- \\
\hline \multirow[t]{7}{*}{$\mathrm{LACUO} 4$} & 7 & -6484 & 2895 & 35 & 116 & 61 \\
\hline & 8 & -3258 & 2756 & 68 & 142 & 42 \\
\hline & 9 & -1604 & 1654 & 31 & 12 & 31 \\
\hline & 10 & -704 & 900 & 51 & $<10$ & 26 \\
\hline & 11 & 7 & 711 & 67 & $<10$ & 23 \\
\hline & 15 & -9379 & -- & -- & -- & - \\
\hline & 16 & -6014 & 470 & 5 & -- & -- \\
\hline
\end{tabular}


Table 4--Layer number; altitude of top, thickness, and sand percentage of layer: and average dissolved-solids concentration and temperature of water in layer; by log number--Continued.

\begin{tabular}{|c|c|c|c|c|c|c|}
\hline $\begin{array}{l}\text { Log } \\
\text { number }\end{array}$ & $\begin{array}{l}\text { Layer } \\
\text { number }\end{array}$ & $\begin{array}{r}\text { Altitude } \\
\text { of top } \\
\text { (feet) }\end{array}$ & $\begin{array}{r}\text { Thick- } \\
\text { ness } \\
(\text { feet })\end{array}$ & $\begin{array}{r}\text { Sand } \\
\text { per- } \\
\text { centage }\end{array}$ & $\begin{array}{l}\text { Dis - } \\
\text { solved } \\
\text { solids }\end{array}$ & $\begin{array}{l}\text { Tem- } \\
\text { per- } \\
\text { ature }\end{array}$ \\
\hline \multirow[t]{3}{*}{ LADSOI } & 2 & -75 & 20 & 50 & -- & 23 \\
\hline & 3 & 225 & 300 & 51 & $<10$ & 20 \\
\hline & 12 & -95 & 759 & 1 & -- & - \\
\hline \multirow{3}{*}{ LADSO 2} & 2 & 55 & 20 & 50 & -- & 21 \\
\hline & 3 & 320 & 265 & 66 & $<10$ & 20 \\
\hline & 12 & 35 & 798 & 0 & -- & - \\
\hline \multirow[t]{3}{*}{ LADSO 3} & 2 & -48 & 20 & 50 & -- & 22 \\
\hline & 3 & 227 & 275 & 81 & $<10$ & 20 \\
\hline & 12 & -68 & 880 & 1 & -- & -- \\
\hline \multirow[t]{8}{*}{ LAEBOI } & 3 & -10096 & 1210 & 17 & -- & 85 \\
\hline & 7 & -4619 & 3387 & 49 & 63 & 62 \\
\hline & 8 & -1946 & 2673 & 53 & 66 & 47 \\
\hline & 9 & -1246 & 700 & 57 & -- & 32 \\
\hline & 10 & -543 & 703 & 64 & -- & 26 \\
\hline & 11 & 68 & 611 & 50 & -- & 20 \\
\hline & 12 & -11306 & 3772 & 1 & -- & -- \\
\hline & 15 & -8006 & 2090 & 1 & -- & -- \\
\hline \multirow[t]{6}{*}{$L A E B O 2$} & 7 & -6656 & 3232 & 30 & 94 & 66 \\
\hline & 8 & -3051 & 3605 & 55 & 91 & 46 \\
\hline & 9 & -2126 & 925 & 25 & $<10$ & 34 \\
\hline & 10 & -1026 & 1100 & 41 & $<10$ & 28 \\
\hline & 11 & 23 & 1049 & 52 & $<10$ & 22 \\
\hline & 15 & -9888 & 2170 & 1 & -- & -- \\
\hline \multirow[t]{8}{*}{ LAECOI } & 2 & -2044 & 340 & 40 & $<10$ & 40 \\
\hline & 3 & -1530 & 514 & 31 & -- & 36 \\
\hline & 4 & -1491 & 39 & 99 & $<10$ & 34 \\
\hline & 5 & -252 & 935 & 47 & $<10$ & 26 \\
\hline & 6 & -7 & 245 & 95 & $<10$ & 20 \\
\hline & 11 & 113 & 120 & -- & -- & 18 \\
\hline & 12 & -2384 & 480 & 0 & -- & - \\
\hline & 13 & -1187 & 304 & 5 & -- & -- \\
\hline \multirow[t]{9}{*}{ L AECO2 } & 2 & -2196 & 378 & 92 & 25 & 40 \\
\hline & 3 & -1614 & 582 & 43 & 10 & 36 \\
\hline & 4 & -1513 & 101 & 99 & $<10$ & 33 \\
\hline & 5 & -549 & 605 & 50 & $<10$ & 26 \\
\hline & 6 & -19 & 398 & 73 & $<10$ & 20 \\
\hline & 11 & 90 & 109 & -- & - - & 18 \\
\hline & 12 & -2574 & 329 & 0 & -- & -- \\
\hline & 13 & -1154 & 359 & 6 & -- & -- \\
\hline & 14 & -417 & 132 & 31 & -- & -- \\
\hline \multirow[t]{10}{*}{ LAEFOI } & 2 & -9145 & -- & -- & -- & -- \\
\hline & 3 & -6540 & 2605 & 39 & 43 & 63 \\
\hline & 5 & -5495 & 466 & 32 & -- & 52 \\
\hline & 7 & -2565 & 2307 & 49 & - & 40 \\
\hline & 8 & -965 & 1600 & 52 & $<10$ & 29 \\
\hline & 9 & -325 & 640 & 25 & $<10$ & 23 \\
\hline & 10 & 75 & 400 & 49 & $<10$ & 20 \\
\hline & 11 & 260 & 185 & 33 & $<10$ & 19 \\
\hline & 13 & -5961 & 579 & 6 & -- & -- \\
\hline & 15 & -4872 & 623 & 7 & -- & -- \\
\hline
\end{tabular}


Table 4--Layer number; altitude of top, thickness, and sand percentage of layer; and average dissolved-solids concentration and temperature of water in layer; by log number--Continued.

\begin{tabular}{|c|c|c|c|c|c|c|}
\hline $\begin{array}{l}\text { Log } \\
\text { number }\end{array}$ & $\begin{array}{r}\text { Layer } \\
\text { number }\end{array}$ & $\begin{array}{r}\text { Altitude } \\
\text { of top } \\
\text { (feet) }\end{array}$ & $\begin{array}{r}\text { Thick- } \\
\text { ness } \\
(\text { feet })\end{array}$ & $\begin{array}{r}\text { Sand } \\
\text { per- } \\
\text { centage }\end{array}$ & $\begin{array}{l}\text { Dis- } \\
\text { solved } \\
\text { solids }\end{array}$ & $\begin{array}{l}\text { Tem- } \\
\text { per- } \\
\text { ature }\end{array}$ \\
\hline \multirow[t]{9}{*}{ LAEFO 2} & 2 & -10515 & 895 & 26 & 100 & 90 \\
\hline & 3 & -7947 & 2568 & 22 & 55 & 77 \\
\hline & 7 & -3349 & 2892 & 36 & 65 & 45 \\
\hline & 8 & -1328 & 2021 & 49 & 29 & 32 \\
\hline & 9 & -671 & 657 & 40 & $<10$ & 25 \\
\hline & 10 & -42 & 629 & 50 & $<10$ & 22 \\
\hline & 11 & 199 & 241 & 85 & $<10$ & 19 \\
\hline & 12 & -11410 & 1123 & 1 & -- & -- \\
\hline & 15 & -6241 & 1706 & 2 & -- & - \\
\hline \multirow[t]{11}{*}{ I AEVO I } & 3 & -9577 & -- & -- & -- & - \\
\hline & 5 & -8631 & 208 & 37 & 22 & 65 \\
\hline & 6 & -7411 & 367 & 16 & 51 & 60 \\
\hline & 7 & -3538 & 2836 & 57 & 68 & 50 \\
\hline & 8 & -1673 & 1865 & 55 & 52 & 41 \\
\hline & 9 & -690 & 983 & 34 & $<10$ & 31 \\
\hline & 10 & -38 & 652 & 29 & $<10$ & 23 \\
\hline & 11 & 105 & 143 & 85 & $<10$ & 18 \\
\hline & 13 & -8839 & 738 & 0 & -- & -- \\
\hline & 14 & -7778 & 853 & 2 & -- & -- \\
\hline & 15 & -6374 & 1037 & 12 & -- & - \\
\hline \multirow[t]{12}{*}{ LAEVO 2} & 3 & -9596 & 3432 & 30 & 43 & 94 \\
\hline & 5 & -8438 & 390 & 23 & 80 & 78 \\
\hline & 6 & -7396 & 265 & 25 & 74 & 72 \\
\hline & 7 & -3529 & 2997 & 48 & 89 & 57 \\
\hline & 8 & -1699 & 1830 & 42 & 61 & 41 \\
\hline & 9 & -661 & 1038 & 40 & $<10$ & 29 \\
\hline & 10 & -108 & 553 & 18 & -- & 23 \\
\hline & 11 & 60 & 168 & -- & -- & 20 \\
\hline & 12 & -13028 & 2345 & 0 & -- & - \\
\hline & 13 & -8828 & 768 & 5 & -- & -- \\
\hline & 14 & -7661 & 777 & 4 & -- & -- \\
\hline & 15 & -6526 & 870 & 0 & -- & -- \\
\hline \multirow[t]{9}{*}{ LAEVO 3} & 3 & -11433 & -- & -- & -- & - \\
\hline & 5 & -10248 & 319 & 32 & 54 & 78 \\
\hline & 7 & -4439 & 3596 & 46 & 53 & 63 \\
\hline & 8 & -2127 & 2312 & 55 & 57 & 49 \\
\hline & 9 & -950 & 1177 & 48 & $<10$ & 33 \\
\hline & 10 & -213 & 737 & 44 & $<10$ & 25 \\
\hline & 11 & 66 & 279 & -- & $<10$ & 20 \\
\hline & 13 & -10567 & 866 & 0 & - & $\ldots$ \\
\hline & 15 & -8035 & 2213 & 2 & -- & -- \\
\hline \multirow[t]{6}{*}{ LAEVO 4} & 7 & -5374 & 2590 & 37 & 43 & 59 \\
\hline & 8 & -2777 & 2597 & 43 & 40 & 47 \\
\hline & 9 & -1527 & 1250 & 30 & $<10$ & 38 \\
\hline & 10 & -354 & 1173 & 47 & $<10$ & 28 \\
\hline & 11 & 51 & 405 & 84 & $<10$ & 21 \\
\hline & 15 & -7964 & 2844 & 3 & - & - \\
\hline
\end{tabular}


Table 4--Layer number; altitude of top, thickness, and sand percentage of layer; and average dissolved-solids concentration and temperature of water in layer; by log number--Continued.

\begin{tabular}{|c|c|c|c|c|c|c|}
\hline $\begin{array}{l}\text { Log } \\
\text { number }\end{array}$ & $\begin{array}{r}\text { Layer } \\
\text { number }\end{array}$ & $\begin{array}{r}\text { Altitude } \\
\text { of top } \\
\text { (feet) }\end{array}$ & $\begin{array}{r}\text { Thlck- } \\
\text { ness } \\
\text { (feet) }\end{array}$ & $\begin{array}{r}\text { Sand } \\
\text { per- } \\
\text { centage }\end{array}$ & $\begin{array}{l}\text { Dis- } \\
\text { solved } \\
\text { solids }\end{array}$ & $\begin{array}{l}\text { Tem- } \\
\text { per- } \\
\text { ature }\end{array}$ \\
\hline \multirow[t]{10}{*}{ LAFROI } & 2 & -4250 & 588 & 43 & 25 & 52 \\
\hline & 3 & -2682 & 1568 & 61 & 41 & 44 \\
\hline & 4 & -2500 & 182 & 91 & 50 & 38 \\
\hline & 5 & -1300 & 830 & 56 & 33 & 32 \\
\hline & 6 & -870 & 260 & 69 & 15 & 27 \\
\hline & 11 & 64 & 114 & - & -- & 20 \\
\hline & 12 & -4838 & 562 & 0 & -- & - \\
\hline & 13 & -2130 & 370 & 0 & -- & -- \\
\hline & 14 & -1130 & 170 & 0 & -- & -- \\
\hline & 15 & -50 & 820 & 9 & -- & -- \\
\hline \multirow[t]{9}{*}{$\mathrm{LAFRO2}$} & 2 & -2839 & 660 & 45 & 28 & 41 \\
\hline & 3 & -2159 & 680 & 56 & 27 & 37 \\
\hline & 4 & -1839 & 320 & 76 & -- & 33 \\
\hline & 5 & -813 & 716 & 64 & 23 & 28 \\
\hline & 6 & -19 & 630 & - & 19 & 22 \\
\hline & 11 & 70 & 89 & -- & -- & 20 \\
\hline & 12 & -3499 & 571 & 0 & -- & -- \\
\hline & 13 & -1529 & 310 & 1 & - & -- \\
\hline & 14 & -649 & 164 & 0 & -- & - \\
\hline \multirow[t]{10}{*}{ LAFRO3 } & 2 & -3913 & 537 & 86 & 78 & 67 \\
\hline & 3 & -2769 & 1144 & 57 & 51 & 58 \\
\hline & 4 & -2468 & 301 & 83 & 54 & 49 \\
\hline & 5 & -1273 & 820 & 58 & 45 & 39 \\
\hline & 6 & -465 & 676 & 51 & 12 & 29 \\
\hline & 11 & 68 & 102 & 62 & $<10$ & 20 \\
\hline & 12 & -4450 & 523 & 2 & - & $\ldots$ \\
\hline & 13 & -2093 & 375 & 2 & -- & - \\
\hline & 14 & -1141 & 132 & 0 & -- & $\ldots$ \\
\hline & 15 & -34 & 431 & 0 & -- & -- \\
\hline \multirow[t]{10}{*}{ LAFRO 4} & 2 & -2976 & 198 & 89 & 38 & 42 \\
\hline & 3 & -2046 & 930 & 57 & 24 & 38 \\
\hline & 4 & -1914 & 132 & 99 & 24 & 34 \\
\hline & 5 & -774 & 800 & 80 & 24 & 28 \\
\hline & 6 & -154 & 493 & 63 & $<10$ & 23 \\
\hline & 11 & 88 & 122 & -- & $\cdots$ & 20 \\
\hline & 12 & -3174 & 447 & 0 & -- & - \\
\hline & 13 & -1574 & 340 & 0 & -- & -- \\
\hline & 14 & -647 & 127 & 0 & -- & -- \\
\hline & 15 & -34 & 120 & -- & -- & -- \\
\hline \multirow[t]{9}{*}{ LAGRO 1} & 3 & -3157 & -- & -- & -- & -- \\
\hline & 4 & -3004 & 153 & 70 & -- & -- \\
\hline & 5 & -2144 & 670 & 48 & -- & -- \\
\hline & 6 & -1274 & 680 & 33 & -- & -- \\
\hline & 7 & -94 & 616 & 30 & -- & -- \\
\hline & 8 & 100 & 194 & -- & -- & -- \\
\hline & 13 & -2814 & 190 & 0 & -- & -- \\
\hline & 14 & -1954 & 190 & 2 & -- & -- \\
\hline & 15 & -710 & 564 & 2 & -- & - \\
\hline
\end{tabular}


Table 4--Layer number; altitude of top, thickness, and sand percentage of layer; and average dissolved-solids concentration and temperature of water in layer; by log number--Continued.

\begin{tabular}{|c|c|c|c|c|c|c|}
\hline $\begin{array}{l}\text { Log } \\
\text { number }\end{array}$ & $\begin{array}{r}\text { Layer } \\
\text { number }\end{array}$ & $\begin{array}{r}\text { Altitude } \\
\text { of top } \\
\text { (feet) }\end{array}$ & $\begin{array}{r}\text { Thick- } \\
\text { ness } \\
(\text { feet) }\end{array}$ & $\begin{array}{r}\text { Sand } \\
\text { per- } \\
\text { centage }\end{array}$ & $\begin{array}{l}\text { Dis- } \\
\text { solved } \\
\text { solids }\end{array}$ & $\begin{array}{l}\text { Tem- } \\
\text { per- } \\
\text { ature }\end{array}$ \\
\hline \multirow[t]{11}{*}{ L AGRO 2} & 2 & -6339 & 298 & 62 & 43 & 71 \\
\hline & 3 & -3497 & 2842 & 40 & 40 & 62 \\
\hline & 4 & -3277 & 220 & 99 & 54 & 49 \\
\hline & 5 & -2347 & 634 & 59 & 24 & 43 \\
\hline & 6 & -1387 & 752 & 43 & $<10$ & 35 \\
\hline & 7 & -67 & 712 & 24 & $<10$ & 24 \\
\hline & 8 & 138 & 205 & 54 & $<10$ & 20 \\
\hline & 12 & -6637 & 790 & 0 & -- & $\cdots$ \\
\hline & 13 & -2981 & 296 & 0 & -- & - \\
\hline & 14 & -2139 & 208 & 2 & -- & -- \\
\hline & 15 & -779 & 608 & 0 & -- & -- \\
\hline \multirow[t]{9}{*}{$\mathrm{LAGRO} 3$} & 2 & -4089 & -- & -- & -- & -- \\
\hline & 3 & -1889 & 2200 & 60 & - & -- \\
\hline & 4 & -1818 & 71 & 99 & -- & -- \\
\hline & 5 & -1115 & 500 & 70 & -- & - \\
\hline & 6 & -369 & 633 & 22 & -- & $\cdots$ \\
\hline & 7 & 225 & 227 & -- & -- & -- \\
\hline & 13 & -1615 & 203 & 0 & -- & -- \\
\hline & 14 & -1002 & 113 & 6 & -- & - \\
\hline & 15 & -2 & 367 & 0 & -- & - \\
\hline \multirow[t]{9}{*}{ L AG RO 4} & 2 & -3777 & 486 & 50 & 36 & 49 \\
\hline & 3 & -1740 & 2037 & 51 & 34 & 40 \\
\hline & 4 & -1530 & 210 & 81 & 42 & 32 \\
\hline & 5 & -813 & 533 & 62 & 23 & 28 \\
\hline & 6 & -5 & 667 & 21 & $<10$ & 23 \\
\hline & 12 & -4263 & 752 & 0 & - - & -- \\
\hline & 13 & -1346 & 184 & 0 & -- & -- \\
\hline & 14 & -672 & 141 & 3 & -- & -- \\
\hline & 15 & 190 & 195 & -- & -- & -- \\
\hline \multirow[t]{4}{*}{ LAIAO 1} & 8 & -7257 & 4133 & 66 & 213 & 72 \\
\hline & 9 & -3837 & 3420 & 50 & 287 & 46 \\
\hline & 10 & -1053 & 2784 & 54 & 45 & 28 \\
\hline & 11 & 3 & 1056 & 51 & $<10$ & 22 \\
\hline \multirow[t]{5}{*}{$\mathrm{LAIAO} 2$} & 8 & -7215 & 4750 & 57 & 157 & 70 \\
\hline & 9 & -3785 & 3430 & 63 & 131 & 55 \\
\hline & 10 & -1006 & 2779 & 65 & 55 & 43 \\
\hline & 11 & 18 & 1024 & 71 & $<10$ & 22 \\
\hline & 16 & -11965 & 517 & 8 & -- & -- \\
\hline \multirow[t]{4}{*}{$\mathrm{LAIAO} 3$} & 8 & -10566 & 4416 & 10 & 101 & 92 \\
\hline & 9 & -5506 & 5060 & 46 & 97 & 63 \\
\hline & 10 & -1123 & 4383 & 52 & 37 & 35 \\
\hline & 11 & 1 & 1124 & 73 & $<10$ & 23 \\
\hline
\end{tabular}


Table 4--Layer number; altitude of top, thickness, and sand percentage of layer; and average dissolved-solids concentration and temperature of water in layer; by log number--Continued.

\begin{tabular}{|c|c|c|c|c|c|c|}
\hline $\begin{array}{l}\log \\
\text { number }\end{array}$ & $\begin{array}{r}\text { Layer } \\
\text { number }\end{array}$ & $\begin{array}{r}\text { Altitude } \\
\text { of top } \\
(f \in e t)\end{array}$ & $\begin{array}{r}\text { Thick- } \\
\text { ness } \\
\text { (feet) }\end{array}$ & $\begin{array}{r}\text { Sand } \\
\text { per- } \\
\text { centage }\end{array}$ & $\begin{array}{l}\text { Dis- } \\
\text { solved } \\
\text { solids }\end{array}$ & $\begin{array}{l}\text { Tem- } \\
\text { per- } \\
\text { ature }\end{array}$ \\
\hline \multirow[t]{4}{*}{ I A I AO 4} & 8 & -8659 & 4018 & 55 & 123 & 74 \\
\hline & 9 & -4549 & 4110 & 53 & 91 & 56 \\
\hline & 10 & -1057 & 3492 & 59 & 59 & 39 \\
\hline & 11 & 0 & 1057 & 62 & $<10$ & 23 \\
\hline \multirow[t]{5}{*}{ L AIBO 1} & 8 & -6500 & 4800 & 53 & 165 & 59 \\
\hline & 9 & -3632 & 2868 & 63 & 154 & 42 \\
\hline & 10 & -1030 & 2602 & 49 & 86 & 30 \\
\hline & 11 & 2 & 1032 & 78 & $<10$ & 22 \\
\hline & 16 & -11300 & 309 & 0 & - & $\cdots$ \\
\hline \multirow[t]{6}{*}{ LAIBO2 } & 7 & -10555 & 3226 & 12 & 111 & 78 \\
\hline & 8 & -5650 & 4000 & 63 & 79 & 66 \\
\hline & 9 & -3150 & 2500 & 50 & 55 & 57 \\
\hline & 10 & -929 & 2221 & 41 & 25 & 41 \\
\hline & 11 & 12 & 941 & 84 & $<10$ & 23 \\
\hline & 16 & -9650 & 905 & 14 & -- & $\cdots$ \\
\hline \multirow[t]{5}{*}{ LAI $B O 3$} & 7 & -8126 & -- & -- & -- & - \\
\hline & 8 & -4194 & 3932 & 66 & 136 & 54 \\
\hline & 9 & -2548 & 1646 & 65 & 124 & 41 \\
\hline & 10 & -975 & 1573 & 22 & $<10$ & 33 \\
\hline & 11 & 10 & 985 & 67 & $<10$ & 22 \\
\hline \multirow[t]{7}{*}{ LAJAO 1} & 3 & -1098 & 506 & 40 & $<10$ & 34 \\
\hline & 4 & -950 & 148 & 72 & $<10$ & 31 \\
\hline & 5 & -24 & 668 & 53 & $<10$ & 24 \\
\hline & 6 & 180 & 34 & - & -- & 19 \\
\hline & 12 & -1604 & 662 & 2 & -- & - \\
\hline & 13 & -692 & 258 & 0 & -- & -- \\
\hline & 14 & 146 & 170 & 0 & -- & - \\
\hline \multirow[t]{6}{*}{ LAJAO2 } & 2 & -1584 & 82 & 99 & 18 & 34 \\
\hline & 3 & -634 & 950 & 44 & 11 & 30 \\
\hline & 4 & -489 & 145 & 68 & $<10$ & 26 \\
\hline & 5 & 130 & 364 & 55 & $<10$ & 22 \\
\hline & 12 & -1666 & 690 & 0 & 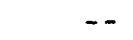 & - \\
\hline & 13 & -234 & 255 & 0 & -- & - \\
\hline \multirow[t]{5}{*}{ LAJAO3 } & 4 & -1022 & -- & -- & -. & - \\
\hline & 5 & -73 & 705 & 70 & $<10$ & 26 \\
\hline & 6 & 254 & 171 & 38 & - & 15 \\
\hline & 13 & -778 & 244 & 0 & -- & - \\
\hline & 14 & 83 & 156 & 0 & -- & - \\
\hline \multirow[t]{8}{*}{$\mathrm{LAJAO} 4$} & 2 & -2037 & 305 & 74 & 22 & 39 \\
\hline & 3 & -1148 & 889 & 60 & 18 & 34 \\
\hline & 4 & -1004 & 144 & 96 & 25 & 30 \\
\hline & 5 & -64 & 700 & 67 & $<10$ & 25 \\
\hline & 6 & 260 & 168 & - & - & 20 \\
\hline & 12 & -2342 & 736 & 0 & -- & $\ldots$ \\
\hline & 13 & -764 & 240 & 0 & -- & $\cdots$ \\
\hline & 14 & 92 & 156 & $\ldots$ & -- & $\ldots$ \\
\hline
\end{tabular}


Table 4--Layer number; altitude of top, thickness, and sand percentage of layer; and average dissolved-solids concentration and temperature of water in layer; by log number--Continued.

\begin{tabular}{|c|c|c|c|c|c|c|}
\hline $\begin{array}{l}\mathrm{L} \circ \mathrm{g} \\
\text { number }\end{array}$ & $\begin{array}{r}\text { Layer } \\
\text { number }\end{array}$ & $\begin{array}{r}\text { Altitude } \\
\text { of top } \\
(f \in e t)\end{array}$ & $\begin{array}{r}\text { Thlck- } \\
\text { ness } \\
(\text { feet) }\end{array}$ & $\begin{array}{r}\text { Sand } \\
\text { per- } \\
\text { centage }\end{array}$ & $\begin{array}{l}\text { Dis- } \\
\text { solved } \\
\text { solids }\end{array}$ & $\begin{array}{l}\text { Tem- } \\
\text { per- } \\
\text { ature }\end{array}$ \\
\hline \multirow[t]{7}{*}{ LAJDO 1} & 7 & -9523 & 898 & 38 & -- & 72 \\
\hline & 8 & -4137 & 3839 & 59 & 166 & 56 \\
\hline & 9 & -2186 & 1951 & 55 & 143 & 43 \\
\hline & 10 & -866 & 1320 & 55 & 103 & 34 \\
\hline & 11 & 24 & 890 & 90 & -- & 24 \\
\hline & 15 & -10421 & 1483 & 8 & -- & -- \\
\hline & 16 & -7976 & 1547 & 6 & -- & -- \\
\hline \multirow[t]{7}{*}{ LAJDO 2} & 7 & -8298 & 1595 & 39 & 121 & 68 \\
\hline & 8 & -3969 & 3756 & 48 & 120 & 51 \\
\hline & 9 & -2069 & 1900 & 67 & 87 & 36 \\
\hline & 10 & -778 & 1291 & -- & 108 & 28 \\
\hline & 11 & 25 & 803 & -- & -- & 23 \\
\hline & 15 & -9893 & 73 & 0 & -- & -- \\
\hline & 16 & -7725 & 573 & 6 & -- & -- \\
\hline \multirow[t]{5}{*}{ LAJDO 3} & 8 & -4912 & 4201 & 61 & 107 & 65 \\
\hline & 9 & -2413 & 2499 & 56 & 63 & 50 \\
\hline & 10 & -887 & 1526 & 65 & 37 & 34 \\
\hline & 11 & 7 & 894 & 60 & $<10$ & 23 \\
\hline & 16 & -9113 & 1970 & 1 & -- & - \\
\hline \multirow[t]{3}{*}{$\mathrm{LAJDO} 4$} & 9 & -2077 & -- & -- & -- & -- \\
\hline & 10 & -786 & 1291 & 44 & $<10$ & 28 \\
\hline & 11 & 27 & 813 & 67 & $<10$ & 23 \\
\hline \multirow[t]{5}{*}{$\angle A J F 01$} & 8 & -4489 & 3690 & 51 & 93 & 63 \\
\hline & 9 & -2589 & 1900 & 36 & 40 & 50 \\
\hline & 10 & -789 & 1800 & 49 & 15 & 34 \\
\hline & 11 & 0 & 789 & 58 & $<10$ & 22 \\
\hline & 16 & -8179 & 2693 & 0 & -- & -- \\
\hline \multirow[t]{11}{*}{$\operatorname{LALAOI}$} & 3 & -2984 & 2688 & 40 & 59 & 47 \\
\hline & 4 & -2942 & 42 & 79 & -- & 39 \\
\hline & 5 & -2162 & 680 & 35 & 55 & 36 \\
\hline & 6 & -1562 & 470 & 34 & 51 & 32 \\
\hline & 7 & -372 & 728 & 41 & 10 & 25 \\
\hline & 8 & -52 & 320 & -- & -- & 22 \\
\hline & 11 & 51 & 103 & -- & -- & 20 \\
\hline & 12 & -5672 & 613 & 0 & -- & -- \\
\hline & 13 & -2842 & 100 & 0 & -- & -- \\
\hline & 14 & -2032 & 130 & 7 & -- & -- \\
\hline & 15 & -1100 & 462 & 3 & -- & -- \\
\hline \multirow[t]{8}{*}{$\mathrm{LALAO} 2$} & 2 & -2658 & 1090 & 63 & 22 & 50 \\
\hline & 3 & -1528 & 1130 & 46 & 18 & 40 \\
\hline & 4 & -1465 & 63 & 99 & 20 & 34 \\
\hline & 5 & -612 & 606 & 50 & $<10$ & 29 \\
\hline & 6 & 102 & 544 & 76 & $<10$ & 22 \\
\hline & 12 & -3748 & -- & -- & -- & -- \\
\hline & 13 & -1218 & 247 & 9 & -- & -- \\
\hline & 14 & -442 & 170 & 13 & -- & -- \\
\hline
\end{tabular}


Table 4--Layer number; altitude of top, thickness, and sand percentage of layer; and average dissolved-solids concentration and temperature of water in layer; by log number--Continued.

\begin{tabular}{|c|c|c|c|c|c|c|}
\hline $\begin{array}{l}\log \\
\text { number }\end{array}$ & $\begin{array}{r}\text { Layer } \\
\text { number }\end{array}$ & $\begin{array}{r}\text { Altitude } \\
\text { of top } \\
(f e e t)\end{array}$ & $\begin{array}{r}\text { Thick- } \\
\text { ness } \\
\text { (feet) }\end{array}$ & $\begin{array}{r}\text { Sand } \\
\text { per- } \\
\text { centage }\end{array}$ & $\begin{array}{l}\text { Dis- } \\
\text { solved } \\
\text { solids }\end{array}$ & $\begin{array}{l}\text { Tem- } \\
\text { per- } \\
\text { ature }\end{array}$ \\
\hline \multirow[t]{10}{*}{$\mathrm{LALAO} 3$} & 2 & -4033 & 649 & 49 & 53 & 57 \\
\hline & 3 & -2190 & 1843 & 34 & 29 & 48 \\
\hline & 4 & -2084 & 106 & 99 & 43 & 41 \\
\hline & 5 & -1309 & 668 & 61 & 19 & 36 \\
\hline & 6 & -634 & 532 & 39 & $<10$ & 29 \\
\hline & 7 & 166 & 288 & 30 & $<10$ & 20 \\
\hline & 12 & -4682 & 629 & 0 & - & -- \\
\hline & 13 & -1977 & 107 & 0 & $\cdots$ & $\cdots$ \\
\hline & 14 & -1166 & 143 & 0 & -- & -- \\
\hline & 15 & -122 & 512 & 2 & -- & -- \\
\hline \multirow[t]{5}{*}{ LALFOI } & 8 & -4936 & 4704 & 63 & 70 & 59 \\
\hline & 9 & -2456 & 2480 & 55 & 63 & 44 \\
\hline & 10 & -944 & 1512 & 54 & 20 & 33 \\
\hline & 11 & 26 & 970 & 91 & $<10$ & 23 \\
\hline & 16 & -9640 & 1786 & 5 & - & - \\
\hline \multirow[t]{8}{*}{ LALIOI } & 3 & -9248 & 2738 & 6 & -- & 77 \\
\hline & 7 & -4485 & 3334 & 47 & 81 & 53 \\
\hline & 8 & -1542 & 2943 & 53 & 27 & 36 \\
\hline & 9 & -867 & 675 & 36 & $<10$ & 27 \\
\hline & 10 & -229 & 638 & 71 & $<10$ & 23 \\
\hline & 11 & 75 & 304 & -- & $<10$ & 21 \\
\hline & 12 & -11986 & 1618 & 0 & - & -- \\
\hline & 15 & -7819 & 1429 & 1 & -- & -- \\
\hline \multirow[t]{6}{*}{$\mathrm{LAL} I 02$} & 7 & -8477 & 1910 & 6 & -- & 73 \\
\hline & 8 & -4429 & 4048 & 53 & 73 & 57 \\
\hline & 9 & -2740 & 1689 & 50 & 42 & 41 \\
\hline & 10 & -900 & 1840 & 47 & 24 & 30 \\
\hline & 11 & 0 & 900 & 71 & $<10$ & 23 \\
\hline & 15 & -10387 & - & $\cdots$ & -- & -- \\
\hline \multirow[t]{6}{*}{$\mathrm{LALIO} 3$} & 7 & -8594 & 1934 & 16 & 34 & 91 \\
\hline & 8 & -4514 & 4080 & 49 & 57 & 74 \\
\hline & 9 & -2798 & 1716 & 32 & 43 & 57 \\
\hline & 10 & -955 & 1843 & 51 & $<10$ & 47 \\
\hline & 11 & 1 & 956 & 64 & $<10$ & 23 \\
\hline & 15 & -10528 & -- & -- & -- & - \\
\hline \multirow[t]{8}{*}{ LAL I 04} & 3 & -10211 & 492 & 11 & 48 & 87 \\
\hline & 7 & -6075 & 2276 & 22 & 104 & 65 \\
\hline & 8 & -2706 & 3369 & 57 & 68 & 45 \\
\hline & 9 & -1861 & 845 & 30 & $<10$ & 33 \\
\hline & 10 & -733 & 1128 & 44 & $<10$ & 27 \\
\hline & 11 & 4 & 737 & 75 & $<10$ & 22 \\
\hline & 12 & -10703 & 3330 & 0 & - & - \\
\hline & 15 & -8351 & 1860 & 0 & $\ldots$ & -- \\
\hline
\end{tabular}


Table 4--Layer number; altitude of top, thickness, and sand percentage of layer; and average dissolved-solids concentration and temperature of water in layer; by log number--Continued.

\begin{tabular}{|c|c|c|c|c|c|c|}
\hline $\begin{array}{l}\text { Log } \\
\text { number }\end{array}$ & $\begin{array}{r}\text { Layer } \\
\text { number }\end{array}$ & $\begin{array}{r}\text { Altitude } \\
\text { of top } \\
\text { ( } f \text { eet) }\end{array}$ & $\begin{array}{r}\text { Thick- } \\
\text { ness } \\
\text { (feet) }\end{array}$ & $\begin{array}{r}\text { Sand } \\
\text { per- } \\
\text { centage }\end{array}$ & $\begin{array}{l}\text { Dis- } \\
\text { solved } \\
\text { solids }\end{array}$ & $\begin{array}{l}\text { Tem- } \\
\text { Per- } \\
\text { ature }\end{array}$ \\
\hline \multirow[t]{6}{*}{ LALIOS } & 7 & -6545 & 2220 & 18 & -- & 65 \\
\hline & 8 & -2854 & 3691 & 58 & -- & 48 \\
\hline & 9 & -1981 & 873 & 51 & $<10$ & 34 \\
\hline & 10 & -830 & 1151 & 37 & $<10$ & 28 \\
\hline & 11 & 12 & 842 & 43 & $<10$ & 22 \\
\hline & 15 & -8765 & -- & -- & -- & - \\
\hline \multirow[t]{8}{*}{ LALNOI } & 2 & -1060 & 90 & 39 & - & 34 \\
\hline & 3 & -757 & 303 & 24 & -- & 32 \\
\hline & 4 & -737 & 20 & 50 & -- & 30 \\
\hline & 5 & 97 & 610 & 63 & $<10$ & 22 \\
\hline & 6 & 315 & 42 & -- & - & 16 \\
\hline & 12 & -1150 & 643 & 0 & - - & - - \\
\hline & 13 & -513 & 224 & 0 & $\ldots$ & -- \\
\hline & 14 & 273 & 176 & 26 & -- & -- \\
\hline \multirow[t]{8}{*}{ LALNO2 } & 2 & -1291 & 20 & 50 & -- & 34 \\
\hline & 3 & -659 & 632 & 44 & $<10$ & 31 \\
\hline & 4 & -639 & 20 & 50 & - & 27 \\
\hline & 5 & 52 & 473 & 59 & $<10$ & 22 \\
\hline & 6 & 315 & 136 & -- & -- & 18 \\
\hline & 12 & -1311 & 633 & 0 & -- & -- \\
\hline & 13 & -421 & 218 & 0 & $\ldots$ & -- \\
\hline & 14 & 179 & 127 & 12 & -- & -- \\
\hline \multirow[t]{4}{*}{ LALROI } & 8 & -7184 & 3864 & 31 & 70 & 62 \\
\hline & 9 & -3615 & 3569 & 50 & 94 & 45 \\
\hline & 10 & -953 & 2662 & 55 & 21 & 30 \\
\hline & 11 & 2 & 955 & 51 & $<10$ & 22 \\
\hline \multirow[t]{4}{*}{ LALRO 2} & 8 & -8078 & -- & -- & -- & -- \\
\hline & 9 & -3990 & 4088 & 56 & 83 & 50 \\
\hline & 10 & -978 & 3012 & 48 & 22 & 34 \\
\hline & 11 & 0 & 978 & 68 & $<10$ & 22 \\
\hline \multirow[t]{4}{*}{ LALRO3 } & 8 & -9480 & 2128 & 20 & 104 & 66 \\
\hline & 9 & -4899 & 4581 & 62 & 129 & 50 \\
\hline & 10 & -1005 & 3894 & 37 & 50 & 32 \\
\hline & 11 & 0 & 1005 & 41 & 10 & 22 \\
\hline \multirow[t]{4}{*}{$\mathrm{LALRO}_{4}$} & 8 & -8381 & 4773 & 28 & 114 & 73 \\
\hline & 9 & -4176 & 4205 & 42 & - & 50 \\
\hline & 10 & -1021 & 3155 & 47 & - & 32 \\
\hline & 11 & 3 & 1024 & 52 & $<10$ & 22 \\
\hline \multirow[t]{4}{*}{ LALROS } & 8 & -10396 & 2595 & 22 & 62 & 84 \\
\hline & 9 & -5457 & 4939 & 57 & 121 & 63 \\
\hline & 10 & -1009 & 4448 & 51 & 66 & 37 \\
\hline & 11 & 0 & 1009 & 77 & $<10$ & 22 \\
\hline
\end{tabular}


Table 4--Layer number; altitude of top, thickness, and sand percentage of layer; and average dissolved-solids concentration and temperature of water in layer; by log number--Continued.

\begin{tabular}{|c|c|c|c|c|c|c|}
\hline $\begin{array}{l}\text { Log } \\
\text { number }\end{array}$ & $\begin{array}{r}\text { Layer } \\
\text { number }\end{array}$ & $\begin{array}{r}\text { Altude } \\
\text { of top } \\
(f e e t)\end{array}$ & $\begin{array}{r}\text { Thlck- } \\
\text { ness } \\
(\text { feet })\end{array}$ & $\begin{array}{r}\text { Sand } \\
\text { per- } \\
\text { centage }\end{array}$ & $\begin{array}{l}\text { Dis- } \\
\text { solved } \\
\text { solids }\end{array}$ & $\begin{array}{l}\text { Tem- } \\
\text { per- } \\
\text { ature }\end{array}$ \\
\hline \multirow[t]{4}{*}{ L ALRO 6} & 8 & -10826 & 7576 & 23 & 97 & 103 \\
\hline & 9 & -5527 & 5299 & 46 & 86 & 64 \\
\hline & 10 & -1006 & 4521 & 43 & 41 & 37 \\
\hline & 11 & 0 & 1006 & 37 & 23 & 22 \\
\hline \multirow[t]{4}{*}{ LALRO 7} & 8 & -11931 & 2020 & 30 & 181 & 89 \\
\hline & 9 & -6176 & 5755 & 44 & 118 & 74 \\
\hline & 10 & -1036 & 5140 & 51 & 71 & 45 \\
\hline & 11 & 0 & 1036 & 53 & 33 & 23 \\
\hline \multirow[t]{4}{*}{ LALROB } & 8 & -8347 & 4944 & 31 & 135 & 76 \\
\hline & 9 & -4147 & 4200 & 41 & 53 & 47 \\
\hline & 10 & -1072 & 3075 & 51 & 29 & 31 \\
\hline & 11 & 6 & 1078 & 59 & $<10$ & 22 \\
\hline \multirow[t]{10}{*}{ L AMA 01} & 2 & -2984 & 506 & 73 & 52 & 43 \\
\hline & 3 & -2162 & 822 & 60 & 24 & 39 \\
\hline & 4 & -2025 & 137 & 47 & -- & 35 \\
\hline & 5 & -883 & 722 & 67 & $<10$ & 29 \\
\hline & 6 & -217 & 484 & 38 & $<10$ & 23 \\
\hline & 11 & 82 & 144 & 58 & $<10$ & 20 \\
\hline & 12 & -3490 & 482 & 0 & -- & -- \\
\hline & 13 & -1605 & 420 & 3 & - & - \\
\hline & 14 & -701 & 182 & 0 & $\cdots$ & -- \\
\hline & 15 & -62 & 155 & 0 & -- & - \\
\hline \multirow[t]{10}{*}{$\mathrm{L} A M A O 2$} & 2 & -4628 & 506 & 84 & -- & 64 \\
\hline & 3 & -3128 & 1500 & 58 & 132 & 58 \\
\hline & 4 & -2810 & 318 & 79 & 92 & 53 \\
\hline & 5 & -1382 & 979 & 67 & 13 & 43 \\
\hline & 6 & -571 & 605 & 57 & $<10$ & 30 \\
\hline & 11 & 82 & 223 & -- & $<10$ & 19 \\
\hline & 12 & -5134 & 623 & 0 & - & - \\
\hline & 13 & -2361 & 449 & 0 & -- & -- \\
\hline & 14 & -1176 & 206 & 4 & -- & - \\
\hline & 15 & -141 & 430 & 0 & -- & -- \\
\hline \multirow[t]{8}{*}{ LAMOO I } & 2 & -2001 & 121 & 78 & 28 & 35 \\
\hline & 3 & -1541 & 460 & 54 & 12 & 33 \\
\hline & 4 & -1414 & 127 & 96 & 20 & 31 \\
\hline & 5 & -164 & 935 & 57 & $<10$ & 25 \\
\hline & 6 & 31 & 195 & 52 & $<10$ & 21 \\
\hline & 11 & 161 & 130 & -- & -- & 19 \\
\hline & 12 & -2122 & 457 & 0 & -- & - \\
\hline & 13 & -1099 & 315 & 4 & -- & -- \\
\hline \multirow[t]{8}{*}{ L. AMOO 2} & 2 & -1595 & 20 & 50 & -- & 32 \\
\hline & 3 & -1179 & 416 & 40 & $<10$ & 31 \\
\hline & 4 & -1154 & 25 & 99 & $<10$ & 29 \\
\hline & 5 & -165 & 680 & 73 & $<10$ & 24 \\
\hline & 6 & -32 & 133 & -- & -- & 21 \\
\hline & 11 & 60 & 92 & -- & -- & 20 \\
\hline & 12 & -1615 & 474 & 0 & -- & -- \\
\hline & 13 & -845 & 309 & 2 & -- & - \\
\hline
\end{tabular}


Table 4--Layer number; altitude of top, thickness, and sand percentage of layer; and average dissolved-solids concentration and temperature of water in layer; by log number--Continued.

\begin{tabular}{|c|c|c|c|c|c|c|}
\hline $\begin{array}{l}\text { Log } \\
\text { number }\end{array}$ & $\begin{array}{r}\text { Layer } \\
\text { number }\end{array}$ & $\begin{array}{r}\text { Altitude } \\
\text { of top } \\
\text { (feet) }\end{array}$ & $\begin{array}{r}\text { Thick- } \\
\text { ness } \\
\text { (feet) }\end{array}$ & $\begin{array}{r}\text { Sand } \\
\text { per- } \\
\text { centage }\end{array}$ & $\begin{array}{l}\text { Dis- } \\
\text { solved } \\
\text { solids }\end{array}$ & $\begin{array}{r}\text { Tem- } \\
\text { per- } \\
\text { ature }\end{array}$ \\
\hline \multirow[t]{8}{*}{ LAMOO 3} & 2 & -1792 & 20 & 50 & -- & 32 \\
\hline & 3 & -1264 & 528 & 78 & $<10$ & 30 \\
\hline & 4 & -1219 & 45 & 56 & -- & 28 \\
\hline & 5 & -169 & 764 & 59 & $<10$ & 24 \\
\hline & 6 & -39 & 130 & -- & -- & 21 \\
\hline & 11 & 76 & 115 & -- & -- & 20 \\
\hline & 12 & -1812 & 455 & 0 & -- & -- \\
\hline & 13 & -933 & 286 & 0 & -- & - \\
\hline \multirow[t]{6}{*}{ L AN AO 1} & 2 & -1093 & 430 & 48 & $<10$ & 36 \\
\hline & 3 & -628 & 465 & 40 & -- & 31 \\
\hline & 4 & -533 & 95 & 46 & $<10$ & 27 \\
\hline & 5 & 158 & 363 & 40 & $<10$ & 21 \\
\hline & 12 & -1523 & 753 & 0 & -- & -- \\
\hline & 13 & -205 & 328 & 4 & -- & -- \\
\hline \multirow[t]{6}{*}{$\mathrm{LANAO} 2$} & 2 & -2484 & 443 & 40 & 25 & 46 \\
\hline & 3 & -572 & 1912 & 35 & $<10$ & 35 \\
\hline & 4 & -441 & 131 & 73 & $<10$ & 26 \\
\hline & 5 & 102 & 321 & 55 & $<10$ & 21 \\
\hline & 12 & -2927 & 767 & 0 & -- & -- \\
\hline & 13 & -219 & 222 & 0 & -- & -- \\
\hline \multirow[t]{3}{*}{ LANAO3 } & 2 & -1332 & 500 & 56 & $<10$ & 39 \\
\hline & 3 & 303 & 1635 & 45 & $<10$ & 28 \\
\hline & 12 & -1832 & 752 & 0 & -- & -- \\
\hline \multirow[t]{10}{*}{ LANAO 4} & 2 & -4815 & 660 & 58 & 48 & 71 \\
\hline & 3 & -2500 & 2315 & 45 & 46 & 57 \\
\hline & 4 & -2327 & 173 & 39 & 34 & 45 \\
\hline & 5 & -1532 & 653 & 62 & 12 & 39 \\
\hline & 6 & -595 & 717 & 19 & -- & 30 \\
\hline & 7 & 160 & 81 & -- & -- & 20 \\
\hline & 12 & -5475 & -- & -- & -- & -- \\
\hline & 13 & -2185 & 142 & 0 & -- & -- \\
\hline & 14 & -1312 & 220 & 0 & -- & -- \\
\hline & 15 & 79 & 674 & 1 & -- & -- \\
\hline \multirow[t]{5}{*}{ L AORO 1} & 8 & -4388 & 3636 & 54 & 155 & 73 \\
\hline & 9 & -2560 & 1828 & 53 & 161 & 49 \\
\hline & 10 & -800 & 1760 & 45 & 80 & 33 \\
\hline & 11 & 0 & 800 & 42 & $<10$ & 22 \\
\hline & 16 & -8024 & 3029 & 4 & -- & - \\
\hline \multirow[t]{8}{*}{ LAOUO 1} & 2 & -1510 & 130 & 49 & 21 & 33 \\
\hline & 3 & -1140 & 370 & 55 & -- & 31 \\
\hline & 4 & -1070 & 70 & 24 & -- & 29 \\
\hline & 5 & -107 & 681 & 51 & $<10$ & 24 \\
\hline & 6 & 174 & 94 & -- & -- & 19 \\
\hline & 12 & -1640 & 644 & 0 & -- & -- \\
\hline & 13 & -788 & 282 & 3 & -- & -- \\
\hline & 14 & 80 & 187 & 11 & -- & -- \\
\hline
\end{tabular}


Table 4--Layer number; altitude of top, thickness, and sand percentage of layer; and average dissolved-solids concentration and temperature of water in layer; by log number--Continued.

\begin{tabular}{|c|c|c|c|c|c|c|}
\hline $\begin{array}{l}\text { Log } \\
\text { number }\end{array}$ & $\begin{array}{r}\text { Layer } \\
\text { number }\end{array}$ & $\begin{array}{r}\text { Altitude } \\
\text { of top } \\
(f e t)\end{array}$ & $\begin{array}{r}\text { Thlck- } \\
\text { ness } \\
\text { (feet) }\end{array}$ & $\begin{array}{r}\text { Sand } \\
\text { per- } \\
\text { centage }\end{array}$ & $\begin{array}{l}\text { Dis- } \\
\text { solved } \\
\text { solids }\end{array}$ & $\begin{array}{l}\text { Tem- } \\
\text { per- } \\
\text { ature }\end{array}$ \\
\hline \multirow[t]{8}{*}{ L AOU 02} & 2 & -1701 & 20 & 50 & -- & 33 \\
\hline & 3 & -1113 & 588 & 42 & 11 & 31 \\
\hline & 4 & -985 & 128 & 90 & 34 & 28 \\
\hline & 5 & -133 & 591 & 62 & $<10$ & 23 \\
\hline & 6 & 92 & 75 & -- & -- & 20 \\
\hline & 12 & -1721 & 724 & 0 & -- & -- \\
\hline & 13 & -724 & 261 & 0 & -- & -- \\
\hline & 14 & 17 & 150 & -- & -- & -- \\
\hline \multirow[t]{9}{*}{ L AOUO 3} & 2 & -1736 & 20 & 50 & -- & 35 \\
\hline & 3 & -1196 & 540 & 50 & $<10$ & 33 \\
\hline & 4 & -1136 & 60 & 92 & 12 & 30 \\
\hline & 5 & -206 & 678 & 89 & $<10$ & 25 \\
\hline & 6 & -16 & 30 & -- & - - & 20 \\
\hline & 11 & 57 & 73 & -- & -- & 20 \\
\hline & 12 & -1756 & 484 & 0 & -- & - \\
\hline & 13 & -884 & 252 & 4 & -- & -- \\
\hline & 14 & -46 & 160 & 0 & -- & -- \\
\hline \multirow[t]{8}{*}{$\mathrm{LAPCO} 1$} & 3 & -9892 & 2745 & 30 & 56 & 84 \\
\hline & 7 & -4142 & 3235 & 46 & 115 & 50 \\
\hline & 8 & -1882 & 2260 & 62 & 59 & 34 \\
\hline & 9 & -942 & 940 & 37 & $<10$ & 27 \\
\hline & 10 & -215 & 727 & 32 & $<10$ & 24 \\
\hline & 11 & 26 & 241 & $\ldots$ & $<10$ & 22 \\
\hline & 12 & -12637 & 2770 & 1 & -- & -- \\
\hline & 15 & -7377 & 2515 & 3 & -- & -- \\
\hline \multirow[t]{10}{*}{$\mathrm{LAPCO} 2$} & 3 & -10170 & 2070 & 14 & -- & 85 \\
\hline & 5 & -9143 & 292 & 23 & -- & 74 \\
\hline & 7 & -4340 & 3677 & 48 & 76 & 56 \\
\hline & 8 & -1860 & 2480 & 52 & 27 & 38 \\
\hline & 9 & -1075 & 785 & 41 & $<10$ & 29 \\
\hline & 10 & -285 & 790 & 50 & $<10$ & 24 \\
\hline & 11 & 29 & 314 & 99 & $<10$ & 21 \\
\hline & 12 & -12240 & 2950 & 1 & -- & -- \\
\hline & 13 & -9435 & 735 & 0 & $\ldots$ & - - \\
\hline & 15 & -8017 & 1126 & 2 & - & - \\
\hline \multirow[t]{6}{*}{$\mathrm{LAPCO}_{3}$} & 7 & -5073 & 3762 & 42 & 105 & 63 \\
\hline & 8 & -2515 & 2558 & 72 & 53 & 44 \\
\hline & 9 & -1497 & 1018 & 50 & $<10$ & 33 \\
\hline & 10 & -380 & 1117 & 35 & $<10$ & 26 \\
\hline & 11 & 23 & 403 & -- & $<10$ & 21 \\
\hline & 15 & -8835 & 2830 & 2 & -- & -- \\
\hline \multirow[t]{5}{*}{ LAPLO 1} & 8 & -6481 & 4172 & 59 & 106 & 69 \\
\hline & 9 & -3416 & 3065 & 62 & 106 & 49 \\
\hline & 10 & -976 & 2440 & 63 & 36 & 33 \\
\hline & 11 & 0 & 976 & 74 & $<10$ & 23 \\
\hline & 16 & -10653 & 118 & 0 & - - & -- \\
\hline
\end{tabular}


Table 4--Layer number; altitude of top, thickness, and sand percentage of layer; and average dissolved-solids concentration and temperature of water in layer; by log number--Continued.

\begin{tabular}{|c|c|c|c|c|c|c|}
\hline $\begin{array}{l}\text { Log } \\
\text { number }\end{array}$ & $\begin{array}{r}\text { Layer } \\
\text { number }\end{array}$ & $\begin{array}{l}\text { Altitude } \\
\text { of top } \\
\text { (feet) }\end{array}$ & $\begin{array}{r}\text { Thick- } \\
\text { ness } \\
\text { (feet) }\end{array}$ & $\begin{array}{r}\text { Sand } \\
\text { per- } \\
\text { centage }\end{array}$ & $\begin{array}{l}\text { Dis- } \\
\text { solved } \\
\text { solids }\end{array}$ & $\begin{array}{l}\text { Tem- } \\
\text { per- } \\
\text { ature }\end{array}$ \\
\hline \multirow[t]{5}{*}{$\mathrm{LAPLO} 2$} & 8 & -8031 & 6575 & 35 & 77 & 79 \\
\hline & 9 & -4031 & 4000 & 54 & 87 & 53 \\
\hline & 10 & -1031 & 3000 & 35 & 27 & 35 \\
\hline & 11 & 0 & 1031 & 27 & $<10$ & 24 \\
\hline & 16 & -14606 & 964 & 2 & -- & - \\
\hline \multirow[t]{4}{*}{$\mathrm{LAPLO} 3$} & 8 & -6688 & -- & -- & -- & -- \\
\hline & 9 & -3557 & 3131 & 54 & 165 & 53 \\
\hline & 10 & -1027 & 2530 & 70 & 68 & 35 \\
\hline & 11 & 0 & 1027 & 46 & $<10$ & 24 \\
\hline \multirow[t]{4}{*}{$\mathrm{LAPLO} 4$} & 8 & -9776 & -- & -- & - & - \\
\hline & 9 & -5108 & 4668 & 51 & 106 & 70 \\
\hline & 10 & -1099 & 4009 & 50 & 47 & 41 \\
\hline & 11 & 0 & 1099 & 31 & $<10$ & 24 \\
\hline \multirow[t]{4}{*}{ LAPLO5 } & 8 & -7959 & -- & -- & -- & -- \\
\hline & 9 & -3963 & 3996 & 50 & 68 & 50 \\
\hline & 10 & -1040 & 2923 & 57 & 32 & 37 \\
\hline & 11 & 0 & 1040 & 29 & $<10$ & 24 \\
\hline \multirow[t]{4}{*}{ LAPLO 6} & 8 & -9869 & 5537 & 21 & 107 & 82 \\
\hline & 9 & -5170 & 4699 & 45 & 88 & 60 \\
\hline & 10 & -1155 & 4015 & 46 & 22 & 39 \\
\hline & 11 & 0 & 1155 & 8 & $<10$ & 24 \\
\hline \multirow[t]{4}{*}{ LAPLO 07} & 8 & -8277 & 3703 & 32 & 88 & 77 \\
\hline & 9 & -4084 & 4193 & 47 & 88 & 56 \\
\hline & 10 & -1006 & 3078 & 49 & 34 & 35 \\
\hline & 11 & 0 & 1006 & 6 & -- & 23 \\
\hline \multirow[t]{4}{*}{ L AP LO 8} & 8 & -10981 & 364 & -- & -- & -- \\
\hline & 9 & -5681 & 5300 & 17 & 47 & 65 \\
\hline & 10 & -1178 & 4503 & 36 & 36 & 39 \\
\hline & 11 & 0 & 1178 & -- & -- & 24 \\
\hline \multirow[t]{4}{*}{ LAPLO9 } & 8 & -10080 & 376 & 23 & 132 & 72 \\
\hline & 9 & -5275 & 4805 & 29 & 136 & 59 \\
\hline & 10 & -1170 & 4105 & 37 & 62 & 37 \\
\hline & 11 & 0 & 1170 & 5 & -- & 24 \\
\hline \multirow[t]{4}{*}{ L AP L 10} & 8 & -10179 & 1070 & - & - & -- \\
\hline & 9 & -5323 & 4856 & 29 & 82 & 68 \\
\hline & 10 & -1175 & 4148 & 28 & 93 & 40 \\
\hline & 11 & 0 & 1175 & 24 & 16 & 24 \\
\hline \multirow[t]{10}{*}{ L ARAO 1} & 3 & -3710 & 3438 & 51 & 39 & 74 \\
\hline & 4 & -3478 & 232 & 85 & 81 & 53 \\
\hline & 5 & -2590 & 552 & 74 & 76 & 45 \\
\hline & 6 & -1514 & 806 & 49 & 39 & 34 \\
\hline & 7 & -120 & 573 & 18 & $<10$ & 23 \\
\hline & 8 & 267 & 387 & 25 & $<10$ & 20 \\
\hline & 12 & -7148 & 892 & 0 & - & -- \\
\hline & 13 & -3142 & 336 & 0 & -- & - \\
\hline & 14 & -2320 & 270 & 1 & -- & -- \\
\hline & 15 & -693 & 821 & 3 & -- & - \\
\hline
\end{tabular}


Table 4--Layer number; altitude of top, thickness, and sand percentage of layer; and average dissolved-solids concentration and temperature of water in layer; by log number--Continued.

\begin{tabular}{|c|c|c|c|c|c|c|}
\hline $\begin{array}{l}\text { Log } \\
\text { number }\end{array}$ & $\begin{array}{r}\text { Layer } \\
\text { number }\end{array}$ & $\begin{array}{r}\text { Altitude } \\
\text { of top } \\
\text { (feet) }\end{array}$ & $\begin{array}{r}\text { Thick - } \\
\text { ness } \\
\text { (feet) }\end{array}$ & $\begin{array}{r}\text { Sand } \\
\text { per- } \\
\text { centage }\end{array}$ & $\begin{array}{l}\text { Dis- } \\
\text { solved } \\
\text { solids }\end{array}$ & $\begin{array}{l}\text { Tem- } \\
\text { per- } \\
\text { ature }\end{array}$ \\
\hline \multirow[t]{11}{*}{$\mathrm{LARAO} 2$} & 3 & -5585 & - & -- & $\ldots$ & $\ldots$ \\
\hline & 4 & -5383 & 202 & 75 & -- & 58 \\
\hline & 5 & -4416 & 534 & 53 & -- & 53 \\
\hline & 6 & -3230 & 885 & 45 & -- & 46 \\
\hline & 7 & -1170 & 1259 & 39 & $<10$ & 33 \\
\hline & 8 & -290 & 880 & 47 & $<10$ & 25 \\
\hline & 9 & 110 & 400 & - & - & 21 \\
\hline & 10 & 222 & 112 & -- & -- & 19 \\
\hline & 13 & -4950 & 433 & 1 & -- & - \\
\hline & 14 & -4115 & 301 & 1 & -- & -- \\
\hline & 15 & -2429 & 801 & 2 & - & -- \\
\hline \multirow[t]{12}{*}{ LARAO3 } & 3 & -7793 & 3250 & 42 & 44 & 72 \\
\hline & 5 & -6818 & 485 & 41 & 36 & 62 \\
\hline & 6 & -5603 & 808 & 39 & 36 & 55 \\
\hline & 7 & -2543 & 2206 & 47 & 59 & 42 \\
\hline & 8 & -1073 & 1470 & 32 & $<10$ & 31 \\
\hline & 9 & -358 & 715 & 25 & $<10$ & 25 \\
\hline & 10 & 17 & 375 & 35 & $<10$ & 22 \\
\hline & 11 & 136 & 119 & - & - & 20 \\
\hline & 12 & -11043 & 1790 & 5 & -- & $\ldots$ \\
\hline & 13 & -7303 & 490 & 0 & -- & - \\
\hline & 14 & -6411 & 407 & 0 & -- & -- \\
\hline & 15 & -4749 & 854 & 1 & -- & $\cdots$ \\
\hline \multirow[t]{11}{*}{$\mathrm{LARAO} 4$} & 3 & -5334 & - & -- & -- & -- \\
\hline & 4 & -5149 & 185 & 85 & 88 & 49 \\
\hline & 5 & -4175 & 637 & 43 & 59 & 45 \\
\hline & 6 & -3147 & 718 & 41 & 32 & 40 \\
\hline & 7 & -1317 & 1210 & 32 & 36 & 31 \\
\hline & 8 & -467 & 850 & 12 & -- & 26 \\
\hline & 9 & -57 & 410 & - & - & 22 \\
\hline & 11 & 71 & 128 & -- & -- & 21 \\
\hline & 13 & -4812 & 337 & 1 & -- & $\ldots$ \\
\hline & 14 & -3865 & 310 & 6 & -- & -- \\
\hline & 15 & -2527 & 620 & 0 & -- & -- \\
\hline \multirow[t]{11}{*}{ LARAO 5} & 3 & -7132 & - & -- & -- & -- \\
\hline & 5 & -6036 & 650 & 28 & 38 & 62 \\
\hline & 6 & -4974 & 740 & 36 & -- & 54 \\
\hline & 7 & -2486 & 1755 & 47 & -- & 41 \\
\hline & 8 & -1224 & 1262 & 25 & 20 & 32 \\
\hline & 9 & -404 & 820 & -- & -- & 26 \\
\hline & 10 & -54 & 350 & -- & -- & 22 \\
\hline & 11 & 65 & 119 & -- & -- & 21 \\
\hline & 13 & -6686 & 446 & 1 & -- & - \\
\hline & 14 & -5714 & 322 & 0 & $\cdots$ & $\cdots$ \\
\hline & 15 & -4241 & 733 & 3 & -- & - \\
\hline
\end{tabular}


Table 4--Layer number; altitude of top, thickness, and sand percentage of layer; and average dissolved-solids concentration and temperature of water in layer; by log number--Continued.

\begin{tabular}{|c|c|c|c|c|c|c|}
\hline $\begin{array}{l}\text { Log } \\
\text { number }\end{array}$ & $\begin{array}{l}\text { Layer } \\
\text { number }\end{array}$ & $\begin{array}{r}\text { Altitude } \\
\text { of top } \\
\text { ( feet) }\end{array}$ & $\begin{array}{r}\text { Thlck- } \\
\text { ness } \\
\text { (feet) }\end{array}$ & $\begin{array}{r}\text { Sand } \\
\text { per- } \\
\text { centage }\end{array}$ & $\begin{array}{l}\text { Dis- } \\
\text { solved } \\
\text { solids }\end{array}$ & $\begin{array}{r}\text { Tem- } \\
\text { per- } \\
\text { ature }\end{array}$ \\
\hline \multirow[t]{9}{*}{ LARIOI } & 2 & -1803 & 20 & 50 & $\cdots$ & 34 \\
\hline & 3 & -1275 & 528 & 43 & 14 & 32 \\
\hline & 4 & -1058 & 217 & 91 & -- & 29 \\
\hline & 5 & -168 & 671 & 75 & $<10$ & 24 \\
\hline & 6 & -13 & 55 & 99 & $<10$ & 20 \\
\hline & 11 & 70 & 83 & -- & $<10$ & 19 \\
\hline & 12 & -1823 & 495 & 0 & -- & -- \\
\hline & 13 & -839 & 219 & 2 & -- & -- \\
\hline & 14 & -68 & 100 & 7 & -- & - \\
\hline \multirow[t]{9}{*}{$\mathrm{LARIO2}$} & 2 & -2169 & 20 & 50 & -- & 41 \\
\hline & 3 & -1540 & 629 & 45 & 12 & 38 \\
\hline & 4 & -1424 & 116 & 99 & -- & 34 \\
\hline & 5 & -482 & 641 & 66 & $<10$ & 27 \\
\hline & 6 & -27 & 316 & 22 & $<10$ & 21 \\
\hline & 11 & 85 & 112 & -- & -- & 19 \\
\hline & 12 & -2189 & 458 & 0 & -- & -- \\
\hline & 13 & -1123 & 301 & 1 & -- & -- \\
\hline & 14 & -343 & 139 & 9 & -- & - \\
\hline \multirow[t]{3}{*}{ LARROI } & 2 & -103 & 20 & 50 & -- & 22 \\
\hline & 3 & 221 & 324 & 25 & -- & 20 \\
\hline & 12 & -123 & 773 & 0 & -- & -- \\
\hline \multirow[t]{3}{*}{ L ARRO 2} & 2 & -249 & 20 & 50 & -- & -- \\
\hline & 3 & 118 & 367 & 59 & -- & -- \\
\hline & 12 & -269 & 790 & 0 & -- & -- \\
\hline \multirow[t]{3}{*}{ LASAO 1} & 2 & -1223 & 20 & 50 & -- & 32 \\
\hline & 3 & 265 & 1488 & 59 & $<10$ & 26 \\
\hline & 12 & -1243 & 859 & 0 & - & - \\
\hline \multirow[t]{3}{*}{ LASAO2 } & 2 & -763 & 20 & 50 & -- & 29 \\
\hline & 3 & 240 & 1003 & 63 & $<10$ & 24 \\
\hline & 12 & -783 & 842 & 0 & -- & -- \\
\hline \multirow[t]{7}{*}{ L ASAO3 } & 3 & -406 & 2799 & 41 & 11 & 36 \\
\hline & 4 & -365 & 41 & 99 & $<10$ & 25 \\
\hline & 5 & -83 & 204 & 81 & $<10$ & 23 \\
\hline & 6 & 338 & 243 & -- & - & 20 \\
\hline & 12 & -3205 & -- & -- & -- & -- \\
\hline & 13 & -287 & 78 & 17 & -- & - \\
\hline & 14 & 95 & 178 & 14 & -- & -- \\
\hline \multirow[t]{7}{*}{ IASAO 4} & 3 & -554 & 3185 & 53 & -- & -- \\
\hline & 4 & -497 & 57 & 99 & -- & -- \\
\hline & 5 & -164 & 244 & 70 & -- & -- \\
\hline & 6 & 280 & 284 & 57 & -- & -- \\
\hline & 12 & -3739 & 935 & 0 & -- & -- \\
\hline & 13 & -408 & 89 & 0 & -- & -- \\
\hline & 14 & -4 & 160 & 9 & $\cdots$ & $\cdots$ \\
\hline
\end{tabular}


Table 4--Layer number; altitude of top, thickness, and sand percentage of layer; and average dissolved-solids concentration and temperature of water in layer; by log number--Continued.

\begin{tabular}{|c|c|c|c|c|c|c|}
\hline $\begin{array}{l}\text { Log } \\
\text { number }\end{array}$ & $\begin{array}{r}\text { Layer } \\
\text { number }\end{array}$ & $\begin{array}{r}\text { Altitude } \\
\text { of top } \\
\text { (feet) }\end{array}$ & $\begin{array}{r}\text { Thlck- } \\
\text { ness } \\
(\text { feet) }\end{array}$ & $\begin{array}{r}\text { Sand } \\
\text { per- } \\
\text { centage }\end{array}$ & $\begin{array}{l}\text { Dis- } \\
\text { solved } \\
\text { solids }\end{array}$ & $\begin{array}{r}\text { Tem- } \\
\text { per- } \\
\text { ature }\end{array}$ \\
\hline \multirow[t]{10}{*}{ LASBOI } & 3 & -6704 & 462 & 26 & 40 & 66 \\
\hline & 5 & -5812 & 451 & -- & -- & - \\
\hline & 7 & -4272 & 891 & 63 & 114 & 52 \\
\hline & 8 & -2659 & 1613 & 72 & 76 & 44 \\
\hline & 9 & -1799 & 860 & 56 & $<10$ & 35 \\
\hline & 10 & -819 & 980 & 38 & $<10$ & 29 \\
\hline & 11 & 0 & 819 & 64 & $<10$ & 23 \\
\hline & 12 & -7166 & 1633 & 1 & - & - \\
\hline & 13 & -6263 & 441 & 8 & -- & - \\
\hline & 15 & -5163 & 649 & 5 & -- & -- \\
\hline \multirow[t]{7}{*}{ LASBOL 2} & 3 & -8695 & 350 & 33 & 50 & 79 \\
\hline & 7 & -7233 & 285 & 20 & -- & 70 \\
\hline & 8 & -4279 & 2954 & 56 & 92 & 67 \\
\hline & 9 & -2465 & 1814 & 52 & 58 & 63 \\
\hline & 10 & -1000 & 1465 & 46 & 34 & 47 \\
\hline & 11 & 0 & 1000 & 61 & $<10$ & 24 \\
\hline & 15 & -7518 & 1177 & 2 & - & - \\
\hline \multirow[t]{4}{*}{ LASBO3 } & 8 & -6423 & -- & -- & -- & -- \\
\hline & 9 & -3423 & 3000 & 65 & 25 & 48 \\
\hline & 10 & -1031 & 2392 & 56 & 26 & 34 \\
\hline & 11 & 0 & 1031 & 46 & $<10$ & 24 \\
\hline \multirow[t]{5}{*}{ LASBO 4} & 8 & -4972 & 3727 & 61 & 110 & 63 \\
\hline & 9 & -2899 & 2073 & 57 & 78 & 45 \\
\hline & 10 & -1007 & 1892 & 62 & 42 & 33 \\
\hline & 11 & 0 & 1007 & 69 & $<10$ & 24 \\
\hline & 16 & -8699 & 2775 & 6 & -- & -- \\
\hline \multirow[t]{5}{*}{ LASCOI } & 8 & -5929 & 4390 & 50 & 117 & 59 \\
\hline & 9 & -3195 & 2734 & 63 & 105 & 42 \\
\hline & 10 & -904 & 2291 & 46 & 23 & 30 \\
\hline & 11 & 8 & 912 & 58 & $<10$ & 23 \\
\hline & 16 & -10319 & 327 & 0 & -- & -- \\
\hline \multirow[t]{5}{*}{ LASCO2 } & 8 & -6674 & 4396 & 39 & 82 & 78 \\
\hline & 9 & -3479 & 3195 & 40 & 67 & 55 \\
\hline & 10 & -924 & 2555 & 60 & 22 & 35 \\
\hline & 11 & 0 & 924 & 47 & $<10$ & 23 \\
\hline & 16 & -11070 & 1395 & 3 & -- & -- \\
\hline \multirow[t]{5}{*}{$\mathrm{LASCO}_{3}$} & 8 & -6880 & 3520 & 48 & 93 & 64 \\
\hline & 9 & -3586 & 3294 & 52 & 79 & 47 \\
\hline & 10 & -945 & 2641 & 48 & 38 & 32 \\
\hline & 11 & 1 & 946 & 68 & $<10$ & 23 \\
\hline & 16 & -10400 & 2061 & 4 & $\ldots$ & $\ldots$ \\
\hline
\end{tabular}


Table 4--Layer number; altitude of top, thickness, and sand percentage of layer; and average dissolved-solids concentration and temperature of water in layer; by log number--Continued.

\begin{tabular}{|c|c|c|c|c|c|c|}
\hline $\begin{array}{l}\text { Log } \\
\text { number }\end{array}$ & $\begin{array}{r}\text { Layer } \\
\text { number }\end{array}$ & $\begin{array}{r}\text { Altitude } \\
\text { of top } \\
\text { (feet) }\end{array}$ & $\begin{array}{r}\text { Thick- } \\
\text { ness } \\
\text { (feet) }\end{array}$ & $\begin{array}{r}\text { Sand } \\
\text { per- } \\
\text { centage }\end{array}$ & $\begin{array}{l}\text { Dis- } \\
\text { solved } \\
\text { solids }\end{array}$ & $\begin{array}{l}\text { Tem- } \\
\text { per- } \\
\text { ature }\end{array}$ \\
\hline \multirow[t]{11}{*}{ LASBOI } & 2 & -8971 & 855 & 44 & $\cdots$ & 85 \\
\hline & 3 & -6564 & 2407 & 26 & -- & 74 \\
\hline & 5 & -5501 & 290 & - & $\cdots$ & $\cdots$ \\
\hline & 7 & -2560 & 2310 & 64 & 34 & 48 \\
\hline & 8 & -1011 & 1549 & 46 & $<10$ & 33 \\
\hline & 9 & -391 & 620 & 42 & $<10$ & 24 \\
\hline & 10 & -1 & 390 & 33 & $<10$ & 20 \\
\hline & 11 & 193 & 194 & $\ldots$ & - - & 17 \\
\hline & 12 & -9826 & 1095 & 0 & $\cdots$ & -- \\
\hline & 13 & -5791 & 773 & 0 & $\cdots$ & $\cdots$ \\
\hline & 15 & -4870 & 631 & 6 & -- & - \\
\hline \multirow[t]{10}{*}{ LASHO 2} & 2 & -7820 & 765 & 78 & 40 & 75 \\
\hline & 3 & -5545 & 2275 & 28 & 48 & 68 \\
\hline & 5 & -4538 & 330 & 18 & 23 & 59 \\
\hline & 7 & -1960 & 2109 & 49 & 26 & 51 \\
\hline & 8 & -530 & 1430 & 44 & $<10$ & 38 \\
\hline & 9 & -30 & 500 & 72 & $<10$ & 18 \\
\hline & 10 & 296 & 326 & $\ldots$ & - & $\cdots$ \\
\hline & 12 & -8585 & 1112 & 8 & -- & $\cdots$ \\
\hline & 13 & -4868 & 677 & 11 & -- & $\cdots$ \\
\hline & 15 & -4069 & 469 & 4 & - & $\cdots$ \\
\hline \multirow[t]{11}{*}{ LASBO3 } & 2 & -8868 & 464 & 42 & 65 & 77 \\
\hline & 3 & -6726 & 2142 & 24 & 31 & 66 \\
\hline & 5 & -5723 & 311 & $\cdots$ & -- & -- \\
\hline & 7 & -2860 & 2563 & 50 & 67 & 44 \\
\hline & 8 & -1089 & 1771 & 65 & $<10$ & 31 \\
\hline & 9 & -461 & 628 & 57 & $<10$ & 24 \\
\hline & 10 & 0 & 461 & 16 & $<10$ & 21 \\
\hline & 11 & 224 & 224 & 99 & $<10$ & 19 \\
\hline & 12 & -9332 & $\ldots$ & - & - & $\ldots$ \\
\hline & 13 & -6034 & 692 & 6 & - & - \\
\hline & 15 & -5423 & 300 & 16 & -- & $\cdots$ \\
\hline \multirow[t]{10}{*}{ LASHO 4} & 3 & -7433 & 2708 & 24 & 34 & 73 \\
\hline & 5 & -6501 & 320 & $\cdots$ & $\ldots$ & 60 \\
\hline & 7 & -3329 & 2902 & 52 & 50 & 48 \\
\hline & 8 & -1291 & 2038 & 56 & 14 & 33 \\
\hline & 9 & -651 & 640 & $\ldots$ & - & 25 \\
\hline & 10 & -151 & 500 & -- & -- & 22 \\
\hline & 11 & 112 & 263 & -- & $\cdots$ & 19 \\
\hline & 12 & -10141 & - & -- & -- & $\cdots$ \\
\hline & 13 & -6821 & 612 & 10 & -- & - \\
\hline & 15 & -6231 & 270 & 0 & -- & $\cdots$ \\
\hline \multirow[t]{5}{*}{ LASJOI } & 8 & -6500 & 4093 & 45 & 114 & 72 \\
\hline & 9 & -3420 & 3080 & 53 & 121 & 53 \\
\hline & 10 & -980 & 2440 & 43 & 36 & 36 \\
\hline & 11 & 10 & 990 & 74 & $<10$ & 23 \\
\hline & 16 & -10593 & 1336 & 1 & -- & -- \\
\hline
\end{tabular}


Table 4--Layer number; altitude of top, thickness, and sand percentage of layer; and average dissolved-solids concentration and temperature of water in layer; by log number--Continued.

\begin{tabular}{|c|c|c|c|c|c|c|}
\hline $\begin{array}{l}\text { Log } \\
\text { number }\end{array}$ & $\begin{array}{r}\text { Layer } \\
\text { number }\end{array}$ & $\begin{array}{r}\text { Alt itude } \\
\text { of top } \\
(f e e t)\end{array}$ & $\begin{array}{r}\text { Thlck- } \\
\text { ness } \\
\text { (feet) }\end{array}$ & $\begin{array}{r}\text { Sand } \\
\text { per- } \\
\text { centage }\end{array}$ & $\begin{array}{l}\text { Dis- } \\
\text { solved } \\
\text { solids }\end{array}$ & $\begin{array}{l}\text { Tem- } \\
\text { per- } \\
\text { ature }\end{array}$ \\
\hline \multirow[t]{9}{*}{ LASLOI } & 3 & -11431 & -- & - & -- & -- \\
\hline & 5 & -10123 & 473 & 27 & 75 & 86 \\
\hline & 7 & -4578 & 3560 & 41 & 108 & 60 \\
\hline & 8 & -2235 & 2343 & 54 & 97 & 48 \\
\hline & 9 & -1038 & 1197 & 36 & 11 & 36 \\
\hline & 10 & -268 & 770 & 40 & $<10$ & 25 \\
\hline & 11 & 26 & 294 & 76 & $<10$ & 19 \\
\hline & 13 & -10596 & 835 & 0 & -- & -- \\
\hline & 15 & -8138 & 1985 & 2 & -- & -- \\
\hline \multirow[t]{6}{*}{ LASLO2 } & 7 & -6838 & 3000 & 36 & 144 & 57 \\
\hline & 8 & -3848 & 2990 & 63 & 148 & 49 \\
\hline & 9 & -2048 & 1800 & 48 & 64 & 43 \\
\hline & 10 & -583 & 1465 & 44 & $<10$ & 30 \\
\hline & 11 & 25 & 608 & 87 & $<10$ & 21 \\
\hline & 15 & -9838 & 1147 & 2 & -- & -- \\
\hline \multirow{7}{*}{$\mathrm{LASLO} 3$} & 3 & -12145 & 1032 & 21 & 61 & 112 \\
\hline & 7 & -5484 & 3815 & 37 & 135 & 64 \\
\hline & 8 & -2868 & 2616 & 65 & 118 & 45 \\
\hline & 9 & -1578 & 1290 & 41 & $<10$ & 33 \\
\hline & 10 & -405 & 1173 & 31 & $<10$ & 26 \\
\hline & 11 & 20 & 425 & 69 & $<10$ & 21 \\
\hline & 15 & -9299 & 2846 & 2 & -- & -- \\
\hline \multirow[t]{6}{*}{$\mathrm{LASLO} 4$} & 7 & -5551 & 2542 & 42 & 86 & 55 \\
\hline & 8 & -3039 & 2512 & 52 & 66 & 42 \\
\hline & 9 & -1718 & 1321 & 50 & 24 & 32 \\
\hline & 10 & -584 & 1134 & 37 & $<10$ & 26 \\
\hline & 11 & 55 & 639 & 84 & $<10$ & 22 \\
\hline & 15 & -8093 & -- & $\cdots$ & -- & - \\
\hline \multirow[t]{4}{*}{ LASMOL } & 8 & -9848 & 3760 & 35 & 99 & 87 \\
\hline & 9 & -5120 & 4728 & 71 & 148 & 57 \\
\hline & 10 & -1111 & 4009 & 50 & 77 & 34 \\
\hline & 11 & 2 & 1113 & 82 & $<10$ & 23 \\
\hline \multirow[t]{4}{*}{ LASMO2 } & 8 & -10870 & -- & -- & -- & -- \\
\hline & 9 & -5579 & 5291 & 48 & 83 & 64 \\
\hline & 10 & -1128 & 4451 & 48 & 50 & 39 \\
\hline & 11 & 0 & 1128 & 68 & $<10$ & 23 \\
\hline \multirow[t]{4}{*}{ LASMO 3} & 8 & -9715 & 5747 & 39 & 158 & 80 \\
\hline & 9 & -5038 & 4677 & 56 & 104 & 61 \\
\hline & 10 & -1070 & 3968 & 53 & 56 & 46 \\
\hline & 11 & 0 & 1070 & 51 & $<10$ & 24 \\
\hline \multirow[t]{4}{*}{ LASMO 4} & 8 & -9128 & 4708 & 48 & 117 & 83 \\
\hline & 9 & -4632 & 4496 & 52 & 63 & 58 \\
\hline & 10 & -1070 & 3562 & 60 & 35 & 37 \\
\hline & 11 & 8 & 1078 & 64 & $<10$ & 23 \\
\hline
\end{tabular}


Table 4--Layer number; altitude of top, thickness, and sand percentage of layer: and average dissolved-solids concentration and temperature of water in layer; by log number--Continued.

\begin{tabular}{|c|c|c|c|c|c|c|}
\hline $\begin{array}{l}\text { Log } \\
\text { number }\end{array}$ & $\begin{array}{r}\text { Layer } \\
\text { number }\end{array}$ & $\begin{array}{r}\text { Altitude } \\
\text { of top } \\
\text { (feet) }\end{array}$ & $\begin{array}{c}\text { Thick- } \\
\text { ness } \\
\text { (feet) }\end{array}$ & $\begin{array}{r}\text { Sand } \\
\text { per- } \\
\text { centage }\end{array}$ & $\begin{array}{l}\text { Dis- } \\
\text { solved } \\
\text { solids }\end{array}$ & $\begin{array}{l}\text { Tem- } \\
\text { per- } \\
\text { ature }\end{array}$ \\
\hline \multirow[t]{4}{*}{ LASNO1 } & 8 & -8069 & 4903 & 42 & 106 & 76 \\
\hline & 9 & -4059 & 4010 & 47 & 64 & 56 \\
\hline & 10 & -1059 & 3000 & 38 & 33 & 40 \\
\hline & 11 & 0 & 1059 & 67 & $<10$ & 23 \\
\hline \multirow[t]{5}{*}{ LASNO2 } & 8 & -5742 & 4306 & 62 & 68 & 60 \\
\hline & 9 & -3192 & 2550 & 65 & 48 & 42 \\
\hline & 10 & -964 & 2228 & 59 & 23 & 30 \\
\hline & 11 & 19 & 983 & 66 & $<10$ & 22 \\
\hline & 16 & -10048 & -- & -- & -- & -- \\
\hline \multirow[t]{11}{*}{ LASTO 1} & 2 & -7695 & 256 & 99 & -- & 66 \\
\hline & 3 & -5435 & 2260 & 3 & 53 & 59 \\
\hline & 5 & -4740 & 505 & 14 & -- & 50 \\
\hline & 7 & -3163 & 1477 & 63 & 68 & 48 \\
\hline & 8 & -1950 & 1213 & 61 & $<10$ & 45 \\
\hline & 9 & -1255 & 695 & 90 & $<10$ & 36 \\
\hline & 10 & -568 & 687 & 58 & $<10$ & 29 \\
\hline & 11 & 10 & 578 & 43 & $<10$ & 22 \\
\hline & 12 & -7951 & 711 & 0 & -- & -- \\
\hline & 13 & -5245 & 190 & 8 & -- & -- \\
\hline & 15 & -4640 & 100 & 0 & -- & -- \\
\hline \multirow[t]{9}{*}{ LASTO2 } & 3 & -7152 & -- & -- & -- & -- \\
\hline & 5 & -6392 & 530 & -- & -- & -- \\
\hline & 7 & -3593 & 2637 & 59 & 60 & 50 \\
\hline & 8 & -1951 & 1642 & 53 & $<10$ & 38 \\
\hline & 9 & -1242 & 709 & 47 & $<10$ & 31 \\
\hline & 10 & -492 & 750 & -- & - & 27 \\
\hline & 11 & 47 & 539 & -- & -- & 23 \\
\hline & 13 & -6922 & 230 & 0 & -- & -- \\
\hline & 15 & -6230 & 162 & 19 & -- & -- \\
\hline \multirow[t]{7}{*}{ LASTO3 } & 5 & -8093 & -- & -- & -- & -- \\
\hline & 7 & -5431 & 2372 & 33 & 95 & 58 \\
\hline & 8 & -2471 & 2960 & 66 & 31 & 44 \\
\hline & 9 & -1615 & 856 & 69 & $<10$ & 33 \\
\hline & 10 & -508 & 1107 & -- & -- & 28 \\
\hline & 11 & 0 & 508 & -- & -- & 23 \\
\hline & 15 & -7803 & 290 & 0 & -- & -- \\
\hline \multirow[t]{3}{*}{ LASTO 4} & 9 & -1619 & -- & -- & -- & -- \\
\hline & 10 & -512 & 1107 & 55 & -- & -- \\
\hline & 11 & 6 & 518 & 53 & -- & -- \\
\hline \multirow[t]{11}{*}{ LASTO5 } & 2 & -7285 & 842 & 23 & -- & 81 \\
\hline & 3 & -6061 & 1224 & 6 & -- & 73 \\
\hline & 5 & -5145 & 590 & 12 & -- & 63 \\
\hline & 7 & -2896 & 2141 & 44 & 52 & 52 \\
\hline & 8 & -1615 & 1281 & 78 & $<10$ & 38 \\
\hline & 9 & -965 & 650 & -- & -- & 31 \\
\hline & 10 & -315 & 650 & -- & -- & 26 \\
\hline & 11 & 124 & 439 & -- & -- & 21 \\
\hline & 12 & -8127 & 1301 & 4 & -- & -- \\
\hline & 13 & -5735 & 326 & 5 & -- & -- \\
\hline & 15 & -5037 & 108 & 89 & -- & -- \\
\hline
\end{tabular}


Table 4--Layer number; altitude of top, thickness, and sand percentage of layer: and average dissolved-solids concentration and temperature of water in layer; by log number--Continued.

\begin{tabular}{|c|c|c|c|c|c|c|}
\hline $\begin{array}{l}\text { Log } \\
\text { number }\end{array}$ & $\begin{array}{r}\text { Layer } \\
\text { number }\end{array}$ & $\begin{array}{r}\text { Altitude } \\
\text { of top } \\
(\text { feet) }\end{array}$ & $\begin{array}{r}\text { Thick- } \\
\text { ness } \\
(\text { feet) }\end{array}$ & $\begin{array}{r}\text { Sand } \\
\text { per- } \\
\text { centage }\end{array}$ & $\begin{array}{l}\text { Dis- } \\
\text { solved } \\
\text { solids }\end{array}$ & $\begin{array}{r}\text { Tem- } \\
\text { per- } \\
\text { ature }\end{array}$ \\
\hline \multirow[t]{7}{*}{ LASTO 6} & 5 & -6823 & - & -- & -- & $\cdots$ \\
\hline & 7 & -3750 & 2935 & 35 & 40 & 48 \\
\hline & 8 & -1743 & 2007 & 51 & $<10$ & 36 \\
\hline & 9 & -1043 & 700 & 33 & $<10$ & 29 \\
\hline & 10 & -343 & 700 & -- & - & 25 \\
\hline & 11 & 45 & 388 & -- & -- & 23 \\
\hline & 15 & -6685 & 138 & 0 & -- & - \\
\hline \multirow[t]{6}{*}{ LASTO 7} & 7 & -5027 & 1121 & 32 & 45 & 54 \\
\hline & 8 & -3056 & 1971 & 83 & 51 & 45 \\
\hline & 9 & -2063 & 993 & 56 & $<10$ & 36 \\
\hline & 10 & -828 & 1235 & 39 & $<10$ & 30 \\
\hline & 11 & 2 & 830 & 44 & $<10$ & 24 \\
\hline & 15 & -6148 & 5079 & 0 & - & -- \\
\hline \multirow[t]{6}{*}{ LASTOB } & 7 & -6754 & 1440 & 10 & 50 & 62 \\
\hline & 8 & -3613 & 3141 & 52 & 56 & 50 \\
\hline & 9 & -2372 & 1241 & 65 & $<10$ & 38 \\
\hline & 10 & -799 & 1573 & 24 & $<10$ & 30 \\
\hline & 11 & 0 & 799 & 64 & $<10$ & 24 \\
\hline & 15 & -8194 & 6575 & 1 & -- & - \\
\hline \multirow[t]{11}{*}{$\mathrm{LATAO} 1$} & 2 & -8318 & 650 & 39 & 36 & 79 \\
\hline & 3 & -5868 & 2450 & 28 & 26 & 68 \\
\hline & 5 & -5018 & 430 & 10 & -- & 55 \\
\hline & 7 & -2543 & 2255 & 40 & 26 & 44 \\
\hline & 8 & -938 & 1605 & 46 & $<10$ & 31 \\
\hline & 9 & -338 & 600 & -- & -- & 23 \\
\hline & 10 & 32 & 370 & $=$ & $\cdots$ & 20 \\
\hline & 11 & 220 & 188 & -- & $\cdots$ & 18 \\
\hline & 12 & -8968 & 910 & 0 & -- & $\cdots$ \\
\hline & 13 & -5448 & 420 & 3 & -- & -- \\
\hline & 15 & -4798 & 220 & 0 & -- & - \\
\hline \multirow[t]{11}{*}{$\mathrm{LATAO} 2$} & 2 & -8156 & 862 & 26 & 60 & 77 \\
\hline & 3 & -6266 & 1890 & 31 & 99 & 63 \\
\hline & 5 & -5576 & 310 & 20 & -- & 53 \\
\hline & 7 & -2864 & 2543 & 48 & 167 & 44 \\
\hline & 8 & -1196 & 1668 & 48 & 13 & 33 \\
\hline & 9 & -571 & 625 & 35 & $<10$ & 25 \\
\hline & 10 & -126 & 445 & 52 & $<10$ & 21 \\
\hline & 11 & 130 & 256 & 61 & $<10$ & 19 \\
\hline & 12 & -9018 & -- & -- & -- & - \\
\hline & 13 & -5886 & 380 & 0 & -- & - \\
\hline & 15 & -5407 & 169 & 6 & -- & - \\
\hline \multirow[t]{6}{*}{$\mathrm{LATAO} 3$} & 7 & -6325 & 2275 & 18 & 48 & 62 \\
\hline & 8 & -2815 & 3510 & 58 & 51 & 47 \\
\hline & 9 & -1917 & 898 & 27 & $<10$ & 34 \\
\hline & 10 & -742 & 1175 & 63 & $<10$ & 27 \\
\hline & 11 & 2 & 744 & 33 & $<10$ & 21 \\
\hline & 15 & -8600 & 2753 & 0 & - & -- \\
\hline
\end{tabular}


Table 4--Layer number; altitude of top, thickness, and sand percentage of layer; and average dissolved-solids concentration and temperature of water in layer; by log number--Continued.

\begin{tabular}{|c|c|c|c|c|c|c|}
\hline $\begin{array}{l}\text { Log } \\
\text { number }\end{array}$ & $\begin{array}{r}\text { Layer } \\
\text { number }\end{array}$ & $\begin{array}{r}\text { Altitude } \\
\text { of top } \\
\text { (feet) }\end{array}$ & $\begin{array}{r}\text { Thick- } \\
\text { ness } \\
\text { (feet) }\end{array}$ & $\begin{array}{r}\text { Sand } \\
\text { per- } \\
\text { centage }\end{array}$ & $\begin{array}{l}\text { Dis- } \\
\text { solved } \\
\text { solids }\end{array}$ & $\begin{array}{l}\text { Tem- } \\
\text { per- } \\
\text { ature }\end{array}$ \\
\hline \multirow[t]{4}{*}{ LATEOI } & 8 & -9849 & 2775 & 23 & 112 & 73 \\
\hline & 9 & -5179 & 4670 & 48 & 157 & 56 \\
\hline & 10 & -1108 & 4071 & 43 & 61 & 35 \\
\hline & 11 & 0 & 1108 & 75 & $<10$ & 23 \\
\hline \multirow[t]{4}{*}{ LATEO2 } & 8 & -9580 & 3723 & 19 & 101 & 74 \\
\hline & 9 & -5007 & 4573 & 50 & 211 & 53 \\
\hline & 10 & -1122 & 3885 & 48 & 116 & 34 \\
\hline & 11 & 0 & 1122 & 89 & $<10$ & 23 \\
\hline \multirow[t]{3}{*}{$\mathrm{LATEO} 3$} & 9 & -6287 & 5840 & 54 & 122 & 67 \\
\hline & 10 & -1158 & 5129 & 45 & 74 & 39 \\
\hline & 11 & 0 & 1158 & -- & -- & 23 \\
\hline \multirow[t]{4}{*}{ LATEO 4} & 8 & -11381 & 3092 & 22 & 114 & 94 \\
\hline & 9 & -5851 & 5530 & 52 & 97 & 60 \\
\hline & 10 & -1087 & 4764 & 29 & 69 & 53 \\
\hline & 11 & 0 & 1087 & 72 & 27 & 25 \\
\hline \multirow[t]{4}{*}{ LATEOS } & 8 & -10588 & 3888 & 34 & 134 & 84 \\
\hline & 9 & -5576 & 5012 & 48 & 69 & 69 \\
\hline & 10 & -1142 & 4434 & 50 & 34 & 52 \\
\hline & 11 & 1 & 1143 & 73 & $<10$ & 25 \\
\hline \multirow[t]{4}{*}{ LATEO 6} & 8 & -11878 & 5059 & 26 & 68 & 106 \\
\hline & 9 & -6103 & 5775 & 37 & 98 & 74 \\
\hline & 10 & -1158 & 4945 & 39 & 61 & 42 \\
\hline & 11 & 0 & 1158 & 53 & 31 & 23 \\
\hline \multirow[t]{4}{*}{ LATEOT } & 8 & -11100 & 6767 & 9 & 53 & 103 \\
\hline & 9 & -5776 & 5324 & 40 & 92 & 69 \\
\hline & 10 & -1140 & 4636 & 52 & 42 & 40 \\
\hline & 11 & 0 & 1140 & 63 & $<10$ & 23 \\
\hline \multirow[t]{4}{*}{ LATEO 8} & 8 & -11118 & 1016 & 24 & 72 & 89 \\
\hline & 9 & -5776 & 5342 & 36 & 134 & 75 \\
\hline & 10 & -1145 & 4631 & 41 & 55 & 54 \\
\hline & 11 & 0 & 1145 & 62 & $<10$ & 25 \\
\hline \multirow[t]{11}{*}{ LATSO 1} & 2 & -5710 & 570 & 62 & 51 & 67 \\
\hline & 3 & -3710 & 2000 & 61 & 51 & 63 \\
\hline & 4 & -3390 & 320 & 88 & 31 & 59 \\
\hline & 5 & -1814 & 966 & 75 & 36 & 46 \\
\hline & 6 & -942 & 668 & 57 & $<10$ & 34 \\
\hline & 7 & -45 & 310 & 34 & -- & 22 \\
\hline & 11 & 80 & 125 & -- & -- & 19 \\
\hline & 12 & -6280 & 880 & 0 & -- & - \\
\hline & 13 & -2780 & 610 & 0 & -- & -- \\
\hline & 14 & -1610 & 204 & 1 & -- & -- \\
\hline & 15 & -355 & 587 & 0 & -- & -- \\
\hline
\end{tabular}


Table 4--Layer number; altitude of top, thickness, and sand percentage of layer; and average dissolved-solids concentration and temperature of water in layer; by log number--Continued.

\begin{tabular}{|c|c|c|c|c|c|c|}
\hline $\begin{array}{l}\text { Log } \\
\text { number }\end{array}$ & $\begin{array}{r}\text { Layer } \\
\text { number }\end{array}$ & $\begin{array}{r}\text { Alt } 1 \text { tude } \\
\text { of top } \\
(\text { feet })\end{array}$ & $\begin{array}{r}\text { Thlck- } \\
\text { ness } \\
\text { (feet) }\end{array}$ & $\begin{array}{r}\text { Sand } \\
\text { per- } \\
\text { centage }\end{array}$ & $\begin{array}{l}\text { DIs- } \\
\text { solved } \\
\text { solids }\end{array}$ & $\begin{array}{l}\text { Tem- } \\
\text { per- } \\
\text { ature }\end{array}$ \\
\hline \multirow[t]{11}{*}{ LATSO2 } & 2 & -5258 & 663 & 76 & 67 & 65 \\
\hline & 3 & -3365 & 1893 & 64 & 65 & 54 \\
\hline & 4 & -3105 & 260 & 83 & 55 & 46 \\
\hline & 5 & -1620 & 1028 & 60 & 39 & 37 \\
\hline & 6 & -910 & 535 & 45 & 11 & 29 \\
\hline & 7 & -90 & 200 & 32 & $\cdots$ & 21 \\
\hline & 11 & 62 & 152 & 99 & $<10$ & 19 \\
\hline & 12 & -5921 & 664 & 0 & - & - \\
\hline & 13 & -2648 & 457 & 0 & -- & -- \\
\hline & 14 & -1445 & 175 & 0 & -- & -- \\
\hline & 15 & -290 & 620 & 0 & -- & $\ldots$ \\
\hline \multirow[t]{10}{*}{ LATSO3 } & 2 & -5316 & -- & -- & -- & - \\
\hline & 3 & -3252 & 2064 & 54 & 31 & 50 \\
\hline & 4 & -3081 & 171 & 87 & 46 & 42 \\
\hline & 5 & -1704 & 961 & 74 & 20 & 35 \\
\hline & 6 & -966 & 535 & 47 & -- & 28 \\
\hline & 7 & -89 & 408 & - & -- & 21 \\
\hline & 11 & 60 & 149 & -- & -- & 19 \\
\hline & 13 & -2665 & 416 & 0 & -- & $\cdots$ \\
\hline & 14 & -1501 & 203 & 0 & $\ldots$ & -- \\
\hline & 15 & -497 & 469 & 0 & $-\infty$ & $\cdots$ \\
\hline \multirow[t]{9}{*}{ LATSO 4} & 3 & -3481 & -- & -- & - & - \\
\hline & 5 & -2142 & 856 & 73 & 30 & 37 \\
\hline & 6 & -1348 & 598 & 72 & 15 & 31 \\
\hline & 7 & -141 & 795 & 36 & $<10$ & 23 \\
\hline & 8 & -96 & 45 & $\ldots$ & - & 20 \\
\hline & 11 & 73 & 169 & -- & - & 19 \\
\hline & 13 & -2998 & 483 & 0 & -- & $\ldots$ \\
\hline & 14 & -1946 & 196 & 0 & -- & -- \\
\hline & 15 & -936 & 412 & 0 & -- & - \\
\hline \multirow[t]{11}{*}{ LATSOS } & 2 & -5496 & 719 & 63 & 47 & 60 \\
\hline & 3 & -3546 & 1950 & 40 & 49 & 52 \\
\hline & 4 & -3267 & 279 & 34 & -- & 45 \\
\hline & 5 & -1918 & 938 & 47 & 24 & 39 \\
\hline & 6 & -1109 & 620 & 50 & - & 32 \\
\hline & 7 & -110 & 562 & 52 & $<10$ & 23 \\
\hline & 11 & 60 & 170 & 35 & $<10$ & 20 \\
\hline & 12 & -6215 & 1032 & 0 & - & - \\
\hline & 13 & -2856 & 411 & 0 & -- & - \\
\hline & 14 & -1729 & 189 & 3 & -- & -- \\
\hline & 15 & -672 & 437 & 0 & - & -- \\
\hline \multirow[t]{8}{*}{ LAUNO 1} & 2 & -1199 & 91 & 99 & 37 & 35 \\
\hline & 3 & -955 & 244 & 32 & $\ldots$ & 33 \\
\hline & 4 & -935 & 20 & 50 & - & 31 \\
\hline & 5 & -30 & 605 & 56 & $<10$ & 23 \\
\hline & 6 & 208 & 153 & - & - & 16 \\
\hline & 12 & -1290 & 535 & 0 & $\ldots$ & - \\
\hline & 13 & -635 & 300 & 13 & -- & -- \\
\hline & 14 & 55 & 85 & 12 & - & $\ldots$ \\
\hline
\end{tabular}


Table 4--Layer number; altitude of top, thickness, and sand percentage of layer; and average dissolved-solids concentration and temperature of water in layer; by log number--Continued.

\begin{tabular}{|c|c|c|c|c|c|c|}
\hline $\begin{array}{l}\text { Log } \\
\text { number }\end{array}$ & $\begin{array}{r}\text { Layer } \\
\text { number }\end{array}$ & $\begin{array}{r}\text { Altitude } \\
\text { of top } \\
\text { (feet) }\end{array}$ & $\begin{array}{r}\text { Thfck- } \\
\text { ness } \\
\text { (feet) }\end{array}$ & $\begin{array}{r}\text { Sand } \\
\text { per- } \\
\text { centage }\end{array}$ & $\begin{array}{r}\text { Dis- } \\
\text { solved } \\
\text { solids }\end{array}$ & $\begin{array}{l}\text { Tem- } \\
\text { per- } \\
\text { ature }\end{array}$ \\
\hline \multirow[t]{8}{*}{$\mathrm{L} A U N O 2$} & 2 & -1172 & 55 & 73 & -- & 32 \\
\hline & 3 & -909 & 263 & 35 & -- & 30 \\
\hline & 4 & -884 & 25 & 99 & $<10$ & 29 \\
\hline & 5 & -119 & 494 & 54 & $<10$ & 23 \\
\hline & 6 & 134 & 93 & -- & -- & 19 \\
\hline & 12 & -1227 & 582 & 4 & -- & -- \\
\hline & 13 & -613 & 271 & 6 & -- & -- \\
\hline & 14 & 41 & 160 & 18 & -- & -- \\
\hline \multirow[t]{8}{*}{ LAUNO3 } & 2 & -1158 & 97 & 75 & 15 & 29 \\
\hline & 3 & -910 & 248 & 22 & -- & 28 \\
\hline & 4 & -883 & 27 & 99 & $<10$ & 27 \\
\hline & 5 & -8 & 623 & 78 & $<10$ & 23 \\
\hline & 6 & 180 & 143 & 76 & $<10$ & 20 \\
\hline & 12 & -1255 & 643 & 0 & -- & -- \\
\hline & 13 & -631 & 252 & 18 & -- & -- \\
\hline & 14 & 37 & 45 & 11 & -- & -- \\
\hline \multirow[t]{8}{*}{ LAUNO 4} & 2 & -1227 & 130 & 99 & 35 & 30 \\
\hline & 3 & -935 & 292 & 26 & -- & 29 \\
\hline & 4 & -867 & 68 & 99 & 37 & 27 \\
\hline & 5 & -27 & 583 & 43 & $<10$ & 23 \\
\hline & 6 & 195 & 114 & -- & -- & 20 \\
\hline & 12 & -1357 & 620 & 0 & -- & -- \\
\hline & 13 & -610 & 257 & 12 & -- & -- \\
\hline & 14 & 81 & 108 & 5 & -- & -- \\
\hline \multirow[t]{8}{*}{ L AUNO5 } & 2 & -1217 & 20 & 50 & -- & 31 \\
\hline & 3 & -935 & 282 & 23 & -- & 30 \\
\hline & 4 & -880 & 55 & 99 & $<10$ & 28 \\
\hline & 5 & -20 & 510 & 55 & $<10$ & 23 \\
\hline & 6 & 210 & 90 & -- & -- & 19 \\
\hline & 12 & -1237 & 543 & 0 & -- & -- \\
\hline & 13 & -530 & 350 & 7 & -- & -- \\
\hline & 14 & 120 & 140 & 45 & -- & -- \\
\hline \multirow[t]{7}{*}{ LAUNO6 } & 2 & -1321 & 118 & 66 & $<10$ & 32 \\
\hline & 3 & -1046 & 275 & 44 & $<10$ & 30 \\
\hline & 4 & -1026 & 20 & 50 & -- & 29 \\
\hline & 5 & -29 & 723 & 60 & $<10$ & 24 \\
\hline & 6 & 110 & 139 & -- & $<10$ & 20 \\
\hline & 12 & -1439 & 539 & 0 & -- & -- \\
\hline & 13 & -752 & 274 & 0 & -- & -- \\
\hline \multirow[t]{4}{*}{ LAVEO 1} & 8 & -7121 & 5260 & 55 & 103 & 70 \\
\hline & 9 & -3671 & 3450 & 58 & 68 & 49 \\
\hline & 10 & -1005 & 2666 & 55 & 45 & 34 \\
\hline & 11 & 8 & 1013 & 77 & $<10$ & 23 \\
\hline
\end{tabular}


Table 4--Layer number; altitude of top, thickness, and sand percentage of layer; and average dissolved-solids concentration and temperature of water in layer; by log number--Continued.

\begin{tabular}{|c|c|c|c|c|c|c|}
\hline $\begin{array}{l}\text { Log } \\
\text { number }\end{array}$ & $\begin{array}{r}\text { Layer } \\
\text { number }\end{array}$ & $\begin{array}{r}\text { Altitude } \\
\text { of top } \\
\text { (feet) }\end{array}$ & $\begin{array}{r}\text { Thick- } \\
\text { ness } \\
\text { (feet) }\end{array}$ & $\begin{array}{r}\text { Sand } \\
\text { per- } \\
\text { centage }\end{array}$ & $\begin{array}{l}\text { Dis- } \\
\text { solved } \\
\text { solids }\end{array}$ & $\begin{array}{l}\text { Tem- } \\
\text { per- } \\
\text { ature }\end{array}$ \\
\hline \multirow[t]{4}{*}{ LAVEO2 } & 8 & -8483 & 2498 & 50 & 155 & 70 \\
\hline & 9 & -4439 & 4044 & 54 & 68 & 60 \\
\hline & 10 & -1055 & 3384 & 50 & 49 & 37 \\
\hline & 11 & 0 & 1055 & 82 & $<10$ & 24 \\
\hline \multirow[t]{4}{*}{ LAVEO3 } & 8 & -9395 & 6081 & 33 & 123 & 93 \\
\hline & 9 & -5040 & 4355 & 50 & 123 & 62 \\
\hline & 10 & -1058 & 3982 & 52 & 65 & 37 \\
\hline & 11 & 0 & 1058 & 52 & $<10$ & 23 \\
\hline \multirow[t]{4}{*}{ LAVEO 4} & 8 & -9771 & 3523 & 24 & 88 & 84 \\
\hline & 9 & -5286 & 4485 & 41 & 92 & 62 \\
\hline & 10 & -1066 & 4220 & 49 & 48 & 38 \\
\hline & 11 & 3 & 1069 & 79 & $<10$ & 23 \\
\hline \multirow[t]{4}{*}{ LAVEOS } & 8 & -9636 & 4344 & 19 & 69 & 80 \\
\hline & 9 & -5269 & 4367 & 35 & 106 & 61 \\
\hline & 10 & -1068 & 4201 & 56 & 45 & 44 \\
\hline & 11 & 3 & 1071 & 46 & $<10$ & 24 \\
\hline \multirow[t]{5}{*}{ LAVEO 6} & 8 & -6788 & 4061 & 50 & 128 & 68 \\
\hline & 9 & -3523 & 3265 & 71 & 118 & 48 \\
\hline & 10 & -973 & 2550 & 54 & 39 & 33 \\
\hline & 11 & 15 & 988 & 77 & $<10$ & 23 \\
\hline & 16 & -10849 & 1609 & 0 & - & $\ldots$ \\
\hline \multirow[t]{10}{*}{ LAVNOI } & 3 & -4115 & 3690 & 44 & 68 & 61 \\
\hline & 4 & -3958 & 157 & 81 & 98 & 48 \\
\hline & 5 & -3073 & 521 & 60 & 47 & 43 \\
\hline & 6 & -1861 & 986 & 43 & 12 & 37 \\
\hline & 7 & -350 & 758 & 26 & $<10$ & 26 \\
\hline & 8 & 334 & 684 & 30 & $<10$ & 22 \\
\hline & 12 & -7805 & - - & - & -- & - \\
\hline & 13 & -3594 & 364 & 0 & -- & -- \\
\hline & 14 & -2847 & 226 & 9 & -- & -- \\
\hline & 15 & -1108 & 753 & 0 & -- & - \\
\hline \multirow[t]{9}{*}{ LAVNO2 } & 3 & -4040 & 4060 & 45 & 79 & 61 \\
\hline & 5 & -3323 & 377 & 44 & 76 & 52 \\
\hline & 6 & -1980 & 1045 & 34 & 49 & 49 \\
\hline & 7 & -490 & 741 & 17 & $<10$ & 31 \\
\hline & 8 & 335 & 825 & 27 & $<10$ & 21 \\
\hline & 12 & -8100 & 1056 & 0 & -- & - \\
\hline & 13 & -3700 & 340 & 0 & - & - \\
\hline & 14 & -3025 & 298 & 0 & -- & -- \\
\hline & 15 & -1231 & 749 & 0 & -- & -- \\
\hline \multirow[t]{10}{*}{ LAVNO3 } & 3 & -5991 & 3617 & 40 & 32 & 78 \\
\hline & 5 & -5271 & 271 & 49 & 86 & 58 \\
\hline & 6 & -3701 & 1057 & 38 & 35 & 48 \\
\hline & 7 & -1147 & 1644 & 26 & $<10$ & 34 \\
\hline & 8 & -11 & 1136 & 39 & $<10$ & 25 \\
\hline & 9 & 230 & 241 & -- & -- & 21 \\
\hline & 12 & -9608 & 243 & 0 & -- & -- \\
\hline & 13 & -5542 & 449 & 0 & -- & -- \\
\hline & 14 & -4758 & 513 & 0 & -- & -- \\
\hline & 15 & -2791 & 910 & 1 & -- & -- \\
\hline
\end{tabular}


Table 4--Layer number; altitude of top, thickness, and sand percentage of layer; and average dissolved-solids concentration and temperature of water in layer; by log number--Continued.

\begin{tabular}{|c|c|c|c|c|c|c|}
\hline $\begin{array}{l}\text { Log } \\
\text { number }\end{array}$ & $\begin{array}{r}\text { Layer } \\
\text { number }\end{array}$ & $\begin{array}{r}\text { Altitude } \\
\text { of top } \\
(f e e t)\end{array}$ & $\begin{array}{r}\text { Thick- } \\
\text { ness } \\
(\text { feet) }\end{array}$ & $\begin{array}{r}\text { Sand } \\
\text { per- } \\
\text { centage }\end{array}$ & $\begin{array}{l}\text { Dis- } \\
\text { solved } \\
\text { solids }\end{array}$ & $\begin{array}{l}\text { Tem- } \\
\text { per- } \\
\text { ature }\end{array}$ \\
\hline \multirow[t]{11}{*}{ L A VNO 4} & 3 & -7119 & 3448 & 37 & 68 & 80 \\
\hline & 5 & -6422 & 285 & 33 & 54 & 76 \\
\hline & 6 & -4734 & 998 & 40 & 48 & 68 \\
\hline & 7 & -1662 & 2140 & 42 & 21 & 54 \\
\hline & 8 & -242 & 1420 & 41 & $<10$ & 35 \\
\hline & 9 & 48 & 290 & 40 & $<10$ & 21 \\
\hline & 10 & 220 & 172 & 99 & $<10$ & 17 \\
\hline & 12 & -10567 & 429 & 0 & - & -- \\
\hline & 13 & -6707 & 412 & 0 & -- & -- \\
\hline & 14 & -5732 & 690 & 0 & -- & -- \\
\hline & 15 & -3802 & 932 & 1 & -- & -- \\
\hline \multirow[t]{10}{*}{ LAVNO5 } & 3 & -6666 & -- & -- & -- & -- \\
\hline & 5 & -5755 & 559 & 21 & -- & 62 \\
\hline & 6 & -4359 & 1085 & 24 & 41 & 55 \\
\hline & 7 & -1663 & 1738 & 40 & 29 & 39 \\
\hline & 8 & -264 & 1399 & -- & $<10$ & 28 \\
\hline & 9 & 161 & 425 & -- & -- & 22 \\
\hline & 10 & 220 & 59 & -- & -- & 20 \\
\hline & 13 & -6314 & 352 & 0 & -- & -- \\
\hline & 14 & -5444 & 311 & 0 & -- & - \\
\hline & 15 & -3401 & 958 & 1 & -- & - \\
\hline \multirow[t]{10}{*}{ LAWAO 1} & 2 & -7193 & 500 & 49 & 56 & 73 \\
\hline & 3 & -4818 & 2375 & 31 & 43 & 62 \\
\hline & 5 & -4118 & 240 & -- & -- & - \\
\hline & 7 & -1888 & 1980 & 43 & 21 & 39 \\
\hline & 8 & -628 & 1260 & -- & -- & 27 \\
\hline & 9 & -128 & 500 & -- & -- & 20 \\
\hline & 10 & 160 & 288 & -- & -- & 17 \\
\hline & 12 & -7693 & 993 & 0 & -- & -- \\
\hline & 13 & -4358 & 460 & 1 & -- & -- \\
\hline & 15 & -3868 & 250 & 8 & -- & - \\
\hline \multirow[t]{9}{*}{ L AWA 02} & 2 & -4995 & 810 & 22 & 31 & 62 \\
\hline & 3 & -3062 & 1933 & 30 & 27 & 54 \\
\hline & 5 & -2330 & 295 & 5 & -- & 44 \\
\hline & 7 & -841 & 1314 & 56 & $<10$ & 36 \\
\hline & 8 & -63 & 778 & 28 & $<10$ & 21 \\
\hline & 9 & 102 & 165 & 40 & $<10$ & 14 \\
\hline & 12 & -5805 & 816 & 5 & -- & -- \\
\hline & 13 & -2625 & 437 & 0 & -- & -- \\
\hline & 15 & -2155 & 175 & 0 & -- & -- \\
\hline \multirow[t]{10}{*}{ L AWAO 3} & 2 & -6048 & 792 & 33 & 54 & 63 \\
\hline & 3 & -4052 & 1996 & 32 & 50 & 54 \\
\hline & 5 & -3428 & 200 & -- & -- & - \\
\hline & 7 & -1384 & 1844 & 58 & $<10$ & 39 \\
\hline & 8 & -386 & 998 & 61 & $<10$ & 25 \\
\hline & 9 & 57 & 443 & 55 & $<10$ & 18 \\
\hline & 10 & 294 & 237 & -- & -- & 14 \\
\hline & 12 & -6840 & 848 & 1 & -- & -- \\
\hline & 13 & -3628 & 424 & 0 & -- & -- \\
\hline & 15 & -3228 & 200 & 23 & -- & - \\
\hline
\end{tabular}


Table 4--Layer number; altitude of top, thickness, and sand percentage of layer: and average dissolved-solids concentration and temperature of water in layer: by log number--Continued.

\begin{tabular}{|c|c|c|c|c|c|c|}
\hline $\begin{array}{l}\text { Log } \\
\text { number }\end{array}$ & $\begin{array}{r}\text { Layer } \\
\text { number }\end{array}$ & $\begin{array}{r}\text { Altitude } \\
\text { of top } \\
(f \in e t)\end{array}$ & $\begin{array}{r}\text { Thick- } \\
\text { ness } \\
\text { (feet) }\end{array}$ & $\begin{array}{r}\text { Sand } \\
\text { per- } \\
\text { centage }\end{array}$ & $\begin{array}{l}\text { Dis- } \\
\text { solved } \\
\text { solids }\end{array}$ & $\begin{array}{l}\text { Tem- } \\
\text { per- } \\
\text { ature }\end{array}$ \\
\hline \multirow[t]{11}{*}{$\mathrm{LAWAO} 4$} & 2 & -7125 & 760 & 49 & 32 & 60 \\
\hline & 3 & -5303 & 1822 & 22 & $\cdots$ & 50 \\
\hline & 5 & -4665 & 255 & - & -- & $\cdots$ \\
\hline & 7 & -2445 & 2125 & 42 & 31 & 36 \\
\hline & 8 & -1075 & 1370 & 63 & $<10$ & 27 \\
\hline & 9 & -470 & 605 & 70 & $<10$ & 23 \\
\hline & 10 & -93 & 377 & 36 & $<10$ & 20 \\
\hline & 11 & 111 & 204 & 85 & $<10$ & 19 \\
\hline & 12 & -7885 & 1186 & 6 & - & $\cdots$ \\
\hline & 13 & -4920 & 383 & 0 & - & -- \\
\hline & 15 & -4570 & 95 & 0 & -- & -- \\
\hline \multirow[t]{10}{*}{ LAWAO5 5} & 2 & -5397 & 803 & 38 & 29 & 61 \\
\hline & 3 & -3699 & 1698 & 24 & 28 & 51 \\
\hline & 5 & -2842 & 585 & -- & - & -- \\
\hline & 7 & -1272 & 1405 & 52 & $<10$ & 32 \\
\hline & 8 & -472 & 800 & $\cdots$ & -- & 24 \\
\hline & 9 & -47 & 425 & -- & $\cdots$ & 19 \\
\hline & 10 & 87 & 134 & - & $\cdots$ & 17 \\
\hline & 12 & -6200 & 940 & 3 & - & $\cdots$ \\
\hline & 13 & -3427 & 272 & 0 & -- & $\cdots$ \\
\hline & 15 & -2677 & 165 & 0 & - & $\cdots$ \\
\hline \multirow[t]{8}{*}{$\mathrm{L} A W B O 1$} & 3 & -10765 & 1210 & 7 & -- & 94 \\
\hline & 7 & -4763 & 3817 & 43 & 102 & 68 \\
\hline & 8 & -2195 & 2568 & 57 & 40 & 49 \\
\hline & 9 & -1450 & 745 & 42 & $<10$ & 34 \\
\hline & 10 & -615 & 835 & 24 & $<10$ & 27 \\
\hline & 11 & 40 & 655 & 64 & $<10$ & 20 \\
\hline & 12 & -11975 & 2800 & 0 & - & $\cdots$ \\
\hline & 15 & -8580 & 2185 & 0 & -- & - \\
\hline \multirow[t]{8}{*}{ LAWCOI } & 2 & -1936 & 340 & 69 & 28 & 46 \\
\hline & 3 & -1584 & 352 & 54 & $<10$ & 44 \\
\hline & 4 & -1512 & 72 & 99 & $<10$ & 44 \\
\hline & 5 & -273 & 880 & 45 & $<10$ & 34 \\
\hline & 6 & -9 & 264 & 45 & $<10$ & 21 \\
\hline & 11 & 100 & 109 & $\cdots$ & - & 17 \\
\hline & 12 & -2276 & 421 & 0 & -- & $\cdots$ \\
\hline & 13 & -1153 & 359 & 7 & -- & -- \\
\hline \multirow[t]{9}{*}{ L AWCO2 } & 2 & -1898 & 250 & 54 & 12 & 30 \\
\hline & 3 & -1524 & 374 & 48 & -- & 29 \\
\hline & 4 & -1484 & 40 & 38 & -- & 28 \\
\hline & 5 & -494 & 664 & 55 & $<10$ & 24 \\
\hline & 6 & -23 & 353 & 80 & $<10$ & 21 \\
\hline & 11 & 97 & 120 & $\cdots$ & -- & 20 \\
\hline & 12 & -2148 & 356 & 0 & - & $\cdots$ \\
\hline & 13 & -1158 & 326 & 0 & - & $\cdots$ \\
\hline & 14 & -376 & 118 & 12 & -- & $\cdots$ \\
\hline
\end{tabular}


Table 4--Layer number; altitude of top, thickness, and sand percentage of layer; and average dissolved-solids concentration and temperature of water in layer; by log number--Continued.

\begin{tabular}{|c|c|c|c|c|c|c|}
\hline $\begin{array}{l}\text { Log } \\
\text { number }\end{array}$ & $\begin{array}{r}\text { Layer } \\
\text { number }\end{array}$ & $\begin{array}{r}\text { Altitude } \\
\text { of top } \\
(f e e t)\end{array}$ & $\begin{array}{r}\text { Thick- } \\
\text { ness } \\
\text { (feet) }\end{array}$ & $\begin{array}{r}\text { Sand } \\
\text { per- } \\
\text { centage }\end{array}$ & $\begin{array}{l}\text { Dis- } \\
\text { solved } \\
\text { solids }\end{array}$ & $\begin{array}{l}\text { Tem- } \\
\text { per- } \\
\text { ature }\end{array}$ \\
\hline \multirow[t]{7}{*}{ L AWE 01} & 2 & -891 & 93 & و 9 & $<10$ & 29 \\
\hline & 3 & -434 & 457 & 50 & $<10$ & 27 \\
\hline & 4 & -414 & 20 & 50 & -- & 25 \\
\hline & 5 & 124 & 365 & 63 & $<10$ & 21 \\
\hline & 12 & -984 & 635 & 0 & -- & -- \\
\hline & 13 & -241 & 173 & 16 & -- & -- \\
\hline & 14 & 265 & 141 & -- & -- & -- \\
\hline \multirow[t]{7}{*}{ L AWEO 2} & 2 & -996 & 72 & 81 & $<10$ & 30 \\
\hline & 3 & -633 & 363 & 35 & $<10$ & 28 \\
\hline & 4 & -522 & 111 & 76 & $<10$ & 26 \\
\hline & 5 & 127 & 337 & 57 & $<10$ & 21 \\
\hline & 12 & -1068 & 664 & 0 & - - & -- \\
\hline & 13 & -210 & 312 & 12 & -- & - \\
\hline & 14 & 208 & 81 & -- & -- & -- \\
\hline \multirow[t]{5}{*}{ LAWEO 3} & 2 & -313 & 20 & 50 & -- & 24 \\
\hline & 3 & 70 & 383 & 27 & -- & 22 \\
\hline & 4 & 104 & 34 & 99 & $<10$ & 20 \\
\hline & 12 & -333 & 789 & 0 & - - & -- \\
\hline & 13 & 205 & 101 & -- & -- & -- \\
\hline \multirow[t]{10}{*}{ LAWF 01} & 3 & -8046 & 3232 & 43 & 78 & 86 \\
\hline & 5 & -7156 & 202 & 64 & 89 & 70 \\
\hline & 7 & -3325 & 2611 & 52 & 70 & 53 \\
\hline & 8 & -1491 & 1834 & 52 & 29 & 37 \\
\hline & 9 & -652 & 839 & 35 & $<10$ & 27 \\
\hline & 10 & -128 & 524 & 48 & $<10$ & 22 \\
\hline & 11 & 40 & 168 & -- & - - & 19 \\
\hline & 12 & -11278 & 2227 & 0 & -- & - \\
\hline & 13 & -7358 & 688 & 3 & -- & $\cdots$ \\
\hline & 15 & -5936 & 1220 & 6 & -- & -- \\
\hline \multirow[t]{11}{*}{ L AWF 02} & 3 & -7137 & -- & -- & -- & -- \\
\hline & 5 & -6290 & 500 & 19 & 19 & 62 \\
\hline & 6 & -5613 & 284 & 27 & 29 & 57 \\
\hline & 7 & -2620 & 2450 & 46 & 75 & 45 \\
\hline & 8 & -990 & 1630 & 30 & 37 & 31 \\
\hline & 9 & -290 & 700 & -- & -- & 24 \\
\hline & 10 & 150 & 440 & -- & -- & 20 \\
\hline & 11 & 295 & 145 & -- & -- & 18 \\
\hline & 13 & -6790 & 347 & 1 & -- & -- \\
\hline & 14 & -5897 & 393 & 2 & - & -- \\
\hline & 15 & $-50>0$ & 543 & 0 & -- & -- \\
\hline \multirow[t]{9}{*}{ LAWF 03} & 3 & -7890 & -- & -- & - & -- \\
\hline & 5 & -6940 & 242 & 6 & -- & 55 \\
\hline & 7 & -3225 & 2722 & 51 & 44 & 44 \\
\hline & 8 & -1410 & 1815 & 47 & 20 & 32 \\
\hline & 9 & -660 & 750 & 46 & $<10$ & 26 \\
\hline & 10 & -60 & 600 & -- & -- & 22 \\
\hline & 11 & 230 & 290 & -- & -- & 20 \\
\hline & 13 & -7182 & 708 & 3 & - & -- \\
\hline & 15 & -5947 & 993 & 5 & - & -- \\
\hline
\end{tabular}


Table 4--Layer number; altitude of top, thickness, and sand percentage of layer; and average dissolved-solids concentration and temperature of water in layer; by log number--Continued.

\begin{tabular}{|c|c|c|c|c|c|c|}
\hline $\begin{array}{l}\text { Log } \\
\text { number }\end{array}$ & $\begin{array}{r}\text { Layer } \\
\text { number }\end{array}$ & $\begin{array}{r}\text { Altitude } \\
\text { of top } \\
\text { (feet) }\end{array}$ & $\begin{array}{r}\text { Thick- } \\
\text { ness } \\
\text { (feet) }\end{array}$ & $\begin{array}{r}\text { Sand } \\
\text { per- } \\
\text { centage }\end{array}$ & $\begin{array}{l}\text { Dis- } \\
\text { solved } \\
\text { solids }\end{array}$ & $\begin{array}{r}\text { Tem- } \\
\text { per- } \\
\text { ature }\end{array}$ \\
\hline \multirow[t]{7}{*}{ L AWIO 1} & 2 & -1661 & 670 & 52 & 17 & 40 \\
\hline & 3 & -716 & 945 & 37 & $<10$ & 33 \\
\hline & 4 & -636 & 80 & 79 & $<10$ & 27 \\
\hline & 5 & 59 & 530 & 45 & $<10$ & 23 \\
\hline & 12 & -2331 & 830 & 0 & -- & - \\
\hline & 13 & -471 & 165 & 0 & -- & -- \\
\hline & 14 & 169 & 110 & 12 & -- & -- \\
\hline \multirow[t]{6}{*}{ LAWIO 2} & 3 & -1161 & -- & -- & -- & -- \\
\hline & 4 & -1053 & 108 & 99 & 13 & 30 \\
\hline & 5 & -161 & 673 & 59 & $<10$ & 25 \\
\hline & 6 & 180 & 211 & -- & -- & 20 \\
\hline & 13 & -834 & 219 & 6 & -- & -- \\
\hline & 14 & -31 & 130 & 9 & -- & - \\
\hline \multirow[t]{4}{*}{270001} & 9 & -4359 & 3150 & 21 & 58 & 60 \\
\hline & 10 & -1059 & 3300 & 48 & 52 & 37 \\
\hline & 11 & -53 & 1006 & 47 & 12 & 26 \\
\hline & 17 & -7509 & 1849 & 7 & -- & - \\
\hline \multirow[t]{5}{*}{170002} & 8 & -6217 & 3825 & 49 & 172 & 69 \\
\hline & 9 & -3005 & 3212 & 62 & 141 & 46 \\
\hline & 10 & -922 & 2083 & 17 & 70 & 33 \\
\hline & 11 & -13 & 909 & 36 & -- & 25 \\
\hline & 16 & -10042 & 1040 & 4 & -- & - \\
\hline \multirow[t]{4}{*}{170101} & 9 & -3371 & 2905 & 19 & -- & 57 \\
\hline & 10 & -1006 & 2365 & 44 & 48 & 39 \\
\hline & 11 & -59 & 947 & 48 & 32 & 28 \\
\hline & 17 & -6276 & 470 & 3 & -- & - \\
\hline \multirow[t]{4}{*}{ L 70102} & 9 & -4878 & 2965 & 10 & 85 & 55 \\
\hline & 10 & -1113 & 3765 & 48 & 90 & 34 \\
\hline & 11 & -102 & 1011 & 20 & 59 & 30 \\
\hline & 17 & -7843 & 14 & 0 & -- & -- \\
\hline \multirow[t]{3}{*}{ L 70201} & 9 & -5471 & 5590 & 21 & -- & -- \\
\hline & 10 & -1151 & 4320 & -- & -- & - \\
\hline & 11 & -152 & 999 & -- & -- & -- \\
\hline \multirow[t]{3}{*}{ L 70202} & 9 & -5640 & 771 & -- & -- & -- \\
\hline & 10 & -1190 & 4450 & 33 & 21 & 45 \\
\hline & 11 & -297 & 893 & -- & -- & 27 \\
\hline \multirow[t]{3}{*}{270301} & 9 & -5590 & 5345 & 31 & -- & -- \\
\hline & 10 & -1140 & 4450 & 43 & 46 & 45 \\
\hline & 11 & -102 & 1038 & 85 & 34 & 28 \\
\hline
\end{tabular}


Table 4--Layer number; altitude of top, thickness, and sand percentage of layer; and average dissolved-solids concentration and temperature of water in layer; by log number--Continued.

\begin{tabular}{|c|c|c|c|c|c|c|}
\hline $\begin{array}{l}\text { Log } \\
\text { number }\end{array}$ & $\begin{array}{r}\text { Layer } \\
\text { number }\end{array}$ & $\begin{array}{r}\text { Altide } \\
\text { of top } \\
(\text { feet) }\end{array}$ & $\begin{array}{r}\text { Thick- } \\
\text { ness } \\
(\text { feet) }\end{array}$ & $\begin{array}{r}\text { Sand } \\
\text { per- } \\
\text { centage }\end{array}$ & $\begin{array}{l}\text { Dis- } \\
\text { solved } \\
\text { solids }\end{array}$ & $\begin{array}{r}\text { Tem- } \\
\text { Per- } \\
\text { ature }\end{array}$ \\
\hline \multirow[t]{3}{*}{$L 70302$} & 9 & -5412 & 3265 & 24 & -- & $\ldots$ \\
\hline & 10 & -1092 & 4320 & 64 & -- & -- \\
\hline & 11 & -53 & 1039 & -- & -- & -- \\
\hline \multirow[t]{4}{*}{$L 70303$} & 8 & -9440 & 2372 & 11 & 93 & 91 \\
\hline & 9 & -5160 & 4280 & 20 & 60 & 71 \\
\hline & 10 & -1065 & 4095 & 54 & -- & 44 \\
\hline & 11 & -17 & 1048 & -- & -- & 27 \\
\hline \multirow[t]{3}{*}{ L 70401} & 9 & -5802 & - & -- & -- & - \\
\hline & 10 & -1142 & 4660 & 34 & 42 & 50 \\
\hline & 11 & -162 & 980 & 63 & -- & 27 \\
\hline \multirow[t]{3}{*}{ L 70402} & 9 & -5963 & 1255 & -- & -- & -- \\
\hline & 10 & -1213 & 4750 & 34 & 30 & 46 \\
\hline & 11 & -294 & 919 & 37 & -- & 30 \\
\hline \multirow[t]{4}{*}{ L 70501} & 8 & -9709 & 3760 & 21 & 236 & 83 \\
\hline & 9 & -5309 & 4400 & 30 & 199 & 69 \\
\hline & 10 & -1069 & 4240 & 54 & 116 & 54 \\
\hline & 11 & 0 & 1069 & 43 & $<10$ & 27 \\
\hline \multirow[t]{3}{*}{ L70502 } & 9 & -5733 & 3690 & 31 & 107 & 62 \\
\hline & 10 & -1148 & 4585 & 48 & 71 & 44 \\
\hline & 11 & -99 & 1049 & 25 & 14 & 27 \\
\hline \multirow[t]{4}{*}{$L 70503$} & 8 & -10416 & 273 & -- & -- & -- \\
\hline & 9 & -5516 & 4900 & 21 & 90 & 75 \\
\hline & 10 & -1086 & 4430 & 32 & 68 & 51 \\
\hline & 11 & -7 & 1079 & 33 & 21 & 28 \\
\hline \multirow[t]{3}{*}{$L 70601$} & 9 & -5901 & 4664 & 26 & 87 & 70 \\
\hline & 10 & -1141 & 4760 & 44 & 64 & 47 \\
\hline & 11 & -152 & 989 & -- & -- & 26 \\
\hline \multirow[t]{2}{*}{$L 70602$} & 10 & -1185 & 4803 & 21 & 29 & -- \\
\hline & 11 & -244 & 941 & -- & - & 26 \\
\hline \multirow[t]{4}{*}{$L 70701$} & 8 & -10173 & 1250 & 20 & -- & -- \\
\hline & 9 & -5373 & 4800 & 42 & -- & -- \\
\hline & 10 & -1073 & 4300 & 51 & -- & -- \\
\hline & 11 & -17 & 1056 & -- & -- & -- \\
\hline \multirow[t]{4}{*}{ L 70702} & 8 & -11869 & 1790 & 25 & -- & -- \\
\hline & 9 & -5924 & 5945 & 30 & 78 & 73 \\
\hline & 10 & -1139 & 4785 & 37 & 62 & 43 \\
\hline & 11 & -102 & 1037 & 31 & 32 & 26 \\
\hline
\end{tabular}


Table 4--Layer number; altitude of top, thickness, and sand percentage of layer; and average dissolved-solids concentration and temperature of water in layer; by log number--Continued.

\begin{tabular}{|c|c|c|c|c|c|c|}
\hline $\begin{array}{l}\text { Log } \\
\text { number }\end{array}$ & $\begin{array}{r}\text { Layer } \\
\text { number }\end{array}$ & $\begin{array}{r}\text { Altitude } \\
\text { of top } \\
\text { (feet) }\end{array}$ & $\begin{array}{r}\text { Thlek- } \\
\text { ness } \\
\text { (feet) }\end{array}$ & $\begin{array}{r}\text { Sand } \\
\text { per- } \\
\text { centage }\end{array}$ & $\begin{array}{l}\text { Dis- } \\
\text { solved } \\
\text { solids }\end{array}$ & $\begin{array}{l}\text { Tem- } \\
\text { per- } \\
\text { ature }\end{array}$ \\
\hline \multirow[t]{4}{*}{ L. 70703} & 8 & -11110 & - - & -- & $\ldots$ & $\ldots$ \\
\hline & 9 & -5725 & 5385 & 35 & 100 & 69 \\
\hline & 10 & -1145 & 4580 & 43 & 58 & 51 \\
\hline & 11 & -46 & 1099 & 0 & -- & 27 \\
\hline \multirow[t]{2}{*}{ L. 70801} & 10 & -1223 & 4524 & 9 & 82 & 49 \\
\hline & 11 & -251 & 972 & -- & - & 29 \\
\hline \multirow[t]{3}{*}{ L 70802} & 9 & -6268 & 3300 & 24 & 69 & 67 \\
\hline & 10 & -1268 & 5000 & 30 & -- & 45 \\
\hline & 11 & -175 & 1093 & 37 & -- & 29 \\
\hline \multirow[t]{3}{*}{ L 70901} & 9 & -6573 & 3300 & 22 & 139 & 60 \\
\hline & 10 & -1223 & 5350 & 38 & 101 & 41 \\
\hline & 11 & -69 & 1154 & -- & - & 27 \\
\hline \multirow[t]{4}{*}{ L 70902} & 8 & -11123 & 200 & 73 & 107 & 82 \\
\hline & 9 & -5743 & 5380 & 39 & 124 & 64 \\
\hline & 10 & -1143 & 4600 & 48 & 48 & 43 \\
\hline & 11 & -33 & 1110 & 89 & $<10$ & 27 \\
\hline \multirow[t]{4}{*}{ L 70903} & 8 & -10713 & 1010 & -- & -- & -- \\
\hline & 9 & -5513 & 5200 & 41 & 48 & 62 \\
\hline & 10 & -1083 & 4430 & 47 & 32 & 40 \\
\hline & 11 & -7 & 1076 & 53 & $<10$ & 27 \\
\hline \multirow[t]{4}{*}{ L 70904} & 8 & -12456 & 2435 & 26 & 196 & 93 \\
\hline & 9 & -6381 & 6075 & 35 & 194 & 71 \\
\hline & 10 & -1191 & 5190 & 54 & 139 & 42 \\
\hline & 11 & -79 & 1112 & -- & - & 28 \\
\hline \multirow[t]{3}{*}{ L. 71001} & 9 & -6663 & 1620 & 7 & 62 & 60 \\
\hline & 10 & -1263 & 5400 & 44 & 56 & 46 \\
\hline & 11 & -198 & 1065 & 29 & 21 & 26 \\
\hline \multirow[t]{2}{*}{ L 71002} & 10 & -1277 & 2950 & 21 & 39 & 48 \\
\hline & 11 & -304 & 973 & -- & -- & 28 \\
\hline \multirow[t]{4}{*}{ L 71101} & 8 & -11918 & -- & -- & -- & - \\
\hline & 9 & -6108 & 5810 & 53 & 55 & 74 \\
\hline & 10 & -1108 & 5000 & 55 & 45 & 45 \\
\hline & 11 & -3 & 1105 & 26 & 16 & 29 \\
\hline \multirow[t]{4}{*}{ L 71102} & 8 & -12328 & 540 & 19 & -- & -- \\
\hline & 9 & -6348 & 5980 & 46 & 136 & 71 \\
\hline & 10 & -1143 & 5205 & 44 & 77 & 60 \\
\hline & 11 & -13 & 1130 & 55 & 17 & 29 \\
\hline
\end{tabular}


Table 4--Layer number; altitude of top, thickness, and sand percentage of layer; and average dissolved-solids concentration and temperature of water in layer; by log number--Continued.

\begin{tabular}{|c|c|c|c|c|c|c|}
\hline $\begin{array}{l}\text { Log } \\
\text { number }\end{array}$ & $\begin{array}{l}\text { Layer } \\
\text { number }\end{array}$ & $\begin{array}{r}\text { Altitude } \\
\text { of top } \\
\text { (feet) }\end{array}$ & $\begin{array}{r}\text { Thick- } \\
\text { ness } \\
\text { (feet) }\end{array}$ & $\begin{array}{r}\text { Sand } \\
\text { per- } \\
\text { centage }\end{array}$ & $\begin{array}{l}\text { Dis- } \\
\text { solved } \\
\text { solids }\end{array}$ & $\begin{array}{l}\text { Tem- } \\
\text { per- } \\
\text { ature }\end{array}$ \\
\hline \multirow[t]{3}{*}{171103} & 9 & -6732 & -- & -- & -- & -- \\
\hline & 10 & -1277 & 5455 & 31 & 53 & 47 \\
\hline & 11 & -53 & 1224 & 64 & 30 & 29 \\
\hline \multirow[t]{4}{*}{271104} & 8 & -11193 & -- & -- & -- & -- \\
\hline & 9 & -5803 & 5390 & 46 & 110 & 65 \\
\hline & 10 & -1103 & 4700 & 52 & 85 & 38 \\
\hline & 11 & -3 & 1100 & 49 & -- & 28 \\
\hline \multirow[t]{3}{*}{171201} & 9 & -6929 & 3565 & 19 & -- & 68 \\
\hline & 10 & -1279 & 5650 & 25 & 42 & 42 \\
\hline & 11 & -162 & 1117 & -- & -- & 23 \\
\hline \multirow[t]{3}{*}{ L71202 } & 9 & -6744 & 2155 & 18 & 99 & -- \\
\hline & 10 & -1239 & 5505 & 29 & 73 & 47 \\
\hline & 11 & -182 & 1057 & -- & -- & 24 \\
\hline \multirow[t]{2}{*}{ L71203 } & 10 & -1287 & 4520 & 24 & -- & -- \\
\hline & 11 & -383 & 904 & -- & -- & 23 \\
\hline \multirow[t]{3}{*}{ L71301 } & 9 & -6397 & 5855 & 39 & -- & 74 \\
\hline & 10 & -1142 & 5255 & 40 & 30 & 51 \\
\hline & 11 & -13 & 1129 & -- & 34 & 29 \\
\hline \multirow[t]{3}{*}{171501} & 9 & -6338 & -- & -- & -- & -- \\
\hline & 10 & -1093 & 5245 & 56 & 120 & 42 \\
\hline & 11 & -3 & 1090 & 40 & 29 & 27 \\
\hline \multirow[t]{4}{*}{ L 71502} & 8 & -12305 & 450 & 19 & -- & -- \\
\hline & 9 & -6385 & 5920 & 36 & 78 & 69 \\
\hline & 10 & -1135 & 5250 & 37 & 51 & 42 \\
\hline & 11 & -46 & 1089 & -- & -- & 27 \\
\hline \multirow[t]{4}{*}{171503} & 8 & -12366 & 600 & 18 & -- & -- \\
\hline & 9 & -6366 & 6000 & 44 & 106 & 76 \\
\hline & 10 & -1161 & 5205 & 35 & 65 & 54 \\
\hline & 11 & -3 & 1158 & 33 & -- & 27 \\
\hline \multirow[t]{3}{*}{171504} & 9 & -6785 & 6330 & 27 & 82 & 70 \\
\hline & 10 & -1235 & 5550 & 20 & 36 & 42 \\
\hline & 11 & -66 & 1169 & -- & 32 & 27 \\
\hline \multirow[t]{3}{*}{ L 71505} & 9 & -6711 & 5400 & 26 & 104 & 98 \\
\hline & 10 & -1211 & 5500 & 15 & 71 & 55 \\
\hline & 11 & -132 & 1079 & 27 & 26 & 33 \\
\hline \multirow[t]{3}{*}{ L71601 } & 9 & -6928 & 500 & -- & -- & -- \\
\hline & 10 & -1278 & 5650 & 15 & 27 & -- \\
\hline & 11 & -185 & 1093 & 26 & 28 & 23 \\
\hline
\end{tabular}


Table 4--Layer number; altitude of top, thickness, and sand percentage of layer; and average dissolved-solids concentration and temperature of water in layer; by log number--Continued.

\begin{tabular}{|c|c|c|c|c|c|c|}
\hline $\begin{array}{l}\text { Log } \\
\text { number }\end{array}$ & $\begin{array}{r}\text { Layer } \\
\text { number }\end{array}$ & $\begin{array}{r}\text { Alt } 1 \text { tude } \\
\text { of top } \\
(\text { feet) }\end{array}$ & $\begin{array}{r}\text { Thick- } \\
\text { ness } \\
(\text { feet })\end{array}$ & $\begin{array}{r}\text { Sand } \\
\text { per- } \\
\text { centage }\end{array}$ & $\begin{array}{l}\text { Dis- } \\
\text { solved } \\
\text { solids }\end{array}$ & $\begin{array}{l}\text { Tem- } \\
\text { per- } \\
\text { ature }\end{array}$ \\
\hline \multirow[t]{2}{*}{171602} & 10 & -1293 & 4115 & 23 & -- & 43 \\
\hline & 11 & -396 & 897 & - & -- & 26 \\
\hline \multirow[t]{2}{*}{171603} & 10 & -1246 & 4560 & 9 & 22 & 41 \\
\hline & 11 & -251 & 995 & 16 & -- & 24 \\
\hline \multirow[t]{4}{*}{271701} & 8 & -12108 & 700 & 42 & 54 & 90 \\
\hline & 9 & -6298 & 5810 & 26 & 52 & 68 \\
\hline & 10 & -1133 & 5165 & 44 & 52 & 40 \\
\hline & 11 & -99 & 1034 & 17 & 32 & 27 \\
\hline \multirow[t]{3}{*}{271901} & 9 & -5669 & 5190 & 26 & 95 & 67 \\
\hline & 10 & -1154 & 4515 & 47 & 74 & 39 \\
\hline & 11 & -56 & 1098 & -- & 15 & 30 \\
\hline \multirow[t]{4}{*}{$L 71902$} & 8 & -12117 & -- & -- & -- & $\cdots$ \\
\hline & 9 & -6302 & 5815 & 28 & 134 & 74 \\
\hline & 10 & -1142 & 5160 & 38 & 59 & 45 \\
\hline & 11 & -139 & 1003 & -- & -- & 30 \\
\hline \multirow[t]{4}{*}{172001} & 8 & -12547 & -- & -- & -- & - \\
\hline & 9 & -6537 & 6010 & 5 & 94 & 81 \\
\hline & 10 & -1197 & 5340 & 0 & -- & 47 \\
\hline & 11 & -396 & 801 & -- & -- & 31 \\
\hline \multirow[t]{4}{*}{ L72101 } & 8 & -10013 & 1760 & 10 & 120 & 98 \\
\hline & 9 & -5213 & 4800 & 30 & 95 & 66 \\
\hline & 10 & -1103 & 4110 & 28 & 65 & 46 \\
\hline & 11 & -3 & 1100 & 27 & 12 & 28 \\
\hline \multirow[t]{4}{*}{172102} & 8 & -10963 & 210 & -- & -- & - \\
\hline & 9 & -5643 & 5320 & 14 & 69 & 64 \\
\hline & 10 & -1133 & 4510 & 17 & 38 & 44 \\
\hline & 11 & -3 & 1130 & 0 & -- & 29 \\
\hline \multirow[t]{4}{*}{172103} & 8 & -10773 & 850 & -- & -- & - \\
\hline & 9 & -5573 & 5200 & 17 & 147 & 70 \\
\hline & 10 & -1123 & 4450 & 26 & 145 & 40 \\
\hline & 11 & -3 & 1120 & 9 & -- & 30 \\
\hline \multirow[t]{4}{*}{172104} & 8 & -10929 & 1040 & -- & -- & $\ldots$ \\
\hline & 9 & -5679 & 5250 & 10 & 41 & 66 \\
\hline & 10 & -1179 & 4500 & 8 & 95 & 44 \\
\hline & 11 & -323 & 856 & -- & -- & 31 \\
\hline \multirow[t]{4}{*}{ L72201 } & 8 & -12193 & 2820 & 21 & 77 & 97 \\
\hline & 9 & -6333 & 5860 & 12 & 72 & 75 \\
\hline & 10 & -1183 & 5150 & -- & -- & 47 \\
\hline & 11 & -370 & 813 & -- & - & 31 \\
\hline
\end{tabular}


Table 4--Layer number; altitude of top, thickness, and sand percentage of layer; and average dissolved-solids concentration and temperature of water in layer; by log number--Continued.

\begin{tabular}{|c|c|c|c|c|c|c|}
\hline $\begin{array}{l}\text { Log } \\
\text { number }\end{array}$ & $\begin{array}{r}\text { Layer } \\
\text { number }\end{array}$ & $\begin{array}{r}\text { Altitude } \\
\text { of top } \\
(\text { feet) }\end{array}$ & $\begin{array}{r}\text { Thick- } \\
\text { ness } \\
(\text { feet })\end{array}$ & $\begin{array}{r}\text { Sand } \\
\text { per- } \\
\text { centage }\end{array}$ & $\begin{array}{l}\text { Dis- } \\
\text { solved } \\
\text { solids }\end{array}$ & $\begin{array}{l}\text { Tem- } \\
\text { per- } \\
\text { ature }\end{array}$ \\
\hline \multirow[t]{3}{*}{$L 72301$} & 9 & -5212 & 4695 & 13 & 122 & 68 \\
\hline & 10 & -1097 & 4115 & 12 & 116 & 41 \\
\hline & 11 & -50 & 1047 & -- & $\ldots$ & 30 \\
\hline \multirow[t]{4}{*}{272401} & 8 & -5615 & 1285 & -- & -- & -- \\
\hline & 9 & -3115 & 2500 & 15 & 56 & 54 \\
\hline & 10 & -900 & 2215 & 26 & 29 & 40 \\
\hline & 11 & -210 & 690 & -- & 13 & 29 \\
\hline \multirow[t]{4}{*}{ L 72402} & 8 & -5450 & 960 & -- & -- & -- \\
\hline & 9 & -3050 & 2400 & 1 & -- & -- \\
\hline & 10 & -896 & 2154 & 31 & 32 & 37 \\
\hline & 11 & -300 & 596 & -- & -- & 30 \\
\hline \multirow[t]{5}{*}{$L 72501$} & 8 & -6413 & 4000 & 18 & -- & 66 \\
\hline & 9 & -3413 & 3000 & 59 & 45 & 46 \\
\hline & 10 & -958 & 2455 & 45 & 37 & 36 \\
\hline & 11 & 0 & 958 & 31 & -- & 29 \\
\hline & 16 & -10413 & 610 & 4 & -- & -- \\
\hline \multirow[t]{4}{*}{$L 72502$} & 8 & -6739 & 1770 & -- & -- & -- \\
\hline & 9 & -3839 & 3900 & 19 & 56 & -- \\
\hline & 10 & -929 & 2910 & 29 & 31 & 41 \\
\hline & 11 & -60 & 869 & -- & -- & 28 \\
\hline \multirow[t]{4}{*}{ L 72601 } & 8 & -8118 & 2500 & 13 & 73 & 76 \\
\hline & 9 & -3983 & 4135 & 43 & 36 & 54 \\
\hline & 10 & -943 & 3040 & 56 & 24 & 38 \\
\hline & 11 & -3 & 940 & 37 & $<10$ & 29 \\
\hline \multirow[t]{5}{*}{ L 72701} & 8 & -5225 & 3830 & 24 & 119 & 56 \\
\hline & 9 & -2975 & 2250 & 53 & -- & 49 \\
\hline & 10 & -950 & 2025 & 35 & -- & 40 \\
\hline & 11 & -15 & 935 & 1 & -- & 27 \\
\hline & 16 & -9055 & 495 & 2 & -- & -- \\
\hline \multirow[t]{5}{*}{ MODUO 1} & 1 & -417 & -- & -- & -- & -- \\
\hline & 2 & 53 & 95 & 99 & -- & -- \\
\hline & 3 & 146 & 93 & -- & $\ldots$ & -- \\
\hline & 4 & 380 & 234 & -- & -- & -- \\
\hline & 12 & -42 & 375 & 0 & - & -- \\
\hline \multirow[t]{6}{*}{ MODUO2 } & 1 & -465 & -- & -- & -- & -- \\
\hline & 2 & -15 & 108 & -- & -- & -- \\
\hline & 3 & 22 & 37 & -- & -- & -- \\
\hline & 4 & 272 & 250 & 46 & -- & - \\
\hline & 11 & 305 & 33 & 97 & -- & -- \\
\hline & 12 & -123 & 342 & -- & -- & -- \\
\hline
\end{tabular}


Table 4--Layer number; altitude of top, thickness, and sand percentage of layer; and average dissolved-solids concentration and temperature of water in layer; by log number--Continued.

\begin{tabular}{|c|c|c|c|c|c|c|}
\hline $\begin{array}{l}\text { Log } \\
\text { number }\end{array}$ & $\begin{array}{r}\text { Layer } \\
\text { number }\end{array}$ & $\begin{array}{r}\text { Altitude } \\
\text { of top } \\
(f e e t)\end{array}$ & $\begin{array}{r}\text { Thick- } \\
\text { ness } \\
(\text { feet) }\end{array}$ & $\begin{array}{r}\text { Sand } \\
\text { per- } \\
\text { centage }\end{array}$ & $\begin{array}{l}\text { Dis- } \\
\text { solved } \\
\text { solids }\end{array}$ & $\begin{array}{r}\text { Tem- } \\
\text { per- } \\
\text { ature }\end{array}$ \\
\hline \multirow[t]{6}{*}{ MODUO3 } & 1 & -1179 & -- & -- & -- & -- \\
\hline & 2 & -378 & 255 & 99 & $<10$ & 24 \\
\hline & 3 & -315 & 63 & -- & -- & 22 \\
\hline & 4 & 91 & 406 & 79 & $<10$ & 19 \\
\hline & 11 & 247 & 156 & -- & $<10$ & 15 \\
\hline & 12 & -633 & 546 & 0 & -- & - \\
\hline \multirow[t]{6}{*}{ MODUO 4} & 2 & -512 & 155 & 99 & -- & -- \\
\hline & 3 & -422 & 90 & 99 & -- & -. \\
\hline & 4 & -147 & 275 & 31 & -- & - \\
\hline & 5 & 43 & 190 & 99 & -- & -- \\
\hline & 11 & 267 & 224 & -- & -- & - \\
\hline & 12 & -667 & -- & -- & -- & -- \\
\hline \multirow[t]{8}{*}{ MODUO5 } & 1 & -1552 & -- & -- & -- & $\cdots$ \\
\hline & 2 & -754 & 270 & 96 & -- & - \\
\hline & 3 & -608 & 146 & 21 & -- & -- \\
\hline & 4 & -284 & 324 & 99 & -- & -- \\
\hline & 5 & -79 & 205 & 55 & -- & -- \\
\hline & 11 & 246 & 174 & 99 & -- & $\cdots$ \\
\hline & 12 & -1024 & 528 & 0 & -- & -- \\
\hline & 14 & 72 & 151 & 0 & -- & - \\
\hline \multirow[t]{7}{*}{ MOMSO 1} & 1 & -860 & 246 & 99 & -- & $\cdots$ \\
\hline & 2 & -402 & 122 & 99 & -- & -- \\
\hline & 3 & -336 & 66 & 18 & -- & -- \\
\hline & 4 & -156 & 180 & 88 & -- & -- \\
\hline & 5 & 12 & 168 & 12 & -- & -- \\
\hline & 11 & 293 & 281 & 99 & -- & -- \\
\hline & 12 & -524 & 336 & 0 & -- & -- \\
\hline \multirow[t]{6}{*}{ MOMSO2 } & 1 & -420 & -- & -- & -- & -- \\
\hline & 2 & -146 & 28 & 99 & -- & -- \\
\hline & 3 & -88 & 58 & 24 & -- & -- \\
\hline & 4 & 200 & 288 & 83 & -- & - \\
\hline & 11 & 315 & 115 & -- & -- & -- \\
\hline & 12 & -174 & 246 & 0 & -- & - \\
\hline \multirow[t]{6}{*}{ MONMO 1} & 1 & -744 & 275 & 93 & -- & $\cdots$ \\
\hline & 2 & -362 & 108 & 99 & -- & -- \\
\hline & 3 & -248 & 124 & 79 & -- & - \\
\hline & 4 & 95 & 343 & 42 & -- & -- \\
\hline & 11 & 269 & 174 & -- & -- & -- \\
\hline & 12 & $-4>0$ & 274 & 0 & -- & -- \\
\hline \multirow[t]{9}{*}{ MONMO 2} & 1 & -1402 & 332 & 87 & -- & -- \\
\hline & 2 & -906 & 150 & 99 & -- & -- \\
\hline & 3 & -766 & 140 & 43 & -- & -- \\
\hline & 4 & -461 & 305 & 99 & -- & -- \\
\hline & 5 & -311 & 150 & 99 & -- & -- \\
\hline & 6 & 154 & 333 & 49 & -- & -- \\
\hline & 11 & 284 & 130 & -- & -- & -- \\
\hline & 12 & -1056 & 346 & 0 & -- & -- \\
\hline & 14 & -179 & 132 & 0 & -- & -- \\
\hline
\end{tabular}


Table 4--Layer number; altitude of top, thickness, and sand percentage of layer; and average dissolved-solids concentration and temperature of water in layer; by log number--Continued.

\begin{tabular}{|c|c|c|c|c|c|c|}
\hline $\begin{array}{l}\text { Log } \\
\text { number }\end{array}$ & $\begin{array}{r}\text { Layer } \\
\text { number }\end{array}$ & $\begin{array}{r}\text { Altitude } \\
\text { of top } \\
(f e e t)\end{array}$ & $\begin{array}{r}\text { Thick- } \\
\text { ness } \\
\text { (feet) }\end{array}$ & $\begin{array}{r}\text { Sand } \\
\text { per- } \\
\text { centage }\end{array}$ & $\begin{array}{l}\text { Dis- } \\
\text { solved } \\
\text { solids }\end{array}$ & $\begin{array}{l}\text { Tem- } \\
\text { per- } \\
\text { ature }\end{array}$ \\
\hline \multirow[t]{9}{*}{ MONMO 3} & 1 & -966 & 284 & 87 & -- & -- \\
\hline & 2 & -565 & 127 & 84 & -- & -- \\
\hline & 3 & -440 & 125 & 62 & -- & -- \\
\hline & 4 & -223 & 217 & 76 & -- & -- \\
\hline & 5 & -33 & 190 & 25 & $\cdots$ & $\cdots$ \\
\hline & 6 & 170 & 85 & 99 & $\ldots$ & - \\
\hline & 11 & 302 & 132 & -- & -- & -- \\
\hline & 12 & -692 & 274 & 0 & -- & -- \\
\hline & 14 & 85 & 118 & 0 & -- & -- \\
\hline \multirow[t]{9}{*}{ MOPEOI } & 1 & -1858 & -- & -- & -- & -- \\
\hline & 2 & -933 & 405 & 99 & -- & -- \\
\hline & 3 & -776 & 157 & 16 & -- & -- \\
\hline & 4 & -400 & 376 & 76 & -- & -- \\
\hline & 5 & -263 & 137 & 87 & -- & -- \\
\hline & 6 & 80 & 184 & 44 & -- & -- \\
\hline & 11 & 255 & 175 & -- & $\cdots$ & -- \\
\hline & 12 & -1338 & 520 & 0 & -- & -- \\
\hline & 14 & -104 & 159 & 0 & $\cdots$ & -- \\
\hline \multirow[t]{8}{*}{ MOPEO 2} & 1 & -1364 & 426 & 85 & -- & -- \\
\hline & 2 & -601 & 332 & 99 & $\cdots$ & - \\
\hline & 3 & -463 & 138 & 32 & -- & - \\
\hline & 4 & -59 & 404 & 77 & -- & -- \\
\hline & 5 & 11 & 70 & -- & -- & -- \\
\hline & 11 & 266 & 210 & -- & -- & $\cdots$ \\
\hline & 12 & -933 & 431 & 0 & -- & -- \\
\hline & 14 & 56 & 45 & -- & -- & -- \\
\hline \multirow[t]{5}{*}{ MOPEO3 } & 4 & -410 & -- & -- & -- & -- \\
\hline & 5 & -210 & 200 & 63 & -- & -- \\
\hline & 6 & 117 & 209 & 49 & $\cdots$ & -- \\
\hline & 11 & 276 & 159 & -- & -- & -- \\
\hline & 14 & -92 & 118 & 0 & -- & -- \\
\hline \multirow[t]{5}{*}{ MOSCOL } & 2 & 3 & 62 & 99 & -- & - \\
\hline & 3 & 55 & 52 & 27 & -- & -- \\
\hline & 4 & 121 & 66 & 99 & -- & - \\
\hline & 11 & 325 & 204 & 99 & -- & - \\
\hline & 12 & -59 & -- & -- & -- & -- \\
\hline MOSTO1 & 11 & 348 & -- & -- & -- & -- \\
\hline \multirow[t]{3}{*}{ MOSTO2 } & 1 & 10 & 242 & -- & -- & -- \\
\hline & 11 & 300 & 52 & -- & -- & -- \\
\hline & 12 & 248 & 238 & - & -- & -- \\
\hline \multirow[t]{2}{*}{ MOSTO3 } & 1 & 226 & 138 & 75 & -- & -- \\
\hline & 12 & 354 & 128 & 0 & -- & - \\
\hline
\end{tabular}


Table 4--Layer number; altitude of top, thickness, and sand percentage of layer; and average dissolved-solids concentration and temperature of water in layer; by log number--Continued.

\begin{tabular}{|c|c|c|c|c|c|c|}
\hline $\begin{array}{l}\text { Log } \\
\text { number }\end{array}$ & $\begin{array}{r}\text { Layer } \\
\text { number }\end{array}$ & $\begin{array}{r}\text { Altitude } \\
\text { of top } \\
\text { (feet) }\end{array}$ & $\begin{array}{r}\text { Thick- } \\
\text { ness } \\
(\text { feet) }\end{array}$ & $\begin{array}{r}\text { Sand } \\
\text { per- } \\
\text { centage }\end{array}$ & $\begin{array}{l}\text { Dis- } \\
\text { solved } \\
\text { solids }\end{array}$ & $\begin{array}{l}\text { Tem- } \\
\text { per- } \\
\text { ature }\end{array}$ \\
\hline \multirow[t]{11}{*}{ MSADO 1} & 2 & -6922 & 480 & 44 & 28 & 53 \\
\hline & 3 & -4002 & 2920 & 25 & 56 & 49 \\
\hline & 5 & -2752 & 815 & 45 & 56 & 39 \\
\hline & 6 & -2042 & 530 & 38 & 22 & 34 \\
\hline & 7 & -627 & 895 & 33 & $<10$ & 26 \\
\hline & 8 & -52 & 575 & 45 & $<10$ & 21 \\
\hline & 9 & 208 & 260 & 70 & $<10$ & 18 \\
\hline & 12 & -7402 & 950 & 0 & -- & - \\
\hline & 13 & -3567 & 435 & 0 & -- & - \\
\hline & 14 & -2572 & 180 & 0 & -- & -- \\
\hline & 15 & -1522 & 520 & 0 & -- & - \\
\hline \multirow[t]{12}{*}{ MSAMO 1} & 2 & -7501 & 297 & 76 & 51 & 72 \\
\hline & 3 & -4611 & 2890 & 43 & 51 & 65 \\
\hline & 5 & -3478 & 610 & 41 & 44 & 54 \\
\hline & 6 & -3078 & 260 & 37 & 39 & 51 \\
\hline & 7 & -1218 & 1480 & 27 & 17 & 44 \\
\hline & 8 & -228 & 990 & 47 & $<10$ & 26 \\
\hline & 9 & 107 & 335 & 34 & $<10$ & 16 \\
\hline & 10 & 299 & 192 & 36 & $<10$ & -- \\
\hline & 12 & -7798 & 1515 & 1 & -- & -- \\
\hline & 13 & -4088 & 523 & 0 & -- & -- \\
\hline & 14 & -3338 & 140 & 0 & -- & -- \\
\hline & 15 & -2698 & 380 & 0 & -- & - \\
\hline \multirow[t]{12}{*}{ MSAMO2 } & 2 & -7373 & 870 & 45 & -- & 105 \\
\hline & 3 & -5083 & 2290 & 22 & 31 & 87 \\
\hline & 5 & -4118 & 735 & 18 & -- & 68 \\
\hline & 6 & -3913 & 80 & 38 & -- & 61 \\
\hline & 7 & -1693 & 1920 & 45 & 20 & 47 \\
\hline & 8 & -393 & 1300 & 47 & $<10$ & 28 \\
\hline & 9 & 52 & 445 & 59 & $<10$ & 19 \\
\hline & 10 & 341 & 289 & 61 & $<10$ & 14 \\
\hline & 12 & -8243 & 1020 & 5 & -- & -- \\
\hline & 13 & -4853 & 230 & 0 & -- & -- \\
\hline & 14 & -3993 & 125 & 4 & -- & -- \\
\hline & 15 & -3613 & 300 & 0 & -- & -- \\
\hline \multirow[t]{10}{*}{ MSAMO 3} & 3 & -5681 & -- & -- & -- & -- \\
\hline & 5 & -4551 & 570 & 19 & 50 & 50 \\
\hline & 6 & -4241 & 145 & 45 & 52 & 46 \\
\hline & 7 & -1816 & 2155 & 31 & 26 & 37 \\
\hline & 8 & -396 & 1420 & 67 & $<10$ & 25 \\
\hline & 9 & 39 & 435 & 1 & -- & 19 \\
\hline & 10 & 369 & 330 & 21 & -- & 17 \\
\hline & 13 & -5121 & 560 & 0 & -- & -- \\
\hline & 14 & -4386 & 165 & 0 & -- & - \\
\hline & 15 & -3971 & 270 & 0 & -- & -- \\
\hline
\end{tabular}


Table 4--Layer number; altitude of top, thickness, and sand percentage of layer; and average dissolved-solids concentration and temperature of water in layer; by log number--Continued.

\begin{tabular}{|c|c|c|c|c|c|c|}
\hline $\begin{array}{l}\text { Log } \\
\text { number }\end{array}$ & $\begin{array}{r}\text { Layer } \\
\text { number }\end{array}$ & $\begin{array}{r}\text { Altitude } \\
\text { of top } \\
\text { (feet) }\end{array}$ & $\begin{array}{r}\text { Thick- } \\
\text { ness } \\
(f e e t)\end{array}$ & $\begin{array}{r}\text { Sand } \\
\text { per- } \\
\text { centage }\end{array}$ & $\begin{array}{l}\text { Dis- } \\
\text { solved } \\
\text { solids }\end{array}$ & $\begin{array}{l}\text { Tem- } \\
\text { per- } \\
\text { ature }\end{array}$ \\
\hline \multirow[t]{12}{*}{$\mathrm{MSAMO}_{4}$} & 2 & -7143 & 270 & 43 & -- & 72 \\
\hline & 3 & -4123 & 3020 & 30 & 75 & 60 \\
\hline & 5 & -3003 & 720 & 30 & 62 & 43 \\
\hline & 6 & -2743 & 155 & 45 & 55 & 39 \\
\hline & 7 & -973 & 1480 & 42 & $<10$ & 31 \\
\hline & 8 & 7 & 980 & 27 & - - & 22 \\
\hline & 9 & 212 & 205 & 9 & -- & 17 \\
\hline & 10 & 405 & 193 & 74 & -- & 16 \\
\hline & 12 & -7413 & 1150 & 0 & -- & -- \\
\hline & 13 & -3723 & 400 & 0 & -- & - \\
\hline & 14 & -2898 & 105 & 10 & -- & - \\
\hline & 15 & -2453 & 290 & 0 & -- & -- \\
\hline \multirow[t]{10}{*}{ MSAMO5 } & 3 & -4070 & -- & -- & -- & -- \\
\hline & 5 & -2940 & 720 & 32 & 16 & 45 \\
\hline & 6 & -2660 & 165 & 61 & 13 & 40 \\
\hline & 7 & -915 & 1445 & 27 & $<10$ & 31 \\
\hline & 8 & 40 & 955 & 28 & $<10$ & 21 \\
\hline & 9 & 240 & 200 & 10 & -- & 17 \\
\hline & 10 & 432 & 192 & 85 & -- & 15 \\
\hline & 13 & -3660 & 410 & 0 & -- & $\ldots$ \\
\hline & 14 & -2825 & 115 & 9 & -- & - - \\
\hline & 15 & -2360 & 300 & 0 & -- & -- \\
\hline \multirow[t]{6}{*}{ MSATOI } & 2 & -834 & 260 & 50 & $<10$ & 26 \\
\hline & 3 & -159 & 675 & 21 & $<10$ & 23 \\
\hline & 4 & 201 & 360 & 24 & $<10$ & 20 \\
\hline & 5 & 319 & 58 & 57 & $<10$ & 18 \\
\hline & 12 & -1094 & 1065 & 0 & - & $\ldots$ \\
\hline & 13 & 261 & 60 & 17 & -- & - \\
\hline \multirow[t]{4}{*}{ MSATO2 } & 2 & -247 & 255 & 94 & -- & - \\
\hline & 3 & 363 & 610 & 24 & -- & - \\
\hline & 4 & 503 & 140 & 50 & -- & -- \\
\hline & 12 & -502 & 765 & 0 & -- & - \\
\hline \multirow[t]{4}{*}{ MSATO 3} & 2 & -542 & 435 & 80 & $<10$ & 31 \\
\hline & 3 & -227 & 315 & 51 & $<10$ & 26 \\
\hline & 4 & 278 & 505 & 36 & $<10$ & 20 \\
\hline & 12 & -977 & 735 & 0 & -- & -- \\
\hline \multirow[t]{3}{*}{ MSATO 4} & 2 & -64 & 220 & 55 & -- & -- \\
\hline & 3 & 485 & 549 & 9 & -- & - \\
\hline & 12 & -284 & 810 & 0 & -- & - \\
\hline \multirow[t]{5}{*}{ MSBEOI } & 1 & -123 & 184 & 92 & - & - \\
\hline & 2 & 279 & 57 & 53 & -- & - \\
\hline & 3 & 465 & 186 & 5 & -- & -- \\
\hline & 4 & 613 & 148 & 93 & -- & -- \\
\hline & 12 & 222 & 345 & 3 & -- & -- \\
\hline
\end{tabular}


Table 4--Layer number; altitude of top, thickness, and sand percentage of layer; and average dissolved-solids concentration and temperature of water in layer; by log number-Continued.

\begin{tabular}{|c|c|c|c|c|c|c|}
\hline $\begin{array}{l}\text { Log } \\
\text { number }\end{array}$ & $\begin{array}{r}\text { Layer } \\
\text { number }\end{array}$ & $\begin{array}{r}\text { Altitude } \\
\text { of top } \\
\text { (feet) }\end{array}$ & $\begin{array}{r}\text { Thtck- } \\
\text { ness } \\
(\text { feet })\end{array}$ & $\begin{array}{r}\text { Sand } \\
\text { per- } \\
\text { centage }\end{array}$ & $\begin{array}{l}\text { Dis- } \\
\text { solved } \\
\text { solids }\end{array}$ & $\begin{array}{l}\text { Tem- } \\
\text { per- } \\
\text { ature }\end{array}$ \\
\hline \multirow[t]{9}{*}{ MSBO01 } & 2 & -2103 & 430 & 51 & $<10$ & 33 \\
\hline & 3 & -1613 & 490 & 26 & $<10$ & 30 \\
\hline & 4 & -883 & 730 & 49 & $<10$ & 26 \\
\hline & 5 & -143 & 640 & 68 & $<10$ & 21 \\
\hline & 6 & 27 & 100 & 50 & -- & 18 \\
\hline & 11 & 155 & 128 & 86 & -- & 17 \\
\hline & 12 & -2533 & 855 & 0 & - & - \\
\hline & 13 & -783 & 100 & 10 & -- & -- \\
\hline & 14 & -73 & 70 & 0 & - & - \\
\hline \multirow[t]{9}{*}{$\mathrm{MSBOO} 2$} & 2 & -2246 & 430 & 49 & 14 & 39 \\
\hline & 3 & -1656 & 590 & 25 & $<10$ & 35 \\
\hline & 4 & -996 & 660 & 57 & $<10$ & 29 \\
\hline & 5 & -266 & 670 & 82 & $<10$ & 22 \\
\hline & 6 & 24 & 220 & 9 & -- & 17 \\
\hline & 11 & 144 & 120 & 85 & -- & 16 \\
\hline & 12 & -2676 & 780 & 0 & $\ldots$ & -- \\
\hline & 13 & -936 & 60 & 17 & -- & -. \\
\hline & 14 & -196 & 70 & 0 & -- & - \\
\hline \multirow[t]{9}{*}{$M S B O 03$} & 2 & -2464 & 460 & 42 & 25 & 35 \\
\hline & 3 & -1984 & 480 & 19 & - & 32 \\
\hline & 4 & -1044 & 940 & 47 & $<10$ & 28 \\
\hline & 5 & -254 & 680 & 42 & $<10$ & 22 \\
\hline & 6 & -14 & 130 & 38 & - & 18 \\
\hline & 11 & 128 & 142 & 87 & -- & 17 \\
\hline & 12 & -2924 & 870 & 0 & -- & -- \\
\hline & 13 & -934 & 110 & 9 & -- & $\ldots$ \\
\hline & 14 & -144 & 110 & 18 & -- & -- \\
\hline $\mathrm{MSCAO} 1$ & 12 & 316 & 290 & 0 & -- & - \\
\hline \multirow[t]{6}{*}{ MSCKO1 } & 2 & -773 & 400 & 89 & -- & 25 \\
\hline & 3 & 62 & 835 & 33 & -- & 20 \\
\hline & 4 & 317 & 255 & 41 & -- & 16 \\
\hline & 5 & 417 & 60 & 29 & $\ldots$ & 14 \\
\hline & 12 & -1173 & 720 & 3 & -- & - \\
\hline & 13 & 357 & 40 & 25 & -- & -- \\
\hline \multirow[t]{9}{*}{$\mathrm{MSCKO} 2$} & 2 & -1782 & 320 & 94 & $<10$ & 31 \\
\hline & 3 & -572 & 1210 & 36 & $<10$ & 26 \\
\hline & 4 & -297 & 275 & 18 & $<10$ & 21 \\
\hline & 5 & -22 & 200 & 42 & $<10$ & 19 \\
\hline & 6 & 148 & 100 & 20 & - & 18 \\
\hline & 12 & -2102 & 890 & 1 & -- & $\ldots$ \\
\hline & 13 & -222 & 75 & 7 & -- & -- \\
\hline & 14 & 48 & 70 & 0 & -- & -- \\
\hline & 15 & 266 & 118 & 4 & -- & -- \\
\hline
\end{tabular}


Table 4--Layer number; altitude of top, thickness, and sand percentage of layer; and average dissolved-solids concentration and temperature of water in layer; by log number--Continued.

\begin{tabular}{|c|c|c|c|c|c|c|}
\hline $\begin{array}{l}\text { Log } \\
\text { number }\end{array}$ & $\begin{array}{r}\text { Layer } \\
\text { number }\end{array}$ & $\begin{array}{r}\text { Altitude } \\
\text { of top } \\
\text { (feet) }\end{array}$ & $\begin{array}{r}\text { Thick- } \\
\text { ness } \\
\text { (feet) }\end{array}$ & $\begin{array}{r}\text { Sand } \\
\text { per- } \\
\text { centage }\end{array}$ & $\begin{array}{l}\text { Dis- } \\
\text { solved } \\
\text { solids }\end{array}$ & $\begin{array}{l}\text { Tem- } \\
\text { per- } \\
\text { ature }\end{array}$ \\
\hline \multirow[t]{11}{*}{ MSCLOI } & 2 & -5647 & 850 & 68 & 45 & 62 \\
\hline & 3 & -3597 & 2050 & 32 & 27 & 59 \\
\hline & 4 & -3327 & 270 & 89 & 19 & 50 \\
\hline & 5 & -1697 & 950 & 47 & $<10$ & 40 \\
\hline & 6 & -937 & 570 & 32 & $<10$ & 31 \\
\hline & 7 & 173 & 580 & 18 & $<10$ & 20 \\
\hline & 8 & 313 & 140 & 5 & $\ldots$ & 16 \\
\hline & 12 & -6497 & 980 & 0 & $\ldots$ & $\ldots$ \\
\hline & 13 & -2647 & 680 & 1 & -- & $\cdots$ \\
\hline & 14 & -1507 & 190 & 0 & -- & -. \\
\hline & 15 & -407 & 530 & 2 & -- & - \\
\hline \multirow[t]{7}{*}{ MSCOOI } & 2 & -1512 & 170 & 94 & $<10$ & 31 \\
\hline & 3 & -1002 & 510 & 24 & $<10$ & 28 \\
\hline & 4 & -362 & 640 & 70 & $<10$ & 23 \\
\hline & 5 & 8 & 270 & 74 & $<10$ & 18 \\
\hline & 11 & 163 & 155 & 89 & - & 16 \\
\hline & 12 & -1682 & 945 & 0 & $\ldots$ & $\ldots$ \\
\hline & 13 & -262 & 100 & 0 & -- & -- \\
\hline \multirow[t]{7}{*}{ MSCOO2 } & 2 & -1290 & 420 & 99 & $<10$ & 35 \\
\hline & 3 & -1050 & 240 & 13 & - & 29 \\
\hline & 4 & -300 & 750 & 58 & $<10$ & 21 \\
\hline & 5 & -30 & 180 & 99 & $<10$ & $\cdots$ \\
\hline & 11 & 166 & 196 & 91 & $<10$ & - \\
\hline & 12 & -1710 & 850 & 0 & -- & -- \\
\hline & 13 & -210 & 90 & 22 & -- & -- \\
\hline \multirow[t]{11}{*}{ MSCPO 1} & 2 & -5531 & 920 & 52 & 88 & 66 \\
\hline & 3 & -3496 & 2035 & 45 & 84 & 59 \\
\hline & 4 & -3156 & 340 & 56 & 37 & 54 \\
\hline & 5 & -1631 & 875 & 47 & $<10$ & 48 \\
\hline & 6 & -1091 & 460 & 20 & $<10$ & 39 \\
\hline & 7 & 44 & 680 & 22 & $<10$ & 18 \\
\hline & 8 & 359 & 315 & 16 & $<10$ & -- \\
\hline & 12 & -6451 & 970 & 0 & -- & $\cdots$ \\
\hline & 13 & -2506 & 650 & 0 & -- & -- \\
\hline & 14 & -1551 & 80 & 0 & -- & -- \\
\hline & 15 & -636 & 455 & 0 & -- & - \\
\hline \multirow[t]{11}{*}{ MSCPO2 } & 2 & -5811 & 610 & 30 & 53 & 61 \\
\hline & 3 & -3406 & 2405 & 31 & 61 & 50 \\
\hline & 4 & -3106 & 300 & 33 & $<10$ & 41 \\
\hline & 5 & -1686 & 800 & 38 & $<10$ & 33 \\
\hline & 6 & -1201 & 405 & 25 & $<10$ & 28 \\
\hline & 7 & -91 & 690 & 22 & $<10$ & 21 \\
\hline & 8 & 219 & 310 & 32 & -- & 18 \\
\hline & 12 & -6421 & 850 & 0 & $\cdots$ & -- \\
\hline & 13 & -2486 & 620 & 0 & -- & -- \\
\hline & 14 & -1606 & 80 & 0 & $\cdots$ & -- \\
\hline & 15 & -781 & 420 & 0 & $\cdots$ & -- \\
\hline
\end{tabular}


Table 4--Layer number; altitude of top, thickness, and sand percentage of layer; and average dissolved-solids concentration and temperature of water in layer; by log number--Continued.

\begin{tabular}{|c|c|c|c|c|c|c|}
\hline $\begin{array}{l}\text { Log } \\
\text { number }\end{array}$ & $\begin{array}{r}\text { Layer } \\
\text { number }\end{array}$ & $\begin{array}{r}\text { Altitude } \\
\text { of top } \\
\text { ( } f e e t)\end{array}$ & $\begin{array}{c}\text { Thlok- } \\
\text { ness } \\
\text { (feet) }\end{array}$ & $\begin{array}{r}\text { Sand } \\
\text { per- } \\
\text { centage }\end{array}$ & $\begin{array}{l}\text { Dis- } \\
\text { solved } \\
\text { solids }\end{array}$ & $\begin{array}{l}\text { Tem- } \\
\text { per- } \\
\text { ature }\end{array}$ \\
\hline \multirow[t]{11}{*}{$\mathrm{MSCPO} 3$} & 2 & -5855 & 810 & 54 & 69 & 63 \\
\hline & 3 & -3680 & 2175 & 58 & 70 & 52 \\
\hline & 4 & -3345 & 335 & 64 & 25 & 43 \\
\hline & 5 & -1685 & 1000 & 34 & $<10$ & 34 \\
\hline & 6 & -1015 & 575 & 37 & $<10$ & 27 \\
\hline & 7 & -5 & 600 & 9 & - & 20 \\
\hline & 8 & 255 & 260 & 54 & $<10$ & 17 \\
\hline & 12 & -6665 & 960 & 0 & - & -- \\
\hline & 13 & -2685 & 660 & 0 & -- & - \\
\hline & 14 & -1590 & 95 & 0 & -- & -- \\
\hline & 15 & -605 & 410 & 0 & -- & -- \\
\hline \multirow[t]{10}{*}{$\mathrm{MSCPO} 4$} & 2 & -5851 & 840 & 25 & -- & 62 \\
\hline & 3 & -3321 & 2530 & 29 & 28 & 50 \\
\hline & 5 & -1861 & 920 & 28 & $<10$ & 34 \\
\hline & 6 & -1326 & 455 & 33 & $<10$ & 29 \\
\hline & 7 & -11 & 905 & 67 & $<10$ & 22 \\
\hline & 8 & 365 & 376 & 34 & $<10$ & 17 \\
\hline & 12 & -6691 & 840 & 0 & -- & - \\
\hline & 13 & -2781 & 540 & 0 & -- & -- \\
\hline & 14 & -1781 & 80 & 13 & -- & -- \\
\hline & 15 & -916 & 410 & 2 & -- & - \\
\hline \multirow[t]{4}{*}{ MSCRO1 } & 2 & -444 & 435 & 55 & $<10$ & 33 \\
\hline & 3 & -29 & 415 & 39 & $<10$ & -- \\
\hline & 4 & 325 & 354 & 47 & $<10$ & - \\
\hline & 12 & -879 & 755 & 0 & -- & -- \\
\hline \multirow[t]{6}{*}{$\mathrm{MSCRO2}$} & 2 & -935 & 470 & 87 & $<10$ & 26 \\
\hline & 3 & -405 & 530 & 70 & $<10$ & 22 \\
\hline & 4 & 75 & 480 & 94 & $<10$ & 18 \\
\hline & 5 & 405 & 280 & 24 & $<10$ & 14 \\
\hline & 12 & -1405 & 800 & 0 & -- & - \\
\hline & 13 & 125 & 50 & 0 & -- & -- \\
\hline \multirow[t]{7}{*}{$\mathrm{MSCRO}_{3}$} & 2 & -1539 & 340 & 56 & $<10$ & 30 \\
\hline & 3 & -989 & 550 & 15 & -- & 26 \\
\hline & 4 & -299 & 690 & 30 & $<10$ & 22 \\
\hline & 5 & 31 & 230 & 28 & - & 18 \\
\hline & 11 & 121 & 90 & 80 & -- & 16 \\
\hline & 12 & -1879 & 820 & 0 & -- & - \\
\hline & 13 & -199 & 100 & 0 & -- & - \\
\hline \multirow[t]{6}{*}{$\mathrm{MSCRO}_{4}$} & 2 & -936 & 315 & 54 & $<10$ & 25 \\
\hline & 3 & -351 & 585 & 50 & $<10$ & 22 \\
\hline & 4 & 109 & 460 & 23 & $<10$ & 18 \\
\hline & 5 & 252 & 103 & 64 & - & 16 \\
\hline & 12 & -1251 & 810 & 1 & -- & -- \\
\hline & 13 & 149 & 40 & 0 & -- & - \\
\hline
\end{tabular}


Table 4--Layer number; altitude of top, thickness, and sand percentage of layer; and average dissolved-solids concentration and temperature of water in layer; by log number--Continued.

\begin{tabular}{|c|c|c|c|c|c|c|}
\hline $\begin{array}{l}\text { Log } \\
\text { number }\end{array}$ & $\begin{array}{r}\text { Layer } \\
\text { number }\end{array}$ & $\begin{array}{r}\text { Altitude } \\
\text { of top } \\
(f e e t)\end{array}$ & $\begin{array}{c}\text { Thick- } \\
\text { ness } \\
(\text { feet })\end{array}$ & $\begin{array}{r}\text { Sand } \\
\text { per- } \\
\text { centage }\end{array}$ & $\begin{array}{l}\text { Dis- } \\
\text { solved } \\
\text { solids }\end{array}$ & $\begin{array}{l}\text { Tem- } \\
\text { per- } \\
\text { ature }\end{array}$ \\
\hline \multirow[t]{3}{*}{ MSCTO1 } & 2 & -50 & 110 & 82 & $<10$ & 21 \\
\hline & 3 & 370 & 420 & 5 & -- & 20 \\
\hline & 12 & -160 & 665 & 0 & -- & -- \\
\hline \multirow[t]{3}{*}{ MSCTO2 } & 2 & 307 & 160 & 69 & $<10$ & 19 \\
\hline & 3 & 447 & 140 & 10 & - & 18 \\
\hline & 12 & 147 & 650 & 0 & -- & -- \\
\hline \multirow[t]{11}{*}{ MSCVO 1} & 2 & -4496 & 930 & 48 & 77 & 51 \\
\hline & 3 & -2706 & 1790 & 27 & 22 & 41 \\
\hline & 4 & -2126 & 580 & 26 & $<10$ & 34 \\
\hline & 5 & -1316 & 600 & 48 & $<10$ & 29 \\
\hline & 6 & -946 & 295 & 25 & $<10$ & 26 \\
\hline & 7 & 84 & 690 & 72 & $<10$ & 21 \\
\hline & 8 & 396 & 312 & 82 & $<10$ & 17 \\
\hline & 12 & -5426 & 820 & 1 & -- & - \\
\hline & 13 & -1916 & 210 & 0 & -- & -- \\
\hline & 14 & -1241 & 75 & 0 & -- & -- \\
\hline & 15 & -606 & 340 & 1 & -- & - \\
\hline \multirow[t]{10}{*}{$M S C V O 2$} & 2 & -4452 & 793 & 37 & 48 & 45 \\
\hline & 3 & -2445 & 2007 & 24 & 39 & 40 \\
\hline & 5 & -1460 & 520 & 33 & $<10$ & 32 \\
\hline & 6 & -1090 & 300 & 53 & $<10$ & 28 \\
\hline & 7 & -55 & 790 & 31 & $<10$ & 22 \\
\hline & 8 & 285 & 340 & 25 & -- & 17 \\
\hline & 12 & -5245 & 920 & 0 & -- & -- \\
\hline & 13 & -1980 & 465 & 6 & -- & -- \\
\hline & 14 & -1390 & 70 & 21 & -- & -- \\
\hline & 15 & -845 & 245 & 0 & -- & -- \\
\hline \multirow[t]{8}{*}{ MSDSO 1} & 1 & -2315 & 150 & 99 & $<10$ & 34 \\
\hline & 2 & -1110 & 375 & 87 & $<10$ & 30 \\
\hline & 3 & -810 & 300 & 25 & $<10$ & 29 \\
\hline & 4 & -260 & 550 & 89 & $<10$ & 25 \\
\hline & 5 & 20 & 280 & 95 & $<10$ & 18 \\
\hline & 11 & 210 & 150 & 88 & $<10$ & 14 \\
\hline & 12 & -1485 & 830 & 0 & -- & -- \\
\hline & 14 & 60 & 40 & 0 & -- & - \\
\hline \multirow[t]{6}{*}{ MSDSO 2} & 2 & -1102 & 215 & 81 & -- & -- \\
\hline & 3 & -679 & 423 & 13 & -- & -- \\
\hline & 4 & -125 & 554 & 74 & -- & -- \\
\hline & 5 & 183 & 308 & 75 & -- & -- \\
\hline & 12 & -1317 & -- & -- & -- & -- \\
\hline & 14 & 243 & 60 & 0 & -- & -- \\
\hline \multirow[t]{9}{*}{ MSFOO1 } & 2 & -4233 & 690 & 48 & 48 & 46 \\
\hline & 3 & -2333 & 1900 & 22 & 47 & 39 \\
\hline & 5 & -1673 & 365 & 20 & 16 & 31 \\
\hline & 7 & -438 & 1085 & 41 & $<10$ & 26 \\
\hline & 8 & 52 & 490 & 64 & $<10$ & 21 \\
\hline & 9 & 165 & 113 & 4 & -- & 19 \\
\hline & 12 & -4923 & 840 & 2 & -- & - \\
\hline & 13 & -2038 & 295 & 5 & -- & -- \\
\hline & 15 & -1523 & 150 & 0 & -- & -- \\
\hline
\end{tabular}


Table 4--Layer number; altitude of top, thickness, and sand percentage of layer: and average dissolved-solids concentration and temperature of water in layer; by log number--Continued.

\begin{tabular}{|c|c|c|c|c|c|c|}
\hline $\begin{array}{l}\text { Log } \\
\text { number }\end{array}$ & $\begin{array}{r}\text { Layer } \\
\text { number }\end{array}$ & $\begin{array}{r}\text { Altitude } \\
\text { of top } \\
\text { (feet) }\end{array}$ & $\begin{array}{r}\text { Thlck- } \\
\text { ness } \\
\text { (feet) }\end{array}$ & $\begin{array}{r}\text { Sand } \\
\text { per- } \\
\text { centage }\end{array}$ & $\begin{array}{l}\text { Dis- } \\
\text { solved } \\
\text { solids }\end{array}$ & $\begin{array}{l}\text { Tem- } \\
\text { per- } \\
\text { ature }\end{array}$ \\
\hline \multirow[t]{8}{*}{ MSFOO2 } & 2 & -4043 & 835 & 58 & 32 & 52 \\
\hline & 3 & -1958 & 2085 & 23 & 22 & 43 \\
\hline & 5 & -1198 & 340 & - & -- & -- \\
\hline & 7 & -178 & 810 & 61 & $<10$ & 24 \\
\hline & 8 & 182 & 360 & 47 & $<10$ & 18 \\
\hline & 12 & -4878 & 850 & 0 & - & -- \\
\hline & 13 & -1538 & 420 & 10 & -- & -- \\
\hline & 15 & -988 & 210 & 0 & $-\infty$ & - \\
\hline \multirow[t]{8}{*}{ MSFOO3 } & 2 & -3751 & 860 & 38 & 104 & 58 \\
\hline & 3 & -1851 & 1900 & 23 & 43 & 50 \\
\hline & 5 & -1091 & 340 & 44 & -- & 35 \\
\hline & 7 & -191 & 745 & 50 & $<10$ & 24 \\
\hline & 8 & 229 & 420 & 57 & $<10$ & 14 \\
\hline & 12 & -4611 & 775 & 0 & - & $\cdots$ \\
\hline & 13 & -1431 & 420 & 12 & -- & - \\
\hline & 15 & -936 & 155 & 0 & -- & - \\
\hline \multirow[t]{11}{*}{ MSERO1 } & 2 & -6985 & 310 & 47 & 44 & 68 \\
\hline & 3 & -4075 & 2910 & 37 & 39 & 57 \\
\hline & 5 & -2785 & 870 & 39 & 40 & 41 \\
\hline & 6 & -2350 & 325 & 43 & 22 & 36 \\
\hline & 7 & -665 & 1290 & 24 & $<10$ & 27 \\
\hline & 8 & 35 & 700 & 7 & - & 20 \\
\hline & 9 & 210 & 175 & 39 & -- & 17 \\
\hline & 12 & -7295 & 1430 & 2 & -- & - \\
\hline & 13 & -3655 & 420 & 0 & -- & -- \\
\hline & 14 & -2675 & 110 & 0 & -- & -- \\
\hline & 15 & -1955 & 395 & 0 & -- & -- \\
\hline \multirow[t]{9}{*}{ MSFRO2 } & 3 & -3993 & -- & -- & -- & - \\
\hline & 5 & -2673 & 860 & 63 & 67 & 42 \\
\hline & 6 & -2233 & 330 & 33 & -- & 37 \\
\hline & 7 & -613 & 1235 & 25 & $<10$ & 27 \\
\hline & 8 & 47 & 660 & 8 & -- & 20 \\
\hline & 9 & 205 & 158 & 44 & -- & 17 \\
\hline & 13 & -3533 & 460 & 2 & -- & - \\
\hline & 14 & -2563 & 110 & 0 & -- & -- \\
\hline & 15 & -1848 & 385 & 0 & -- & -- \\
\hline \multirow[t]{10}{*}{ MSGEO1 } & 2 & -4186 & 350 & 71 & -- & 46 \\
\hline & 3 & -2661 & 1525 & 7 & 20 & 40 \\
\hline & 4 & -2226 & 435 & 37 & 19 & 35 \\
\hline & 5 & -1796 & 220 & -- & -- & -- \\
\hline & 7 & -491 & 1155 & 29 & $<10$ & 26 \\
\hline & 8 & 29 & 520 & 23 & -- & 21 \\
\hline & 9 & 92 & 63 & 5 & -- & 19 \\
\hline & 12 & -4536 & 810 & 3 & -- & - \\
\hline & 13 & -2016 & 210 & 10 & -- & -- \\
\hline & 15 & -1646 & 150 & 7 & -- & - \\
\hline
\end{tabular}


Table 4--Layer number; altitude of top, thickness, and sand percentage of layer: and average dissolved-solids concentration and temperature of water in layer; by log number--Continued.

\begin{tabular}{|c|c|c|c|c|c|c|}
\hline $\begin{array}{l}\text { Log } \\
\text { number }\end{array}$ & $\begin{array}{r}\text { Layer } \\
\text { number }\end{array}$ & $\begin{array}{r}\text { Altide } \\
\text { of top } \\
\text { (feet) }\end{array}$ & $\begin{array}{r}\text { Thlck- } \\
\text { ness } \\
(\text { feet) }\end{array}$ & $\begin{array}{r}\text { Sand } \\
\text { per- } \\
\text { centage }\end{array}$ & $\begin{array}{l}\text { Dis- } \\
\text { solved } \\
\text { solids }\end{array}$ & $\begin{array}{l}\text { Tem- } \\
\text { per- } \\
\text { 2ture }\end{array}$ \\
\hline \multirow[t]{10}{*}{$M S G E O 2$} & 2 & -4298 & 240 & 42 & 56 & 37 \\
\hline & 3 & -2728 & 1570 & 8 & 25 & 51 \\
\hline & 4 & -2258 & 470 & 37 & 28 & 45 \\
\hline & 5 & -1848 & 210 & -- & - & -- \\
\hline & 7 & -433 & 1170 & 29 & $<10$ & 33 \\
\hline & 8 & 112 & 545 & 8 & - & 18 \\
\hline & 9 & 182 & 70 & 9 & $\ldots$ & - \\
\hline & 12 & -4538 & 780 & 4 & $\ldots$ & -- \\
\hline & 13 & -2058 & 200 & 0 & - & $\ldots$ \\
\hline & 15 & -1603 & 245 & 4 & - & -- \\
\hline \multirow[t]{10}{*}{ MSGEO3 } & 2 & -4326 & 200 & 93 & $\ldots$ & 56 \\
\hline & 3 & -2946 & 1380 & 6 & $\ldots$ & 51 \\
\hline & 4 & -2636 & 310 & 35 & 23 & 47 \\
\hline & 5 & -2246 & 155 & $\ldots$ & 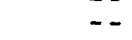 & $\ldots$ \\
\hline & 7 & -846 & 1330 & 21 & $<10$ & 39 \\
\hline & 8 & -166 & 680 & 40 & -- & 26 \\
\hline & 9 & 66 & 232 & 80 & $\ldots$ & $\ldots$ \\
\hline & 12 & -4526 & 985 & 3 & $\ldots$ & $\ldots$ \\
\hline & 13 & -2401 & 235 & 4 & $\ldots$ & $\ldots$ \\
\hline & 15 & -2176 & 70 & 0 & - & - \\
\hline \multirow[t]{9}{*}{ MSGNO1 } & 2 & -3798 & 330 & 44 & 31 & 45 \\
\hline & 3 & -1988 & 1810 & 13 & 21 & 38 \\
\hline & 4 & -1923 & 65 & 62 & $<10$ & 32 \\
\hline & 5 & -893 & 655 & 34 & - & 27 \\
\hline & 7 & 2 & 830 & 76 & $<10$ & 22 \\
\hline & 8 & 274 & 272 & 11 & -- & 18 \\
\hline & 12 & -4128 & 740 & 4 & -- & $\ldots$ \\
\hline & 13 & -1548 & 375 & 7 & $\ldots$ & $\ldots$ \\
\hline & 15 & -828 & 65 & 0 & - & - \\
\hline \multirow[t]{9}{*}{ MSGNO2 } & 2 & -3907 & 370 & 53 & 25 & 45 \\
\hline & 3 & -2377 & 1530 & 8 & 17 & 39 \\
\hline & 4 & -2217 & 160 & 44 & 18 & 34 \\
\hline & 5 & -1347 & 380 & $\therefore$ & - & - \\
\hline & 7 & -137 & 1070 & 33 & $<10$ & 23 \\
\hline & 8 & 313 & 450 & 65 & $<10$ & 18 \\
\hline & 12 & -4277 & 700 & 0 & - - & -- \\
\hline & 13 & -1727 & 490 & 8 & $\ldots$ & -- \\
\hline & 15 & -1207 & 140 & 0 & -- & - \\
\hline \multirow[t]{9}{*}{$\mathrm{MSGNO}_{3}$} & 2 & -3780 & 800 & 37 & 59 & 46 \\
\hline & 3 & -2435 & 1345 & 21 & - & 39 \\
\hline & 4 & -1820 & 615 & 33 & $<10$ & 33 \\
\hline & 5 & -995 & 595 & 28 & - & 27 \\
\hline & 7 & $-1>0$ & 720 & 28 & $<10$ & 22 \\
\hline & 8 & 200 & 370 & 11 & - & 19 \\
\hline & 12 & -4580 & 600 & 10 & -- & $\ldots$ \\
\hline & 13 & -1590 & 230 & 0 & -- & - \\
\hline & 15 & -890 & 105 & 10 & -- & $\ldots$ \\
\hline
\end{tabular}


Table 4--Layer number; altitude of top, thickness, and sand percentage of layer; and average dissolved-solids concentration and temperature of water in layer; by log number--Continued.

\begin{tabular}{|c|c|c|c|c|c|c|}
\hline $\begin{array}{l}\log \\
\text { number }\end{array}$ & $\begin{array}{r}\text { Layer } \\
\text { number }\end{array}$ & $\begin{array}{r}\text { Altitude } \\
\text { of top } \\
\text { (feet) }\end{array}$ & $\begin{array}{r}\text { Thick- } \\
\text { ness } \\
\text { (feet) }\end{array}$ & $\begin{array}{r}\text { Sand } \\
\text { per- } \\
\text { centage }\end{array}$ & $\begin{array}{l}\text { Dis- } \\
\text { solved } \\
\text { solids }\end{array}$ & $\begin{array}{l}\text { Tem- } \\
\text { per- } \\
\text { ature }\end{array}$ \\
\hline \multirow[t]{4}{*}{ MSGRO 1} & 2 & -35 & 370 & 62 & $<10$ & 21 \\
\hline & 3 & 245 & 280 & 7 & -- & 18 \\
\hline & 4 & 315 & 70 & 25 & -- & 16 \\
\hline & 12 & -405 & 670 & 0 & -- & -- \\
\hline \multirow[t]{6}{*}{ MSGRO 2} & 2 & -869 & 300 & 43 & $<10$ & 27 \\
\hline & 3 & -459 & 410 & 44 & $<10$ & 24 \\
\hline & 4 & 111 & 570 & 43 & $<10$ & 20 \\
\hline & 5 & 271 & 40 & 40 & - & 17 \\
\hline & 12 & -1169 & 745 & 0 & -- & - \\
\hline & 13 & 231 & 120 & 0 & -- & -- \\
\hline \multirow[t]{11}{*}{ MSAAO 1} & 2 & -5549 & 260 & 75 & -- & 62 \\
\hline & 3 & -4149 & 1400 & 9 & -- & 56 \\
\hline & 5 & -3629 & 315 & 49 & -- & 48 \\
\hline & 7 & -2129 & 1460 & 57 & $<10$ & 42 \\
\hline & 8 & -1269 & 860 & 68 & $<10$ & 33 \\
\hline & 9 & -629 & 640 & 48 & $<10$ & 27 \\
\hline & 10 & -164 & 465 & 23 & $<10$ & 23 \\
\hline & 11 & 52 & 216 & 10 & -- & 21 \\
\hline & 12 & -5809 & 1160 & 9 & -- & -- \\
\hline & 13 & -3944 & 205 & 0 & -- & -- \\
\hline & 15 & -3589 & 40 & 0 & -- & -- \\
\hline \multirow[t]{10}{*}{ MSBIOI } & 2 & -2893 & 325 & 46 & -- & 42 \\
\hline & 3 & -1948 & 945 & 35 & -- & 38 \\
\hline & 4 & -1783 & 165 & 67 & -- & 34 \\
\hline & 5 & -853 & 490 & 29 & -- & 28 \\
\hline & 6 & -278 & 390 & 41 & -- & 24 \\
\hline & 7 & 357 & 235 & 31 & -- & 19 \\
\hline & 12 & -3218 & 150 & 0 & -- & -- \\
\hline & 13 & -1343 & 440 & 0 & -- & -- \\
\hline & 14 & -668 & 185 & 0 & -- & -- \\
\hline & 15 & 122 & 400 & 0 & -- & -- \\
\hline \multirow[t]{10}{*}{ MSBIO 2} & 2 & -4972 & 570 & 61 & 58 & 65 \\
\hline & 3 & -2917 & 2055 & 49 & 21 & 63 \\
\hline & 4 & -2792 & 125 & 96 & $<10$ & 61 \\
\hline & 5 & -1267 & 895 & 56 & $<10$ & 47 \\
\hline & 6 & -567 & 535 & 28 & $<10$ & 28 \\
\hline & 7 & 198 & 305 & 32 & $<10$ & -- \\
\hline & 12 & -5542 & 1010 & 3 & -- & -- \\
\hline & 13 & -2162 & 630 & 0 & -- & -- \\
\hline & 14 & -1102 & 165 & 3 & -- & -- \\
\hline & 15 & -107 & 460 & 0 & -- & -- \\
\hline \multirow[t]{9}{*}{ MSHIO 3} & 2 & -2158 & 350 & 64 & $<10$ & 39 \\
\hline & 3 & -1338 & 820 & 34 & $<10$ & 34 \\
\hline & 4 & -1233 & 105 & 71 & $<10$ & 30 \\
\hline & 5 & -418 & 470 & 76 & $<10$ & 25 \\
\hline & 6 & 52 & 315 & 52 & $<10$ & 21 \\
\hline & 12 & -2508 & 135 & 0 & -- & -- \\
\hline & 13 & -888 & 345 & 0 & -- & -- \\
\hline & 14 & -263 & 155 & 6 & -- & -- \\
\hline & 15 & 322 & 270 & 4 & -- & -- \\
\hline
\end{tabular}


Table 4--Layer number; altitude of top, thickness, and sand percentage of layer; and average dissolved-solids concentration and temperature of water in layer; by log number--Continued.

\begin{tabular}{|c|c|c|c|c|c|c|}
\hline $\begin{array}{l}\text { Log } \\
\text { number }\end{array}$ & $\begin{array}{r}\text { Layer } \\
\text { number }\end{array}$ & $\begin{array}{l}\text { Altitude } \\
\text { of top } \\
\text { ( } f \text { e t } t)\end{array}$ & $\begin{array}{r}\text { Thick- } \\
\text { ness } \\
\text { (feet) }\end{array}$ & $\begin{array}{r}\text { Sand } \\
\text { per- } \\
\text { centage }\end{array}$ & $\begin{array}{l}\text { Dis- } \\
\text { solved } \\
\text { solids }\end{array}$ & $\begin{array}{r}\text { Tem- } \\
\text { per- } \\
\text { ature }\end{array}$ \\
\hline \multirow[t]{11}{*}{ MSHIO 4} & 2 & -4671 & 985 & 60 & 61 & 58 \\
\hline & 3 & -2881 & 1790 & 42 & 36 & 48 \\
\hline & 4 & -2621 & 260 & 99 & $<10$ & 41 \\
\hline & 5 & -1296 & 815 & 27 & $<10$ & 33 \\
\hline & 6 & -691 & 480 & 27 & -- & 27 \\
\hline & 7 & 159 & 440 & 26 & -- & 21 \\
\hline & 8 & 437 & 278 & 16 & -- & 18 \\
\hline & 12 & -5656 & 895 & 0 & -- & -- \\
\hline & 13 & -2111 & 510 & 0 & -- & -- \\
\hline & 14 & -1171 & 125 & 0 & -- & -- \\
\hline & 15 & -281 & 410 & 0 & -- & -- \\
\hline \multirow[t]{6}{*}{ MSHOOI } & 2 & -1380 & 245 & 78 & $<10$ & 28 \\
\hline & 3 & -630 & 750 & 43 & $<10$ & 24 \\
\hline & 4 & -120 & 510 & 65 & $<10$ & 20 \\
\hline & 5 & 271 & 241 & 36 & -- & 16 \\
\hline & 12 & -1625 & 1065 & 3 & -- & -- \\
\hline & 13 & 30 & 150 & 7 & -- & -- \\
\hline \multirow[t]{8}{*}{ MSEOO2 } & 2 & -2175 & 280 & 32 & -- & -- \\
\hline & 3 & -1455 & 720 & 28 & -- & -- \\
\hline & 4 & -675 & 780 & 57 & -- & -- \\
\hline & 5 & -125 & 450 & 41 & -- & -- \\
\hline & 6 & 155 & 160 & 18 & -- & -- \\
\hline & 12 & -2455 & 1160 & 1 & -- & -- \\
\hline & 13 & -575 & 100 & 0 & -- & -- \\
\hline & 14 & -5 & 120 & 17 & -- & -- \\
\hline \multirow[t]{8}{*}{ MSHOO3 } & 2 & -2582 & 665 & 63 & $<10$ & 41 \\
\hline & 3 & -1572 & 1010 & 38 & $<10$ & 34 \\
\hline & 4 & -987 & 585 & 53 & $<10$ & 27 \\
\hline & 5 & -92 & 750 & 77 & $<10$ & 20 \\
\hline & 6 & 318 & 410 & 12 & -- & 15 \\
\hline & 12 & -3247 & 910 & 0 & -- & -- \\
\hline & 13 & -842 & 145 & 0 & -- & -- \\
\hline & 15 & 358 & 40 & 0 & -- & -- \\
\hline \multirow[t]{10}{*}{ MSBRO 1} & 3 & -4630 & 300 & 5 & -- & 60 \\
\hline & 5 & -4130 & 325 & -- & -- & -- \\
\hline & 7 & -2350 & 1610 & 62 & $<10$ & 49 \\
\hline & 8 & -1425 & 925 & 42 & $<10$ & 37 \\
\hline & 9 & -770 & 655 & 29 & $<10$ & 29 \\
\hline & 10 & -230 & 540 & 40 & $<10$ & 23 \\
\hline & 11 & 4 & 234 & 6 & -- & 19 \\
\hline & 12 & -4930 & 2380 & 3 & -- & -- \\
\hline & 13 & -4455 & 175 & 0 & -- & -- \\
\hline & 15 & -3960 & 170 & 0 & -- & -- \\
\hline
\end{tabular}


Table 4--Layer number; altitude of top, thickness, and sand percentage of layer; and average dissolved-solids concentration and temperature of water in layer; by log number--Continued.

\begin{tabular}{|c|c|c|c|c|c|c|}
\hline $\begin{array}{l}\text { Log } \\
\text { number }\end{array}$ & $\begin{array}{r}\text { Layer } \\
\text { number }\end{array}$ & $\begin{array}{r}\text { Altide } \\
\text { of top } \\
(f e e t)\end{array}$ & $\begin{array}{r}\text { Thick- } \\
\text { ness } \\
(\text { feet) }\end{array}$ & $\begin{array}{r}\text { Sand } \\
\text { per- } \\
\text { centage }\end{array}$ & $\begin{array}{l}\text { Dis- } \\
\text { solved } \\
\text { solids }\end{array}$ & $\begin{array}{l}\text { Tem- } \\
\text { per- } \\
\text { ature }\end{array}$ \\
\hline \multirow[t]{10}{*}{ MSHRO 2} & 2 & -5051 & 230 & 91 & -- & 55 \\
\hline & 3 & -3376 & 1675 & 11 & -- & 51 \\
\hline & 5 & -2941 & 330 & -- & -- & -- \\
\hline & 7 & -1456 & 1390 & 29 & $<10$ & 37 \\
\hline & 8 & -666 & 790 & 27 & $<10$ & 28 \\
\hline & 9 & -106 & 560 & 45 & $<10$ & 23 \\
\hline & 10 & 100 & 206 & 11 & - & 20 \\
\hline & 12 & -5281 & 945 & 2 & -- & - \\
\hline & 13 & -3271 & 105 & 0 & -- & -- \\
\hline & 15 & -2846 & 95 & 0 & -- & -- \\
\hline \multirow[t]{10}{*}{ MSHRO 3} & 2 & -5199 & 200 & 60 & -- & 62 \\
\hline & 3 & -3609 & 1590 & 10 & -- & 55 \\
\hline & 5 & -3339 & 200 & -- & -- & - \\
\hline & 7 & -1629 & 1640 & 29 & $<10$ & 39 \\
\hline & 8 & -779 & 850 & 5 & -- & 29 \\
\hline & 9 & -179 & 600 & 26 & -- & 23 \\
\hline & 10 & 57 & 236 & 19 & -- & 20 \\
\hline & 12 & -5399 & 845 & 2 & -- & -- \\
\hline & 13 & -3539 & 70 & 0 & -- & -- \\
\hline & 15 & -3269 & 70 & 14 & -- & -- \\
\hline \multirow[t]{9}{*}{ MSHUO 1} & 2 & -3366 & 340 & 59 & 43 & 50 \\
\hline & 3 & -2076 & 1290 & 35 & $<10$ & 42 \\
\hline & 4 & -1426 & 650 & 48 & $<10$ & 33 \\
\hline & 5 & -546 & 670 & 71 & $<10$ & 24 \\
\hline & 6 & -236 & 310 & 60 & $<10$ & 19 \\
\hline & 11 & 103 & 219 & 83 & $<10$ & 15 \\
\hline & 12 & -3706 & 1020 & 2 & -- & $\ldots$ \\
\hline & 13 & -1216 & 210 & 2 & -- & -- \\
\hline & 15 & -116 & 120 & 0 & -- & -- \\
\hline \multirow[t]{8}{*}{ MSHUO 2} & 2 & -2585 & 320 & 99 & 11 & 42 \\
\hline & 3 & -1655 & 930 & 24 & $<10$ & 36 \\
\hline & 4 & -1075 & 580 & 43 & $<10$ & 29 \\
\hline & 5 & -375 & 550 & 40 & $<10$ & 22 \\
\hline & 6 & -15 & 360 & 51 & $<10$ & 17 \\
\hline & 11 & 115 & 130 & 86 & -- & 15 \\
\hline & 12 & -2905 & 1020 & 2 & -- & -- \\
\hline & 13 & -925 & 150 & 0 & -- & -- \\
\hline \multirow[t]{9}{*}{ MSISO1 } & 2 & -2060 & 425 & 73 & -- & -- \\
\hline & 3 & -1565 & 495 & 12 & -- & - - \\
\hline & 4 & -1435 & 130 & 54 & -- & - - \\
\hline & 5 & -455 & 650 & 52 & -- & -- \\
\hline & 6 & -85 & 170 & 44 & -- & -- \\
\hline & 11 & 105 & 190 & 90 & -- & -- \\
\hline & 12 & -2485 & 480 & 0 & -- & -- \\
\hline & 13 & -1105 & 330 & 0 & -- & -- \\
\hline & 14 & -255 & 200 & 15 & -- & -- \\
\hline
\end{tabular}


Table 4--Layer number; altitude of top, thickness, and sand percentage of layer; and average dissolved-solids concentration and temperature of water in layer; by log number--Continued.

\begin{tabular}{|c|c|c|c|c|c|c|}
\hline $\begin{array}{l}\text { Log } \\
\text { number } x\end{array}$ & $\begin{array}{r}\text { Layer } \\
\text { number }\end{array}$ & $\begin{array}{r}\text { Altitude } \\
\text { of top } \\
\text { (feet) }\end{array}$ & $\begin{array}{c}\text { Thick- } \\
\text { ness } \\
\text { (feet) }\end{array}$ & $\begin{array}{r}\text { Sand } \\
\text { per- } \\
\text { centage }\end{array}$ & $\begin{array}{l}\text { Dis- } \\
\text { solved } \\
\text { solids }\end{array}$ & $\begin{array}{l}\text { Tem- } \\
\text { per- } \\
\text { 2ture }\end{array}$ \\
\hline \multirow[t]{10}{*}{ MSI SO2 } & 2 & -2407 & 465 & 63 & 26 & 42 \\
\hline & 3 & -1892 & 515 & 23 & -- & 37 \\
\hline & 4 & -1732 & 160 & 97 & $<10$ & 34 \\
\hline & 5 & -717 & 670 & 38 & $<10$ & 26 \\
\hline & 6 & -117 & 420 & 35 & $<10$ & 18 \\
\hline & 11 & 93 & 95 & 81 & -- & 14 \\
\hline & 12 & -2872 & 450 & 0 & -- & -- \\
\hline & 13 & -1387 & 345 & 3 & -- & -- \\
\hline & 14 & -537 & 180 & 17 & $\cdots$ & $\cdots$ \\
\hline & 15 & -2 & 115 & 4 & -- & -- \\
\hline \multirow[t]{10}{*}{ MSISO 3} & 2 & -4441 & 495 & 49 & -- & -- \\
\hline & 3 & -3101 & 1340 & 38 & -- & $\cdots$ \\
\hline & 4 & -2856 & 245 & 99 & -- & -- \\
\hline & 5 & -1296 & 1085 & 59 & -- & -- \\
\hline & 6 & -541 & 595 & 45 & -- & -- \\
\hline & 11 & 89 & 160 & 88 & $-\cdots$ & -- \\
\hline & 12 & -4936 & 515 & 0 & $=$ & -- \\
\hline & 13 & -2381 & 475 & 0 & -- & -- \\
\hline & 14 & -1136 & 160 & 0 & -- & -- \\
\hline & 15 & -71 & 470 & 0 & -- & -- \\
\hline \multirow[t]{10}{*}{ MSJAO 1} & 2 & -4852 & 130 & 85 & -- & $\cdots$ \\
\hline & 3 & -3162 & 1690 & 24 & -- & - \\
\hline & 5 & -2872 & 140 & -- & -- & -- \\
\hline & 7 & -1257 & 1455 & 45 & -- & -- \\
\hline & 8 & -422 & 835 & 26 & -- & -- \\
\hline & 9 & -22 & 400 & 69 & -- & $\cdots$ \\
\hline & 10 & 108 & 130 & 19 & -- & -- \\
\hline & 12 & -4982 & 870 & 6 & -- & -- \\
\hline & 13 & -3012 & 150 & 0 & -- & -- \\
\hline & 15 & -2712 & 160 & 0 & -- & -- \\
\hline \multirow[t]{9}{*}{$\mathrm{MSJAO} 2$} & 3 & -3922 & 1330 & 11 & -- & 56 \\
\hline & 5 & -3572 & 200 & -- & -- & -- \\
\hline & 7 & -1742 & 1720 & 26 & 20 & 40 \\
\hline & 8 & -642 & 1100 & 8 & $<10$ & 29 \\
\hline & 9 & -152 & 490 & 5 & -- & 23 \\
\hline & 10 & 30 & 182 & 50 & -- & 20 \\
\hline & 12 & -5252 & 1090 & 0 & -- & -- \\
\hline & 13 & -3772 & 150 & 0 & -- & -- \\
\hline & 15 & -3462 & 110 & 0 & $\cdots$ & -- \\
\hline \multirow[t]{10}{*}{$\mathrm{MSJAO} 3$} & 2 & -5153 & 90 & 67 & -- & 57 \\
\hline & 3 & -3753 & 1400 & 12 & - & 53 \\
\hline & 5 & -3358 & 206 & -- & -- & -- \\
\hline & 7 & -1528 & 1700 & 20 & 16 & 42 \\
\hline & 8 & -478 & 1050 & 5 & - & 30 \\
\hline & 9 & -78 & 400 & 39 & $<10$ & 20 \\
\hline & 10 & 10 & 88 & 7 & - & 16 \\
\hline & 12 & -5243 & 895 & 4 & -- & -- \\
\hline & 13 & -3564 & 189 & 0 & -- & $\cdots$ \\
\hline & 15 & -3228 & 130 & 0 & -- & -- \\
\hline
\end{tabular}


Table 4--Layer number; altitude of top, thickness, and sand percentage of layer; and average dissolved-solids concentration and temperature of water in layer; by log number--Continued.

\begin{tabular}{|c|c|c|c|c|c|c|}
\hline $\begin{array}{l}\text { Log } \\
\text { number }\end{array}$ & $\begin{array}{r}\text { Layer } \\
\text { number }\end{array}$ & $\begin{array}{r}\text { Altitude } \\
\text { of top } \\
\text { (feet) }\end{array}$ & $\begin{array}{r}\text { Thick- } \\
\text { ness } \\
\text { (feet) }\end{array}$ & $\begin{array}{r}\text { Sand } \\
\text { per- } \\
\text { centage }\end{array}$ & $\begin{array}{l}\text { Dis- } \\
\text { solved } \\
\text { solids }\end{array}$ & $\begin{array}{l}\text { Tem- } \\
\text { per- } \\
\text { ature }\end{array}$ \\
\hline \multirow[t]{10}{*}{$\mathrm{MSJAO}_{4}$} & 3 & -4916 & 1320 & 28 & 49 & 56 \\
\hline & 5 & -4656 & 150 & -- & -- & - \\
\hline & 7 & -2791 & 1785 & 31 & 59 & 44 \\
\hline & 8 & -1271 & 1520 & 76 & 24 & 34 \\
\hline & 9 & -571 & 700 & 27 & $<10$ & 26 \\
\hline & 10 & -176 & 395 & 32 & $<10$ & 23 \\
\hline & 11 & 6 & 182 & 5 & - & 21 \\
\hline & 12 & -6236 & 940 & 1 & -. & $\ldots$ \\
\hline & 13 & -4806 & 110 & 0 & -- & $\ldots$ \\
\hline & 15 & -4576 & 80 & 0 & -- & $\ldots$ \\
\hline \multirow[t]{10}{*}{ MSJAOS } & 2 & -4671 & 130 & 99 & -- & 56 \\
\hline & 3 & -3191 & 1480 & 7 & -- & 50 \\
\hline & 4 & -3031 & 160 & 50 & -- & 44 \\
\hline & 5 & -2471 & 220 & $\ldots$ & - & $\ldots$ \\
\hline & 7 & -911 & 1360 & 41 & $<10$ & 32 \\
\hline & 8 & -191 & 720 & 30 & - & 24 \\
\hline & 9 & 49 & 240 & 10 & - & 20 \\
\hline & 12 & -4801 & 860 & 0 & $\ldots$ & $\ldots$ \\
\hline & 13 & -2691 & 340 & 12 & -- & $\cdots$ \\
\hline & 15 & -2271 & 200 & 0 & -- & $\cdots$ \\
\hline \multirow[t]{11}{*}{ MSJDOI } & 2 & -4773 & 840 & 56 & 51 & 107 \\
\hline & 3 & -2593 & 2180 & 17 & 40 & 81 \\
\hline & 5 & -1628 & 425 & 32 & $<10$ & 49 \\
\hline & 6 & -1273 & 290 & 24 & $<10$ & 41 \\
\hline & 7 & -73 & 900 & 25 & $<10$ & 25 \\
\hline & 8 & 377 & 450 & 72 & $<10$ & $\ldots$ \\
\hline & 9 & 437 & 60 & 64 & - & $\ldots$ \\
\hline & 12 & -5613 & 975 & 4 & -- & $\cdots$ \\
\hline & 13 & -2053 & 540 & 4 & - & $\ldots$ \\
\hline & 14 & -1563 & 65 & 15 & $\ldots$ & $\ldots$ \\
\hline & 15 & -973 & 300 & 0 & -. & $\ldots$ \\
\hline \multirow[t]{11}{*}{ MSJDO2 } & 2 & -5284 & 600 & 32 & 68 & 49 \\
\hline & 3 & -2774 & 2510 & 32 & 45 & 41 \\
\hline & 5 & -1904 & 470 & 20 & $<10$ & 31 \\
\hline & 6 & -1629 & 245 & 12 & - & 29 \\
\hline & 7 & -354 & 930 & 28 & $<10$ & 24 \\
\hline & 8 & 136 & 490 & 66 & $<10$ & 20 \\
\hline & 9 & 280 & 144 & 4 & - & 18 \\
\hline & 12 & -5884 & 950 & 0 & -. & $\ldots$ \\
\hline & 13 & -2374 & 400 & 7 & -- & -- \\
\hline & 14 & -1874 & 30 & 0 & -- & $\ldots$ \\
\hline & 15 & -1284 & 345 & 0 & -- & $\cdots$ \\
\hline \multirow[t]{11}{*}{ MSJDO3 } & 2 & -4209 & 970 & 48 & 60 & 59 \\
\hline & 3 & -2589 & 1620 & 46 & 38 & 47 \\
\hline & 4 & -2254 & 335 & 40 & $<10$ & 39 \\
\hline & 5 & -1314 & 445 & 30 & $<10$ & 31 \\
\hline & 6 & -929 & 320 & 28 & $<10$ & 28 \\
\hline & 7 & 171 & 790 & 43 & $<10$ & 20 \\
\hline & 8 & 502 & 331 & 63 & $<10$ & 15 \\
\hline & 12 & -5179 & 920 & 1 & - & - \\
\hline & 13 & -1759 & 495 & 2 & $\ldots$ & $\ldots$ \\
\hline & 14 & -1249 & 65 & 0 & -- & -- \\
\hline & 15 & -619 & 310 & 0 & $\ldots$ & -- \\
\hline
\end{tabular}


Table 4--Layer number; altitude of top, thickness, and sand percentage of layer; and average dissolved-solids concentration and temperature of water in layer; by log number--Continued.

\begin{tabular}{|c|c|c|c|c|c|c|}
\hline $\begin{array}{l}\text { Log } \\
\text { number }\end{array}$ & $\begin{array}{r}\text { Layer } \\
\text { number }\end{array}$ & $\begin{array}{r}\text { Altitude } \\
\text { of top } \\
(f \in e t)\end{array}$ & $\begin{array}{r}\text { Thlok- } \\
\text { ness } \\
\text { (feet) }\end{array}$ & $\begin{array}{r}\text { Sand } \\
\text { per- } \\
\text { centage }\end{array}$ & $\begin{array}{l}\text { Dis- } \\
\text { solved } \\
\text { solids }\end{array}$ & $\begin{array}{l}\text { Tem- } \\
\text { per- } \\
\text { 2ture }\end{array}$ \\
\hline \multirow[t]{10}{*}{ MSJEOI } & 2 & -5875 & 880 & 35 & 53 & 69 \\
\hline & 3 & -3445 & 2430 & 43 & 36 & 56 \\
\hline & 5 & -2105 & 850 & 65 & 30 & 40 \\
\hline & 6 & -1375 & 570 & 26 & 20 & 33 \\
\hline & 7 & 5 & 900 & 16 & $<10$ & 23 \\
\hline & 8 & 195 & 190 & 7 & -- & 19 \\
\hline & 12 & -6755 & 970 & 0 & -- & -- \\
\hline & 13 & -2955 & 490 & 2 & -- & $\cdots$ \\
\hline & 14 & -1945 & 160 & 0 & -- & -- \\
\hline & 15 & -895 & 480 & 0 & -- & -- \\
\hline \multirow[t]{10}{*}{ MSJEO2 } & 2 & -6145 & 760 & 32 & 96 & 69 \\
\hline & 3 & -3615 & 2530 & 35 & 85 & 58 \\
\hline & 5 & -2335 & 850 & 56 & 63 & 44 \\
\hline & 6 & -1610 & 490 & 41 & 36 & 38 \\
\hline & 7 & -225 & 960 & 40 & $<10$ & 27 \\
\hline & 8 & 175 & 400 & 26 & $<10$ & 19 \\
\hline & 12 & -6905 & 1150 & 0 & -- & $\cdots$ \\
\hline & 13 & -3185 & 430 & 0 & -- & -- \\
\hline & 14 & -2100 & 235 & 0 & -- & - \\
\hline & 15 & -1185 & 425 & 0 & -- & - \\
\hline \multirow[t]{11}{*}{$M S J E 03$} & 2 & -6312 & 800 & 34 & 58 & 68 \\
\hline & 3 & -3692 & 2620 & 27 & 44 & 56 \\
\hline & 5 & -2362 & 880 & 63 & 59 & 40 \\
\hline & 6 & -1742 & 420 & 42 & 32 & 34 \\
\hline & 7 & -262 & 1015 & 21 & $<10$ & 25 \\
\hline & 8 & 238 & 500 & 38 & -- & 20 \\
\hline & 9 & 478 & 240 & 52 & $\cdots$ & 17 \\
\hline & 12 & -7112 & 1060 & 0 & -- & -- \\
\hline & 13 & -3242 & 450 & 0 & -- & $\cdots$ \\
\hline & 14 & -2162 & 200 & 0 & $\cdots$ & $\cdots$ \\
\hline & 15 & -1277 & 465 & 0 & -- & -- \\
\hline \multirow[t]{9}{*}{$\mathrm{MSJEO} 4$} & 3 & -3778 & -- & -- & -- & -- \\
\hline & 5 & -2438 & 870 & 50 & -- & - \\
\hline & 6 & -1813 & 400 & 38 & -- & $\cdots$ \\
\hline & 7 & -358 & 1030 & 24 & -- & -- \\
\hline & 8 & 162 & 520 & 38 & -- & $\cdots$ \\
\hline & 9 & 402 & 240 & 52 & -- & -- \\
\hline & 13 & -3308 & 470 & 0 & -- & -- \\
\hline & 14 & -2213 & 225 & 0 & -- & - \\
\hline & 15 & -1388 & 425 & 0 & -- & -- \\
\hline \multirow[t]{11}{*}{ MSJEOS } & 2 & -5885 & 750 & 61 & 60 & 71 \\
\hline & 3 & -3665 & 2220 & 48 & 52 & 61 \\
\hline & 4 & -3415 & 250 & 84 & 88 & 52 \\
\hline & 5 & -2055 & 850 & 50 & 51 & 45 \\
\hline & 6 & -1325 & 560 & 33 & 27 & 39 \\
\hline & 7 & 65 & 880 & 23 & $<10$ & 23 \\
\hline & 8 & 185 & 120 & 8 & -- & 16 \\
\hline & 12 & -6635 & 1000 & 0 & -- & -- \\
\hline & 13 & -2905 & 510 & 2 & -- & -- \\
\hline & 14 & -1885 & 170 & 0 & $\cdots$ & -- \\
\hline & 15 & -815 & 510 & 0 & -- & $\cdots$ \\
\hline
\end{tabular}


Table 4--Layer number; altitude of top, thickness, and sand percentage of layer; and average dissolved-solids concentration and temperature of water in layer; by log number--Continued.

\begin{tabular}{|c|c|c|c|c|c|c|}
\hline $\begin{array}{l}\text { Log } \\
\text { number }\end{array}$ & $\begin{array}{l}\text { Layer } \\
\text { number }\end{array}$ & $\begin{array}{r}\text { Altitude } \\
\text { of top } \\
\text { (feet) }\end{array}$ & $\begin{array}{r}\text { Thick- } \\
\text { ness } \\
\text { (feet) }\end{array}$ & $\begin{array}{r}\text { Sand } \\
\text { per- } \\
\text { centage }\end{array}$ & $\begin{array}{l}\text { Dis- } \\
\text { solved } \\
\text { solids }\end{array}$ & $\begin{array}{l}\text { Tem- } \\
\text { per- } \\
\text { ature }\end{array}$ \\
\hline \multirow[t]{11}{*}{ MSJEO 6} & 2 & -6172 & 790 & 21 & 26 & 92 \\
\hline & 3 & -3572 & 2600 & 40 & 42 & 73 \\
\hline & 5 & -2167 & 935 & 60 & 19 & 48 \\
\hline & 6 & -1592 & 415 & 41 & 11 & 39 \\
\hline & 7 & -142 & 1020 & 25 & $<10$ & 26 \\
\hline & 8 & 308 & 450 & 29 & $<10$ & 18 \\
\hline & 9 & 428 & 120 & 64 & - & 14 \\
\hline & 12 & -6962 & 1105 & 1 & -- & -- \\
\hline & 13 & -3102 & 470 & 1 & -- & -- \\
\hline & 14 & -2007 & 160 & 0 & -- & -- \\
\hline & 15 & -1152 & 440 & 0 & -- & -- \\
\hline \multirow[t]{11}{*}{ MSJEOT } & 2 & -5963 & 730 & 59 & 42 & 65 \\
\hline & 3 & -3673 & 2290 & 34 & 66 & 59 \\
\hline & 4 & -3383 & 290 & 69 & 52 & 49 \\
\hline & 5 & -1978 & 965 & 33 & 38 & 40 \\
\hline & 6 & -1218 & 585 & 45 & $<10$ & 32 \\
\hline & 7 & 72 & 885 & 23 & $<10$ & 23 \\
\hline & 8 & 197 & 125 & 5 & -- & 18 \\
\hline & 12 & -6693 & 970 & 0 & -- & -- \\
\hline & 13 & -2943 & 440 & 0 & -- & -- \\
\hline & 14 & -1803 & 175 & 0 & -- & $=-$ \\
\hline & 15 & -813 & 405 & 0 & -- & -- \\
\hline \multirow[t]{10}{*}{ MSJOOI } & 2 & -3897 & 840 & 37 & 47 & 51 \\
\hline & 3 & -1812 & 2085 & 29 & 21 & 40 \\
\hline & 5 & -1337 & 80 & -- & -- & 29 \\
\hline & 6 & -1037 & 230 & 50 & $<10$ & 27 \\
\hline & 7 & -127 & 730 & 41 & $<10$ & 22 \\
\hline & 8 & 293 & 420 & 40 & $<10$ & 18 \\
\hline & 12 & -4737 & 490 & 0 & - & - \\
\hline & 13 & -1417 & 395 & 11 & -- & -- \\
\hline & 14 & -1267 & 70 & 0 & -- & -- \\
\hline & 15 & -857 & 180 & 0 & -- & -- \\
\hline \multirow[t]{10}{*}{ MSJOO2 } & 2 & -3753 & 985 & 38 & -- & -- \\
\hline & 3 & -1858 & 1895 & 21 & -- & -- \\
\hline & 5 & -1248 & 405 & 21 & -- & -- \\
\hline & 6 & -908 & 280 & 32 & -- & -- \\
\hline & 7 & -48 & 570 & 58 & -- & -- \\
\hline & 8 & 252 & 300 & 45 & -- & - \\
\hline & 12 & -4738 & 840 & 0 & -- & -- \\
\hline & 13 & -1653 & 205 & 0 & -- & -- \\
\hline & 14 & -1188 & 60 & 0 & -- & -- \\
\hline & 15 & -618 & 290 & 7 & -- & -- \\
\hline \multirow[t]{10}{*}{ MSJOO3 } & 2 & -2906 & 480 & 56 & 14 & 52 \\
\hline & 3 & -1486 & 1420 & 20 & $<10$ & 42 \\
\hline & 4 & -1086 & 400 & 16 & $<10$ & 32 \\
\hline & 5 & -586 & 430 & 21 & $<10$ & 26 \\
\hline & 6 & -336 & 185 & 51 & $<10$ & 22 \\
\hline & 7 & 314 & 345 & 27 & $<10$ & 16 \\
\hline & 12 & -3386 & 500 & 18 & - - & -- \\
\hline & 13 & -1016 & 70 & 0 & -- & -- \\
\hline & 14 & -521 & 65 & 0 & -- & -- \\
\hline & 15 & -31 & 305 & 3 & -- & -- \\
\hline
\end{tabular}


Table 4--Layer number; altitude of top, thickness, and sand percentage of layer; and average dissolved-solids concentration and temperature of water in layer; by log number--Continued.

\begin{tabular}{|c|c|c|c|c|c|c|}
\hline $\begin{array}{l}\text { Log } \\
\text { number }\end{array}$ & $\begin{array}{r}\text { Layer } \\
\text { number }\end{array}$ & $\begin{array}{r}\text { Alt itude } \\
\text { of top } \\
\text { (feet) }\end{array}$ & $\begin{array}{r}\text { Thlck- } \\
\text { ness } \\
\text { (feet) }\end{array}$ & $\begin{array}{r}\text { Sand } \\
\text { per- } \\
\text { centage }\end{array}$ & $\begin{array}{l}\text { Dis- } \\
\text { solved } \\
\text { solids }\end{array}$ & $\begin{array}{l}\text { Tem- } \\
\text { per- } \\
\text { ature }\end{array}$ \\
\hline \multirow[t]{11}{*}{ MSJ004 } & 2 & -3466 & 750 & 48 & -- & - \\
\hline & 3 & -1736 & 1730 & 22 & -- & -- \\
\hline & 4 & -1406 & 330 & 21 & -- & -- \\
\hline & 5 & -866 & 395 & 25 & -- & -- \\
\hline & 6 & -596 & 190 & 42 & - & -- \\
\hline & 7 & -26 & 260 & 67 & -- & - \\
\hline & 8 & 221 & 247 & 48 & -- & -- \\
\hline & 12 & -4216 & 720 & 0 & $\ldots$ & $\ldots$ \\
\hline & 13 & -1261 & 145 & 0 & -- & -- \\
\hline & 14 & -786 & 80 & 0 & $\ldots$ & - \\
\hline & 15 & -286 & 310 & 6 & -- & -- \\
\hline \multirow[t]{11}{*}{ MSJOOS } & 2 & -2928 & 840 & 38 & -- & -- \\
\hline & 3 & -1513 & 1415 & 28 & - & -- \\
\hline & 4 & -1213 & 300 & 10 & -- & -- \\
\hline & 5 & -993 & 65 & 99 & -- & - \\
\hline & 6 & -743 & 195 & 23 & $\ldots$ & - \\
\hline & 7 & 147 & 590 & 64 & -- & -- \\
\hline & 8 & 307 & 160 & 26 & - & - \\
\hline & 12 & -3768 & 745 & 0 & - & - \\
\hline & 13 & -1058 & 155 & 0 & -- & -- \\
\hline & 14 & -938 & 55 & 0 & -- & -- \\
\hline & 15 & -443 & 300 & 3 & -- & -- \\
\hline \multirow[t]{9}{*}{ MSJSOI } & 2 & -1817 & 585 & 94 & $<10$ & 36 \\
\hline & 3 & -477 & 1340 & 19 & $<10$ & 28 \\
\hline & 4 & -387 & 90 & 11 & $\ldots$ & 22 \\
\hline & 5 & -17 & 270 & 69 & $<10$ & 19 \\
\hline & 6 & 163 & 130 & 69 & $<10$ & 17 \\
\hline & 12 & -2402 & 780 & 0 & - - & -- \\
\hline & 13 & -287 & 100 & 0 & $\ldots$ & -- \\
\hline & 14 & 33 & 50 & 0 & -- & - \\
\hline & 15 & 485 & 322 & 3 & -- & - \\
\hline \multirow[t]{9}{*}{ MSJSO2 } & 2 & -2395 & 380 & 82 & $<10$ & 41 \\
\hline & 3 & -1255 & 1140 & 19 & $<10$ & 35 \\
\hline & 4 & -785 & 470 & 43 & $<10$ & 27 \\
\hline & 5 & -275 & 390 & 56 & $<10$ & 22 \\
\hline & 6 & -35 & 150 & 60 & $<10$ & 18 \\
\hline & 12 & -2775 & 1160 & 0 & - & $\cdots$ \\
\hline & 13 & -665 & 120 & 17 & -- & - \\
\hline & 14 & -185 & 90 & 11 & -- & -- \\
\hline & 15 & 325 & 360 & 3 & -- & $\cdots$ \\
\hline MSKEOI & 12 & 235 & 510 & 0 & -- & -- \\
\hline \multirow[t]{2}{*}{ MSKEO2 } & 2 & 532 & 470 & 69 & $<10$ & 14 \\
\hline & 12 & 62 & 545 & 0 & $\ldots$ & $\ldots$ \\
\hline
\end{tabular}


Table 4--Layer number; altitude of top, thickness, and sand percentage of layer; and average dissolved-solids concentration and temperature of water in layer; by log number--Continued.

\begin{tabular}{|c|c|c|c|c|c|c|}
\hline $\begin{array}{l}\text { Log } \\
\text { number }\end{array}$ & $\begin{array}{r}\text { Layer } \\
\text { number }\end{array}$ & $\begin{array}{r}\text { Alt } t \text { tude } \\
\text { of top } \\
(f e e t)\end{array}$ & $\begin{array}{r}\text { Thick- } \\
\text { ness } \\
(\text { feet })\end{array}$ & $\begin{array}{r}\text { Sand } \\
\text { per- } \\
\text { centage }\end{array}$ & $\begin{array}{l}\text { Dis- } \\
\text { solved } \\
\text { solids }\end{array}$ & $\begin{array}{r}\text { Tem- } \\
\text { per- } \\
\text { 2ture }\end{array}$ \\
\hline \multirow[t]{3}{*}{ MSLAO 1} & 2 & 379 & 100 & 50 & -- & - \\
\hline & 3 & 569 & 190 & 25 & -- & - \\
\hline & 12 & 279 & 530 & 0 & -- & - \\
\hline \multirow[t]{5}{*}{$\mathrm{MSLAO} 2$} & 1 & -650 & 95 & 37 & -- & -- \\
\hline & 2 & 265 & 325 & 51 & -- & - \\
\hline & 3 & 430 & 165 & 30 & -- & $\cdots$ \\
\hline & 4 & 490 & 60 & 71 & -- & - \\
\hline & 12 & -60 & 590 & 0 & -- & -- \\
\hline \multirow[t]{4}{*}{ MSLAO3 } & 2 & -153 & 200 & 55 & -. & -- \\
\hline & 3 & 157 & 310 & 29 & -- & - \\
\hline & 4 & 427 & 270 & 18 & -- & -- \\
\hline & 12 & -353 & 480 & 0 & -- & -- \\
\hline \multirow{4}{*}{$\mathrm{MSLAO} 4$} & 1 & -302 & 300 & 40 & $<10$ & 23 \\
\hline & 2 & 228 & 110 & 50 & $<10$ & -- \\
\hline & 3 & 374 & 146 & 13 & -- & -- \\
\hline & 12 & 118 & 420 & 0 & -- & -- \\
\hline \multirow[t]{4}{*}{ MSLDO1 } & 2 & -526 & 215 & 99 & -- & -- \\
\hline & 3 & 254 & 780 & 15 & -- & -- \\
\hline & 4 & 444 & 190 & 45 & -- & -- \\
\hline & 12 & -741 & 740 & 3 & -- & -- \\
\hline \multirow[t]{4}{*}{ MSLDO2 } & 2 & -105 & 245 & 76 & $<10$ & 24 \\
\hline & 3 & 425 & 530 & 9 & -- & 21 \\
\hline & 4 & 465 & 40 & 20 & -- & 19 \\
\hline & 12 & -350 & 655 & 2 & -- & - \\
\hline \multirow[t]{3}{*}{ MSLDO 3} & 2 & -52 & 250 & 66 & -- & 24 \\
\hline & 3 & 418 & 470 & 13 & -- & 21 \\
\hline & 12 & -302 & 490 & 0 & -- & $\cdots$ \\
\hline \multirow[t]{11}{*}{ MSLIO 1} & 2 & -6235 & 720 & 22 & 64 & 60 \\
\hline & 3 & -3525 & 2710 & 46 & 44 & 49 \\
\hline & 5 & -2150 & 805 & 40 & $<10$ & 33 \\
\hline & 6 & -1725 & 270 & 19 & $<10$ & 28 \\
\hline & 7 & -165 & 1190 & 20 & -- & 21 \\
\hline & 8 & 325 & 490 & 61 & -- & 15 \\
\hline & 9 & 475 & 150 & 76 & -- & -- \\
\hline & 12 & -6955 & 920 & 0 & -- & -. \\
\hline & 13 & -2955 & 570 & 4 & -- & -- \\
\hline & 14 & -1995 & 155 & 0 & -- & -- \\
\hline & 15 & -1355 & 370 & 3 & -- & -- \\
\hline \multirow[t]{11}{*}{ MSLIO 2} & 2 & -6511 & 530 & 35 & 84 & 63 \\
\hline & 3 & -3431 & 3080 & 45 & 66 & 50 \\
\hline & 5 & -2156 & 865 & 47 & 11 & 34 \\
\hline & 6 & -1721 & 280 & 21 & $<10$ & 29 \\
\hline & 7 & -191 & 1160 & 21 & $<10$ & 21 \\
\hline & 8 & 299 & 490 & 61 & $<10$ & 15 \\
\hline & 9 & 434 & 135 & 69 & $<10$ & -- \\
\hline & 12 & -7041 & 920 & 0 & -- & - \\
\hline & 13 & -3021 & 410 & 2 & -- & -- \\
\hline & 14 & -2001 & 155 & 0 & -- & -- \\
\hline & 15 & -1351 & 370 & 3 & -- & -- \\
\hline
\end{tabular}


Table 4--Layer number; altitude of top, thickness, and sand percentage of layer; and average dissolved-solids concentration and temperature of water in layer; by log number--Continued.

\begin{tabular}{|c|c|c|c|c|c|c|}
\hline $\begin{array}{l}\text { Log } \\
\text { number }\end{array}$ & $\begin{array}{r}\text { Layex } \\
\text { number }\end{array}$ & $\begin{array}{r}\text { Alt itude } \\
\text { of top } \\
(f e e t)\end{array}$ & $\begin{array}{r}\text { Thick- } \\
\text { ness } \\
(\text { feet) }\end{array}$ & $\begin{array}{r}\text { Sand } \\
\text { per- } \\
\text { centage }\end{array}$ & $\begin{array}{l}\text { Dis- } \\
\text { solved } \\
\text { solids }\end{array}$ & $\begin{array}{l}\text { Tem- } \\
\text { per- } \\
\text { 2ture }\end{array}$ \\
\hline \multirow[t]{11}{*}{ MSLIO3 } & 2 & -6073 & 510 & 37 & 62 & 66 \\
\hline & 3 & -3503 & 2570 & 19 & 61 & 54 \\
\hline & 5 & -2388 & 535 & 19 & 40 & 37 \\
\hline & 6 & -2083 & 150 & 37 & $<10$ & 33 \\
\hline & 7 & -443 & 1340 & 26 & $<10$ & 24 \\
\hline & 8 & 252 & 695 & 9 & $<10$ & 16 \\
\hline & 9 & 415 & 163 & 37 & -- & -- \\
\hline & 12 & -6583 & 1220 & 0 & -- & -- \\
\hline & 13 & -2923 & 580 & 2 & -- & -- \\
\hline & 14 & -2233 & 155 & 0 & -- & -- \\
\hline & 15 & -1783 & 300 & 0 & -- & -- \\
\hline \multirow[t]{4}{*}{ MSLKO1 } & 2 & -617 & 490 & 63 & $<10$ & 25 \\
\hline & 3 & 93 & 710 & 23 & $<10$ & 19 \\
\hline & 4 & 473 & 380 & 41 & $<10$ & 14 \\
\hline & 12 & -1107 & 685 & 0 & -- & -- \\
\hline \multirow[t]{6}{*}{ MSLKO 2} & 2 & -761 & 415 & 87 & $<10$ & 25 \\
\hline & 3 & -81 & 680 & 43 & $<10$ & 21 \\
\hline & 4 & 254 & 335 & 40 & $<10$ & 17 \\
\hline & 5 & 404 & 80 & 56 & -- & 14 \\
\hline & 12 & -1176 & 905 & 2 & -- & - \\
\hline & 13 & 324 & 70 & 0 & -- & - \\
\hline \multirow[t]{8}{*}{ MSLKO3 } & 2 & -1370 & 450 & 84 & $<10$ & 29 \\
\hline & 3 & -940 & 430 & 12 & -- & 26 \\
\hline & 4 & -140 & 800 & 34 & -- & 21 \\
\hline & 5 & 230 & 220 & 95 & -- & 17 \\
\hline & 6 & 450 & 90 & 50 & -- & 15 \\
\hline & 12 & -1820 & 1070 & 2 & -- & -- \\
\hline & 13 & 10 & 150 & 13 & -- & -- \\
\hline & 14 & 360 & 130 & 23 & -- & -- \\
\hline \multirow[t]{9}{*}{ MSLMO 1} & 2 & -4498 & 690 & 49 & 72 & 53 \\
\hline & 3 & -2388 & 2110 & 28 & 64 & 43 \\
\hline & 5 & -1628 & 380 & 14 & -- & 31 \\
\hline & 7 & -318 & 1165 & 64 & $<10$ & 24 \\
\hline & 8 & 272 & 590 & 32 & $<10$ & 18 \\
\hline & 9 & 376 & 104 & 43 & -- & 15 \\
\hline & 12 & -5188 & 870 & 2 & -- & - \\
\hline & 13 & -2008 & 380 & 5 & -- & -- \\
\hline & 15 & -1483 & 145 & 0 & -- & -- \\
\hline \multirow[t]{11}{*}{ MSLMO2 } & 2 & -4684 & 515 & 45 & -- & -- \\
\hline & 3 & -2854 & 1830 & 22 & -- & -- \\
\hline & 5 & -2164 & 200 & -- & -- & -- \\
\hline & 6 & -2054 & 30 & 99 & -- & -- \\
\hline & 7 & -674 & 1220 & 37 & $<10$ & 35 \\
\hline & 8 & 86 & 760 & 66 & $<10$ & 20 \\
\hline & 9 & 204 & 118 & 32 & $<10$ & -- \\
\hline & 12 & -5199 & 1075 & 1 & -- & - \\
\hline & 13 & -2364 & 490 & 8 & -- & -- \\
\hline & 14 & -2084 & 80 & 0 & -- & -- \\
\hline & 15 & -1894 & 160 & 6 & -- & -- \\
\hline
\end{tabular}


Table 4--Layer number; altitude of top, thickness, and sand percentage of layer; and average dissolved-solids concentration and temperature of water in layer; by log number--Continued.

\begin{tabular}{|c|c|c|c|c|c|c|}
\hline $\begin{array}{l}\text { Log } \\
\text { number }\end{array}$ & $\begin{array}{r}\text { Layer } \\
\text { number }\end{array}$ & $\begin{array}{r}\text { Altitude } \\
\text { of top } \\
\text { (feet) }\end{array}$ & $\begin{array}{r}\text { Thick- } \\
\text { ness } \\
\text { (feet) }\end{array}$ & $\begin{array}{r}\text { Sand } \\
\text { per- } \\
\text { centage }\end{array}$ & $\begin{array}{l}\text { Dis- } \\
\text { solved } \\
\text { solids }\end{array}$ & $\begin{array}{r}\text { Tem- } \\
\text { per- } \\
\text { ature }\end{array}$ \\
\hline \multirow[t]{9}{*}{ MSLMO3 } & . 2 & -4615 & 810 & 27 & 30 & 61 \\
\hline & 3 & -2415 & 2200 & 20 & 19 & 55 \\
\hline & 5 & -1435 & 680 & 18 & $<10$ & 49 \\
\hline & 7 & -250 & 920 & 35 & $<10$ & 30 \\
\hline & 8 & 230 & 480 & 71 & $<10$ & -- \\
\hline & 9 & 365 & 135 & 50 & -- & -- \\
\hline & 12 & -5425 & 850 & 1 & -- & -- \\
\hline & 13 & -2115 & 300 & 3 & -- & -- \\
\hline & 15 & $-11>0$ & 265 & 0 & -- & -- \\
\hline \multirow[t]{12}{*}{ MSLWO 1} & 2 & -5673 & 570 & 47 & 95 & 65 \\
\hline & 3 & -3233 & 2440 & 33 & 107 & 58 \\
\hline & 4 & -2983 & 250 & 48 & $<10$ & 49 \\
\hline & 5 & -1663 & 780 & 41 & $<10$ & 38 \\
\hline & 6 & -1213 & 385 & 34 & $<10$ & 31 \\
\hline & 7 & -3 & 790 & 32 & $<10$ & 19 \\
\hline & 8 & 357 & 360 & 73 & $<10$ & -- \\
\hline & 9 & 441 & 84 & 80 & -- & -- \\
\hline & 12 & -6243 & 910 & 0 & -- & - \\
\hline & 13 & -2443 & 540 & 4 & -- & -- \\
\hline & 14 & -1598 & 65 & 0 & -- & -- \\
\hline & 15 & -793 & 420 & 2 & -- & -- \\
\hline \multirow[t]{9}{*}{ MSMAO 1} & 2 & -2535 & 385 & 86 & $<10$ & 45 \\
\hline & 3 & -1550 & 985 & 38 & $<10$ & 38 \\
\hline & 4 & -1415 & 135 & 70 & $<10$ & 32 \\
\hline & 5 & -485 & 550 & 83 & $<10$ & 24 \\
\hline & 6 & 0 & 325 & 92 & $<10$ & 17 \\
\hline & 12 & -2920 & 135 & 0 & -- & -- \\
\hline & 13 & -1035 & 380 & 3 & -- & -- \\
\hline & 14 & -325 & 160 & 3 & -- & -- \\
\hline & 15 & 340 & 340 & 3 & -- & -- \\
\hline \multirow[t]{9}{*}{$M S M A O 2$} & 2 & -2610 & 410 & 56 & $<10$ & 34 \\
\hline & 3 & -1980 & 630 & 14 & -- & 32 \\
\hline & 4 & -1055 & 925 & 58 & $<10$ & 28 \\
\hline & 5 & -300 & 580 & 66 & $<10$ & 24 \\
\hline & 6 & 190 & 350 & 63 & $<10$ & 21 \\
\hline & 12 & -3020 & 1350 & 7 & -- & -- \\
\hline & 13 & -880 & 175 & 14 & -- & -- \\
\hline & 14 & -160 & 140 & 7 & -- & - \\
\hline & 15 & 260 & 70 & 0 & -- & -- \\
\hline \multirow[t]{8}{*}{ MSMAO3 } & 2 & -2062 & 420 & 98 & $<10$ & 36 \\
\hline & 3 & -1462 & 600 & 27 & $<10$ & 32 \\
\hline & 4 & -592 & 870 & 50 & $<10$ & 26 \\
\hline & 5 & -42 & 470 & 99 & $<10$ & 20 \\
\hline & 6 & 280 & 202 & 19 & -- & 17 \\
\hline & 12 & -2482 & 1180 & 5 & -- & - \\
\hline & 13 & -512 & 80 & 0 & -- & -- \\
\hline & 14 & 78 & 120 & 17 & -- & -- \\
\hline
\end{tabular}


Table 4--Layer number; altitude of top, thickness, and sand percentage of layer; and average dissolved-solids concentration and temperature of water in layer; by log number--Continued.

\begin{tabular}{|c|c|c|c|c|c|c|}
\hline $\begin{array}{l}\text { Log } \\
\text { number }\end{array}$ & $\begin{array}{r}\text { Layer } \\
\text { number }\end{array}$ & $\begin{array}{r}\text { Altitude } \\
\text { of top } \\
\text { (feet) }\end{array}$ & $\begin{array}{r}\text { Thlck- } \\
\text { ness } \\
\text { (feet) }\end{array}$ & $\begin{array}{r}\text { Sand } \\
\text { per- } \\
\text { centage }\end{array}$ & $\begin{array}{l}\text { Dis- } \\
\text { solved } \\
\text { solids }\end{array}$ & $\begin{array}{l}\text { Tem- } \\
\text { per- } \\
\text { ature }\end{array}$ \\
\hline \multirow[t]{9}{*}{$\mathrm{MSMAO} 4$} & 2 & -3311 & 420 & 37 & $<10$ & 48 \\
\hline & 3 & -2036 & 1275 & 26 & $<10$ & 41 \\
\hline & 4 & -1801 & 235 & 81 & $<10$ & 34 \\
\hline & 5 & -641 & 675 & 59 & $<10$ & 26 \\
\hline & 6 & -61 & 450 & 41 & $<10$ & 20 \\
\hline & 12 & -3731 & 450 & 0 & -- & -- \\
\hline & 13 & -1316 & 485 & 2 & -- & -- \\
\hline & 14 & -511 & 130 & 0 & -- & -- \\
\hline & 15 & 199 & 260 & 0 & -- & -- \\
\hline \multirow[t]{4}{*}{ MSMNO 1} & 2 & -319 & 180 & 92 & $<10$ & 23 \\
\hline & 3 & 321 & 640 & 14 & $<10$ & 18 \\
\hline & 4 & 441 & 120 & 35 & -- & -- \\
\hline & 12 & -499 & 680 & 0 & -- & -- \\
\hline \multirow[t]{11}{*}{ MSMRO 1} & 2 & -5055 & 895 & 30 & 73 & 56 \\
\hline & 3 & -3130 & 1925 & 22 & 65 & 47 \\
\hline & 5 & -2285 & 485 & 12 & 58 & 36 \\
\hline & 6 & -2155 & 55 & 45 & 10 & 34 \\
\hline & 7 & -745 & 1200 & 41 & $<10$ & 28 \\
\hline & 8 & -5 & 740 & 19 & $<10$ & 22 \\
\hline & 9 & 167 & 172 & 64 & $<10$ & 19 \\
\hline & 12 & -5950 & 835 & 1 & -- & -- \\
\hline & 13 & -2770 & 360 & 3 & -- & -- \\
\hline & 14 & -2210 & 75 & 13 & -- & - \\
\hline & 15 & -1945 & 210 & 0 & -- & -- \\
\hline \multirow[t]{5}{*}{ MSMSO 1} & 1 & -730 & 115 & 91 & $<10$ & 24 \\
\hline & 2 & -90 & 210 & 24 & $<10$ & 21 \\
\hline & 3 & 120 & 210 & 5 & -- & 20 \\
\hline & 4 & 386 & 266 & 71 & -- & 18 \\
\hline & 12 & -300 & 430 & 0 & -- & -- \\
\hline \multirow[t]{6}{*}{ MSMSO 2} & 1 & -1186 & 82 & 83 & -- & -- \\
\hline & 2 & -728 & 56 & 99 & -- & -- \\
\hline & 3 & -268 & 460 & 18 & -- & -- \\
\hline & 4 & 77 & 345 & 99 & -- & -- \\
\hline & 5 & 360 & 283 & 53 & -- & -- \\
\hline & 12 & -784 & 402 & 2 & -- & -- \\
\hline \multirow[t]{5}{*}{ MSMSO3 } & 1 & -643 & 82 & 99 & -- & -- \\
\hline & 2 & -121 & 94 & 94 & -- & -- \\
\hline & 3 & 137 & 258 & 19 & -- & -- \\
\hline & 4 & 513 & 376 & 65 & -- & -- \\
\hline & 12 & -215 & 428 & 0 & -- & -- \\
\hline \multirow[t]{4}{*}{ MSNEO1 } & 2 & -142 & 450 & 79 & $<10$ & 24 \\
\hline & 3 & 268 & 410 & 73 & $<10$ & 20 \\
\hline & 4 & 446 & 178 & 63 & $<10$ & 17 \\
\hline & 12 & -592 & 635 & 0 & -- & -- \\
\hline
\end{tabular}


Table 4--Layer number; altitude of top, thickness, and sand percentage of layer; and average dissolved-solids concentration and temperature of water in layer; by log number--Continued.

\begin{tabular}{|c|c|c|c|c|c|c|}
\hline $\begin{array}{l}\text { Log } \\
\text { number }\end{array}$ & $\begin{array}{r}\text { Layer } \\
\text { number }\end{array}$ & $\begin{array}{r}\text { Altitude } \\
\text { of top } \\
\text { (feet) }\end{array}$ & $\begin{array}{r}\text { Thick- } \\
\text { ness } \\
\text { (feet) }\end{array}$ & $\begin{array}{r}\text { Sand } \\
\text { per- } \\
\text { centage }\end{array}$ & $\begin{array}{l}\text { Dis- } \\
\text { solved } \\
\text { solids }\end{array}$ & $\begin{array}{l}\text { Tem- } \\
\text { per- } \\
\text { ature }\end{array}$ \\
\hline \multirow[t]{3}{*}{ MSNEO2 } & 2 & 146 & 375 & 96 & $<10$ & 21 \\
\hline & 3 & 495 & 349 & 34 & $<10$ & 17 \\
\hline & 12 & -229 & 570 & 0 & -- & -- \\
\hline \multirow[t]{4}{*}{ MSNEO3 } & 2 & -395 & 465 & 84 & $<10$ & 25 \\
\hline & 3 & -35 & 360 & 51 & $<10$ & 22 \\
\hline & 4 & 470 & 505 & 20 & $<10$ & 19 \\
\hline & 12 & -860 & 635 & 0 & -- & - \\
\hline \multirow[t]{6}{*}{ MSNWOI } & 2 & -824 & 435 & 92 & $<10$ & 34 \\
\hline & 3 & 51 & 875 & 11 & $<10$ & 26 \\
\hline & 4 & 256 & 205 & 39 & $<10$ & 19 \\
\hline & 5 & 381 & 90 & 70 & - & 17 \\
\hline & 12 & -1259 & 750 & 0 & -- & - - \\
\hline & 13 & 291 & 35 & 0 & -- & - \\
\hline \multirow[t]{8}{*}{ MSNWO 2} & 2 & -1398 & 365 & 59 & $<10$ & 30 \\
\hline & 3 & -393 & 1005 & 7 & -- & 26 \\
\hline & 4 & -33 & 360 & 18 & $<10$ & 22 \\
\hline & 5 & 177 & 165 & 79 & $<10$ & 20 \\
\hline & 6 & 369 & 32 & 20 & - & 18 \\
\hline & 12 & -1763 & 800 & 0 & -- & - \\
\hline & 13 & 12 & 45 & 0 & -- & -- \\
\hline & 14 & 337 & 160 & 13 & -- & - \\
\hline \multirow[t]{7}{*}{ MSNWO 3} & 2 & -828 & 420 & 92 & -- & -- \\
\hline & 3 & -43 & 785 & 24 & -- & - \\
\hline & 4 & 292 & 335 & 37 & $\cdots$ & - \\
\hline & 5 & 492 & 165 & 52 & -- & -- \\
\hline & 12 & -1248 & 795 & 13 & -- & -- \\
\hline & 13 & 327 & 35 & 0 & -- & -- \\
\hline & 14 & 522 & 30 & 25 & -- & -- \\
\hline MSNXOI & 12 & 250 & 67 & 0 & -- & - \\
\hline MSOKOI & 12 & 450 & 510 & 0 & $-\infty$ & $\ldots$ \\
\hline \multirow[t]{6}{*}{ MSPAOI } & 1 & -1530 & 185 & 76 & $-\infty$ & $\ldots$ \\
\hline & 2 & -640 & 190 & 66 & -- & -- \\
\hline & 3 & -370 & 270 & 46 & -- & -- \\
\hline & 4 & 180 & 550 & 71 & -- & - \\
\hline & 5 & 370 & 190 & 85 & $-\infty$ & - \\
\hline & 12 & -830 & 700 & 0 & -- & -- \\
\hline \multirow[t]{5}{*}{ MSPAO2 } & 2 & -600 & 200 & 45 & $<10$ & 24 \\
\hline & 3 & -250 & 350 & 34 & $<10$ & 22 \\
\hline & 4 & 100 & 350 & 34 & $<10$ & 19 \\
\hline & 5 & 350 & 250 & 77 & -- & 16 \\
\hline & 12 & -800 & 650 & 0 & -- & -- \\
\hline
\end{tabular}


Table 4--Layer number; altitude of top, thickness, and sand percentage of layer; and average dissolved-solids concentration and temperature of water in layer; by log number--Continued.

\begin{tabular}{|c|c|c|c|c|c|c|}
\hline $\begin{array}{l}\text { Log } \\
\text { number }\end{array}$ & $\begin{array}{r}\text { Layer } \\
\text { number }\end{array}$ & $\begin{array}{r}\text { Altitude } \\
\text { of top } \\
\text { (feet) }\end{array}$ & $\begin{array}{r}\text { Thick- } \\
\text { ness } \\
\text { (feet) }\end{array}$ & $\begin{array}{r}\text { Sand } \\
\text { per- } \\
\text { centage }\end{array}$ & $\begin{array}{l}\text { Dis- } \\
\text { solved } \\
\text { solids }\end{array}$ & $\begin{array}{r}\text { Tem- } \\
\text { per- } \\
\text { ature }\end{array}$ \\
\hline \multirow[t]{9}{*}{ MSPEO1 } & 2 & -4654 & 285 & 46 & -- & 54 \\
\hline & 3 & -2384 & 2270 & 10 & - & 46 \\
\hline & 5 & -1804 & 265 & 25 & -- & 34 \\
\hline & 7 & -584 & 1060 & 36 & $<10$ & 27 \\
\hline & 8 & 26 & 610 & 34 & $<10$ & 20 \\
\hline & 9 & 107 & 81 & 55 & $<10$ & 17 \\
\hline & 12 & -4939 & 835 & 4 & - & - \\
\hline & 13 & -2069 & 315 & 13 & - & -- \\
\hline & 15 & -1644 & 160 & 0 & -- & -- \\
\hline \multirow[t]{9}{*}{ MSPEO2 } & 2 & -3966 & 590 & 29 & -- & 48 \\
\hline & 3 & -2211 & 1755 & 17 & -- & 40 \\
\hline & 5 & -1391 & 295 & 54 & -- & 29 \\
\hline & 7 & -346 & 985 & 38 & $<10$ & 24 \\
\hline & 8 & 134 & 480 & 69 & $<10$ & 19 \\
\hline & 9 & 236 & 102 & 5 & -- & 17 \\
\hline & 12 & -4556 & 990 & 1 & - & - \\
\hline & 13 & -1686 & 525 & 18 & - & -- \\
\hline & 15 & -1331 & 60 & 0 & -- & -- \\
\hline \multirow[t]{9}{*}{ MSPEO3 } & 2 & -3961 & 550 & 33 & 23 & 51 \\
\hline & 3 & -2501 & 1460 & 8 & -- & 43 \\
\hline & 4 & -2171 & 330 & 44 & $<10$ & 37 \\
\hline & 5 & -1151 & 450 & 41 & -- & 29 \\
\hline & 7 & -291 & 760 & 31 & $<10$ & 23 \\
\hline & 8 & 94 & 385 & 59 & $<10$ & 19 \\
\hline & 12 & -4511 & 1020 & 11 & -- & - \\
\hline & 13 & -1601 & 570 & 3 & -- & -- \\
\hline & 15 & -1051 & 100 & 10 & -- & -- \\
\hline \multirow[t]{8}{*}{$\mathrm{MSPEO} 4$} & 2 & -3661 & 680 & 25 & 47 & 51 \\
\hline & 3 & -2066 & 1595 & 12 & $<10$ & 46 \\
\hline & 5 & -1041 & 400 & 35 & - & 30 \\
\hline & 7 & -191 & 740 & 34 & $<10$ & 23 \\
\hline & 8 & 177 & 368 & 20 & $<10$ & 17 \\
\hline & 12 & -4341 & 765 & 0 & -- & - - \\
\hline & 13 & -1441 & 625 & 0 & - & -- \\
\hline & 15 & -931 & 110 & 0 & -- & -- \\
\hline \multirow[t]{12}{*}{ MSPIOI } & 2 & -6413 & 790 & 44 & 29 & 64 \\
\hline & 3 & -4143 & 2270 & 30 & 18 & 54 \\
\hline & 5 & -3293 & 160 & - & $\ldots$ & -- \\
\hline & 6 & -3068 & 85 & 59 & 33 & 39 \\
\hline & 7 & -1103 & 1690 & 34 & $<10$ & 31 \\
\hline & 8 & -113 & 990 & 22 & $<10$ & 22 \\
\hline & 9 & 127 & 240 & 99 & $<10$ & 18 \\
\hline & 10 & 337 & 210 & 57 & $<10$ & 17 \\
\hline & 12 & -7203 & 1060 & 2 & -- & - \\
\hline & 13 & -3453 & 690 & 4 & -- & -- \\
\hline & 14 & -3153 & 140 & 11 & -- & -- \\
\hline & 15 & -2793 & 275 & 24 & - & -- \\
\hline
\end{tabular}


Table 4--Layer number; altitude of top, thickness, and sand percentage of layer; and average dissolved-solids concentration and temperature of water in layer; by log number--Continued.

\begin{tabular}{|c|c|c|c|c|c|c|}
\hline $\begin{array}{l}\text { Log } \\
\text { number }\end{array}$ & $\begin{array}{r}\text { Layer } \\
\text { number }\end{array}$ & $\begin{array}{r}\text { Altitude } \\
\text { of top } \\
\text { (feet) }\end{array}$ & $\begin{array}{r}\text { Thick- } \\
\text { ness } \\
\text { (feet) }\end{array}$ & $\begin{array}{r}\text { Sand } \\
\text { per- } \\
\text { centage }\end{array}$ & $\begin{array}{l}\text { Dis- } \\
\text { solved } \\
\text { solids }\end{array}$ & $\begin{array}{l}\text { Tem- } \\
\text { Per- } \\
\text { ature }\end{array}$ \\
\hline \multirow[t]{2}{*}{ MSPOO1 } & 1 & -3 & 50 & 40 & -- & 16 \\
\hline & 12 & 340 & 343 & 0 & -- & -- \\
\hline \multirow[t]{10}{*}{ MSPRO 1} & 2 & -4815 & 830 & 43 & 44 & 62 \\
\hline & 3 & -3135 & 1680 & 22 & 25 & 53 \\
\hline & 5 & -2305 & 540 & 33 & 25 & 43 \\
\hline & 7 & -825 & 1375 & 77 & $<10$ & 34 \\
\hline & 8 & -45 & 780 & 35 & $<10$ & 24 \\
\hline & 9 & 165 & 210 & 7 & - - & 19 \\
\hline & 10 & 170 & 5 & 5 & -- & 18 \\
\hline & 12 & -5645 & 840 & 2 & -- & -- \\
\hline & 13 & -2845 & 290 & 0 & -- & -- \\
\hline & 15 & -2200 & 105 & 0 & -- & -- \\
\hline \multirow[t]{11}{*}{ MSPRO 2} & 2 & -5552 & 690 & 20 & 33 & 63 \\
\hline & 3 & -4422 & 1130 & 9 & -- & 56 \\
\hline & 5 & -3782 & 400 & 30 & 50 & 49 \\
\hline & 7 & -1737 & 1925 & 61 & $<10$ & 40 \\
\hline & 8 & -897 & 840 & 23 & - - & 30 \\
\hline & 9 & -272 & 625 & 36 & -- & 25 \\
\hline & 10 & 68 & 340 & 27 & -- & 22 \\
\hline & 11 & 222 & 154 & 39 & -- & 20 \\
\hline & 12 & -6242 & 1265 & 3 & -- & -- \\
\hline & 13 & -4182 & 240 & 8 & -- & -- \\
\hline & 15 & -3662 & 120 & 0 & -- & -- \\
\hline \multirow[t]{7}{*}{ MSQUO1 } & 2 & -1107 & 398 & 31 & -- & -- \\
\hline & 3 & -737 & 370 & 15 & -- & -- \\
\hline & 4 & -163 & 574 & 32 & -- & -- \\
\hline & 5 & -23 & 48 & 21 & -- & -- \\
\hline & 11 & 163 & 186 & 78 & -- & - \\
\hline & 12 & -1505 & 562 & 0 & -- & -- \\
\hline & 13 & -71 & 92 & 54 & -- & -- \\
\hline \multirow[t]{9}{*}{ MSRAO 1} & 2 & -3314 & 635 & 80 & 37 & 65 \\
\hline & 3 & -1704 & 1610 & 48 & $<10$ & 50 \\
\hline & 4 & -1494 & 210 & 88 & $<10$ & 37 \\
\hline & 5 & -434 & 595 & 56 & $<10$ & 25 \\
\hline & 6 & -94 & 245 & 37 & $<10$ & 18 \\
\hline & 12 & -3949 & 730 & 0 & -- & - \\
\hline & 13 & -1029 & 465 & 9 & -- & - \\
\hline & 14 & -339 & 95 & 0 & -- & -- \\
\hline & 15 & 426 & 520 & 9 & -- & -- \\
\hline \multirow[t]{10}{*}{$\mathrm{MSRAO} 2$} & 2 & -3175 & 740 & 66 & -- & -- \\
\hline & 3 & -2115 & 1060 & 35 & -- & -- \\
\hline & 4 & -1795 & 320 & 80 & -- & -- \\
\hline & 5 & -695 & 675 & 51 & -- & -- \\
\hline & 6 & -285 & 335 & 57 & -- & -- \\
\hline & 7 & 415 & 130 & 50 & -- & -- \\
\hline & 12 & -3915 & 670 & 0 & -- & -- \\
\hline & 13 & -1370 & 425 & 0 & -- & -- \\
\hline & 14 & -620 & 75 & 0 & -- & -- \\
\hline & 15 & 285 & 570 & 7 & -- & - \\
\hline
\end{tabular}


Table 4--Layer number; altitude of top, thickness, and sand percentage of layer; and average dissolved-solids concentration and temperature of water in layer; by log number--Continued.

\begin{tabular}{|c|c|c|c|c|c|c|}
\hline $\begin{array}{l}\text { Log } \\
\text { number }\end{array}$ & $\begin{array}{r}\text { Layer } \\
\text { number }\end{array}$ & $\begin{array}{r}\text { Alt itude } \\
\text { of top } \\
(f e e t)\end{array}$ & $\begin{array}{r}\text { Thick- } \\
\text { ness } \\
(\text { feet })\end{array}$ & $\begin{array}{r}\text { Sand } \\
\text { per- } \\
\text { centage }\end{array}$ & $\begin{array}{l}\text { Dis- } \\
\text { solved } \\
\text { solids }\end{array}$ & $\begin{array}{l}\text { Tem- } \\
\text { per- } \\
\text { ature }\end{array}$ \\
\hline \multirow[t]{9}{*}{ MSRAO 3} & 2 & -2564 & 395 & 58 & $<10$ & 41 \\
\hline & 3 & -1439 & 1125 & 26 & $<10$ & 35 \\
\hline & 4 & -1179 & 260 & 96 & $<10$ & 30 \\
\hline & 5 & -289 & 480 & 58 & $<10$ & 24 \\
\hline & 6 & 196 & 330 & 92 & $<10$ & 20 \\
\hline & 12 & -2959 & 1220 & 7 & -- & - \\
\hline & 13 & -769 & 410 & 2 & -- & -- \\
\hline & 14 & -134 & 155 & 0 & - - & -- \\
\hline & 15 & 371 & 175 & 0 & -- & -- \\
\hline \multirow[t]{9}{*}{ MSSCOI } & 2 & -1919 & 540 & 70 & $<10$ & 35 \\
\hline & 3 & -1009 & 910 & 14 & $<10$ & 29 \\
\hline & 4 & -499 & 510 & 51 & $<10$ & 23 \\
\hline & 5 & -14 & 330 & 67 & $<10$ & 18 \\
\hline & 6 & 371 & 265 & 49 & $<10$ & 15 \\
\hline & 12 & -2459 & 1040 & 3 & - - & -- \\
\hline & 13 & -344 & 155 & 0 & -- & -- \\
\hline & 14 & 106 & 120 & 8 & -- & -- \\
\hline & 15 & 411 & 40 & 0 & -- & -- \\
\hline \multirow[t]{9}{*}{ MSSCO2 } & 2 & -2052 & 540 & 70 & $<10$ & 38 \\
\hline & 3 & -952 & 1100 & 18 & $<10$ & 30 \\
\hline & 4 & -562 & 390 & 44 & $<10$ & 23 \\
\hline & 5 & -52 & 435 & 62 & $<10$ & 19 \\
\hline & 6 & 278 & 290 & 52 & $<10$ & 15 \\
\hline & 12 & -2592 & 730 & 10 & - - & -- \\
\hline & 13 & -487 & 75 & 0 & -- & -- \\
\hline & 14 & -12 & 40 & 0 & -- & -- \\
\hline & 15 & 398 & 120 & 0 & -- & -- \\
\hline \multirow[t]{10}{*}{ MSSHOI } & 2 & -3447 & 480 & 69 & 22 & 55 \\
\hline & 3 & -2437 & 1010 & 48 & 17 & 47 \\
\hline & 4 & -2162 & 275 & 84 & $<10$ & 40 \\
\hline & 5 & -927 & 750 & 75 & $<10$ & 30 \\
\hline & 6 & -237 & 590 & 36 & $<10$ & 22 \\
\hline & 11 & 103 & 150 & 88 & - & 16 \\
\hline & 12 & -3927 & 665 & 0 & -- & -- \\
\hline & 13 & -1677 & 485 & 4 & -- & -- \\
\hline & 14 & -827 & 100 & 15 & -- & -- \\
\hline & 15 & -47 & 190 & 3 & -- & -- \\
\hline \multirow[t]{9}{*}{ MSSHO2 } & 2 & -2253 & 650 & 54 & 26 & 41 \\
\hline & 3 & -1693 & 560 & 30 & $<10$ & 35 \\
\hline & 4 & -1543 & 150 & 97 & $<10$ & 32 \\
\hline & 5 & -343 & 830 & 56 & $<10$ & 24 \\
\hline & 6 & -43 & 170 & 29 & -- & 18 \\
\hline & 11 & 97 & 140 & 80 & $<10$ & 17 \\
\hline & 12 & -2903 & 615 & 0 & - - & -- \\
\hline & 13 & -1173 & 370 & 0 & -- & -- \\
\hline & 14 & -213 & 130 & 8 & - & -- \\
\hline
\end{tabular}


Table 4--Layer number; altitude of top, thickness, and sand percentage of layer; and average dissolved-solids concentration and temperature of water in layer; by log number--Continued.

\begin{tabular}{|c|c|c|c|c|c|c|}
\hline $\begin{array}{l}\text { Log } \\
\text { number }\end{array}$ & $\begin{array}{r}\text { Layer } \\
\text { number }\end{array}$ & $\begin{array}{r}\text { Alt } 1 \text { tude } \\
\text { of top } \\
(f e e t)\end{array}$ & $\begin{array}{r}\text { Thick- } \\
\text { ness } \\
(\text { feet) }\end{array}$ & $\begin{array}{r}\text { Sand } \\
\text { per- } \\
\text { centage }\end{array}$ & $\begin{array}{l}\text { Dis- } \\
\text { solved } \\
\text { solids }\end{array}$ & $\begin{array}{l}\text { Tem- } \\
\text { per- } \\
\text { ature }\end{array}$ \\
\hline \multirow[t]{11}{*}{ MSSI01 } & 2 & -4397 & 1055 & 53 & 33 & 54 \\
\hline & 3 & -2752 & 1645 & 25 & 14 & 44 \\
\hline & 4 & -2437 & 315 & 73 & $<10$ & 37 \\
\hline & 5 & -1172 & 635 & 38 & $<10$ & 29 \\
\hline & 6 & -687 & 395 & 41 & $<10$ & 25 \\
\hline & 7 & 228 & 510 & 24 & $<10$ & 18 \\
\hline & 8 & 478 & 250 & 66 & $<10$ & 15 \\
\hline & 12 & -5452 & 775 & 0 & - & -- \\
\hline & 13 & -1807 & 630 & 0 & -- & -- \\
\hline & 14 & -1082 & 90 & 6 & -- & -- \\
\hline & 15 & -282 & 405 & 0 & -- & - \\
\hline \multirow[t]{11}{*}{ MSSIO2 } & 2 & -4112 & 1290 & 57 & 54 & 50 \\
\hline & 3 & -2672 & 1440 & 37 & 28 & 41 \\
\hline & 4 & -2412 & 260 & 42 & $<10$ & 35 \\
\hline & 5 & -1132 & 650 & 37 & $<10$ & 28 \\
\hline & 6 & -682 & 350 & 63 & $<10$ & 24 \\
\hline & 7 & 148 & 520 & 40 & $<10$ & 19 \\
\hline & 8 & 351 & 203 & 53 & -- & 17 \\
\hline & 12 & -5402 & 710 & 0 & -- & - \\
\hline & 13 & -1782 & 630 & 0 & -- & - - \\
\hline & 14 & -1032 & 100 & 0 & -- & -- \\
\hline & 15 & -372 & 310 & 0 & -- & -- \\
\hline \multirow[t]{10}{*}{ MSSMOI } & 2 & -2695 & 410 & 70 & $<10$ & 53 \\
\hline & 3 & -1345 & 1350 & 26 & $<10$ & 48 \\
\hline & 4 & -935 & 410 & 22 & $<10$ & 37 \\
\hline & 5 & -320 & 505 & 79 & $<10$ & 27 \\
\hline & 6 & -10 & 225 & 67 & $<10$ & 19 \\
\hline & 7 & 425 & 170 & 28 & $<10$ & -- \\
\hline & 12 & -3105 & 1140 & 7 & -- & -- \\
\hline & 13 & -825 & 110 & 0 & -- & -- \\
\hline & 14 & -235 & 85 & 0 & -- & -- \\
\hline & 15 & 255 & 265 & 0 & -- & -- \\
\hline \multirow[t]{11}{*}{ MSSMO2 } & 2 & -3461 & 1280 & 64 & 52 & 49 \\
\hline & 3 & -2261 & 1200 & 23 & $<10$ & 41 \\
\hline & 4 & -1741 & 520 & 16 & $<10$ & 34 \\
\hline & 5 & -916 & 535 & 55 & $<10$ & 29 \\
\hline & 6 & -561 & 255 & 45 & $<10$ & 25 \\
\hline & 7 & 294 & 515 & 41 & $<10$ & 20 \\
\hline & 8 & 489 & 195 & 85 & $<10$ & 17 \\
\hline & 12 & -4741 & 750 & 0 & -- & - \\
\hline & 13 & -1451 & 290 & 10 & -- & -- \\
\hline & 14 & -816 & 100 & 15 & -- & -- \\
\hline & 15 & -221 & 340 & 3 & -- & -- \\
\hline \multirow[t]{11}{*}{ MSSMO 3} & 2 & -3782 & 850 & 39 & 34 & 52 \\
\hline & 3 & -2102 & 1680 & 26 & 17 & 44 \\
\hline & 4 & -1612 & 490 & 36 & $<10$ & 38 \\
\hline & 5 & -952 & 510 & 64 & $<10$ & 31 \\
\hline & 6 & -622 & 265 & 74 & $<10$ & 27 \\
\hline & 7 & 108 & 440 & 45 & $<10$ & 20 \\
\hline & 8 & 255 & 147 & 44 & $<10$ & 17 \\
\hline & 12 & -4632 & 620 & 0 & -- & - \\
\hline & 13 & -1462 & 150 & 0 & -- & -- \\
\hline & 14 & -887 & 65 & 0 & -- & -- \\
\hline & 15 & -332 & 290 & 3 & -- & -- \\
\hline
\end{tabular}


Table 4--Layer number; altitude of top, thickness, and sand percentage of layer; and average dissolved-solids concentration and temperature of water in layer; by log number--Continued.

\begin{tabular}{|c|c|c|c|c|c|c|}
\hline $\begin{array}{l}\text { Log } \\
\text { number }\end{array}$ & $\begin{array}{r}\text { Layer } \\
\text { number }\end{array}$ & $\begin{array}{r}\text { Altitude } \\
\text { of top } \\
(f e e t)\end{array}$ & $\begin{array}{r}\text { Thick- } \\
\text { ness } \\
(\text { feet })\end{array}$ & $\begin{array}{r}\text { Sand } \\
\text { per- } \\
\text { centage }\end{array}$ & $\begin{array}{l}\text { Dis- } \\
\text { solved } \\
\text { solids }\end{array}$ & $\begin{array}{l}\text { Tem- } \\
\text { per- } \\
\text { ature }\end{array}$ \\
\hline \multirow[t]{11}{*}{ MSSTO1 } & 2 & -4554 & 395 & 65 & 47 & 58 \\
\hline & 3 & -3119 & 1435 & 4 & -- & 51 \\
\hline & 4 & -2819 & 300 & 55 & 42 & 44 \\
\hline & 5 & -2429 & 260 & -- & -- & - \\
\hline & 7 & -994 & 1385 & 40 & $<10$ & 33 \\
\hline & 8 & -224 & 770 & 35 & $<10$ & 24 \\
\hline & 9 & 111 & 335 & 43 & $<10$ & 20 \\
\hline & 10 & 235 & 124 & 16 & -- & 18 \\
\hline & 12 & -4949 & 835 & 11 & -- & $\ldots$ \\
\hline & 13 & -2689 & 130 & 0 & -- & $\cdots$ \\
\hline & 15 & -2379 & 50 & 0 & -- & - \\
\hline \multirow[t]{11}{*}{$\mathrm{MSSTO} 2$} & 2 & -4555 & 180 & 22 & -- & 51 \\
\hline & 3 & -3205 & 1350 & 9 & 18 & 46 \\
\hline & 4 & -2855 & 350 & 54 & 29 & 41 \\
\hline & 5 & -2490 & 235 & -- & - & -- \\
\hline & 7 & -1030 & 1365 & 52 & $<10$ & 32 \\
\hline & 8 & -295 & 735 & 67 & $<10$ & 25 \\
\hline & 9 & 30 & 325 & 84 & $<10$ & 21 \\
\hline & 10 & 147 & 117 & 5 & -- & 20 \\
\hline & 12 & -4735 & 930 & 2 & -- & - \\
\hline & 13 & -2725 & 130 & 27 & -- & $\cdots$ \\
\hline & 15 & -2395 & 95 & 21 & -- & - \\
\hline \multirow[t]{7}{*}{ MSSUO 1} & 2 & -1817 & 350 & 56 & $<10$ & 30 \\
\hline & 3 & -1407 & 410 & 15 & -- & 28 \\
\hline & 4 & -652 & 755 & 66 & $<10$ & 24 \\
\hline & 5 & -37 & 550 & 45 & $<10$ & 19 \\
\hline & 11 & 133 & 170 & 89 & - & 17 \\
\hline & 12 & -2167 & 965 & 1 & -- & - \\
\hline & 13 & -587 & 65 & 0 & -- & $\ldots$ \\
\hline \multirow[t]{8}{*}{ MSSUO2 } & 2 & -2228 & 370 & 45 & $<10$ & 36 \\
\hline & 3 & -1478 & 750 & 34 & $<10$ & 31 \\
\hline & 4 & -813 & 665 & 55 & $<10$ & 25 \\
\hline & 5 & -288 & 420 & 48 & $<10$ & 20 \\
\hline & 6 & 12 & 300 & 82 & $<10$ & 17 \\
\hline & 11 & 114 & 102 & 83 & -- & 15 \\
\hline & 12 & -2598 & 980 & 0 & -- & - \\
\hline & 13 & -708 & 105 & 0 & -- & - \\
\hline \multirow[t]{7}{*}{ MSSUO3 } & 2 & -1931 & 320 & 50 & $<10$ & 32 \\
\hline & 3 & -1221 & 710 & 30 & $<10$ & 28 \\
\hline & 4 & -571 & 650 & 36 & $<10$ & 23 \\
\hline & 5 & -46 & 465 & 33 & -- & 19 \\
\hline & 11 & 114 & 160 & 89 & -- & 16 \\
\hline & 12 & -2251 & 960 & 1 & -- & - \\
\hline & 13 & -511 & 60 & 0 & -- & -- \\
\hline
\end{tabular}


Table 4--Layer number; altitude of top, thickness, and sand percentage of layer; and average dissolved-solids concentration and temperature of water in layer; by log number--Continued.

\begin{tabular}{|c|c|c|c|c|c|c|}
\hline $\begin{array}{l}\text { Log } \\
\text { number }\end{array}$ & $\begin{array}{r}\text { Layer } \\
\text { number }\end{array}$ & $\begin{array}{r}\text { Altitude } \\
\text { of top } \\
\text { (feet) }\end{array}$ & $\begin{array}{r}\text { Thick- } \\
\text { ness } \\
\text { (feet) }\end{array}$ & $\begin{array}{r}\text { Sand } \\
\text { per- } \\
\text { centage }\end{array}$ & $\begin{array}{l}\text { Dis- } \\
\text { solved } \\
\text { solids }\end{array}$ & $\begin{array}{l}\text { Tem- } \\
\text { per- } \\
\text { ature }\end{array}$ \\
\hline \multirow[t]{7}{*}{ MSTAO 1} & 2 & -1494 & 360 & 49 & -- & $\cdots$ \\
\hline & 3 & -1124 & 370 & 24 & $\ldots$ & $\ldots$ \\
\hline & 4 & -429 & 695 & 44 & $\cdots$ & $\cdots$ \\
\hline & 5 & -54 & 330 & 65 & -- & $\cdots$ \\
\hline & 11 & 126 & 180 & 89 & -- & $\cdots$ \\
\hline & 12 & -1854 & 900 & 2 & $\ldots$ & $\cdots$ \\
\hline & 13 & -384 & 45 & 0 & -- & $\cdots$ \\
\hline \multirow[t]{6}{*}{ MSTAO 2} & 2 & -722 & 305 & 51 & -- & -- \\
\hline & 3 & -287 & 435 & 11 & -- & -- \\
\hline & 4 & 73 & 360 & 32 & -- & $\ldots$ \\
\hline & 5 & 348 & 210 & 45 & $\ldots$ & - \\
\hline & 12 & -1027 & 695 & 0 & -- & $\ldots$ \\
\hline & 13 & 138 & 65 & 0 & -- & $\cdots$ \\
\hline \multirow[t]{6}{*}{ MSTAO3 } & 2 & -553 & 375 & 41 & $<10$ & 22 \\
\hline & 3 & -253 & 300 & 33 & $<10$ & 20 \\
\hline & 4 & 2 & 255 & 59 & $<10$ & 18 \\
\hline & 5 & 322 & 190 & 42 & - & 16 \\
\hline & 12 & -928 & 710 & 0 & -- & - \\
\hline & 13 & 132 & 130 & 8 & -- & $\cdots$ \\
\hline \multirow[t]{7}{*}{$\mathrm{MSTAO} 4$} & 2 & -1357 & 275 & 29 & -- & $\cdots$ \\
\hline & 3 & -757 & 600 & 22 & - & -- \\
\hline & 4 & -187 & 570 & 51 & $\ldots$ & - \\
\hline & 5 & -37 & 100 & 99 & -- & - \\
\hline & 11 & 136 & 173 & 89 & -- & -- \\
\hline & 12 & -1632 & 795 & 0 & -- & -- \\
\hline & 13 & -137 & 50 & 20 & -- & $\cdots$ \\
\hline \multirow[t]{6}{*}{ MSTEO 1} & 2 & -1091 & 375 & 52 & $<10$ & 28 \\
\hline & 3 & -801 & 290 & 69 & $<10$ & 24 \\
\hline & 4 & -131 & 670 & 84 & $<10$ & 20 \\
\hline & 5 & 139 & 270 & 7 & - & 16 \\
\hline & 12 & -1466 & 665 & 0 & - & $\ldots$ \\
\hline & 14 & 239 & 100 & 0 & -- & $\cdots$ \\
\hline \multirow[t]{6}{*}{ MSTEO2 } & 1 & -1637 & 75 & 99 & $<10$ & 29 \\
\hline & 2 & -777 & 265 & 53 & $<10$ & 24 \\
\hline & 3 & -447 & 330 & 15 & -- & 22 \\
\hline & 4 & 53 & 500 & 63 & $<10$ & 19 \\
\hline & 5 & 263 & 210 & 0 & - & 16 \\
\hline & 12 & -1042 & 595 & 0 & -- & $\ldots$ \\
\hline \multirow[t]{4}{*}{ MSTIO 1} & 1 & -380 & 255 & 67 & $<10$ & 32 \\
\hline & 2 & 285 & 260 & 75 & $<10$ & 15 \\
\hline & 3 & 506 & 221 & 11 & $<10$ & -- \\
\hline & 12 & 25 & 405 & 0 & - & - \\
\hline
\end{tabular}


Table 4--Layer number; altitude of top, thickness, and sand percentage of layer; and average dissolved-solids concentration and temperature of water in layer; by log number--Continued.

\begin{tabular}{|c|c|c|c|c|c|c|}
\hline $\begin{array}{l}\text { Log } \\
\text { number }\end{array}$ & $\begin{array}{r}\text { Layer } \\
\text { number }\end{array}$ & $\begin{array}{r}\text { Altitude } \\
\text { of top } \\
\text { (feet) }\end{array}$ & $\begin{array}{r}\text { Thlck- } \\
\text { ness } \\
\text { (feet) }\end{array}$ & $\begin{array}{r}\text { Sand } \\
\text { per- } \\
\text { centage }\end{array}$ & $\begin{array}{l}\text { Dis- } \\
\text { solved } \\
\text { solids }\end{array}$ & $\begin{array}{l}\text { Tem- } \\
\text { per- } \\
\text { ature }\end{array}$ \\
\hline \multirow[t]{7}{*}{ MSTUOI } & 2 & -1377 & 430 & 91 & $<10$ & 27 \\
\hline & 3 & -1077 & 300 & 7 & -- & 25 \\
\hline & 4 & -397 & 680 & 82 & - & $\ldots$ \\
\hline & 5 & -47 & 350 & -- & $<10$ & 22 \\
\hline & 11 & 193 & 160 & 88 & - & 17 \\
\hline & 12 & -1807 & 940 & 0 & -- & $\ldots$ \\
\hline & 14 & 33 & 80 & 0 & - & $\ldots$ \\
\hline \multirow[t]{7}{*}{ MSTUO2 } & 2 & -1379 & 280 & 99 & $<10$ & 28 \\
\hline & 3 & -989 & 390 & 6 & - - & 25 \\
\hline & 4 & -749 & 240 & 94 & $<10$ & 23 \\
\hline & 5 & 71 & 480 & 78 & $<10$ & 18 \\
\hline & 11 & 213 & 142 & 88 & - & 15 \\
\hline & 12 & -1659 & 970 & 0 & $\ldots$ & $\ldots$ \\
\hline & 13 & -409 & 340 & 15 & -- & -- \\
\hline \multirow[t]{2}{*}{ MSUNO 1} & 1 & -8 & 110 & 99 & -- & - \\
\hline & 12 & 390 & 398 & 1 & -- & -- \\
\hline \multirow[t]{9}{*}{ MSWAO 1} & 2 & -5526 & 920 & 42 & 65 & 66 \\
\hline & 3 & -3376 & 2150 & 30 & 24 & 54 \\
\hline & 5 & -2266 & 420 & -- & -- & - \\
\hline & 7 & -566 & 1440 & 30 & $<10$ & 29 \\
\hline & 8 & 184 & 750 & 74 & $<10$ & 21 \\
\hline & 9 & 354 & 170 & 68 & $<10$ & 17 \\
\hline & 12 & -6446 & 930 & 1 & - & $\ldots$ \\
\hline & 13 & -2686 & 690 & 0 & -- & $\ldots$ \\
\hline & 15 & -2006 & 260 & 0 & -- & - \\
\hline \multirow[t]{12}{*}{ MSWA 02} & 2 & -5713 & 900 & 26 & 121 & 72 \\
\hline & 3 & -3688 & 2025 & 16 & 78 & 60 \\
\hline & 5 & -2903 & 200 & -- & -- & - \\
\hline & 6 & -2783 & 30 & 50 & - - & 46 \\
\hline & 7 & -1088 & 1535 & 31 & $<10$ & 39 \\
\hline & 8 & -173 & 915 & 35 & $<10$ & 25 \\
\hline & 9 & 227 & 400 & 99 & $<10$ & 18 \\
\hline & 10 & 387 & 160 & 82 & $<10$ & 15 \\
\hline & 12 & -6613 & 860 & 0 & -- & - \\
\hline & 13 & -3103 & 585 & 3 & -- & -- \\
\hline & 14 & -2813 & 90 & 0 & -- & - \\
\hline & 15 & -2623 & 160 & 0 & -- & - \\
\hline \multirow[t]{3}{*}{ MSWEO 1} & 2 & 202 & 90 & 83 & $<10$ & 18 \\
\hline & 3 & 302 & 100 & 18 & -- & 17 \\
\hline & 12 & 112 & 650 & 0 & -- & -- \\
\hline \multirow[t]{2}{*}{ MSWEO2 } & 2 & 520 & 40 & 50 & -- & - \\
\hline & 12 & 480 & 550 & 0 & -- & - \\
\hline
\end{tabular}


Table 4--Layer number; altitude of top, thickness, and sand percentage of layer; and average dissolved-solids concentration and temperature of water in layer; by log number--Continued.

\begin{tabular}{|c|c|c|c|c|c|c|}
\hline $\begin{array}{l}\log \\
\text { number }\end{array}$ & $\begin{array}{r}\text { Layer } \\
\text { number }\end{array}$ & $\begin{array}{r}\text { Altitude } \\
\text { of top } \\
\text { (feet) }\end{array}$ & $\begin{array}{r}\text { Thick- } \\
\text { ness } \\
\text { (feet) }\end{array}$ & $\begin{array}{r}\text { Sand } \\
\text { per- } \\
\text { cent2ge }\end{array}$ & $\begin{array}{l}\text { Dis- } \\
\text { solved } \\
\text { solids }\end{array}$ & $\begin{array}{l}\text { Tem- } \\
\text { per- } \\
\text { ature }\end{array}$ \\
\hline \multirow[t]{3}{*}{ MSWIOI } & 8 & -410 & -- & -- & -- & -. \\
\hline & 9 & 25 & 435 & 13 & -- & -- \\
\hline & 10 & 364 & 339 & 33 & -- & -- \\
\hline \multirow[t]{12}{*}{ MSWIO 2} & 2 & -7844 & 480 & 39 & 25 & 82 \\
\hline & 3 & -4674 & 3170 & 36 & 58 & 70 \\
\hline & 5 & -3364 & 940 & 41 & 60 & 50 \\
\hline & 6 & -2909 & 285 & 46 & 35 & 44 \\
\hline & 7 & -1169 & 1295 & 33 & 40 & 33 \\
\hline & 8 & -294 & 875 & 56 & $\ldots$ & 24 \\
\hline & 9 & 6 & 300 & 55 & -- & 20 \\
\hline & 10 & 203 & 197 & 3 & -- & 17 \\
\hline & 12 & -8324 & 1040 & 0 & -- & -- \\
\hline & 13 & -4304 & 370 & 0 & -- & -- \\
\hline & 14 & -3194 & 170 & 12 & -- & -. \\
\hline & 15 & -2464 & 445 & 0 & -- & -- \\
\hline \multirow[t]{10}{*}{ MSWIO 3} & 3 & -4673 & -- & -- & -- & - \\
\hline & 5 & -3403 & 920 & 30 & -- & 42 \\
\hline & 6 & -2983 & 340 & 41 & 19 & 38 \\
\hline & 7 & -1188 & 1305 & 42 & 15 & 30 \\
\hline & 8 & -313 & 875 & 57 & $<10$ & 24 \\
\hline & 9 & -13 & 300 & 55 & -- & 20 \\
\hline & 10 & 187 & 200 & 3 & -- & 19 \\
\hline & 13 & -4323 & 350 & 1 & -- & -- \\
\hline & 14 & -3323 & 80 & 0 & -- & -- \\
\hline & 15 & -2493 & 490 & 0 & -- & -- \\
\hline \multirow[t]{12}{*}{ MSWIO5 } & 2 & -9138 & 750 & 33 & 34 & 80 \\
\hline & 3 & -6418 & 2720 & 23 & 39 & 69 \\
\hline & 5 & -5248 & 800 & 28 & 49 & 56 \\
\hline & 6 & -4838 & 140 & 39 & -- & 51 \\
\hline & 7 & -2083 & 2345 & 39 & 62 & 40 \\
\hline & 8 & -588 & 1495 & 51 & $<10$ & 28 \\
\hline & 9 & 12 & 600 & 21 & -- & 21 \\
\hline & 10 & 346 & 334 & 17 & -- & 18 \\
\hline & 12 & -9888 & 1050 & 0 & -- & -. \\
\hline & 13 & -6048 & 370 & 0 & -- & -- \\
\hline & 14 & -4978 & 270 & 9 & -- & -- \\
\hline & 15 & -4428 & 410 & 0 & -- & - \\
\hline \multirow[t]{2}{*}{ MSWNO1 } & 2 & 468 & 188 & 60 & -- & 19 \\
\hline & 12 & 280 & 535 & 0 & -- & $\cdots$ \\
\hline \multirow[t]{10}{*}{ MSWRO 1} & 2 & -4719 & 795 & 66 & 52 & 55 \\
\hline & 3 & -3314 & 1405 & 51 & 45 & 47 \\
\hline & 4 & -2909 & 405 & 98 & $<10$ & 41 \\
\hline & 5 & -1364 & 960 & 61 & $<10$ & 33 \\
\hline & 6 & -614 & 520 & 57 & $<10$ & 26 \\
\hline & 7 & 353 & 442 & 42 & $<10$ & 19 \\
\hline & 12 & -5514 & 935 & 0 & -- & - \\
\hline & 13 & -2324 & 585 & 0 & -- & -- \\
\hline & 14 & -1134 & 230 & 0 & -- & -- \\
\hline & 15 & -89 & 525 & 0 & -- & -- \\
\hline
\end{tabular}


Table 4--Layer number; altitude of top, thickness, and sand percentage of layer; and average dissolved-solids concentration and temperature of water in layer; by log number--Continued.

\begin{tabular}{|c|c|c|c|c|c|c|}
\hline $\begin{array}{l}\text { Log } \\
\text { number }\end{array}$ & $\begin{array}{r}\text { Layer } \\
\text { number }\end{array}$ & $\begin{array}{l}\text { Altitude } \\
\text { of top } \\
(f \in e t)\end{array}$ & $\begin{array}{r}\text { Thick- } \\
\text { ness } \\
(\text { feet })\end{array}$ & $\begin{array}{r}\text { Sand } \\
\text { per- } \\
\text { centage }\end{array}$ & $\begin{array}{l}\text { Dis- } \\
\text { solved } \\
\text { solids }\end{array}$ & $\begin{array}{r}\text { Tem- } \\
\text { per- } \\
\text { ature }\end{array}$ \\
\hline \multirow[t]{10}{*}{ MSWRO 2} & 2 & -5313 & 730 & 58 & 57 & 68 \\
\hline & 3 & -3353 & 1960 & 44 & 33 & 63 \\
\hline & 4 & -3098 & 255 & 92 & $<10$ & 48 \\
\hline & 5 & -1503 & 1030 & 43 & $<10$ & 36 \\
\hline & 6 & -688 & 650 & 50 & $<10$ & 27 \\
\hline & 7 & 257 & 505 & 27 & $<10$ & 17 \\
\hline & 12 & -6043 & 970 & 0 & - & $\ldots$ \\
\hline & 13 & -2533 & 565 & 0 & -- & $\ldots$ \\
\hline & 14 & -1338 & 165 & 0 & -- & -- \\
\hline & 15 & -248 & 440 & 0 & -- & -- \\
\hline \multirow[t]{10}{*}{ MSWSOI } & 2 & -2765 & 370 & 70 & -- & -- \\
\hline & 3 & -1965 & 800 & 22 & -- & -- \\
\hline & 4 & -1425 & 540 & 51 & -- & -- \\
\hline & 5 & -665 & 570 & 56 & -- & -- \\
\hline & 6 & -245 & 330 & 61 & -- & -- \\
\hline & 11 & 135 & 90 & 50 & -- & - \\
\hline & 12 & -3135 & 710 & 0 & -- & - \\
\hline & 13 & -1235 & 190 & 11 & -- & -- \\
\hline & 14 & -575 & 90 & 22 & -- & $\ldots$ \\
\hline & 15 & 45 & 290 & 3 & -- & -- \\
\hline \multirow[t]{10}{*}{ MSWSO 2} & 2 & -2356 & 410 & 59 & 14 & 39 \\
\hline & 3 & -1826 & 530 & 44 & $<10$ & 35 \\
\hline & 4 & -1656 & 170 & 99 & $<10$ & 31 \\
\hline & 5 & -651 & 485 & 41 & $<10$ & 24 \\
\hline & 6 & -216 & 400 & 27 & $<10$ & 19 \\
\hline & 11 & 107 & 153 & 88 & $<10$ & 15 \\
\hline & 12 & -2766 & 770 & 0 & -- & $\ldots$ \\
\hline & 13 & -1136 & 520 & 8 & -- & - \\
\hline & 14 & -616 & 35 & 0 & -- & - \\
\hline & 15 & -46 & 170 & 6 & -- & -- \\
\hline \multirow[t]{10}{*}{ MSWSO 3} & 2 & -2268 & 340 & 84 & 22 & 37 \\
\hline & 3 & -1568 & 700 & 32 & $<10$ & 32 \\
\hline & 4 & -1488 & 80 & 88 & $<10$ & 29 \\
\hline & 5 & -518 & 570 & 34 & $<10$ & 23 \\
\hline & 6 & -128 & 330 & 45 & -- & 18 \\
\hline & 11 & 110 & 88 & 70 & -- & 15 \\
\hline & 12 & -2608 & 490 & 0 & -- & - \\
\hline & 13 & -1088 & 400 & 0 & -- & -- \\
\hline & 14 & -458 & 60 & 0 & -- & - \\
\hline & 15 & 22 & 150 & 7 & -- & -- \\
\hline \multirow[t]{9}{*}{$\mathrm{MSWSO}_{4}$} & 2 & -2353 & 335 & 60 & $<10$ & 38 \\
\hline & 3 & -1568 & 785 & 22 & $<10$ & 33 \\
\hline & 4 & -1398 & 170 & 99 & $<10$ & 29 \\
\hline & 5 & -408 & 550 & 66 & $<10$ & 22 \\
\hline & 6 & -108 & 300 & 22 & $<10$ & 18 \\
\hline & 11 & 102 & 170 & 78 & -- & 15 \\
\hline & 12 & -2688 & 710 & 0 & -- & -- \\
\hline & 13 & -958 & 440 & 5 & -- & -- \\
\hline & 15 & -68 & 40 & 0 & -- & -- \\
\hline
\end{tabular}


Table 4--Layer number; altitude of top, thickness, and sand percentage of layer; and average dissolved-solids concentration and temperature of water in layer; by log number--Continued.

\begin{tabular}{|c|c|c|c|c|c|c|}
\hline $\begin{array}{l}\text { Log } \\
\text { number }\end{array}$ & $\begin{array}{r}\text { Layer } \\
\text { number }\end{array}$ & $\begin{array}{r}\text { Altitude } \\
\text { of top } \\
\text { (feet) }\end{array}$ & $\begin{array}{r}\text { Thick- } \\
\text { ness } \\
\text { (feet) }\end{array}$ & $\begin{array}{r}\text { Sand } \\
\text { per- } \\
\text { centage }\end{array}$ & $\begin{array}{l}\text { Dis- } \\
\text { solved } \\
\text { solids }\end{array}$ & $\begin{array}{l}\text { Tem- } \\
\text { per- } \\
\text { ature }\end{array}$ \\
\hline \multirow[t]{10}{*}{ MSWYO 1} & 2 & -3110 & 720 & 48 & 18 & 48 \\
\hline & 3 & -1490 & 1620 & 20 & $<10$ & 39 \\
\hline & 4 & -1260 & 230 & 76 & $<10$ & 31 \\
\hline & 5 & -935 & 65 & 85 & -- & 27 \\
\hline & 6 & -750 & 120 & 79 & -- & 26 \\
\hline & 7 & 170 & 720 & 32 & - - & 21 \\
\hline & 12 & -3830 & 660 & 0 & - & -- \\
\hline & 13 & -1000 & 260 & 0 & -- & -- \\
\hline & 14 & -870 & 65 & 0 & - & -- \\
\hline & 15 & -550 & 200 & 50 & -- & -- \\
\hline \multirow[t]{10}{*}{ MSWYO 2} & 2 & -2562 & 665 & 42 & 10 & 44 \\
\hline & 3 & -1067 & 1495 & 14 & $<10$ & 38 \\
\hline & 4 & -867 & 200 & 27 & -- & 29 \\
\hline & 5 & -557 & 60 & 83 & $<10$ & 25 \\
\hline & 6 & -257 & 240 & 29 & $<10$ & 23 \\
\hline & 7 & 163 & 265 & 42 & $<10$ & 18 \\
\hline & 12 & -3227 & 610 & 0 & -- & - \\
\hline & 13 & -617 & 250 & 12 & -- & -- \\
\hline & 14 & -497 & 60 & 0 & -- & -- \\
\hline & 15 & -102 & 155 & 0 & -- & -- \\
\hline \multirow[t]{10}{*}{ MSWYO 3} & 2 & -2895 & 810 & 46 & 23 & 45 \\
\hline & 3 & -1465 & 1430 & 20 & 16 & 36 \\
\hline & 4 & -1050 & 415 & 20 & $<10$ & 29 \\
\hline & 5 & -785 & 75 & 99 & $<10$ & 26 \\
\hline & 6 & -515 & 220 & 20 & -- & 24 \\
\hline & 7 & 277 & 612 & 71 & $<10$ & 20 \\
\hline & 12 & -3705 & 650 & 0 & -- & -- \\
\hline & 13 & -860 & 190 & 0 & -- & -- \\
\hline & 14 & -735 & 50 & 0 & -- & -- \\
\hline & 15 & -335 & 180 & 0 & -- & -- \\
\hline \multirow[t]{10}{*}{ MSWYO 4} & 2 & -2432 & 715 & 47 & $<10$ & 38 \\
\hline & 3 & -1112 & 1320 & 26 & $<10$ & 32 \\
\hline & 4 & -812 & 300 & 25 & $<10$ & 26 \\
\hline & 5 & -462 & 210 & 50 & $<10$ & 24 \\
\hline & 6 & -267 & 125 & 48 & $<10$ & 22 \\
\hline & 7 & 298 & 330 & 38 & -- & 19 \\
\hline & 12 & -3147 & 635 & 0 & $\ldots$ & $\ldots$ \\
\hline & 13 & -672 & 140 & 0 & - & -- \\
\hline & 14 & -392 & 70 & 0 & -- & -- \\
\hline & 15 & -32 & 235 & 0 & -- & -- \\
\hline \multirow[t]{5}{*}{ MSYAOI } & 2 & -471 & 295 & 64 & $<10$ & 24 \\
\hline & 3 & -121 & 350 & 49 & $<10$ & 21 \\
\hline & 4 & 164 & 285 & 60 & $<10$ & 17 \\
\hline & 5 & 334 & 170 & 43 & $<10$ & 15 \\
\hline & 12 & -766 & 585 & 0 & -- & - \\
\hline
\end{tabular}


Table 4--Layer number; altitude of top, thickness, and sand percentage of layer; and average dissolved-solids concentration and temperature of water in layer; by log number--Continued.

\begin{tabular}{|c|c|c|c|c|c|c|}
\hline $\begin{array}{l}\text { Log } \\
\text { number }\end{array}$ & $\begin{array}{r}\text { Layer } \\
\text { number }\end{array}$ & $\begin{array}{r}\text { Alt itude } \\
\text { of top } \\
\text { (feet) }\end{array}$ & $\begin{array}{r}\text { Thlck- } \\
\text { ness } \\
\text { (feet) }\end{array}$ & $\begin{array}{r}\text { Sand } \\
\text { per- } \\
\text { centage }\end{array}$ & $\begin{array}{l}\text { Dis- } \\
\text { solved } \\
\text { solids }\end{array}$ & $\begin{array}{l}\text { Tem- } \\
\text { per- } \\
\text { ature }\end{array}$ \\
\hline \multirow[t]{4}{*}{$\mathrm{MSYAO} 2$} & 2 & -212 & 295 & 44 & -- & -- \\
\hline & 3 & 98 & 310 & 42 & -- & -- \\
\hline & 4 & 268 & 170 & 67 & -- & -- \\
\hline & 12 & -507 & 550 & 0 & -- & -- \\
\hline \multirow[t]{10}{*}{ MSYZOI } & 2 & -2779 & 780 & 47 & -- & -- \\
\hline & 3 & -1919 & 860 & 21 & -- & -- \\
\hline & 4 & -1619 & 300 & 53 & -- & -- \\
\hline & 5 & -609 & 610 & 51 & -- & -- \\
\hline & 6 & -49 & 360 & 33 & -- & -- \\
\hline & 11 & 261 & 90 & 80 & -- & -- \\
\hline & 12 & -3559 & 985 & 0 & -- & -- \\
\hline & 13 & -1219 & 400 & 6 & -- & -- \\
\hline & 14 & -409 & 200 & 10 & -- & - \\
\hline & 15 & 171 & 220 & 0 & -- & -- \\
\hline \multirow[t]{9}{*}{ MSYZO2 } & 2 & -3071 & 810 & 60 & $<10$ & 45 \\
\hline & 3 & -2066 & 1005 & 38 & $<10$ & 38 \\
\hline & 4 & -1361 & 705 & 62 & $<10$ & 31 \\
\hline & 5 & -556 & 535 & 87 & $<10$ & 24 \\
\hline & 6 & -56 & 440 & 76 & $<10$ & 20 \\
\hline & 12 & -3881 & 875 & 0 & -- & -- \\
\hline & 13 & -1091 & 270 & 13 & -- & - \\
\hline & 14 & -496 & 60 & 8 & -- & -- \\
\hline & 15 & 354 & 410 & 2 & -- & -- \\
\hline \multirow[t]{3}{*}{ INCKOI } & 4 & 40 & -- & -- & -- & -- \\
\hline & 5 & 262 & 222 & 87 & -- & -- \\
\hline & 14 & 350 & 88 & 2 & -- & -- \\
\hline \multirow[t]{3}{*}{$\mathrm{TNCRO} 2$} & 5 & 84 & -- & -- & -- & -- \\
\hline & 6 & 368 & 208 & 33 & -- & -- \\
\hline & 14 & 160 & 76 & 0 & -- & -- \\
\hline \multirow[t]{4}{*}{ TNCRO1 } & 1 & -162 & 372 & 76 & -- & -- \\
\hline & 2 & 222 & 98 & 99 & -- & -- \\
\hline & 4 & 422 & 200 & 78 & -- & -- \\
\hline & 12 & 124 & 286 & 28 & -- & -- \\
\hline \multirow[t]{4}{*}{ INCRO 2} & 2 & 390 & 31 & 99 & -- & -- \\
\hline & 3 & 425 & 35 & 34 & -- & -- \\
\hline & 4 & 510 & 85 & 59 & -- & -- \\
\hline & 12 & 359 & -- & -- & -- & -- \\
\hline \multirow[t]{4}{*}{ TNCRO3 } & 1 & 415 & -- & -- & -- & -- \\
\hline & 2 & 526 & 31 & 99 & -- & -- \\
\hline & 3 & 630 & 104 & 57 & -- & -- \\
\hline & 12 & 495 & 80 & 0 & -- & -- \\
\hline
\end{tabular}


Table 4--Layer number; altitude of top, thickness, and sand percentage of layer; and average dissolved-solids concentration and temperature of water in layer; by log number--Continued.

\begin{tabular}{|c|c|c|c|c|c|c|}
\hline $\begin{array}{l}\text { L०g } \\
\text { number }\end{array}$ & $\begin{array}{r}\text { Layer } \\
\text { number }\end{array}$ & $\begin{array}{r}\text { Altitude } \\
\text { of top } \\
\text { (feet) }\end{array}$ & $\begin{array}{c}\text { Thick- } \\
\text { ness } \\
\text { (feet) }\end{array}$ & $\begin{array}{r}\text { Sand } \\
\text { per- } \\
\text { centage }\end{array}$ & $\begin{array}{l}\text { Dis- } \\
\text { solved } \\
\text { solids }\end{array}$ & $\begin{array}{l}\text { Tem- } \\
\text { per- } \\
\text { ature }\end{array}$ \\
\hline \multirow[t]{9}{*}{ TNDYOI } & 1 & -1828 & -- & -- & -- & -- \\
\hline & 2 & -1008 & 260 & 99 & -- & - \\
\hline & 3 & -778 & 230 & 17 & -- & -- \\
\hline & 4 & -473 & 305 & 53 & -- & - \\
\hline & 5 & -293 & 180 & 83 & -- & - \\
\hline & 6 & 197 & 197 & -- & -- & -- \\
\hline & 11 & 258 & 61 & -- & -- & -- \\
\hline & 12 & -1268 & 560 & 13 & -- & - \\
\hline & 14 & 0 & 293 & 20 & -- & -- \\
\hline \multirow[t]{3}{*}{ TNDY 02} & 5 & -128 & -- & -- & -- & -- \\
\hline & 6 & 291 & 359 & 79 & -- & -- \\
\hline & 14 & -68 & 60 & 0 & -- & -- \\
\hline \multirow[t]{8}{*}{ T NF AOI } & 1 & -1331 & 366 & 95 & -- & -- \\
\hline & 2 & -584 & 149 & 99 & -- & -- \\
\hline & 3 & -327 & 257 & 19 & $\cdots$ & -- \\
\hline & 4 & -4 & 323 & 99 & -- & -- \\
\hline & 5 & 199 & 203 & 85 & -- & -- \\
\hline & 6 & 318 & 42 & -- & -- & -- \\
\hline & 12 & -733 & 598 & 3 & -- & -- \\
\hline & 14 & 276 & 77 & -- & -- & -- \\
\hline \multirow[t]{5}{*}{ TNFA 02} & 1 & -399 & 360 & 96 & $<10$ & 28 \\
\hline & 2 & 50 & 62 & 99 & $<10$ & 19 \\
\hline & 3 & 265 & 215 & 61 & $<10$ & 17 \\
\hline & 4 & 381 & 116 & -- & -- & 15 \\
\hline & 12 & -12 & 387 & 4 & -- & - \\
\hline \multirow[t]{6}{*}{ TNFAO3 } & 1 & -748 & 360 & 95 & -- & -- \\
\hline & 2 & -203 & 150 & 99 & -- & -- \\
\hline & 3 & -43 & 160 & 51 & -- & -- \\
\hline & 4 & 257 & 300 & 96 & -- & -- \\
\hline & 5 & 420 & 163 & -- & -- & -- \\
\hline & 12 & -353 & 395 & 0 & -- & - \\
\hline \multirow[t]{6}{*}{ TNFAO 4} & 1 & -1061 & -- & -- & -- & -- \\
\hline & 2 & -424 & 79 & 99 & $<10$ & 22 \\
\hline & 3 & -157 & 267 & 11 & -- & 20 \\
\hline & 4 & 148 & 305 & 99 & -- & 18 \\
\hline & 5 & 431 & 283 & 41 & -- & 16 \\
\hline & 12 & -503 & 558 & 8 & -- & - \\
\hline \multirow[t]{7}{*}{ TNFAOS } & 1 & -1014 & -- & -- & -- & -- \\
\hline & 2 & -339 & 161 & 98 & $<10$ & 19 \\
\hline & 3 & -192 & 147 & 43 & $<10$ & 18 \\
\hline & 4 & 158 & 350 & 96 & -- & 17 \\
\hline & 5 & 298 & 140 & 89 & -- & 17 \\
\hline & 12 & -500 & 514 & 6 & -- & -- \\
\hline & 14 & 356 & 58 & -- & -- & -- \\
\hline
\end{tabular}


Table 4--Layer number; altitude of top, thickness, and sand percentage of layer; and average dissolved-solids concentration and temperature of water in layer; by log number--Continued.

\begin{tabular}{|c|c|c|c|c|c|c|}
\hline $\begin{array}{l}\text { Log } \\
\text { number }\end{array}$ & $\begin{array}{r}\text { Layer } \\
\text { number }\end{array}$ & $\begin{array}{r}\text { Altitude } \\
\text { of top } \\
(\text { feet) }\end{array}$ & $\begin{array}{r}\text { Thick- } \\
\text { ness } \\
(\text { feet) }\end{array}$ & $\begin{array}{r}\text { Sand } \\
\text { per- } \\
\text { centage }\end{array}$ & $\begin{array}{l}\text { Dis- } \\
\text { solved } \\
\text { solids }\end{array}$ & $\begin{array}{r}\text { Tem- } \\
\text { per- } \\
\text { ature }\end{array}$ \\
\hline \multirow[t]{3}{*}{ TNFAO 6} & 3 & -66 & -- & -- & -- & -- \\
\hline & 4 & 226 & 292 & 99 & -- & - \\
\hline & 5 & 395 & 169 & 61 & -- & -- \\
\hline \multirow[t]{7}{*}{ TNGBO 1} & 1 & -1058 & 371 & 82 & -- & -- \\
\hline & 2 & -490 & 163 & 88 & -- & -- \\
\hline & 3 & -416 & 74 & 27 & -- & -- \\
\hline & 4 & -20 & 396 & -- & -- & -- \\
\hline & 5 & 218 & 238 & -- & -- & - \\
\hline & 12 & -653 & 405 & 29 & -- & -- \\
\hline & 14 & 316 & 98 & -- & -- & -- \\
\hline \multirow[t]{4}{*}{ TNHNO 1} & 2 & 474 & 114 & 67 & -- & - \\
\hline & 3 & 491 & 17 & 0 & -- & -- \\
\hline & 4 & 570 & 79 & 70 & -- & -- \\
\hline & 12 & 360 & -- & -- & -- & -- \\
\hline \multirow[t]{4}{*}{ TNHNO2 } & 2 & 419 & 99 & 99 & -- & - \\
\hline & 3 & 475 & 56 & 99 & -- & -- \\
\hline & 4 & 605 & 130 & 26 & -- & -- \\
\hline & 12 & 320 & -- & -- & -- & -- \\
\hline \multirow[t]{3}{*}{ TNENO3 } & 1 & 401 & 181 & 96 & -- & -- \\
\hline & 2 & 570 & 21 & 48 & -- & - \\
\hline & 12 & 549 & 148 & 0 & -- & -- \\
\hline \multirow[t]{3}{*}{ TNHNO4 } & 1 & 405 & 230 & 91 & -- & -- \\
\hline & 2 & 525 & 40 & 72 & -- & -- \\
\hline & 12 & 485 & 80 & 0 & -- & -- \\
\hline \multirow[t]{4}{*}{ TNHRO 1} & 2 & 225 & 60 & 99 & -- & - \\
\hline & 3 & 363 & 138 & 0 & -- & -- \\
\hline & 4 & 431 & 68 & 64 & -- & -- \\
\hline & 12 & 165 & -- & -- & -- & -- \\
\hline \multirow[t]{4}{*}{ TNHRO 2} & 2 & 296 & 43 & 99 & -- & -- \\
\hline & 3 & 418 & 122 & 80 & -- & -- \\
\hline & 4 & 500 & 82 & 75 & -- & -- \\
\hline & 12 & 253 & -- & -- & -- & -- \\
\hline \multirow[t]{4}{*}{ TNHRO 3} & 1 & 253 & -- & -- & -- & -- \\
\hline & 2 & 538 & 54 & 99 & -- & -- \\
\hline & 3 & 570 & 32 & -- & -- & -- \\
\hline & 12 & 484 & 231 & 13 & -- & - \\
\hline \multirow[t]{2}{*}{ TNHRO 4} & 1 & 306 & 290 & 99 & -- & -- \\
\hline & 12 & 433 & 127 & -- & -- & -- \\
\hline
\end{tabular}


Table 4--Layer number; altitude of top, thickness, and sand percentage of layer; and average dissolved-solids concentration and temperature of water in layer; by log number--Continued.

\begin{tabular}{|c|c|c|c|c|c|c|}
\hline $\begin{array}{l}\log \\
\text { number }\end{array}$ & $\begin{array}{r}\text { Layer } \\
\text { number }\end{array}$ & $\begin{array}{r}\text { Altitude } \\
\text { of top } \\
\text { (feet) }\end{array}$ & $\begin{array}{r}\text { Thick- } \\
\text { ness } \\
\text { (feet) }\end{array}$ & $\begin{array}{r}\text { Sand } \\
\text { per- } \\
\text { centage }\end{array}$ & $\begin{array}{l}\text { Dis- } \\
\text { solved } \\
\text { solids }\end{array}$ & $\begin{array}{l}\text { Tem- } \\
\text { per- } \\
\text { ature }\end{array}$ \\
\hline \multirow[t]{8}{*}{ TNLDOI } & 1 & -1920 & 388 & 96 & $<10$ & 33 \\
\hline & 2 & -1068 & 163 & 99 & $<10$ & 26 \\
\hline & 3 & -872 & 196 & 15 & - - & 25 \\
\hline & 4 & -418 & 454 & 99 & $<10$ & 22 \\
\hline & 5 & -173 & 245 & 86 & $<10$ & 20 \\
\hline & 6 & 437 & 412 & 89 & $<10$ & 16 \\
\hline & 12 & -1231 & 689 & 6 & -- & -- \\
\hline & 14 & 25 & 198 & 0 & -- & -- \\
\hline \multirow{8}{*}{ TNLDO2 } & 1 & -1550 & 470 & 93 & -- & - \\
\hline & 2 & -835 & 296 & 95 & -- & -- \\
\hline & 3 & -680 & 155 & 26 & - & -- \\
\hline & 4 & -185 & 495 & 97 & $\ldots$ & - \\
\hline & 5 & -23 & 162 & 69 & -- & $\ldots$ \\
\hline & 6 & 330 & 255 & - & -- & -- \\
\hline & 12 & -1131 & 419 & 0 & -- & - - \\
\hline & 14 & 75 & 98 & -- & -- & -- \\
\hline \multirow[t]{4}{*}{ TNLDO3 } & 4 & -115 & -- & -- & -- & -- \\
\hline & 5 & 82 & 197 & 99 & -- & -- \\
\hline & 6 & 430 & 288 & 59 & -- & -- \\
\hline & 14 & 142 & 60 & 0 & -- & -- \\
\hline \multirow[t]{9}{*}{ TNLKO1 } & 1 & -1567 & 395 & 59 & $<10$ & 42 \\
\hline & 2 & -799 & 328 & 99 & $<10$ & 31 \\
\hline & 3 & -719 & 80 & 56 & $<10$ & 28 \\
\hline & 4 & -355 & 364 & 78 & $<10$ & 25 \\
\hline & 5 & -124 & 231 & 65 & $<10$ & 21 \\
\hline & 6 & 113 & 37 & -- & -- & 17 \\
\hline & 11 & 290 & 177 & -- & -- & 16 \\
\hline & 12 & -1127 & 440 & 5 & -- & $\ldots$ \\
\hline & 14 & 76 & 200 & 0 & -- & -- \\
\hline \multirow[t]{9}{*}{$\mathrm{INLKO} 2$} & 1 & -1761 & 392 & 82 & -- & -- \\
\hline & 2 & -999 & 309 & 99 & -- & -- \\
\hline & 3 & -853 & 146 & 34 & -- & -- \\
\hline & 4 & -501 & 352 & 93 & -- & -- \\
\hline & 5 & -256 & 245 & 78 & -- & - \\
\hline & 6 & 104 & 157 & 79 & -- & -. \\
\hline & 11 & 284 & 180 & -- & -- & -- \\
\hline & 12 & -1308 & 453 & 7 & -- & -- \\
\hline & 14 & -53 & 203 & 0 & -- & -- \\
\hline \multirow[t]{4}{*}{ TNMDOI } & 1 & -214 & -- & -- & -- & -- \\
\hline & 2 & 234 & 144 & 99 & $<10$ & 15 \\
\hline & 4 & 508 & 274 & 77 & $<10$ & 14 \\
\hline & 12 & 90 & 304 & 10 & - & $\therefore$ \\
\hline
\end{tabular}


Table 4--Layer number; altitude of top, thickness, and sand percentage of layer: and average dissolved-solids concentration and temperature of water in layer; by log number--Continued.

\begin{tabular}{|c|c|c|c|c|c|c|}
\hline $\begin{array}{l}\text { Log } \\
\text { number }\end{array}$ & $\begin{array}{r}\text { Layer } \\
\text { number }\end{array}$ & $\begin{array}{r}\text { Altitude } \\
\text { of top } \\
(f e e t)\end{array}$ & $\begin{array}{r}\text { Thick - } \\
\text { ness } \\
\text { (feet) }\end{array}$ & $\begin{array}{r}\text { Sand } \\
\text { per- } \\
\text { centage }\end{array}$ & $\begin{array}{l}\text { Dis- } \\
\text { solved } \\
\text { solids }\end{array}$ & $\begin{array}{l}\text { Tem- } \\
\text { per- } \\
\text { ature }\end{array}$ \\
\hline \multirow[t]{4}{*}{ TNMDO 2} & 1 & 175 & 365 & 84 & $<10$ & 20 \\
\hline & 2 & 487 & 70 & 99 & $<10$ & $\cdots$ \\
\hline & 4 & 563 & 76 & -- & -- & -- \\
\hline & 12 & 417 & 242 & 35 & $\cdots$ & $\sim$ \\
\hline \multirow[t]{4}{*}{ TNMDO 3} & 1 & -152 & 354 & 81 & -- & -- \\
\hline & 2 & 279 & 139 & 99 & -- & $\cdots$ \\
\hline & 4 & 361 & 82 & -- & -- & - \\
\hline & 12 & 140 & 292 & 3 & $\cdots$ & - \\
\hline \multirow[t]{2}{*}{ TNMDO 4} & 1 & 266 & 364 & 73 & - & $\cdots$ \\
\hline & 12 & 390 & 124 & 28 & -- & -- \\
\hline \multirow[t]{4}{*}{ TNOBO 1} & 4 & -141 & - & -- & -- & -- \\
\hline & 5 & 116 & 257 & 46 & -- & - \\
\hline & 6 & 346 & 164 & 53 & -- & -- \\
\hline & 14 & 182 & 66 & 0 & -- & - \\
\hline \multirow[t]{7}{*}{ TNS B 01} & 1 & -1651 & 346 & 55 & $\cdots$ & $\sim$ \\
\hline & 2 & -742 & 210 & 91 & -- & $\cdots$ \\
\hline & 3 & -457 & 285 & 14 & -- & - \\
\hline & 4 & -65 & 392 & 88 & -- & - \\
\hline & 5 & 245 & 310 & -- & $-\cdots$ & - \\
\hline & 12 & -952 & 699 & 1 & -- & - \\
\hline & 14 & 392 & 147 & -- & $\cdots$ & - \\
\hline \multirow[t]{8}{*}{ TNSHO 2} & 1 & -1855 & 375 & 71 & -- & -- \\
\hline & 2 & -925 & 240 & 99 & $\cdots$ & $\cdots$ \\
\hline & 3 & -730 & 195 & 51 & -- & - \\
\hline & 4 & -205 & 525 & 99 & -- & -- \\
\hline & 5 & 65 & 270 & -- & -- & - \\
\hline & 6 & 242 & 90 & - & -- & - \\
\hline & 12 & -1165 & 690 & 6 & -- & - \\
\hline & 14 & 152 & 87 & -- & $\cdots$ & - \\
\hline \multirow[t]{8}{*}{ TNSHO3 } & 1 & -2141 & 419 & 64 & $<10$ & 31 \\
\hline & 2 & -1149 & 225 & 99 & $<10$ & 25 \\
\hline & 3 & -904 & 245 & 8 & -- & 24 \\
\hline & 4 & -284 & 620 & 92 & $<10$ & 21 \\
\hline & 5 & -53 & 231 & 55 & $<10$ & 19 \\
\hline & 6 & 391 & 358 & - & - & 17 \\
\hline & 12 & -1374 & 767 & 0 & -- & -- \\
\hline & 14 & 33 & 86 & 0 & -- & -- \\
\hline \multirow[t]{7}{*}{ TNSHO 4} & 2 & -1002 & 234 & 99 & -- & - \\
\hline & 3 & -810 & 192 & 64 & -- & - \\
\hline & 4 & -337 & 473 & 99 & $\cdots$ & $\cdots$ \\
\hline & 5 & 73 & 410 & 93 & -- & - \\
\hline & 6 & 248 & 127 & -- & -- & - \\
\hline & 12 & -1236 & - & -- & -- & $\cdots$ \\
\hline & 14 & 121 & 48 & 0 & -- & -- \\
\hline
\end{tabular}


Table 4--Layer number; altitude of top, thickness, and sand percentage of layer; and average dissolved-solids concentration and temperature of water in layer; by log number--Continued.

\begin{tabular}{|c|c|c|c|c|c|c|}
\hline $\begin{array}{l}\text { Log } \\
\text { number }\end{array}$ & $\begin{array}{l}\text { Layer } \\
\text { number }\end{array}$ & $\begin{array}{r}\text { Altitude } \\
\text { of top } \\
(f e e t)\end{array}$ & $\begin{array}{r}\text { Thick- } \\
\text { ness } \\
\text { (feet) }\end{array}$ & $\begin{array}{r}\text { Sand } \\
\text { per- } \\
\text { centage }\end{array}$ & $\begin{array}{l}\text { Dis- } \\
\text { solved } \\
\text { solids }\end{array}$ & $\begin{array}{l}\text { Tem- } \\
\text { per- } \\
\text { ature }\end{array}$ \\
\hline \multirow[t]{6}{*}{ TNSHO 5} & 2 & -1052 & -- & -- & -- & - \\
\hline & 3 & -814 & 238 & 34 & -- & 25 \\
\hline & 4 & -247 & 567 & 97 & -- & 22 \\
\hline & 5 & 72 & 319 & 77 & -- & 19 \\
\hline & 6 & 310 & 178 & -- & -- & 17 \\
\hline & 14 & 132 & 60 & 17 & -- & -- \\
\hline \multirow[t]{5}{*}{ TNSHO 6} & 3 & -873 & -- & -- & -- & -- \\
\hline & 4 & -361 & 512 & 83 & $<10$ & 32 \\
\hline & 5 & -55 & 306 & 96 & $<10$ & 25 \\
\hline & 6 & 287 & 293 & 95 & $<10$ & 18 \\
\hline & 14 & -6 & 49 & 31 & -- & -- \\
\hline \multirow[t]{7}{*}{ TNSHO 7} & 2 & -957 & 208 & 99 & -- & 29 \\
\hline & 3 & -720 & 237 & 15 & -- & 27 \\
\hline & 4 & -270 & 450 & 98 & -- & 23 \\
\hline & 5 & 88 & 358 & 93 & -- & 19 \\
\hline & 6 & 260 & 112 & -- & -- & 17 \\
\hline & 12 & -1165 & - & -- & -- & -- \\
\hline & 14 & 148 & 60 & 0 & -- & -- \\
\hline \multirow[t]{3}{*}{ TNTPOI } & 5 & -65 & -- & -- & -- & -- \\
\hline & 6 & 385 & 310 & 62 & -- & -- \\
\hline & 14 & 75 & 140 & 7 & -- & -- \\
\hline \multirow[t]{3}{*}{ TNTPO2 } & 5 & -84 & -- & -- & -- & -- \\
\hline & 6 & 328 & 215 & 27 & -- & -- \\
\hline & 14 & 113 & 197 & 0 & -- & -- \\
\hline \multirow[t]{5}{*}{ TNWKOI } & 2 & -94 & 13 & 99 & -- & -- \\
\hline & 3 & 66 & 160 & 28 & -- & -- \\
\hline & 4 & 366 & 300 & 99 & -- & -- \\
\hline & 5 & 430 & 64 & 27 & -- & -- \\
\hline & 12 & -107 & 253 & 6 & -- & -- \\
\hline \multirow[t]{6}{*}{ TNWKO2 } & 1 & -222 & -- & -- & -- & -- \\
\hline & 2 & 113 & 87 & 99 & -- & 18 \\
\hline & 3 & 180 & 67 & 0 & -- & 16 \\
\hline & 4 & 458 & 278 & 99 & -- & 14 \\
\hline & 5 & 465 & 7 & -- & -- & -- \\
\hline & 12 & 26 & 248 & 23 & -- & -- \\
\hline \multirow[t]{5}{*}{ TXADOI } & 3 & -79 & 1310 & 62 & $<10$ & 31 \\
\hline & 4 & 61 & 140 & 99 & -- & 22 \\
\hline & 5 & 529 & 368 & 84 & $<10$ & 18 \\
\hline & 12 & -1389 & 1470 & 1 & -- & -- \\
\hline & 13 & 161 & 100 & 17 & -- & -- \\
\hline
\end{tabular}


Table 4--Layer number; altitude of top, thickness, and sand percentage of layer; and average dissolved-solids concentration and temperature of water in layer; by log number--Continued.

\begin{tabular}{|c|c|c|c|c|c|c|}
\hline $\begin{array}{l}\text { Log } \\
\text { number }\end{array}$ & $\begin{array}{r}\text { Layer } \\
\text { number }\end{array}$ & $\begin{array}{r}\text { Altitude } \\
\text { of top } \\
\text { (feet) }\end{array}$ & $\begin{array}{r}\text { Thick- } \\
\text { ness } \\
(\text { feet ) }\end{array}$ & $\begin{array}{r}\text { Sand } \\
\text { per- } \\
\text { centage }\end{array}$ & $\begin{array}{l}\text { Dis- } \\
\text { solved } \\
\text { solids }\end{array}$ & $\begin{array}{l}\text { Tem- } \\
\text { per- } \\
\text { 2ture }\end{array}$ \\
\hline \multirow[t]{5}{*}{ TXADO2 } & 3 & 0 & 1810 & 80 & $<10$ & 30 \\
\hline & 4 & 100 & 100 & 99 & $<10$ & 22 \\
\hline & 5 & 490 & 190 & 77 & $<10$ & 20 \\
\hline & 12 & -1810 & 1710 & 0 & -- & -- \\
\hline & 13 & 300 & 200 & 17 & -- & -- \\
\hline \multirow[t]{4}{*}{ TXADO 3} & 3 & 36 & 1285 & 56 & $<10$ & 28 \\
\hline & 4 & 166 & 130 & 99 & $<10$ & 22 \\
\hline & 12 & -1249 & 795 & 3 & - & - \\
\hline & 13 & 352 & 186 & 25 & -- & -- \\
\hline \multirow[t]{6}{*}{ TXAGO1 } & 3 & -1222 & 3180 & 69 & -- & -- \\
\hline & 5 & -632 & 230 & 87 & -- & -- \\
\hline & 6 & 278 & 500 & 45 & -- & -- \\
\hline & 12 & -4402 & $\ldots$ & -- & -- & - - \\
\hline & 13 & -862 & 360 & 7 & -- & -- \\
\hline & 14 & -222 & 410 & 0 & -- & - \\
\hline \multirow[t]{8}{*}{ TXARO 1} & 7 & -7042 & 1800 & 30 & -- & 79 \\
\hline & 8 & -4592 & 1640 & 40 & 38 & 53 \\
\hline & 9 & -1597 & 1765 & 27 & -- & 40 \\
\hline & 10 & -582 & 1015 & 22 & -- & 30 \\
\hline & 11 & 5 & 587 & 32 & -- & 25 \\
\hline & 15 & -8842 & 240 & 0 & -- & -- \\
\hline & 16 & -6232 & 810 & 0 & -- & -- \\
\hline & 17 & -3362 & 1230 & 2 & -- & -- \\
\hline \multirow[t]{7}{*}{ TXARO 2} & 7 & -5742 & 2360 & 76 & 66 & 67 \\
\hline & 8 & -2777 & 2170 & 44 & 22 & 49 \\
\hline & 9 & -1207 & 1570 & 38 & -- & 39 \\
\hline & 10 & -557 & 650 & 42 & $<10$ & 30 \\
\hline & 11 & 17 & 574 & 23 & $<10$ & 25 \\
\hline & 15 & -8102 & 415 & 0 & -- & - \\
\hline & 16 & -4947 & 795 & 0 & -- & -- \\
\hline \multirow[t]{6}{*}{ TXAR 03} & 7 & -7988 & 610 & 44 & -- & -- \\
\hline & 8 & -4238 & 1800 & 32 & 144 & 66 \\
\hline & 9 & -1938 & 2300 & 21 & 121 & 45 \\
\hline & 10 & -648 & 1290 & 28 & 18 & 31 \\
\hline & 11 & 15 & 663 & 39 & $<10$ & 25 \\
\hline & 16 & -6038 & 1950 & 0 & -- & -- \\
\hline \multirow[t]{7}{*}{ TXAT01 } & 3 & -2148 & 2210 & 63 & $<10$ & 56 \\
\hline & 4 & -1438 & 710 & 92 & $<10$ & 47 \\
\hline & 5 & -148 & 920 & 70 & $<10$ & 31 \\
\hline & 6 & 402 & 50 & -- & -- & 17 \\
\hline & 12 & -4358 & 1960 & 1 & -- & -- \\
\hline & 13 & -1068 & 370 & 8 & -- & - \\
\hline & 14 & 352 & 500 & 6 & -- & -- \\
\hline
\end{tabular}


Table 4--Layer number; altitude of top, thickness, and sand percentage of layer; and average dissolved-solids concentration and temperature of water in layer; by log number--Continued.

\begin{tabular}{|c|c|c|c|c|c|c|}
\hline $\begin{array}{l}\text { L } \circ 8 \\
\text { number }\end{array}$ & $\begin{array}{r}\text { Layer } \\
\text { number }\end{array}$ & $\begin{array}{r}\text { Altitude } \\
\text { of top } \\
(f e e t)\end{array}$ & $\begin{array}{r}\text { Thick- } \\
\text { ness } \\
(f \text { et })\end{array}$ & $\begin{array}{r}\text { Sand } \\
\text { per- } \\
\text { centage }\end{array}$ & $\begin{array}{l}\text { DIs- } \\
\text { solved } \\
\text { solids }\end{array}$ & $\begin{array}{l}\text { Tem- } \\
\text { per- } \\
\text { ature }\end{array}$ \\
\hline \multirow[t]{2}{*}{ TXATO2 } & 3 & 662 & 343 & 57 & $<10$ & 20 \\
\hline & 12 & 319 & 1500 & 0 & -- & - \\
\hline \multirow[t]{6}{*}{ TXATO3 } & 3 & -1908 & 1740 & 71 & $<10$ & 43 \\
\hline & 4 & -1208 & 700 & 99 & $<10$ & 36 \\
\hline & 5 & 142 & 1120 & 65 & $<10$ & 28 \\
\hline & 12 & -3648 & 750 & 5 & -- & -- \\
\hline & 13 & -978 & 230 & 22 & -- & -- \\
\hline & 14 & 402 & 260 & 28 & -- & -- \\
\hline \multirow[t]{10}{*}{ TXAU01 } & 3 & -6119 & 4505 & 68 & 64 & 86 \\
\hline & 5 & -4634 & 240 & 63 & -- & 60 \\
\hline & 6 & -2854 & 1185 & 57 & $<10$ & 49 \\
\hline & 7 & -1034 & 650 & 46 & $<10$ & 32 \\
\hline & 8 & 106 & 1140 & 24 & $<10$ & 25 \\
\hline & 9 & 293 & 187 & 45 & $<10$ & 20 \\
\hline & 12 & -10624 & -- & -- & -- & -- \\
\hline & 13 & -4874 & 1245 & 2 & -- & -- \\
\hline & 14 & -4039 & 595 & 2 & -- & -- \\
\hline & 15 & -1684 & 1170 & 3 & -- & -- \\
\hline \multirow[t]{9}{*}{ TXAUO2 } & 3 & -6280 & 2990 & 60 & 28 & 72 \\
\hline & 6 & -3910 & 730 & 64 & 26 & 51 \\
\hline & 7 & -2040 & 700 & 33 & $<10$ & 38 \\
\hline & 8 & -730 & 1310 & 39 & $<10$ & 31 \\
\hline & 9 & -70 & 660 & 58 & $<10$ & 24 \\
\hline & 10 & 140 & 210 & 56 & $<10$ & 21 \\
\hline & 12 & -9270 & 970 & 3 & -- & -- \\
\hline & 14 & -4640 & 1640 & 0 & -- & -- \\
\hline & 15 & -2740 & 1170 & 2 & -- & -- \\
\hline \multirow[t]{8}{*}{ TXAUO3 } & 3 & -7714 & -- & -- & -- & -- \\
\hline & 6 & -4534 & 1065 & 61 & 49 & 61 \\
\hline & 7 & -2434 & 860 & 54 & 34 & 55 \\
\hline & 8 & -884 & 1550 & 39 & $<10$ & 47 \\
\hline & 9 & 21 & 905 & 39 & $<10$ & 27 \\
\hline & 10 & 250 & 229 & 76 & $<10$ & 18 \\
\hline & 14 & -5599 & 2115 & 0 & -- & -- \\
\hline & 15 & -3294 & 1240 & 0 & -- & -- \\
\hline \multirow[t]{8}{*}{ TXAUO 4} & 6 & -6452 & 985 & 29 & 115 & 70 \\
\hline & 7 & -4052 & 1055 & 48 & 85 & 55 \\
\hline & 8 & -2112 & 1940 & 40 & 24 & 45 \\
\hline & 9 & -812 & 1300 & 40 & $<10$ & 33 \\
\hline & 10 & -152 & 660 & 87 & $<10$ & 25 \\
\hline & 11 & 138 & 290 & 73 & $<10$ & 21 \\
\hline & 14 & -7437 & 1905 & 0 & -- & -- \\
\hline & 15 & -5107 & 1345 & 0 & -- & -- \\
\hline
\end{tabular}


Table 4--Layer number; altitude of top, thickness, and sand percentage of layer; and average dissolved-solids concentration and temperature of water in layer; by log number--Continued.

\begin{tabular}{|c|c|c|c|c|c|c|}
\hline $\begin{array}{l}\text { Log } \\
\text { number }\end{array}$ & $\begin{array}{r}\text { Layer } \\
\text { number }\end{array}$ & $\begin{array}{r}\text { Altitude } \\
\text { of top } \\
\text { (feet) }\end{array}$ & $\begin{array}{r}\text { Thick- } \\
\text { ness } \\
\text { (feet) }\end{array}$ & $\begin{array}{r}\text { Sand } \\
\text { per- } \\
\text { centage }\end{array}$ & $\begin{array}{l}\text { Dis - } \\
\text { solved } \\
\text { solids }\end{array}$ & $\begin{array}{l}\text { Tem- } \\
\text { per- } \\
\text { arure }\end{array}$ \\
\hline \multirow[t]{2}{*}{$\mathrm{TXBAO} 1$} & 3 & 500 & 215 & 46 & -- & -- \\
\hline & 12 & 285 & 1450 & 0 & -- & -- \\
\hline \multirow[t]{2}{*}{$\mathrm{TXBAO} 2$} & 3 & 465 & 1145 & 57 & $<10$ & 26 \\
\hline & 12 & -680 & 525 & 0 & -- & - \\
\hline \multirow[t]{2}{*}{$\mathrm{T} \times \mathrm{BAO} 3$} & 3 & 573 & 742 & 73 & -- & 23 \\
\hline & 12 & -169 & 1550 & 0 & -- & -- \\
\hline \multirow[t]{5}{*}{$\mathrm{TXBAO}_{4}$} & 3 & -498 & 2490 & 56 & -- & -- \\
\hline & 4 & -208 & 290 & 76 & -- & -- \\
\hline & 5 & 550 & 548 & 66 & -- & -- \\
\hline & 12 & -2988 & 1920 & 1 & -- & -- \\
\hline & 13 & 2 & 210 & 11 & -- & -- \\
\hline \multirow[t]{7}{*}{ TXBEOI } & 6 & -4010 & 850 & 33 & 71 & 60 \\
\hline & 7 & -1930 & 25 & 8 & -- & 40 \\
\hline & 8 & -160 & 715 & 37 & $<10$ & 29 \\
\hline & 9 & 346 & 506 & 33 & -- & 24 \\
\hline & 14 & -4860 & 2365 & 0 & -- & -- \\
\hline & 15 & -1955 & 2055 & 3 & -- & -- \\
\hline & 16 & -875 & 1055 & 7 & -- & -- \\
\hline \multirow[t]{7}{*}{ TXBEO 2} & 3 & -5557 & 1980 & 43 & 37 & 73 \\
\hline & 6 & -1042 & 2065 & 43 & 18 & 40 \\
\hline & 7 & -257 & 25 & 0 & -- & 27 \\
\hline & 8 & 342 & 199 & 45 & $<10$ & 23 \\
\hline & 14 & -3107 & 2450 & 2 & -- & -- \\
\hline & 15 & -282 & 760 & 11 & -- & -- \\
\hline & 16 & 143 & 400 & 5 & -- & - \\
\hline \multirow[t]{6}{*}{$\mathrm{TXBEO} 3$} & 7 & -3019 & 2040 & 31 & 39 & 62 \\
\hline & 8 & -1139 & 1500 & 29 & 18 & 42 \\
\hline & 9 & -124 & 1015 & 59 & $<10$ & 31 \\
\hline & 10 & 99 & 223 & 63 & $<10$ & 25 \\
\hline & 15 & -5059 & 420 & 8 & - - & -- \\
\hline & 16 & -2639 & 380 & 3 & -- & - \\
\hline \multirow[t]{6}{*}{ TXBKOI } & 7 & -3928 & 3270 & 47 & 76 & 66 \\
\hline & 8 & -2193 & 1735 & 30 & 16 & 54 \\
\hline & 9 & -898 & 1295 & 45 & $<10$ & 41 \\
\hline & 10 & -328 & 570 & 51 & $<10$ & 31 \\
\hline & 11 & 214 & 542 & 91 & $<10$ & 25 \\
\hline & 15 & -7198 & 270 & 0 & -- & -- \\
\hline \multirow[t]{6}{*}{$\mathrm{TXBKO} 2$} & 7 & -4362 & 3650 & 37 & 24 & 70 \\
\hline & 8 & -2472 & 1890 & 44 & 36 & 50 \\
\hline & 9 & -1047 & 1425 & 30 & 30 & 38 \\
\hline & 10 & -457 & 590 & 66 & $<10$ & 31 \\
\hline & 11 & 95 & 552 & 56 & $<10$ & 27 \\
\hline & 15 & -8012 & 480 & 2 & - - & -- \\
\hline
\end{tabular}


Table 4--Layer number; altitude of top, thickness, and sand percentage of layer; and average dissolved-solids concentration and temperature of water in layer; by log number--Continued.

\begin{tabular}{|c|c|c|c|c|c|c|}
\hline $\begin{array}{l}\text { Log } \\
\text { number }\end{array}$ & $\begin{array}{r}\text { Layer } \\
\text { number }\end{array}$ & $\begin{array}{r}\text { Altitude } \\
\text { of top } \\
\text { (feet) }\end{array}$ & $\begin{array}{r}\text { Thick- } \\
\text { ness } \\
\text { (feet) }\end{array}$ & $\begin{array}{r}\text { Sand } \\
\text { per- } \\
\text { centage }\end{array}$ & $\begin{array}{l}\text { Dis- } \\
\text { solved } \\
\text { solids }\end{array}$ & $\begin{array}{l}\text { Tem- } \\
\text { per- } \\
\text { ature }\end{array}$ \\
\hline \multirow[t]{7}{*}{ TXBRO 1} & 7 & -7050 & 2180 & 45 & 70 & 66 \\
\hline & 8 & -4765 & 935 & 50 & 103 & 59 \\
\hline & 9 & -1555 & 2315 & 34 & 17 & 39 \\
\hline & 10 & -540 & 1015 & 70 & $<10$ & 31 \\
\hline & 11 & 42 & 582 & 47 & $<10$ & 27 \\
\hline & 16 & -5700 & 1350 & 0 & - & $\cdots$ \\
\hline & 17 & -3870 & 895 & 13 & -- & -- \\
\hline \multirow[t]{7}{*}{$\mathrm{TXBRO2}$} & 7 & -10880 & 970 & 37 & 75 & 90 \\
\hline & 8 & -5355 & 1545 & 64 & 119 & 58 \\
\hline & 9 & -1605 & 2865 & 48 & 41 & 40 \\
\hline & 10 & -545 & 1060 & 43 & - & 31 \\
\hline & 11 & 29 & 574 & 55 & $<10$ & 27 \\
\hline & 16 & -6900 & 3980 & 1 & -- & -- \\
\hline & 17 & -4470 & 885 & 11 & - & -- \\
\hline \multirow[t]{7}{*}{ IXBRO 3} & 7 & -9040 & 515 & 20 & -- & - \\
\hline & 8 & -4825 & 1970 & 58 & 52 & 50 \\
\hline & 9 & -1585 & 2605 & 56 & 41 & 38 \\
\hline & 10 & -555 & 1030 & 60 & - & 30 \\
\hline & 11 & 24 & 579 & 80 & $<10$ & 27 \\
\hline & 16 & -6795 & 2245 & 0 & -- & - \\
\hline & 17 & -4190 & 635 & 13 & -- & -- \\
\hline \multirow[t]{6}{*}{$\mathrm{TXBRO} 4$} & 8 & -4780 & 1710 & 68 & 43 & 67 \\
\hline & 9 & -1635 & 2560 & 42 & 24 & 46 \\
\hline & 10 & -570 & 1065 & 51 & $\ldots$ & 33 \\
\hline & 11 & 3 & 573 & 46 & $\ldots$ & 26 \\
\hline & 16 & -6490 & 530 & 0 & -- & -- \\
\hline & 17 & -4195 & 585 & 42 & -- & - \\
\hline \multirow[t]{6}{*}{ TXBRO5 } & 8 & -4820 & 1310 & 64 & -- & 55 \\
\hline & 9 & -1640 & 2730 & 50 & -- & 42 \\
\hline & 10 & -580 & 1060 & 34 & -- & 31 \\
\hline & 11 & 0 & 580 & 68 & $\ldots$ & 27 \\
\hline & 16 & -6130 & 1190 & 0 & $\ldots$ & -- \\
\hline & 17 & -4370 & 450 & 17 & -- & -- \\
\hline \multirow[t]{7}{*}{ TXBRO 6} & 7 & -5877 & 1985 & 68 & 71 & 57 \\
\hline & 8 & -2897 & 2180 & 30 & - & 47 \\
\hline & 9 & -1287 & 1610 & 55 & $<10$ & 38 \\
\hline & 10 & -497 & 790 & 86 & $<10$ & 30 \\
\hline & 11 & 58 & 555 & 25 & $<10$ & 26 \\
\hline & 15 & -7862 & - & -- & - & - \\
\hline & 16 & -5077 & 800 & 0 & -- & $\cdots$ \\
\hline \multirow[t]{5}{*}{ TXBUO1 } & 3 & -675 & 2255 & 78 & - & -- \\
\hline & 4 & -350 & 325 & 85 & $=$ & -- \\
\hline & 5 & 410 & 640 & 68 & -- & - \\
\hline & 12 & -2930 & -- & - & - & - \\
\hline & 13 & -230 & 120 & 0 & - & - \\
\hline
\end{tabular}


Table 4--Layer number; altitude of top, thickness, and sand percentage of layer; and average dissolved-solids concentration and temperature of water in layer; by log number--Continued.

\begin{tabular}{|c|c|c|c|c|c|c|}
\hline $\begin{array}{l}\text { Log } \\
\text { number }\end{array}$ & $\begin{array}{r}\text { Layer } \\
\text { number }\end{array}$ & $\begin{array}{r}\text { Altitude } \\
\text { of top } \\
\text { (feet) }\end{array}$ & $\begin{array}{r}\text { Thick- } \\
\text { ness } \\
\text { (feet) }\end{array}$ & $\begin{array}{r}\text { Sand } \\
\text { per- } \\
\text { centage }\end{array}$ & $\begin{array}{l}\text { DIs- } \\
\text { solved } \\
\text { solids }\end{array}$ & $\begin{array}{l}\text { Tem- } \\
\text { per- } \\
\text { ature }\end{array}$ \\
\hline \multirow[t]{8}{*}{$\mathrm{TXBUO2}$} & 3 & -2234 & 2990 & 68 & 16 & 50 \\
\hline & 4 & -2009 & 225 & 87 & $<10$ & 39 \\
\hline & 5 & -964 & 680 & 65 & $<10$ & 33 \\
\hline & 6 & 256 & 850 & 44 & $<10$ & 24 \\
\hline & 12 & -5224 & 2430 & 0 & -- & - \\
\hline & 13 & -1644 & 365 & 2 & - - & $\ldots$ \\
\hline & 14 & -594 & 370 & 5 & $\cdots$ & -- \\
\hline & 15 & 294 & 38 & 0 & -- & -- \\
\hline \multirow[t]{7}{*}{ TXB 201} & 3 & -1681 & 2790 & 73 & 10 & 44 \\
\hline & 4 & -1531 & 150 & 93 & $<10$ & 35 \\
\hline & 5 & -521 & 700 & 46 & $<10$ & 30 \\
\hline & 6 & 354 & 505 & 38 & $<10$ & 24 \\
\hline & 12 & -4471 & -- & -- & -- & -- \\
\hline & 13 & -1221 & 310 & 0 & -- & -- \\
\hline & 14 & -151 & 370 & 0 & -- & - \\
\hline \multirow[t]{7}{*}{$\mathrm{TXB} 202$} & 3 & -3681 & 4050 & 76 & 52 & 61 \\
\hline & 5 & -2491 & 825 & 50 & $<10$ & 41 \\
\hline & 6 & -1361 & 610 & 36 & $<10$ & 33 \\
\hline & 12 & -7731 & -- & -- & -- & -- \\
\hline & 13 & -3316 & 365 & 0 & -- & -- \\
\hline & 14 & -1971 & 520 & 2 & -- & -- \\
\hline & 15 & 225 & 1586 & 10 & -- & - \\
\hline \multirow[t]{7}{*}{$\mathrm{TXB} 203$} & 3 & -1349 & 2520 & 64 & $<10$ & 48 \\
\hline & 4 & -1089 & 260 & 92 & $<10$ & 35 \\
\hline & 5 & -169 & 780 & 60 & $<10$ & 29 \\
\hline & 6 & 352 & 171 & 58 & $<10$ & 20 \\
\hline & 12 & -3869 & 3225 & 0 & -- & -- \\
\hline & 13 & -949 & 140 & 7 & -- & -- \\
\hline & 14 & 181 & 350 & 1 & -- & -- \\
\hline \multirow[t]{4}{*}{$\mathrm{TXCAO} 1$} & 3 & -65 & 1570 & 25 & $<10$ & 32 \\
\hline & 4 & 435 & 500 & 78 & $<10$ & 24 \\
\hline & 12 & -1635 & - & -- & -- & -- \\
\hline & 13 & 488 & 53 & 0 & -- & -- \\
\hline \multirow[t]{6}{*}{$\mathrm{TXCHO} 1$} & 7 & -7730 & 1640 & 34 & 87 & 79 \\
\hline & 8 & -3670 & 2820 & 55 & 143 & 56 \\
\hline & 9 & -1720 & 1950 & 44 & 81 & 40 \\
\hline & 10 & -635 & 1085 & 38 & $<10$ & 29 \\
\hline & 11 & 13 & 648 & 50 & $<10$ & 23 \\
\hline & 16 & -6490 & 1240 & 5 & -- & -- \\
\hline \multirow[t]{6}{*}{$\mathrm{TXCHO} 2$} & 7 & -9019 & 1050 & 51 & -- & -- \\
\hline & 8 & -4219 & 2965 & 67 & -- & -- \\
\hline & 9 & -2104 & 2115 & 44 & -- & -- \\
\hline & 10 & -819 & 1285 & 51 & -- & -- \\
\hline & 11 & 11 & 830 & 67 & -- & -- \\
\hline & 16 & -7184 & 1835 & 0 & -- & -- \\
\hline
\end{tabular}


Table 4--Layer number; altitude of top, thickness, and sand percentage of layer; and average dissolved-solids concentration and temperature of water in layer; by log number--Continued.

\begin{tabular}{|c|c|c|c|c|c|c|}
\hline $\begin{array}{l}\text { Log } \\
\text { number }\end{array}$ & $\begin{array}{r}\text { Layer } \\
\text { number }\end{array}$ & $\begin{array}{r}\text { Altitude } \\
\text { of top } \\
(f e e t)\end{array}$ & $\begin{array}{r}\text { Thlek- } \\
\text { ness } \\
(\text { feet })\end{array}$ & $\begin{array}{r}\text { Sand } \\
\text { per- } \\
\text { centage }\end{array}$ & $\begin{array}{l}\text { DIs- } \\
\text { solved } \\
\text { solids }\end{array}$ & $\begin{array}{l}\text { Tem- } \\
\text { per- } \\
\text { ature }\end{array}$ \\
\hline \multirow[t]{7}{*}{$\mathrm{TXCHO} 3$} & 7 & -5972 & 2180 & 51 & 93 & 70 \\
\hline & 8 & -3182 & 2290 & 25 & 70 & 51 \\
\hline & 9 & -1397 & 1785 & 44 & 24 & 37 \\
\hline & 10 & -497 & 900 & 69 & -- & 28 \\
\hline & 11 & 46 & 543 & 65 & -- & 23 \\
\hline & 15 & -8152 & 790 & 0 & -- & -- \\
\hline & 16 & -5472 & 500 & 0 & -- & -- \\
\hline \multirow[t]{5}{*}{ TXCKO 1} & 3 & -8 & 1400 & 50 & $<10$ & 30 \\
\hline & 4 & 107 & 115 & 99 & $<10$ & 22 \\
\hline & 5 & 479 & 222 & 66 & $<10$ & 18 \\
\hline & 12 & -1408 & 1550 & 1 & -- & -- \\
\hline & 13 & 257 & 150 & 11 & -- & -- \\
\hline \multirow[t]{5}{*}{$\mathrm{TXCKO} 2$} & 3 & 27 & 1890 & 66 & -- & -- \\
\hline & 4 & 162 & 135 & 99 & -- & -- \\
\hline & 5 & 440 & 138 & 47 & -- & -- \\
\hline & 12 & -1863 & 1585 & 0 & -- & $-\cdot$ \\
\hline & 13 & 302 & 140 & 14 & -- & -- \\
\hline \multirow{5}{*}{$\mathrm{TXCKO} 3$} & 3 & -146 & 2285 & 63 & -- & -- \\
\hline & 4 & 9 & 155 & 97 & $\cdots$ & -- \\
\hline & 5 & 340 & 91 & 90 & -- & -- \\
\hline & 12 & -2431 & 1490 & 1 & -- & -- \\
\hline & 13 & 249 & 240 & 0 & -- & - \\
\hline \multirow[t]{6}{*}{ TXCLO1 } & 8 & -4618 & 1305 & 34 & 42 & 60 \\
\hline & 9 & -1328 & 1580 & 41 & 34 & 38 \\
\hline & 10 & -568 & 760 & 39 & $<10$ & 29 \\
\hline & 11 & 11 & 579 & 33 & $<10$ & 24 \\
\hline & 16 & -5923 & 2650 & 1 & -- & -- \\
\hline & 17 & -2908 & 1710 & 0 & -- & - \\
\hline \multirow[t]{6}{*}{ TXCLO2 } & 8 & -5236 & 880 & 52 & -- & 59 \\
\hline & 9 & -1751 & 1590 & 61 & -- & 39 \\
\hline & 10 & -621 & 1130 & 35 & 38 & 30 \\
\hline & 11 & 13 & 634 & 48 & $<10$ & 25 \\
\hline & 16 & -6116 & 3300 & 0 & -- & -- \\
\hline & 17 & -3341 & 1895 & 0 & -- & -- \\
\hline \multirow[t]{7}{*}{ TXCMO1 } & 7 & -8709 & 480 & 67 & -- & -- \\
\hline & 8 & -3529 & 4640 & 53 & -- & -- \\
\hline & 9 & -1579 & 1435 & 59 & -- & 37 \\
\hline & 10 & -544 & 1035 & 71 & $<10$ & 31 \\
\hline & 11 & 31 & 575 & 63 & $<10$ & 26 \\
\hline & 16 & -8169 & 540 & 0 & -- & - \\
\hline & 17 & -3014 & 515 & 6 & -- & -- \\
\hline
\end{tabular}


Table 4--Layer number; altitude of top, thickness, and sand percentage of layer; and average dissolved-solids concentration and temperature of water in layer; by log number--Continued.

\begin{tabular}{|c|c|c|c|c|c|c|}
\hline $\begin{array}{l}\text { Log } \\
\text { number }\end{array}$ & $\begin{array}{l}\text { Layer } \\
\text { number }\end{array}$ & $\begin{array}{r}\text { Altitude } \\
\text { of top } \\
\text { (feet) }\end{array}$ & $\begin{array}{r}\text { Thick- } \\
\text { ness } \\
\text { (feet) }\end{array}$ & $\begin{array}{r}\text { Sand } \\
\text { per- } \\
\text { centage }\end{array}$ & $\begin{array}{l}\text { Dis- } \\
\text { solved } \\
\text { solids }\end{array}$ & $\begin{array}{l}\text { Tem- } \\
\text { per- } \\
\text { ature }\end{array}$ \\
\hline \multirow[t]{6}{*}{$\operatorname{TXCMO2}$} & 7 & -7718 & 695 & 29 & -- & -- \\
\hline & 8 & -3173 & 3800 & 45 & -- & -- \\
\hline & 9 & -1448 & 1725 & 48 & -- & - \\
\hline & 10 & -513 & 935 & 54 & -- & -- \\
\hline & 11 & 48 & 561 & 57 & -- & -- \\
\hline & 16 & -6973 & 745 & 19 & -- & - \\
\hline \multirow[t]{4}{*}{$\operatorname{TXCMO3}$} & 9 & -2470 & 3140 & 6 & -- & 63 \\
\hline & 10 & -740 & 1730 & 25 & 19 & 44 \\
\hline & 11 & 0 & 740 & 33 & 18 & 22 \\
\hline & 17 & -5610 & 1962 & 1 & -- & -- \\
\hline \multirow[t]{8}{*}{$\operatorname{Txc001}$} & 3 & -6430 & 4390 & 60 & -- & -- \\
\hline & 6 & -3460 & 1045 & 72 & -- & -- \\
\hline & 7 & -1390 & 745 & 49 & -- & -- \\
\hline & 8 & -120 & 1270 & 41 & -- & -- \\
\hline & 9 & 295 & 415 & 73 & -- & -- \\
\hline & 12 & -10820 & -- & -- & -- & -- \\
\hline & 14 & -4505 & 1925 & 0 & -- & -- \\
\hline & 15 & -2135 & 1325 & 0 & -- & -- \\
\hline \multirow[t]{7}{*}{$\mathrm{TXCOO2}$} & 3 & -6693 & -- & -- & -- & -- \\
\hline & 6 & -3653 & 955 & 50 & 31 & 57 \\
\hline & 7 & -1623 & 830 & 28 & 20 & 40 \\
\hline & 8 & -193 & 1430 & 16 & -- & 30 \\
\hline & 9 & 260 & 453 & 59 & $<10$ & 20 \\
\hline & 14 & -4608 & 2085 & 0 & -- & -- \\
\hline & 15 & -2453 & 1200 & 0 & -- & -- \\
\hline \multirow[t]{8}{*}{$\operatorname{Ixc} 003$} & 3 & -6387 & 4175 & 53 & 93 & 95 \\
\hline & 6 & -3522 & 1040 & 58 & 34 & 56 \\
\hline & 7 & -1402 & 760 & 27 & 17 & 37 \\
\hline & 8 & -97 & 1305 & 37 & -- & 28 \\
\hline & 9 & 322 & 419 & 47 & -- & 20 \\
\hline & 12 & -10562 & -- & -- & -- & -- \\
\hline & 14 & -4562 & 1825 & 0 & -- & -- \\
\hline & 15 & -2162 & 1360 & 0 & -- & -- \\
\hline \multirow[t]{9}{*}{ IXCO0 4} & 3 & -8480 & -- & -- & -- & -- \\
\hline & 6 & -5845 & 565 & 23 & 12 & 66 \\
\hline & 7 & -3270 & 915 & 37 & 13 & 49 \\
\hline & 8 & -1580 & 1690 & 26 & $<10$ & 39 \\
\hline & 9 & -480 & 1100 & 37 & $<10$ & 29 \\
\hline & 10 & -55 & 425 & 47 & $<10$ & 23 \\
\hline & 11 & 190 & 245 & 87 & $<10$ & 21 \\
\hline & 14 & -6410 & 2070 & 0 & -- & -- \\
\hline & 15 & -4185 & 1660 & 1 & -- & -- \\
\hline
\end{tabular}


Table 4--Layer number; altitude of top, thickness, and sand percentage of layer; and average dissolved-solids concentration and temperature of water in layer; by log number--Continued.

\begin{tabular}{|c|c|c|c|c|c|c|}
\hline $\begin{array}{l}\text { Log } \\
\text { number }\end{array}$ & $\begin{array}{r}\text { Layer } \\
\text { number }\end{array}$ & $\begin{array}{r}\text { Altitude } \\
\text { of top } \\
\text { (feet) }\end{array}$ & $\begin{array}{r}\text { Th1ck- } \\
\text { ness } \\
(\text { feet) }\end{array}$ & $\begin{array}{r}\text { Sand } \\
\text { per- } \\
\text { centage }\end{array}$ & $\begin{array}{l}\text { Dis- } \\
\text { solved } \\
\text { solids }\end{array}$ & $\begin{array}{l}\text { Tem- } \\
\text { per- } \\
\text { ature }\end{array}$ \\
\hline \multirow[t]{5}{*}{$\operatorname{TXCSO} 1$} & 3 & 8 & 430 & 62 & $<10$ & 25 \\
\hline & 4 & 158 & 150 & 97 & $<10$ & 22 \\
\hline & 5 & 321 & 103 & 83 & $<10$ & 20 \\
\hline & 12 & -422 & 920 & 0 & -- & $\cdots$ \\
\hline & 13 & 218 & 60 & 0 & -- & $\cdots$ \\
\hline \multirow[t]{5}{*}{$\operatorname{TXCSO} 2$} & 3 & -302 & 530 & 60 & $<10$ & 27 \\
\hline & 4 & -92 & 210 & 93 & $<10$ & 24 \\
\hline & 5 & 308 & 380 & 78 & $<10$ & 22 \\
\hline & 12 & -832 & 910 & 0 & -- & -- \\
\hline & 13 & -72 & 20 & 0 & -- & -- \\
\hline \multirow[t]{5}{*}{$\operatorname{TXCs03}$} & 3 & -21 & 440 & 53 & $<10$ & 25 \\
\hline & 4 & 39 & 60 & 99 & $<10$ & 23 \\
\hline & 5 & 207 & 118 & 69 & $<10$ & 21 \\
\hline & 12 & -461 & 875 & 0 & -- & -- \\
\hline & 13 & 89 & 50 & 0 & -- & -- \\
\hline \multirow[t]{2}{*}{$\mathrm{TXCSO}_{4}$} & 3 & 227 & 313 & 44 & $<10$ & 22 \\
\hline & 12 & -86 & 910 & 0 & -- & -- \\
\hline \multirow[t]{2}{*}{ TXDIOI } & 3 & 755 & 694 & 40 & $<10$ & 26 \\
\hline & 12 & 61 & 375 & 5 & -- & -- \\
\hline \multirow[t]{5}{*}{ TXDIO 2} & 3 & -774 & 1355 & 63 & $<10$ & 39 \\
\hline & 4 & -479 & 295 & 86 & $<10$ & 34 \\
\hline & 5 & 575 & 954 & 31 & $<10$ & 29 \\
\hline & 12 & -2129 & 715 & 0 & -- & -- \\
\hline & 13 & -379 & 100 & 0 & -- & -- \\
\hline \multirow[t]{5}{*}{ TXDIO 3} & 3 & -272 & 1070 & 57 & $<10$ & 35 \\
\hline & 4 & -82 & 190 & 87 & $<10$ & 31 \\
\hline & 5 & 701 & 733 & 28 & -- & 28 \\
\hline & 12 & -1342 & 350 & 0 & -- & -- \\
\hline & 13 & -32 & 30 & 0 & -- & -- \\
\hline \multirow[t]{7}{*}{ TXDU01 } & 5 & -4614 & 1500 & 62 & 17 & 77 \\
\hline & 6 & -1599 & 1815 & 45 & 14 & 51 \\
\hline & 7 & 266 & 490 & 31 & -- & 27 \\
\hline & 8 & 702 & 436 & 39 & $<10$ & 23 \\
\hline & 13 & -6114 & 470 & 0 & -- & -- \\
\hline & 14 & -3414 & 1200 & 0 & -- & -- \\
\hline & 15 & -224 & 1375 & 7 & -- & -- \\
\hline \multirow[t]{8}{*}{ TXDU02 } & 5 & -5969 & 530 & 31 & -- & -- \\
\hline & 6 & -1849 & 2490 & 62 & -- & -- \\
\hline & 7 & -1129 & 70 & 93 & -- & -- \\
\hline & 8 & 411 & 410 & 67 & -- & -- \\
\hline & 9 & 580 & 169 & 77 & -- & -- \\
\hline & 14 & -4339 & 1630 & 0 & -- & -- \\
\hline & 15 & -1199 & 650 & 11 & -- & -- \\
\hline & 16 & 1 & 1130 & 3 & -- & -- \\
\hline
\end{tabular}


Table 4--Layer number; altitude of top, thickness, and sand percentage of layer; and average dissolved-solids concentration and temperature of water in layer; by log number--Continued.

\begin{tabular}{|c|c|c|c|c|c|c|}
\hline $\begin{array}{l}\text { Log } \\
\text { number }\end{array}$ & $\begin{array}{r}\text { Layer } \\
\text { number }\end{array}$ & $\begin{array}{r}\text { Altitude } \\
\text { of top } \\
\text { (feet) }\end{array}$ & $\begin{array}{r}\text { Thick- } \\
\text { ness } \\
\text { (feet) }\end{array}$ & $\begin{array}{r}\text { Sand } \\
\text { per- } \\
\text { centage }\end{array}$ & $\begin{array}{l}\text { Dis- } \\
\text { solved } \\
\text { solids }\end{array}$ & $\begin{array}{l}\text { Tem- } \\
\text { per- } \\
\text { ature }\end{array}$ \\
\hline \multirow[t]{6}{*}{ TXDUO3 3} & 7 & -3221 & 1325 & 33 & $<10$ & 56 \\
\hline & 8 & -561 & 1105 & 33 & $<10$ & 35 \\
\hline & 9 & 189 & 750 & 55 & $<10$ & 29 \\
\hline & 10 & 330 & 141 & 73 & $<10$ & 25 \\
\hline & 15 & -4546 & 915 & 19 & -- & - \\
\hline & 16 & -1666 & 1555 & 2 & -- & -- \\
\hline \multirow[t]{6}{*}{ TXDU0 4} & 6 & -4178 & 1150 & 53 & -- & -- \\
\hline & 7 & -1618 & 1360 & 24 & $<10$ & 44 \\
\hline & 8 & -423 & 1195 & 29 & $<10$ & 35 \\
\hline & 9 & 287 & 710 & 15 & $<10$ & 28 \\
\hline & 10 & 461 & 174 & 83 & $<10$ & 25 \\
\hline & 15 & -2978 & 1200 & 15 & -- & -- \\
\hline \multirow[t]{6}{*}{ TXDWO 1} & 3 & -7406 & 1400 & 47 & 34 & 92 \\
\hline & 7 & -2431 & 995 & 41 & 12 & 48 \\
\hline & 8 & -406 & 1310 & 42 & $<10$ & 33 \\
\hline & 9 & 175 & 581 & 52 & $<10$ & 25 \\
\hline & 15 & -3426 & 3980 & 0 & -- & -- \\
\hline & 16 & -1716 & 715 & 0 & -- & -- \\
\hline \multirow[t]{5}{*}{ TXDWO 2} & 3 & -7290 & 2050 & 49 & -- & -- \\
\hline & 7 & -2040 & 1000 & 14 & -- & -- \\
\hline & 8 & -550 & 1490 & 27 & -- & -- \\
\hline & 9 & 191 & 741 & 48 & -- & -- \\
\hline & 15 & -3040 & 4250 & 0 & -- & -- \\
\hline \multirow[t]{7}{*}{ TXDWO 3} & 3 & -7022 & 2100 & 46 & 33 & 84 \\
\hline & 6 & -4207 & 590 & 45 & 28 & 58 \\
\hline & 7 & -1932 & 940 & 18 & 14 & 40 \\
\hline & 8 & -512 & 1420 & 43 & $<10$ & 33 \\
\hline & 9 & 166 & 678 & 40 & $<10$ & 26 \\
\hline & 14 & -4797 & 2225 & 0 & -- & -- \\
\hline & 15 & -2872 & 1335 & 0 & -- & -- \\
\hline \multirow[t]{8}{*}{ TXDWO 4} & 3 & -5950 & 3340 & 62 & 46 & 84 \\
\hline & 6 & -3130 & 690 & 68 & 30 & 52 \\
\hline & 7 & -620 & 625 & 48 & $<10$ & 32 \\
\hline & 8 & 145 & 765 & 50 & $<10$ & 26 \\
\hline & 9 & 378 & 233 & 73 & $<10$ & 22 \\
\hline & 12 & -9290 & 2610 & 0 & -- & -- \\
\hline & 14 & -3820 & 2130 & 0 & -- & -- \\
\hline & 15 & -1245 & 1885 & 6 & -- & -- \\
\hline \multirow[t]{7}{*}{ TXDWO 5} & 3 & -6027 & 3090 & 54 & 72 & 87 \\
\hline & 6 & -2897 & 830 & 67 & 31 & 52 \\
\hline & 7 & -147 & 280 & 64 & $<10$ & 27 \\
\hline & 8 & 403 & 550 & 55 & $<10$ & 23 \\
\hline & 12 & -9117 & 460 & 0 & -- & -- \\
\hline & 14 & -3727 & 2300 & 0 & -- & -- \\
\hline & 15 & -427 & 2470 & 17 & -- & -- \\
\hline
\end{tabular}


Table 4--Layer number; altitude of top, thickness, and sand percentage of layer; and average dissolved-solids concentration and temperature of water in layer; by log number--Continued.

\begin{tabular}{|c|c|c|c|c|c|c|}
\hline $\begin{array}{l}\mathrm{L} \circ \mathrm{g} \\
\text { number }\end{array}$ & $\begin{array}{r}\text { Layer } \\
\text { number }\end{array}$ & $\begin{array}{r}\text { Altitude } \\
\text { of top } \\
(f e e t)\end{array}$ & $\begin{array}{r}\text { Thick- } \\
\text { ness } \\
\text { (feet) }\end{array}$ & $\begin{array}{r}\text { Sand } \\
\text { per- } \\
\text { centage }\end{array}$ & $\begin{array}{l}\text { Dis- } \\
\text { solved } \\
\text { solids }\end{array}$ & $\begin{array}{l}\text { Tem- } \\
\text { per- } \\
\text { ature }\end{array}$ \\
\hline \multirow[t]{7}{*}{ TXFBO1 } & 6 & -7243 & 635 & 27 & -- & - \\
\hline & 7 & -4578 & 1340 & 37 & -- & -- \\
\hline & 8 & -2488 & 2090 & 28 & $-\cdots$ & -- \\
\hline & 9 & -1048 & 1440 & 21 & -- & -- \\
\hline & 10 & -328 & 720 & 41 & -- & -- \\
\hline & 11 & 100 & 428 & 67 & $\ldots$ & - - \\
\hline & 15 & -5918 & 1325 & 0 & -- & -- \\
\hline \multirow[t]{6}{*}{$\mathrm{TXFBO} 2$} & 7 & -4860 & 1725 & 44 & 134 & 64 \\
\hline & 8 & -2730 & 2130 & 50 & 43 & 51 \\
\hline & 9 & -1220 & 1510 & 47 & $<10$ & 36 \\
\hline & 10 & -450 & 770 & 69 & $<10$ & 27 \\
\hline & 11 & 80 & 530 & 76 & $<10$ & 21 \\
\hline & 15 & -6585 & 1120 & 1 & -- & - \\
\hline \multirow[t]{5}{*}{ TXFOOI } & 3 & -1333 & 1390 & 63 & $<10$ & 41 \\
\hline & 4 & -568 & 765 & 80 & $<10$ & 35 \\
\hline & 5 & 559 & 1022 & 42 & $<10$ & 29 \\
\hline & 12 & -2723 & 1410 & 0 & -- & -- \\
\hline & 13 & -463 & 105 & 0 & - & - \\
\hline \multirow[t]{3}{*}{$T \times F 002$} & 3 & 364 & 565 & 60 & $<10$ & 25 \\
\hline & 4 & 673 & 309 & 70 & $<10$ & 20 \\
\hline & 12 & -201 & $\ldots$ & $\ldots$ & $\ldots$ & $\ldots$ \\
\hline \multirow[t]{2}{*}{ TXFROI } & 3 & 440 & 798 & 69 & $<10$ & 22 \\
\hline & 12 & -358 & 860 & 0 & -- & -- \\
\hline \multirow[t]{2}{*}{ TXFSOI } & 3 & 400 & 1041 & 48 & $<10$ & 24 \\
\hline & 12 & -641 & 1800 & 1 & - & - \\
\hline \multirow[t]{2}{*}{ TXFSO 2} & 3 & 256 & 490 & 51 & -- & 22 \\
\hline & 12 & -234 & 1800 & 1 & - & -- \\
\hline \multirow[t]{2}{*}{$\mathrm{TXFSO} 3$} & 3 & 513 & 325 & 48 & -- & - \\
\hline & 12 & 188 & 960 & 0 & -- & - \\
\hline \multirow[t]{6}{*}{ TXFYOI } & 3 & -1839 & -- & -- & -- & -- \\
\hline & 4 & -1129 & 710 & 75 & $<10$ & 38 \\
\hline & 5 & -59 & 680 & 58 & $<10$ & 29 \\
\hline & 6 & 460 & 119 & 62 & $<10$ & 22 \\
\hline & 13 & -739 & 390 & 3 & -- & $\Rightarrow$ \\
\hline & 14 & 341 & 400 & 0 & -- & -- \\
\hline \multirow[t]{7}{*}{ TXFYO2 } & 3 & -2084 & - & - & -- & -. \\
\hline & 4 & -1709 & 375 & 79 & -- & $\ldots$ \\
\hline & 5 & -774 & 835 & 68 & - & -- \\
\hline & 6 & 326 & 700 & 56 & - & - \\
\hline & 13 & -1609 & 100 & 0 & -- & -- \\
\hline & 14 & -374 & 400 & 4 & $\ldots$ & - \\
\hline & 15 & 365 & 39 & 0 & 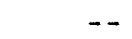 & -- \\
\hline
\end{tabular}


Table 4--Layer number; altitude of top, thickness, and sand percentage of layer; and average dissolved-solids concentration and temperature of water in layer; by log number--Continued.

\begin{tabular}{|c|c|c|c|c|c|c|}
\hline $\begin{array}{l}\text { Log } \\
\text { number }\end{array}$ & $\begin{array}{r}\text { Layer } \\
\text { number }\end{array}$ & $\begin{array}{r}\text { Altitude } \\
\text { of top } \\
(\text { feet) }\end{array}$ & $\begin{array}{r}\text { Thick- } \\
\text { ness } \\
(\text { feet })\end{array}$ & $\begin{array}{r}\text { Sand } \\
\text { per- } \\
\text { centage }\end{array}$ & $\begin{array}{l}\text { Dis- } \\
\text { solved } \\
\text { solids }\end{array}$ & $\begin{array}{l}\text { Tem- } \\
\text { per- } \\
\text { ature }\end{array}$ \\
\hline \multirow[t]{9}{*}{ TXFYO3 } & 3 & -3485 & 3700 & 70 & 77 & 65 \\
\hline & 4 & -3150 & 335 & 79 & $<10$ & 50 \\
\hline & 5 & -2055 & 850 & 36 & $<10$ & 44 \\
\hline & 6 & -715 & 950 & 53 & $<10$ & 34 \\
\hline & 7 & 450 & 195 & 32 & -- & 23 \\
\hline & 12 & -7185 & 2620 & 0 & -- & - - \\
\hline & 13 & -2905 & 245 & 0 & -- & -- \\
\hline & 14 & -1665 & 390 & 0 & -- & -- \\
\hline & 15 & 255 & 970 & 11 & -- & -- \\
\hline \multirow[t]{9}{*}{ TXFYO 4} & 3 & -5295 & -- & -- & -- & -- \\
\hline & 4 & -4915 & 380 & 84 & -- & -- \\
\hline & 5 & -3555 & 870 & 30 & -- & -- \\
\hline & 6 & -2245 & 810 & 58 & -- & -- \\
\hline & 7 & -760 & 635 & 23 & -- & -- \\
\hline & 8 & 366 & 1126 & 43 & -- & -- \\
\hline & 13 & -4425 & 490 & 0 & -- & -- \\
\hline & 14 & -3055 & 500 & 0 & -- & -- \\
\hline & 15 & -1395 & 850 & 0 & -- & -- \\
\hline \multirow[t]{6}{*}{$\mathrm{TXGAOI}$} & 7 & -8296 & 2370 & 42 & 107 & 80 \\
\hline & 8 & -4291 & 2400 & 39 & 95 & 56 \\
\hline & 9 & -1576 & 2715 & 48 & 95 & 40 \\
\hline & 10 & -571 & 1005 & 66 & 13 & 29 \\
\hline & 11 & 17 & 588 & 41 & $<10$ & 24 \\
\hline & 16 & -6691 & 1605 & 0 & -- & -- \\
\hline \multirow[t]{5}{*}{$T \times G G 01$} & 3 & -73 & 670 & 34 & $<10$ & 26 \\
\hline & 4 & 72 & 145 & 79 & $<10$ & 22 \\
\hline & 5 & 276 & 114 & 76 & $<10$ & 20 \\
\hline & 12 & -743 & 1510 & 1 & - & -- \\
\hline & 13 & 162 & 90 & 0 & -- & -- \\
\hline \multirow[t]{6}{*}{ TXG001 } & 7 & -2610 & 1080 & 44 & 21 & 47 \\
\hline & 8 & -690 & 1530 & 20 & $<10$ & 35 \\
\hline & 9 & 140 & 830 & 63 & $<10$ & 27 \\
\hline & 10 & 209 & 69 & 17 & -- & 23 \\
\hline & 15 & -3690 & 5070 & 0 & -- & -- \\
\hline & 16 & -2220 & 390 & 0 & -- & -- \\
\hline \multirow[t]{6}{*}{$T \times G 002$} & 7 & -3000 & 440 & 68 & 23 & 51 \\
\hline & 8 & -580 & 960 & 27 & $<10$ & 33 \\
\hline & 9 & 110 & 690 & 49 & $<10$ & 26 \\
\hline & 10 & 200 & 90 & 24 & -- & 23 \\
\hline & 15 & -3440 & 2350 & 0 & -- & -- \\
\hline & 16 & -1540 & 1460 & 0 & -- & -- \\
\hline \multirow[t]{7}{*}{ TXGRO 1} & 3 & -2886 & 4600 & 69 & 86 & 77 \\
\hline & 5 & -1856 & 730 & 49 & $<10$ & 39 \\
\hline & 6 & -326 & 1000 & 43 & $<10$ & 29 \\
\hline & 12 & -7486 & -- & -- & -- & -- \\
\hline & 13 & -2586 & 300 & 0 & -- & -- \\
\hline & 14 & -1326 & 530 & 0 & -- & -- \\
\hline & 15 & 380 & 706 & 11 & -- & -- \\
\hline
\end{tabular}


Table 4--Layer number; altitude of top, thickness, and sand percentage of layer: and average dissolved-solids concentration and temperature of water in layer; by log number--Continued.

\begin{tabular}{|c|c|c|c|c|c|c|}
\hline $\begin{array}{l}\text { Log } \\
\text { number }\end{array}$ & $\begin{array}{r}\text { Layer } \\
\text { number }\end{array}$ & $\begin{array}{r}\text { Altitude } \\
\text { of top } \\
(f \in e t)\end{array}$ & $\begin{array}{r}\text { Thick- } \\
\text { ness } \\
\text { (feet) }\end{array}$ & $\begin{array}{r}\text { Sand } \\
\text { per- } \\
\text { centage }\end{array}$ & $\begin{array}{l}\text { Dis- } \\
\text { solved } \\
\text { solids }\end{array}$ & $\begin{array}{l}\text { Tem- } \\
\text { per- } \\
\text { ature }\end{array}$ \\
\hline \multirow[t]{9}{*}{ TXGRO 2} & 3 & -5017 & 5165 & 68 & 98 & 65 \\
\hline & 5 & -3887 & 800 & 38 & 38 & 51 \\
\hline & 6 & -2252 & 1125 & 47 & 14 & 45 \\
\hline & 7 & -202 & 835 & 24 & $<10$ & 31 \\
\hline & 8 & 315 & 517 & 42 & $<10$ & 23 \\
\hline & 12 & -10182 & -- & -- & - & -- \\
\hline & 13 & -4687 & 330 & 0 & -- & -- \\
\hline & 14 & -3377 & 510 & 0 & -- & -- \\
\hline & 15 & -1037 & 1215 & 6 & -- & - \\
\hline \multirow[t]{6}{*}{ IXGRO3 } & 3 & -2184 & -- & -- & -- & - \\
\hline & 5 & -1294 & 660 & 66 & $<10$ & 37 \\
\hline & 6 & 166 & 1010 & 38 & $<10$ & 26 \\
\hline & 13 & -1954 & 230 & 4 & - & - \\
\hline & 14 & -844 & 450 & 7 & -- & -- \\
\hline & 15 & 355 & 189 & 0 & -- & $\cdots$ \\
\hline \multirow[t]{3}{*}{ IXGUO1 } & 3 & 490 & 995 & 42 & $<10$ & 26 \\
\hline & 4 & 639 & 149 & 99 & $<10$ & 21 \\
\hline & 12 & -505 & 1320 & 0 & -- & -- \\
\hline \multirow[t]{7}{*}{$\mathrm{TXGZO1}$} & 3 & -2428 & 2360 & 49 & 11 & 51 \\
\hline & 4 & -1708 & 720 & 81 & $<10$ & 40 \\
\hline & 5 & -528 & 770 & 31 & $<10$ & 32 \\
\hline & 6 & 290 & 258 & 70 & $<10$ & 24 \\
\hline & 12 & -4788 & -- & -- & - & -- \\
\hline & 13 & -1298 & 410 & 4 & -- & -- \\
\hline & 14 & 32 & 560 & 4 & -- & -- \\
\hline \multirow[t]{9}{*}{ IXGZO2 } & 3 & -4440 & 2900 & 65 & 58 & 75 \\
\hline & 4 & -3730 & 710 & 84 & 37 & 62 \\
\hline & 5 & -2450 & 900 & 16 & 11 & 54 \\
\hline & 6 & -260 & 1475 & 55 & 10 & 37 \\
\hline & 7 & 332 & 277 & 42 & $<10$ & 19 \\
\hline & 12 & -7340 & 2935 & 0 & -- & -- \\
\hline & 13 & -3350 & 380 & 5 & -- & -- \\
\hline & 14 & -1735 & 715 & 3 & -- & -- \\
\hline & 15 & 55 & 315 & 11 & -- & - \\
\hline \multirow[t]{4}{*}{$\mathrm{TXGZO3}$} & 3 & -48 & 1450 & 48 & $<10$ & 34 \\
\hline & 4 & 297 & 345 & 99 & $<10$ & 19 \\
\hline & 12 & -1498 & 1680 & 0 & -- & -- \\
\hline & 13 & 382 & 85 & 5 & -- & -- \\
\hline \multirow[t]{5}{*}{$\mathrm{TXGZO4}$} & 3 & -1437 & 1725 & 43 & $<10$ & 38 \\
\hline & 4 & -507 & 930 & 91 & $<10$ & 31 \\
\hline & 5 & 331 & 688 & 64 & $<10$ & 27 \\
\hline & 12 & -3162 & 2080 & 0 & -- & -- \\
\hline & 13 & -357 & 150 & 0 & -- & - \\
\hline
\end{tabular}


Table 4--Layer number; altitude of top, thickness, and sand percentage of layer; and average dissolved-solids concentration and temperature of water in layer; by log number--Continued.

\begin{tabular}{|c|c|c|c|c|c|c|}
\hline $\begin{array}{l}\text { Log } \\
\text { number }\end{array}$ & $\begin{array}{r}\text { Layer } \\
\text { number }\end{array}$ & $\begin{array}{r}\text { Altitude } \\
\text { of top } \\
\text { (feet) }\end{array}$ & $\begin{array}{r}\text { Thick- } \\
\text { ness } \\
\text { (feet) }\end{array}$ & $\begin{array}{r}\text { Sand } \\
\text { per- } \\
\text { centage }\end{array}$ & $\begin{array}{l}\text { Dis- } \\
\text { solved } \\
\text { solids }\end{array}$ & $\begin{array}{l}\text { Tem- } \\
\text { per- } \\
\text { ature }\end{array}$ \\
\hline \multirow[t]{8}{*}{$\mathrm{TXHAO} 1$} & 6 & -7346 & 1310 & 68 & 93 & 77 \\
\hline & 7 & -3456 & 2290 & 61 & 118 & 50 \\
\hline & 8 & -1656 & 1800 & 49 & $<10$ & 36 \\
\hline & 9 & -896 & 760 & 44 & $<10$ & 29 \\
\hline & 10 & -346 & 550 & 71 & $<10$ & 25 \\
\hline & 11 & 65 & 411 & 92 & $<10$ & 23 \\
\hline & 14 & -8656 & 875 & 0 & -- & -- \\
\hline & 15 & -5746 & 1600 & 0 & -- & - \\
\hline \multirow[t]{8}{*}{$\mathrm{TXHAO} 2$} & 6 & -6277 & 1215 & 61 & 71 & 68 \\
\hline & 7 & -2642 & 2200 & 50 & 45 & 50 \\
\hline & 8 & -1012 & 1630 & 44 & $<10$ & 36 \\
\hline & 9 & -462 & 550 & 67 & $<10$ & 27 \\
\hline & 10 & -97 & 365 & 65 & $<10$ & 23 \\
\hline & 11 & 82 & 179 & 76 & $<10$ & 20 \\
\hline & 14 & -7492 & 1400 & 0 & -- & -- \\
\hline & 15 & -4842 & 1435 & 0 & -- & - \\
\hline \multirow[t]{2}{*}{ TXHEO 1} & 3 & 444 & 804 & 63 & $<10$ & 23 \\
\hline & 12 & -360 & 1645 & 2 & -- & $\ldots$ \\
\hline \multirow[t]{5}{*}{ ТXHEO2 } & 3 & 298 & 980 & 73 & $<10$ & 25 \\
\hline & 4 & 368 & 70 & 99 & $<10$ & 20 \\
\hline & 5 & 448 & 15 & 40 & -- & 19 \\
\hline & 12 & -682 & 1620 & 0 & -- & - \\
\hline & 13 & 433 & 65 & 18 & -- & -- \\
\hline \multirow[t]{6}{*}{ TXHI01 } & 7 & -3432 & 2775 & 33 & 96 & 63 \\
\hline & 8 & -1832 & 1600 & 5 & - & 46 \\
\hline & 9 & -632 & 1200 & 33 & 13 & 35 \\
\hline & 10 & -102 & 530 & 48 & - & 28 \\
\hline & 11 & 298 & 400 & 60 & $<10$ & 24 \\
\hline & 15 & -6207 & 580 & 0 & - & $\cdots$ \\
\hline \multirow[t]{5}{*}{ TXHIO 2} & 7 & -6323 & 3690 & 35 & 92 & 79 \\
\hline & 8 & -3223 & 3100 & 17 & 55 & 58 \\
\hline & 9 & -1298 & 1925 & 43 & 10 & 41 \\
\hline & 10 & -443 & 855 & 61 & $<10$ & 32 \\
\hline & 11 & 72 & 515 & 47 & $<10$ & 27 \\
\hline \multirow[t]{5}{*}{ TXHIO3 } & 7 & -5442 & 2845 & 36 & -- & 81 \\
\hline & 8 & -2697 & 2745 & 6 & -- & 58 \\
\hline & 9 & -1032 & 1665 & 33 & 12 & 40 \\
\hline & 10 & -312 & 720 & 59 & $<10$ & 31 \\
\hline & 11 & 94 & 406 & 55 & $<10$ & 26 \\
\hline \multirow[t]{6}{*}{ TXHIO 4} & 7 & -7280 & 845 & 76 & -- & $\ldots$ \\
\hline & 8 & -2945 & 3695 & 52 & - & - \\
\hline & 9 & -1285 & 1660 & 55 & -- & - - \\
\hline & 10 & -480 & 805 & 41 & $<10$ & 32 \\
\hline & 11 & 56 & 536 & 58 & $<10$ & 27 \\
\hline & 16 & -6640 & 640 & 15 & - & - \\
\hline
\end{tabular}


Table 4--Layer number; altitude of top, thickness, and sand percentage of layer; and average dissolved-solids concentration and temperature of water in layer; by log number--Continued.

\begin{tabular}{|c|c|c|c|c|c|c|}
\hline $\begin{array}{l}\text { Log } \\
\text { number }\end{array}$ & $\begin{array}{r}\text { Layer } \\
\text { number }\end{array}$ & $\begin{array}{r}\text { Altitude } \\
\text { of top } \\
(\text { feet) }\end{array}$ & $\begin{array}{r}\text { Thick- } \\
\text { ness } \\
\text { (feet) }\end{array}$ & $\begin{array}{r}\text { Sand } \\
\text { per- } \\
\text { centage }\end{array}$ & $\begin{array}{l}\text { Dis- } \\
\text { solved } \\
\text { solids }\end{array}$ & $\begin{array}{l}\text { Tem- } \\
\text { per- } \\
\text { ature }\end{array}$ \\
\hline \multirow[t]{5}{*}{ TXHNO 1} & 3 & 110 & 670 & 36 & $<10$ & 27 \\
\hline & 4 & 260 & 150 & 87 & $<10$ & 22 \\
\hline & 5 & 368 & 28 & 38 & -- & 20 \\
\hline & 12 & -560 & 1330 & 3 & -- & -- \\
\hline & 13 & 340 & 80 & 4 & -- & -- \\
\hline \multirow[t]{2}{*}{ TXHNO2 } & 3 & 309 & 300 & 74 & $<10$ & 22 \\
\hline & 12 & 9 & 1270 & 2 & -- & - \\
\hline \multirow[t]{5}{*}{ TXHNO3 } & 3 & 60 & 660 & 56 & $<10$ & 26 \\
\hline & 4 & 280 & 220 & 95 & $<10$ & 22 \\
\hline & 5 & 372 & 72 & 56 & $<10$ & 21 \\
\hline & 12 & -600 & 1480 & 2 & -- & - \\
\hline & 13 & 300 & 20 & 0 & -- & - \\
\hline \multirow[t]{2}{*}{$\mathrm{TXHNO}_{4}$} & 3 & 338 & 350 & 58 & -- & -- \\
\hline & 12 & -12 & 1280 & 1 & -- & - \\
\hline TXHOO & 12 & 406 & 564 & 6 & -- & -- \\
\hline \multirow[t]{9}{*}{ IXHRO 1} & 6 & -6795 & 1135 & 32 & 50 & 72 \\
\hline & 7 & -4575 & 925 & 38 & 65 & 57 \\
\hline & 8 & -2125 & 1815 & 24 & -- & 43 \\
\hline & 9 & -900 & 1225 & 49 & $<10$ & 32 \\
\hline & 10 & -295 & 605 & 77 & $<10$ & 25 \\
\hline & 11 & 96 & 391 & 61 & $<10$ & 21 \\
\hline & 14 & -7930 & 750 & 0 & - - & -- \\
\hline & 15 & -5500 & 1295 & 0 & -- & - \\
\hline & 16 & -3940 & 635 & 7 & -- & -- \\
\hline \multirow[t]{9}{*}{ TXHRO 2} & 3 & -8966 & 750 & 49 & -- & -- \\
\hline & 6 & -5726 & 1065 & 51 & -- & -- \\
\hline & 7 & -3211 & 875 & 36 & -- & -. \\
\hline & 8 & -1576 & 1635 & 22 & -- & -- \\
\hline & 9 & -476 & 1100 & 30 & -- & - \\
\hline & 10 & 14 & 490 & 80 & -- & -- \\
\hline & 11 & 161 & 147 & 37 & -- & -- \\
\hline & 14 & -6791 & 2175 & 0 & -- & - \\
\hline & 15 & -4086 & 1640 & 0 & -- & -- \\
\hline \multirow[t]{9}{*}{ TXHRO 3} & 6 & -6535 & 1060 & 31 & 50 & 65 \\
\hline & 7 & -3945 & 1030 & 44 & 43 & 49 \\
\hline & 8 & -2015 & 1680 & 16 & 13 & 39 \\
\hline & 9 & -875 & 1140 & 47 & 10 & 30 \\
\hline & 10 & -280 & 595 & 78 & -- & 25 \\
\hline & 11 & 91 & 371 & 78 & -- & 22 \\
\hline & 14 & -7595 & 1900 & 0 & -- & -- \\
\hline & 15 & -4975 & 1560 & 0 & -- & -- \\
\hline & 16 & -3695 & 250 & 7 & -- & -- \\
\hline
\end{tabular}


Table 4--Layer number; altitude of top, thickness, and sand percentage of layer: and average dissolved-solids concentration and temperature of water in layer; by log number--Continued.

\begin{tabular}{|c|c|c|c|c|c|c|}
\hline $\begin{array}{l}\text { L } \circ 8 \\
\text { number }\end{array}$ & $\begin{array}{r}\text { Layer } \\
\text { number }\end{array}$ & $\begin{array}{r}\text { Altitude } \\
\text { of top } \\
(f e e t)\end{array}$ & $\begin{array}{r}\text { Thick- } \\
\text { ness } \\
(\text { feet })\end{array}$ & $\begin{array}{r}\text { Sand } \\
\text { per- } \\
\text { centage }\end{array}$ & $\begin{array}{l}\text { Dis- } \\
\text { solved } \\
\text { solids }\end{array}$ & $\begin{array}{l}\text { Tem- } \\
\text { per- } \\
\text { ature }\end{array}$ \\
\hline \multirow[t]{8}{*}{$\mathrm{TXHRO}_{4}$} & 6 & -8293 & 235 & 38 & -- & -- \\
\hline & 7 & -5413 & 1115 & 35 & 65 & 59 \\
\hline & 8 & -2488 & 2020 & 42 & 22 & 43 \\
\hline & 9 & -1128 & 1360 & 35 & $<10$ & 33 \\
\hline & 10 & -393 & 735 & 57 & $<10$ & 26 \\
\hline & 11 & 61 & 454 & 51 & $<10$ & 22 \\
\hline & 15 & -6528 & 1765 & 1 & -- & -- \\
\hline & 16 & -4508 & 905 & 10 & -- & -- \\
\hline \multirow[t]{5}{*}{ TXHSOI } & 3 & -217 & 2390 & 51 & $<10$ & 35 \\
\hline & 4 & -42 & 175 & 99 & $<10$ & 24 \\
\hline & 5 & 262 & 139 & 60 & $<10$ & 21 \\
\hline & 12 & -2607 & 1295 & 0 & -- & -- \\
\hline & 13 & 123 & 165 & 8 & -- & -- \\
\hline \multirow[t]{5}{*}{ TXESO2 } & 3 & -570 & 2775 & 51 & -- & 36 \\
\hline & 4 & -365 & 205 & 98 & $<10$ & 26 \\
\hline & 5 & 214 & 379 & 73 & $<10$ & 23 \\
\hline & 12 & -3345 & 1170 & 0 & -- & -- \\
\hline & 13 & -165 & 200 & 9 & -- & -- \\
\hline \multirow[t]{6}{*}{$\mathrm{TXHSO} 3$} & 3 & -985 & 3180 & 56 & $<10$ & 48 \\
\hline & 4 & -735 & 250 & 99 & $<10$ & 31 \\
\hline & 5 & 85 & 610 & 71 & $<10$ & 25 \\
\hline & 12 & -4165 & 1520 & 0 & -- & -- \\
\hline & 13 & -525 & 210 & 0 & -- & -- \\
\hline & 14 & 375 & 290 & 11 & -- & -- \\
\hline \multirow[t]{7}{*}{$\mathrm{TXJAOI}$} & 7 & -5208 & -- & -- & -- & -- \\
\hline & 8 & -2683 & 1740 & 39 & 37 & 43 \\
\hline & 9 & -763 & 1180 & 39 & $<10$ & 30 \\
\hline & 10 & -323 & 440 & 65 & $<10$ & 26 \\
\hline & 11 & 77 & 400 & 56 & $<10$ & 23 \\
\hline & 16 & -4423 & 785 & 11 & -- & -- \\
\hline & 17 & -1943 & 740 & 7 & -- & -- \\
\hline \multirow[t]{7}{*}{ TXJAO2 } & 7 & -5182 & 3585 & 64 & 69 & 61 \\
\hline & 8 & -3797 & 780 & 48 & 59 & 46 \\
\hline & 9 & -1062 & 1400 & 41 & 25 & 32 \\
\hline & 10 & -467 & 595 & 63 & $<10$ & 27 \\
\hline & 11 & 35 & 502 & 50 & $<10$ & 24 \\
\hline & 16 & -4577 & 605 & 0 & -- & -- \\
\hline & 17 & -2462 & 1335 & 12 & -- & -- \\
\hline \multirow[t]{5}{*}{ TXJEO1 } & 8 & -3965 & 3060 & 57 & 91 & 51 \\
\hline & 9 & -2045 & 1920 & 42 & 54 & 38 \\
\hline & 10 & -800 & 1245 & 50 & 30 & 29 \\
\hline & 11 & 13 & 813 & 47 & -- & 24 \\
\hline & 16 & -7025 & 750 & 2 & -- & -- \\
\hline
\end{tabular}


Table 4--Layer number; altitude of top, thickness, and sand percentage of layer; and average dissolved-solids concentration and temperature of water in layer; by log number--Continued.

\begin{tabular}{|c|c|c|c|c|c|c|}
\hline $\begin{array}{l}\text { Log } \\
\text { number }\end{array}$ & $\begin{array}{r}\text { Layer } \\
\text { number }\end{array}$ & $\begin{array}{r}\text { Altitude } \\
\text { of top } \\
(f e e t)\end{array}$ & $\begin{array}{r}\text { Thick- } \\
\text { ness } \\
\text { (feet) }\end{array}$ & $\begin{array}{r}\text { Sand } \\
\text { per- } \\
\text { centage }\end{array}$ & $\begin{array}{l}\text { Dis- } \\
\text { solved } \\
\text { solids }\end{array}$ & $\begin{array}{l}\text { Tem- } \\
\text { per- } \\
\text { ature }\end{array}$ \\
\hline \multirow[t]{6}{*}{ TXJHOI } & 5 & -5170 & 990 & 45 & -- & -- \\
\hline & 6 & -1665 & 2005 & 54 & -- & - \\
\hline & 7 & 415 & 1105 & 28 & $<10$ & 14 \\
\hline & 8 & 819 & 404 & 43 & $<10$ & -- \\
\hline & 14 & -3670 & 1500 & 0 & -- & -- \\
\hline & 15 & -690 & 975 & 0 & -- & $\cdots$ \\
\hline \multirow[t]{5}{*}{ TXJHO2 } & 7 & -2057 & 1750 & 37 & $<10$ & 49 \\
\hline & 8 & -807 & 1250 & 27 & $<10$ & 37 \\
\hline & 9 & 133 & 940 & 75 & $<10$ & 28 \\
\hline & 10 & 442 & 309 & 63 & $<10$ & 24 \\
\hline & 15 & -3807 & 740 & 8 & -- & -- \\
\hline \multirow[t]{8}{*}{ TXJSOI } & 3 & -3205 & 3840 & 57 & -- & -- \\
\hline & 5 & -2595 & 235 & 74 & -- & -- \\
\hline & 6 & -1070 & 1150 & 48 & -- & -- \\
\hline & 7 & 109 & 289 & 58 & -- & -- \\
\hline & 12 & -7045 & 835 & 0 & -- & -- \\
\hline & 13 & -2830 & 375 & 11 & -- & $\cdots$ \\
\hline & 14 & -2220 & 375 & 10 & -- & -- \\
\hline & 15 & -180 & 890 & 0 & -- & -- \\
\hline \multirow[t]{8}{*}{ TXJSO2 } & 3 & -5148 & -- & -- & -- & $\cdots$ \\
\hline & 5 & -4468 & 225 & 53 & 46 & 55 \\
\hline & 6 & -2808 & 1290 & 59 & 26 & 46 \\
\hline & 7 & -818 & 1205 & 28 & 13 & 31 \\
\hline & 8 & 170 & 988 & 81 & $<10$ & 22 \\
\hline & 13 & -4693 & 455 & 3 & - & -- \\
\hline & 14 & -4098 & 370 & 0 & -- & -- \\
\hline & 15 & -2023 & 785 & 0 & -- & -- \\
\hline \multirow[t]{8}{*}{$\mathrm{TXJSO} 3$} & 6 & -6786 & 870 & 47 & -- & 68 \\
\hline & 7 & -2901 & 2275 & 63 & 47 & 44 \\
\hline & 8 & -1171 & 1730 & 60 & $<10$ & 32 \\
\hline & 9 & -549 & 622 & 74 & $<10$ & 26 \\
\hline & 10 & -126 & 423 & 94 & $<10$ & 23 \\
\hline & 11 & 90 & 216 & 51 & $<10$ & 21 \\
\hline & 14 & -7656 & -- & -- & -- & -- \\
\hline & 15 & -5176 & 1610 & 3 & -- & - \\
\hline \multirow[t]{7}{*}{ TXJSO 4} & 7 & -5095 & 1735 & 62 & 50 & 41 \\
\hline & 8 & -2405 & 2335 & 72 & 37 & 32 \\
\hline & 9 & -1215 & 1190 & 41 & $<10$ & 27 \\
\hline & 10 & -455 & 760 & 70 & $<10$ & 24 \\
\hline & 11 & 35 & 490 & 69 & $<10$ & 23 \\
\hline & 15 & -6830 & $\cdots$ & $\cdots$ & - & -- \\
\hline & 16 & -4740 & 355 & 5 & -- & -- \\
\hline
\end{tabular}


Table 4--Layer number; altitude of top, thickness, and sand percentage of layer; and average dissolved-solids concentration and temperature of water in layer: by log number--Continued.

\begin{tabular}{|c|c|c|c|c|c|c|}
\hline $\begin{array}{l}\log \\
\text { number }\end{array}$ & $\begin{array}{r}\text { Layer } \\
\text { number }\end{array}$ & $\begin{array}{r}\text { Altide } \\
\text { of top } \\
(\text { feet) }\end{array}$ & $\begin{array}{r}\text { Thick- } \\
\text { ness } \\
(\text { feet) }\end{array}$ & $\begin{array}{r}\text { Sand } \\
\text { per- } \\
\text { centage }\end{array}$ & $\begin{array}{l}\text { Dis- } \\
\text { solved } \\
\text { solids }\end{array}$ & $\begin{array}{l}\text { Tem- } \\
\text { per- } \\
\text { ature }\end{array}$ \\
\hline \multirow[t]{6}{*}{ TXJWOI } & 7 & -2866 & 1130 & 11 & 13 & 54 \\
\hline & 8 & -706 & 1200 & 23 & $<10$ & 36 \\
\hline & 9 & 159 & 865 & 61 & $<10$ & 25 \\
\hline & 10 & 302 & 143 & 59 & $<10$ & 19 \\
\hline & 15 & -3996 & 890 & 7 & -- & - \\
\hline & 16 & -1906 & 960 & 13 & -- & -- \\
\hline \multirow[t]{6}{*}{ TXJWO 2} & 7 & -3938 & 3055 & 30 & -- & -- \\
\hline & 8 & -1653 & 1420 & 26 & -- & - \\
\hline & 9 & -668 & 985 & 17 & -- & - \\
\hline & 10 & -203 & 465 & 98 & -- & -- \\
\hline & 11 & 129 & 332 & 42 & -- & -- \\
\hline & 16 & -3073 & 865 & 9 & -- & -- \\
\hline \multirow[t]{7}{*}{ TXKAO1 } & 3 & -5848 & 1975 & 51 & -- & -- \\
\hline & 6 & -1583 & 2045 & 40 & -- & 44 \\
\hline & 7 & -673 & 15 & 20 & -- & 32 \\
\hline & 8 & 258 & 611 & 53 & $<10$ & 28 \\
\hline & 14 & -3628 & 2220 & 1 & - & -- \\
\hline & 15 & -688 & 895 & 6 & -- & -- \\
\hline & 16 & -353 & 320 & 17 & -- & -- \\
\hline \multirow[t]{8}{*}{$\mathrm{TXKAO} 2$} & 3 & -6449 & -- & -- & -- & -- \\
\hline & 6 & -1839 & 2055 & 30 & 17 & 48 \\
\hline & 7 & -914 & 10 & 20 & -- & 34 \\
\hline & 8 & 76 & 655 & 46 & $<10$ & 29 \\
\hline & 9 & 311 & 235 & 5 & -- & 26 \\
\hline & 14 & -3894 & 2555 & 4 & -- & - \\
\hline & 15 & -924 & 915 & 7 & -- & -- \\
\hline & 16 & -579 & 335 & 15 & -- & -- \\
\hline \multirow[t]{7}{*}{ TXKEO1 } & 7 & -8442 & 2040 & 56 & -- & - \\
\hline & 8 & -3562 & 3880 & 54 & -- & -- \\
\hline & 9 & -1697 & 1295 & 34 & -- & -- \\
\hline & 10 & -592 & 1105 & 44 & -- & -- \\
\hline & 11 & -10 & 582 & 28 & -- & -- \\
\hline & 16 & -7442 & 1000 & 4 & -- & - \\
\hline & 17 & -2992 & 570 & 2 & -- & -- \\
\hline \multirow[t]{6}{*}{ TXKEO 2} & 7 & -6520 & 4450 & 48 & 52 & 83 \\
\hline & 8 & -2970 & 3550 & 49 & 40 & 59 \\
\hline & 9 & -1400 & 1120 & 60 & -- & 41 \\
\hline & 10 & -580 & 820 & 35 & $<10$ & 32 \\
\hline & 11 & 10 & 590 & 60 & $<10$ & 25 \\
\hline & 17 & -2520 & 450 & 7 & -- & - \\
\hline \multirow[t]{5}{*}{ IXKEO3 } & 7 & -6183 & 3765 & 46 & -- & 79 \\
\hline & 8 & -3348 & 2835 & 40 & 25 & 61 \\
\hline & 9 & -1318 & 2030 & 34 & -- & 47 \\
\hline & 10 & -518 & 800 & 45 & $<10$ & 32 \\
\hline & 11 & 31 & 549 & 64 & $<10$ & 24 \\
\hline
\end{tabular}


Table 4--Layer number; altitude of top, thickness, and sand percentage of layer; and average dissolved-solids concentration and temperature of water in layer; by log number--Continued.

\begin{tabular}{|c|c|c|c|c|c|c|}
\hline $\begin{array}{l}\log \\
\text { number }\end{array}$ & $\begin{array}{r}\text { Layer } \\
\text { number }\end{array}$ & $\begin{array}{r}\text { Altitude } \\
\text { of top } \\
(f e e t)\end{array}$ & $\begin{array}{c}\text { Thlck- } \\
\text { ness } \\
\text { (feet) }\end{array}$ & $\begin{array}{r}\text { Sand } \\
\text { per- } \\
\text { centage }\end{array}$ & $\begin{array}{l}\text { Dis- } \\
\text { solved } \\
\text { solids }\end{array}$ & $\begin{array}{r}\text { Tem- } \\
\text { per- } \\
\text { ature }\end{array}$ \\
\hline \multirow[t]{5}{*}{$\mathrm{TXKEO}_{4}$} & 7 & -5467 & 3825 & 39 & 83 & 74 \\
\hline & 8 & -3102 & 2365 & 52 & 52 & 54 \\
\hline & 9 & -1177 & 1925 & 42 & 33 & 42 \\
\hline & 10 & -517 & 660 & 62 & $<10$ & 31 \\
\hline & 11 & 43 & 560 & 37 & $<10$ & 25 \\
\hline \multirow[t]{7}{*}{ TXKE05 } & 7 & -6924 & 3960 & 63 & 74 & 84 \\
\hline & 8 & -3244 & 3080 & 54 & 57 & 61 \\
\hline & 9 & -1529 & 1285 & 65 & 27 & 46 \\
\hline & 10 & -629 & 900 & 42 & $<10$ & 35 \\
\hline & 11 & 5 & 634 & 48 & 12 & 24 \\
\hline & 16 & -6324 & 600 & 5 & -- & -- \\
\hline & 17 & -2814 & 430 & 6 & -- & -- \\
\hline TXKFO1 & 12 & 360 & 784 & 5 & -- & -- \\
\hline \multirow[t]{7}{*}{ TXKL01 } & 7 & -7898 & 1080 & 52 & -- & - \\
\hline & 8 & -4328 & 2600 & 55 & -- & - \\
\hline & 9 & -1623 & 2155 & 37 & 37 & - \\
\hline & 10 & -628 & 995 & 49 & 34 & 32 \\
\hline & 11 & 0 & 628 & 61 & -- & 25 \\
\hline & 16 & -6928 & 970 & 0 & -- & -. \\
\hline & 17 & -3778 & 550 & 26 & -- & $\cdots$ \\
\hline \multirow[t]{6}{*}{ TXKLO2 } & 7 & -5659 & 3790 & 39 & -- & -- \\
\hline & 8 & -3079 & 2380 & 51 & -- & -- \\
\hline & 9 & -1224 & 1855 & 13 & -- & -. \\
\hline & 10 & -539 & 685 & 51 & -- & -. \\
\hline & 11 & 32 & 571 & 18 & -- & -- \\
\hline & 16 & -5459 & 200 & 0 & -- & -- \\
\hline \multirow[t]{5}{*}{ TXLEOI } & 3 & -452 & 2570 & 52 & $<10$ & 34 \\
\hline & 4 & -162 & 290 & 72 & $<10$ & 26 \\
\hline & 5 & 453 & 515 & 52 & $<10$ & 23 \\
\hline & 12 & -3022 & 2095 & 0 & - & $\therefore$ \\
\hline & 13 & -62 & 100 & 0 & -- & -- \\
\hline \multirow[t]{9}{*}{ TXLIO1 } & 3 & -9026 & -- & -. & -- & - - \\
\hline & 6 & -5796 & 1180 & 79 & 73 & 64 \\
\hline & 7 & -2636 & 1885 & 32 & 43 & 48 \\
\hline & 8 & -1116 & 1520 & 33 & $<10$ & 38 \\
\hline & 9 & -346 & 770 & 18 & $<10$ & 26 \\
\hline & 10 & -26 & 320 & 70 & $<10$ & 20 \\
\hline & 11 & 140 & 166 & 67 & - & 17 \\
\hline & 14 & -6976 & 2050 & 0 & -- & - - \\
\hline & 15 & -4521 & 1275 & 0 & -- & -- \\
\hline \multirow[t]{7}{*}{ TXLIO2 } & 7 & -6722 & 1990 & 66 & 86 & 69 \\
\hline & 8 & -3412 & 2760 & 47 & 65 & 57 \\
\hline & 9 & -1522 & 1890 & 52 & 30 & 44 \\
\hline & 10 & -572 & 950 & 41 & $<10$ & 29 \\
\hline & 11 & 33 & 605 & 65 & $<10$ & 21 \\
\hline & 15 & -8712 & 440 & 0 & -- & -- \\
\hline & 16 & -6172 & 550 & 0 & -- & -- \\
\hline
\end{tabular}


Table 4--Layer number; altitude of top, thickness, and sand percentage of layer; and average dissolved-solids concentration and temperature of water in layer; by log number--Continued.

\begin{tabular}{|c|c|c|c|c|c|c|}
\hline $\begin{array}{l}\text { Log } \\
\text { number }\end{array}$ & $\begin{array}{r}\text { Layer } \\
\text { number }\end{array}$ & $\begin{array}{r}\text { Altitude } \\
\text { of top } \\
\text { (feet) }\end{array}$ & $\begin{array}{r}\text { Thick- } \\
\text { ness } \\
\text { (feet) }\end{array}$ & $\begin{array}{r}\text { Sand } \\
\text { per- } \\
\text { centage }\end{array}$ & $\begin{array}{l}\text { Dis- } \\
\text { solved } \\
\text { solids }\end{array}$ & $\begin{array}{l}\text { Tem- } \\
\text { per- } \\
\text { ature }\end{array}$ \\
\hline \multirow[t]{9}{*}{$\mathrm{TXLIO} 3$} & 3 & -9306 & 660 & 64 & -- & -- \\
\hline & 6 & -5846 & 1355 & 64 & -- & -- \\
\hline & 7 & -2996 & 1650 & 23 & -- & -- \\
\hline & 8 & -1396 & 1600 & 40 & $<10$ & -- \\
\hline & 9 & -501 & 895 & 23 & $<10$ & 33 \\
\hline & 10 & -86 & 415 & 94 & $<10$ & 19 \\
\hline & 11 & 123 & 209 & 48 & $<10$ & - \\
\hline & 14 & -7201 & 2105 & 0 & -- & -- \\
\hline & 15 & -4646 & 1200 & 0 & -- & -- \\
\hline \multirow[t]{6}{*}{$\mathrm{TXIO}_{4}$} & 7 & -4170 & 1900 & 43 & 91 & 62 \\
\hline & 8 & -2230 & 1940 & 35 & 33 & 53 \\
\hline & 9 & -995 & 1235 & 48 & $<10$ & 41 \\
\hline & 10 & -365 & 630 & 62 & $<10$ & 26 \\
\hline & 11 & 72 & 437 & 38 & $<10$ & 17 \\
\hline & 15 & -6070 & 1845 & 0 & -- & -- \\
\hline \multirow[t]{7}{*}{ TXLIOS } & 7 & -4967 & 1970 & 74 & 111 & 65 \\
\hline & 8 & -2487 & 2190 & 37 & 58 & 52 \\
\hline & 9 & -1187 & 1300 & 20 & $<10$ & 38 \\
\hline & 10 & -437 & 750 & 82 & $<10$ & 27 \\
\hline & 11 & 46 & 483 & 40 & $<10$ & 20 \\
\hline & 15 & -6937 & 500 & 3 & -- & -- \\
\hline & 16 & -4677 & 290 & 4 & -- & -- \\
\hline \multirow[t]{2}{*}{ TXLMOI } & 3 & 396 & 653 & 59 & $<10$ & 22 \\
\hline & 12 & -257 & 900 & 3 & -- & -- \\
\hline \multirow[t]{5}{*}{ TXLNO1 } & 3 & 54 & 1800 & 75 & $<10$ & 30 \\
\hline & 4 & 219 & 165 & 91 & $<10$ & 22 \\
\hline & 5 & 460 & 101 & 78 & $<10$ & 20 \\
\hline & 12 & -1746 & 1280 & 0 & -- & -- \\
\hline & 13 & 359 & 140 & 13 & -- & -- \\
\hline \multirow[t]{6}{*}{ TXLNO2 } & 3 & -992 & 2965 & 66 & $<10$ & 45 \\
\hline & 4 & -757 & 235 & 99 & $<10$ & 31 \\
\hline & 5 & 158 & 675 & 70 & $<10$ & 25 \\
\hline & 12 & -3957 & 1850 & 0 & -- & -- \\
\hline & 13 & -517 & 240 & 0 & -- & -- \\
\hline & 14 & 251 & 93 & 0 & -- & -- \\
\hline \multirow[t]{5}{*}{$\mathrm{TXLNO}_{3}$} & 3 & -578 & 2420 & 63 & $<10$ & 40 \\
\hline & 4 & -338 & 240 & 90 & $<10$ & 27 \\
\hline & 5 & 479 & 557 & 73 & $<10$ & 21 \\
\hline & 12 & -2998 & 940 & 0 & -- & -- \\
\hline & 13 & -78 & 260 & 17 & -- & -- \\
\hline \multirow[t]{10}{*}{ TXLOOO1 } & 3 & -6001 & -- & -- & -- & $=$ \\
\hline & 4 & -5386 & 615 & 75 & $<10$ & 64 \\
\hline & 5 & -3976 & 520 & 34 & -- & 53 \\
\hline & 6 & -481 & 2105 & 49 & $<10$ & 37 \\
\hline & 7 & -281 & 30 & 13 & -- & 28 \\
\hline & 8 & 160 & 216 & 12 & -- & 25 \\
\hline & 13 & -4496 & 890 & 0 & -- & -- \\
\hline & 14 & -2586 & 1390 & 0 & -- & -- \\
\hline & 15 & -311 & 170 & 0 & -- & -- \\
\hline & 16 & -56 & 225 & 12 & -- & -- \\
\hline
\end{tabular}


Table 4--Layer number; altitude of top, thickness, and sand percentage of layer; and average dissolved-solids concentration and temperature of water in layer; by log number--Continued.

\begin{tabular}{|c|c|c|c|c|c|c|}
\hline $\begin{array}{l}\text { Log } \\
\text { number }\end{array}$ & $\begin{array}{r}\text { Layer } \\
\text { number }\end{array}$ & $\begin{array}{r}\text { Altitude } \\
\text { of top } \\
\text { ( } f \in e t)\end{array}$ & $\begin{array}{r}\text { Thick- } \\
\text { ness } \\
\text { (feet) }\end{array}$ & $\begin{array}{r}\text { Sand } \\
\text { per- } \\
\text { centage }\end{array}$ & $\begin{array}{l}\text { Dis- } \\
\text { solved } \\
\text { solids }\end{array}$ & $\begin{array}{l}\text { Tem- } \\
\text { per- } \\
\text { ature }\end{array}$ \\
\hline \multirow[t]{8}{*}{$\mathrm{TXLO02}$} & 4 & -8118 & 590 & 50 & -- & - \\
\hline & 6 & -2558 & 2120 & 33 & -- & 61 \\
\hline & 7 & -1628 & 40 & 7 & $\ldots$ & 42 \\
\hline & 8 & 2 & 610 & 65 & $<10$ & 29 \\
\hline & 9 & 211 & 209 & 34 & -- & 25 \\
\hline & 14 & -4678 & 3440 & 0 & -- & - \\
\hline & 15 & -1668 & 890 & 4 & -- & - \\
\hline & 16 & -608 & 1020 & 8 & -- & -- \\
\hline \multirow[t]{7}{*}{ TXLO03 } & 6 & -2238 & 2270 & 47 & 58 & 56 \\
\hline & 7 & -1168 & 20 & 0 & -- & 41 \\
\hline & 8 & 202 & 565 & 59 & $<10$ & 27 \\
\hline & 9 & 392 & 190 & 24 & - & 20 \\
\hline & 14 & -4508 & 1480 & 0 & -- & -- \\
\hline & 15 & -1188 & 1050 & 0 & - & -. \\
\hline & 16 & -363 & 805 & 2 & -- & - \\
\hline \multirow[t]{6}{*}{ TXLSO 1} & 3 & -1467 & 1510 & 60 & $<10$ & 44 \\
\hline & 4 & -747 & 720 & 73 & $<10$ & 37 \\
\hline & 5 & 453 & 1102 & 42 & $<10$ & 31 \\
\hline & 12 & -2977 & 1420 & 2 & -- & -- \\
\hline & 13 & -649 & 98 & 15 & -- & - \\
\hline & 14 & 544 & 91 & 35 & -- & -- \\
\hline \multirow[t]{6}{*}{$\mathrm{TXLSO} 2$} & 3 & -2404 & 1970 & 46 & $<10$ & 53 \\
\hline & 4 & -1304 & 1100 & 79 & $<10$ & 45 \\
\hline & 5 & 261 & 445 & 79 & $<10$ & 29 \\
\hline & 12 & -4374 & -- & -- & -- & $\cdots$ \\
\hline & 13 & -184 & 1120 & 12 & -- & -- \\
\hline & 14 & 496 & 235 & 0 & -- & - \\
\hline \multirow[t]{6}{*}{$\operatorname{TXLSO3}$} & 3 & -2685 & 1520 & 55 & $<10$ & 48 \\
\hline & 4 & -1785 & 900 & 76 & $<10$ & 42 \\
\hline & 5 & -75 & 1435 & 62 & $<10$ & 34 \\
\hline & 12 & -4205 & 1200 & 1 & -- & -- \\
\hline & 13 & -1510 & 275 & 42 & -- & -- \\
\hline & 14 & 435 & 510 & 10 & -- & -- \\
\hline \multirow[t]{7}{*}{$\mathrm{TXLSO} 4$} & 3 & -3331 & 1900 & 37 & $<10$ & 57 \\
\hline & 4 & -2551 & 780 & 88 & $<10$ & 48 \\
\hline & 5 & -1421 & 1045 & 83 & $<10$ & 42 \\
\hline & 6 & 382 & 653 & 30 & $<10$ & 30 \\
\hline & 12 & -5231 & - & -- & -- & -- \\
\hline & 13 & -2466 & 85 & 0 & -- & -- \\
\hline & 14 & -271 & 1150 & 18 & -- & - \\
\hline \multirow[t]{7}{*}{ TXLV01 } & 3 & -4790 & 3155 & 52 & 75 & 66 \\
\hline & 6 & -1625 & 1450 & 40 & $<10$ & 39 \\
\hline & 7 & -40 & 875 & 29 & $<10$ & 27 \\
\hline & 8 & 360 & 400 & 28 & $<10$ & 22 \\
\hline & 12 & -7945 & - & -- & -- & -- \\
\hline & 14 & -3075 & 1715 & 3 & -- & - \\
\hline & 15 & -915 & 710 & 11 & -- & - \\
\hline
\end{tabular}


Table 4--Layer number; altitude of top, thickness, and sand percentage of layer; and average dissolved-solids concentration and temperature of water in layer; by log number--Continued.

\begin{tabular}{|c|c|c|c|c|c|c|}
\hline $\begin{array}{l}\text { Log } \\
\text { number }\end{array}$ & $\begin{array}{r}\text { Layer } \\
\text { number }\end{array}$ & $\begin{array}{r}\text { Altitude } \\
\text { of top } \\
\text { (feet) }\end{array}$ & $\begin{array}{r}\text { Thick- } \\
\text { ness } \\
\text { (feet) }\end{array}$ & $\begin{array}{r}\text { Sand } \\
\text { per- } \\
\text { centage }\end{array}$ & $\begin{array}{l}\text { Dis- } \\
\text { solved } \\
\text { solids }\end{array}$ & $\begin{array}{l}\text { Tem- } \\
\text { per- } \\
\text { ature }\end{array}$ \\
\hline \multirow[t]{8}{*}{ TXLVO2 } & 3 & -6106 & 3180 & 58 & 53 & 77 \\
\hline & 6 & -3236 & 885 & 31 & $<10$ & 56 \\
\hline & 7 & -1511 & 610 & 13 & -- & 46 \\
\hline & 8 & -226 & 1285 & 26 & $<10$ & 35 \\
\hline & 9 & 200 & 426 & 36 & $<10$ & 22 \\
\hline & 12 & -9286 & -- & -- & -- & -- \\
\hline & 14 & -4121 & 1985 & 0 & -- & -- \\
\hline & 15 & -2121 & 1115 & 2 & -- & - \\
\hline \multirow[t]{9}{*}{ TXLV03 } & 3 & -6482 & 3785 & 61 & 48 & 80 \\
\hline & 6 & -3847 & 750 & 21 & -- & 50 \\
\hline & 7 & -2132 & 725 & 0 & -- & 39 \\
\hline & 8 & -482 & 1350 & 20 & $<10$ & 31 \\
\hline & 9 & 220 & 702 & 47 & $<10$ & 24 \\
\hline & 12 & -10267 & -- & -- & -- & -- \\
\hline & 14 & -4597 & 1885 & 0 & -- & -- \\
\hline & 15 & -2857 & 990 & 33 & -- & -- \\
\hline & 16 & -1832 & 300 & 0 & -- & -- \\
\hline \multirow[t]{8}{*}{ TXLVO 4} & 3 & -8270 & 720 & 72 & -- & -- \\
\hline & 6 & -5180 & 705 & 27 & -- & - \\
\hline & 7 & -3068 & 750 & 36 & $\cdots$ & - \\
\hline & 8 & -1368 & 1700 & 12 & $<10$ & 44 \\
\hline & 9 & -180 & 1188 & 31 & $<10$ & 30 \\
\hline & 10 & 199 & 379 & 66 & $<10$ & 23 \\
\hline & 14 & -5885 & 2385 & 0 & -- & -- \\
\hline & 15 & -3818 & 1362 & 0 & -- & - \\
\hline \multirow[t]{7}{*}{ TXLVO 5} & 3 & -8236 & 880 & 68 & -- & - \\
\hline & 7 & -3381 & 705 & 39 & -- & - \\
\hline & 8 & -1096 & 1450 & 19 & $<10$ & 38 \\
\hline & 9 & -151 & 945 & 70 & $<10$ & 28 \\
\hline & 10 & 144 & 295 & 75 & $<10$ & 23 \\
\hline & 15 & -4086 & 4150 & 0 & -- & -- \\
\hline & 16 & -2546 & 835 & 6 & -- & - \\
\hline \multirow[t]{6}{*}{ TXMGOI } & 8 & -4934 & 960 & 68 & 100 & 58 \\
\hline & 9 & -1724 & 2640 & 32 & 41 & 42 \\
\hline & 10 & -584 & 1140 & 57 & 27 & 30 \\
\hline & 11 & 1 & 585 & 51 & $<10$ & 24 \\
\hline & 16 & -5894 & 985 & 0 & - - & -- \\
\hline & 17 & -4364 & 570 & 11 & -- & -- \\
\hline \multirow[t]{6}{*}{ TXMGO2 } & 8 & -4432 & 1720 & 74 & 105 & 59 \\
\hline & 9 & -1577 & 2380 & 41 & 90 & 45 \\
\hline & 10 & -542 & 1035 & 42 & -- & 33 \\
\hline & 11 & 10 & 552 & 37 & $<10$ & 23 \\
\hline & 16 & -6152 & 520 & 0 & -- & - \\
\hline & 17 & -3957 & 475 & 11 & -- & -- \\
\hline
\end{tabular}


Table 4--Layer number; altitude of top, thickness, and sand percentage of layer; and average dissolved-solids concentration and temperature of water in layer; by log number--Continued.

\begin{tabular}{|c|c|c|c|c|c|c|}
\hline $\begin{array}{l}\text { Log } \\
\text { number }\end{array}$ & $\begin{array}{r}\text { Layer } \\
\text { number }\end{array}$ & $\begin{array}{r}\text { Altitude } \\
\text { of top } \\
(f e e t)\end{array}$ & $\begin{array}{r}\text { Thick- } \\
\text { ness } \\
(\text { feet })\end{array}$ & $\begin{array}{r}\text { Sand } \\
\text { per- } \\
\text { centage }\end{array}$ & $\begin{array}{l}\text { Dis- } \\
\text { solved } \\
\text { solids }\end{array}$ & $\begin{array}{l}\text { Tem- } \\
\text { per- } \\
\text { ature }\end{array}$ \\
\hline \multirow[t]{7}{*}{ TXMGO3 } & 7 & -7308 & 1770 & 36 & -- & - \\
\hline & 8 & -5023 & 760 & 38 & -- & -- \\
\hline & 9 & -1613 & 2330 & 28 & 15 & 39 \\
\hline & 10 & -558 & 1055 & 48 & $<10$ & 29 \\
\hline & 11 & 10 & 568 & 32 & $<10$ & 24 \\
\hline & 16 & -5783 & 1525 & 0 & - & - \\
\hline & 17 & -3943 & 1080 & 13 & -- & -- \\
\hline \multirow[t]{6}{*}{ TXMG 04} & 8 & -4633 & 1740 & 59 & 87 & 59 \\
\hline & 9 & -1558 & 2315 & 44 & 87 & 40 \\
\hline & 10 & -523 & 1035 & 42 & 35 & 29 \\
\hline & 11 & 24 & 547 & 37 & $<10$ & 24 \\
\hline & 16 & -6373 & 680 & 0 & -- & -- \\
\hline & 17 & -3873 & 760 & 19 & -- & - \\
\hline \multirow[t]{7}{*}{ TXMGOS } & 7 & -7112 & 1865 & 42 & 78 & 70 \\
\hline & 8 & -5322 & 560 & 27 & 50 & 58 \\
\hline & 9 & -1577 & 2295 & 22 & 18 & 43 \\
\hline & 10 & -547 & 1030 & 53 & $<10$ & 30 \\
\hline & 11 & 10 & 557 & 52 & $<10$ & 24 \\
\hline & 16 & -5882 & 1230 & 0 & -- & - \\
\hline & 17 & $-38>2$ & 1450 & 10 & -- & -- \\
\hline \multirow[t]{2}{*}{ IXMIO 1} & 3 & 496 & 759 & 62 & -- & -- \\
\hline & 12 & -263 & 645 & 2 & -- & -- \\
\hline \multirow[t]{7}{*}{ TXMMO 1} & 3 & -3208 & 2320 & 43 & $<10$ & 54 \\
\hline & 4 & -2563 & 645 & 93 & $<10$ & 45 \\
\hline & 5 & -908 & 1275 & 85 & $<10$ & 37 \\
\hline & 6 & 321 & 834 & 27 & $<10$ & 27 \\
\hline & 12 & -5528 & $\ldots$ & - & -- & - \\
\hline & 13 & -2183 & 380 & 7 & -- & $\ldots$ \\
\hline & 14 & -513 & 395 & 0 & -- & -- \\
\hline \multirow[t]{10}{*}{ TXMMO2 } & 3 & -6159 & 1185 & 32 & 18 & 81 \\
\hline & 4 & -5654 & 505 & 78 & $<10$ & 74 \\
\hline & 5 & -4024 & 805 & 35 & -- & 61 \\
\hline & 6 & -614 & 2210 & 48 & $<10$ & 37 \\
\hline & 7 & -254 & 20 & 0 & -- & 28 \\
\hline & 8 & 244 & 278 & 47 & $<10$ & 26 \\
\hline & 13 & -4829 & 825 & 0 & -- & -- \\
\hline & 14 & -2824 & 1200 & 0 & -- & -- \\
\hline & 15 & -274 & 340 & 0 & -- & -- \\
\hline & 16 & -34 & 220 & 0 & -- & -- \\
\hline \multirow[t]{9}{*}{ TXMMO 3} & 3 & -5549 & 1305 & 39 & 26 & 79 \\
\hline & 4 & -4999 & 550 & 71 & 12 & 71 \\
\hline & 5 & -3234 & 1240 & 50 & 43 & 59 \\
\hline & 6 & -1334 & 920 & 51 & 10 & 41 \\
\hline & 8 & 288 & 92 & -- & -- & 24 \\
\hline & 12 & -6854 & 350 & 0 & -- & - \\
\hline & 13 & -4474 & 525 & 0 & -- & -- \\
\hline & 14 & -2254 & 980 & 0 & -- & -- \\
\hline & 15 & 196 & 1530 & 5 & -- & -- \\
\hline
\end{tabular}


Table 4--Layer number; altitude of top, thickness, and sand percentage of layer; and average dissolved-solids concentration and temperature of water in layer; by log number--Continued.

\begin{tabular}{|c|c|c|c|c|c|c|}
\hline $\begin{array}{l}\text { Log } \\
\text { number }\end{array}$ & $\begin{array}{r}\text { Layer } \\
\text { number }\end{array}$ & $\begin{array}{r}\text { Altitude } \\
\text { of top } \\
\text { (feet) }\end{array}$ & $\begin{array}{r}\text { Thick- } \\
\text { ness } \\
\text { (feet) }\end{array}$ & $\begin{array}{r}\text { Sand } \\
\text { per- } \\
\text { centage }\end{array}$ & $\begin{array}{l}\text { Dis- } \\
\text { solved } \\
\text { solids }\end{array}$ & $\begin{array}{l}\text { Tem- } \\
\text { Per- } \\
\text { ature }\end{array}$ \\
\hline \multirow[t]{7}{*}{ TXMMO 4} & 3 & -5491 & 1225 & 20 & -- & -- \\
\hline & 4 & -4196 & 1295 & 80 & -- & -- \\
\hline & 5 & -2166 & 1500 & 70 & -- & -- \\
\hline & 6 & -1216 & 400 & 74 & -- & -- \\
\hline & 13 & -3666 & 530 & 0 & -- & -- \\
\hline & 14 & -1616 & 550 & 0 & -- & - \\
\hline & 15 & 255 & 1471 & 7 & -- & -- \\
\hline \multirow[t]{7}{*}{ TXMMO 5} & 5 & -5527 & 840 & 23 & -- & -- \\
\hline & 6 & -1417 & 2470 & 56 & - & -- \\
\hline & 7 & -407 & 30 & 99 & $<10$ & 35 \\
\hline & 8 & 418 & 225 & 44 & $<10$ & 20 \\
\hline & 14 & -3887 & 1640 & 0 & -- & $\ldots$ \\
\hline & 15 & -437 & 980 & 3 & -- & -- \\
\hline & 16 & 193 & 600 & 0 & -- & - \\
\hline \multirow[t]{4}{*}{ TXMOOO } & 3 & 150 & 395 & 62 & $<10$ & 29 \\
\hline & 4 & 335 & 185 & 95 & $<10$ & -- \\
\hline & 12 & -245 & 960 & 0 & -- & -- \\
\hline & 13 & 374 & 39 & 0 & -- & -- \\
\hline \multirow[t]{10}{*}{ TXMYO 1} & 3 & -5942 & 4720 & 72 & 43 & 87 \\
\hline & 5 & -4932 & 720 & 25 & 38 & 64 \\
\hline & 6 & -3102 & 1060 & 57 & $<10$ & 51 \\
\hline & 7 & -1217 & 705 & 48 & $<10$ & 34 \\
\hline & 8 & -117 & 1100 & 47 & $<10$ & 27 \\
\hline & 9 & 222 & 339 & 34 & $<10$ & 21 \\
\hline & 12 & -10662 & 1340 & 0 & - - & -- \\
\hline & 13 & -5652 & 290 & 0 & -- & -- \\
\hline & 14 & -4162 & 770 & 0 & -- & -- \\
\hline & 15 & -1922 & 1180 & 4 & -- & -- \\
\hline \multirow[t]{9}{*}{ TXMYO2 } & 3 & -7312 & 4409 & 53 & 41 & 92 \\
\hline & 6 & -4122 & 1560 & 55 & 55 & 66 \\
\hline & 7 & -1662 & 1260 & 21 & $<10$ & 46 \\
\hline & 8 & -412 & 1250 & 56 & $<10$ & 32 \\
\hline & 9 & 78 & 490 & 69 & $<10$ & 23 \\
\hline & 10 & 264 & 186 & 72 & $<10$ & 19 \\
\hline & 12 & -11721 & -- & -- & -- & -- \\
\hline & 14 & -5682 & 1630 & 0 & -- & -- \\
\hline & 15 & -2922 & 1200 & 3 & -- & -- \\
\hline \multirow[t]{8}{*}{ TXMYO 3} & 3 & -8102 & -- & -- & -- & -- \\
\hline & 6 & -5042 & 1300 & 62 & 47 & 62 \\
\hline & 7 & -2512 & 990 & 40 & 16 & 52 \\
\hline & 8 & -1042 & 1470 & 34 & $<10$ & 40 \\
\hline & 9 & -132 & 910 & 71 & $<10$ & 27 \\
\hline & 10 & 169 & 301 & 59 & $<10$ & 21 \\
\hline & 14 & -6342 & 1760 & 0 & -- & -- \\
\hline & 15 & -3502 & 1540 & 3 & -- & $\ldots$ \\
\hline
\end{tabular}


Table 4--Layer number; altitude of top, thickness, and sand percentage of layer; and average dissolved-solids concentration and temperature of water in layer; by log number--Continued.

\begin{tabular}{|c|c|c|c|c|c|c|}
\hline $\begin{array}{l}\text { Log } \\
\text { number }\end{array}$ & $\begin{array}{r}\text { Layer } \\
\text { number }\end{array}$ & $\begin{array}{r}\text { Alt } 1 \text { tude } \\
\text { of top } \\
(\text { feet) }\end{array}$ & $\begin{array}{r}\text { Thick- } \\
\text { ness } \\
(\text { feet) }\end{array}$ & $\begin{array}{r}\text { Sand } \\
\text { per- } \\
\text { centage }\end{array}$ & $\begin{array}{l}\text { Dis- } \\
\text { solved } \\
\text { solids }\end{array}$ & $\begin{array}{l}\text { Tem- } \\
\text { per- } \\
\text { ature }\end{array}$ \\
\hline \multirow[t]{8}{*}{ TXMYO 4} & 3 & -8290 & 3985 & 66 & -- & -- \\
\hline & 6 & -5125 & 1270 & 67 & -- & -- \\
\hline & 7 & -2675 & 900 & 22 & -- & -- \\
\hline & 8 & -1090 & 1585 & 19 & -- & -- \\
\hline & 9 & -125 & 965 & 43 & -- & -- \\
\hline & 10 & 203 & 328 & 50 & -- & -- \\
\hline & 14 & -6395 & 1895 & 0 & -- & - \\
\hline & 15 & -3575 & 1550 & 0 & -- & -- \\
\hline \multirow[t]{5}{*}{ TXNAOI } & 3 & -46 & 2085 & 65 & $<10$ & 34 \\
\hline & 4 & 169 & 215 & 93 & $<10$ & 22 \\
\hline & 5 & 345 & 76 & 70 & $<10$ & 19 \\
\hline & 12 & -2131 & 1010 & 0 & - & -- \\
\hline & 13 & 269 & 100 & 0 & -- & -- \\
\hline \multirow[t]{5}{*}{$\mathrm{TXNAO} 2$} & 3 & 73 & 2105 & 62 & $<10$ & 29 \\
\hline & 4 & 288 & 215 & 99 & $<10$ & 21 \\
\hline & 5 & 525 & 112 & 68 & $<10$ & 19 \\
\hline & 12 & -2032 & 975 & 0 & -- & - \\
\hline & 13 & 413 & 125 & 0 & -- & -- \\
\hline \multirow[t]{9}{*}{ TXNEO1 } & 3 & -4792 & 4370 & 59 & 79 & -- \\
\hline & 5 & -4242 & 205 & 59 & 41 & 62 \\
\hline & 6 & -2552 & 1156 & 53 & 22 & 50 \\
\hline & 7 & -772 & 1027 & 42 & $<10$ & 31 \\
\hline & 8 & 108 & 880 & 76 & $<10$ & 22 \\
\hline & 12 & -9162 & -- & $\ldots$ & -- & -- \\
\hline & 13 & -4447 & 345 & 4 & -- & -- \\
\hline & 14 & -3708 & 534 & 6 & -- & -- \\
\hline & 15 & -1799 & 753 & 3 & -- & -- \\
\hline \multirow[t]{9}{*}{ TXNEO2 } & 3 & -4431 & 4225 & 48 & 44 & 73 \\
\hline & 5 & -3916 & 195 & 41 & 64 & 52 \\
\hline & 6 & -2341 & 1105 & 39 & 46 & 43 \\
\hline & 7 & -516 & 1035 & 40 & -- & 28 \\
\hline & 8 & 330 & 846 & 66 & -- & 20 \\
\hline & 12 & -8656 & 1175 & 0 & -- & -- \\
\hline & 13 & -4111 & 320 & 1 & -- & -- \\
\hline & 14 & -3446 & 470 & 3 & -- & -- \\
\hline & 15 & -1551 & 790 & 3 & -- & - \\
\hline \multirow[t]{9}{*}{ TXNEO3 } & 3 & -5053 & 4580 & 47 & 37 & 86 \\
\hline & 5 & -4513 & 220 & 41 & 45 & 65 \\
\hline & 6 & -2993 & 1042 & 53 & 49 & 57 \\
\hline & 7 & -633 & 1100 & 30 & $<10$ & 35 \\
\hline & 8 & 297 & 930 & 77 & - - & 19 \\
\hline & 12 & -9633 & 1180 & 1 & -- & -- \\
\hline & 13 & -4733 & 320 & 2 & -- & -- \\
\hline & 14 & -4035 & 478 & 4 & -- & -- \\
\hline & 15 & -1733 & 1260 & 6 & -- & - \\
\hline
\end{tabular}


Table 4--Layer number; altitude of top, thickness, and sand percentage of layer; and average dissolved-solids concentration and temperature of water in layer; by log number--Continued.

\begin{tabular}{|c|c|c|c|c|c|c|}
\hline $\begin{array}{l}\text { Log } \\
\text { number }\end{array}$ & $\begin{array}{r}\text { Layer } \\
\text { number }\end{array}$ & $\begin{array}{r}\text { Altitude } \\
\text { of top } \\
(\text { feet) }\end{array}$ & $\begin{array}{r}\text { Thlck- } \\
\text { ness } \\
\text { (feet) }\end{array}$ & $\begin{array}{r}\text { Sand } \\
\text { per- } \\
\text { centage }\end{array}$ & $\begin{array}{l}\text { Dis- } \\
\text { solved } \\
\text { solids }\end{array}$ & $\begin{array}{l}\text { Tem- } \\
\text { per- } \\
\text { ature }\end{array}$ \\
\hline \multirow[t]{8}{*}{$\mathrm{TXNEO}_{4}$} & 3 & -7830 & - - & -- & - - & -- \\
\hline & 6 & -5130 & 1265 & 56 & 105 & 59 \\
\hline & 7 & -1870 & 2095 & 43 & 10 & 40 \\
\hline & 8 & -410 & 1460 & 65 & $<10$ & 27 \\
\hline & 9 & 0 & 410 & 76 & $<10$ & 21 \\
\hline & 10 & 80 & 80 & 99 & $<10$ & 19 \\
\hline & 14 & -6395 & 1435 & 2 & - & - \\
\hline & 15 & -3965 & 1165 & 6 & -- & - \\
\hline \multirow[t]{9}{*}{ TXNEO 5} & 3 & -9087 & -- & -- & -- & - \\
\hline & 6 & -6427 & 1180 & 45 & 31 & 61 \\
\hline & 7 & -2812 & 2245 & 53 & 50 & 43 \\
\hline & 8 & -1127 & 1685 & 62 & $<10$ & 32 \\
\hline & 9 & -537 & 590 & 31 & $<10$ & 25 \\
\hline & 10 & -138 & 399 & 39 & $<10$ & 22 \\
\hline & 11 & 60 & 198 & 81 & $<10$ & 20 \\
\hline & 14 & -7607 & 1480 & 1 & - & - \\
\hline & 15 & -5057 & 1370 & 1 & -- & -- \\
\hline \multirow[t]{6}{*}{ TXNUO 1} & 7 & -3310 & 2540 & 10 & 20 & 57 \\
\hline & 8 & -1165 & 1215 & 37 & $<10$ & 37 \\
\hline & 9 & -215 & 950 & 62 & $<10$ & 30 \\
\hline & 10 & 99 & 314 & 70 & $<10$ & 25 \\
\hline & 15 & -5850 & -- & -- & -- & -- \\
\hline & 16 & -2380 & 930 & 0 & -- & -- \\
\hline \multirow[t]{8}{*}{ TXNUO2 } & 7 & -5574 & 4430 & 71 & 78 & 76 \\
\hline & 8 & -3444 & 1625 & 59 & 62 & 53 \\
\hline & 9 & -1114 & 1530 & 38 & 33 & 38 \\
\hline & 10 & -494 & 620 & 75 & 12 & 31 \\
\hline & 11 & 36 & 530 & 48 & $<10$ & 27 \\
\hline & 15 & -10004 & 440 & 0 & - - & -- \\
\hline & 16 & -5069 & 505 & 8 & -- & - \\
\hline & 17 & -2644 & 800 & 10 & -- & -- \\
\hline \multirow[t]{8}{*}{ TXNUO3 } & 7 & -8057 & 195 & 41 & -- & -- \\
\hline & 8 & -4722 & 1735 & 66 & -- & - \\
\hline & 9 & -1617 & 2030 & 56 & -- & -- \\
\hline & 10 & -617 & 1000 & 18 & -- & -- \\
\hline & 11 & 56 & 673 & 25 & -- & -- \\
\hline & 15 & -8252 & 150 & 0 & -- & - \\
\hline & 16 & -6457 & 1600 & 0 & -- & -- \\
\hline & 17 & -3647 & 1075 & 5 & -- & -- \\
\hline TXNVO 1 & 12 & 375 & 548 & 11 & -- & - \\
\hline \multirow[t]{5}{*}{ TXORO 1} & 8 & -4045 & 3125 & 59 & 141 & 56 \\
\hline & 9 & -2045 & 2000 & 46 & 102 & 40 \\
\hline & 10 & -825 & 1220 & 55 & 65 & 29 \\
\hline & 11 & 4 & 829 & 49 & $<10$ & 23 \\
\hline & 16 & -7170 & 1805 & 13 & - & - \\
\hline
\end{tabular}


Table 4--Layer number; altitude of top, thickness, and sand percentage of layer; and average dissolved-solids concentration and temperature of water in layer; by log number--Continued.

\begin{tabular}{|c|c|c|c|c|c|c|}
\hline $\begin{array}{l}\log \\
\text { number }\end{array}$ & $\begin{array}{r}\text { Layer } \\
\text { number }\end{array}$ & $\begin{array}{r}\text { Altitude } \\
\text { of top } \\
(\text { feet) }\end{array}$ & $\begin{array}{r}\text { Thick- } \\
\text { ness } \\
\text { (feet) }\end{array}$ & $\begin{array}{r}\text { Sand } \\
\text { per- } \\
\text { centage }\end{array}$ & $\begin{array}{l}\text { Dis- } \\
\text { solved } \\
\text { solids }\end{array}$ & $\begin{array}{l}\text { Tem- } \\
\text { per- } \\
\text { ature }\end{array}$ \\
\hline \multirow[t]{6}{*}{ TXORO2 } & 7 & -7051 & 1265 & 60 & -- & - \\
\hline & 8 & -3551 & 2960 & 65 & -- & - \\
\hline & 9 & -1716 & 1835 & 37 & -- & -- \\
\hline & 10 & -661 & 1055 & 61 & -- & -- \\
\hline & 11 & 12 & 673 & 76 & -- & -- \\
\hline & 16 & -6511 & 540 & 6 & -- & - \\
\hline \multirow[t]{2}{*}{ TXPAOI } & 3 & 307 & 518 & 72 & $<10$ & 18 \\
\hline & 12 & -211 & 900 & 1 & - & - \\
\hline \multirow[t]{2}{*}{$\mathrm{TXPAO} 2$} & 3 & 294 & 369 & 66 & -- & 21 \\
\hline & 12 & -75 & 890 & 0 & -- & - \\
\hline \multirow[t]{10}{*}{ TXPO01 } & 3 & -5933 & 4520 & 47 & 18 & 88 \\
\hline & 5 & -5223 & 130 & 50 & -- & 65 \\
\hline & 6 & -3283 & 1360 & 57 & 25 & 54 \\
\hline & 7 & -1023 & 1160 & 28 & $<10$ & 34 \\
\hline & 8 & -53 & 970 & 52 & $<10$ & 26 \\
\hline & 9 & 284 & 337 & 0 & -- & 20 \\
\hline & 12 & -10453 & - & -- & -- & -- \\
\hline & 13 & -5353 & 580 & 0 & - & - \\
\hline & 14 & -4643 & 580 & 5 & -- & $\cdots$ \\
\hline & 15 & -2183 & 1100 & 3 & -- & - \\
\hline \multirow[t]{7}{*}{ TXPOO2 } & 3 & -6622 & -- & -- & -- & - \\
\hline & 6 & -3707 & 1400 & 59 & 70 & 50 \\
\hline & 7 & -1367 & 1255 & 10 & $<10$ & 34 \\
\hline & 8 & -332 & 1035 & 52 & $<10$ & 27 \\
\hline & 9 & 126 & 458 & 29 & $<10$ & 22 \\
\hline & 14 & -5107 & 1515 & 0 & -- & -- \\
\hline & 15 & -2622 & 1085 & 0 & -- & - \\
\hline \multirow[t]{7}{*}{$\mathrm{TXPOO3}$} & 3 & -3244 & 4020 & 69 & 56 & 56 \\
\hline & 5 & -2534 & 200 & 82 & -- & 39 \\
\hline & 6 & -1104 & 980 & 50 & $<10$ & 32 \\
\hline & 12 & -7264 & 1530 & 0 & - & - \\
\hline & 13 & -2734 & 510 & 0 & -- & -- \\
\hline & 14 & -2084 & 450 & 0 & -- & - \\
\hline & 15 & 267 & 1371 & 6 & -- & -- \\
\hline \multirow[t]{8}{*}{ TXPOO 4} & 3 & -7714 & 2640 & 46 & 60 & 84 \\
\hline & 6 & -4764 & 1340 & 59 & 29 & 61 \\
\hline & 7 & -1984 & 1680 & 25 & 15 & 46 \\
\hline & 8 & -549 & 1435 & 38 & $<10$ & 34 \\
\hline & 9 & -29 & 520 & 71 & $<10$ & 24 \\
\hline & 10 & 165 & 194 & 57 & $<10$ & 20 \\
\hline & 14 & -6104 & 1610 & 0 & -- & $\cdots$ \\
\hline & 15 & -3664 & 1100 & 0 & -- & -- \\
\hline
\end{tabular}


Table 4--Layer number; altitude of top, thickness, and sand percentage of layer; and average dissolved-solids concentration and temperature of water in layer; by log number--Continued.

\begin{tabular}{|c|c|c|c|c|c|c|}
\hline $\begin{array}{l}\text { Log } \\
\text { number }\end{array}$ & $\begin{array}{r}\text { Layer } \\
\text { number }\end{array}$ & $\begin{array}{r}\text { Altitude } \\
\text { of top } \\
(f \in e t)\end{array}$ & $\begin{array}{r}\text { Thick- } \\
\text { ness } \\
\text { (feet) }\end{array}$ & $\begin{array}{r}\text { Sand } \\
\text { per- } \\
\text { centage }\end{array}$ & $\begin{array}{l}\text { Dis- } \\
\text { solved } \\
\text { solids }\end{array}$ & $\begin{array}{l}\text { Tem- } \\
\text { per- } \\
\text { ature }\end{array}$ \\
\hline \multirow[t]{2}{*}{ TXRAO 1} & 3 & 460 & 475 & 63 & - & -- \\
\hline & 12 & -15 & 1115 & 0 & -- & -- \\
\hline \multirow[t]{2}{*}{ TXRAO 2} & 3 & 470 & 158 & 62 & $<10$ & - \\
\hline & 12 & 312 & 1180 & 4 & $\ldots$ & -- \\
\hline \multirow[t]{6}{*}{ TXREO 1} & 7 & -4763 & 3430 & 46 & 72 & 61 \\
\hline & 8 & -2633 & 2130 & 55 & 57 & 51 \\
\hline & 9 & -1073 & 1560 & 12 & 24 & 45 \\
\hline & 10 & -443 & 630 & 60 & $<10$ & 28 \\
\hline & 11 & 43 & 486 & 49 & $<10$ & $\ldots$ \\
\hline & 15 & -8193 & -- & -- & -- & - \\
\hline \multirow[t]{2}{*}{ TXROO 1} & 3 & 296 & 896 & 58 & $<10$ & 14 \\
\hline & 12 & -600 & 1190 & 2 & - & - \\
\hline \multirow[t]{2}{*}{ TXROO 2} & 3 & 359 & 88 & $\sim$ & -- & 17 \\
\hline & 12 & 271 & 300 & 0 & -- & -- \\
\hline \multirow[t]{3}{*}{ TXRU 01} & 3 & 340 & 975 & 74 & $<10$ & 23 \\
\hline & 4 & 400 & 60 & $\approx$ & - - & 18 \\
\hline & 12 & -635 & 1155 & 1 & -- & -- \\
\hline \multirow[t]{2}{*}{ TXRU 02} & 3 & 417 & 835 & 59 & $<10$ & 21 \\
\hline & 12 & -418 & 1170 & 0 & - & - \\
\hline \multirow[t]{4}{*}{ TXRUO3 } & 3 & 133 & 1265 & 62 & -- & - \\
\hline & 4 & 313 & 180 & 99 & -- & -- \\
\hline & 12 & -1132 & 1090 & 0 & -- & -- \\
\hline & 13 & 417 & 104 & 0 & -- & -- \\
\hline \multirow[t]{5}{*}{ TXRUO 4} & 3 & 47 & 1405 & 24 & $<10$ & 28 \\
\hline & 4 & 197 & 150 & 99 & $<10$ & 20 \\
\hline & 5 & 581 & 234 & 60 & $<10$ & 17 \\
\hline & 12 & -1358 & 1480 & 2 & - - & -- \\
\hline & 13 & 347 & 150 & 9 & -- & - \\
\hline \multirow[t]{5}{*}{ TXSAOI } & 3 & -473 & 2925 & 65 & 12 & 37 \\
\hline & 5 & -18 & 290 & 60 & $<10$ & 26 \\
\hline & 12 & -3398 & 1030 & 0 & - & - \\
\hline & 13 & -308 & 165 & 6 & -- & $\ldots$ \\
\hline & 14 & 252 & 270 & 3 & -- & - \\
\hline \multirow[t]{2}{*}{$T \times S B O 1$} & 3 & 286 & 2075 & 60 & $<10$ & 30 \\
\hline & 12 & -1789 & 845 & 0 & - & - \\
\hline \multirow[t]{7}{*}{ TXSBO2 } & 3 & -1419 & 3340 & 62 & 27 & 50 \\
\hline & 5 & -999 & 305 & 44 & $<10$ & 33 \\
\hline & 6 & 171 & 890 & 60 & - & 26 \\
\hline & 12 & -4759 & 1050 & 0 & - - & - \\
\hline & 13 & -1304 & 115 & 0 & -- & - \\
\hline & 14 & -719 & 280 & 6 & -- & - \\
\hline & 15 & 221 & 50 & 0 & -- & -- \\
\hline
\end{tabular}


Table 4--Layer number; altitude of top, thickness, and sand percentage of layer; and average dissolved-solids concentration and temperature of water in layer; by log number--Continued.

\begin{tabular}{|c|c|c|c|c|c|c|}
\hline $\begin{array}{l}\log \\
\text { number }\end{array}$ & $\begin{array}{r}\text { Layer } \\
\text { number }\end{array}$ & $\begin{array}{r}\text { Altide } \\
\text { of top } \\
(\text { feet) }\end{array}$ & $\begin{array}{r}\text { Thlck- } \\
\text { ness } \\
(\text { feet) }\end{array}$ & $\begin{array}{r}\text { Sand } \\
\text { per- } \\
\text { centage }\end{array}$ & $\begin{array}{l}\text { Dis- } \\
\text { solved } \\
\text { solids }\end{array}$ & $\begin{array}{l}\text { Tem- } \\
\text { per- } \\
\text { ature }\end{array}$ \\
\hline \multirow[t]{6}{*}{$\operatorname{TXSBO3}$} & 3 & -707 & 3300 & 65 & 21 & 41 \\
\hline & 5 & -407 & 240 & 46 & $<10$ & 28 \\
\hline & 6 & 216 & 333 & 59 & $<10$ & 24 \\
\hline & 12 & -4007 & - & -. & -- & -- \\
\hline & 13 & -647 & 60 & 0 & -- & -- \\
\hline & 14 & -117 & 290 & 4 & -- & - \\
\hline \multirow[t]{2}{*}{ TXSHO 1} & 3 & 347 & 1090 & 62 & $<10$ & 26 \\
\hline & 12 & -743 & 1280 & 1 & -- & - \\
\hline \multirow[t]{2}{*}{ TXSHO2 } & 3 & 245 & 910 & 54 & $<10$ & 25 \\
\hline & 12 & -665 & 730 & 0 & -- & -- \\
\hline \multirow[t]{9}{*}{ TXSJ01 } & 3 & -4852 & 4730 & 66 & 44 & 78 \\
\hline & 5 & -3962 & 590 & 38 & 38 & 54 \\
\hline & 6 & -2202 & 1250 & 41 & 16 & 43 \\
\hline & 7 & -242 & 810 & 38 & $<10$ & 26 \\
\hline & 8 & 250 & 492 & 70 & $<10$ & 21 \\
\hline & 12 & -9582 & 1750 & 0 & - & - \\
\hline & 13 & -4552 & 300 & 0 & $\ldots$ & $\ldots$ \\
\hline & 14 & -3452 & 510 & 0 & -- & -- \\
\hline & 15 & -1052 & 1150 & 5 & -- & -- \\
\hline \multirow[t]{8}{*}{ TXSJO2 } & 3 & -7606 & 3130 & 54 & 50 & 76 \\
\hline & 6 & -4296 & 1580 & 66 & 59 & 57 \\
\hline & 7 & -1796 & 1310 & 25 & $<10$ & 44 \\
\hline & 8 & -446 & 1350 & 42 & $<10$ & 32 \\
\hline & 9 & 54 & 500 & 41 & $<10$ & 22 \\
\hline & 10 & 254 & 200 & 82 & $<10$ & 18 \\
\hline & 14 & $-58>6$ & 1730 & 0 & - - & -. \\
\hline & 15 & -3106 & 1190 & 0 & -- & -. \\
\hline \multirow[t]{5}{*}{ TXSMOI } & 3 & -333 & 1165 & 46 & $<10$ & 34 \\
\hline & 4 & -68 & 265 & 96 & $<10$ & 26 \\
\hline & 5 & 569 & 567 & 74 & $<10$ & 21 \\
\hline & 12 & -1498 & 1490 & 1 & -- & - \\
\hline & 13 & 2 & 70 & 7 & -- & - \\
\hline \multirow[t]{5}{*}{$\operatorname{TXSMO} 2$} & 3 & 75 & 760 & 59 & $<10$ & 27 \\
\hline & 4 & 165 & 90 & 99 & $<10$ & 23 \\
\hline & 5 & 398 & 133 & 75 & $<10$ & 21 \\
\hline & 12 & -685 & 1770 & 2 & -- & -- \\
\hline & 13 & 265 & 100 & 17 & -- & -- \\
\hline \multirow[t]{6}{*}{ TXSPO1 } & 7 & -2726 & 2145 & 16 & 71 & 52 \\
\hline & 8 & -971 & 1140 & 44 & 32 & 35 \\
\hline & 9 & -81 & 890 & 78 & $<10$ & 27 \\
\hline & 10 & 149 & 230 & 40 & $\ldots$ & 23 \\
\hline & 15 & -4871 & 760 & 3 & -- & - \\
\hline & 16 & -2111 & 615 & 7 & -- & -- \\
\hline
\end{tabular}


Table 4--Layer number; altitude of top, thickness, and sand percentage of layer; and average dissolved-solids concentration and temperature of water in layer; by log number--Continued.

\begin{tabular}{|c|c|c|c|c|c|c|}
\hline $\begin{array}{l}\text { Log } \\
\text { number }\end{array}$ & $\begin{array}{r}\text { Layer } \\
\text { number }\end{array}$ & $\begin{array}{r}\text { Altitude } \\
\text { of top } \\
(f e e t)\end{array}$ & $\begin{array}{r}\text { Thick- } \\
\text { ness } \\
(\text { feet) }\end{array}$ & $\begin{array}{r}\text { Sand } \\
\text { per- } \\
\text { centage }\end{array}$ & $\begin{array}{l}\text { Dis- } \\
\text { solved } \\
\text { solids }\end{array}$ & $\begin{array}{l}\text { Tem- } \\
\text { per- } \\
\text { ature }\end{array}$ \\
\hline \multirow[t]{6}{*}{ IXSPO2 } & 7 & -3722 & 4400 & 38 & -- & -- \\
\hline & 8 & -1662 & 1350 & 30 & -- & -- \\
\hline & 9 & -667 & 995 & 55 & -- & -- \\
\hline & 10 & -262 & 405 & 61 & -- & -- \\
\hline & 11 & 54 & 316 & 41 & -- & -- \\
\hline & 16 & -3012 & 710 & 19 & -- & - \\
\hline \multirow[t]{7}{*}{ IXSPO3 } & 7 & -6638 & 2140 & 60 & -- & -- \\
\hline & 8 & -4063 & 1660 & 48 & -- & -- \\
\hline & 9 & -1433 & 1650 & 44 & -- & -- \\
\hline & 10 & -563 & 870 & 38 & -- & -- \\
\hline & 11 & 4 & 567 & 40 & -- & -- \\
\hline & 16 & -5723 & 915 & 2 & -- & -- \\
\hline & 17 & -3083 & 980 & 4 & -- & - \\
\hline \multirow[t]{6}{*}{ IXST01 } & 7 & -3822 & 3250 & 26 & 67 & 52 \\
\hline & 8 & -2102 & 1720 & 12 & $<10$ & 54 \\
\hline & 9 & -837 & 1265 & 52 & $<10$ & 46 \\
\hline & 10 & -252 & 585 & 31 & $<10$ & 36 \\
\hline & 11 & 247 & 499 & 50 & $<10$ & 29 \\
\hline & 15 & -7072 & 170 & 4 & - - & - \\
\hline \multirow[t]{4}{*}{ IXSTO2 } & 5 & -3898 & 1330 & 27 & -- & -- \\
\hline & 6 & -858 & 1410 & 43 & -- & -- \\
\hline & 14 & -2268 & 1630 & 0 & -- & -- \\
\hline & 15 & 355 & 1213 & 9 & -- & -- \\
\hline \multirow[t]{5}{*}{ IXSTO3 } & 7 & -1625 & 1475 & 33 & $<10$ & 45 \\
\hline & 8 & -620 & 1005 & 17 & - & 37 \\
\hline & 9 & 260 & 880 & 17 & -- & 30 \\
\hline & 10 & 527 & 267 & 80 & $<10$ & 26 \\
\hline & 15 & -3100 & 1360 & 24 & -- & -- \\
\hline \multirow[t]{2}{*}{ TXTIOI } & 3 & 420 & 321 & 67 & $<10$ & 20 \\
\hline & 12 & 99 & 850 & 0 & - & - \\
\hline \multirow[t]{5}{*}{ TXTIO2 } & 3 & -19 & 620 & 47 & -- & -- \\
\hline & 4 & 181 & 200 & 97 & -- & -- \\
\hline & 5 & 299 & 88 & 80 & -- & -- \\
\hline & 12 & -639 & 970 & 0 & -- & -- \\
\hline & 13 & 211 & 30 & 0 & -- & -- \\
\hline \multirow[t]{7}{*}{ TXTRO1 } & 3 & -2138 & 4240 & 75 & 49 & 62 \\
\hline & 5 & -1278 & 675 & 57 & $<10$ & 38 \\
\hline & 6 & 132 & 970 & 60 & $<10$ & 26 \\
\hline & 12 & -6378 & 1410 & 0 & -- & -- \\
\hline & 13 & -1953 & 185 & 0 & -- & -- \\
\hline & 14 & -838 & 440 & 6 & -- & -- \\
\hline & 15 & 170 & 38 & 0 & -- & -- \\
\hline
\end{tabular}


Table 4--Layer number; altitude of top, thickness, and sand percentage of layer; and average dissolved-solids concentration and temperature of water in layer; by log number--Continued.

\begin{tabular}{|c|c|c|c|c|c|c|}
\hline $\begin{array}{l}\text { L०8 } \\
\text { number }\end{array}$ & $\begin{array}{r}\text { Layer } \\
\text { number }\end{array}$ & $\begin{array}{r}\text { Altitude } \\
\text { of top } \\
\text { (feet) }\end{array}$ & $\begin{array}{r}\text { Thick- } \\
\text { ness } \\
\text { (feet) }\end{array}$ & $\begin{array}{r}\text { Sand } \\
\text { per- } \\
\text { centage }\end{array}$ & $\begin{array}{l}\text { DIs } \\
\text { solved } \\
\text { solids }\end{array}$ & $\begin{array}{l}\text { Tem- } \\
\text { per- } \\
\text { ature }\end{array}$ \\
\hline \multirow[t]{7}{*}{ TXTRO2 } & 3 & -1300 & 3265 & 68 & 18 & 49 \\
\hline & 4 & -1055 & 245 & 98 & $<10$ & 33 \\
\hline & 5 & -321 & 534 & 68 & $<10$ & 28 \\
\hline & 6 & 285 & 256 & 56 & $<10$ & 22 \\
\hline & 12 & -4565 & 1200 & 0 & - & -- \\
\hline & 13 & -855 & 200 & 5 & -- & -- \\
\hline & 14 & 29 & 350 & 13 & -- & $\cdots$ \\
\hline \multirow[t]{9}{*}{ TXTYOI } & 3 & -5171 & 4484 & 56 & 48 & 102 \\
\hline & 5 & -4422 & 309 & 44 & 54 & 69 \\
\hline & 6 & -2657 & 1368 & 65 & 44 & 55 \\
\hline & 7 & -555 & 1040 & 37 & $<10$ & 31 \\
\hline & 8 & 294 & 849 & 53 & $<10$ & 22 \\
\hline & 12 & -9655 & 1490 & 0 & - & - \\
\hline & 13 & -4731 & 440 & 2 & $-\sim$ & $\cdots$ \\
\hline & 14 & -4025 & 397 & 2 & $-\sim$ & $\sim$ \\
\hline & 15 & -1595 & 1062 & 4 & -- & -- \\
\hline \multirow[t]{10}{*}{ TXTYO2 } & 3 & -5975 & 4260 & 62 & 41 & 84 \\
\hline & 5 & -5255 & 115 & 39 & 92 & 64 \\
\hline & 6 & -3275 & 1470 & 63 & 47 & 55 \\
\hline & 7 & -1055 & 1320 & 39 & 17 & 39 \\
\hline & 8 & 40 & 1095 & 42 & $<10$ & 26 \\
\hline & 9 & 305 & 265 & 39 & $<10$ & 19 \\
\hline & 12 & -10235 & 1920 & 0 & - & $\ldots$ \\
\hline & 13 & -5370 & 605 & 7 & $\ldots$ & -- \\
\hline & 14 & -4745 & 510 & 0 & -- & -- \\
\hline & 15 & -2375 & 900 & 6 & -- & $\sim$ \\
\hline \multirow[t]{10}{*}{ TXTYO3 } & 3 & -6514 & 4415 & 59 & 40 & 78 \\
\hline & 5 & -5809 & 170 & 44 & -- & 61 \\
\hline & 6 & -3769 & 1520 & 72 & 39 & 53 \\
\hline & 7 & -1329 & 1490 & 46 & $<10$ & 37 \\
\hline & 8 & -174 & 1155 & 55 & $<10$ & 27 \\
\hline & 9 & 270 & 444 & 45 & $<10$ & 21 \\
\hline & 12 & -10929 & 1760 & 0 & - & - \\
\hline & 13 & -5979 & 535 & 6 & $\ldots$ & $\cdots$ \\
\hline & 14 & -5289 & 520 & 0 & -- & - \\
\hline & 15 & -2819 & 950 & 9 & - & $\cdots$ \\
\hline \multirow[t]{5}{*}{ TXUPO 1} & 3 & -123 & 510 & 66 & $<10$ & 25 \\
\hline & 4 & 7 & 130 & 73 & $<10$ & 22 \\
\hline & 5 & 330 & 233 & 58 & $<10$ & 20 \\
\hline & 12 & -633 & 945 & 0 & $\ldots$ & $\ldots$ \\
\hline & 13 & 97 & 90 & 13 & -- & - \\
\hline \multirow[t]{6}{*}{ TXVIOI } & 7 & -2730 & 1005 & 21 & 43 & 52 \\
\hline & 8 & -815 & 1550 & 20 & $<10$ & 37 \\
\hline & 9 & 35 & 850 & 48 & $<10$ & 25 \\
\hline & 10 & 131 & 96 & 95 & $<10$ & 20 \\
\hline & 15 & -3735 & 4570 & 0 & - & - \\
\hline & 16 & -2365 & 365 & 2 & -- & $\ldots$ \\
\hline
\end{tabular}


Table 4--Layer number; altitude of top, thickness, and sand percentage of layer; and average dissolved-solids concentration and temperature of water in layer: by log number--Continued.

\begin{tabular}{|c|c|c|c|c|c|c|}
\hline $\begin{array}{l}\text { Log } \\
\text { number }\end{array}$ & $\begin{array}{r}\text { Layer } \\
\text { number }\end{array}$ & $\begin{array}{l}\text { Altitude } \\
\text { of top } \\
(\text { feet) }\end{array}$ & $\begin{array}{r}\text { Thlek- } \\
\text { ness } \\
(\text { feet) }\end{array}$ & $\begin{array}{r}\text { Sand } \\
\text { per- } \\
\text { centage }\end{array}$ & $\begin{array}{l}\text { Dis- } \\
\text { solved } \\
\text { solids }\end{array}$ & $\begin{array}{r}\text { Tem- } \\
\text { per- } \\
\text { ature }\end{array}$ \\
\hline \multirow[t]{7}{*}{ TXVIO2 } & 7 & -4816 & 1090 & 7 & -- & 56 \\
\hline & 8 & -2186 & 2000 & 46 & 57 & 42 \\
\hline & 9 & -841 & 1345 & 31 & $<10$ & 32 \\
\hline & 10 & -356 & 485 & 82 & $<10$ & 26 \\
\hline & 11 & 82 & 438 & 56 & $<10$ & 23 \\
\hline & 15 & -5906 & - & -- & - & - \\
\hline & 16 & -4186 & 630 & 7 & -- & - \\
\hline \multirow[t]{8}{*}{ TXVIO 3} & 7 & -4756 & 2725 & 43 & 63 & 67 \\
\hline & 8 & -3561 & 815 & 59 & 35 & 51 \\
\hline & 9 & -1056 & 1560 & 34 & $<10$ & 35 \\
\hline & 10 & -446 & 610 & 60 & $<10$ & 27 \\
\hline & 11 & 43 & 489 & 42 & $<10$ & 23 \\
\hline & 15 & -7481 & 560 & 0 & -- & -- \\
\hline & 16 & -4376 & 380 & 0 & -- & - \\
\hline & 17 & -2616 & 945 & 4 & -- & -- \\
\hline \multirow[t]{2}{*}{$\operatorname{TXV} 201$} & 3 & 414 & 578 & 84 & -- & -- \\
\hline & 12 & -164 & 1975 & 3 & -- & -- \\
\hline \multirow[t]{2}{*}{$\mathrm{TXVZO} 2$} & 3 & 445 & 185 & 56 & $<10$ & 18 \\
\hline & 12 & 260 & 1165 & 4 & -- & - \\
\hline \multirow[t]{7}{*}{ TXWAO 1} & 3 & -2687 & 4580 & 65 & 71 & 66 \\
\hline & 5 & -1797 & 695 & 37 & $<10$ & 47 \\
\hline & 6 & -297 & 1010 & 37 & $<10$ & 32 \\
\hline & 12 & -7267 & 1560 & 0 & -- & - \\
\hline & 13 & -2492 & 195 & 8 & -- & $\cdots$ \\
\hline & 14 & -1307 & 490 & 0 & $\cdots$ & -- \\
\hline & 15 & 168 & 465 & 4 & -- & - \\
\hline \multirow[t]{10}{*}{ TXWAO2 } & 3 & -5332 & 5205 & 50 & -- & 72 \\
\hline & 5 & -4862 & 170 & 47 & $<10$ & 53 \\
\hline & 6 & -2622 & 1160 & 32 & $<10$ & 41 \\
\hline & 7 & -882 & 590 & 47 & $<10$ & 31 \\
\hline & 8 & 68 & 950 & 37 & $<10$ & 27 \\
\hline & 9 & 350 & 282 & 72 & $<10$ & 24 \\
\hline & 12 & -10537 & 1705 & 0 & - & - \\
\hline & 13 & -5032 & 300 & 0 & -- & -- \\
\hline & 14 & -3782 & 1080 & 5 & -- & -- \\
\hline & 15 & -1472 & 1150 & 7 & -- & -- \\
\hline \multirow[t]{5}{*}{ TXWEO 1} & 3 & -295 & 2050 & 58 & $<10$ & 35 \\
\hline & 4 & -15 & 280 & 61 & $<10$ & 28 \\
\hline & 5 & 744 & 529 & 24 & $<10$ & 25 \\
\hline & 12 & -2345 & 770 & 0 & - - & - \\
\hline & 13 & 215 & 230 & 0 & -- & -- \\
\hline
\end{tabular}


Table 4--Layer number; altitude of top, thickness, and sand percentage of layer; and average dissolved-solids concentration and temperature of water in layer; by log number--Continued.

\begin{tabular}{|c|c|c|c|c|c|c|}
\hline $\begin{array}{l}\log \\
\text { number }\end{array}$ & $\begin{array}{r}\text { Layer } \\
\text { number }\end{array}$ & $\begin{array}{r}\text { Altitude } \\
\text { of top } \\
\text { ( } f \in e t)\end{array}$ & $\begin{array}{r}\text { Thick- } \\
\text { ness } \\
\text { (feet) }\end{array}$ & $\begin{array}{r}\text { Sand } \\
\text { per- } \\
\text { centage }\end{array}$ & $\begin{array}{l}\text { Dis- } \\
\text { solved } \\
\text { solids }\end{array}$ & $\begin{array}{l}\text { Tem- } \\
\text { per- } \\
\text { ature }\end{array}$ \\
\hline \multirow[t]{6}{*}{ TXWEO2 } & 3 & -1318 & 3750 & 67 & $<10$ & 50 \\
\hline & 4 & -1108 & 210 & 83 & $<10$ & 36 \\
\hline & 5 & 552 & 945 & 80 & $<10$ & 26 \\
\hline & 12 & -5068 & 1200 & 0 & -- & -- \\
\hline & 13 & -393 & 715 & 5 & -- & $\cdots$ \\
\hline & 14 & 701 & 149 & 6 & -- & -- \\
\hline \multirow[t]{6}{*}{ TXWEO3 } & 3 & -2643 & 2240 & 64 & 42 & 56 \\
\hline & 5 & -273 & 2030 & 75 & 22 & 36 \\
\hline & 6 & 610 & 263 & 53 & -- & 23 \\
\hline & 12 & -4883 & 2080 & 3 & -- & - \\
\hline & 13 & -2303 & 340 & 0 & -- & -- \\
\hline & 14 & 347 & 620 & 0 & -- & -- \\
\hline \multirow[t]{6}{*}{ TXWE 04} & 3 & -4514 & 2370 & 53 & -- & 65 \\
\hline & 5 & -2144 & 2020 & 67 & -- & 48 \\
\hline & 6 & -1114 & 410 & 67 & $<10$ & 36 \\
\hline & 13 & -4164 & 350 & 0 & -- & -- \\
\hline & 14 & -1524 & 620 & 3 & -- & -- \\
\hline & 15 & 497 & 1611 & 4 & -- & -- \\
\hline \multirow[t]{7}{*}{ TXWEOS } & 3 & -5423 & 1690 & 38 & -- & -- \\
\hline & 4 & -4853 & 570 & 68 & -- & -- \\
\hline & 5 & -2593 & 1690 & 72 & -- & -- \\
\hline & 6 & -1543 & 480 & 80 & -- & -- \\
\hline & 13 & -4283 & 570 & 0 & -- & -- \\
\hline & 14 & -2023 & 570 & 0 & -- & $\cdots$ \\
\hline & 15 & 326 & 1869 & 5 & -- & -- \\
\hline \multirow[t]{5}{*}{ TXWEO 6} & 3 & -881 & 4020 & 49 & $<10$ & 55 \\
\hline & 5 & 139 & 685 & 47 & $<10$ & 27 \\
\hline & 12 & -4901 & 1010 & 0 & -- & -- \\
\hline & 13 & -546 & 335 & 12 & -- & - \\
\hline & 14 & 480 & 341 & 0 & -- & -- \\
\hline \multirow[t]{5}{*}{ TXWEO 7} & 3 & -4070 & 2490 & 40 & -- & -- \\
\hline & 5 & -1150 & 2390 & 64 & -- & -- \\
\hline & 6 & 422 & 1132 & 30 & -- & -- \\
\hline & 13 & -3540 & 530 & 5 & -- & $\cdots$ \\
\hline & 14 & -710 & 440 & 20 & -- & -- \\
\hline \multirow[t]{7}{*}{ TXWE0 8} & 5 & -4127 & 1240 & 64 & 28 & 69 \\
\hline & 6 & -1722 & 1310 & 42 & $<10$ & 48 \\
\hline & 7 & 463 & 1050 & 0 & -- & 26 \\
\hline & 8 & 848 & 385 & 0 & -- & 20 \\
\hline & 13 & -5367 & 270 & 9 & -- & -- \\
\hline & 14 & -3032 & 1095 & 1 & -- & -- \\
\hline & 15 & -587 & 1135 & 25 & -- & -- \\
\hline
\end{tabular}


Table 4--Layer number; altitude of top, thickness, and sand percentage of layer; and average dissolved-solids concentration and temperature of water in layer; by log number--Continued.

\begin{tabular}{|c|c|c|c|c|c|c|}
\hline $\begin{array}{l}\log \\
\text { number }\end{array}$ & $\begin{array}{r}\text { Layer } \\
\text { number }\end{array}$ & $\begin{array}{r}\text { Altitude } \\
\text { of top } \\
\text { ( } f e \in t)\end{array}$ & $\begin{array}{c}\text { Thlck- } \\
\text { ness } \\
\text { (feet) }\end{array}$ & $\begin{array}{r}\text { Sand } \\
\text { per- } \\
\text { centage }\end{array}$ & $\begin{array}{l}\text { Dis- } \\
\text { solved } \\
\text { solids }\end{array}$ & $\begin{array}{l}\text { Tem- } \\
\text { per- } \\
\text { 2ture }\end{array}$ \\
\hline \multirow[t]{7}{*}{ ТXWHO1 } & 7 & -5852 & 1426 & 50 & 46 & 56 \\
\hline & 8 & -4187 & 610 & 63 & 25 & 45 \\
\hline & 9 & -1197 & 1615 & 29 & $<10$ & 33 \\
\hline & 10 & -437 & 760 & 64 & $<10$ & 27 \\
\hline & 11 & 81 & 518 & 70 & $<10$ & 24 \\
\hline & 16 & -4797 & 1055 & 4 & -- & -- \\
\hline & 17 & -2812 & 1375 & 19 & -- & -- \\
\hline \multirow[t]{7}{*}{ TXWHO2 } & 7 & -7151 & 365 & 42 & -- & -- \\
\hline & 8 & -5946 & 580 & 69 & -- & -- \\
\hline & 9 & -1516 & 2755 & 37 & $<10$ & -- \\
\hline & 10 & -541 & 975 & 57 & $<10$ & 35 \\
\hline & 11 & 70 & 611 & 73 & $<10$ & 25 \\
\hline & 16 & -6526 & 625 & 0 & -- & -- \\
\hline & 17 & -4271 & 1675 & 28 & -- & -- \\
\hline \multirow[t]{8}{*}{ TXWHO3 } & 6 & -6614 & 850 & 21 & 37 & 70 \\
\hline & 7 & -4154 & 1060 & 32 & 89 & 54 \\
\hline & 8 & -2209 & 1945 & 30 & 28 & 44 \\
\hline & 9 & -899 & 1310 & 31 & $<10$ & 33 \\
\hline & 10 & -204 & 695 & 52 & $<10$ & 26 \\
\hline & 11 & 116 & 320 & 66 & $<10$ & 23 \\
\hline & 14 & -7464 & 300 & 0 & -- & -- \\
\hline & 15 & -5214 & 1400 & 2 & -- & -- \\
\hline \multirow[t]{7}{*}{ TXWHO4 } & 6 & -6460 & 780 & 17 & -- & - \\
\hline & 7 & -3960 & 995 & 23 & -- & -- \\
\hline & 8 & -2105 & 1855 & 33 & -- & -- \\
\hline & 9 & -855 & 1250 & 31 & -- & - \\
\hline & 10 & -180 & 675 & 86 & -- & - \\
\hline & 11 & 138 & 318 & 58 & -- & -- \\
\hline & 15 & -4955 & 1505 & 1 & -- & - \\
\hline \multirow[t]{7}{*}{ TXWIO 1} & 7 & -9313 & 260 & 54 & -- & - \\
\hline & 8 & -3813 & 4640 & 58 & -- & -- \\
\hline & 9 & -1828 & 1445 & 67 & -- & - \\
\hline & 10 & -588 & 1240 & 65 & 10 & 35 \\
\hline & 11 & 9 & 597 & 84 & 11 & 19 \\
\hline & 16 & -8453 & 860 & 0 & -- & -- \\
\hline & 17 & -3273 & 540 & 6 & -- & -- \\
\hline \multirow[t]{6}{*}{ TXWIO 2} & 7 & -7635 & 2775 & 52 & -- & -- \\
\hline & 8 & -3315 & 3705 & 50 & -- & - \\
\hline & 9 & -1610 & 1705 & 49 & -- & -- \\
\hline & 10 & -530 & 1080 & 58 & -- & -- \\
\hline & 11 & 20 & 550 & 51 & -- & -- \\
\hline & 16 & -7020 & 615 & 3 & -- & -- \\
\hline
\end{tabular}


Table 4--Layer number; altitude of top, thickness, and sand percentage of layer; and average dissolved-solids concentration and temperature of water in layer; by log number--Continued.

\begin{tabular}{|c|c|c|c|c|c|c|}
\hline $\begin{array}{l}\text { Log } \\
\text { number }\end{array}$ & $\begin{array}{r}\text { Layer } \\
\text { number }\end{array}$ & $\begin{array}{r}\text { Altitude } \\
\text { of top } \\
\text { (feet) }\end{array}$ & $\begin{array}{r}\text { Thlck- } \\
\text { ness } \\
\text { (feet) }\end{array}$ & $\begin{array}{r}\text { Sand } \\
\text { per- } \\
\text { centage }\end{array}$ & $\begin{array}{l}\text { Dis- } \\
\text { solved } \\
\text { solids }\end{array}$ & $\begin{array}{l}\text { Tem- } \\
\text { per- } \\
\text { ature }\end{array}$ \\
\hline \multirow[t]{9}{*}{ TXWLOI } & 3 & -6756 & 4640 & 65 & 74 & 93 \\
\hline & 6 & -3716 & 1250 & 50 & 67 & 53 \\
\hline & 7 & -1856 & 715 & 41 & $<10$ & 36 \\
\hline & 8 & -566 & 1290 & 48 & $<10$ & 29 \\
\hline & 9 & 44 & 610 & 57 & $<10$ & 23 \\
\hline & 10 & 235 & 191 & 31 & $<10$ & 20 \\
\hline & 12 & -11396 & 1550 & 0 & $\ldots$ & -- \\
\hline & 14 & -4966 & 1790 & 2 & -- & -- \\
\hline & 15 & -2571 & 1145 & 3 & -- & -- \\
\hline \multirow[t]{8}{*}{ TXWLO2 } & 3 & -7544 & -- & -- & -- & $\cdots$ \\
\hline & 6 & -4282 & 1400 & 70 & 100 & 78 \\
\hline & 7 & -2362 & 750 & 28 & 24 & 44 \\
\hline & 8 & -872 & 1490 & 23 & $<10$ & 33 \\
\hline & 9 & 58 & 930 & 47 & $<10$ & 24 \\
\hline & 10 & 264 & 206 & 62 & $<10$ & 19 \\
\hline & 14 & -5682 & 1862 & 0 & -- & -- \\
\hline & 15 & -3112 & 1170 & 0 & -- & -- \\
\hline \multirow[t]{9}{*}{ TXWLO3 } & 3 & -10184 & 1605 & 42 & 11 & 103 \\
\hline & 6 & -6454 & 1405 & 52 & 76 & 75 \\
\hline & 7 & -3939 & 990 & 45 & 73 & 56 \\
\hline & 8 & -2019 & 1920 & 25 & 31 & 46 \\
\hline & 9 & -719 & 1300 & 49 & $<10$ & 32 \\
\hline & 10 & -109 & 610 & 64 & $<10$ & 24 \\
\hline & 11 & 192 & 301 & 67 & $<10$ & 20 \\
\hline & 14 & -7859 & 2325 & 0 & -- & -- \\
\hline & 15 & -4929 & 1525 & 0 & -- & -- \\
\hline \multirow[t]{9}{*}{ TXWLO 04} & 3 & -10838 & 770 & 45 & 44 & 106 \\
\hline & 6 & -6928 & 1130 & 44 & 92 & 78 \\
\hline & 7 & -4313 & 1150 & 29 & 101 & 59 \\
\hline & 8 & -2383 & 1930 & 17 & -- & 47 \\
\hline & 9 & -993 & 1390 & 38 & $<10$ & 34 \\
\hline & 10 & -288 & 705 & 51 & $<10$ & 26 \\
\hline & 11 & 120 & 408 & 75 & $<10$ & 21 \\
\hline & 14 & -8058 & 2780 & 0 & -- & -- \\
\hline & 15 & -5463 & 1465 & 0 & -- & -- \\
\hline \multirow[t]{3}{*}{ TXWNO 1} & 3 & 128 & 980 & 70 & $<10$ & 30 \\
\hline & 4 & 451 & 323 & 99 & $<10$ & 24 \\
\hline & 12 & -852 & 1300 & 3 & -- & - \\
\hline \multirow[t]{7}{*}{ TXWNO2 } & 3 & -2619 & 2030 & 67 & $<10$ & 49 \\
\hline & 4 & -1619 & 1000 & 89 & $<10$ & 40 \\
\hline & 5 & -389 & 1030 & 59 & $<10$ & 33 \\
\hline & 6 & 367 & 336 & 64 & $<10$ & 26 \\
\hline & 12 & -4649 & -- & -- & -- & -- \\
\hline & 13 & -1419 & 200 & 0 & -- & -- \\
\hline & 14 & 31 & 420 & 0 & -- & -- \\
\hline
\end{tabular}


Table 4--Layer number; altitude of top, thickness, and sand percentage of layer; and average dissolved-solids concentration and temperature of water in layer; by log number--Continued.

\begin{tabular}{|c|c|c|c|c|c|c|}
\hline $\begin{array}{l}\text { Log } \\
\text { number }\end{array}$ & $\begin{array}{r}\text { Layer } \\
\text { number }\end{array}$ & $\begin{array}{r}\text { Alt itude } \\
\text { of top } \\
\text { (feet) }\end{array}$ & $\begin{array}{r}\text { Thick- } \\
\text { ness } \\
\text { (feet) }\end{array}$ & $\begin{array}{r}\text { Sand } \\
\text { per- } \\
\text { centage }\end{array}$ & $\begin{array}{l}\text { Dis- } \\
\text { solved } \\
\text { solids }\end{array}$ & $\begin{array}{l}\text { Tem- } \\
\text { per- } \\
\text { ature }\end{array}$ \\
\hline \multirow[t]{8}{*}{ TXWNO3 } & 3 & -3451 & 2210 & 52 & 12 & 54 \\
\hline & 4 & -2381 & 1070 & 87 & -- & 45 \\
\hline & 5 & -991 & 1090 & 63 & -- & 37 \\
\hline & 6 & 169 & 750 & 43 & -- & 29 \\
\hline & 12 & -5661 & -- & -- & -- & -- \\
\hline & 13 & -2081 & 300 & 0 & -- & -- \\
\hline & 14 & -581 & 410 & 4 & - & -- \\
\hline & 15 & 307 & 138 & 0 & -- & -- \\
\hline \multirow[t]{4}{*}{ TXWNO 4} & 3 & -197 & 1420 & 46 & $<10$ & 33 \\
\hline & 4 & 433 & 630 & 94 & $<10$ & 27 \\
\hline & 12 & -1617 & -- & -- & -- & -- \\
\hline & 13 & 565 & 132 & 5 & -- & - \\
\hline \multirow[t]{5}{*}{ TXW001 } & 3 & -106 & 1020 & 65 & $<10$ & 26 \\
\hline & 4 & -61 & 45 & 99 & $<10$ & 22 \\
\hline & 5 & 402 & 428 & 76 & $<10$ & 21 \\
\hline & 12 & -1126 & 1090 & 0 & -- & -- \\
\hline & 13 & -26 & 35 & 0 & -- & -- \\
\hline \multirow[t]{8}{*}{ TXWSO 1} & 3 & -3190 & 3530 & 73 & 29 & 57 \\
\hline & 4 & -2845 & 345 & 67 & $<10$ & 43 \\
\hline & 5 & -1770 & 700 & 63 & $<10$ & 37 \\
\hline & 6 & -495 & 855 & 41 & $<10$ & 29 \\
\hline & 12 & $-6>20$ & 2310 & 0 & -- & -- \\
\hline & 13 & -2470 & 375 & 0 & -- & -- \\
\hline & 14 & -1350 & 420 & 8 & -- & - \\
\hline & 15 & 314 & 809 & 0 & -- & -- \\
\hline \multirow[t]{9}{*}{ IXWSO 2} & 3 & -4970 & 4630 & 67 & 59 & 79 \\
\hline & 5 & -3610 & 940 & 36 & 17 & 56 \\
\hline & 6 & -1960 & 1120 & 43 & $<10$ & 44 \\
\hline & 7 & -330 & 560 & 31 & $<10$ & 27 \\
\hline & 8 & 349 & 679 & 25 & $<10$ & 22 \\
\hline & 12 & -9600 & 3280 & 1 & - & -- \\
\hline & 13 & -4550 & 420 & 0 & -- & -- \\
\hline & 14 & -3080 & 530 & 0 & -- & -- \\
\hline & 15 & -890 & 1070 & 12 & -- & -- \\
\hline \multirow[t]{5}{*}{ IXZPOI } & 5 & -3890 & 1490 & 53 & -- & 66 \\
\hline & 6 & -780 & 1830 & 44 & $<10$ & 42 \\
\hline & 13 & -5380 & 590 & 0 & -- & -- \\
\hline & 14 & -2610 & 1280 & 2 & -- & -- \\
\hline & 15 & 512 & 1292 & 0 & -- & -- \\
\hline \multirow[t]{5}{*}{$\mathrm{TXZPO} 2$} & 3 & -4693 & 1370 & 50 & -- & -- \\
\hline & 5 & -1583 & 2530 & 46 & -- & -- \\
\hline & 6 & 421 & 1404 & 56 & -- & -- \\
\hline & 13 & -4113 & 580 & 0 & -- & -- \\
\hline & 14 & -983 & 600 & 0 & -- & -- \\
\hline
\end{tabular}


Table 4--Layer number; altitude of top, thickness, and sand percentage of layer: and average dissolved-solids concentration and temperature of water in layer; by log number--Continued.

\begin{tabular}{|c|c|c|c|c|c|c|}
\hline $\begin{array}{l}\text { Log } \\
\text { number }\end{array}$ & $\begin{array}{r}\text { Layex } \\
\text { number }\end{array}$ & $\begin{array}{r}\text { Altide } \\
\text { of top } \\
(f e e t)\end{array}$ & $\begin{array}{r}\text { Thlck- } \\
\text { ness } \\
(\text { feet })\end{array}$ & $\begin{array}{r}\text { Sand } \\
\text { per- } \\
\text { centage }\end{array}$ & $\begin{array}{l}\text { Dis- } \\
\text { solved } \\
\text { solids }\end{array}$ & $\begin{array}{l}\text { Tem- } \\
\text { per- } \\
\text { ature }\end{array}$ \\
\hline \multirow[t]{5}{*}{$\operatorname{T\times 2} 201$} & 3 & -470 & 930 & 66 & $<10$ & 40 \\
\hline & 4 & -50 & 420 & 69 & $<10$ & 32 \\
\hline & 5 & 590 & 490 & 31 & $<10$ & 24 \\
\hline & 12 & -1400 & 340 & 0 & -- & -- \\
\hline & 13 & 100 & 150 & 11 & -- & -- \\
\hline \multirow[t]{5}{*}{$\mathrm{Txz} \times 02$} & 3 & -21 & 665 & 46 & -- & - \\
\hline & 4 & 209 & 230 & 99 & - & $\ldots$ \\
\hline & 5 & 724 & 295 & 28 & -- & $\cdots$ \\
\hline & 12 & -686 & 440 & 10 & -- & -- \\
\hline & 13 & 429 & 220 & 30 & -- & -- \\
\hline \multirow[t]{5}{*}{$\mathrm{TXzV03}$} & 3 & 185 & 695 & 63 & $<10$ & 30 \\
\hline & 4 & 540 & 355 & 75 & $<10$ & 26 \\
\hline & 5 & 800 & 85 & 50 & -- & 22 \\
\hline & 12 & -510 & 500 & 6 & -- & $\ldots$ \\
\hline & 13 & 715 & 175 & 6 & -- & $\cdots$ \\
\hline \multirow[t]{6}{*}{170001} & 8 & -6349 & 3745 & 39 & 108 & 76 \\
\hline & 9 & -2369 & 2955 & 17 & 49 & 48 \\
\hline & 10 & -769 & 1600 & 0 & -- & 32 \\
\hline & 11 & -69 & 700 & -- & -- & 23 \\
\hline & 16 & -10094 & 75 & 16 & -- & - \\
\hline & 17 & -5324 & 1025 & 7 & -- & - \\
\hline \multirow[t]{7}{*}{$T 70002$} & 7 & -9523 & 1190 & 22 & 44 & $\cdots$ \\
\hline & 8 & -6713 & 700 & 17 & 43 & 77 \\
\hline & 9 & -2313 & 2975 & 18 & 50 & 56 \\
\hline & 10 & -713 & 1600 & 0 & -- & 35 \\
\hline & 11 & -53 & 660 & $\cdots$ & -- & 23 \\
\hline & 16 & -7413 & 2110 & 3 & -- & $\ldots$ \\
\hline & 17 & -5288 & 1425 & 4 & -- & - \\
\hline \multirow[t]{5}{*}{ T 70003} & 8 & -6397 & 1500 & -- & -- & - \\
\hline & 9 & -2447 & 3050 & 6 & 41 & 54 \\
\hline & 10 & -747 & 1700 & 7 & 29 & 50 \\
\hline & 11 & -112 & 635 & 32 & 37 & 25 \\
\hline & 17 & -5497 & 900 & 2 & -- & -- \\
\hline \multirow[t]{3}{*}{$\mathrm{T} 70004$} & 9 & -2751 & 3465 & 1 & $\cdots$ & 57 \\
\hline & 10 & -801 & 1950 & 20 & 32 & 52 \\
\hline & 11 & -125 & 676 & 34 & -- & 27 \\
\hline \multirow[t]{6}{*}{$\mathrm{T} 70201$} & 8 & -4597 & 2620 & 24 & 52 & 60 \\
\hline & 9 & -1712 & 2080 & 44 & 36 & 36 \\
\hline & 10 & -617 & 1095 & 18 & 15 & 27 \\
\hline & 11 & -76 & 541 & 19 & 12 & 22 \\
\hline & 16 & -7217 & 1523 & 1 & -- & -- \\
\hline & 17 & -3792 & 805 & 17 & -- & -- \\
\hline \multirow[t]{4}{*}{$T 70202$} & 9 & -2428 & 2905 & 58 & 118 & 51 \\
\hline & 10 & -773 & 1655 & 29 & 63 & 36 \\
\hline & 11 & -139 & 634 & 43 & 34 & 23 \\
\hline & 17 & -5333 & 490 & 6 & -- & -- \\
\hline
\end{tabular}


Table 4--Layer number; altitude of top, thickness, and sand percentage of layer; and average dissolved-solids concentration and temperature of water in layer; by log number--Continued.

\begin{tabular}{|c|c|c|c|c|c|c|}
\hline $\begin{array}{l}L \circ B \\
\text { number }\end{array}$ & $\begin{array}{r}\text { Layer } \\
\text { number }\end{array}$ & $\begin{array}{r}\text { Alt tude } \\
\text { of top } \\
(f e e t)\end{array}$ & $\begin{array}{r}\text { Thick- } \\
\text { ness } \\
(\text { feet) }\end{array}$ & $\begin{array}{r}\text { Sand } \\
\text { per- } \\
\text { centage }\end{array}$ & $\begin{array}{l}\text { Dis- } \\
\text { solved } \\
\text { solids }\end{array}$ & $\begin{array}{l}\text { Tem- } \\
\text { Per- } \\
\text { ature }\end{array}$ \\
\hline \multirow[t]{6}{*}{$T 70203$} & 8 & -4279 & 1860 & 52 & 71 & 53 \\
\hline & 9 & -1719 & 1810 & 62 & 46 & 35 \\
\hline & 10 & -669 & 1050 & 8 & 43 & 27 \\
\hline & 11 & -69 & 600 & 4 & -- & 22 \\
\hline & 16 & -6139 & 1419 & 3 & -- & - \\
\hline & 17 & -3529 & 750 & 20 &.- & -. \\
\hline \multirow[t]{5}{*}{$T 70204$} & 8 & -6207 & 3635 & 22 & - - & 80 \\
\hline & 9 & -2192 & 2215 & 35 & -- & 49 \\
\hline & 10 & -692 & 1500 & 22 & -- & 41 \\
\hline & 11 & -175 & 517 & -- & 40 & 24 \\
\hline & 17 & -4407 & 1800 & 9 & -- & $\ldots$ \\
\hline \multirow[t]{5}{*}{ T70301 } & 8 & -4506 & 3635 & 65 & 52 & 64 \\
\hline & 9 & -2026 & 2480 & 38 & 53 & 47 \\
\hline & 10 & -626 & 1400 & 33 & 35 & 34 \\
\hline & 11 & -79 & 547 & -. & 28 & 27 \\
\hline & 16 & -8141 & 680 & 9 & -- & $\ldots$ \\
\hline \multirow[t]{6}{*}{$T 70302$} & 8 & -4793 & 5137 & 48 & 65 & 78 \\
\hline & 9 & -1968 & 2195 & 24 & 53 & 47 \\
\hline & 10 & -618 & 1350 & 31 & 29 & 34 \\
\hline & 11 & -36 & 582 & - & 15 & 26 \\
\hline & 16 & -9930 & 302 & 7 & -- & - \\
\hline & 17 & -4163 & 630 & 7 & -- & -- \\
\hline \multirow[t]{6}{*}{ T70303 } & 8 & -6386 & 3075 & 23 & 52 & 76 \\
\hline & 9 & -2411 & 2520 & 44 & 104 & 46 \\
\hline & 10 & -731 & 1680 & 27 & 92 & 34 \\
\hline & 11 & -99 & 632 & - & 59 & 27 \\
\hline & 16 & -9461 & 156 & 4 & -- & -- \\
\hline & 17 & -4931 & 1455 & 7 & $\ldots$ & $\ldots$ \\
\hline \multirow[t]{6}{*}{ T 70401} & 8 & -7033 & 1205 & 20 & 58 & 72 \\
\hline & 9 & -2358 & 2865 & 43 & 74 & 48 \\
\hline & 10 & -748 & 1610 & 31 & $\ldots$ & 33 \\
\hline & 11 & -43 & 705 & - & -- & 25 \\
\hline & 16 & -8238 & 23 & 9 & - & -- \\
\hline & 17 & -5223 & 1810 & 7 & -- & - \\
\hline \multirow[t]{5}{*}{$T 70402$} & 8 & -4995 & 3020 & 19 & 79 & 71 \\
\hline & 9 & -1930 & 2195 & 33 & 54 & 46 \\
\hline & 10 & -630 & 1300 & 25 & 17 & 32 \\
\hline & 11 & -33 & 597 & -- & -- & 24 \\
\hline & 17 & -4125 & 870 & 15 & -- & - \\
\hline \multirow[t]{6}{*}{$T 70403$} & 8 & -5617 & 1175 & 14 & 91 & 63 \\
\hline & 9 & -2177 & 2515 & 29 & 77 & 49 \\
\hline & 10 & -757 & 1420 & 29 & $<10$ & 34 \\
\hline & 11 & -46 & 711 & -- & - & 25 \\
\hline & 16 & -6792 & 1029 & 10 & -- & -- \\
\hline & 17 & -4692 & 925 & 14 & -- & -- \\
\hline
\end{tabular}


Table 4--Layer number; altitude of top, thickness, and sand percentage of layer: and average dissolved-solids concentration and temperature of water in layer; by log number--Continued.

\begin{tabular}{|c|c|c|c|c|c|c|}
\hline $\begin{array}{l}\log \\
\text { number }\end{array}$ & $\begin{array}{r}\text { Layer } \\
\text { number }\end{array}$ & $\begin{array}{r}\text { Altitude } \\
\text { of top } \\
(f e e t)\end{array}$ & $\begin{array}{r}\text { Thick - } \\
\text { ness } \\
(\text { feet })\end{array}$ & $\begin{array}{r}\text { Sand } \\
\text { per- } \\
\text { centage }\end{array}$ & $\begin{array}{l}\text { Dis- } \\
\text { solved } \\
\text { solids }\end{array}$ & $\begin{array}{l}\text { Tem- } \\
\text { Per- } \\
\text { ature }\end{array}$ \\
\hline \multirow[t]{6}{*}{ T70404 } & 8 & -6917 & 1995 & 62 & 45 & 80 \\
\hline & 9 & -2702 & 3595 & 17 & -- & 59 \\
\hline & 10 & -802 & 1900 & 19 & 13 & 38 \\
\hline & 11 & -122 & 680 & -- & -- & 26 \\
\hline & 16 & -8912 & 890 & 3 & -- & -- \\
\hline & 17 & -6297 & 620 & 5 & -- & -- \\
\hline \multirow{4}{*}{$T 70405$} & 9 & -3060 & 4340 & 24 & 75 & 68 \\
\hline & 10 & -910 & 2150 & 18 & 15 & 44 \\
\hline & 11 & -112 & 798 & -- & -- & 26 \\
\hline & 17 & -7400 & 810 & 3 & -- & -- \\
\hline \multirow[t]{2}{*}{ T70501 } & 10 & -966 & 2200 & 4 & -- & -- \\
\hline & 11 & -188 & 778 & -- & -- & -- \\
\hline \multirow[t]{6}{*}{ T70601 } & 8 & -6713 & 1395 & 13 & -- & -- \\
\hline & 9 & -2323 & 2880 & 39 & -- & -- \\
\hline & 10 & -723 & 1600 & 22 & -- & -- \\
\hline & 11 & -30 & 693 & 21 & -- & -- \\
\hline & 16 & -8108 & 1851 & 3 & -- & -- \\
\hline & 17 & -5203 & 1510 & 8 & -- & -- \\
\hline \multirow[t]{6}{*}{$T 70602$} & 8 & -8251 & 1245 & 39 & 96 & 91 \\
\hline & 9 & -2771 & 4800 & 20 & 50 & 58 \\
\hline & 10 & -871 & 1900 & 38 & -- & 34 \\
\hline & 11 & -69 & 802 & 27 & -- & 27 \\
\hline & 16 & -9496 & 395 & 18 & -- & -- \\
\hline & 17 & -7571 & 680 & 17 & -- & -- \\
\hline \multirow[t]{3}{*}{ T70603 } & 9 & -2880 & 41 & -- & -- & -- \\
\hline & 10 & -920 & 1960 & 23 & 40 & 34 \\
\hline & 11 & -115 & 805 & -- & -- & 27 \\
\hline \multirow[t]{5}{*}{$T 70604$} & 8 & -7637 & 50 & 90 & 59 & 78 \\
\hline & 9 & -2552 & 3720 & 48 & 71 & 51 \\
\hline & 10 & -852 & 1700 & 24 & 40 & 34 \\
\hline & 11 & -43 & 809 & -- & 22 & 27 \\
\hline & 17 & -6272 & 1365 & 10 & -- & -- \\
\hline \multirow[t]{4}{*}{$T 70605$} & 9 & -2798 & 3685 & 8 & -- & 57 \\
\hline & 10 & -858 & 1940 & 32 & 13 & 37 \\
\hline & 11 & -99 & 759 & -- & -- & 27 \\
\hline & 17 & -6483 & 1530 & 4 & -- & -- \\
\hline \multirow[t]{3}{*}{$T 70701$} & 9 & -3392 & 1768 & -- & -- & -- \\
\hline & 10 & -992 & 2400 & 21 & 30 & 34 \\
\hline & 11 & -185 & 807 & -- & -- & 24 \\
\hline \multirow[t]{3}{*}{ T 70702} & 9 & -3825 & 1420 & -- & -- & -- \\
\hline & 10 & -1025 & 2800 & 4 & 21 & 45 \\
\hline & 11 & -462 & 563 & -- & -- & 28 \\
\hline
\end{tabular}


Table 4--Layer number; altitude of top, thickness, and sand percentage of layer; and average dissolved-solids concentration and temperature of water in layer; by log number--Continued.

\begin{tabular}{|c|c|c|c|c|c|c|}
\hline $\begin{array}{l}\text { Log } \\
\text { number } x\end{array}$ & $\begin{array}{r}\text { Layer } \\
\text { number }\end{array}$ & $\begin{array}{r}\text { Altide } \\
\text { of top } \\
(f e e t)\end{array}$ & $\begin{array}{r}\text { Thick- } \\
\text { ness } \\
(\text { feet })\end{array}$ & $\begin{array}{r}\text { Sand } \\
\text { per- } \\
\text { centage }\end{array}$ & $\begin{array}{l}\text { Dis- } \\
\text { solved } \\
\text { solids }\end{array}$ & $\begin{array}{l}\text { Tem- } \\
\text { per- } \\
\text { ature }\end{array}$ \\
\hline \multirow[t]{5}{*}{ I 70801 } & 8 & -5323 & 3290 & 42 & 116 & 57 \\
\hline & 9 & -2658 & 2665 & 40 & 63 & 43 \\
\hline & 10 & -908 & 1750 & 58 & 27 & 33 \\
\hline & 11 & -10 & 898 & 17 & $<10$ & 27 \\
\hline & 16 & -8613 & 235 & 4 & -- & -- \\
\hline \multirow[t]{5}{*}{ I 70802} & 8 & -9447 & 848 & 23 & 82 & 90 \\
\hline & 9 & -3027 & 4890 & 22 & 47 & 62 \\
\hline & 10 & -977 & 2050 & 29 & 68 & 36 \\
\hline & 11 & -43 & 934 & 24 & 47 & 27 \\
\hline & 17 & -7917 & 1530 & 3 & -- & -- \\
\hline \multirow[t]{4}{*}{ T 70803} & 9 & -3412 & 3198 & 42 & 120 & 54 \\
\hline & 10 & -1025 & 2387 & 42 & 22 & 40 \\
\hline & 11 & -106 & 919 & 37 & -- & 32 \\
\hline & 17 & -6610 & 482 & 7 & -- & -- \\
\hline \multirow[t]{5}{*}{ T 70804} & 8 & -5495 & 3335 & 41 & 63 & 66 \\
\hline & 9 & -2490 & 3005 & 49 & 55 & 49 \\
\hline & 10 & -860 & 1630 & 21 & 28 & 35 \\
\hline & 11 & -20 & 840 & 16 & 15 & 27 \\
\hline & 16 & -8830 & 596 & 10 & -- & -- \\
\hline \multirow[t]{3}{*}{ T70901 } & 9 & -3874 & 1146 & -- & -- & -- \\
\hline & 10 & -1044 & 2830 & 25 & 49 & 37 \\
\hline & 11 & -175 & 869 & -- & 56 & 24 \\
\hline \multirow[t]{3}{*}{ I 70902} & 9 & -3724 & 2195 & 26 & 83 & 62 \\
\hline & 10 & -1019 & 2705 & 19 & -- & 46 \\
\hline & 11 & -165 & 854 & -- & -- & 25 \\
\hline \multirow[t]{3}{*}{ T70903 } & 9 & -4183 & 4655 & 33 & 72 & 56 \\
\hline & 10 & -1083 & 3100 & 12 & 23 & 45 \\
\hline & 11 & -347 & 736 & -- & -- & 26 \\
\hline \multirow[t]{3}{*}{ T70904 } & 9 & -4826 & 2550 & 11 & 54 & 55 \\
\hline & 10 & -1151 & 3675 & 21 & 35 & 44 \\
\hline & 11 & -330 & 821 & -- & -- & 26 \\
\hline \multirow[t]{4}{*}{ T71001 } & 9 & -4141 & 1945 & 14 & 73 & 62 \\
\hline & 10 & -1036 & 3105 & 35 & 60 & 47 \\
\hline & 11 & -112 & 924 & 43 & 32 & 28 \\
\hline & 17 & -6086 & 130 & 10 & -- & -- \\
\hline \multirow[t]{3}{*}{ T71101 } & 9 & -5517 & 3490 & 31 & 119 & 62 \\
\hline & 10 & -1137 & 4380 & 23 & 32 & 38 \\
\hline & 11 & -363 & 774 & -- & -- & 25 \\
\hline \multirow[t]{3}{*}{ T71102 } & 9 & -4613 & 1646 & -- & -- & -- \\
\hline & 10 & -1123 & 3490 & 20 & 53 & 41 \\
\hline & 11 & -201 & 922 & -- & 45 & 24 \\
\hline
\end{tabular}


Explanation of data in table 5:

Layer number--Sequential number of layer, beginning with lowermost aquifer and numbering aquifers upward (1-11), then lowermost confining unit and numbering confining units upward (12-17).

Number of points with valid data--Total number of layer-thickness values entered in file for given layer.

Maximum thickness--Largest value in file for layer thickness for given layer, in feet.

Mean thickness-Mean of thickness values in file for given layer, in feet.

Standard deviation of thickness.- The square root of the mean of the squares of the deviations from the arithmetic mean of the distribution for given layer, in feet.

Maximum altitude of top--Highest altitude in file for the top of given layer relative to sea level, in feet.

Minimum altitude of top--Lowest altitude in file for the top of given layer relative to sea level, in feet.

Mean sand percentage--Mean percentage of the total thickness of sand in a given layer.

Standard deviation of sand percentage--Standard deviation of the percentage of the total thickness of given layer that is composed of sand, in feet. 


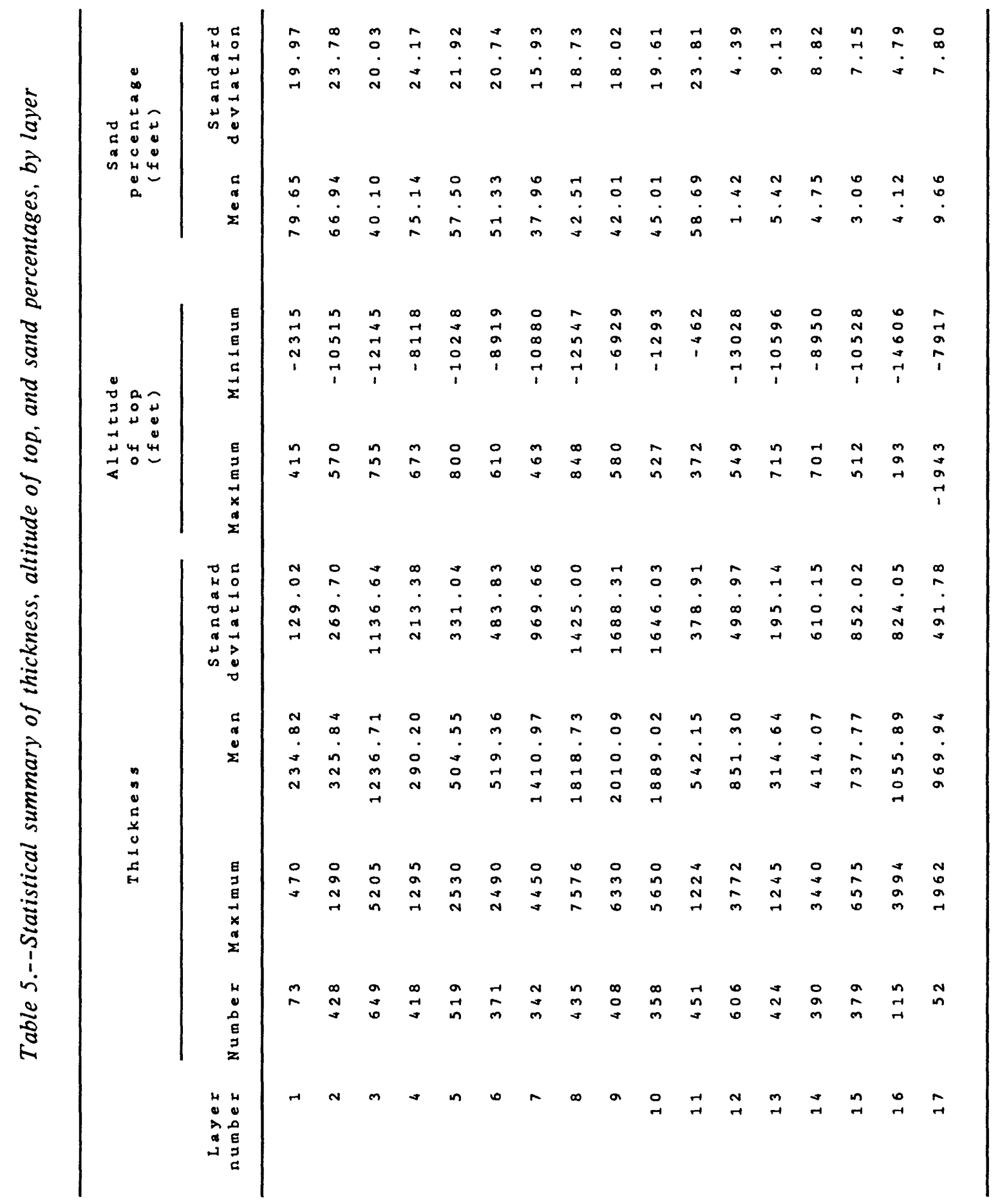




\section{DESCRIPTION OF COMPUTER TAPE FILE}

The geophysical well-log database consists of the data shown in tables 2, 3, and 4 that are contained in two physical files on a magnetic tape in ASCII format. The following parameters are contained in the well log file:

File 1 is described by the following structure:

Field positions

$$
1-3
$$

$$
4-9
$$

$10-17$

$20-27$

$30-35$

$40-45$

$50-55$

$60-75$

$80-99$

$100-146$

$150-198$

\section{$\underline{\text { Parameters }}$}

Log sequence number

Log number

Latitude

Longitude

Land surface altitude

Depth logged from

Depth logged to

State name

County (parish) name

Company name

Well name

File 2 is described by the following structure:

Field positions

$$
\begin{gathered}
1-6 \\
7-8 \\
10-20 \\
20-29 \\
30-39 \\
40-49 \\
50-59
\end{gathered}
$$

\section{Parameters}

Log number

Layer number

Altitude of top

Thickness

Sand percentage

Dissolved solids

Temperature, degrees Celsius

\section{SUMMARY}

Machine-readable files containing geohydrologic data derived from geophysical logs were assembled as part of the Gulf Coast RASA project and are stored on magnetic tape located in the Texas District Office of the U.S. Geological Survey, Water Resources Division.

A copy of the database may be obtained by contacting the computer services office of the U.S. Geological Survey, Water Resources Division in Austin, Texas. Inquires should be addressed to: U.S. Geological Survey, WRD, Federal Building, Room 649, 300 East 8th Street, Austin, Texas 78701 . 


\section{REFERENCES}

Bennett, G.D., 1979, Regional ground-water systems analyses, in Water Spectrum: U.S. Army Corps of Engineers, v. 11, no. 4, p. 30-42.

Grubb, H.F., 1984, Planning report for the Gulf Coast Regional Aquifer System Analysis in the Gulf of Mexico Coastal Plain, United States: U.S. Geological Survey Water-Resources Investigations Report 84-4219, $30 \mathrm{p}$.

Weiss, J.S., 1987, Determining dissolved-solids concentrations in mineralizied ground water of the gulf coast aquifer systems, using electric logs, in, Vecchioli, John, and Johnson, A. Ivan, eds., Aquifers of the Atlantic and Gulf Coastal Plain: American Water Resources Association, Monograph No. 9, p. 136-152. 


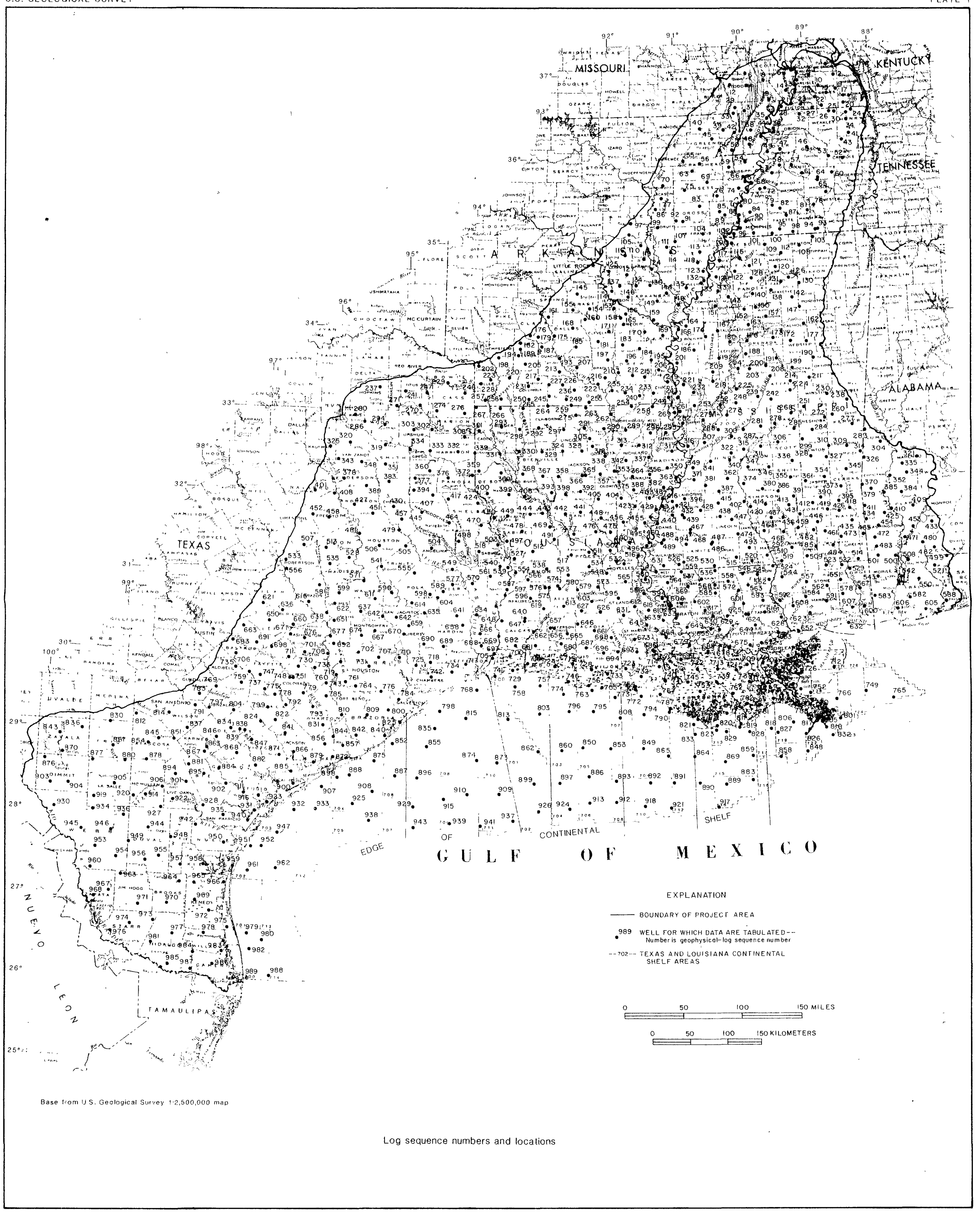

GEOPHYSICAL WELL-LOG DATA BASE FOR THE GULF COAST AQUIFER SYSTEMS, SOUTH-CENTRAL UNITED STATES 